\title{
Pyrazolat-Liganden mit zwei facial tridentaten Koordinationstaschen - Metallkomplexe und erste Reaktivitätsuntersuchungen
}

\author{
Dissertation \\ zur Erlangung des Doktorgrades \\ der Mathematisch-Naturwissenschaftlichen Fakultäten \\ der Georg-August-Universität zu Göttingen \\ vorgelegt von
}

Diplom-Chemiker

Holger Müller

aus Karl-Marx-Stadt (jetzt Chemnitz) 
D7

Referent: Prof. Dr. Franc Meyer

Korreferent: Jun.-Prof. Dr. Carola Schulzke

Tag der mündlichen Prüfung: 01.11.2007 
Ich erkläre hiermit an Eides statt, dass ich diese Arbeit selbständig und ohne unerlaubte Hilfsmittel angefertigt habe. 
Die vorliegende Arbeit entstand im Zeitraum von Oktober 2003 bis August 2007 unter Anleitung von Herrn Prof. Dr. Franc Meyer am Anorganisch-Chemischen Institut der Georg-August-Universität Göttingen. 


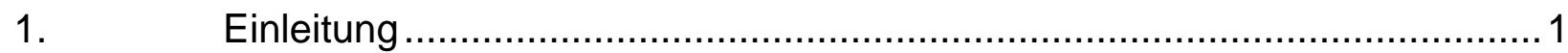

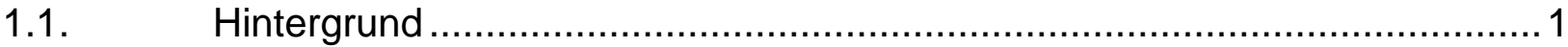

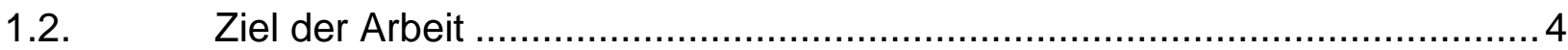

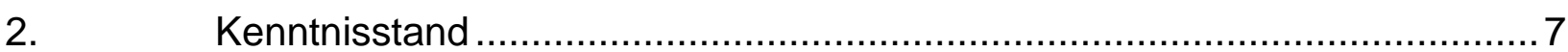

2.1. Sauerstoff und Kohlendioxid in der Natur ...............................................

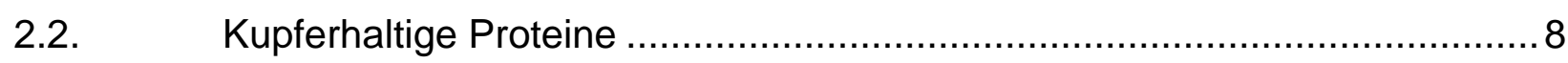

2.3. Modellkomplexe für Tyrosinase und Hämocyanin ................................... 15

2.4. Zweikernkomplexe mit multifunktionellen Pyrazolatliganden .....................24

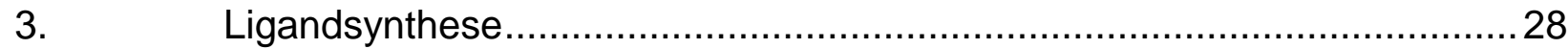

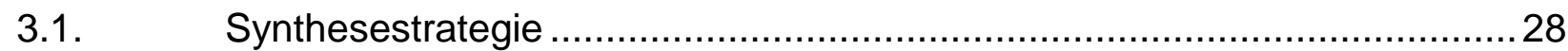

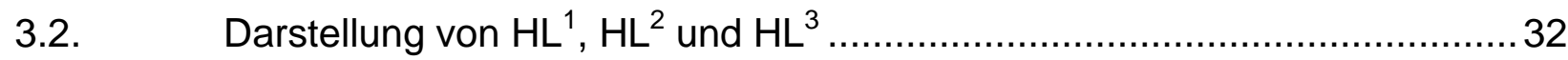

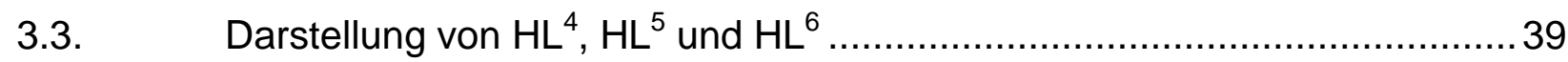

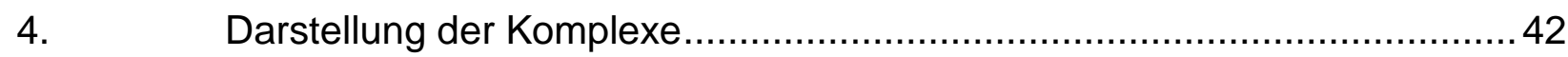

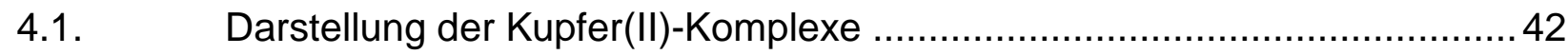

4.2. Synthese der Kupfer(I)-Komplexe ..........................................................

4.3. Darstellung der Nickel(II)-Komplexe ..................................................91

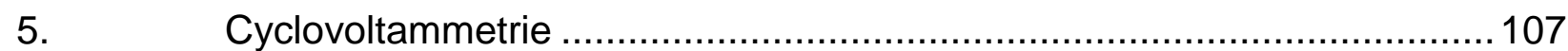

6. Potentiometrische Messungen......................................................110

6.1. Potentiometrie von Kupfer(II)-Komplexen ........................................111

6.2. Untersuchung der Nickel(II)-Komplexe ..............................................115

6.3. Charakterisierung der Zink(II)-Komplexe .............................................118

7. Reaktivitätsuntersuchungen der Kupfer(I)-Komplexe ............................122

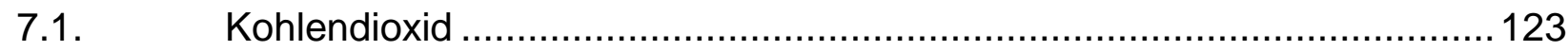

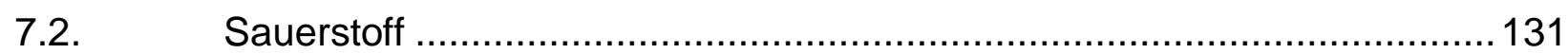

8. Magnetische Eigenschaften der Komplexe ...................................... 135

8.1. Probenvorbereitung und Magnetische Messungen................................. 135

8.2. Auswertung von Suszeptibilitätsmessungen...........................................136

8.3. Anpassung an die experimentellen Daten .............................................. 137

8.4. Ergebnisse der magnetischen Messungen ...........................................138

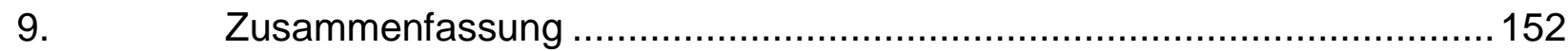

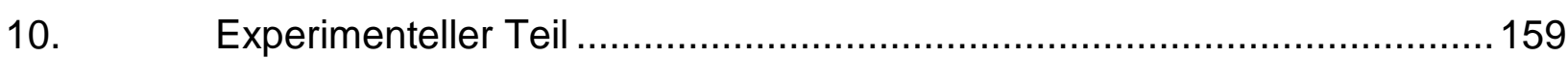

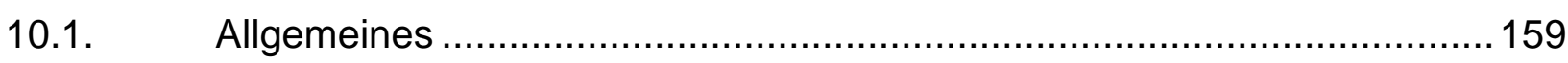

10.2. Darstellung der Liganden ............................................................... 163

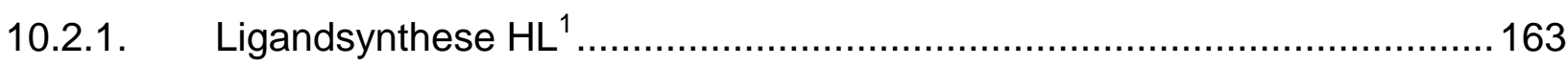


10.2.1.1. 3,5-Bis-[bis-(1-methylimidazol-2-yl)-hydroxymethyl]-1-(tetrahydropyran-2yl)-pyrazol (2a) 163

10.2.1.2. 3,5-Bis-[bis-(1-methylimidazol-2-yl)-methoxymethyl]-1-(tetrahydropyran-2yl)-pyrazol (3a) 164

10.2.1.3. 3,5-Bis-[bis-(1-methylimidazol-2-yl)-methoxymethyl]-pyrazol Hydrochlorid (4a) 166

10.2.1.4. 3,5-Bis-[bis-(1-methylimidazol-2-yl)-methoxymethyl]-pyrazol (5a) 167

10.2.2. Ligandsynthese $\mathrm{HL}^{2}$ 169

10.2.2.1. 3,5-Bis-[bis-(1,4,5-trimethylimidazol-2-yl)-hydroxymethyl]-1(tetrahydropyran-2-yl)-pyrazol (2b) 169

10.2.2.2. 3,5-Bis-[bis-(1,4,5-trimethylimidazol-2-yl)-methoxymethyl]-1(tetrahydropyran-2-yl)-pyrazol (3b)

10.2.2.3. 3,5-Bis-[bis-(1,4,5-trimethylimidazol-2-yl)-methoxymethyl]-pyrazol

Hydrochlorid (4b) 173

10.2.2.4. 3,5-Bis-[bis-(1,4,5-trimethylimidazol-2-yl)-methoxymethyl]-pyrazol (5b) ...174 10.2.3. Ligandsynthese $\mathrm{HL}^{3}$

10.2.3.1. 3,5-Bis-[bis-(4,5-diphenyl-1-methylimidazol-2-yl)-hydroxymethyl]-1(tetrahydropyran-2-yl)-pyrazol (2c)

10.2.3.2. 3,5-Bis-[bis-(4,5-Diphenyl-1-methylimidazol-2-yl)-methoxymethyl]-1-

(tetrahydropyran-2-yl)-pyrazol (3c)

10.2.5.3. 3,5-Bis-[bis-(4,5-diphenyl-1-methylimidazol-2-yl)-methoxymethyl]-pyrazol Hydrochlorid (4c) 178

10.2.5.4. 3,5-Bis-[bis-(4,5-diphenyl-1-methylimidazol-2-yl)-methoxymethyl]-pyrazol (5c)

10.2.4. Ligandsynthese $\mathrm{HL}^{4}$ 181

10.2.4.1. 3,5-Bis-[bis-(1-methylimidazol-2-yl)-hydroxymethyl]-pyrazol Hydrochlorid (6a)

10.2.4.2. Synthese von 3,5-Bis-[bis-(1-methylimidazol-2-yl)-hydroxymethyl]-pyrazol (7a) 182

10.2.5. Ligandsynthese $\mathrm{HL}^{5}$ 183

10.2.5.1. 3,5-Bis-[bis-(1,4,5-trimethylimidazol-2-yl)-hydroxymethyl]-pyrazol Hydrochlorid (6b) 183

10.2.5.2. 3,5-Bis-[bis-(1,4,5-trimethylimidazol-2-yl)-hydroxymethyl]-pyrazol (7b) .... 184 10.2.6. Ligandsynthese $\mathrm{HL}^{6}$ 185 
10.2.6.1. 3,5-Bis-[bis-(4,5-Diphenyl-1-methylimidazol-2-yl)-hydroxymethyl]-pyrazol Hydrochlorid $(6 \mathbf{c})$

10.2.6.2. 3,5-Bis-[bis-(4,5-Diphenyl-1-methylimidazol-2-yl)-hydroxymethyl]-pyrazol (7c) 186

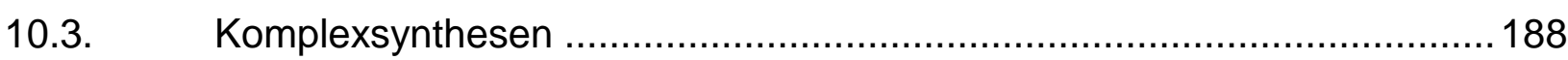

10.3.1. Darstellung von Kupfer(II)-Komplexen ............................................ 188

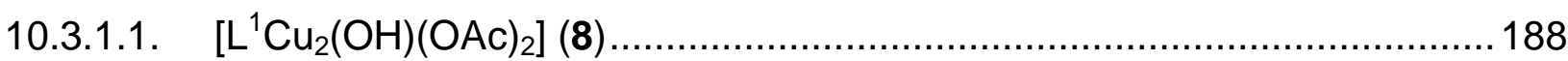

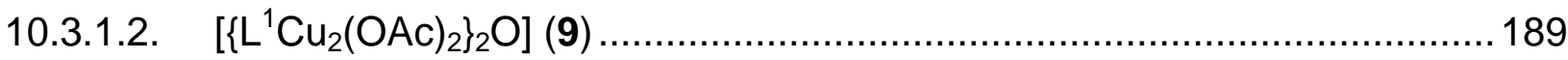

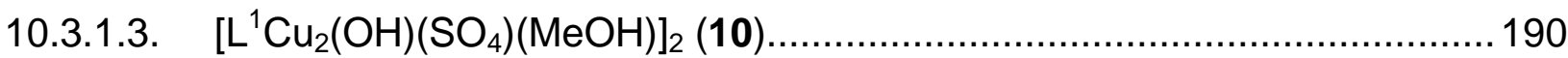

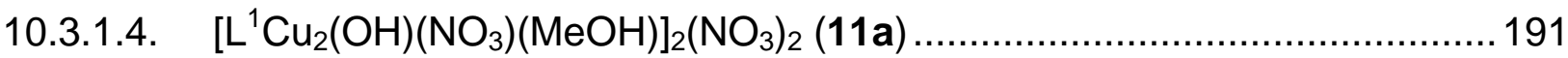

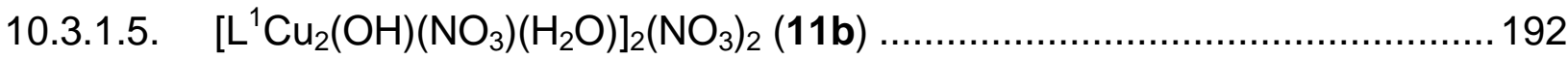

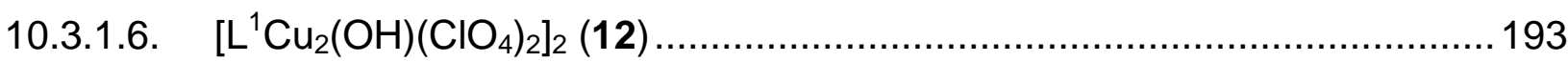

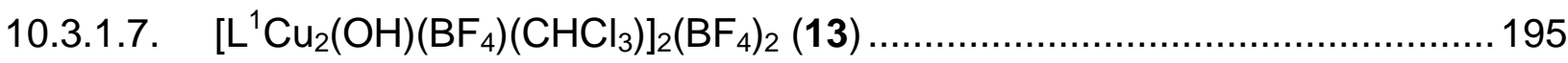

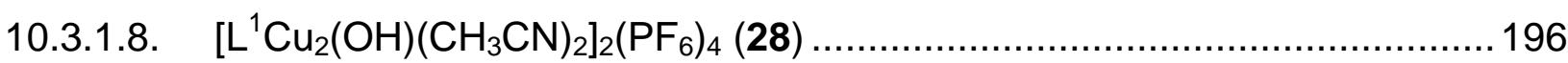

10.3.2. Darstellung von Kupfer(I)-Komplexen .............................................. 197

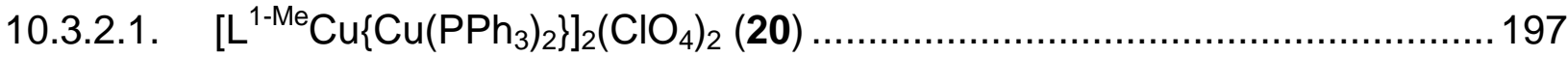

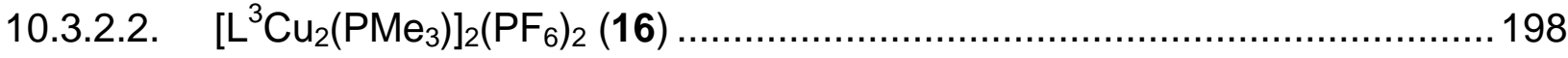

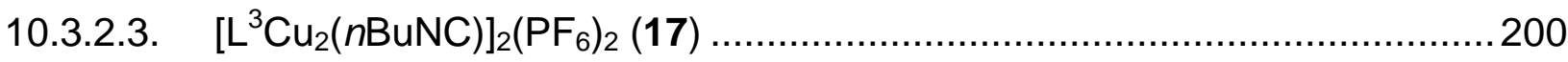

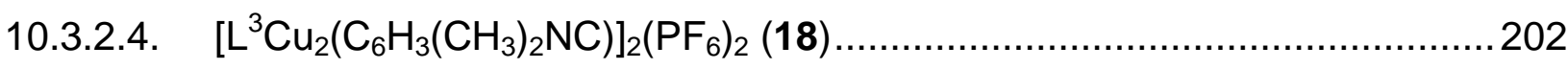

10.3.3. Darstellung von Nickel(II)-Komplexen................................................204

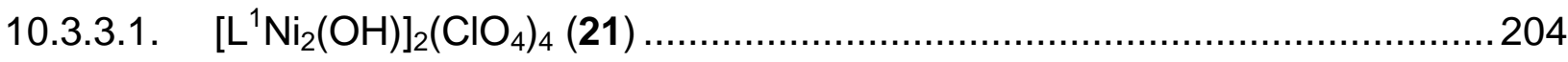

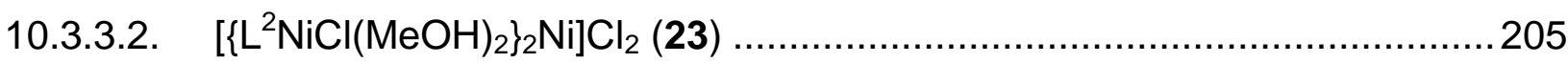

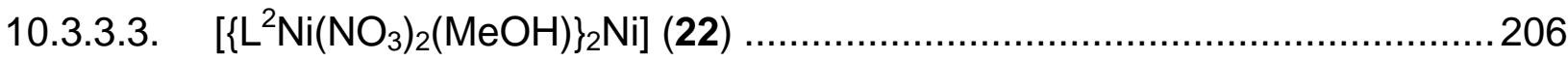

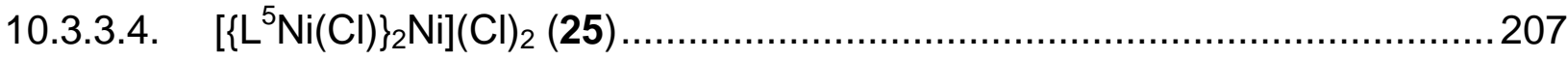

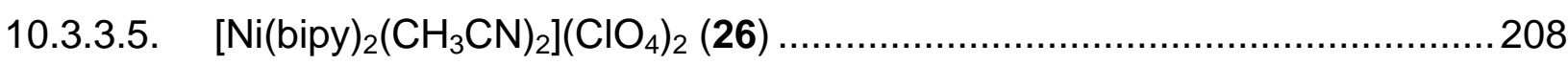

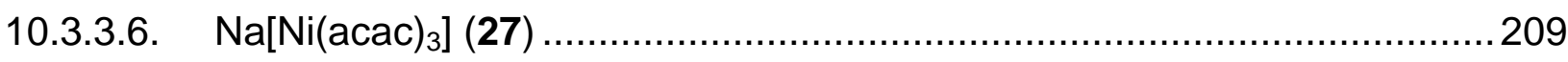

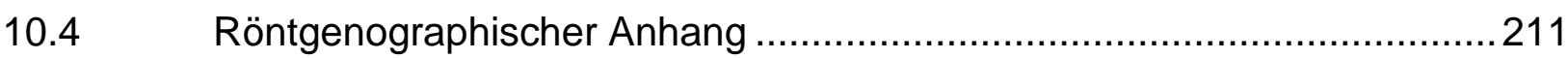

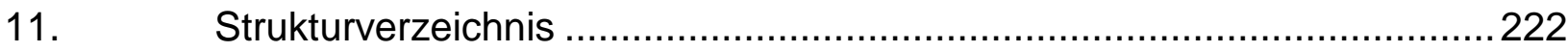

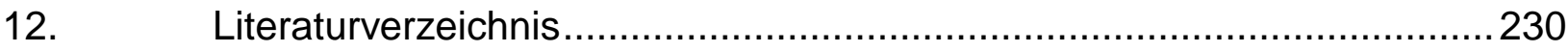




\section{Abkürzungsverzeichnis}

$\AA$

acac

$B$

bipy

n-Bu

n-BuLi

$\mathrm{CHCl}_{3}$

$\mathrm{CH}_{2} \mathrm{Cl}_{2}$

$\chi M$

CV

DCM

$\mathrm{Di}$

DMF

DMSO

$\varepsilon$

EA

EI

ESI

$\mathrm{Et}_{2} \mathrm{O}$

$\mathrm{FAB}$

$g$-Wert

$\mathrm{HL}$

Im

IR

$J$

$\mathrm{K}$

$m$

$\mathrm{Me}$

$\mu_{B}$

$\mu_{\text {eff }}$

$\mathrm{MeCN}$

$\min$
Angstrøm

Acetylacetonat

Magnetisches Feld

2,2'-Bipyridin

$n$-Butyl

$n$-Butyllithium

Chloroform

Dichlormethan

molare Suszeptibilität

Cyclovoltammetrie

Dichlormethan

1-Methyl-4,5-diphenylimidazol

Dimethylformamid

Dimethylsulfoxid

Extinktionskoeffizient

Elementaranalyse

Electron Impact

Electron Spray Ionisation

Diethylether

Fast Atom Bombardment

Landé-Faktor

Ligand

1-Methylimidazol

Infrarot

Kopplungskonstante

Kelvin

Masse

Methyl

Bohr'sches Magneton

effektives magnetisches Moment

Acetonitril

Minute 
Abkürzungsverzeichnis

\begin{tabular}{|c|c|}
\hline MS & Massenspektrometrie \\
\hline NBA & Nitrobenzylalkohol \\
\hline NMR & Nuclear Magnetic Resonance \\
\hline Oe & Oersted \\
\hline $\mathrm{Ph}$ & Phenyl \\
\hline Py & Pyridin \\
\hline $\mathrm{Pz}$ & Pyrazol \\
\hline TACN & Triazacyclononan \\
\hline TFA & Trifluoressigsäure \\
\hline THF & Tetrahydrofuran \\
\hline Thp & tetrahydropyran-2-yl \\
\hline Tri & 1,4,5-Trimethylimidazol \\
\hline$p$-TSA & para-Toluolsulfonsäure \\
\hline UV/Vis & Ultraviolett/Visible \\
\hline$z$ & Ladung \\
\hline
\end{tabular}




\section{Einleitung}

\subsection{Hintergrund}

Seit mehr als 9000 Jahren ist Kupfer dem Menschen als Werkstoff und Schmuckmaterial bekannt. Anfänglich wurde nur elementar vorkommendes Kupfer verarbeitet. Später begann vor allem in der Negev-Wüste und auf Zypern der Kupferbergbau, ehe 922 auch in Frankenberg (Sachsen) der Kupfererz-Bergbau betrieben wurde. Heute liegen die größten Kupfervorkommen in den nord- und südamerikanischen Kordilleren, im kanadischen Schild, in Zentralafrika sowie in den GUS-Staaten. Etwa $0.007 \%$ des obersten, $16 \mathrm{~km}$ dicken Anteils der festen Erdkruste besteht aus Kupfer. In der Häufigkeitsliste aller Elemente nimmt Kupfer damit den 25. Platz ein. ${ }^{[1]}$

Aufgrund seiner hervorragenden Wärmeleitfähigkeit $\left(\lambda=403 \mathrm{~W} \cdot \mathrm{m}^{-1} \cdot \mathrm{K}^{-1} \text { bei } 273 \mathrm{~K}\right)^{[2]}$ findet Kupfer Verwendung bei der Herstellung von Braukesseln, Lötkolben, Destillationsapparaturen, Heiz- und Kühlschlangen. Außerdem wird es aufgrund seines edlen Charakters $\left(E^{0}(\mathrm{Cu} / 1 / \mathrm{Cu})=+0.337 \mathrm{~V}\right)^{[3]}$ für Dachbedeckungen, Münzen, Patronenhülsen, Schiffsbeschläge und Statuen gebraucht. Die Herstellung elektrischer Leitungen begründet sich in der ausgezeichneten elektrischen Leitfähigkeit $\left(\chi=5.959 \cdot 10^{5} \Omega^{-1} \mathrm{~cm}^{-1}\right.$ bei $\left.291 \mathrm{~K}\right) \cdot{ }^{[4]}$ Die Verwendung von Kupfer im Kunstgewerbe beruht auf der leichten Polierbarkeit und Gravierbarkeit des metallischen Kupfers und seiner Legierungen. ${ }^{[5]}$

Kupfer begegnet uns nicht nur im alltäglichen Leben. Immer größerer Beliebtheit in der organischen Synthesechemie erfreuen sich Organokupfer-Verbindungen - die Cuprate. Sie lassen sich sehr einfach durch die Umsetzung von lithiumorganischen Verbindungen mit Kupfer(I)-halogeniden herstellen. Bringt man Cuprate mit Säurechloriden zur Reaktion, so erhält man ausschließlich das entsprechende Keton (Schema 1). ${ }^{[6]}$ 


$$
\mathrm{R}_{2} \mathrm{CuLi}+\mathrm{Cl}_{\mathrm{R}^{\prime}}^{\stackrel{\mathrm{O}}{\mathrm{l}} \longrightarrow \mathrm{R}_{\mathrm{R}^{\prime}}^{\mathrm{O}} \quad \mathrm{R} \text { und } \mathrm{R}^{\prime}=\text { Alkyl, Aryl }}
$$

Schema 1: Ketonsynthese mit Cupraten und Säurechloriden.

Im Gegensatz hierzu würde man beim Einsatz von Grignard- oder OrganolithiumVerbindungen zu den tertiären Alkoholen gelangen. Dieser Unterschied begründet sich in der geringeren Reaktivität der Organokupfer-Verbindungen gegenüber der Carbonylgruppe von Ketonen. Cuprate verhalten sich auch hochselektiv gegenüber verschiedenen funktionellen Gruppen, und diese Selektivität bestimmt im Wesentlichen ihre Nützlichkeit. Sie verhalten sich gegenüber zahlreichen funktionellen Gruppen (z. B. $-\mathrm{COOR},-\mathrm{CN},-\mathrm{NO}_{2}$ ) inert, so dass deren Anwesenheit im Säurechloridmolekül die Ketonsynthese nicht behindert. ${ }^{[6]}$

Zwei weitere interessante Reaktionen zum Aufbau organischer Moleküle sind die Ullmann- und die Sandmeyer-Reaktion. Mit der Ullmann-Reaktion kann man Nucleophile kupfervermittelt an Arylhalogenide kuppeln. Als nucleophile Reaktionspartner finden vor allem Phenolate, aber auch Thiolate und Amide Verwendung. ${ }^{[7]}$ Diazoniumsalze lassen sich mit der Sandmeyer-Reaktion zu den entsprechenden Halogen- bzw. Pseudohalogenverbindungen umsetzen. In der durch Kupfer(I)-Verbindungen katalysierten Reaktion kommt es dabei zu einem Austausch der Diazonium-Gruppe gegen ein (Pseudo-)Halogenid. ${ }^{[8]}$

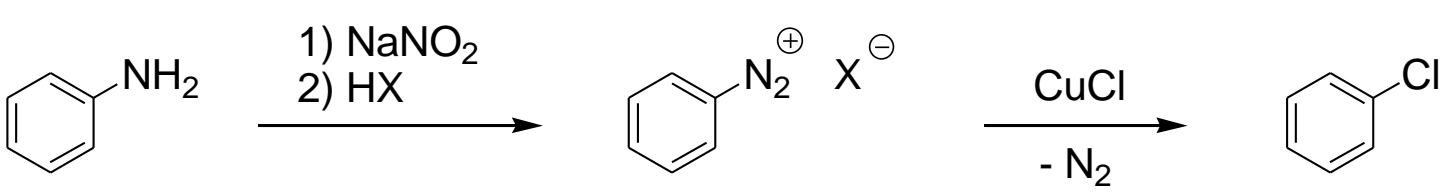

Schema 2: Sandmeyer-Reaktion am Beispiel der Synthese von Chlorbenzol aus Anilin.

Kupfer spielt aber nicht nur in der organischen Chemie, sondern auch in der Bioanorganik eine wichtige Rolle. In der Komplexchemie des Kupfer(II)-lons sind die Koordinationszahlen 4, 5 und 6 am häufigsten anzutreffen. Hierbei kommt es aufgrund des Jahn-Teller-Effekts oftmals zu verzerrten Strukturen, die sich vom Oktaeder ableiten lassen. Die beiden $e_{g}$-Orbitale $\left(d_{z^{2}}\right.$ und $\left.d_{x^{2}-y^{2}}\right)$ im oktaedrischen Kristallfeld sind bei einem $d^{9}$-Ion ungleich besetzt. Die daraus resultierende Dehnung bei fünf- und 
sechsfacher Koordination führt zu einer Verlängerung der apikalen Bindungen im Vergleich zu Bindungen der basalen Liganden. ${ }^{[5]}$ Für Kupfer $(\mathrm{I})$-lonen $\left(\mathrm{d}^{10}\right)$ tritt sehr häufig tetraedrische Geometrie auf. Dies ist zum Beispiel in den Komplexen $\left[\mathrm{Cu}(\mathrm{MeCN})_{4}\right]^{+}$oder $\left[\mathrm{Cu}(\mathrm{CN})_{4}\right]^{3^{-}}$der Fall. Die Koordinationszahlen zwei in $\left[\mathrm{CuCl}_{2}\right]^{-}$oder auch drei in $\left[\mathrm{Cu}(\mathrm{CN})_{3}\right]^{2-}$ sind ebenfalls bekannt. ${ }^{[4]}$

Kupfer ist für den Menschen und andere höhere Lebewesen nur mäßig giftig und stellt erst bei größeren Dosen ein Brechmittel dar. Im Gegensatz dazu wirken Kupferverbindungen für niedere Organismen (Algen, Bakterien) als sehr starkes Gift. Zum Beispiel sterben Bakterien in Wasser, das sich in einem kupfernen Gefäß befindet, rasch ab. Diese Tatsache machte man sich schon im Persien der Antike zunutze, denn Trinkwasser musste dort von Gesetzes wegen in Behältern aus blankem Kupfer aufbewahrt werden. ${ }^{[5]}$

Für den Menschen, höhere Lebewesen und zahlreiche Pflanzen ist Kupfer ein essentielles Spurenelement. So enthält jeder Mensch ca. 80 - 100 mg Kupfer. Es ist ein Bestandteil von Kupfer-Proteinen mit Enzym-Funktion. Dazu zählen z. B. die Cytochrom c-Oxidase, Dopamin- $\beta$-Hydroxylase, Kupfer-Zink-Superoxid-Dismutase oder auch die Tyrosin-Hydroxylase. Letztere ist verantwortlich für die Biosynthese von Melanin, ihr Fehlen führt zum Albinismus. In zahlreichen Schneckenarten, Krebsen oder Tintenfischen findet man Kupfer im Hämocyanin wieder. Dies ist ein Protein, das dem Sauerstofftransport dient. Ein Düngen mit Kupferverbindungen führt zu sattem Pflanzengrün, da es im Plastocyanin vorkommt. Dieses Protein fördert bekanntermaßen die Chlorophyllbildung. ${ }^{[1]}$

Ziel der Bioanorganischen Chemie ist es unter anderem, die Zusammenhänge zwischen Enzymstruktur und katalytischer Aktivität zu verstehen. Desweiteren sollen Funktionsprinzipien auf einfache Modellsysteme übertragen werden. Dazu ist es zunächst notwendig, die Struktur und Funktion des Proteins zu untersuchen. Davon abgeleitet werden einfache Modellsysteme konstruiert, die strukturelle und elektronische Ähnlichkeit mit den Reaktionszentren der natürlichen Vorbilder besitzen. Es gibt aber auch Modellkomplexe, die strukturelle Unterschiede aufweisen und dennoch ähnliche Funktionen erfüllen. Da Enzyme einen langen Optimierungsprozess 
während der Evolutionsgeschichte durchlaufen haben, sollte es möglich sein, effiziente Katalysatoren zu entwickeln, die sich von solchen biomimetischen Modellverbindungen ableiten.

\subsection{Ziel der Arbeit}

Ziel der Arbeit ist es, ein Ligandsystem zu entwickeln, das sich von früheren Arbeiten mit pyrazolbasierten Liganden ${ }^{[9-19]}$ ableitet. Diese Kompartimentliganden haben das Pyrazolfragment als gemeinsames Merkmal. Durch Substitution in der Pyrazol-3- und 5-Position mit verschiedenen aminfunktionalisierten Seitenarmen bilden sich zwei „Taschen“ aus, in denen Metallionen koordinativ gebunden werden können (Abbildung 1).

Beide Koordinationshalbräume können als Analoga eines Chelatliganden auf Aminbasis wie zum Beispiel dem Tris(aminoethyl)amin (TREN) verstanden werden. Fügt man formal zwei Moleküle TREN zusammen, entstehen die in Abbildung 1 (rechts) gezeigten Pyrazolatliganden. Diese besitzen zwei tripodal tetradentate Koordinationstaschen für die Koordination zweier Metallionen.
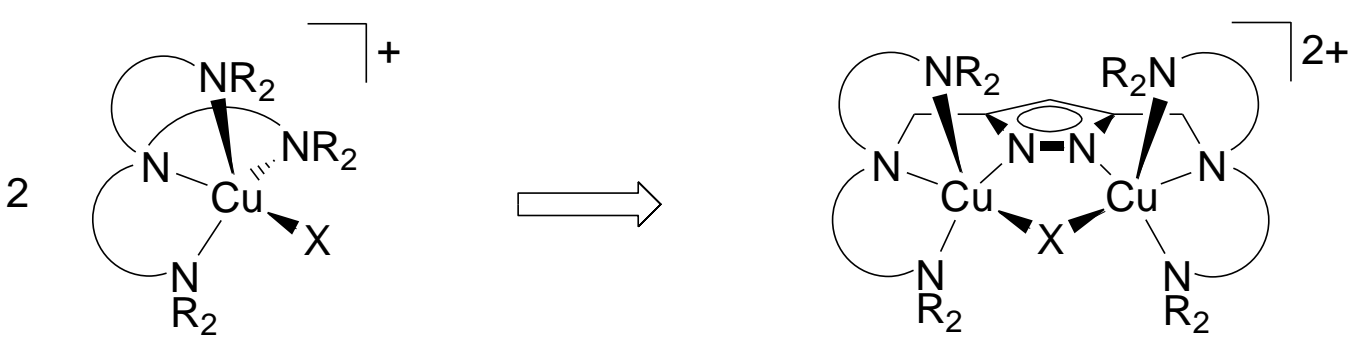

Abbildung 1: Bisher verwendete Pyrazolatliganden (rechts) lassen sich von TREN-Derivaten (links) ableiten.

In biologischen Systemen, wie zum Beispiel im Hämocyanin, lassen sich jedoch vorrangig facial tridentat koordinierte Kupferionen finden. Deshalb soll im Rahmen dieser Arbeit ein abgewandeltes Modellsystem entwickelt werden, dass sich stärker an dem natürlichen Vorbild orientiert. Dazu ist es nötig, das verbrückende Stickstoffatom gegen ein nichtkoordinierendes Kohlenstoffatom auszutauschen. Außerdem sollen die bisher verwendeten Aminseitenarme gegen Heterozyklen ausgetauscht werden, um das biologische Muster der Histidinkoordination noch genauer zu imitieren. Dafür 
könnten sich insbesondere Imidazolringe eignen. Einfache Modellverbindungen, welche die in biologischen Systemen vorkommende facial tridentate Koordination der Kupferzentren imitieren, sind z. B. Tris(imidazolyl)methan ${ }^{[20-25]}$ oder Tris(pyrazolyl)borate. In Abbildung 2 sind zwei Vertreter für derartige Verbindungen abgebildet. Vor allem die Klasse der Tris(pyrazolyl)borate ist sowohl für die Synthese von Kupfer(I)- als auch Kupfer(II)-Komplexen sehr beliebt. ${ }^{\text {[26-29] }}$
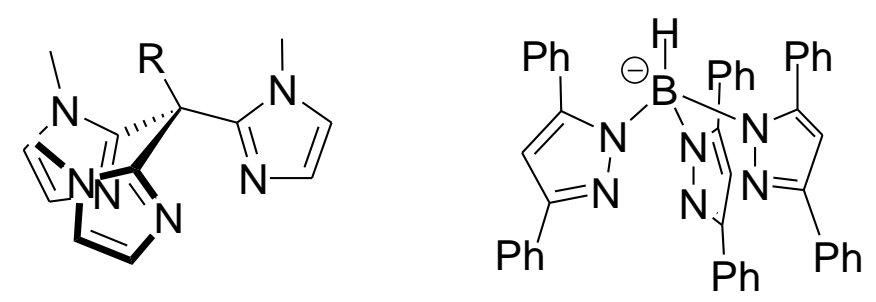

Abbildung 2: Tris(imidazolyl)methan (links) und Tris(pyrazolyl)borat (rechts).

Fügt man erneut zwei der Tris(imidazolyl)methan-Einheiten formal zusammen, erhält man die in Abbildung 3 dargestellte Struktur derartiger pyrazolbasierter Liganden mit zwei facial tridentaten Koordinationstaschen. Auch hier sollte es möglich sein, zwei Metallionen zu binden.

2

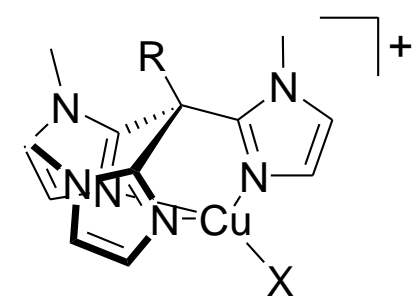
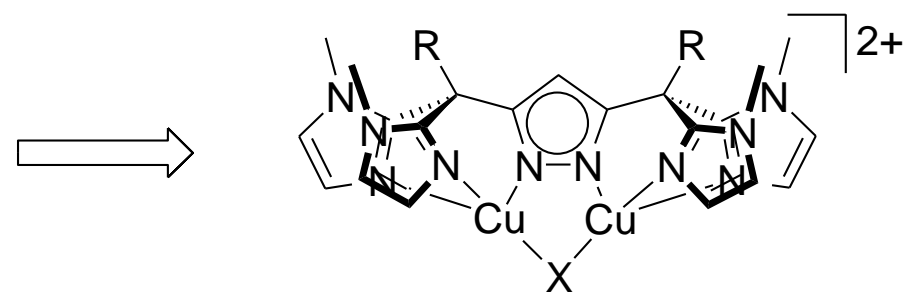

Abbildung 3: Pyrazolbasierte Liganden mit zwei facial tridentaten Koordinationstaschen (rechts), die sich vom Tris(imidazolyl)methan (links) ableiten lassen. 
Schwerpunkt der vorliegenden Arbeit ist es, mit den neu synthetisierten Kompartimentliganden Kupfer(II)-Komplexe darzustellen, diese zu kristallisieren und umfassend zu charakterisieren.

Desweiteren sollen im Rahmen dieser Arbeit Kupfer(I)-Komplexe hergestellt, kristallisiert und analysiert werden. Diese Koordinationsverbindungen werden eingesetzt, um die Reaktivität gegenüber kleinen Molekülen wie zum Beispiel Sauerstoff und Kohlendioxid zu studieren. Das Ziel ist hierbei, erste Einblicke in die ablaufenden Mechanismen zu erhalten und auftretende Zwischenstufen bzw. Folgeprodukte zu charakterisieren.

Um das Koordinationsverhalten der neuen Liganden grundsätzlich zu beleuchten, ist es ein weiterer Bestandteil der vorliegenden Arbeit, die Koordinationschemie des Nickels mit diesen neuartigen Ligandsystemen zu untersuchen. Die dargestellten Komplexverbindungen sollen ebenfalls charakterisiert und analysiert werden. 


\section{Kenntnisstand}

\subsection{Sauerstoff und Kohlendioxid in der Natur}

Sowohl Sauerstoff als auch Kohlendioxid sind essentielle Bestandteile der Atmosphäre. In der heutigen Luft liegt der Anteil des Sauerstoffes bei ungefähr $20.95 \%$ und der des Kohlendioxids bei etwa $0.03 \%$. Damit ist Sauerstoff die zweit- und Kohlendioxid die vierthäufigste Komponente der Luft. ${ }^{[30]}$

Sauerstoff war jedoch kein Bestandteil der „Ursuppe“. Da durch später entwickelte photosynthetisierende Zellen Sauerstoff gebildet wurde, nahm dessen Anteil in der Atmosphäre langsam zu. Zunächst wurde gebildeter Sauerstoff u. a. durch $\mathrm{Fe}^{2+}$ abgefangen. Als dann vor ca. 1700 Millionen Jahren alles verfügbare $\mathrm{Fe}^{2+}$ umgesetzt war, stieg der Sauerstoffgehalt der Atmosphäre auf den heutigen Wert an. Die stetig steigende Verfügbarkeit von $\mathrm{O}_{2}$ wirkte sich stark auf den weiteren Verlauf der Evolution aus. Sauerstoff ist sehr reaktionsfreudig und deshalb auch für viele Lebewesen äußerst giftig. Es folgte eine Entwicklung zahlreicher biochemischer Reaktionen, welche die Zellen vor den schädlichen Wirkungen des Sauerstoffs und anderer reaktionsfähiger Moleküle, die sich im Verlauf der Evolution bilden konnten, schützten. Später entwickelten sich Mechanismen, mit denen Organismen die hohe Reaktionsfähigkeit von Sauerstoff als Antrieb nutzten. Ein Beispiel hierfür ist die vollständige Oxidation von Glucose $\mathrm{zu} \mathrm{H}_{2} \mathrm{O}$ und $\mathrm{CO}_{2} \cdot{ }^{[4,31]}$ Bei der Atmung der meisten Lebewesen spielen Sauerstoff und Kohlendioxid eine entscheidende Rolle. Dabei wird Sauerstoff aufgenommen, zum Ort des Reaktionsgeschehens für diverse Oxidationsprozesse transportiert und das Abfallprodukt Kohlendioxid über die Atemluft ausgeschieden.

Kohlendioxid ist nicht nur an der Atmung von Mensch und Tier entscheidend involviert. Daneben gibt es einen weiteren wichtigen Vorgang mit $\mathrm{CO}_{2}$-Beteiligung. Beim Assimilationsprozess nehmen Pflanzen $\mathrm{CO}_{2}$ auf und wandeln es mit Hilfe von Lichtenergie in Kohlenhydrate um. Der Anteil von $\mathrm{CO}_{2}$ in der Atmosphäre ist für den Wärmehaushalt der Erdoberfläche von großer Bedeutung, da das $\mathrm{CO}_{2}$ der Atmosphäre die von der Erdoberfläche ausgesandten Wärmestrahlen absorbiert, aber die sichtbare Sonnenstrahlung ungehindert passieren lässt. ${ }^{[30]}$ 


\subsection{Kupferhaltige Proteine}

Damit der aus der Luft aufgenommene Sauerstoff für oxidative Reaktionen zur Verfügung steht, muss er von außen an die Stellen in den Zellen transportiert werden, wo er benötigt wird. In niederen Lebewesen kann dies durch Diffusion erfolgen. Bei höheren Lebewesen übernehmen $\mathrm{O}_{2}$-Transportproteine den Transport von Sauerstoff aus dem Atmungsorgan in die Zellen. Diese Aufgaben werden oftmals von Metalloproteinen übernommen, die in ihren aktiven Zentren Eisen oder Kupfer enthalten. Vertreter für eisenhaltige Transportproteine sind das Hämoglobin, Myoglobin oder auch das Hämerythrin. Neben dem kupferhaltigen Transportprotein Hämocyanin gibt es eine Vielzahl von weiteren Enzymen, die in ihrem aktiven Zentrum Kupfer enthalten. ${ }^{[32]}$

Kupferzentren diverser Proteine wurden aufgrund ihres strukturellen Aufbaus und den damit verbundenen Eigenschaften (Magnetismus, ESR, optische Absorption) in drei Klassen unterteilt. Aber diese Einteilung in „Typ-1-“, „Typ-2-“ und „Typ-3-KupferProteine" ist mittlerweile nicht mehr ausreichend, da weitere, nicht in das konventionelle Schema passende Zentren gefunden wurden. Hierzu zählen vor allem Enzyme wie Ascorbat-Oxidase und Laccase, die aus einem Typ(2+3)-Trimer aufgebaut sind, oder auch $\mathrm{N}_{2} \mathrm{O}$-Reduktase und Cytochrom-c-Oxidase, die jeweils ein zweikerniges $\mathrm{Cu}_{\mathrm{A}}$-Zentrum beinhalten. $\mathrm{N}_{2} \mathrm{O}$-Reduktase enthält zudem ein vierkerniges sogenanntes $\mathrm{Cu}_{\mathrm{Z}}$-Zentrum. ${ }^{[33]}$

Alle kupferhaltigen Proteine, die dem „Typ-1“ zugeordnet werden können, sind einkernig und fungieren als Elektronenüberträger. Wichtige Vertreter hierfür sind Azurin und Plastocyanin. In Abbildung 4 ist der generelle Aufbau des Aktivzentrums von Plastocyanin gezeigt. Die stark verzerrte Tetraeder-Geometrie kommt durch wohlkonservierte Aminosäuresequenzen (-His-...-Cys-...-His-...-Met-) zustande. Ebenso wie die Mischung der Liganden (2N und 2S) stellt diese stark verzerrte Anordnung einen Kompromiss (entatischer Zustand) zwischen $\mathrm{Cu}^{\prime}=\mathrm{d}^{10}$ einerseits mit überwiegend tetraedrischer Koordination durch weiche $S$-Liganden und $\mathrm{Cu}^{\prime \prime}=\mathrm{d}^{9}$ andererseits mit bevorzugt quadratisch-planarer oder quadratisch-pyramidaler Geometrie und typischer N-Ligand-Koordination dar. Die verzerrte Anordnung am Metall 
liegt vermutlich nahe an der Übergangszustands-Geometrie zwischen der tetraedrischen und der planaren Vorzugs-(Energieminimums-)Konfiguration der beiden beteiligten Oxidationszustände, wodurch die Geschwindigkeit der Elektronenübertragung erhöht wird. ${ }^{[32]}$

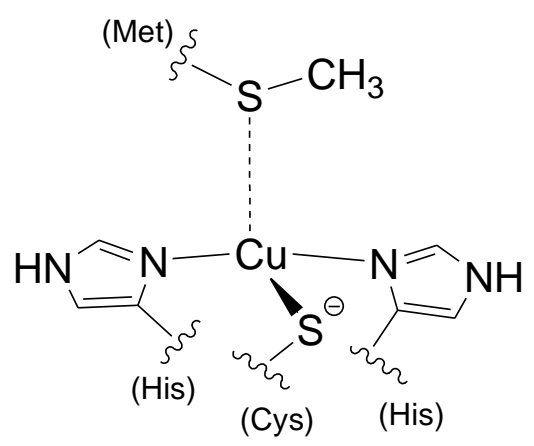

Abbildung 4: Koordination von „Typ-1“-Kupferzentren in "Blauen Kupferproteinen“.

Anhand struktureller, ${ }^{[34]}$ kinetischer $^{[31]}$ und spektroskopischer ${ }^{[35,36]}$ Studien konnte gezeigt werden, dass beide Schwefelliganden ( $\pi$-elektronenreiche Thiolat-Base des Cystein-Anions und als schwacher $\pi$-Akzeptor wirkender Thioether-Rest des Methionins) ebenfalls einen großen Einfluss auf die Elektronenübertragung haben. ${ }^{[33]} \mathrm{Im}$ Kupfer(II)-Zustand gibt es eine starke $\pi$-Wechselwirkung zwischen dem Cysteinat-Ion und dem elektronenarmen Metall-Ion. Darin liegt unter anderem auch die Ursache für die charakteristische intensiv blaue Farbe aller sogenannten "Blauen Kupferproteine“. Diese rührt von einem Ligand-Metall-Charge-Transfer-Übergang (LMCT) her, der im UV/Vis-Spektrum durch eine besonders intensive Bande $\left(\varepsilon>2000 \mathrm{~mol}^{-1} \cdot \mathrm{Icm}^{-1}\right)$ bei ca. $600 \mathrm{~nm}$ zu erkennen ist. ${ }^{[35,36]}$

Die Funktion der „Typ-2“-Kupfer-Proteine ist die $\mathrm{O}_{2}$-Aktivierung im Zusammenwirken mit organischen Coenzymen aus dem $\mathrm{Cu}^{\prime}$-Zustand. Hierzu zählen unter anderem die „Nicht-blauen“ Oxidasen, wie Galactose-Oxidase und Amin-Oxidasen, sowie die zu den Monooxygenasen zählende Dopamin- $\beta$-Monoxygenase. ${ }^{[32]}$ 
Die Amin-Oxidasen besitzen zahlreiche metabolische Funktionen. So sind sie auch für den Aufbau von Bindegewebe (Kollagen) durch vernetzende Polykondensationen zwischen Aminen und Carbonylverbindungen von Bedeutung (Schema 3).

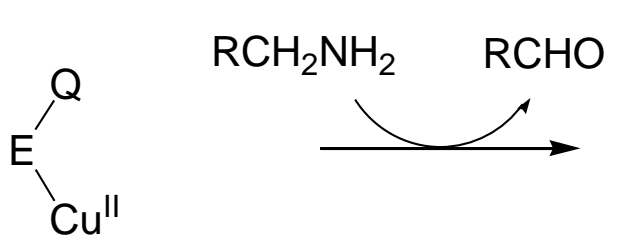

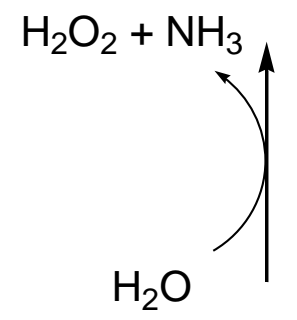

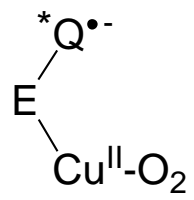

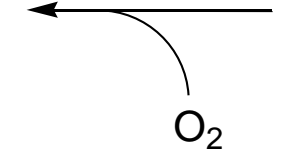<smiles>[GeH2]O[TeH2]</smiles>

E Enzym

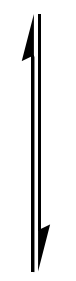

Q Chinon

$Q^{\bullet-}$ Semichinon

$\mathrm{Q}^{2-}$ Catecholat

${ }^{*} \mathrm{Q}^{\mathrm{n}-}$ Imin- bzw. Amin-Fkt.

Schema 3: Oxidation von Aminen durch Amin-Oxidasen.

Mit Hilfe eines Chinons als Co-Enzym, das an das $\mathrm{Cu}(\mathrm{II})$-Zentrum des Enzyms gebunden ist, kommt es zu einer Umwandlung des primären Amins in den entsprechenden Aldehyd. Dabei entsteht aus dem Chinon das Catecholat-lon, welches im Gleichgewicht mit dem Semichinon-Kupfer(I)-Komplex steht. Durch Reaktion mit Sauerstoff entsteht daraus erneut das Kupfer(II)-Zentrum. Zum Schluss bildet sich der Kupfer(II)-Enzym-Chinon-Komplex, indem mit Protonen Ammoniak und Wasserstoffperoxid gebildet wird.

Hierbei spielt eine intraenzymatische Elektronenübertragung vom $\mathrm{Cu}^{11 /} /$ CoenzymCatecholat-Komplex zum Cul/Coenzym-Semichinon-Komplex eine entscheidende Rolle, was anhand des als Coenzym-Ersatz fungierenden 6-Hydroxydopa-Chinon nachgewiesen werden konnte. ${ }^{[37]}$

Zu den „Typ-3“-Kupfer-Proteinen zählen u. a. Phenol-Oxidasen des Tyrosinase-Typs. Diese Enzyme katalysieren die ortho-Hydroxylierung von Phenolen wie Tyrosin sowie 
die Weiteroxidation der resultierenden Catechole zu ortho-Chinonen. Diese Prozesse sind vor allem in der Synthese von Polyphenol-Naturstoffen wichtig. Aber auch beim Aufbau der Insekten-Cuticula, der Bräunung von Früchten und Haut, sowie bei der Wundheilung und Immunantwort spielen diese Enzyme eine große Rolle. ${ }^{[32,33]}$

Im Jahr 2006 ist es erstmals gelungen, die Struktur einer Tyrosinase des Bakteriums Streptomyces castaneoglobisporus in der Oxy- und Deoxy-Form mittels Röntgenstrukturanalyse aufzuklären. Im aktiven Zentrum der Oxy-Form des Enzyms befinden sich zwei Kupfer(II)-Ionen in einem Abstand von etwa $3.4 \AA$ zueinander. Außerdem ist es Matoba et al. gelungen, die kupferfreie Form und die met(I)- sowie met(II)-Form zu kristallisieren. Bei der met(I)-Form befindet sich ein Wasser- bzw. Hydroxidligand zwischen beiden Kupferionen (Abbildung 5). In der met(II)-Form sind beide Kupferionen über zwei Wasser- oder Hydroxidliganden miteinander verbrückt.
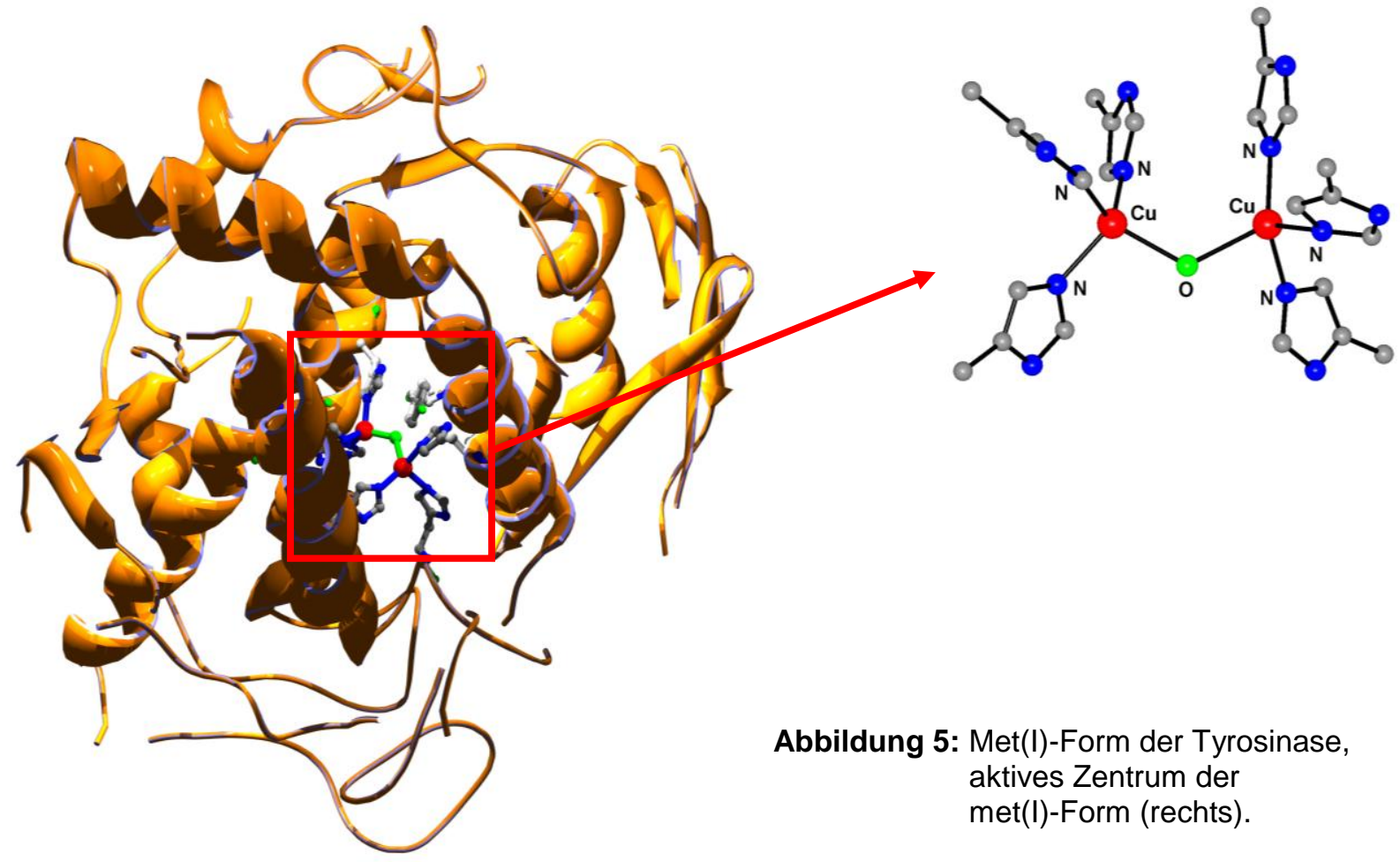

Anhand der in Abbildung 5 gezeigten Struktur wird deutlich, dass beide Kupferzentren von je drei Histidinresten koordiniert sind. ${ }^{[38]}$ Das Enzym zeigt zum Einen beim Wechsel von der Oxy-in die Deoxy-Form mit der einhergehenden Vergrößerung des Cu-Cu-Abstandes von 3.4 zu 4.1 A seine volle Flexibilität. Zum Anderen äußert sich die 
Enzymflexibilität durch die Verkleinerung des Cu-Cu-Abstandes von 3.9 auf $3.3 \AA$, wenn die met(II)- in die met(I)-Form übergeht.

Für die sehr interessante ortho-Hydroxylierung von Phenolen ist der im folgenden Schema 4 abgebildete Reaktionsmechanismus vorgeschlagen worden. Dieser basiert einerseits auf den Kristallstrukturen der Oxy- und Deoxy-Form und andererseits auf der Kenntnis der beiden Strukturen der met(I)- und met(II)-Form.

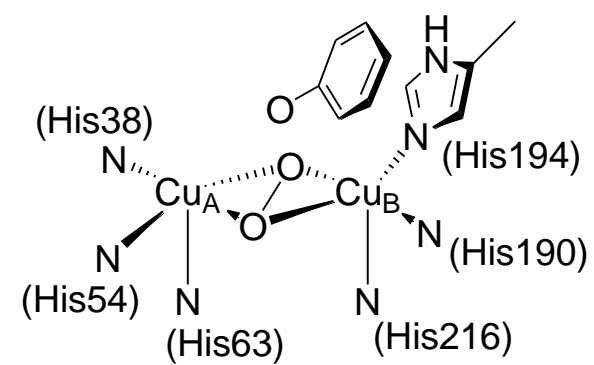

$\longrightarrow$ (His54)

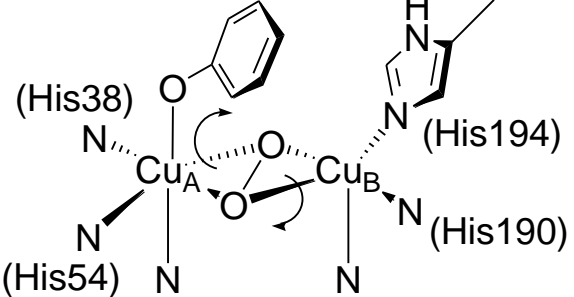

(His63) (His216)

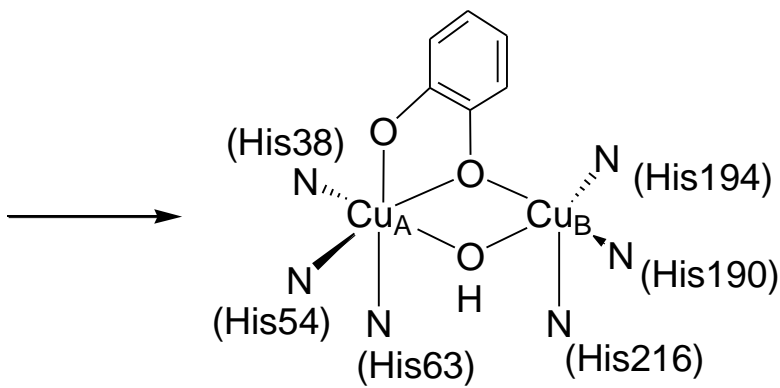

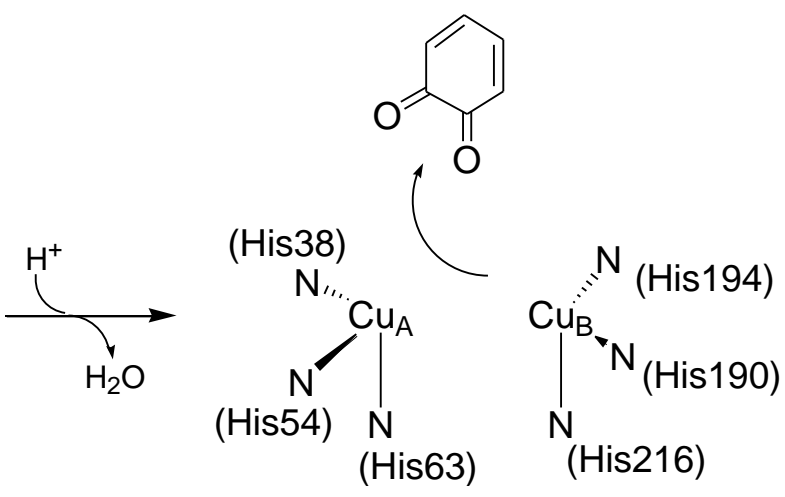

Schema 4: Hydroxylierung von Monophenolsubstraten durch Tyrosinase. 
Nach erfolgter Substratannäherung an das Oxy-Zentrum findet eine Vororientierung aufgrund einer hydrophoben Wechselwirkung mit His194 statt. Dabei zeigt die C-O-Bindung in Richtung des $\mathrm{Cu}_{\mathrm{A}}$. Danach verschiebt sich das Substrat weiter und es findet eine Koordination an das $\mathrm{Cu}_{\mathrm{A}}$ in trans-Stellung zu His63 statt. Damit der aromatische Ring hydroxyliert werden kann, rotiert die O-O-Achse des Peroxoliganden in Richtung des Phenylringes und das Substrat etwas um dessen C-O-Achse. Wegen der räumlichen Nähe der koordinierten Peroxogruppe und der ortho-Position des Phenylringes kann ein elektrophiler Angriff der $\mathrm{Cu}_{2} \mathrm{O}_{2}$-Einheit auf das Aren erfolgen, wobei gleichzeitig die O-O-Bindung gespalten wird. Es bildet sich ein zweizähnig koordiniertes Diphenolintermediat. Aus dieser Zwischenstufe wird im letzten Schritt das Chinon freigesetzt und die Deoxy-Form des Enzyms regeneriert. ${ }^{[39]}$

Ein weiterer Vertreter für das Typ-3-Kupfer-Zentrum ist das Transportprotein Hämocyanin. Es kommt vor allem in Arthropoden und Mollusken vor und ermöglicht auch größeren Tieren wie Hummern oder Tiefseekraken eine effiziente Versorgung mit Sauerstoff. Die Struktur des Hämocyanin aus Panulirus interruptus konnte 1989 erstmals von Volbeda und $\mathrm{Hol}$ mit Hilfe der Kristallstrukturanalyse aufgeklärt werden. Allerdings gelang es Ihnen aufgrund der mäßigen Auflösung (3.2 $\AA$ ) nicht, genauere Aussagen über die Beschaffenheit des aktiven Zentrums zu treffen. ${ }^{[40]}$ So dauerte es bis zum Jahre 1993, dass Einkristalle vom Hämocyanin des Limulus polyphemus mit einer Auflösung von 2.18 $\AA$ gemessen wurden. Der Gruppe um Hazes ist es gelungen, die Deoxy-Form strukturell aufzuklären. Beide Kupferzentren sind demnach von je drei Histidinresten koordiniert. Sie ermittelten außerdem einen Cu-Cu-Abstand von $4.6 \AA^{.}{ }^{[41]}$ Früher durchgeführte EXAFS-Studien zeigten zunächst einen viel kürzeren Abstand zwischen beiden Metallzentren. Ein zusätzlicher Vergleich mit der reduzierten Form der Ascorbat-Oxidase, die spektroskopisch sehr ähnlich ist, ließ zunächst ebenfalls auf einen kürzeren Cu-Cu-Abstand von $3.4 \AA$ schließen. ${ }^{[42-44]}$ Eine weitere Strukturaufklärung der Oxy-Form des Hämocyanins aus Panulirus interruptus von Volbeda et al. ergab einen Cu-Cu-Abstand von $3.5 \AA$ (Abbildung 6). Auch in dieser Form ist jedes Kupferzentrum von drei Histidinresten umgeben. Entgegen früher getroffenen Vorhersagen ändert sich die Koordination um beide Kupferionen nicht, wenn ein Wechsel zwischen der Oxy- und Deoxy-Form stattfindet. ${ }^{[43,45]}$ 


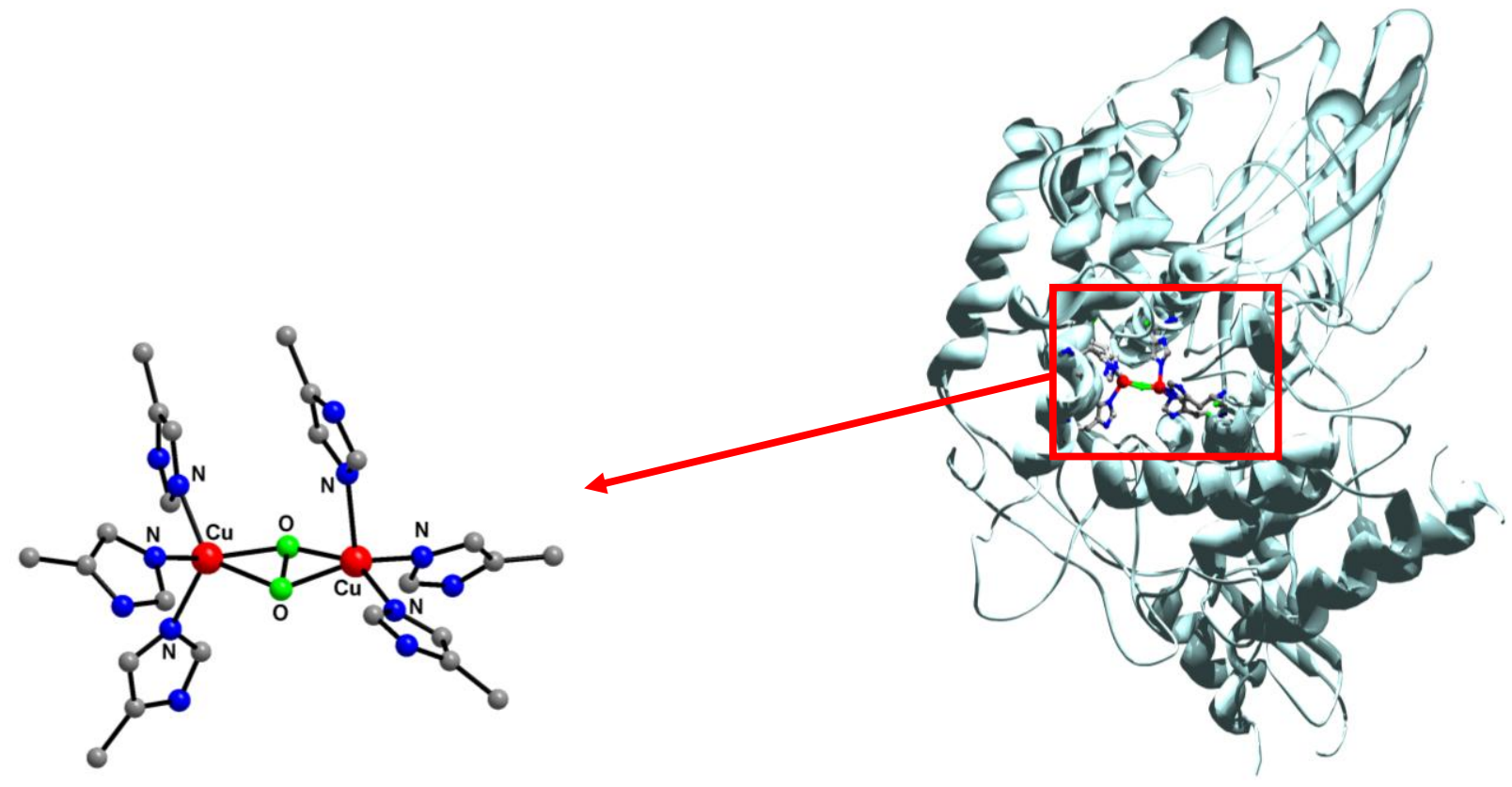

Abbildung 6: Molekül von Hämocyanin, Oxy-Form des aktiven Zentrums (links).

Zu den „Typ-3“-Kupfer-Proteinen gehört auch Catechol-Oxidase. Ihr struktureller Aufbau ist mit dem der beiden bisher vorgestellten Vertretern Hämocyanin und Tyrosinase nahezu identisch. Catechol-Oxidase katalysiert die Weiteroxidation der Brenzkatechine (Catechole) zu ortho-Chinonen (Schema 5).

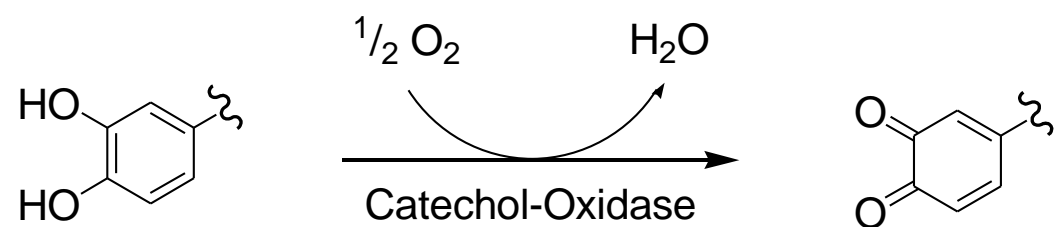

Schema 5: Catechol-Oxidase katalysierte Bildung von ortho-Chinonen aus Catecholen.

Jedes Kupfer(I)-Zentrum der „Typ-3“-Kupfer-Proteine hat eine $\{\text { His }\}_{3}$-Koordination. Diese ordnen sich facial tridentat um das Kupfer-Ion an. Daraus ergeben sich vor allem weitere spektroskopische Gemeinsamkeiten. Aufgrund der antiferromagnetisch gekoppelten Kupfer(II)-Zentren ist der oxidierte Zustand ESR-inaktiv. Nach der Aufnahme von $\mathrm{O}_{2}$ werden intensive Absorptionen bei $350 \mathrm{~nm}\left(\varepsilon=20000 \mathrm{~mol}^{-1} \mid \mathrm{lcm}^{-1}\right)$ und $600 \mathrm{~nm}\left(1000 \mathrm{~mol}^{-1} \cdot \mathrm{lcm}^{-1}\right)$ beobachtet, die LMCT-Übergängen von $\mathrm{O}_{2}{ }^{2-} \rightarrow \mathrm{Cu}(\mathrm{II})$ zuzuordnen sind. ${ }^{[32]}$ 


\subsection{Modellkomplexe für Tyrosinase und Hämocyanin}

Das Verständnis der Prinzipien bei der Sauerstoffanbindung und -aktivierung an Metalloproteinen wie Tyrosinase und Hämocyanin wird für eine gezielte Synthese hocheffizienter Katalysatoren für Oxidationsreaktionen sehr hilfreich sein. Aus diesem Grund sind in der letzten Zeit zahlreiche in struktureller Hinsicht einfache, biomimetische Verbindungen synthetisiert worden, um die Struktur des aktiven Zentrums ihrer natürlichen Vorbilder nachzubilden. Diese sollten neben den strukturellen auch elektronische Gemeinsamkeiten mit den enzymatischen Zentren aufweisen, damit sie ähnliche Funktionen wahrnehmen können.

Die Anbindung des molekularen Sauerstoffes an das Kupferzentrum kann auf verschiedene Arten erfolgen. Eine Vielzahl der in Abbildung 7 gezeigten Bindungsmotive konnte bereits mit Kristallstrukturanalysen aufgeklärt werden. ${ }^{\text {[9,46-70] }}$

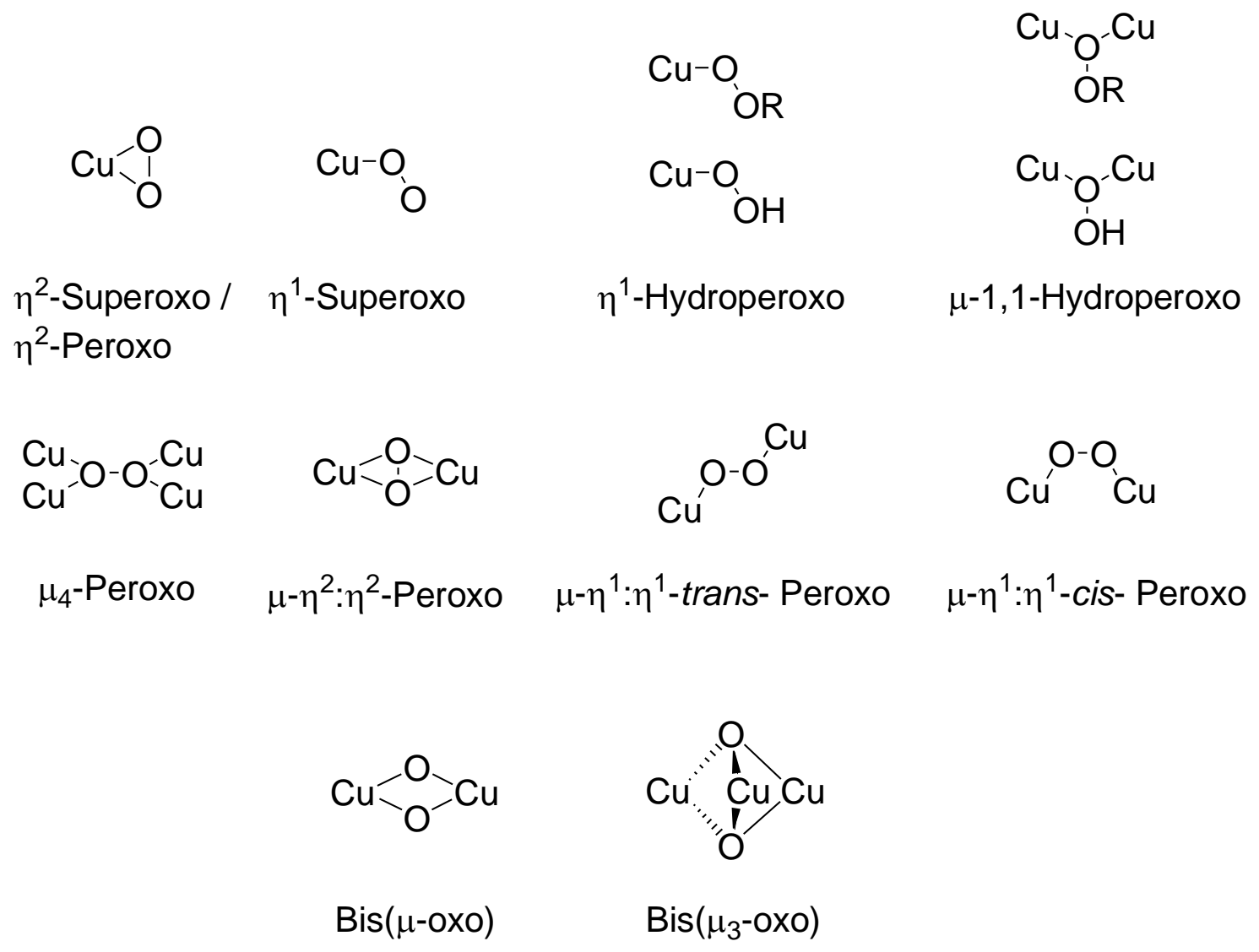

Abbildung 7: Mögliche $\mathrm{O}_{2}$-Anbindungen an Kupferionen. 
In der Regel sind diese Sauerstoff-Addukte hochreaktiv und auch bei tiefen Temperaturen nur eine begrenzte Zeit stabil.

So wurde zum Beispiel im Falle des Oxyhämocyanins diskutiert, in welcher Form molekularer Sauerstoff zwischen beiden Kupferionen gebunden ist. Im Jahre 1988 gelang es Kitajima et al., deutliche Hinweise auf diese Frage zu erhalten, als sie einen $\mu$-Peroxo-Komplex synthetisierten. Sie setzten das Trispyrazolylborat I mit einem Kupfer(I)-Salz um und oxidierten diese Zwischenstufe mit lodosylbenzol zum $\mu$-Oxo-Komplex II (Abbildung 8).

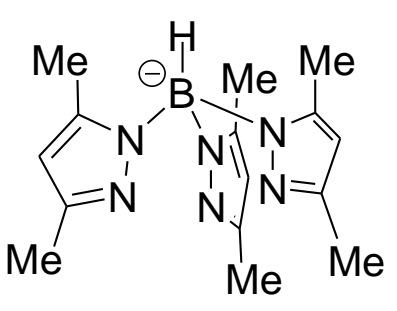

I

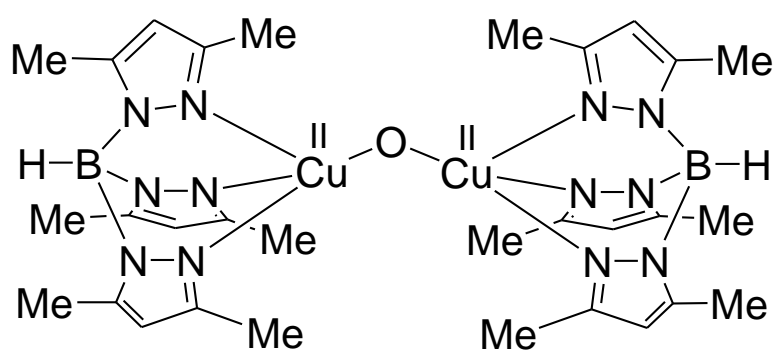

II

Abbildung 8: Verwendeter Ligand I und dazugehöriger $\mu$-Oxo-Komplex II.

Anschließend setzten sie diesen Komplex mit $\mathrm{H}_{2} \mathrm{O}_{2}$ um und erhielten sofort eine purpurfarbene Lösung, aus der sich ein kristalliner Feststoff des Komplexes III (Abbildung 9) bildete. Die daraus gewonnenen spektroskopischen Daten stimmten erstaunlich gut mit denen von Oxyhämocyanin überein. So erhielten sie im ResonanzRaman-Spektrum ein Signal bei $725 \mathrm{~cm}^{-1}$ (Oxyhämocyanin: $744-752 \mathrm{~cm}^{-1}$ ).

Das UV/Vis-Spektrum zeigte Banden bei $530 \mathrm{~nm}\left(\varepsilon=840 \mathrm{~mol}^{-1} \cdot \mathrm{lcm}^{-1}\right)$ und $338 \mathrm{~nm}$ $\left(\varepsilon=20800 \mathrm{~mol}^{-1} \cdot \mathrm{l} \mathrm{cm}^{-1}\right)$. Im Falle des Proteins befinden sich diese Absorptionen bei $570 \mathrm{~nm}\left(\varepsilon \approx 1000 \mathrm{~mol}^{-1} \cdot \mathrm{lcm}{ }^{-1}\right)$ und $345 \mathrm{~nm}\left(\varepsilon \approx 20000 \mathrm{~mol}^{-1} \cdot \mathrm{lcm}^{-1}\right)$. Ebenso zeigte die neue Verbindung kein ESR-Signal und war diamagnetisch, so dass sie trotz irreversibler $\mathrm{O}_{2}$-Anbindung als Modellverbindung für Oxyhämocyanin angesehen werden musste. ${ }^{[71]}$ 


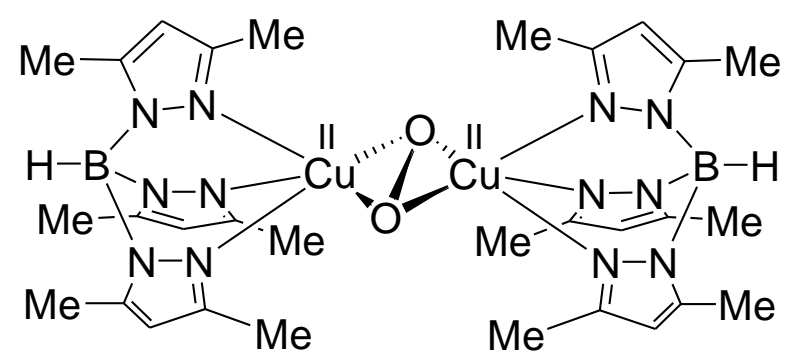

III

Abbildung 9: Erster echter Modellkomplex für Hämocyanin von Kitajima et al.

Die kristallografische Strukturaufklärung gelang Kitajima et al. anhand der Verbindung $\left[\mathrm{Cu}_{2}\left(\mathrm{O}_{2}\right)\left(\mathrm{HB}\left(3,5-\left(\mathrm{Me}_{2} \mathrm{CH}\right)_{2} \mathrm{pz}\right)_{3}\right)_{2}\right]$ - einer dem Komplex III verwandten Verbindung - im Jahre 1989 und lieferte den endgültigen Beweis für die im Oxyhämocyanin zwischen beiden Kupferionen vorliegende $\mu-\eta^{2}: \eta^{2}$-Peroxo-Brücke. ${ }^{[49]}$

Seither haben zahlreiche Gruppen versucht, Systeme herzustellen, die ähnliche spektroskopische Eigenschaften wie Oxyhämocyanin besitzen. Ein weiteres Ziel ist es außerdem, die $\mathrm{O}_{2}$-Anbindung und -aktivierung reversibel ablaufen zu lassen und somit einen entscheidenden Schritt in Richtung katalytisch wirksamer Systeme zu gehen. Dafür werden in der Literatur verschiedene Wege beschrieben, die zu diesem Ergebnis führen könnten. Zum einen ist es möglich, einkernige Kupfer(I)-Komplexe zu synthetisieren und diese dann zu $\mu-\eta^{2}: \eta^{2}$-Peroxo-verbrückten Verbindungen umzusetzen. Ein möglicher Ansatz sind die Systeme aus der Gruppe um Kitajima, die eine ganze Reihe von Komplexen darstellen konnten, die alle auf substituierten Trispyrazolylboraten basieren. ${ }^{[50,72]}$

Die Arbeiten von Tolman et al. basieren zum großen Teil auf Liganden des Triazacyclononans (TACN). Interessant waren deren Beobachtungen, dass bereits bei geringer Variation der Reste an den Stickstoffatomen des TACN-Gerüstes ein Wechsel von Peroxo-verbrückten zu bis( $\mu$-oxo)-verbrückten Kupfer-Komplexen auftritt (Schema 6). Es gelang, spektroskopische Daten von beiden Kupferkomplexen aufzunehmen. Dabei gab es Gemeinsamkeiten, aber auch einige Unterschiede. Beide Verbindungen zeigten ein $\mathrm{Cu}: \mathrm{O}_{2}$-Verhältnis von 2:1 und scharfe Signale im ${ }^{1} \mathrm{H}-\mathrm{NMR}-$ Spektrum. 
Bei Betrachtung der kristallografischen Daten fallen sehr deutliche Unterschiede auf. Der Cu-Cu-Abstand im Fall der Peroxo-verbrückten Spezies IV beträgt $3.56 \AA$, für Komplex V wurde jedoch ein deutlich kürzerer Cu-Cu-Abstand von $2.79 \AA$ beobachtet. Desweiteren ist der O-O-Abstand für den Peroxo-Komplex 1.41 $\mathrm{A}$. Diese Entfernung ist in Komplex $\mathbf{V}$ auf $2.37 \AA$ aufgeweitet, so dass man hier bereits nicht mehr von einer OO-Bindung sprechen kann. Die Cu-O-Bindungen werden von $1.93 \AA$ in Verbindung IV auf $1.80 \AA$ in Komplex $\mathbf{V}$ verkürzt. Beide Verbindungen zeigen ebenfalls Unterschiede im UV/Vis-Spektrum. So zeigt der Peroxo-Komplex IV eine charakteristische Bande bei $366 \mathrm{~nm} \quad\left(\varepsilon=15000 \mathrm{~mol}^{-1} \cdot \mathrm{Icm}^{-1}\right)$ und die $\mu$-Oxo-Verbindung V ruft zwei intensive Absorptionen im UV/Vis-Spektrum bei $320 \mathrm{~nm}\left(\varepsilon=13000 \mathrm{~mol}^{-1} \cdot \mathrm{Icm}^{-1}\right)$ und $430 \mathrm{~nm}$ $\left(\varepsilon=15000 \mathrm{~mol}^{-1} \cdot \mathrm{lcm}^{-1}\right)$ hervor.

2

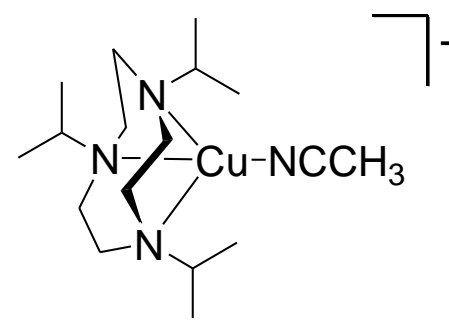

2

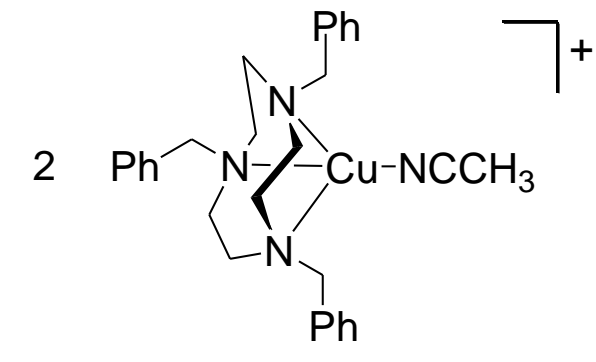

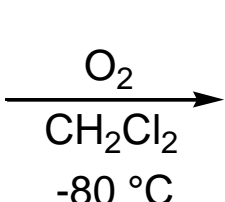

$-80^{\circ} \mathrm{C}$

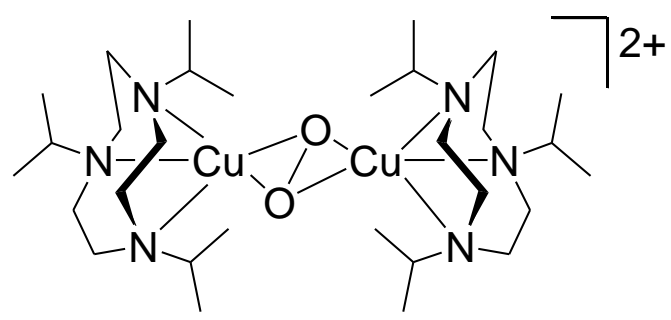

IV

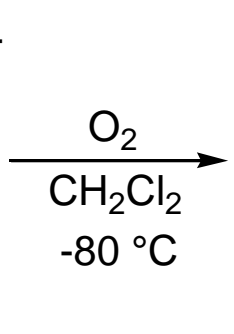

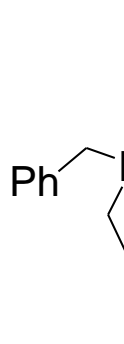

Schema 6: Kontrolle der Art der Sauerstoffanbindung durch Variation der terminalen Reste des Liganden.

Die Auswertung des Resonanz-Raman-Spektrums lieferte für Verbindung $\mathbf{V}$ zwei Banden bei $602 \mathrm{~cm}^{-1}$ und $612 \mathrm{~cm}^{-1}$, die auf eine gemeinsame Bande bei $583 \mathrm{~cm}^{-1}$ zusammenfallen, wenn die Synthese mit ${ }^{18} \mathrm{O}_{2}$ erfolgte. Im Falle von ${ }^{16} \mathrm{O}_{2}$ liegt die Ursache der Banden in einer Aufspaltung der symmetrischen Schwingung in ein FermiDuplett. Im Resonanz-Raman-Spektrum von Komplex IV war hingegen nur eine Bande bei $600 \mathrm{~cm}^{-1} \mathrm{zu}$ sehen, die sich bei Verwendung von ${ }^{18} \mathrm{O}_{2}$ auf $580 \mathrm{~cm}^{-1}$ verschob. Es konnte somit erstmals ein hochvalenter Komplex mit Kupfer(III)-lonen in der 
Kupfer-Sauerstoff-Chemie dargestellt werden. Der Wechsel in der Sauerstoffanbindung hängt eng mit der Natur des $\alpha$-C-Atoms des terminalen Rests am Stickstoffatom zusammen. Sind alle drei C-Atome der Substituenten am TACN tertiär, so kommt es zur Bildung von Peroxo-verbrückten Spezies. Ist jedoch nur eines dieser drei C-Atome geringer substituiert, entstehen $\operatorname{Bis}(\mu-0 \times 0)-K o m p l e x e .{ }^{[60,61]}$

Aufgrund der Beobachtungen und Erkenntnisse aus temperaturabhängigen kinetischen Messungen unter Verwendung der Stopped-Flow-Technik wird als Mechanismus der Oxygenierung einiger mononuklearer Kupfer(I)-Komplexe eine zwischenzeitlich gebildete Superoxo-Kupfer(II)-Spezies postuliert. Durch Reaktion mit einem weiteren Äquivalent des Kupfer(I)-Komplexes bildet sich die $\mu-\eta^{2}: \eta^{2}$-peroxoverbrückte Kupfer(II)Verbindung, die sich im Gleichgewicht mit der isomeren Bis( $\mu$-oxo)-Dikupfer(III)Verbindung befindet (Schema 7). Dieser Mechanismus ist mittlerweile durch Arbeiten mehrer Gruppen bestätigt und allgemein anerkannt. ${ }^{[7,74]}$

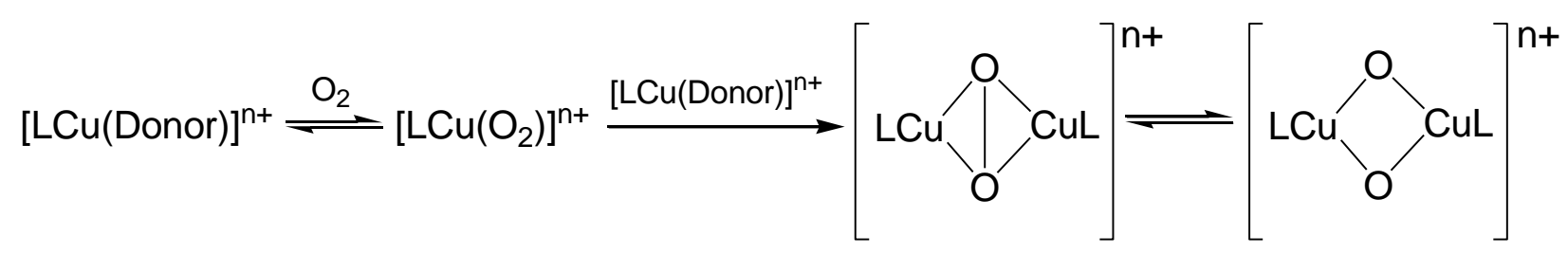
Schema 7: Bildung von $\mu-\eta^{2}: \eta^{2}$-Peroxo-dikupfer(II)- und Bis( $\mu$-oxo)-Dikupfer(III)-Komplexen.

Ein zweiter Ansatz zur Synthese von Modellkomplexen für Hämocyanin ist die Verwendung von Liganden, die zwei über einen Henkel verknüpfte Taschen für die Kupferionen bereitstellen. Somit können die Kupferionen in einem relativ genau definierten Abstand voneinander positioniert werden und außerdem besteht so eher die Möglichkeit, dass beide Kupferzentren gezielt kooperativ zusammenwirken.

Die Gruppe um Tolman hat das oben beschriebene Ligandsystem etwas variiert, indem sie zwei substituierte TACN-Moleküle über einen ortho-Xylyl-Rest verknüpft haben (Schema 8). Beide TACN-Ringe können je ein Kupfer-Ion binden. Wird dieser Kupfer(I)Komplex mit $\mathrm{O}_{2}$ bei $-80^{\circ} \mathrm{C}$ zur Reaktion gebracht, stellt man fest, dass die Art der Sauerstoffanbindung von der Konzentration der Reaktionslösung abhängig ist. Bringt man eine konzentrierte Lösung in Dichlormethan zur Reaktion, entstehen Komplexe des 
Typs VI bzw. Oligomere davon. Diese Verbindungen besitzen zwischen zwei Kupferzentren zwei $\mu$-Oxo-Brücken.

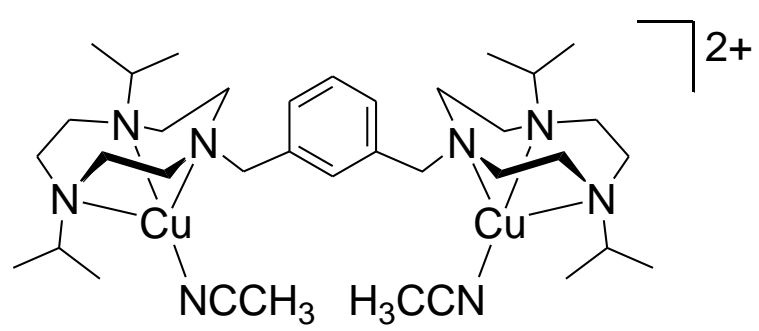

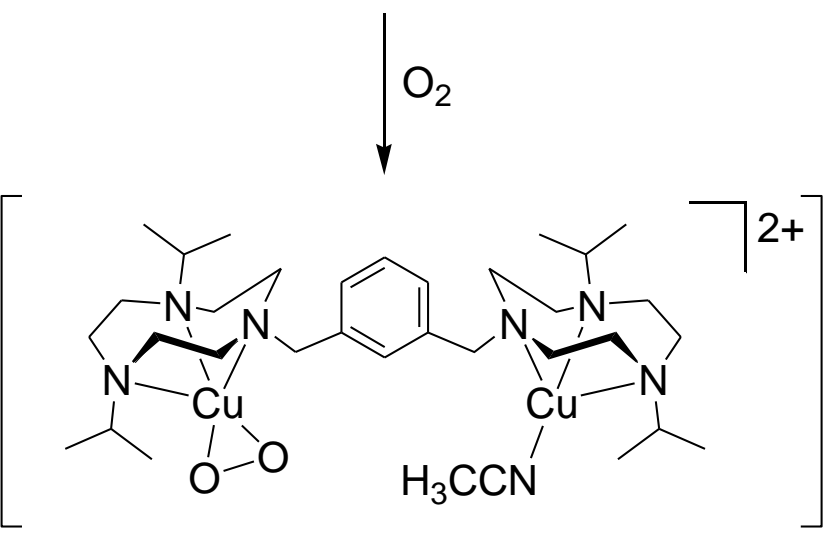

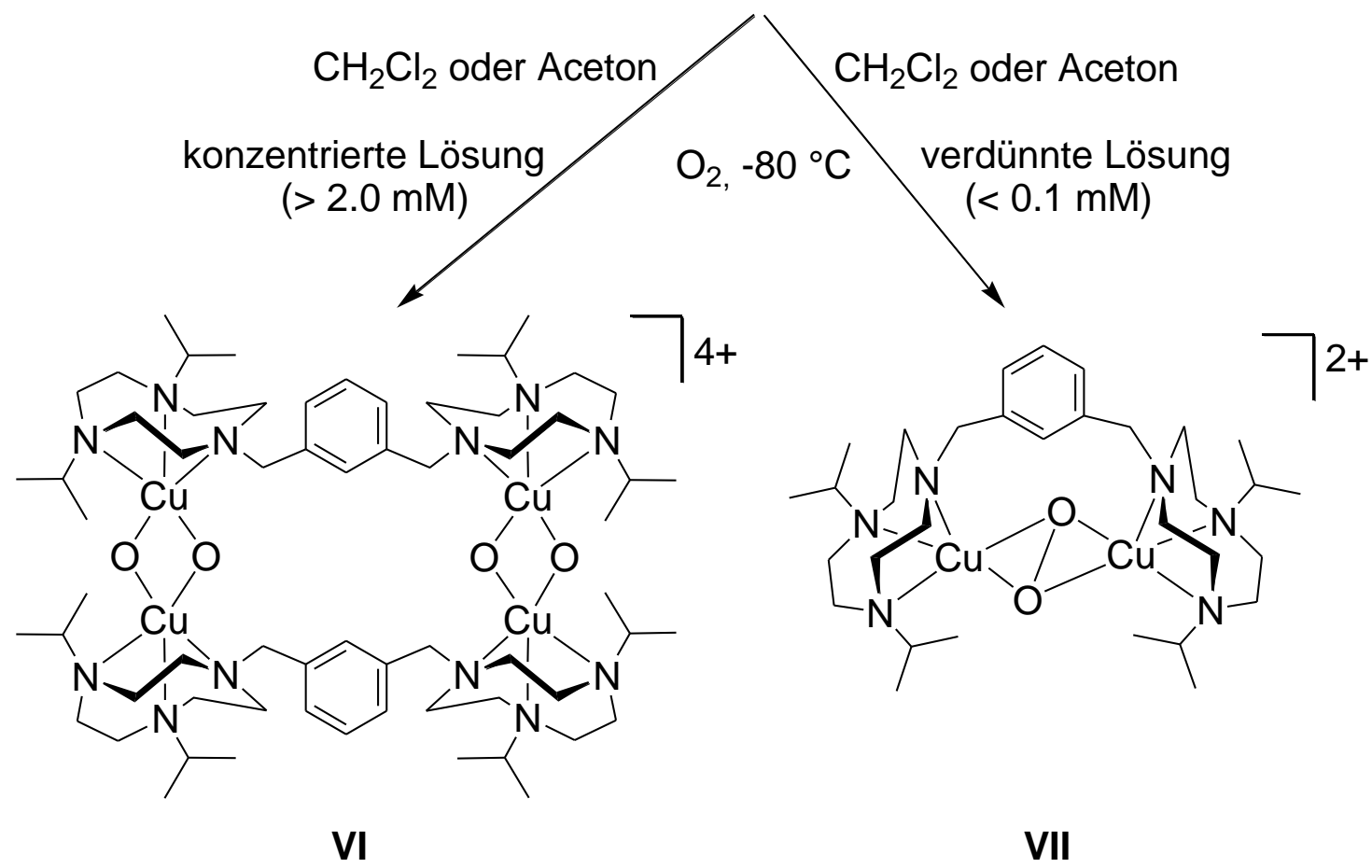

Schema 8: Veränderung der Sauerstoffanbindung durch Konzentrationsänderung.

Wird die gleiche Reaktion bei wesentlich geringerer Konzentration durchgeführt, erhält man fast ausschließlich den peroxo-verbrückten Kupfer-Komplex VII. Anhand der typischen UV/Vis- und Raman-spektroskopischen Eigenschaften lassen sich beide 
Formen zweifelsfrei identifizieren. ${ }^{[70]}$ Dieses Phänomen lässt sich am besten mit dem Auftreten einer Zwischenstufe erklären. Sauerstoff bindet danach erst als $\eta^{2}$-Superoxo an ein Kupferion. Dieses Addukt bildet durch eine intramolekulare Reaktion bei niedrigen Konzentrationen den Peroxo-Komplex VII. Ist die Konzentration der Lösung höher, dominiert der intermolekulare Angriff, der zu den Dimeren VI oder sogar deren Oligomeren mit bis( $\mu$-Oxo)-Einheiten führt. ${ }^{[59]}$

In neueren Arbeiten beschreiben Solomon et al. die Umsetzung eines Kupfer(I)Komplexes mit Sauerstoff in THF bei $-80^{\circ} \mathrm{C}$. Als Liganden sind einfache Diamine eingesetzt worden, die im einen Fall sekundärer und im anderen Fall tertiärer Natur waren (Schema 9). In Abhängigkeit vom gewählten Liganden kommt es unter identischen Reaktionsbedingungen entweder zur Bildung des Peroxo-verbrückten Kupfer(II)-Komplexes oder zu einer 1:1-Mischung der Peroxo-Kupfer(II)- und $\operatorname{Bis}(\mu-0 x 0)-K u p f e r(I I I)-K o m p l e x e$.

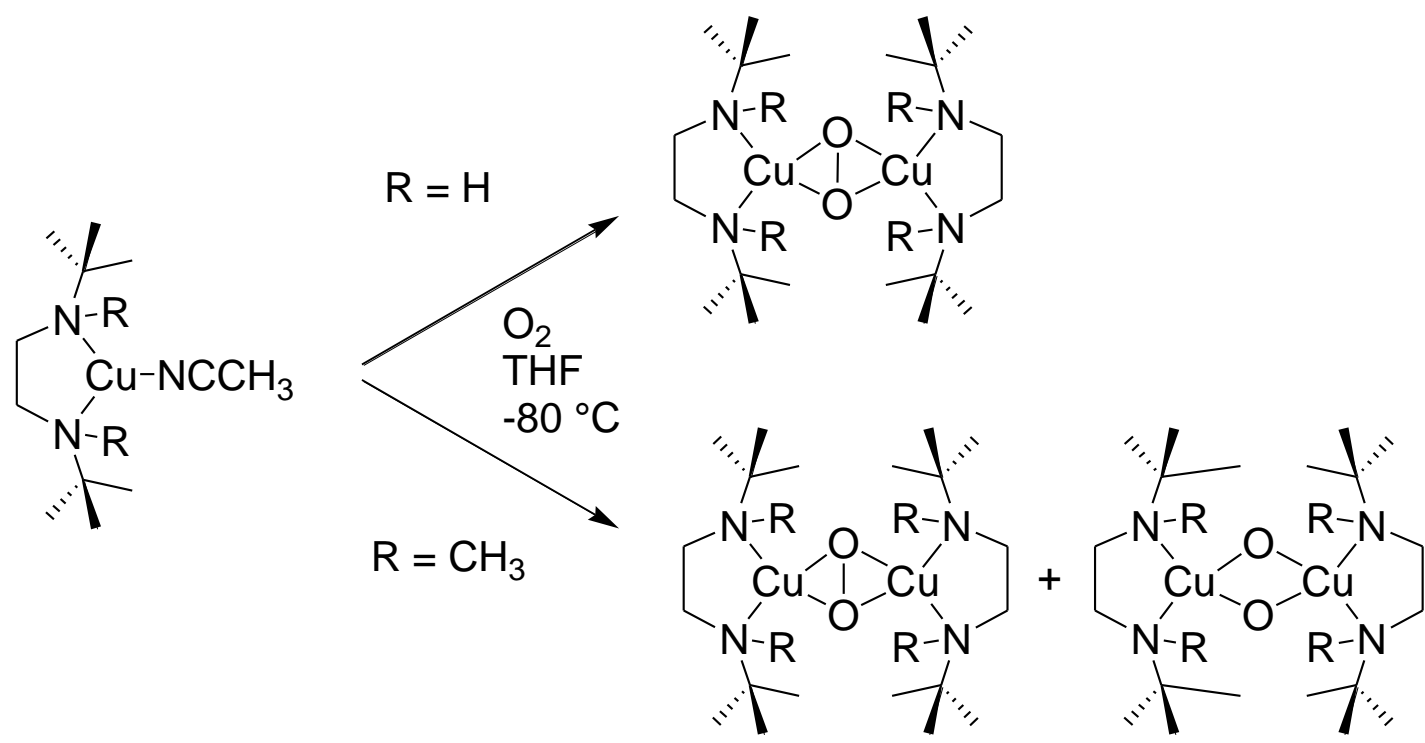

Schema 9: Verschiedene Reaktivität von zwei ähnlichen Liganden gegenüber $\mathrm{O}_{2}$.

Der $\mu-\eta^{2}: \eta^{2}$-peroxoverbrückte Kupfer(II)-Komplex wurde mit einer Vielzahl von exogenen Substraten wie Triphenylphosphin, Benzylalkohol, Benzylamin oder Thiophenol umgesetzt. Dabei kam es zu Substratoxidationen mit Ausbeuten von 70 bis $>95 \%$. Bei Verwendung von 1,4-Cyclohexadien und 9,10-Dihydroanthracen ist allerdings keine Reaktivität beobachtet worden. Die Catecholase-Aktivität dieses Systems konnte durch den Einsatz von 2,4-Di-tert-butyl-catechol nachgewiesen 
werden. Auch hier wurde das entsprechende Oxidationsprodukt (3,5-Di-tert-butylchinon) mit einer Ausbeute von >95\% gewonnen. Die Cresolase-Aktivität des PeroxoKupfer(II)-Komplexes ist mit 2,4-Di-tert-butyl-phenol untersucht worden. Auch nach 6 Stunden konnte keine Umsetzung zum entsprechenden Catechol festgestellt werden. Als allerdings das analoge Phenolat eingesetzt wurde, erfolgte eine Umsetzung, die ein 1:1-Gemisch des Catechols und des Benzochinons in einer Ausbeute von jeweils $30 \%$ ergab. ${ }^{[73,74]}$

In der Literatur sind zahlreiche Modellverbindungen beschrieben, die Tyrosinaseaktivität zeigen. ${ }^{[75-84]}$ Wie anhand des obigen Beispiels von Solomon et al. gezeigt, ist es in einigen Fällen gelungen, gezielt exogene Substrate umzusetzen. ${ }^{[3,74,85,86]}$ Es kommt allerdings auch relativ häufig zu ungewollten Kopplungsreaktionen zwischen den substituierten Phenolen ${ }^{[87-90]}$ oder zur Hydroxylierung des Liganden.

Karlin et al. lieferten ein Beispiel für die Hydroxylierung des Liganden (Schema 10).<smiles>CN1C=CN(Cc2cccc(CN(CCc3ccccn3)CCc3ccccn3)c2)CC1</smiles>

XYL-H<smiles>[TeH][TeH]</smiles>

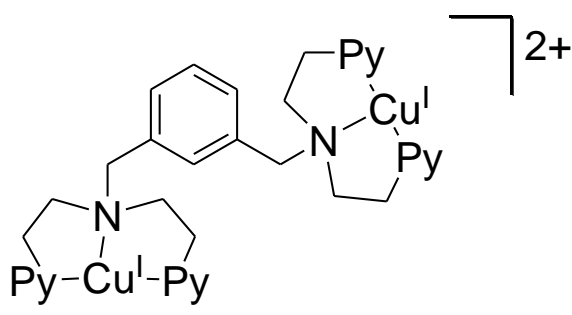<smiles>CN(CCc1ccccn1)CCc1ccccn1</smiles>

\section{XYL-OH}
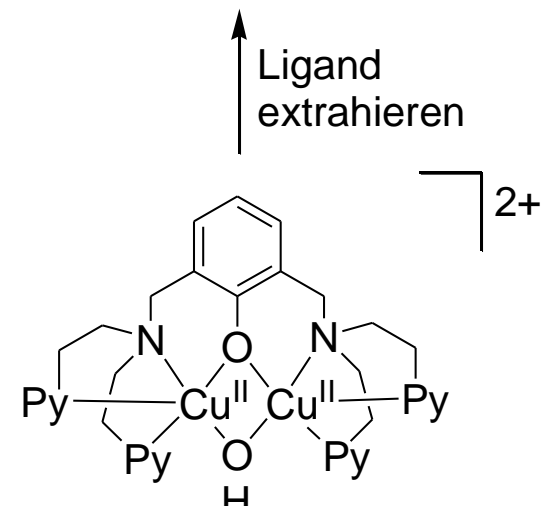

$\mathrm{H}$
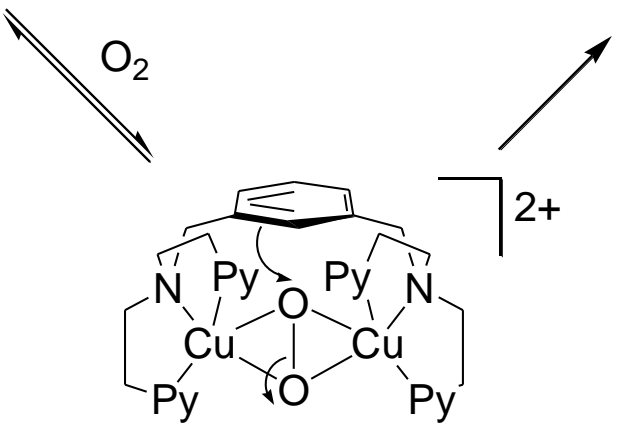

Schema 10: Hydroxylierung des Liganden XYL-H von Karlin et al. 
Bei der Umsetzung des entsprechenden Kupfer(I)-Komplexes von Ligand XYL-H mit Sauerstoff entstand zunächst der gewünschte Peroxo-Komplex. Dieser wandelte sich durch elektrophilen Angriff des Xylyl-Rings an die side-on-gebundene Peroxo-Einheit in den phenoxo-hydroxo-verbrückten Komplex um. Nach der Extraktion des Liganden konnte die hydroxylierte Verbindung XYL-OH isoliert werden.${ }^{[91-93]}$ Dieses TyrosinaseModell ermöglicht die Umwandlung einer nichtaktivierten $\mathrm{C}$-H-Bindung bei sehr milden Bedingungen $\left(-80^{\circ} \mathrm{C}\right)$ unter Verwendung von Sauerstoff.

Zur Modellierung der Tyrosinaseaktivität haben Casella und Mitarbeiter verschiedene Ligandsysteme auf Basis von Polyaminen mit $\mathrm{N}$-Methylbenzimidazolen als Seitenarme entwickelt. Eine kleine Auswahl aus dem umfangreichen Sortiment ist mit VIII und IX in Abbildung 10 gezeigt. Mit den gebildeten Komplexverbindungen war es möglich die Tyrosinaseaktivität zu untersuchen. Als Testsubstrat wurde bei diesen Untersuchungen Methyl-4-Hydroxybenzoat eingesetzt, da der elektronenschiebende Substituent am Phenylring das Kupfer(II)-Catecholat-Addukt stabilisiert und somit eine Weiterreaktion zum Chinon vermieden werden konnte. Für beide Liganden ergaben sich verschiedene Aktivitäten, die sich laut Casella et al. mit einem freien Benzimidazol-Arm von IX begründen lassen. Die Aktivität der Komplexe dieses Liganden ist größer als die des Liganden mit $m$-Xylyl-Spacer VIII. ${ }^{[76]}$

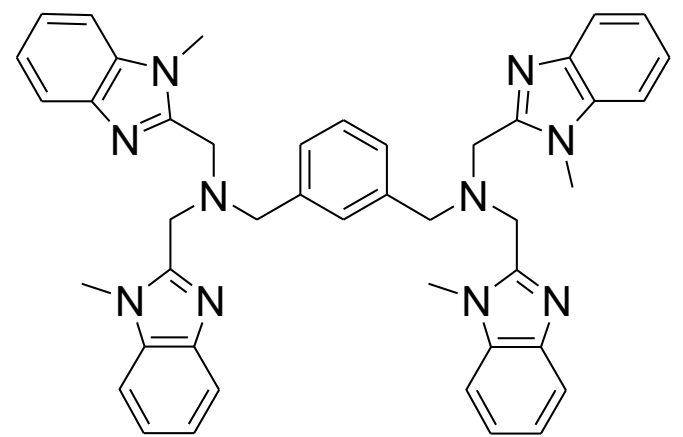

VIII

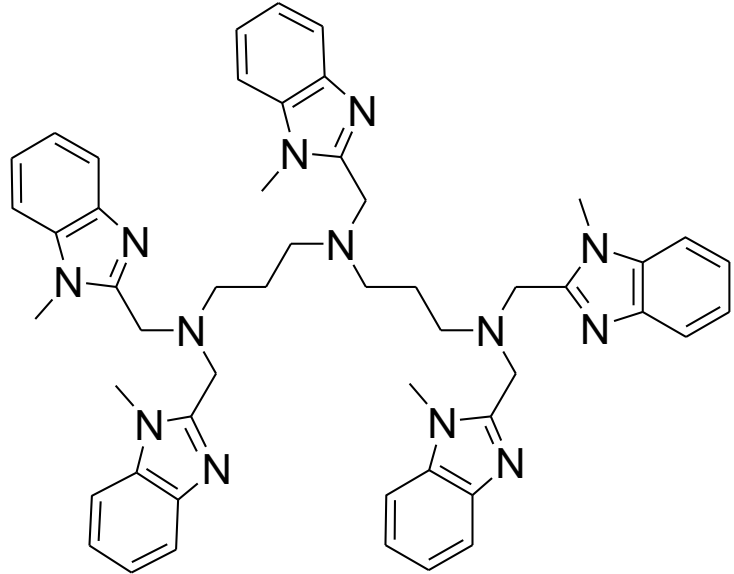

IX

Abbildung 10: Liganden zur Untersuchung der Tyrosinaseaktivität von Casella et al. 
Casella und seine Mitarbeiter fanden bei nahezu allen Komplexen eine CatecholaseAktivität, wohingegen die Cresolase-Aktivität bei vielen Kupferkomplexen eher gering ist. Diese Ansätze lassen schon erahnen, dass bei der Entwicklung geeigneter Verbindungen für solch komplexe Reaktionen zahlreiche Faktoren berücksichtigt werden müssen. So hängt die katalytische Aktivität unter anderem auch vom Abstand der Metallzentren zueinander, der Koordinationsgeometrie, sterischen Behinderungen durch die Ligandreste oder geometrischen Effekten ab.

\subsection{Zweikernkomplexe mit multifunktionellen Pyrazolatliganden}

In den letzten Jahren sind zahlreiche Ligandsysteme entwickelt worden, die es ermöglichen zweikernige Metallkomplexe herzustellen. Die verwendeten Metalle werden dabei in der Regel in einem durch die Ligandgeometrie festgelegten Abstand zueinander gehalten. Um zwei Metallionen mit einem definierten Abstand in einem Ligandsystem zu binden, eignet sich der Pyrazolring ganz besonders. Aufgrund der zwei benachbarten Stickstoff-Donoren können zwei Metallionen gebunden werden. In 3- und 5-Position des Pyrazolrings lassen sich zudem Seitenarme anbringen, die weitere Donoratome aufweisen. Je nach Anzahl und Art dieser Donoratome ist es möglich, Metall-Metall-Abstände von $2.4 \AA$ bis $4.6 \AA$ zu erzeugen. ${ }^{\left[{ }^{[9]}\right.}$ Zudem können mit den chelatisierenden Seitenarmen sowohl elektronische wie auch sterische Eigenschaften der Metallzentren verändert und dadurch die katalytischen Aktivitäten der erhaltenen Komplexe beeinflusst werden. Somit können kooperative Wechselwirkungen zwischen den benachbarten Metallionen und auch kooperative Aktivierung kleiner Moleküle beobachtet werden.

So ist es zum Beispiel möglich Indenyl-, Fluorenyl- oder Cyclopentadienyl-Reste an die 3- und 5-Position des Pyrazolrings anzubringen. Die resultierenden Liganden sind in Abbildung 11 gezeigt. ${ }^{[95-97]}$ Zudem konnten einige Metallkomplexe dieser Liganden strukturell aufgeklärt werden. 


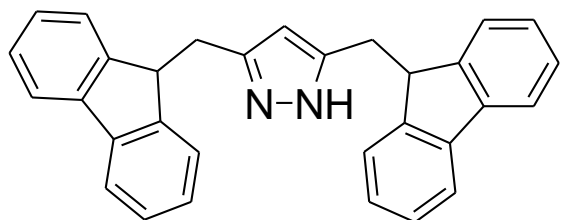

$\mathrm{Xa}$

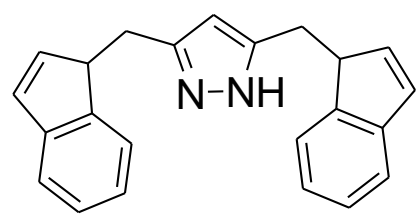

$\mathbf{X b}$

Abbildung 11: Pyrazolatliganden mit Fluorenyl-Seitenarmen (Xa) und Indenyl-Seitenarmen (Xb).

In neueren Arbeiten sind verstärkt Ligandsysteme mit Aminseitenarmen hergestellt und deren Metallkomplexe untersucht worden. Die Aminseitenarme unterscheiden sich zum einen in der Anzahl der N-Donoren, als auch in der Länge der Seitenarme. Außerdem kann zusätzlich die Donorfähigkeit durch Austausch der aliphatischen Aminfunktionen gegen Pyridinringe verändert werden. In zahlreichen Arbeiten ist es gelungen Pyrazolatliganden dieser Art zu synthetisieren (Abbildung 12).<smiles>[R7]NCc1cc(C[NH2+][R7])n[nH]1</smiles><smiles>CN(C)CCN(C)CCN(C)CCCN(C)CCN(C)CCN(C)CCN(C)C</smiles><smiles>CC(C)N1CCNCCN(C(C)C)CC1</smiles>

Abbildung 12: Einige Beispiele für Pyrazolatliganden mit funktionalisierten Aminseitenarmen.

Mit diesen Liganden ist eine Vielzahl von Metallkomplexen hergestellt worden. B. Bauer-Siebenlist und M. Alvariño Gil beschreiben die Synthese einiger Zink(II)Komplexe als Modellverbindungen für Hydrolase-Reaktionen. ${ }^{[13,15,16,98]}$

Außerdem ist es S. Demeshko gelungen, Nickel(II)-Komplexe auf der Basis von Pyrazolatliganden mit funktionalisierten Amin-Seitenarmen herzustellen. Der Unterschied zu allen anderen Arbeiten ist der Einsatz des Azid-lons als Co-Ligand. Dieser ermöglicht abhängig von der beobachteten Koordination an die Nickel(II)-lonen 
sowohl ferromagnetische als auch antiferromagnetische Kopplungen zwischen den Nickelzentren. ${ }^{[14,99,100]}$ Auch der Austausch der Seitenarme mit N-Donoren gegen solche, die Thioether-Funktionen beinhalten (Abbildung 13), lieferte interessante magnetische Eigenschaften. Verwendet man erneut Azid als Co-Liganden, lassen sich sowohl ferromagentische als auch antiferromagnetische Austauschwechselwirkung finden. ${ }^{[101,102]}$

$$
R^{2} R^{1} N N_{N-N H} R^{1} R^{2}
$$

$$
\begin{aligned}
& \mathrm{N}_{\mathrm{R}^{2}}^{\mathrm{R}} \mathrm{R}^{1} \text { : } \\
& \mathrm{N}(\mathrm{SMe})_{2} \\
& \mathrm{~N}(\mathrm{SEt})_{2} \\
& \mathrm{~N}\left(\mathrm{SS}^{\mathrm{i} P r}\right)_{2} \\
& \mathrm{~N}_{\mathrm{Me}}^{\mathrm{SEt}_{2}}
\end{aligned}
$$

Abbildung 13: Pyrazolat-Liganden mit Thioether-Funktionen als Seitenarme.

Die Koordinationsgeometrie der in Abbildung 12 gezeigten Pyrazolatliganden mit zwei tripodal tetradentaten Koordinationstaschen gegenüber Kupfer(II)-lonen ist von J. Ackermann und A. Prokofieva ausgiebig untersucht worden. Dabei konnten zahlreiche dinukleare Kupfer(II)-Komplexe kristallografisch aufgeklärt und vollständig charakterisiert werden. ${ }^{[10-12,103]}$ Zwei beispielhafte Vertreter sind in Abbildung 14 gezeigt.
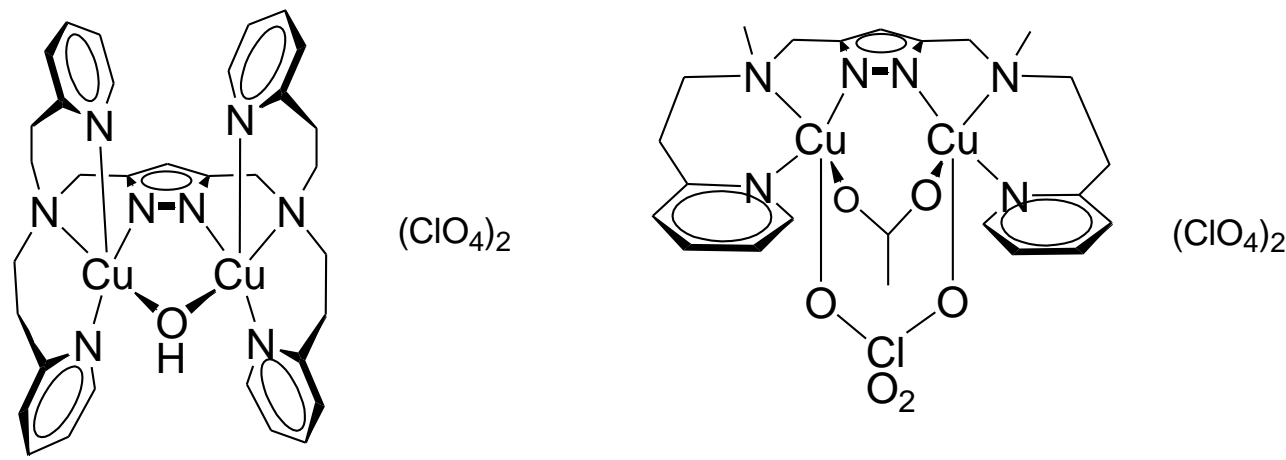

Abbildung 14: Dinukleare Kupfer(II)-Komplexe von J. Ackermann.

Später sind diese Komplexe für kinetische Untersuchungen zur Catecholase-Aktivität verwendet worden. Dabei sind zunächst vor allem Komplexe mit aliphatischen 
Aminseitenarmen zum Einsatz gekommen. Es konnte eine Korrelation zwischen Struktur und Aktivität erhalten werden. So beobachtet man bei geringem $\mathrm{Cu}-\mathrm{Cu}-$ Abstand eine sehr hohe Reaktivität. Mit steigendem Abstand beider Metallionen nimmt die katalytische Wirksamkeit rapide ab. Die Liganden mit endständigen Pyridyl-Resten zeigen in Methanol keine katalytische Aktivität. Erst bei Zugabe von Puffer-Lösungen konnte die Bildung des Chinons im UV/Vis-Spektrum beobachtet werden. Auch für diese Systeme steigt die Aktivität mit abnehmendem Cu-Cu-Abstand stark an. ${ }^{[10,104]}$

Damit ein erster Einblick in den ablaufenden Mechanismus für die Catechol-OxidaseReaktion zu erhalten, wurden die Kupfer(II)-Komplexe mit koordinierten Catecholen hergestellt. Diese ortho-Diphenole sind gegen Polymerisation geschützt, da die vier restlichen Positionen am Phenylring mit Chlor- oder Bromatomen besetzt sind. Anhand der Kristallstrukturen dieser Kupfer(II)-Catecholat-Komplexe zeigt sich, dass die Zweitbrücke zwischen beiden Metallionen abdissoziiert und das Catecholat bidentat an ein Kupfer(II)-Ion bindet.

Weiterhin wurde untersucht, wie sich die Kupfer(I)-Komplexe gegenüber Luft bzw. Sauerstoff verhalten. Dabei kam es auch bei sehr tiefen Temperaturen $\left(-78^{\circ} \mathrm{C}\right)$ in fast allen Fällen zu einer sehr schnellen Reaktion und die Lösung verfärbte sich grün. Lediglich für den makrozyklischen TACN-Ligand wurde zwischenzeitlich eine violette Lösung erhalten, die mittels Raman- und UV/Vis-Spektroskopie untersucht werden konnte. Die Bildung des $\mathrm{O}_{2}$-Adduktes konnte nicht beobachtet werden, da dieser Prozess bereits nach $2.7 \cdot 10^{-3} \mathrm{~s}$ abgeschlossen war. Der Zerfall dieser Zwischenstufe war nach $1 \mathrm{~s}$ beendet. Der Kupfer(I)-Komplex des TACN-haltigen Liganden weist eine viel größere Stabilität gegenüber Sauerstoff auf. Der Zerfall des $\mathrm{O}_{2}$-Adduktes ist erst nach $1124 \mathrm{~s}$ vollzogen und ein Zersetzungsprodukt konnte auch für diese Reaktion beobachtet werden. Trotz der erhöhten Stabilität war es nicht möglich, Kristalle aus der violetten Lösung zu isolieren und eine genaue Strukturaufklärung vorzunehmen. 


\section{Ligandsynthese}

\subsection{Synthesestrategie}

Durch den Austausch der aliphatischen Aminseitenarme in Ligand XI gegen Bis[2-(1-methylimidazolyl)methyl]amin konnte mit Verbindung XII bereits eine bessere Nachahmung der biologischen Donorumgebung erreicht werden (Abbildung 15). Dieser neuartige Ligand XII besitzt wie schon die Liganden mit aliphatischen Aminseitenarmen zwei tripodal tetradentate Koordinationstaschen. ${ }^{[18]}$ Mit diesem Liganden sind ebenfalls Kupfer(II)-Komplexe synthetisiert und die Aktivität für Catechol-Oxidase-Reaktionen studiert worden. Bei Verwendung diverser Phenole sind dabei C-O- und C-C-Kupplungsreaktion aufgetreten, deren Mechanismus ebenfalls untersucht wurde.

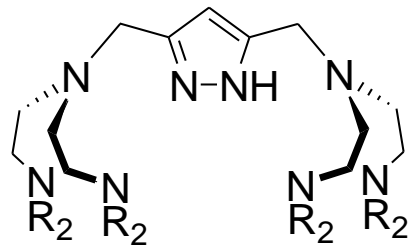

$\mathbf{X I}$

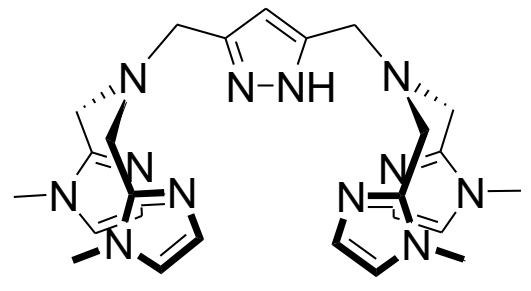

XII

Abbildung 15: Vergleich unterschiedlicher Pyrazolatliganden mit tripodal tetradentater Koordinationsumgebung.

In biologischen Systemen wie etwa im Hämocyanin oder der Tyrosinase findet man aber drei Imidazolringe von Histidin, die an die Kupferionen gebunden sind. Die $\mathrm{N}$-Donoren geben eine facial tridentate Koordinationsumgebung vor. Um dies noch genauer zu imitieren wurde der Ligand XIII entwickelt. Die in 3- und 5-Position des Pyrazolrings befindlichen Methylengruppen sind durch quartäre Kohlenstoffatome ersetzt, die jeweils mit zwei Imidazolringen verknüpft sind.

Liganden des Typs XI und XII lassen sich sehr einfach durch Reaktion des 3,5-Bis(chlormethyl)pyrazols mit dem entsprechenden Amin herstellen. Dafür ist, abhängig vom Aminseitenarm, teilweise keine THP-Schutzgruppe am Pyrazol notwendig. ${ }^{[18,100]}$ Diese Synthesestrategie kann nicht auf die Darstellung von Liganden des Typs XIII übertragen werden. Stattdessen muss die Synthese in Anlehnung an 
literaturbekannte Darstellungen von Bisarylsystemen erfolgen. ${ }^{[105-111]}$ Die beschriebenen Bisarylsysteme besitzen zentrale Benzol- oder Pyridinringe. Für das System mit zentralem Pyrazolbaustein ergibt sich die in Schema 11 gezeigte Retrosynthese.

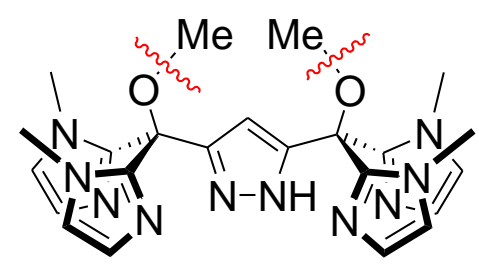

XIII<smiles>[C]1C=C1</smiles>

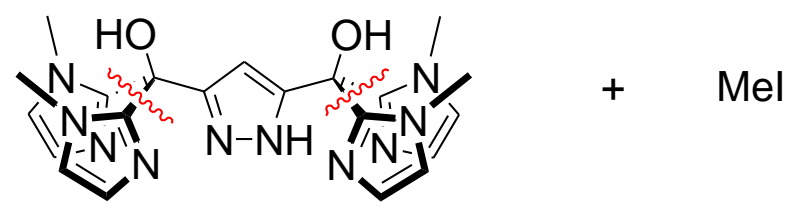<smiles>CCCCCCCC</smiles><smiles>COC(=O)c1cc(C(=O)OC)[nH]n1</smiles>

Schema 11: Retrosynthese zu den hier verwendeten Pyrazolatliganden.

Anhand der Retrosynthese lässt sich erkennen, dass die Reaktion des deprotonierten Imidazols mit 3,5-Pyrazoldicarbonsäuremethylester nur zu dem gewünschten Dialkohol führt, wenn das NH-Proton des Pyrazolrings mit einer Schutzgruppe geschützt wird. Ansonsten kommt es zum Austausch des NH-Protons gegen das Lithiumion vom deprotonierten Imidazol. Das daraus resultierende substituierte Lithium-Pyrazolat lässt sich nicht mit lithiiertem Imidazol zur Reaktion bringen. Die Ursache liegt in der verbesserten Ladungsverteilung bis zur Carbonyl-Einheit. Deshalb ist das Kohlenstoffatom der Esterfunktion weniger bis gar nicht positiviert und ein nucleophiler Angriff des Carbanions vom Imidazol ist nicht möglich. Aufgrund der Notwendigkeit eine Schutzgruppe für das NH-Proton des Pyrazols einzuführen ergeben sich neben dem Reaktionsschritt für das Anbringen der THP-Schutzgruppe zwei weitere Synthesestufen für die Abspaltung der Schutzgruppe. 
Der THP-geschützte 3,5-Pyrazoldicarbonsäuremethylester (1) lässt sich gemäß einer Literaturvorschrift in einer dreistufigen Synthese aus 3,5-Dimethylpyrazol (XIV) herstellen (Schema 12). ${ }^{[112]}$ An die Oxidation beider Methylgruppen mit Kaliumpermanganat und Kohlendioxid in wässriger Lösung im ersten Schritt schließt sich eine Veresterung der 3,5-Pyrazoldicarbonsäure (XV) mit Methanol und Chlorwasserstoffgas zu XVI an. Abschließend wird das NH-Proton mittels 3,4-Dihydro$2 \mathrm{H}$-pyran geschützt und 1 mit einer Ausbeute von $99 \%$ erhalten.<smiles>Cc1cc(C)[nH]n1</smiles>

XIV<smiles>COC(=O)c1cc(C(=O)OC)n(C2CCCCO2)n1</smiles>

1
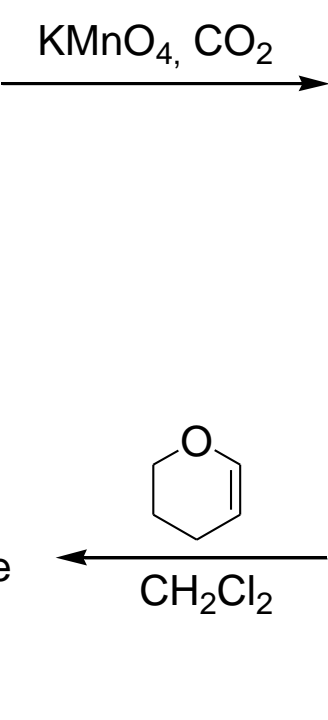<smiles>O=C(O)c1cc(C(=O)O)[nH]n1</smiles>

XV<smiles>[3H]OCO</smiles><smiles>COC(=O)c1cc(C(=O)OC)[nH]n1</smiles>

XVI

Schema 12: Synthese des THP-geschützten Pyrazol-3,5-dicarbonsäuremethylester (1).

Dabei ist wichtig, dass der Ester XVI als Hydrochlorid vorliegt. Zu Beginn der Reaktion mit 3,4-Dihydro-2H-pyran erfolgt offensichtlich zunächst eine Protonierung der Doppelbindung. Dass so positivierte Kohlenstoffatom wird von dem freien Elektronenpaar des Pyrazolstickstoffs angegriffen und das NH-Proton abgespalten. Wenn die Reaktion mit Ester durchgeführt wird, der nicht vollständig als Hydrochlorid vorliegt, findet keine vollständige Umsetzung zu 1 statt. Der Anteil des Esters ohne Hydrochlorid liegt auch nach der Reaktion ohne THP-Schutzgruppe unverändert vor. Durch Umkristallisation aus $n$-Hexan ist es jedoch möglich beide Verbindungen voneinander zu trennen. 
Erstaunlicherweise bringt das Einleiten von Chlorwasserstoffgas in die Reaktionslösung vor Zugabe des Pyrans keine Steigerung der Ausbeute an THP-geschützten Ester. Es wäre eigentlich zu erwarten, dass durch zusätzliches HCl-Gas der Ester XVI vollständig als Hydrochlorid vorliegt und somit auch in nahezu quantitativer Ausbeute zu 1 umgesetzt werden kann. Da die Abspaltung der THP-Schutzgruppe in saurer Lösung erfolgt, ist es denkbar, dass durch überschüssiges $\mathrm{HCl}$-Gas die THP-Schutzgruppe abgespalten wird. 


\subsection{Darstellung von $\mathrm{HL}^{1}, \mathrm{HL}^{2}$ und $\mathrm{HL}^{3}$}<smiles>COC(=O)c1cc(C(=O)OC)n(C2CCCCO2)n1</smiles>

1<smiles>[R]c1ncn(C)c1[R]</smiles>
$\mathrm{R} \quad \mathrm{R}$

$$
\left(\frac{10}{20}\right.
$$<smiles>[R]c1nc(C(OC)(c2cc(C(OC)(c3nc([R])c([R])n3C)c3nc([R])c([R])n3C)[nH]n2)c2nc([R])c([R])n2C)n(C)c1[R]</smiles>

$$
\text { 4b:R }=M e
$$<smiles>[R]c1nc(C(OC)(c2cc(C(OC)(c3nc([R])c([R])n3C)c3nc([R])c([R])n3C)[nH]n2)c2nc([R])c([R])n2C)n(C)c1[R]</smiles>

$$
4 c: R=P h
$$<smiles>[R]c1nc(C(O)(C=[IH])c2nc([R])c([R])n2C)n(C)c1[R]</smiles><smiles>[R]c1nc(C(C)(O)c2ccnn2C2CCCCO2)n(C)c1[R]</smiles>

2a: $\mathrm{R}=\mathrm{H}$

2b:R $=\mathrm{Me}$

2c: $R=P h$

$$
\text { THF 1) } \begin{array}{ll} 
& \mathrm{NaH} \\
\text { 2) } \mathrm{Mel}
\end{array}
$$<smiles>[R]c1nc(C(OC)(c2cc(C(OC)(c3nc([R])c([R])n3C)c3nc([R])c([R])n3C)nn2C2CCCCO2)c2nc([R])c([R])n2C)[nH]c1[R]</smiles>

$3 a: R=H$ $3 b: R=M e$ $3 c: R=P h$

Schema 13: Synthese der Liganden $\mathrm{HL}^{1}(\mathbf{5 a}), \mathrm{HL}^{2}(\mathbf{5 b})$ und $\mathrm{HL}^{3}(\mathbf{5} \mathbf{c})$. 
Gemäß der in Schema 13 gezeigten Reaktionsfolge führt die Umsetzung des THPgeschützten Pyrazol-3,5-dicarbonsäuremethylester (1) mit lithiiertem N-Methylimidazol in absolutem THF zur Verbindung 2a als weißen Feststoff mit einer Ausbeute von $54 \%$. Aus Tetrahydrofuran ist es außerdem möglich diese Substanz in kristalliner Form zu erhalten. Die Kristallstruktur der farblosen blockförmigen Kristalle ist in Abbildung 16 dargestellt.

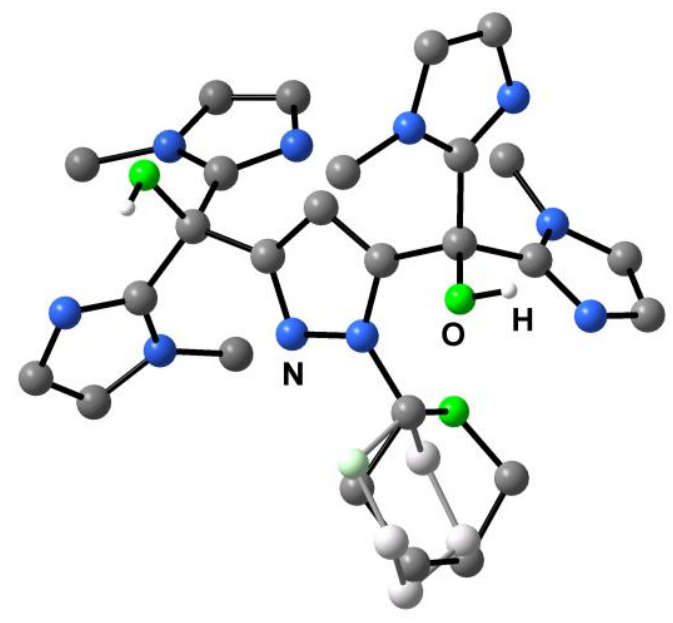

Abbildung 16: Festkörperstruktur von 2a (wegen der Übersichtlichkeit sind alle H-Atome außer denen der $\mathrm{OH}-\mathrm{Gruppen}$ weggelassen, die THP-Gruppe ist fehlgeordnet).

Es wird deutlich, dass die gewünschte Struktur der Ligandvorstufe 2a vorliegt. Auf jeder Seite des Pyrazols befinden sich zwei Imidazolringe. Zudem besteht eine Fehlordnung der THP-Schutzgruppe. Die Bindungslängen und -winkel liegen in dem erwarteten Bereich für derartige Verbindungen. Durch Rotation um die C-C-Einfachbindung zwischen $C^{\mathrm{Pz} 3}$ bzw. $\mathrm{C}^{\mathrm{Pz} 5}$ und dem jeweils benachbarten quartären Kohlenstoffatom ist es möglich, dass sich die Imidazolringe so anordnen, dass nach Abspaltung der THPSchutzgruppe eine Koordination von zwei Metallionen erfolgen kann.

Als nächstes findet eine Veretherung der Alkohol-Funktionen statt. Hierbei werden die Hydroxylgruppen mit Natriumhydrid deprotoniert und in absolutem THF mit Methyliodid zur Reaktion gebracht. Es entsteht die Substanz 3a als weißer Feststoff in $86 \%$ Ausbeute. Die folgende Abspaltung der THP-Schutzgruppe geschieht mit ethanolischer $\mathrm{HCl}$ und das gebildete Hydrochlorid $\mathbf{4 a}$ wird mit absolutem Diethylether ausgefällt. 
Abschließend wird das Hydrochlorid $\mathbf{4 a}$ in ammoniakalischer Dichlormethanlösung zum gewünschten Liganden $\mathrm{HL}^{1}$ (5a) neutralisiert. Für die beiden letzten Stufen der Ligandsynthese liegt die Ausbeute bei $47 \%$. Gegenüber der Neutralisation mit wässriger Natronlauge und anschließender Extraktion mit Dichlormethan hat die Neutralisation mit ammoniakalischer Dichlormethanlösung den entscheidenden Vorteil, dass es sich hierbei um ein nichtwässriges System handelt. Der Ligand ist sehr gut in Wasser löslich und deshalb führt eine Extraktion der wässrigen Lösung mit organischen Lösungsmitteln zu deutlich schlechteren Ausbeuten. Für die Unterscheidung der chemischen Verschiebung für die Protonen und C-Atome in 4- und 5-Position des Imidazolrings erfolgte an Ligand $\mathrm{HL}^{1}$ zum Einen mittels $\mathrm{C}-\mathrm{H}$-Korrelationsexperimenten, damit eine Zuordnung getroffen werden kann, welches Proton an welches C-Atom gebunden ist (Abbildung 17).

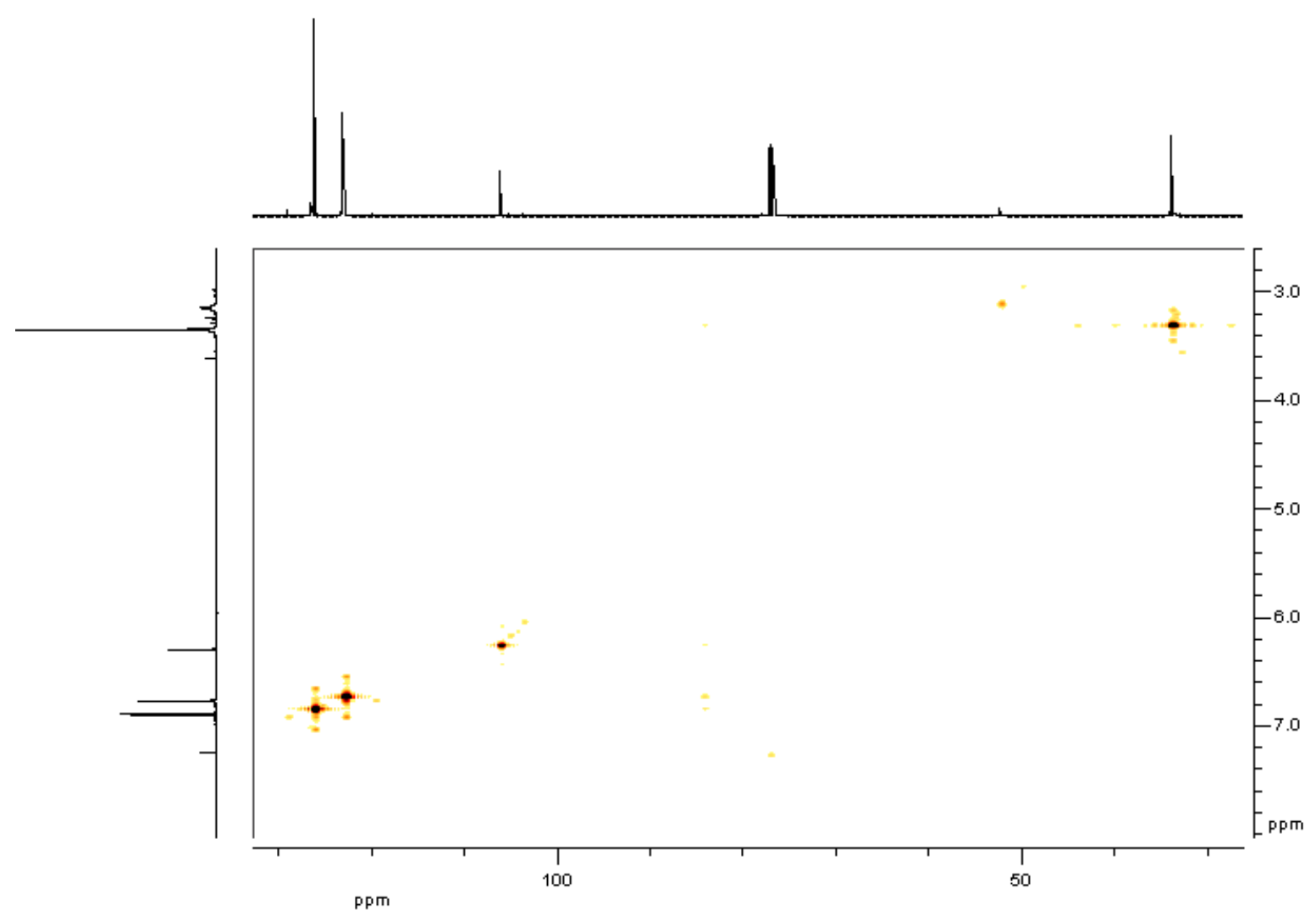

Abbildung 17: NMR-Spektrum der C-H-Korrelation von $\mathrm{HL}^{1}$ in $\mathrm{CDCl}_{3}$.

Zum Anderen wurden ${ }^{13} \mathrm{C}-\mathrm{NMR}$-Spektren aufgenommen, die aufgrund von $\mathrm{C}-\mathrm{H}-K o p p l u n g e n$ verschiedene Aufspaltungen der Signale lieferten. So findet man für das C-Atom in 5-Position neben der Kopplung zum Proton in 4-Position auch eine 
charakteristische Kopplung zu den Protonen der Methylgruppe am benachbarten Stickstoffatom. Für das Kohlenstoffatom in 5-Position konnte keine solche Kopplung festgestellt werden. Die hier gefundene Zuordnung wurde auf alle anderen hier dargestellten Verbindungen übertragen.

Damit der Ligand $\mathrm{HL}^{2} \quad(\mathbf{5 b})$ synthetisiert werden kann, muss zunächst 1,4,5-Trimethylimidazol dargestellt werden. Dies geschieht in Anlehnung an eine Literaturvorschrift ${ }^{[113]}$ über eine Einstufenreaktion aus Diacetyl und wässrigen Lösungen von Ammoniak, Formaldehyd und Methylamin. Die in 1-Propanol durchgeführte Reaktion ist in Schema 14 gezeigt.

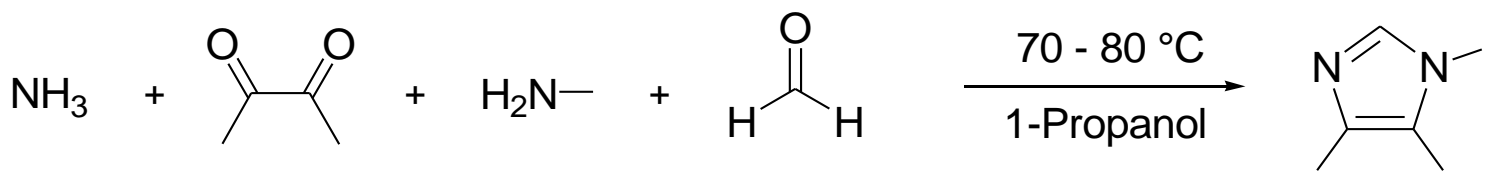

Schema 14: Synthese von 1,4,5-Trimethylimidazol.

Die Aufarbeitung des Reaktionsgemisches ist um einiges aufwendiger als die Durchführung der Reaktion. So müssen zunächst die Lösungsmittel der Reaktion, 1-Propanol und Wasser, destillativ entfernt werden. Die in der Literatur beschriebene Destillation des dunklen Rückstands gestaltet sich jedoch als sehr schwierig, da es sich bei dem gewünschten Imidazol um einen Feststoff handelt, der einen Schmelzpunkt von $25{ }^{\circ} \mathrm{C}$ bis $30^{\circ} \mathrm{C}$ besitzt. Dadurch erstarrt das Produkt auch bei Verwendung einer Feststoff-Destillationsbrücke und muss durch leichtes Erwärmen der Brücke in die Vorlage überführt werden. Das Imidazol wird dabei als gelborange gefärbtes Öl erhalten, das beim Stehen kristallisiert. Die Ausbeute liegt trotz wiederholter Durchführung weit unter dem in der Literatur angegebenen Wert von $57.8 \%$.

Daraufhin wurde nach einer alternativen Aufarbeitungsmethode gesucht. Die Extraktion des dunklen Rückstandes mit $n$-Hexan nach der destillativen Entfernung der Lösungsmittel lieferte keine Verbesserung in der Ausbeute. Die Reinheit des Produktes war ebenfalls schlechter, da ein Teil der Nebenprodukte und andere Verunreinigungen gut in Hexan lösbar sind. Als nächstes wurde versucht, das Rohprodukt nach dem Abtrennen der Lösungsmittel durch Sublimation zu reinigen. Dafür ist auf den Kolben 
mit dem Rohprodukt eine kühlbare Sublimationsbirne mit Vakuumanschluss aufgesetzt worden. Der Kolben wurde nun etwas erwärmt und durch das angelegte Vakuum scheidet sich das Imidazol in der Sublimationsbirne als weißer kristalliner Feststoff ab. Auch wenn man diese Substanz unter Stickstoff aufbewahrt, kann nicht verhindert werden, dass nach einiger Zeit ein Farbwechsel von weiß nach orangebraun eintritt. Außerdem ist die Verbindung extrem hygroskopisch, so dass sich aus dem Feststoff eine ölige Substanz bildet. Deshalb muss das Imidazol vor jedem Gebrauch frisch in die Sublimationsbirne überführt werden.

Der Einsatz des so hergestellten 1,4,5-Trimethylimidazols führt zum Ligand $\mathrm{HL}^{2}$ (5b). Die Umsetzungen erfolgen analog zu den Reaktionen, die zu Ligand $\mathrm{HL}^{1}$ geführt haben. Die oben erwähnte Hygroskopie bringt jedoch einige Probleme mit sich. So ist es schwierig, eine vollständige Deprotonierung der C2-Position des Imidazols mit $n$-BuLi zu erreichen, da stets eine kleine Menge Wasser im Imidazol enthalten ist. Ein größerer Überschuss von Butyllithium beseitigt zwar dieses Problem, jedoch entstehen dabei mehr Nebenprodukte. Diese lassen sich genauso wenig wie das überschüssige Imidazol aus dem Rohprodukt entfernen. Nach dem Lösen der Verbindungen $\mathbf{2 b}$ und $\mathbf{3 b}$ in Dichlormethan bzw. Chloroform kann mittels wässriger Extraktion ein geringer Teil des Trimethylimidazols abgetrennt werden. Jedoch ist es so nicht möglich, das Imidazol vollständig abzutrennen. Auch die Säulenchromatographie mit diversen Lösungsmittelgemischen bringt keine weitere Reinigung des Liganden, da stickstoffreiche Verbindungen dieses Typs keine definierten Spots auf DC-Platten ergeben, sondern über einen großen Bereich verschmieren. Weiterhin unterscheidet sich die Reinigung des Hydrochlorids $\mathbf{4 b}$ von der des Hydrochlorids $\mathbf{4 a}$. So ist eine direkte Fällung von $\mathbf{4 b}$ mit Diethylether aus ethanolischer $\mathrm{HCl}$ nicht möglich. In diesem Fall bildet sich ein zähes dunkelbraunes Öl, das sich dann im Hochvakuum zu einem dunkelbraunen Feststoff trocknen lässt. Nimmt man das entsprechende Rohprodukt in Chloroform auf und tropft diese Lösung in Diethylether, so entsteht das Hydrochlorid als weißer Feststoff. Die anschließende Neutralisation des Hydrochlorids zum Liganden $\mathrm{HL}^{2}$ (5b) lässt sich mit Natriumcarbonat in Acetonitril nur in mäßigen Ausbeuten durchführen. Die Ausbeute lässt sich jedoch bei Neutralisation in ammoniakalischer Dichlormethanlösung auf $65 \%$ steigern. 
Damit der Ligand $\mathrm{HL}^{3}$ (5c) hergestellt werden kann, muss zunächst 1-Methyl-4,5diphenylimidazol in einer Zweistufenreaktion dargestellt werden. ${ }^{[14]}$ Wie in Schema 15 ersichtlich ist, werden im ersten Schritt Benzoin und Formamid bei $170^{\circ} \mathrm{C}$ zur Reaktion gebracht. Nach Aufarbeitung des Rohproduktes wird 4,5-Diphenylimidazol in einer Ausbeute von $97 \%$ erhalten. Anschließend wird mittels Natriumhydrid das azide $\mathrm{NH}$-Proton entfernt und unter Eiskühlung durch Methyliodid in einer exothermen Reaktion das Stickstoffatom methyliert. Das Imidazol kann so in einer Ausbeute von $78 \%$ erhalten werden.

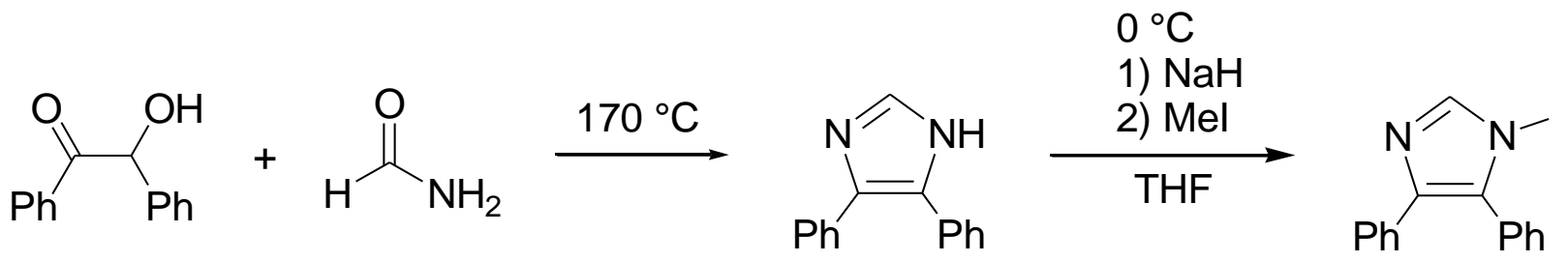

Schema 15: Synthese von 1-Methyl-4,5-diphenylimidazol.

Der Ligand $\mathrm{HL}^{3}$ (5c) entsteht, wenn man ausgehend vom THP-geschützten Pyrazol-3,5dicarbonsäuremethylester (1) die oben beschriebenen Reaktionen unter Verwendung von 1-Methyl-4,5-diphenylimidazol durchführt. Das entstehende Produkt konnte analysenrein erhalten werden. Trotzdem ist es nicht möglich, eine genaue Auswertung der ${ }^{13} \mathrm{C}$-NMR-Spektren vorzunehmen, da die Verbindung 2c viele Signale im Aromatenbereich enthält. Es ist lediglich durch Aufnahme von dept45-Spektren möglich, quartäre Kohlenstoffatome von $\mathrm{CH}$-Gruppen zu unterscheiden. Es lassen sich nur 18 anstelle der erwarteten 22 Signale für quartäre aromatische C-Atome und 18 Signale für aromatische $\mathrm{CH}$-Gruppen statt der erwarteten 24 Signale identifizieren. Die Ursache dafür liegt in dem geringen Unterschied der chemischen Verschiebungen aller Phenylund Imidazol-Signale. Aufgrund der Ähnlichkeit überlagern sich einige der Signale und sind somit nicht voneinander zu unterscheiden. Wie auch schon beim Hydrochlorid des Liganden $\mathrm{HL}^{2}$ lässt sich $4 \mathrm{c}$ ebenso nicht direkt aus der ethanolischen $\mathrm{HCl}$ mit Diethylether ausfällen. Will man das Hydrochlorid $\mathbf{4 c}$ als weißen Feststoff erhalten, ist ein Ausfällen mit Diethylether aus der entsprechenden Chloroform-Lösung notwendig. Die Elementaranalyse dieser Verbindung zeigt einen hohen Chlorid-Gehalt. Dieser Wert lässt den Schluss zu, dass $4.9 \mathrm{HCl}$-Moleküle zusätzlich an Verbindung $\mathbf{4 c}$ gebunden sind. Das gleiche Phänomen kann bei allen in dieser Arbeit synthetisierten 
Hydrochloriden beobachtet werden. Daraus kann man ableiten, dass neben dem Pyrazol auch alle Imidazolringe protoniert vorliegen. Diese Tatsache lässt sich ebenso mit den gewonnenen ${ }^{1} \mathrm{H}$-NMR-Daten belegen. Darin findet man ein sehr breites Signal bei variabler chemischer Verschiebung mit einer Intensität von 6 bis 7 , dass den $\mathrm{NH}$ Protonen zuzuordnen ist. Der Ligand $\mathrm{HL}^{3}$ (5c) lässt sich nach Neutralisation des Hydrochlorides 4c ebenfalls durch Ausfällen aus einer Dichlormethanlösung mit $n$ Hexan als weißgrauer Feststoff erhalten. Auch diese Verbindung lässt sich damit analysenrein herstellen. Obwohl diese Verbindung $C_{2}$-symmetrisch ist und viele Kohlenstoffatome äquivalent werden, ist es nicht möglich, eine exakte Zuordnung für

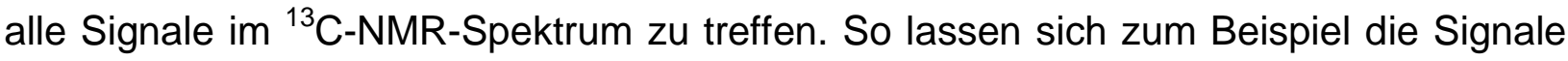
für alle quartären C-Atome der Imidazol- und Phenylringe nicht zuordnen, da es zu einer Überlagerung mit anderen Signalen kommt. 


\subsection{Darstellung von $\mathrm{HL}^{4}, \mathrm{HL}^{5}$ und $\mathrm{HL}^{6}$}

Eine Reihe weiterer Liganden lassen sich nach dem in Schema 16 gezeigten Syntheseplan darstellen. Wenn man auf die Etherbildung verzichtet und die THP-Schutzgruppe auf der Stufe der Alkoholverbindung 2a entfernt, entsteht zunächst das Hydrochlorid 6a. Diese Verbindung lässt sich nach Entfernen der Schutzgruppe mit ethanolischer $\mathrm{HCl}$ und anschließender Fällung mit Diethylether analysenrein gewinnen. Die sich daran anschließende Neutralisation zum Ligand $\mathrm{zu} \mathrm{HL}^{4}(\mathbf{7 a})$ ist aufgrund der hohen Polarität dieser Substanz mit einigen Schwierigkeiten verbunden. Im Gegensatz zu HL ${ }^{1}$ enthält Verbindung 7a anstelle der beiden Methoxyfunktionen jeweils eine Hydroxylgruppe. Vor allem bei der Neutralisation des Hydrochlorides 6a macht sich dies bemerkbar. Führt man diesen letzten Schritt in wässriger Lösung aus, ist es nicht<smiles>[R]c1ncn(C)c1[R]</smiles>

1

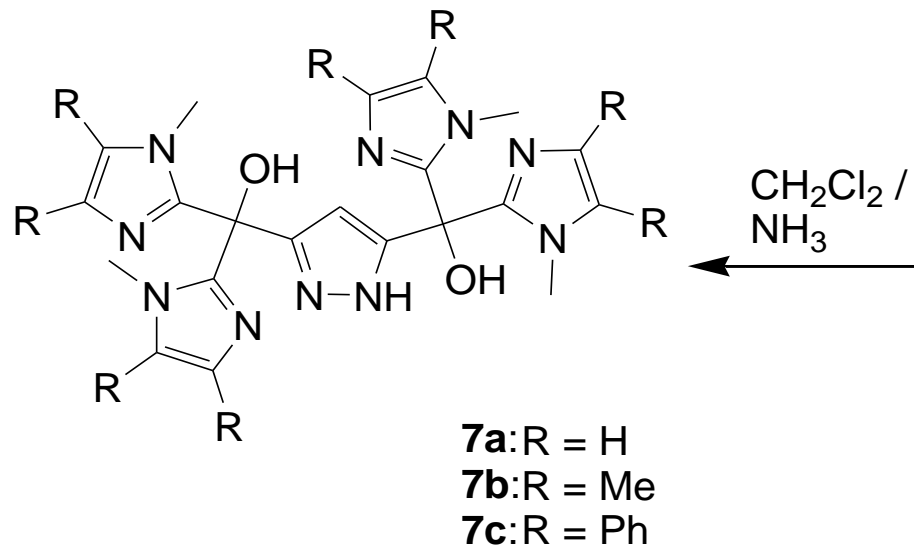

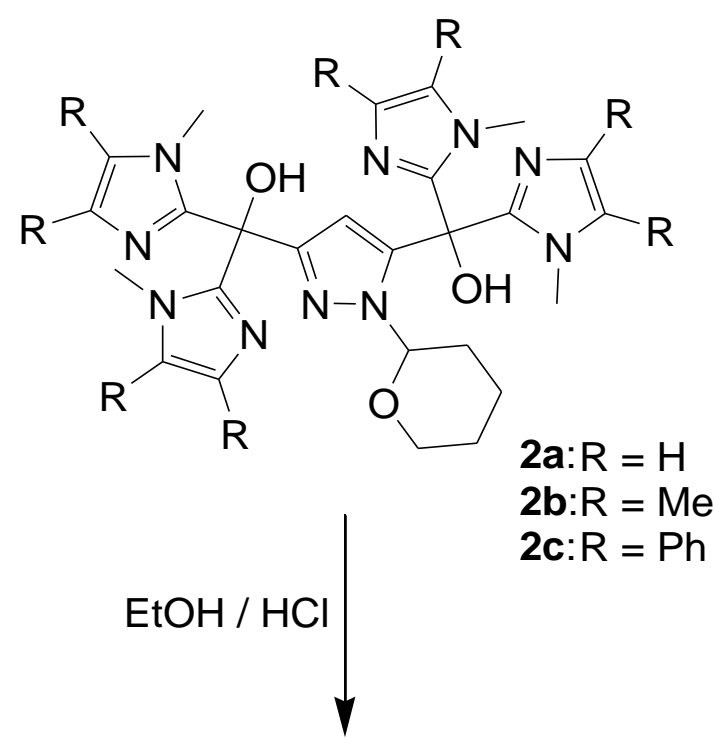<smiles>[R]c1nc(C(O)(c2cc(C(O)(c3nc([R])c([R])n3C)c3nc([R])c([R])n3C)[nH]n2)c2nc([R])c([R])n2C)n(C)c1[R]</smiles>

Schema 16: Darstellung der Liganden $\mathrm{HL}^{4}(7 \mathbf{a}), \mathrm{HL}^{5}(\mathbf{7 b})$ und $\mathrm{HL}^{6}(\mathbf{7 c})$. 
möglich das Produkt mit organischen Lösungsmitteln wie Diethylether, Chloroform oder Dichlormethan zu extrahieren. Aus diesem Grund wurde auch bei dieser Synthese die neuartige Variante der Neutralisation mittels ammoniakalischer Dichlormethanlösung durchgeführt. Trotz der Verbesserung der Reaktionsbedingungen dieser letzten Stufe beträgt die Ausbeute für beide Schritte der THP-Abspaltung $21 \%$. Auch wenn dies sehr wenig erscheint, ist das Ergebnis doch erheblich besser als es bei wässriger Neutralisation zu erzielen ist. Nimmt man von dem neutralisierten Produkt ein ${ }^{1} \mathrm{H}-\mathrm{NMR}$ Spektrum in $\mathrm{CDCl}_{3}$ auf, so erhält man das in Abbildung 18 oben gezeigte Ergebnis.
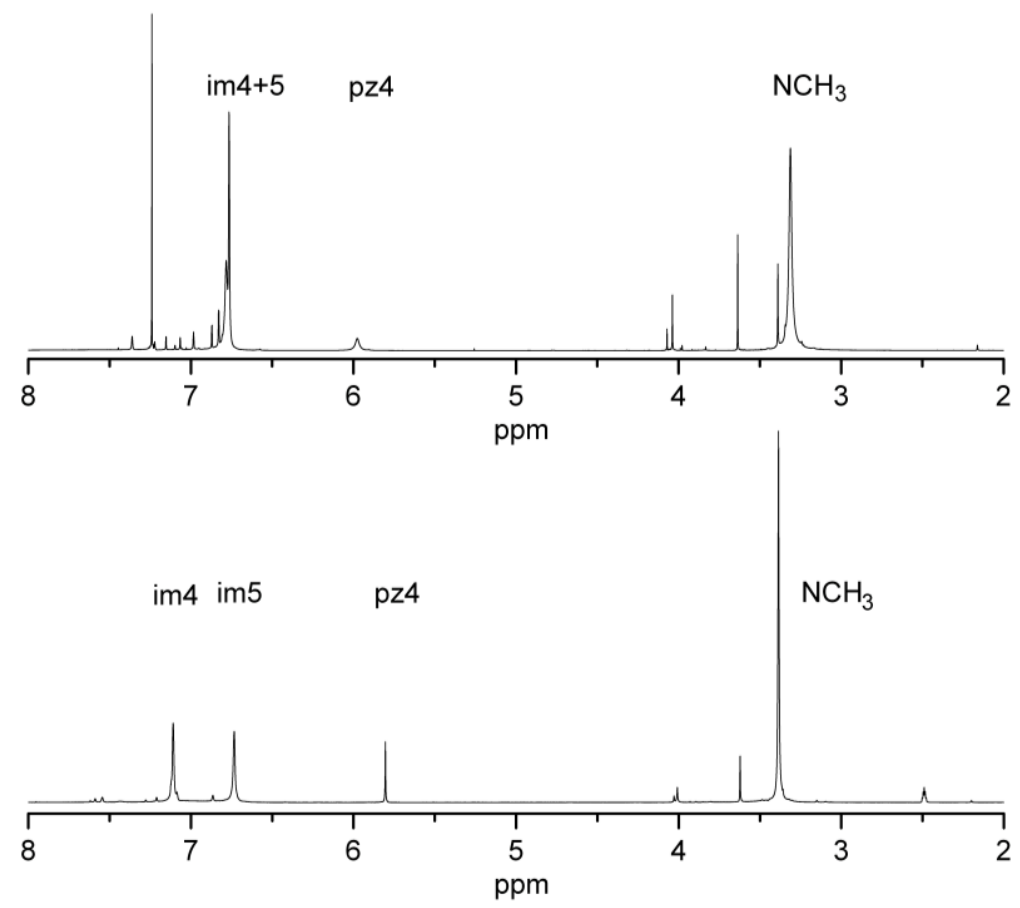

Abbildung 18: ${ }^{1} \mathrm{H}-\mathrm{NMR}$-Spektren von $\mathrm{HL}^{2}$ (7a) in $\mathrm{CDCl}_{3}$ (oben) und $\mathrm{d}_{6}$-DMSO (unten).

Für beide Imidazol-Rückgrat-Protonen erwartet man eigentlich zwei Singuletts, die gegebenenfalls in zwei Dupletts aufgespalten werden durch die Kopplung zum benachbarten H-Atom. Stattdessen wird ein verbreitertes Signal erhalten mit vielen kleinen Signalen im Bereich von $6.80 \mathrm{ppm}$ bis $7.20 \mathrm{ppm}$. Außerdem ist das Signal für das $\mathrm{H}$-Atom in 4-Position des Pyrazolrings sehr breit und das $\mathrm{NH}$-Proton liefert ebenfalls ein sehr breites Signal zwischen $11.50 \mathrm{ppm}$ und $14.00 \mathrm{ppm}$. Aus diesem Grund wurde die gleiche Substanz in $d_{6}$-DMSO vermessen (Abbildung 18 unten). Dabei ergaben sich für die Imidazol-Protonen die erwarteten Singuletts bei 6.73 ppm und $7.11 \mathrm{ppm}$. Auch das H-Atom in 4-Position des Pyrazolrings ergibt ein scharfes Singulett bei $5.80 \mathrm{ppm}$. Ebenso wird auch das Signal für das NH-Proton zwischen $12.00 \mathrm{ppm}$ und 
13.00 ppm deutlich schärfer. Das Signal für beide $\mathrm{OH}$-Protonen lässt sich aber auch in diesem Spektrum nicht erkennen.

Auch die Liganden $\mathrm{HL}^{5}$ (7b) und $\mathrm{HL}^{6}$ (7c) sind durch Abspaltung der THP-Schutzgruppe aus den entsprechenden Vorstufen $\mathbf{2 b}$ und $\mathbf{2 c}$ erhältlich (Schema 15). Ein großer Unterschied zur Synthese von $\mathrm{HL}^{2}$ ist das Ausfällen der Hydrochloride. Entschützt man die Verbindung $\mathbf{2 b}$ mit ethanolischer $\mathrm{HCl}$, ist es anschließend nicht möglich das Hydrochlorid $\mathbf{6 b}$ durch Versetzen mit Diethylether als Feststoff zu erhalten. In diesem Fall entsteht ein braunes Öl. Dieses Öl kann man zwar im Vakuum zu einem hellbraunen Feststoff trocknen, jedoch enthält die Substanz noch einige Verunreinigungen. Deshalb ist es besser, den Rückstand in Chloroform aufzunehmen und das Hydrochlorid mit einem großen Überschuss Diethylether auszufällen. Leider ist es trotzdem nicht möglich, die Verbindung $\mathbf{6} \mathbf{b}$ analysenrein darzustellen. Dies liegt vor allem an Resten von freiem Trimethylimidazol und der Tatsache, dass dieses kleine Molekül sehr hygroskopisch ist. Dadurch kann der Dialkohol $\mathbf{2 b}$ nicht imidazolfrei dargestellt werden - auch nicht durch nachträgliche Reinigung von $\mathbf{2 b}$. Die abschließende Neutralisation mit ammoniakalischer Dichlormethanlösung liefert den mit etwas Trimethylimidazol verunreinigten Liganden $\mathrm{HL}^{5}(\mathbf{7 b})$ mit einer Ausbeute von $58 \%$.

Das Vorgehen zur Darstellung von $\mathrm{HL}^{6}$ (7c) ist identisch mit den bisher vorgestellten Synthesen. Nach Entfernen der THP-Schutzgruppe von Dialkohol 2c kann das Hydrochlorid $\mathbf{6 c}$ durch Fällung mit Diethylether aus der Methanollösung als hellgrauer Feststoff erhalten werden. Die Neutralisation zum Liganden $\mathrm{HL}^{6}(\mathbf{7 c})$ wird erneut mit ammoniakalischer Dichlormethanlösung durchgeführt. Es ist nicht möglich, alle quartären C-Atome im ${ }^{13} \mathrm{C}$-NMR-Spektrum zweifelsfrei zuzuordnen. Außerdem konnte das erwartete breite Signal für beide C-Atome in 3- und 5-Position des Pyrazolrings nicht identifiziert werden. Dennoch können beide Substanzen analysenrein mit einer Ausbeute von $45 \%$ über beide Stufen erhalten werden.

Alle sechs Liganden und deren Vorstufen (2a bis 7c) sind zudem durch Massenspektrometrie und IR-Spektroskopie, sowie teilweise durch Elementaranalyse charakterisiert. 


\section{Darstellung der Komplexe}

\subsection{Darstellung der Kupfer(II)-Komplexe}

Die in dieser Arbeit dargestellten Kupfer(II)-Komplexe wurden stets nach dem gleichen Schema synthetisiert. Der entsprechende Ligand wurde in einem Lösungsmittel (in der Regel Methanol) gelöst und mit zwei Äquivalenten $\mathrm{KO}^{\mathrm{t}} \mathrm{Bu}$ versetzt. Das erste Äquivalent der Base ist notwendig, um das acide NH-Proton der zentralen Pyrazoleinheit zu entfernen. Der deprotonierte Ligand wurde im Anschluss mit zwei Äquivalenten des entsprechenden Kupfer(II)-salzes versetzt. Das in der Lösung vorhandene zweite Äquivalent der Base soll zur Deprotonierung eines Wassermoleküls (aus dem Kristallwasser des Kupfersalzes) dienen, um ein Hydroxid-Ion zu generieren, das die Kupferionen verbrücken kann.

Die gebildeten Komplexe besitzen nur dann ausreichende Reinheit für die Durchführung von Analysen, wenn sie zuvor kristallisiert worden sind. Bei der Synthese bildet sich neben dem entsprechenden Kupferkomplex auch das Kaliumsalz des eingesetzten Anions. In der Regel ist es möglich, das weiße Kaliumsalz von der blaugefärbten Komplexlösung durch Filtration abzutrennen. Die daraus resultierende klare Lösung wird mit einem zweiten Lösungsmittel überschichtet. Dieses zweite Solvens besitzt schlechte Lösungseigenschaften und diffundiert langsam in die gefärbte Komplexlösung. Will man diesen Prozess noch verlangsamen, kann die Kristallbildung auch durch Dampfdiffusion erfolgen. Dabei sollte jedoch das zweite Solvens nicht nur schlechte Lösungseigenschaften besitzen, sondern zusätzlich einen hohen Dampfdruck aufweisen. 
Setzt man den Liganden $\mathrm{HL}^{1}$ (5a) mit diversen Kupfer(II)-Salzen um, so erhält man eine Vielzahl verschiedener Komplexstrukturen. Bei Verwendung von Kupfer(II)-acetat Hydrat entsteht der zweikernige Komplex 8 als violette nadelförmige Kristalle. Wie durch die Stöchiometrie der Reaktion gewünscht, sind zwei Kupferionen an einen Liganden koordiniert. Zwischen beiden Metallatomen bildet sich eine $\mathrm{OH}$-Brücke mit einem $\mathrm{Cu}-\mathrm{O}-\mathrm{Cu}-$ Winkel von $124.89(9)^{\circ}$. Wie man in Abbildung 19 erkennen kann, ist ein Kupferzentrum quadratisch planar umgeben, während das Zweite sehr stark verzerrt quadratisch pyramidal koordiniert ist.

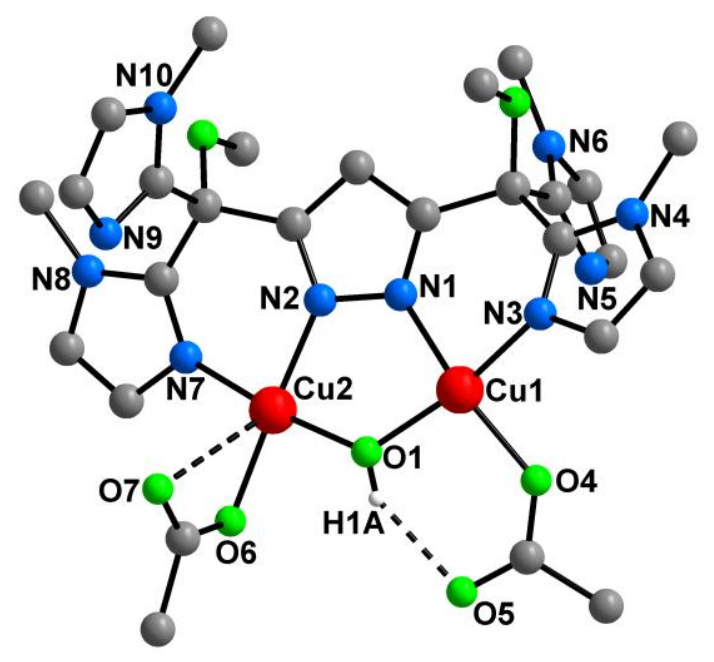

Abbildung 19: Festkörperstruktur von 8 (wegen der Übersichtlichkeit ist nur das $\mathrm{H}$-Atom der OH-Gruppe dargestellt).

Beide Kupferatome werden durch das Pyrazolat in einem Abstand von 3.3367(5) $\AA$ fixiert und sind von jeweils zwei Stickstoffatomen koordiniert. Dabei stammt ein N-Donor vom Pyrazol, den zweiten N-Donor stellt ein Imidazolring zur Verfügung. An jedes Kupferatom koordiniert außerdem das Sauerstoffatom der OH-Brücke. Nur die Koordination des Acetations ist unterschiedlich: Einmal koordiniert nur ein Sauerstoffatom an das Kupferion, das zweite Sauerstoffatom des an Cu1 gebundenen Acetations wird durch eine schwache Wasserstoffbrückenbindung zum Wasserstoffatom der OH-Brücke stabilisiert (2.751(3) ̊̊). Zum zweiten Kupferion binden beide O-Donoren des Acetations. Allerdings fällt auf, dass beide Cu2-O-Bindungen mit

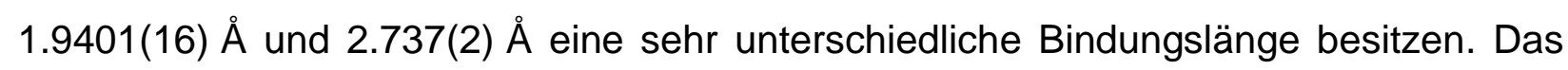
spricht für eine semi-bidentate Bindungsart des Acetat-Ions an Cu2. In Tabelle 1 sind einige wichtige Bindungswinkel und -abstände zusammengefasst. 


\begin{tabular}{|c|c|c|c|}
\hline \multicolumn{4}{|c|}{ Abstände $[\AA]]$} \\
\hline Cu1-N1 & $1.9467(19)$ & Cu2-N2 & $1.9597(18)$ \\
\hline Cu1-N3 & $1.9751(18)$ & Cu2-N7 & $1.978(2)$ \\
\hline Cu1-01 & $1.8726(16)$ & Cu2-O6 & $1.9401(16)$ \\
\hline Cu1-O4 & $1.9371(17)$ & Cu2-O1 & $1.8911(18)$ \\
\hline & & Cu2-O7 & $2.737(2)$ \\
\hline $\mathrm{O} 1-\mathrm{H} 1 \mathrm{~A} \cdots \mathrm{O}$ & $2.751(3)$ & Cu1 ${ }^{\cdots} \mathrm{Cu} 2$ & $3.3367(5)$ \\
\hline \multicolumn{4}{|c|}{ Winkel $\left[^{\circ}\right]$} \\
\hline O4-Cu1-N1 & $164.50(9)$ & O6-Cu2-N2 & $177.67(8)$ \\
\hline O1-Cu1-O4 & $98.21(7)$ & N2-Cu2-N7 & $87.81(8)$ \\
\hline O1-Cu1-N1 & $86.54(7)$ & O1-Cu2-O6 & $90.98(7)$ \\
\hline O1-Cu1-N3 & $165.58(9)$ & O1-Cu2-N2 & $86.84(8)$ \\
\hline O4-Cu1-N3 & $89.63(7)$ & O1-Cu2-N7 & 172.04(8) \\
\hline N1-Cu1-N3 & $89.06(8)$ & O6-Cu2-N7 & $94.28(8)$ \\
\hline Cu1-O1-Cu2 & $124.89(9)$ & & \\
\hline
\end{tabular}

Tabelle 1: Ausgewählte Bindungslängen (in $\AA$ ) und Bindungswinkel (in ${ }^{\circ}$ ) für 8.

Desweiteren kann man in der obigen Struktur deutlich erkennen, dass ein Imidazolring jeder Ligandhälfte von den Kupferionen weggedreht ist und somit nicht an der Koordination beteiligt ist. Diese Tatsache liegt im Design des Liganden begründet. Aufgrund des zentralen, verbrückenden Kohlenstoffatoms neben dem Pyrazol wird eine Bindungstasche gebildet, die eine facial tridentate Koordinationsumgebung um ein Metallion vorgibt. Das Kupfer(II)-Ion bevorzugt jedoch tetragonale Koordinationsgeometrien.

Das UV/Vis-Spektrum dieses Komplexes zeigt drei markante Absorptionen bei $559 \mathrm{~nm}$ $\left(\varepsilon=120 \mathrm{~mol}^{-1} \cdot \mathrm{l} \mathrm{cm}^{-1}\right), 332 \mathrm{~nm}\left(\varepsilon=2860 \mathrm{~mol}^{-1} \cdot \mathrm{lcm}^{-1}\right)$ und $230 \mathrm{~nm}\left(\varepsilon=36000 \mathrm{~mol}^{-1} \mid \mathrm{cm}^{-1}\right)$. 
Diese Werte sind charakteristisch für Kupfer(II)-Komplexe, in denen die Kupfer-Ionen quadratisch planar bzw. quadratisch pyramidal koordiniert sind.

Dagegen ist das erhaltene Massenspektrum nicht so aussagekräftig. Dabei spielt es keine entscheidende Rolle, mit welcher Methode das Massenspektrum aufgenommen wurde. So zeigt das $\mathrm{FAB}^{+}$-Spektrum in den verschiedensten Matrizes eine Vielzahl von Signalen bis zu $\mathrm{m} / \mathrm{z}=1383.8$, was nicht allein von dem dinuklearen Kupfer(II)-Komplex herrühren kann. Da jedoch ein Einkristall vermessen wurde, müssen diese Signale durch Reaktionen unter den Messbedingungen verursacht worden sein. Eine Zuordnung kann nicht für alle Peaks getroffen werden. Das Fragment mit einer Masse von 1141.1 entspricht der Zusammensetzung $\left[\mathrm{L}_{2}^{1} \mathrm{Cu}_{3}\right]^{+}$. Aber auch eindeutige Bestandteile, die den Komplex 8 repräsentieren sind zu finden, wie z. B. $m / z=719.0$ für $\left[\mathrm{L}^{1} \mathrm{Cu}_{2}(\mathrm{OAc})_{2}\right]^{+}$oder auch $\mathrm{m} / \mathrm{z}=659.9$ für $\left[\mathrm{L}^{1} \mathrm{Cu}_{2}(\mathrm{OAc})\right]^{+}$.

Der Komplex 8 kann nur in reiner Form erhalten werden, wenn das Rohprodukt mittels Sephadex-lonenaustauscher gereinigt wird. Ansonsten erhält man einen weiteren türkisfarbenen, acetathaltigen Komplex. Beim Versuch, Komplex 8 durch wiederholtes Umkristallisieren aus Dichlormethan/Hexan zu reinigen, konnte eine Umwandlung des zweikernigen Komplexes 8 in die vierkernige Koordinationsverbindung 9 beobachtet werden. Je öfter diese Prozedur erfolgte und die türkisfarbenen Kristalle von den violetten Nadeln abgetrennt wurden, desto mehr bildete sich vom vierkernigen Kupfer(II)-Komplex $\mathbf{9}$.

Die röntgenographische Strukturanalyse der türkisfarbenen Kristalle von $\mathbf{9}$ ergab, dass sich zwei Äquivalente des Komplexes 8 unter Abspaltung eines Moleküls Wasser zusammengelagert haben und die in Abbildung 20 gezeigte Festkörperstruktur von 9 ergaben. Dabei muss angemerkt werden, dass die Qualität der Kristalle und somit auch die Qualität der erhaltenen Strukturdaten für exakte Vergleiche mit anderen Komplexen unzureichend ist.

Es entsteht ein $\mathrm{Cu}_{4} \mathrm{O}-\mathrm{Kern}$, in dem alle vier Metallionen tetraedrisch um das zentrale Sauerstoffatom gebunden sind. Alle vier Kupferatome sind quadratisch pyramidal umgeben. 


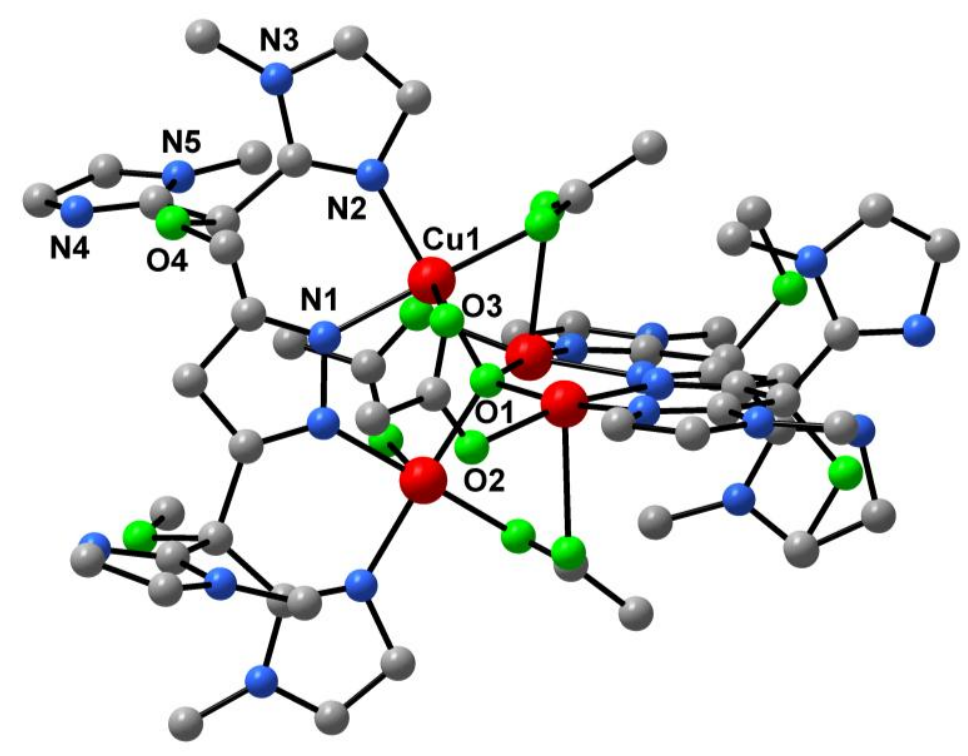

Abbildung 20: Festkörperstruktur von 9 (zur besseren der Übersichtlichkeit sind keine H-Atome dargestellt).

Die Koordinationsgeometrie für fünffach koordinierte Metallzentren kann durch den Parameter $\tau$ quantifiziert werden. Er ergibt sich aus der folgenden Gleichung: $\tau=(\beta-\alpha) / 60^{\circ}$. $\alpha$ und $\beta$ sind die beiden größten Winkel an den fünffach koordinierten Metallzentren, die von den Donoren eingeschlossen werden. Es gilt: $\beta>\alpha$. Für eine ideale trigonale Bipyramide ergibt sich damit der Wert $\tau=\left(180^{\circ}-120^{\circ}\right) / 60^{\circ}=1$ und für eine ideale quadratische Pyramide $\tau=\left(180^{\circ}-180^{\circ}\right) / 60^{\circ}=0 .{ }^{[115]}$

Anhand der Bindungswinkel, die sich aus den kristallographischen Daten ermitteln lassen, kann ein $\tau$-Wert von 0.05 für alle äquivalenten Kupferzentren in $\mathbf{9}$ errechnet werden. Daraus lässt sich schließen, dass es sich hier um eine nahezu perfekte quadratisch-pyramidale Koordinationsumgebung handelt.

Die Basis der quadratischen Pyramide wird von zwei Stickstoffatomen und zwei Sauerstoffatomen gebildet. Der eine N-Donor gehört zum Pyrazolkern und der andere zu einem Imidazolring. Bei den Sauerstoffatomen stammt eins vom zentralen Baustein des Komplexes und der zweite O-Donor ist ein Teil des Acetations. Das zweite Sauerstoffatom des Acetations besetzt die axiale Koordinationsstelle am Kupfer-lon. Aufgrund der tetraedrischen Anordnung um das zentrale Sauerstoffatom stehen beide Liganden senkrecht zueinander. Auch in diesem Komplex ist jeweils nur ein 
Imidazolring an das Kupferion gebunden. Der zweite Imidazolring jeder Ligandseite ist weggedreht und steht nicht für die Koordination zur Verfügung.

Um die vierkernige Struktur 9 gezielt herzustellen, gibt es zahlreiche Varianten. Formal muss ein Äquivalent Wasser aus zwei Molekülen 8 entfernt werden. Dafür sind in der organischen Chemie einige Verfahren entwickelt worden. Zum Einen ist es möglich, die Edukte mit Orthoestern zur Reaktion zu bringen. Dabei entsteht nach Abspaltung von Wasser der entsprechende Alkohol und der dazugehörige Carbonsäureester. Zur Verdeutlichung ist in Schema 17 die Reaktion von Trimethylorthoacetat mit Wasser gezeigt. Als Produkte entstehen bei dieser Hydrolyse Methanol und Essigsäuremethylester. Da die Reaktion in Methanol abläuft, ist die Bildung von Methanol nicht störend. Der Ester lässt sich aufgrund seines niedrigen Siedepunktes von ca. $56{ }^{\circ} \mathrm{C}$ ebenfalls relativ einfach durch Destillation aus dem Reaktionsgemisch entfernen.

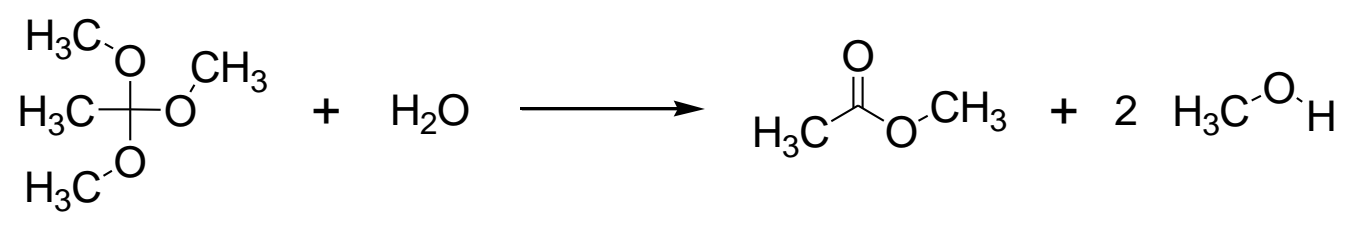

Schema 17: Hydrolyse von Orthoestern.

Wenn man den Komplex 8 mit Trimethylorthoacetat zur Reaktion bringt und das Reaktionsgemisch mehrere Stunden unter Rückfluss sieden lässt, kommt es jedoch nicht zu einer Farbänderung. Die Lösung bleibt violett gefärbt. Daraus lässt sich schlussfolgern, dass keine Reaktion zu Komplexverbindung 9 stattgefunden hat.

Wenn man davon ausgeht, dass für die Kondensation zweier Komplexe $8 \mathrm{zu}$ Verbindung 9 die $\mathrm{OH}$-Brücke eines Komplexes 8 protoniert oder deprotoniert werden muss, um eine aktive Spezies zu erzeugen, besteht außerdem die Möglichkeit, den Komplex 8 mit Säuren oder Basen zur Reaktion zu bringen. Die zwischenzeitlich generierte aktive Spezies kann in einem weiteren Schritt die Kondensation mit dem zweiten Molekül 8 eingehen und somit auch die Abspaltung von Wasser ermöglichen. Dabei bieten sich vor allem Essigsäure und acetathaltige Salze an, da so die Bildung von Komplexen mit anderen Gegenionen oder Brücken ausgeschlossen werden kann. Wenn man die Koordinationsverbindung 8 in Dichlormethan löst und anschließend 
wässrige Natriumacetatlösung zugibt, entfärbt sich die organische Phase, da sich Verbindung 8 viel besser in Wasser löst. Das gleiche Ergebnis erhält man, wenn man wässrige Essigsäurelösungen zur Dichlormethanlösung von $\mathbf{8}$ zusetzt. In beiden Fällen wechselt der Kupfer-Komplex aus der organischen Phase in die wässrige Phase. Es findet jedoch keine Reaktion zu 9 statt.

Ein oft genutztes Mittel, um Gleichgewichts-Reaktionen zu beeinflussen, die Wasser als ein Produkt ergeben, sind Wasserabscheider. Hierfür werden Lösungsmittel benötigt, die sich mit Wasser bei Raumtemperatur nicht mischen, aber ein Azeotrop mit Wasser bilden. Idealerweise sollte der Komplex $\mathbf{8}$ gut in diesem Lösungsmittel löslich sein. Ein Blick in die Literatur zeigt, dass Chloroform all diese Eigenschaften besitzt. Als Säure wird bei solchen Wasserabscheidungsreaktionen standardmäßig Trifluoressigsäure (TFA) oder $p$-Toluolsulfonsäure ( $p$-TSA) eingesetzt. Allerdings hätten beide Säuren den Nachteil, dass sich eventuell Produkte mit anderen Gegenionen oder Brücken bilden können. Ein weiterer Nachteil von $p$-TSA wäre, dass es als Feststoff vorliegt und es somit ziemlich schwierig ist, eine kleine Menge genau zu dosieren. Dieses Problem könnte mit dem Anfertigen einer Stammlösung behoben werden. Jedoch fällt dabei Wasser als Lösungsmittel aus den oben beschriebenen Gründen aus.

Bringt man den in Chloroform gelösten Komplex 8 mit etwas TFA zur Reaktion, kann man weder eine Bildung von Wasser erkennen, die sich im Wasserabscheider über der organischen Phase bildet, noch lässt sich eine Farbänderung des Reaktionsgemisches erkennen. Daraus lässt sich schließen, dass $\mathrm{CHCl}_{3}$ für diese Reaktion ein ungeeignetes Lösungsmittel ist. Üblicherweise werden Wasserabscheidereaktionen in hochsiedenden Lösungsmitteln wie Toluol, Xylol oder höheren Kohlenwasserstoffen durchgeführt. Lösungsmittel wie Xylol oder Chlorbenzol lassen sich aber oftmals nicht restlos aus dem Reaktionsgemisch entfernen. Ein weiteres Argument gegen solch hochsiedende Lösungsmittel ist die mangelnde Löslichkeit des Komplexes 8 in Xylol oder anderen aromatischen Kohlenwasserstoffen. Aus diesem Grund muss man die Verbindung 8 in Toluol suspendieren, ehe TFA zugegeben und zum Rückfluss erhitzt wird. Bereits nach wenigen Augenblicken des Siedens lässt sich die Bildung eines Wassertropfens im Wasserabscheider beobachten. Ein weiteres Indiz für eine erfolgte Reaktion ist, dass die Färbung der Suspension von violett nach türkisfarben übergeht. Damit beim 
anschließenden Kristallisieren des Produktes nicht das Acetat-Ion gegen Trifluoracetat ausgetauscht wird, führt man diese Reaktion ganz analog mit Eisessig anstelle von TFA unter identischen Reaktionsbedingungen durch. Auch in diesem Fall lässt sich die Bildung eines Wassertropfens beobachten. Sowohl Komplex 8 als auch Verbindung 9 sind in Toluol absolut unlöslich. Jedoch ist deutlich erkennbar, dass die Färbung der Suspension sich von violett nach türkisfarben verändert. Abschließend kann die abgekühlte Lösung filtriert, der türkisfarbene Feststoff im Hochvakuum getrocknet und dann aus Dichlormethan/Diethylether oder Dichlormethan/ $n$-Hexan zur Kristallisation gebracht werden.

Anhand der gewonnenen Erkenntnisse, dass die Reaktion säurekatalytisch abläuft, lässt sich folgender Reaktionsmechanismus für die Bildung der $\mathrm{Cu}_{4} \mathrm{O}$-Einheit postulieren (Schema 18).

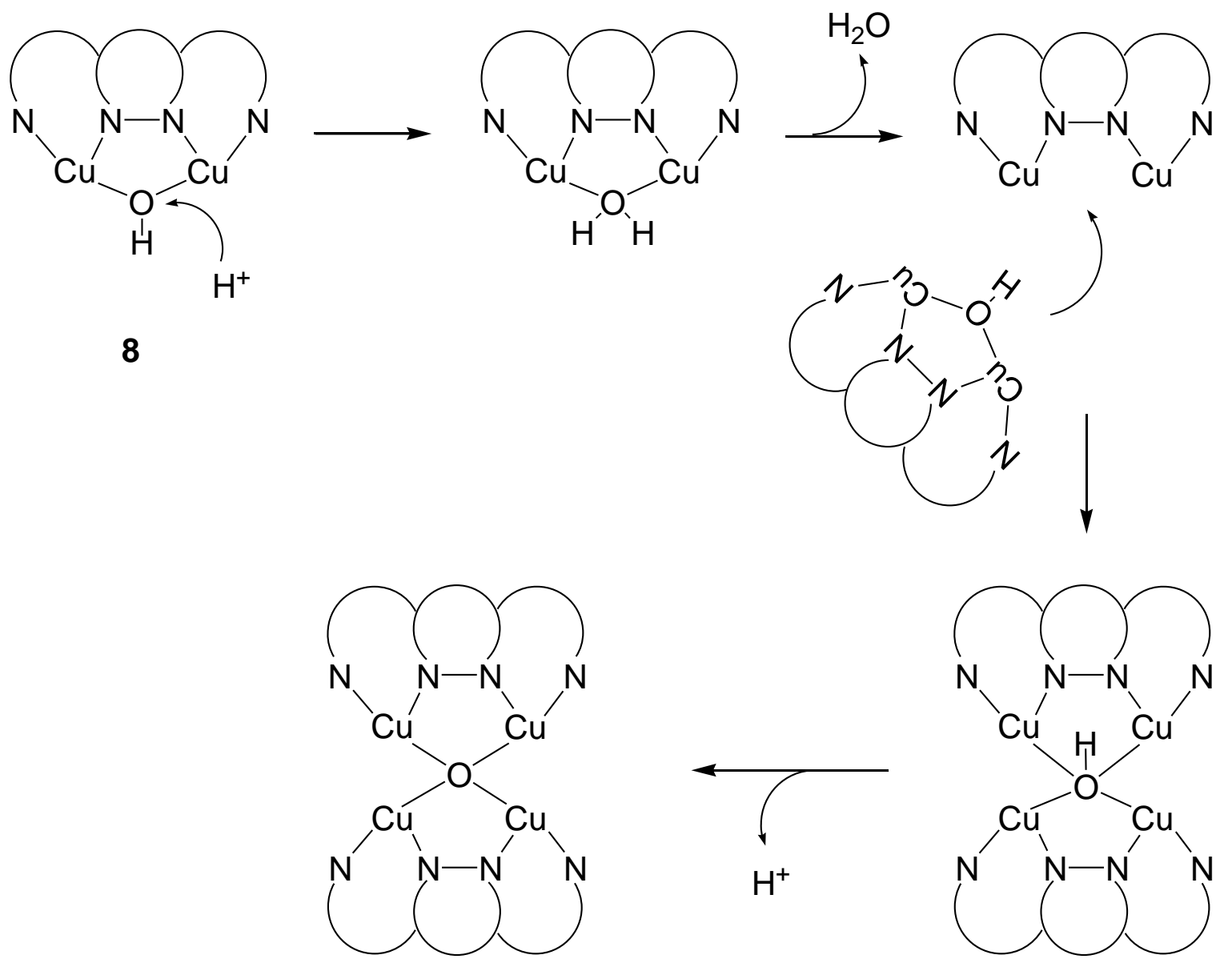

Schema 18: Postulierter, schematischer Ablauf der Komplexkupplung von 8 zu 9 (Ladungen und nichtkoordinierte Imidazolringe sowie Acetat-Coliganden werden aus Gründen der Übersichtlichkeit nicht dargestellt). 
Zuerst kommt es zu einer Protonierung der OH-Brücke im zweikernigen Kupferkomplex 8. Das daraus gebildete Wassermolekül kann danach leicht den Komplex verlassen. Die nun entstandene freie Koordinationsstelle wird durch den Einschub einer zweiten $[\mathrm{Cu}-(\mathrm{OH})-\mathrm{Cu}]$-Einheit gefüllt. Dabei orientiert sich das freie Elektronenpaar des Sauerstoffatoms in Richtung der "halbnackten“ Kupferionen. Nach Koordination kommt es abschließend zur Deprotonierung der $\mathrm{OH}$-Gruppe und es bildet sich das zentrale Oxidion, tetraedrisch koordiniert von vier Kupferionen in Komplex 9.

Außerdem gibt es noch die Möglichkeit, die Umwandlung von $\mathbf{8}$ in $\mathbf{9}$ basenkatalysiert durchzuführen. Wie oben bereits beschrieben entfällt dabei der Einsatz wässriger Lösungen, da sonst der Komplex aus der organischen Phase herausgelöst wird und keine Reaktion stattfinden kann. Bei der Bildung von Komplex 8 aus Ligand $\mathrm{HL}^{1}$, Kalium-tert-butanolat und Kupferacetat Hydrat in Methanol entsteht neben dem gewünschten Komplex auch Kaliumacetat. Ein Blick in ein Tabellenbuch zeigt, dass es sich hierbei um ein schwach basisches Salz handelt. ${ }^{[116]}$ Es liegt also nahe, dass man das tiefblau gefärbte Rohprodukt in Toluol suspendiert und anschließend mit Wasserabscheider zum Rückfluss sieden lässt. Auch hier kann bereits nach kurzer Zeit die Bildung eines Wassertropfens registriert werden. Außerdem verändert sich auch hier die Farbe von dunkelblau violett nach türkis.

Eine Alternative für die basenkatalysierte Reaktionsdurchführung ist die Verwendung von Natriumhydroxid. Dafür wurde das Lösungsmittel von der Reaktionslösung entfernt, in Dichlormethan erneut gelöst und filtriert. Nachdem das Lösungsmittel wieder entfernt wurde, erhielt man einen dunkelblauen Feststoff von Komplex 8. Dieser konnte zusammen mit fein gemörsertem Natriumhydroxid in Toluol suspendiert werden. Da sich bereits nach kurzer Zeit des Siedens zum Rückfluss ein Wassertropfen abgesetzt und sich die Farbe der Suspension von dunkelblau nach türkis verändert hatte, kann ebenfalls eine erfolgte Reaktion festgestellt werden. Die Suspension lässt sich filtrieren und nach Trocknen im Hochvakuum wird das türkisfarbene Rohprodukt in Dichlormethan gelöst und mittels $n$-Hexan zum Kristallisieren gebracht. 
Die in Dichlormethan gemessenen UV/Vis-Spektren der acetathaltigen Komplexe 8 und 9 einen signifikanten Unterschied. Die Bande des d-d-Überganges ist für 8 bei $561 \mathrm{~nm}$ zu finden. In Komplex 9 verschiebt sich dieses Signal zu 596 nm (Abbildung 21).
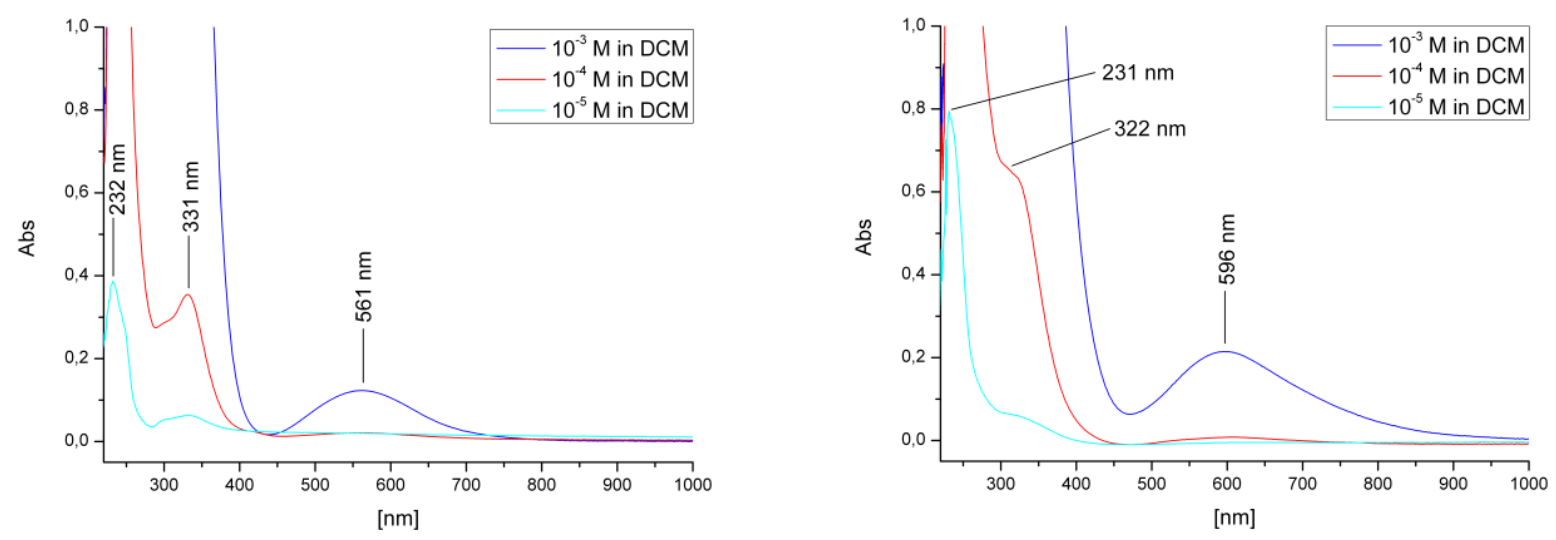

Abbildung 21: Vergleich der UV/Vis-Spektren von 8 (links) und 9 (rechts).

Der stufenförmige tetranukleare Kupfer(II)-Komplex $\mathbf{1 0}$ entsteht, wenn man den deprotonierten Liganden $\mathrm{HL}^{1}$ mit Kupfersulfat Pentahydrat in Methanol zur Reaktion bringt. Dabei handelt es sich um einen vierkernigen Kupfer(II)-Komplex mit einem Inversionszentrum (Abbildung 22). Auch in diesem Fall ist wieder ein Kupferion quadratisch planar koordiniert, da sich einer der äußeren Imidazolringe wegdreht und nicht für die Koordination am Metallzentrum zur Verfügung steht. Die vier Koordinationsstellen der äußeren Kupfer(II)-Ionen werden von zwei N-Donoren und zwei O-Donoren gebildet. Einen N-Donor stellt hierbei der Pyrazolring und den anderen Stickstoffdonor der Imidazolring zur Verfügung. Bei den zwei O-Donoren handelt es sich zum Einen um das Sauerstoffatom der $\mathrm{OH}$-Brücke und zum Anderen um ein Sauerstoffatom des Sulfat-lons. Die zwei inneren Kupferionen sind nahezu ideal quadratisch pyramidal koordiniert $(\tau=0.16)$. 


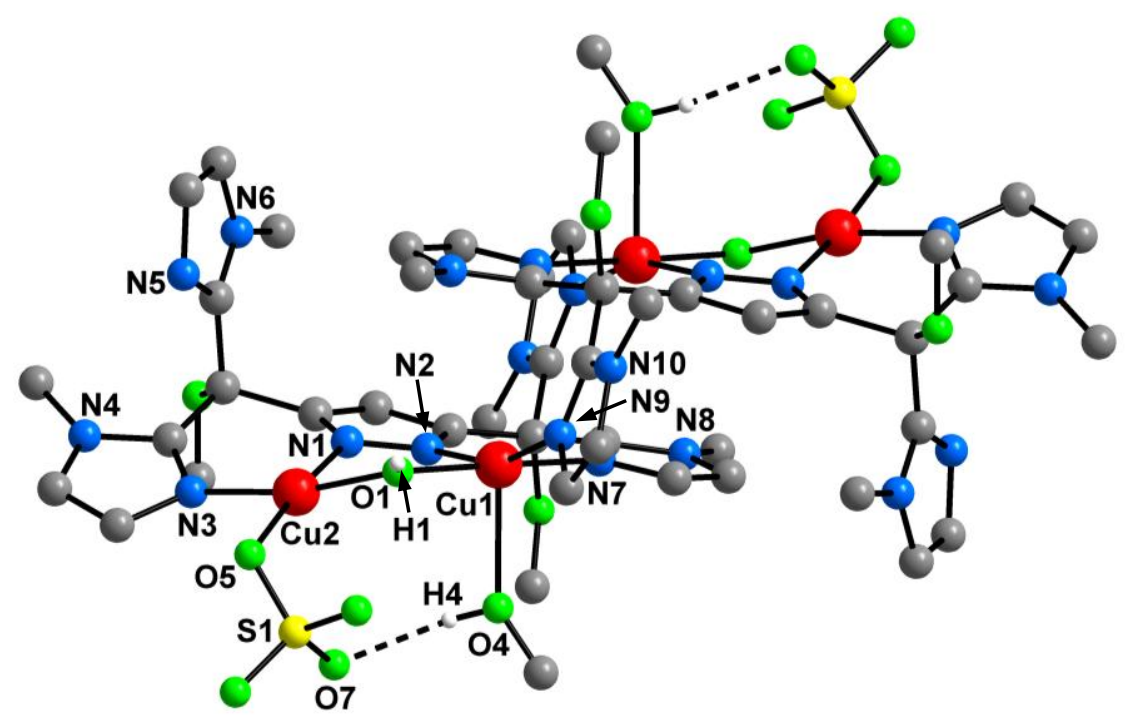

Abbildung 22: Festkörperstruktur von 10 (wegen der Übersichtlichkeit sind alle H-Atome außer den $\mathrm{OH}$-Protonen nicht abgebildet).

Methanol ist jeweils der axiale Ligand. Die Basis bilden ein O-Donor der OH-Brücke und drei N-Donoren: Der Pyrazolring bindet mit einem N-Atom an das Metallzentrum. Einen weiteren $\mathrm{N}$-Donor stellt der Imidazolring zur Verfügung, der an diesem Pyrazolring gebunden ist. Den dritten N-Donor stellt der weggeklappte Imidazolring des jeweils anderen Liganden bereit. Wie schon in Verbindung $\mathbf{8}$ binden die beiden Imidazolringe einer Ligandhälfte nicht an ein und dasselbe Kupferion.

In Tabelle 2 sind ausgewählte Bindungslängen und -winkel für Komplex 8 dargestellt. Darin ist zu sehen, dass die axiale Bindung zum Sauerstoffatom des Methanols wegen des Jahn-Teller-Effekts um ca. 0.4 ppm länger ist als die basalen Bindungen. Es handelt sich somit lediglich um eine schwache koordinative Bindung.

Abstände $[\AA ̊]$

\begin{tabular}{llll}
\hline Cu1-N1 & $1.935(4)$ & Cu2-N2 & $1.970(4)$ \\
Cu1-N3 & $1.966(4)$ & Cu2-N7 & $1.993(4)$ \\
Cu1-O1 & $1.892(3)$ & Cu2-N9' & $2.007(4)$ \\
Cu1-O5 & $1.936(3)$ & Cu2-O1 & $1.922(3)$ \\
O4-H4이 & $1.91(7)$ & Cu1 $\cdots$ Cu2 & $3.2945(8)$
\end{tabular}




\section{Winkel $\left[^{\circ}\right]$}

\begin{tabular}{llll}
\hline O5-Cu1-N1 & $167.78(17)$ & N9'-Cu2-N2 $^{\prime}$ & $165.42(15)$ \\
O1-Cu1-O5 & $95.72(14)$ & N2-Cu2-N7 & $88.40(15)$ \\
O1-Cu1-N1 & $85.65(14)$ & O1-Cu2-N9' & $89.18(14)$ \\
O1-Cu1-N3 & $167.47(16)$ & O1-Cu2-N2 & $86.42(14)$ \\
N1-Cu1-N3 & $89.52(15)$ & O1-Cu2-N7 & $174.81(15)$ \\
O5-Cu1-N3 & $91.44(15)$ & N7-Cu2-N9' & $95.92(15)$ \\
N1-Cu1-O5 & $167.78(17)$ & O1-Cu2-O4 & $86.60(14)$ \\
N2-Cu1-O4 & $97.32(15)$ & N7-Cu2-O4 & $93.79(14)$ \\
N9'-Cu2-O4 & $96.29(15)$ & Cu1-O1-Cu2 & $119.47(18)$ \\
\hline
\end{tabular}

Tabelle 2: Ausgewählte Bindungslängen (in $\AA$ ) und Bindungswinkel (in º für 10.

Im IR-Spektrum des Komplexes 10 lassen sich zahlreiche Banden identifizieren. Neben den üblichen Banden für aromatische und alkylische $\mathrm{C}$-H-Schwingungen zwischen $2933 \mathrm{~cm}^{-1}$ und $3128 \mathrm{~cm}^{-1}$ lässt sich auch die O-H-Schwingung bei $3400 \mathrm{~cm}^{-1}$ ausmachen. Das Massenspektrum zeigt mehrere Peaks, die sich den entsprechenden Fragmenten zuordnen lassen. Der gefundene Molpeak für $\left[\mathrm{L}_{2}^{1} \mathrm{Cu}_{4}(\mathrm{OH})\left(\mathrm{SO}_{4}\right)_{2}\right]^{+}$ist in Abbildung 23 dem berechneten Isotopenmuster für dieses Fragment gegenübergestellt.

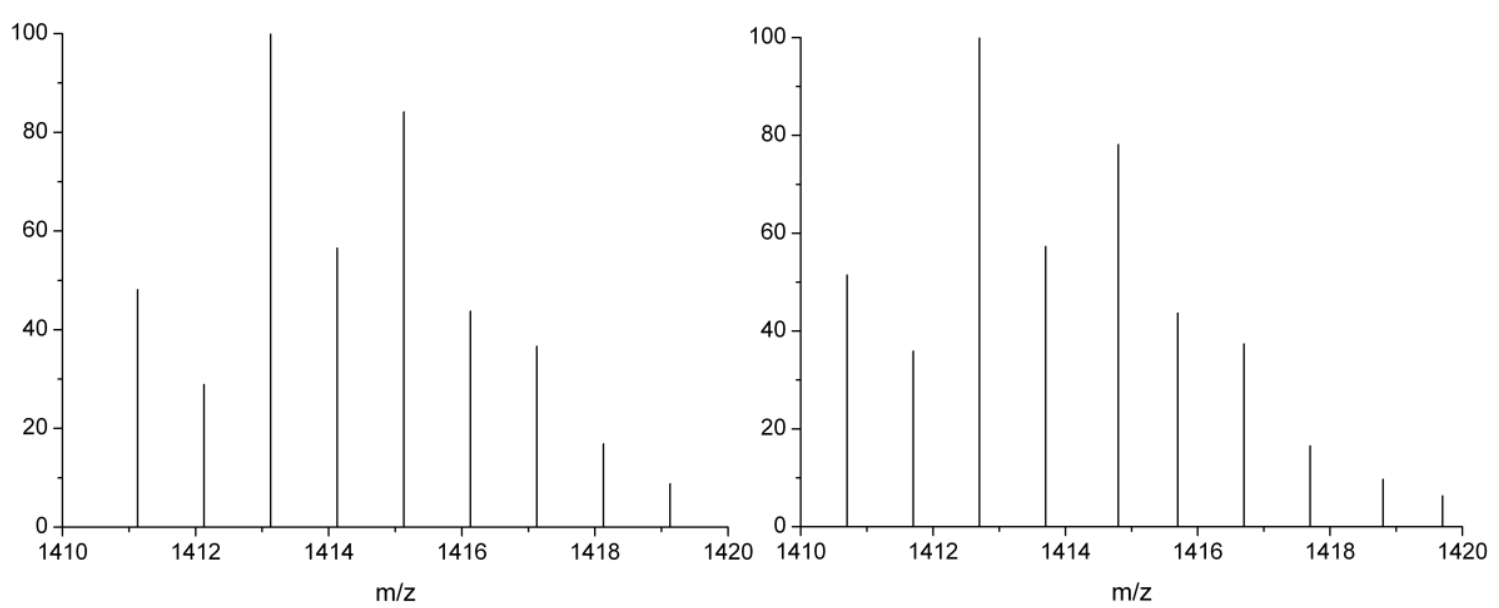

Abbildung 23: Molpeak für 10, gemessen (links) und berechnet (rechts). 
Die Elementaranalyse auch von mehrfach umkristallisierten Proben weist darauf hin, dass pro Komplexmolekül 7 bis 8 Moleküle Kristallwasser in der Verbindung enthalten sind. Das wiederspricht sich allerdings mit den Daten, die von der röntgenografischen Strukturaufklärung erhalten wurden. Möglicherweise verbrennt die Substanz nicht vollständig und liefert somit ungenaue Werte für die $\mathrm{CHN}$-Bestimmung.

Setzt man für die Komplexbildung Kupfer(II)-nitrat Trihydrat ein, bilden sich ebenfalls vierkernige Komplexe. Allerdings sind die Metallatome in den Verbindungen 11a (Abbildung 24) und 11b (Abbildung 25) nicht stufenförmig, sondern rechteckig angeordnet.

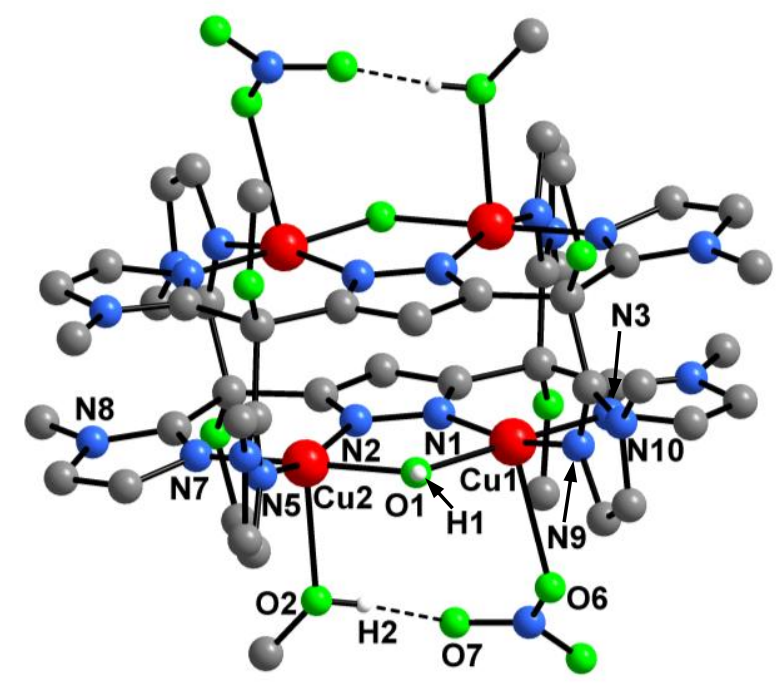

Abbildung 24: Festkörperstruktur des Dikations von 11a (wegen der Übersichtlichkeit sind nur H-Atome der OH-Gruppen abgebildet).

Alle vier Kupferzentren sind quadratisch pyramidal koordiniert. Für Verbindung 11a ergeben sich für die jeweils äquivalenten Kupferzentren $\tau$-Werte von 0.16 (Cu1) und 0.18 (Cu2). Wie aus Abbildung 24 ersichtlich ist, fungieren zweimal Methanol (Cu2) und zweimal das Nitrat-Ion (Cu1) als axiale Liganden. Allerdings sind alle vier Bindungen aufgrund der Jahn-Teller-Elongation relativ lang. So haben die Bindungen zum Sauerstoffatom des Methanols eine Länge von 2.312(4) $\AA$ und die Bindungen zum Sauerstoffatom des Nitrat-lons weisen sogar eine Länge von $2.485(5) \AA$ auf. In den bereits besprochenen Komplexverbindungen 8, 9 und 10 war auffällig, dass die benachbarten Imidazolringe nicht an ein Kupfer-Ion binden. Entweder steht ein 
Imidazolring weg und koordiniert überhaupt nicht, oder beide Imidazolringe koordinieren an verschiedene Kupferatome. Dieses Motiv tritt auch in diesem Komplex wieder auf. Knüpft man formal die freien Imidazolringe des Komplexes $\mathbf{1 0}$ an das Kupfer-lon, das am weitesten von dem jeweiligen Heterozyklus entfernt ist, entsteht eine rechteckige Struktur. Beide Liganden verschränken sich nicht nur in der Mitte des Komplexes, sondern auch an beiden Seiten. Zwischen einem Sauerstoffatom des Nitrat-Ions und dem Wasserstoffatom des Methanols bildet sich eine schwache Wasserstoffbrückenbindung aus $(2.787(6) \AA ̊)$. Die zwei pyrazolverbrückten Kupferatome sind in einem Abstand von 3.3918(9) $\AA$ voneinander separiert (Tabelle 3). Das UV/VisSpektrum einer methanolischen Lösung dieses Komplexes weist zwei charakteristische Banden bei $578 \mathrm{~nm}\left(\varepsilon=238 \mathrm{~mol}^{-1} \mid \mathrm{lcm}^{-1}\right)$ für einen $d$-d-Übergang und $330 \mathrm{~nm}$ $\left(\varepsilon=5200 \mathrm{~mol}^{-1} \cdot \mathrm{cm}^{-1}\right)$ für einen Ligand-Metall-Charge-Transfer auf. Eine weitere Absorption bei etwa $220 \mathrm{~nm}$ lässt sich nur als Schulter erahnen und muss dem $\pi-\pi^{\star}$-Übergang zugeschrieben werden.

Bei dem Versuch, den Komplex 11a mittels Sephadex-lonenaustauscher zu reinigen, sind aus der wässrigen Lösung dunkelblaue blockartige Kristalle gewachsen. Die röntgenographische Strukturaufklärung ergab die in Abbildung 25 dargestellte Struktur von 11b. Wegen des veränderten Kristallisationsmediums sind die axialen Positionen der Kupfer-Ionen nicht mehr mit Methanol- sondern mit Wassermolekülen besetzt.

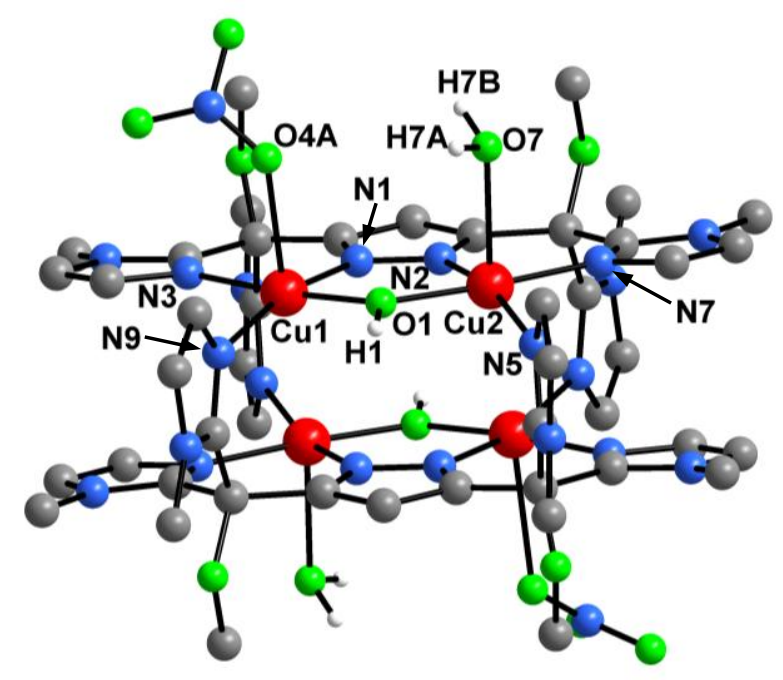

Abbildung 25: Festkörperstruktur des Dikations von 11b (wegen der Übersichtlichkeit sind alle H-Atome außer den $\mathrm{OH}-$ Protonen nicht dargestellt). 
Der Abstand der beiden pyrazolverbrückten Kupferatome ist mit 3.3731(5) Å geringfügig kleiner als in der entsprechenden Struktur mit Methanol als axialem Liganden. Alle vier Kupfer-Ionen haben eine quadratisch-pyramidale Koordinationsumgebung. Diese Tatsache wird durch die Werte für $\tau_{\mathrm{Cu} 1}=0.14$ und $\tau_{\mathrm{Cu} 2}=0.18$ bestätigt. Es fällt auf, dass im Unterschied zu Struktur 11a keine Wasserstoffbrückenbindung zwischen dem koordinierten Wassermolekül und dem ebenfalls axial koordinierten Nitrat-lon vorhanden ist.

Betrachtet man die Kristallstruktur jedoch näher, ist ein kleines Geflecht aus Wasserstoffbrückenbindungen erkennbar. In Abbildung 26 ist ein Ausschnitt aus der Kristallstruktur abgebildet, der die zwei oberen Kupfer(II)-Ionen aus Abbildung 25 mit allen koordinierten Atomen zeigt. Man sieht zum einen die quadratisch-pyramidale Koordinationsumgebung der Kupferionen und zum anderen das sich darüber aufbauende Wasserstoffbrückengebilde zwischen dem fehlgeordneten, koordinierten Nitrat-lon und koordinierten, sowie im Kristall eingelagerten Wassermolekülen. Alle Wasserstoffbrückenbindungen (gestrichelte Linien)

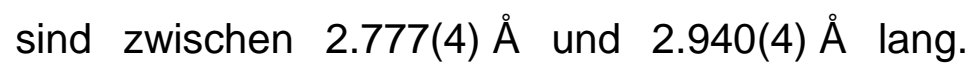
Diese Werte sprechen für eher schwache Wechselwirkungen.

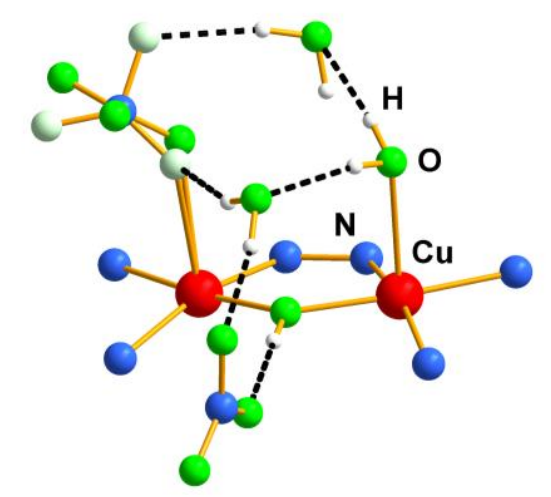

Abbildung 26: Wasserstoffbrücken in Struktur 11b.

In Tabelle 3 sind einige interessante Bindungslängen und Bindungswinkel der Komplexe 11a und 11b gegenübergestellt. In Verbindung 11b sind die axialen Bindungen zum Sauerstoffatom des Nitrat-lons mit 2.439(2) $\AA$ geringfügig kürzer und die axialen Bindungen zum Wassermolekül etwas länger (2.415(2) $\AA$ ). 


\begin{tabular}{|c|c|c|}
\hline \multicolumn{3}{|c|}{ Abstände $[\AA ̊]$} \\
\hline $3.3918(9)$ & $\mathrm{Cu} 1 \cdots \mathrm{Cu} 2$ & $3.3731(5)$ \\
\hline $1.968(4)$ & Cu1-N1 & $1.975(2)$ \\
\hline $1.976(5)$ & Cu1-N3 & $1.974(3)$ \\
\hline $1.909(4)$ & Cu1-O1 & $1.889(2)$ \\
\hline $2.017(5)$ & Cu1-N9' & $2.024(2)$ \\
\hline $2.485(5)$ & Cu1-O6 / Cu1-O4' & $2.439(3)$ \\
\hline $1.981(4)$ & Cu2-N2 & $1.971(2)$ \\
\hline $1.967(5)$ & Cu2-N7 & $1.980(2)$ \\
\hline $1.913(4)$ & Cu2-O1 & $1.899(2)$ \\
\hline $2.027(5)$ & Cu2-N5' & $2.024(2)$ \\
\hline $2.312(4)$ & Cu2-O2 / Cu2-O7 & $2.415(2)$ \\
\hline \multicolumn{3}{|c|}{ Winkel $\left[{ }^{\circ}\right]$} \\
\hline $125.1(2)$ & Cu1-O1-Cu2 & $125.88(11)$ \\
\hline $166.06(19)$ & N9'-Cu1-N1 & $166.13(10)$ \\
\hline $89.79(18)$ & N1-Cu1-N3 & $89.55(10)$ \\
\hline $175.46(18)$ & O1-Cu1-N3 & $174.68(9)$ \\
\hline $86.64(17)$ & O1-Cu1-N1 & $86.36(9)$ \\
\hline $94.37(19)$ & N3-Cu1-N9' & $94.03(10)$ \\
\hline $164.09(18)$ & N5'-Cu2-N2 & $164.71(10)$ \\
\hline $89.38(18)$ & N2-Cu2-N7 & $89.17(9)$ \\
\hline $174.95(18)$ & O1-Cu2-N7 & $175.21(9)$ \\
\hline $86.22(17)$ & O1-Cu2-N2 & $86.36(9)$ \\
\hline $94.39(19)$ & N5'-Cu2-N7 & $94.37(10)$ \\
\hline
\end{tabular}

Tabelle 3: Vergleich ausgewählter Bindungslängen (in $\AA$ ) und Bindungswinkel (in ${ }^{\circ}$ ) von 11a (links) und 11b (rechts).

In dem UV/Vis-Spektrum des Komplexes 11b in wässriger Lösung lassen sich wie schon für 11a zwei eindeutige Banden erkennen. Diese sind denen von 11a sehr ähnlich mit $575 \mathrm{~nm}\left(\varepsilon=323 \mathrm{~mol}^{-1} \cdot \mathrm{lcm}^{-1}\right)$ und $330 \mathrm{~nm}\left(\varepsilon=6610 \mathrm{~mol}^{-1} \cdot \mathrm{cm}^{-1}\right)$. Auch die Schulter bei etwa $230 \mathrm{~nm}$ ist deutlich zu erkennen. Zum Vergleich ist von diesem 
Komplex ein UV/Vis-Spektrum als Feststoff aufgenommen worden. Hier lassen sich aufgrund der Messbedingungen keine Aussagen über Extinktionskoeffizienten treffen. Jedoch kann man die erhaltenen Signale direkt miteinander vergleichen und Aussagen über die vorliegende Koordination treffen. Das Feststoff-UV/Vis-Spektrum von 11b ergab drei deutliche Absorptionen bei $575 \mathrm{~nm}, 334 \mathrm{~nm}$ und $234 \mathrm{~nm}$. Anhand dieser Werte ist klar ersichtlich, dass sich beim Lösen des Komplexes die Koordination um die Kupfer-lonen nicht verändert. Der Komplex liegt in Lösung genauso vor, wie es in der Kristallstruktur zu sehen ist.

Außerdem sind Kupfer(II)-perchlorat Hexahydrat und Kupfer(II)-tetrafluoroborat Tetrahydrat für die Komplexsynthese mit $\mathrm{HL}^{1}$ verwendet worden. In beiden Fällen entstehen die vierkernigen Kupfer(II)-Komplexe 12 und 13 mit rechteckiger Anordnung der Metallatome (Abbildung 27).
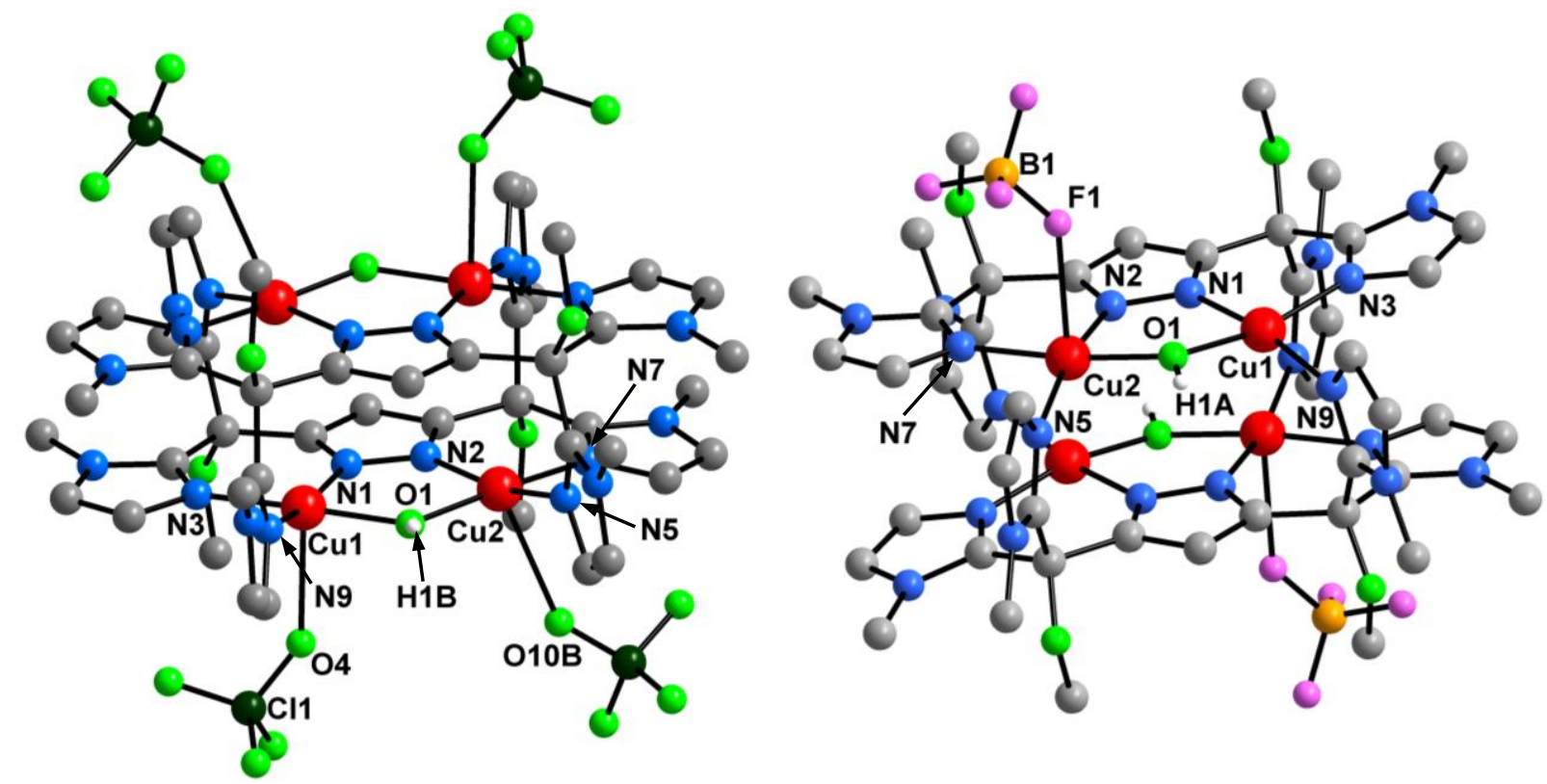

Abbildung 27: Festkörperstruktur von 12 (links) sowie des Dikations 13 (rechts). Wegen der Übersichtlichkeit sind nur die H-Atome der OH-Gruppen dargestellt.

In beiden Komplexen wiederholt sich das schon für 11a und 11b erhaltene Strukturmotiv. Die vier Kupfer(II)-lonen sind jeweils rechteckig angeordnet und unterscheiden sich nur durch die axial koordinierten Co-Liganden. Für 12 ergeben sich vier quadratisch pyramidal koordinierte Kupferionen $\left(\tau_{\mathrm{Cu} 1}=0.13\right.$ und $\left.\tau_{\mathrm{Cu} 2}=0.14\right)$. Die 
axiale Koordinationsstelle wird durch Perchlorat-Ionen besetzt, und der Komplex ist somit nach außen neutral. Allerdings sind die Bindungslängen $\mathrm{Cu}-\mathrm{O}_{\mathrm{ClO}_{4}}$ mit 2.495(7) $\AA$ und 2.72(3) $\AA$ sehr unterschiedlich. Im Falle des Komplexes mit Kupfer(II)tetrafluoroborat 13 sind zwei der vier Kupfer-lonen quadratisch pyramidal umgeben $\left(\tau_{\mathrm{Cu} 1}=0.12\right)$. Auch hier ist das sehr schwach koordinierende Tetrafluoroborat-lon der axiale Ligand für die fünffach koordinierten Kupfer(II)-lonen mit einer Bindungslänge $\mathrm{Cu}-\mathrm{F}_{\mathrm{BF}_{4}}$ von 2.4917(17) $\AA$. Die großen axialen Abstände in 12 und 13 sprechen erneut für eine schwache bis sehr schwache Koordination, wie für ein $d^{9}$-Metallion mit starker Jahn-Teller-Verzerrung zu erwarten.

Es fällt jedoch auf, dass bei Kristallisation dieses Komplexes aus Acetonitril/Diethylether violette Kristalle entstehen, die sich in Acetonitril nur sehr schlecht wieder lösen lassen. Das führt zu Schwierigkeiten bei der Durchführung der Analysen. Dieses Problem lässt sich jedoch beheben, wenn für die Kristallisation ein Gemisch aus Acetonitril und Chloroform verwendet wird. Es entsteht ein Komplex mit stark verbesserter Löslichkeit. Die experimentell gefundene Zusammensetzung stimmt für $12 \cdot \mathrm{CHCl}_{3}$ sehr gut mit den berechneten Werten überein. Das enthaltene Chloroform könnte für die bessere Löslichkeit mit verantwortlich sein. Außerdem ist es denkbar, dass sich hier ein geladener Komplex gebildet hat, wie dies auch schon bei Verbindung 11a und 11b erfolgte. Somit wären erneut zwei Kupferionen quadratisch planar und die anderen beiden Kupferzentren quadratisch pyramidal koordiniert.

Zwei Kupferatome, die von einem Pyrazolring und einer Hydroxid-Einheit verbrückt werden, sind in 12 um 3.377(1) $\AA$ und in 13 um 3.3641(4) $\AA$ separiert. Dieser Wert liegt somit in dem Bereich, der auch schon für die Komplexe $\mathbf{8}$ bis 11b erhalten wurde.

Anhand der in Tabelle 4 abgebildeten interessanten Bindungslängen und -winkel ist erkennbar, dass sich beide Komplexe sehr ähnlich sind. 


\begin{tabular}{|c|c|c|}
\hline \multicolumn{3}{|c|}{ Abstände $[\AA ̊]$} \\
\hline $3.377(1)$ & Cu1 $\cdots \mathrm{Cu} 2$ & $3.3641(4)$ \\
\hline $1.967(5)$ & Cu1-N1 & $1.9595(2)$ \\
\hline $1.965(6)$ & Cu1-N3 & $1.962(2)$ \\
\hline $1.906(5)$ & Cu1-O1 & $1.8950(18)$ \\
\hline $2.014(6)$ & Cu1-N9' & $1.995(2)$ \\
\hline $2.495(7)$ & Cu1-O4 & \\
\hline $1.966(5)$ & Cu2-N2 & $1.9673(19)$ \\
\hline $1.967(6)$ & Cu2-N7 & $1.966(2)$ \\
\hline $1.896(5)$ & Cu2-O1 & $1.9019(19)$ \\
\hline $2.021(6)$ & Cu2-N5' & $1.989(2)$ \\
\hline $2.72(3)$ & Cu2-O10B / Cu2-F1 & $2.4917(17)$ \\
\hline \multicolumn{3}{|c|}{ Winkel $\left[^{\circ}\right]$} \\
\hline $125.3(2)$ & Cu1-O1-Cu2 & $124.75(11)$ \\
\hline $166.8(2)$ & $\mathrm{N} 9^{\prime}-\mathrm{Cu} 1-\mathrm{N} 1$ & $170.03(8)$ \\
\hline $89.9(2)$ & N1-Cu1-N3 & $89.18(8)$ \\
\hline $174.9(2)$ & O1-Cu1-N3 & $167.26(8)$ \\
\hline $86.2(2)$ & O1-Cu1-N1 & $86.62(8)$ \\
\hline $90.3(3)$ & O1-Cu1-N9' & 89.94(8) \\
\hline $94.2(3)$ & N3-Cu1-N9' & $96.08(8)$ \\
\hline $165.3(2)$ & $\mathrm{N} 5^{\prime}-\mathrm{Cu} 2-\mathrm{N} 2$ & $166.25(8)$ \\
\hline $89.5(2)$ & N2-Cu2-N7 & $90.28(8)$ \\
\hline $173.3(2)$ & O1-Cu2-N7 & $173.52(8)$ \\
\hline $86.5(2)$ & O1-Cu2-N2 & $86.19(8)$ \\
\hline $90.0(2)$ & O1-Cu2-N5' & $90.18(8)$ \\
\hline $95.2(3)$ & N5'-Cu2-N7 & $94.49(8)$ \\
\hline
\end{tabular}

Tabelle 4: Ausgewählte Bindungslängen (in $\AA$ ) und Bindungswinkel (in ${ }^{\circ}$ ) von 12 (links) und 13 (rechts). 
Eine weitere Gemeinsamkeit fällt auf, wenn man sowohl die in Acetonitril gemessenen UV/Vis-Spektren als auch die Festkörper-UV/Vis-Spektren von 12 und 13 miteinander vergleicht (Abbildung 28).
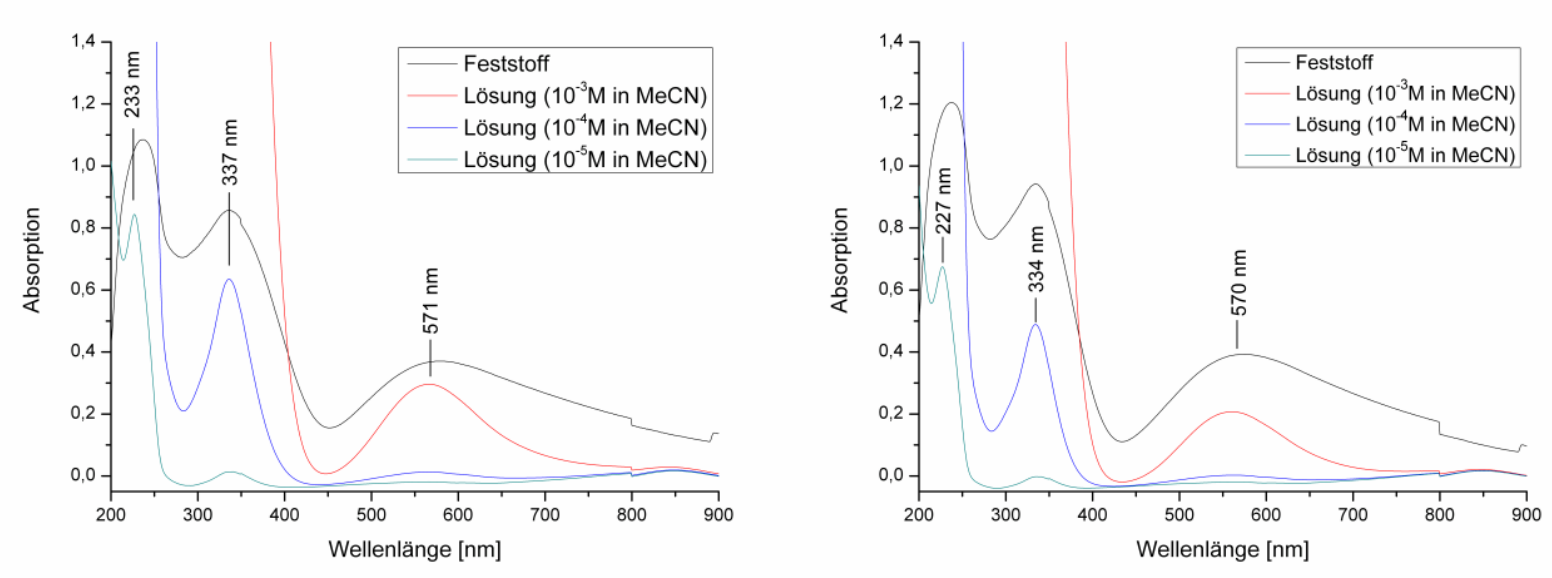

Abbildung 28: UV/Vis-Spektren in Lösung und als Festoff von 12 (links) und 13 (rechts).

Auch für die Komplexe 12 und 13 lassen sich in den aufgenommenen UV/Vis-Spektren die typischen Absorptionsmaxima bei etwa $570 \mathrm{~nm}$ für den d-d-Übergang eines quadratisch pyramidal koordinierten Kupfer(II)-Ion finden. Außerdem sieht man bei $337 \mathrm{~nm}$ bzw. $334 \mathrm{~nm}$ ein Maximum für den LMCT-Übergang, sowie bei $233 \mathrm{~nm}$ bzw. $227 \mathrm{~nm}$ eine Bande für $\pi-\pi^{*}$-Übergänge.

Da die Festkörper-UV/Vis-Spektren mit denen in Lösung übereinstimmen, kann man schließen, dass die durch Röntgenstrukturanalyse ermittelte Struktur auch in Lösung erhalten bleibt, wie dies auch schon für die Komplexe 8 bis 11b beobachtet werden konnte.

Neben der hier vorgestellten Vielzahl von Kupfer(II)-Komplexen des Liganden $\mathrm{HL}^{1}$ wurde ebenfalls versucht, kristalline Kupfer(II)-Komplexe mit den übrigen Liganden zu synthetisieren. Allerdings sind im Falle von $\mathrm{HL}^{3}$ bis $\mathrm{HL}^{6}$ nur Pulver entstanden, die keine ausreichende Reinheit für eine weitere Charakterisierung aufweisen. Aufgrund der $\mathrm{OH}$-Funktionen im Liganden $\mathrm{HL}^{4}$ lassen sich die Kupfer(II)-Komplexe nur in DMF, DMSO oder Wasser lösen. Eine Kristallisation aus diversen Lösungsmitteln war nicht erfolgreich. Bei Verwendung von $\mathrm{HL}^{3}$ und $\mathrm{HL}^{6}$ entstanden Kupfer(II)-Komplexe, die sich zwar alle sehr gut auch in Dichlormethan oder Aceton lösten, jedoch resultierten beim 
Überschichten oder auch bei Dampfdiffusion mit anderen Lösungsmitteln ausschließlich Pulver.

Es ist allerdings gelungen, Kupfer(II)-Komplexe mit dem Liganden $\mathrm{HL}^{2}$ zu synthetisieren. Deprotoniert man den Liganden in Methanol mit Kalium-tert-butanolat und gibt anschließend zwei Äquivalente Kupfer(II)-perchlorat hinzu, entsteht eine grün gefärbte Lösung. Nachdem das Lösungsmittel im Vakuum entfernt und das Rohprodukt in Acetonitril aufgenommen wurde, konnten durch Dampfdiffusion von Diethylether blauviolette Kristalle erhalten werden. Diese waren für die Röntgenstrukturanalyse geeignet und es wurde die in Abbildung 29 gezeigte Struktur von Komplex 14 erhalten.

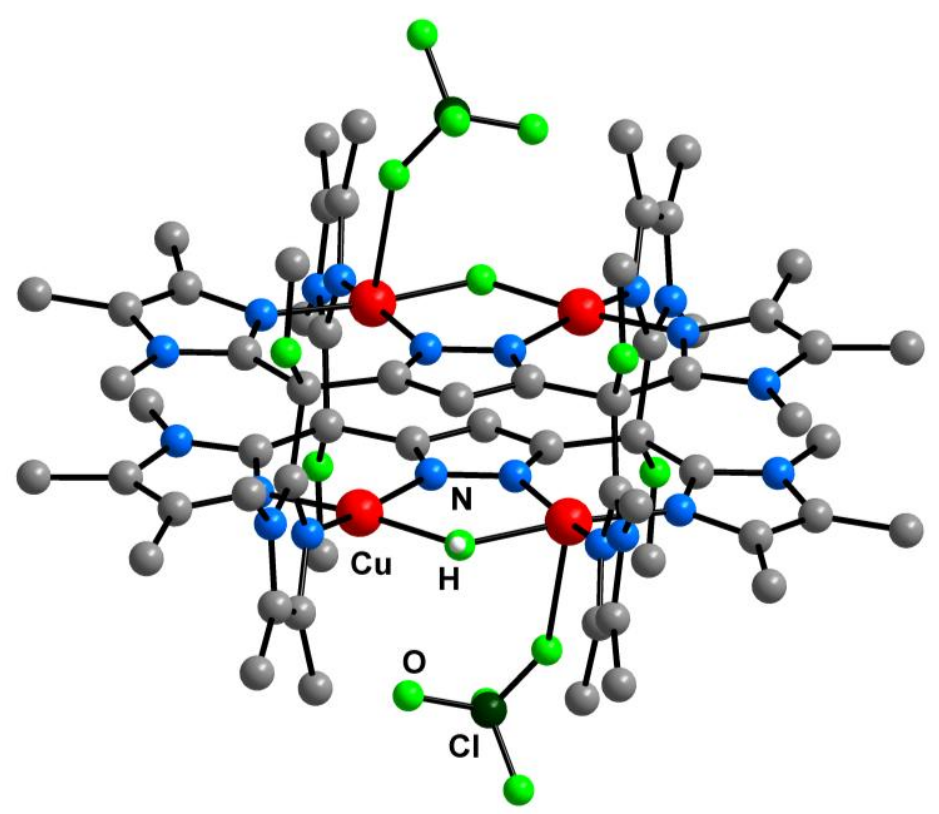

Abbildung 29: Festkörperstruktur des Dikations von 14 (wegen der Übersichtlichkeit sind alle H-Atome außer den $\mathrm{OH}$-Protonen und die nichtkoordinierenden Perchlorat-Ionen nicht abgebildet).

Zu sehen ist ein vierkerniger Komplex, in dem erneut die Kupfer(II)-Ionen rechteckig angeordnet sind. Auch dieser Ligand zeigt das gleiche Bindungsmotiv, wie der bereits vorgestellte Ligand $\mathrm{HL}^{1}$. Ein Imidazolring jeder Ligandseite bindet an das entsprechende Kupfer(II)-lon. Der jeweils andere Imidazolring jeder Ligandhälfte koordiniert mit dem nicht-methylierten Stickstoffatom an ein benachbartes Kupfer-Ion. 
Zwei Kupfer-lonen werden von einem Pyrazolring verbrückt und so in einem Abstand von 3.4094(8) A voneinander gehalten. Zusätzlich sind diese beiden Metallatome über eine $\mathrm{OH}$-Brücke verknüpft. In Tabelle 5 sind einige Bindungslängen und -winkel für Komplex 14 aufgelistet.

\begin{tabular}{|c|c|c|c|}
\hline \multicolumn{4}{|c|}{ Abstände $[\AA ̊]$} \\
\hline Cu1-N1 & $1.953(4)$ & Cu2-N2 & $1.964(3)$ \\
\hline Cu1-N3 & $2.014(4)$ & Cu2-N7 & $2.008(4)$ \\
\hline Cu1-O1 & $1.899(3)$ & Cu2-N5' & $2.004(3)$ \\
\hline Cu1-N9' & $1.980(4)$ & Cu2-O1 & $1.893(3)$ \\
\hline $\mathrm{Cu} 1 \cdots \mathrm{Cu} 2$ & $3.4094(8)$ & Cu2-O8 & $2.780(4)$ \\
\hline \multicolumn{4}{|c|}{ Winkel $\left[^{\circ}\right]$} \\
\hline N9'-Cu1-N1 & $164.31(14)$ & N5'-Cu2-N2 & $163.17(15)$ \\
\hline N3-Cu1-N9' & $99.49(15)$ & N2-Cu2-N7 & $90.89(14)$ \\
\hline O1-Cu1-N1 & $84.07(14)$ & O1-Cu2-N5' & $85.90(14)$ \\
\hline O1-Cu1-N3 & $170.77(14)$ & O1-Cu2-N2 & $84.52(14)$ \\
\hline O1-Cu1-N9' & $86.16(14)$ & O1-Cu2-N7 & $170.27(14)$ \\
\hline N1-Cu1-N3 & $91.86(14)$ & N5'-Cu2-N7 & $100.52(15)$ \\
\hline O1-Cu2-O8 & $85.79(13)$ & N5'-Cu2-O8 & $120.82(14)$ \\
\hline N2-Cu2-O8 & $72.22(13)$ & N7-Cu2-O8 & $84.64(13)$ \\
\hline Cu1-O1-Cu2 & $128.08(17)$ & & \\
\hline
\end{tabular}

Tabelle 5: Ausgewählte Bindungslängen (in $\AA$ ) und Bindungswinkel (in º) von 14.

Beide Cu-O1-Bindungen sind mit 1.899(3) $\AA$ und 1.893(3) $\AA$ deutlich kürzer als alle anderen Bindungen $z u$ den Kupfer(II)-Ionen. Außerdem ist der große Cu1-O1-Cu2-Winkel von 128.08(17) ${ }^{\circ}$ recht auffällig. Es handelt sich um den größten Winkel für diese Brücke, der in den hier vorgestellten Komplexen festgestellt werden konnte. Beide Kupfer(II)-lonen liegen nahezu perfekt in der Ebene des Pyrazolrings. Es 
existiert nur ein geringer Torsionswinkel von 2.027(6) ${ }^{\circ}$. Diese Tatsache wird auch in der seitlichen Ansicht des Komplexes 14 deutlich (Abbildung 30).

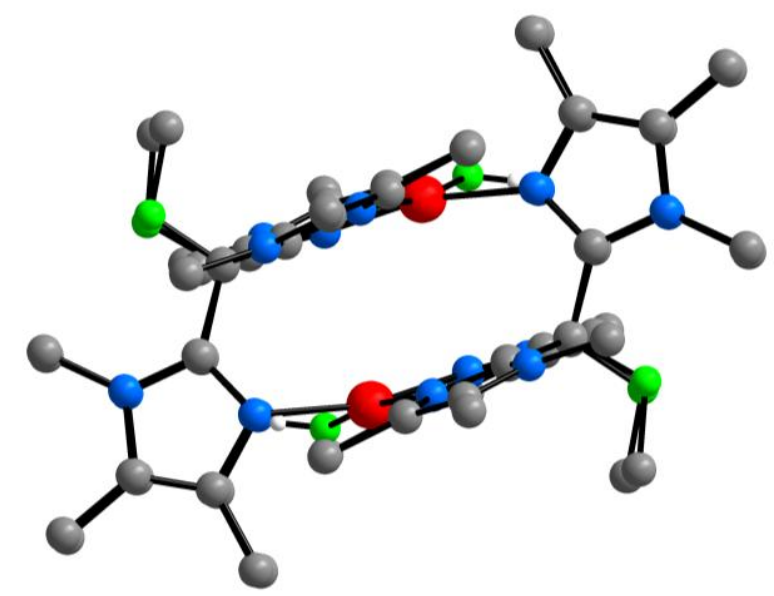

Abbildung 30: Seitliche Ansicht des Tetrakations von 14 (wegen der Übersichtlichkeit sind alle H-Atome außer den $\mathrm{OH}-$ Protonen und die Perchlorat-lonen nicht abgebildet).

Man kann hier ganz besonders gut den gebildeten Hohlraum mit Dimensionen von 6.6127(2) $\AA$ und 3.2721(2) $\AA$ erkennen. Die gegenüberliegenden Ebenen werden aus einem Teil des Liganden - Dikupfer-Pyrazolat-Einheit mit einem Imidazolring jeder Ligandhälfte - gebildet. Die restlichen Imidazolringe stehen sich an den Stirnseiten paarweise gegenüber.

Verwendet man zur Deprotonierung des Liganden Natriummethanolat anstelle von Kalium-tert-butanolat, tritt nach Zugabe von zwei Äquivalenten Kupfer(II)-perchlorat ebenfalls eine Grünfärbung der Lösung ein. Nachdem das Lösungsmittel entfernt wurde, ist das Rohprodukt erneut in Acetonitril aufgenommen worden und anschließend konnten mittels Dampfdiffusion von Diethylether Kristalle erhalten werden, die für eine röntgenografische Strukturaufklärung geeignet waren. In Abbildung 31 ist die dabei erhaltene Struktur des zweikernigen Kupfer(II)-Komplexes 15 gezeigt. 


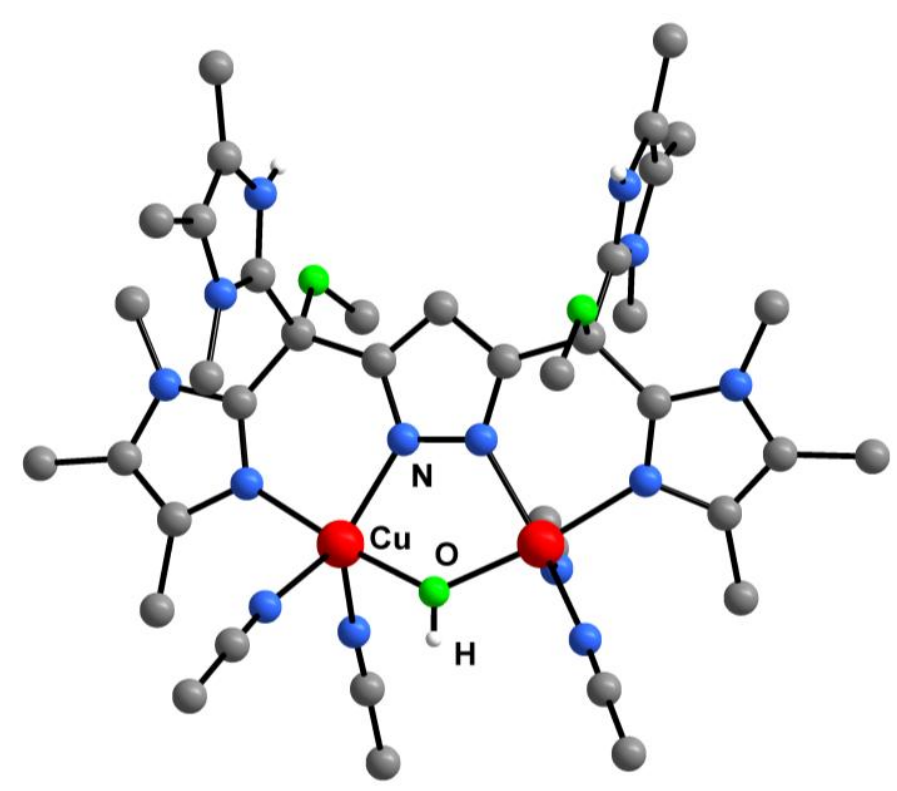

Abbildung 31: Festkörperstruktur des Tetrakations von 15 (wegen der Übersichtlichkeit sind alle $\mathrm{H}$-Atome außer den $\mathrm{NH}$ - sowie $\mathrm{OH}$-Protonen und die Perchlorat-Ionen nicht abgebildet).

Die Struktur ähnelt auf den ersten Blick dem zweikernigen Kupfer(II)-Komplex 8. Auch hier sind zwei Kupfer(II)-lonen sowohl durch den Pyrazolring als auch ein Hydroxid-Ion verbrückt und werden so mit einem Abstand von 3.3440(5) ^ separiert. Der Cu1-O1-Cu2-Winkel von $123.75(10)^{\circ}$ unterscheidet sich nur ganz gering von dem in dem zweikernigen Kupfer(II)-Komplex 8. In Tabelle 6 sind einige Bindungslängen und winkel für Komplex 15 zusammengefasst.

In 15 ist ebenfalls ein Imidazolring jeder Ligandhälfte nicht an der Koordination zum Kupfer(II)-Ion beteiligt. Damit die Kupferzentren koordinativ abgesättigt sind, werden an jedem Kupfer(II)-Ion zwei Moleküle Acetonitril gebunden. Hierbei entsteht allerdings nicht wie in den bisher präsentierten Komplexen eine ziemlich ideale quadratisch pyramidale Koordinationsgeometrie. Stattdessen findet man hier stark verzerrte Geometrien wieder.

Dies kann man sehr gut anhand des Parameters $\tau$ einschätzen. So ergibt sich für Cu1 ein $\tau$-Wert von 0.49 , der exakt zwischen beiden Grenzwerten liegt, so dass es nicht möglich ist, eine Aussage über die genaue Geometrie zu treffen. 


\begin{tabular}{llll}
\multicolumn{5}{c}{ Abstände [Å] } \\
\hline Cu1-N1 & $1.958(2)$ & Cu2-N2 & $1.983(2)$ \\
Cu1-N7 & $1.988(2)$ & Cu2-N3 & $1.991(2)$ \\
Cu1-O1 & $1.8977(19)$ & Cu2-O1 & $1.8940(19)$ \\
Cu1-N13 & $1.996(2)$ & Cu2-N11 & $2.084(3)$ \\
Cu1-N14 & $2.350(3)$ & Cu2-N12 & $2.196(3)$ \\
Cu1-Cu2 & $3.3440(5)$ & & \\
& & Winkel [] & \\
\hline O1-Cu1-N7 & $172.73(9)$ & O1-Cu2-N3 & $174.74(8)$ \\
N1-Cu1-N7 & $88.66(8)$ & N2-Cu2-N3 & $89.74(8)$ \\
O1-Cu1-N1 & $84.49(8)$ & O1-Cu2-N2 & $85.04(8)$ \\
O1-Cu1-N13 & $90.08(9)$ & O1-Cu2-N11 & $89.48(9)$ \\
O1-Cu1-N14 & $93.11(9)$ & O1-Cu2-N12 & $89.01(9)$ \\
N1-Cu1-N13 & $153.48(10)$ & N2-Cu2-N11 & $133.38(9)$ \\
N1-Cu1-N13 & $95.00(9)$ & N2-Cu2-N12 & $122.62(10)$ \\
N7-Cu1-N14 & $90.07(9)$ & N3-Cu2-N11 & $93.58(9)$ \\
N1-Cu1-N14 & $96.48(9)$ & N3-Cu2-N12 & $94.43(9)$ \\
N12-Cu1-N14 & $109.75(10)$ & N11-Cu2-N12 & $103.48(10)$ \\
Cu1-O1-Cu2 & $123.75(10)$ & & \\
\hline
\end{tabular}

Tabelle 6: Ausgewählte Bindungslängen (in $\AA$ ) und Bindungswinkel (in º) von 15.

Etwas eindeutiger ist die Situation für Cu2. In diesem Fall ergibt sich ein $\tau$-Wert von 0.69 und man kann von einer verzerrten trigonalen Bipyramide als Koordinationspolyeder ausgehen. Der Unterschied zu allen bisher erhaltenen Kupfer(II)Komplexen äußert sich auch am beispielhaften Vergleich der UV/Vis-Spektren von 15 mit 8 (Abbildung 32). Die bisher erhaltenen Spektren ähneln dem von 8 sehr stark. Es treten nur geringe Differenzen in den Wellenlängen der einzelnen Absorptionen auf. 
Dabei spielt es keine entscheidende Rolle in welchem Lösungsmittel die UV/VisSpektren gemessen wurden.
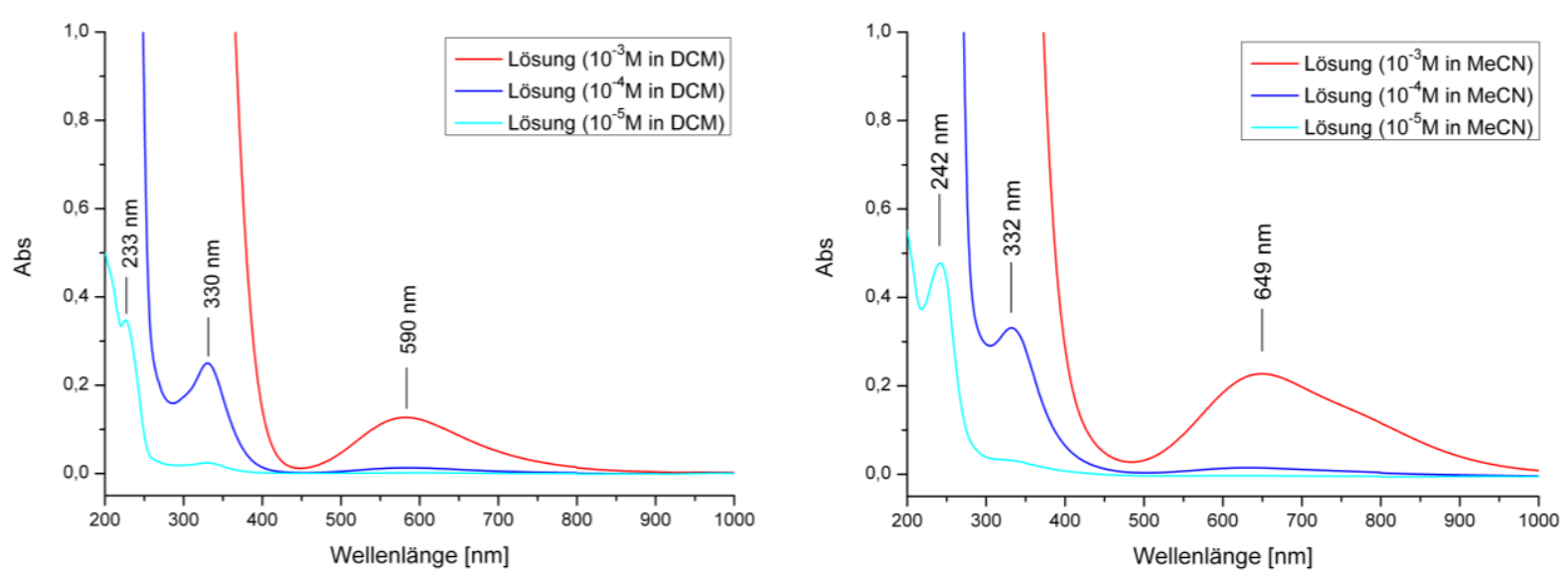

Abbildung 32: Vergleich der UV/Vis-Spektren von 8 (links) mit 15 (rechts).

Während sich für den zweikernigen Kupfer-Acetat-Komplex 8 Absorptionen bei $590 \mathrm{~nm}$ $\left(\varepsilon=120 \mathrm{~mol}^{-1} \cdot \mathrm{lcm}^{-1}\right), 330 \mathrm{~nm}\left(\varepsilon=2860 \mathrm{~mol}^{-1} \cdot \mathrm{lcm}^{-1}\right)$ und $233 \mathrm{~nm}\left(\varepsilon=36000 \mathrm{~mol}^{1} \cdot \mathrm{lcm}^{-1}\right)$ ergeben, erhält man für Verbindung 15 Werte von $649 \mathrm{~nm}\left(\varepsilon=227 \mathrm{~mol}^{-1} \cdot \mathrm{lcm}^{-1}\right), 332 \mathrm{~nm}$ $\left(\varepsilon=3310 \mathrm{~mol}^{-1} \cdot \mathrm{lcm}^{-1}\right)$ und $242 \mathrm{~nm}\left(\varepsilon=47800 \mathrm{~mol}^{-1} \cdot \mathrm{lcm}^{-1}\right)$. Bei etwa $233 \mathrm{~nm}$ finden $\pi \rightarrow \pi^{*}$ - Übergänge statt und die Bande bei ca. $330 \mathrm{~nm}$ ist typisch für $\mathrm{n} \rightarrow \pi^{*}$ Übergänge. Die Unterschiede in den Extinktionskoeffizienten sind dabei unbedeutend. Viel interessanter ist die deutlich unterschiedliche Wellenlänge für den $d_{z^{2}} \rightarrow d_{x^{2}-y^{2}}$ Übergang im Komplex. Diese differiert um $59 \mathrm{~nm}$ beim Wechsel von nahezu ideal quadratisch pyramidaler zu verzerrt trigonal bipyramidaler Geometrie. Für quadratisch planare bzw. quadratisch pyramidale Geometrien sind in der Regel Absorptionen im Bereich von $570 \mathrm{~nm}$ bis $650 \mathrm{~nm}$ zu finden. Diese Verschiebungen können aber auch von den koordinierten Liganden verursacht werden. Nimmt man UV/Vis-Spektren von Kupfer(II)-Komplexen mit nahezu ideal trigonal bipyramidalen Kupfer-Ionen auf, so sind Absorptionen bei etwa $650 \mathrm{~nm}$ und außerdem ein schwaches Signal bei ca. $950 \mathrm{~nm}$ zu finden. ${ }^{[10,11,18,117-119]}$ Die Absorption bei etwa $950 \mathrm{~nm}$ konnte für diese Verbindung jedoch nicht beobachtet werden, so dass man in diesem Fall wohl nicht von trigonal bipyramidaler, sondern eher von stark verzerrt quadratisch pyramidaler Koordination sprechen kann. 
Im Rahmen dieser Arbeit ist es gelungen, die Struktur einiger Kupfer(II)-Komplexe zu bestimmen und diese anschließend spektroskopisch zu charakterisieren. Dabei zeigte sich sowohl für die Kupfer(II)-Komplexe von $\mathrm{HL}^{1}$ als auch für die von $\mathrm{HL}^{2}$ das gleiche Strukturmotiv. In den meisten Fällen lassen sich quadratisch pyramial oder quadratisch planar koordinierte Kupfer(II)-lonen finden. Sofern Lösungsmittel mit guten Donoreigenschaften wie Acetonitril, Wasser oder Methanol zum Kristallisieren verwendet wurden, sind durch Koordination der Lösungsmittel in axialer Position quadratisch pyramidal koordinierte Kupfer(II)-Ionen entstanden. Für alle KupferKomplexe

In Tabelle 7 sind einige Strukturparameter für die kristallinen Kupfer(II)-Komplexe 8 bis 15 zusammengefasst. Dazu gehört der Abstand zweier pyrazolatverbrückter Kupferionen, der $\tau$-Wert für quadratisch pyramidal koordinierte Kupfer(II)-Ionen sowie die beobachteten Absorptionsbanden im UV/Vis.

\begin{tabular}{clll} 
Komplex & $d(\mathrm{Cu} \cdots \mathrm{Cu})[\AA]$ & $\begin{array}{l}\tau \text { (für quadratisch } \\
\text { pyramidale } \mathrm{Cu})\end{array}$ & $\begin{array}{l}\text { Absorptionen im UV/Vis-Spektrum } \\
{\left[\mathrm{nm}\left(\mathrm{mol}^{-1} \cdot \mathrm{cm}^{-1}\right)\right]}\end{array}$ \\
\hline $\mathbf{8}$ & $3.3367(5)$ & --- & $230(36000), 332(2860), 559(120)$ \\
$\mathbf{9}$ & $3.3258(9)$ & --- & $232(70470), 320(6380), 596(214)$ \\
$\mathbf{1 0}$ & $3.2945(8)$ & $0.09 /---$ & $220(44400), 328(4920), 580(211)$ \\
$\mathbf{1 1 a}$ & $3.3918(9)$ & $0.08 / 0.09$ & $330(5200), 578(238)$ \\
$\mathbf{1 1 b}$ & $3.3731(5)$ & $0.09 / 0.09$ & $330(6610), 573(323)$ \\
$\mathbf{1 2}$ & $3.377(1)$ & $0.08 / 0.11$ & $227(84400), 336(6350), 566(296)$ \\
$\mathbf{1 3}$ & $3.3641(4)$ & $---/ 0.11$ & $227(67500), 334(4890), 561(207)$ \\
$\mathbf{1 4}$ & $3.4094(8)$ & $0.04 / 0.16$ & --- \\
$\mathbf{1 5}$ & $3.3440(5)$ & $0.12 / 0.02$ & $242(47650), 332(3340), 649(227)$ \\
\hline
\end{tabular}

Tabelle 7: Zusammenfassung einiger Strukturparameter für die Kupfer(II)-Komplexe 8 bis 15. 
Anhand der $\tau$-Werte ist klar zusehen, dass sich in der Regel fast ideale quadratische Pyramiden bilden. Eine trigonal bipyramidale Anordnung konnte in keinem Fall beobachtet werden. Diese Aussage lässt sich auch mit den aufgenommenen UV/VisSpektren belegen. Je nachdem welche Koordinationsumgebung um das Kupfer-lon vorliegt, verschieben sich die Absorptionsbanden. Für trigonal bipyramidale Anordnungen in Kupfer(II)-Komplexen findet man in der Regel charakteristische Absorptionen im Bereich von etwa 900 bis $950 \mathrm{~nm}$. Prominente Beispiele sind mononukleare Kupfer(II)-Komplexe von Tris[(2-pyridyl)methyl]amin (tmpa). ${ }^{[120-123]}$ Im Gegensatz dazu zeigen die UV/Vis-Spektren von Komplexen mit quadratisch planar oder quadratisch pyramidal koordinierten Kupfer(II)-Ionen charakteristische Banden zwischen 550 und $650 \mathrm{~nm}^{[117-119]}$ Aus diesen spektroskopischen Daten und den aufgenommenen Festkörper-UV/Vis-Spektren lässt sich außerdem ableiten, dass die im Festkörper ermittelte Struktur in Lösung erhalten bleibt.

Der synthetisierte Ligand hat sich als sehr starres System erwiesen. Aufgrund des quartären C-Atoms neben der 3- und 5-Positon des Pyrazols ist eine faciale Koordinationstasche vorgegeben. Daraus ergibt sich, eine tetraedrische bzw. oktaedrische Koordinationsumgebung des Kupfer(I)-Ions. Will man dem System etwas mehr Flexibilität verleihen, ohne dass die bioinspirierte Bindungssituation verloren geht, kann man zum Beispiel eine Methylen-Brücke einfügen (XIII und XIV in Schema 19).

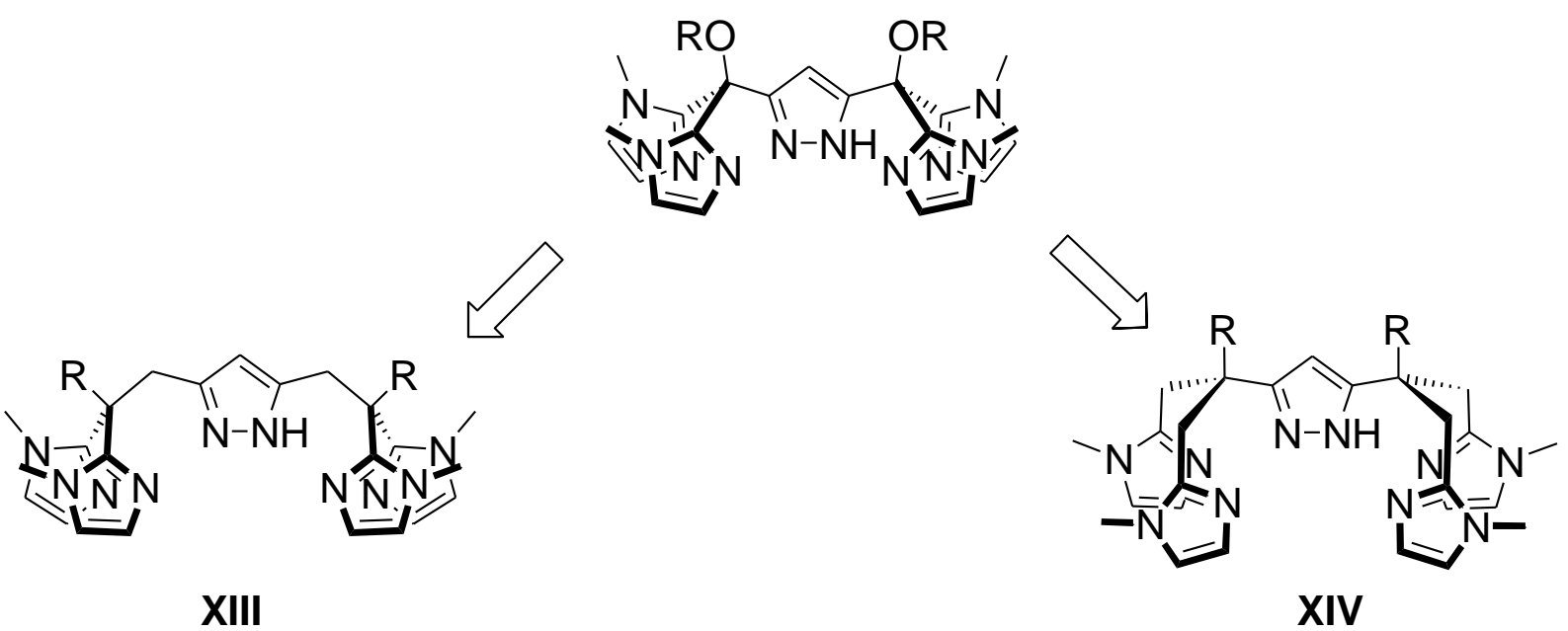

Schema 19: Mögliche Variation des Liganden, damit ein N-Donor des Pyrazolrings und beide Imidazolringe einer Ligandhälfte an ein gemeinsames Kupfer(II)-Ion binden 
Dabei bieten sich zwei Stellen an. Zum Einen zwischen Pyrazolring und quartären Kohlenstoffatom oder zum Anderen zwischen dem Brückenkopf-C-Atom und dem C2Atom des Imidazolrings. Im ersteren Fall (XIII) ist die Flexibilität der gesamten bis(imidazolyl)-einheit erhöht. Die Imidazolringe untereinander sind weiterhin sehr starr verbunden. Deshalb sollte die hier erwähnte zweite Variation (XIV) des Ligandsystems Kupfer(II)-Komplexe nach sich ziehen, in denen beide Imidazolringe jeder Ligandseite an das gleiche Metallion geknüpft sind.

Daraus sollten sich Mehrkernkomplexe bilden, in denen beide Imidazolringe jeder Ligandhälfte an ein und das selbe Kupfer(II)-lon binden. Diese Komplexverbindungen sollten dann auch eine katalytische Aktivität in zahlreichen Reaktionen aufweisen. 


\subsection{Synthese der Kupfer(I)-Komplexe}

Anders als bei der Darstellung der Kupfer(II)-Komplexe ist bei der Synthese der Kupfer(I)-Verbindungen auf strikten Ausschluss von Sauerstoff zu achten. Um dies zu gewährleisten, fanden die im Rahmen dieser Arbeit durchgeführten Synthesen von Kupfer(I)-Komplexen in der Glovebox statt. Dabei wurden die Edukte als Feststoffe in die Glovebox eingeschleust, dort in entgasten und trockenen Lösungsmitteln gelöst und anschließend unter Rühren vereinigt (Schema 20). Im Gegensatz zur Darstellung von Kupfer(II)-Komplexen wird bei der Synthese von Kupfer(I)-Verbindungen nur ein Äquivalent Base benötigt, da hier lediglich der Pyrazolring deprotoniert werden muss. Eine Verbrückung beider Kupfer(I)-Ionen über eine $\mathrm{OH}$-Brücke ist aufgrund der bevorzugten Tetraedergeometrie nicht möglich. Außerdem besitzen harten O-Liganden nur geringe Affinität zum weichen Kupfer(I)-Ion. ${ }^{[80,124]}$ Als mögliche Kupfer(I)-Vorstufen sind diverse Tetrakis(acetonitril)Kupfer(I)-Komplexe verwendet worden. Aber auch andere Kupfer(I)-Verbindungen wie Kupfer(I)-Cyclooctadienyl-Chlorid oder Tris(triphenylphosphan)Kupfer(I)-Phenolat fanden Anwendung.<smiles>COC(c1ccccc1)(c1nc(-c2ccccc2)c(-c2ccccc2)n1C)c1nc(-c2ccccc2)c(-c2ccccc2)n1C(OC)(c1ccccc1)c1nc(-c2ccccc2)c(-c2ccccc2)n1C</smiles>

Schema 20: Synthese von Kupfer(I)-Komplexen am Beispiel des Liganden $\mathrm{HL}^{3}$ mit der erwarteten Struktur der Produkte.

Der Ligand $\mathrm{HL}^{3}$ fand bevorzugt Verwendung bei der Synthese kristalliner Kupfer(I)-Komplexe, da die acht Phenylringe des Liganden die Löslichkeit stark verbessern und somit eine größere Auswahl an Lösungsmitteln für die Kristallisation der Verbindungen gegeben war. Für die Synthese wurde der Ligand in THF gelöst und 
mittels Kalium-tert-butanolat deprotoniert. Anschließend fügt man noch Tetrakisacetonitril-Kupfer(I) Hexafluorophosphat hinzu. Die Farbe der Lösung verändert sich dabei von gelb nach orangebraun. Dabei tritt zwischenzeitlich eine violettbraune Färbung der Lösung auf, wenn mit der Zugabe des Kupfer(I)-Salzes begonnen wird. Ist man bestrebt, die Löslichkeit noch weiter zu erhöhen, kann man dem gebildeten Kupfer(I)-Komplex einen weiteren Co-Liganden zur Verfügung stellen und so das koordinierte Lösungsmittel (in Schema 20: $\mathrm{L}=\mathrm{CH}_{3} \mathrm{CN}$ ) gegen einen besseren Donor austauschen. Dafür eignen sich zum Einen Isonitrile und zum Anderen Phosphine.

Bietet man als Donor für die freie Koordinationsstelle zum Beispiel Trimethylphosphin an, hellt sich die Lösung noch etwas auf. Es wurde aus der Reaktionslösung eine Probe entnommen und ein ESI-Massenspektrum aufgenommen. Das Ergebnis ist in Abbildung 33 gezeigt.

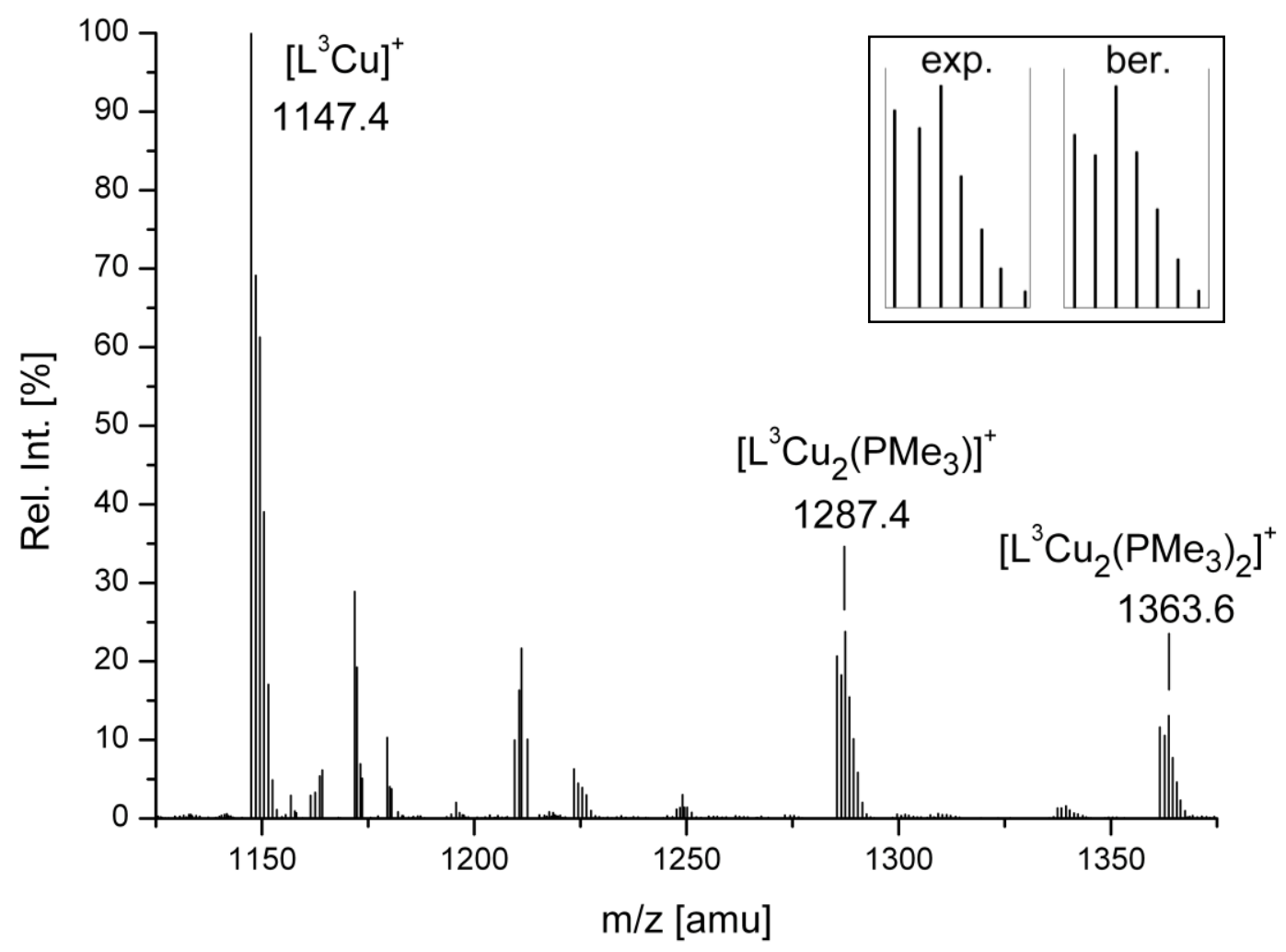

Abbildung 33: ESI-Massenspektrum einer Reaktionslösung zur Synthese von Kupfer(I)-Komplex 16 mit Trimethylphosphin in Acetonitril. Die Einfügung zeigt ein Vergleich des berechneten mit dem experimentell erhaltenen Isotopenmuster für $\left[\mathrm{L}^{3} \mathrm{Cu}_{2}\left(\mathrm{PMe}_{3}\right)_{2}\right]^{+}$. 
In dem Spektrum sind zahlreiche Peaks zu erkennen, die definierten Fragmenten zugeordnet werden können. So lässt sich anhand des Fragments bei $\mathrm{m} / \mathrm{z}=1287.4$ eindeutig belegen, dass das als Co-Ligand zugefügte Phosphin am Kupfer(I)-Ion koordiniert ist. Wenn man sich das Signal bei $\mathrm{m} / \mathrm{z}=1363.6$ ansieht, deutet sogar einiges darauf hin, dass an jedem Kupfer-Ion ein Trimethylphosphin-Molekül koordiniert ist. Der Peak für $\left[\mathrm{L}^{3} \mathrm{Cu}_{2}\left(\mathrm{PMe}_{3}\right)_{2}\right]^{+}$bei $\mathrm{m} / \mathrm{z}=1363.6$ entspricht der in Schema 16 gezeigten erwarteten Zusammensetzung. Es ist jedoch keine Aussage möglich, welche Koordination an den Kupfer(I)-lonen vorliegt.

Nach Kristallisation aus Dichlormethan/Diethylether erhält man fast farblose Kristalle. Die röntgenographische Analyse ergab die in Abbildung 34 gezeigte Struktur eines vierkernigen Kupfer(I)-Komplexes 16.

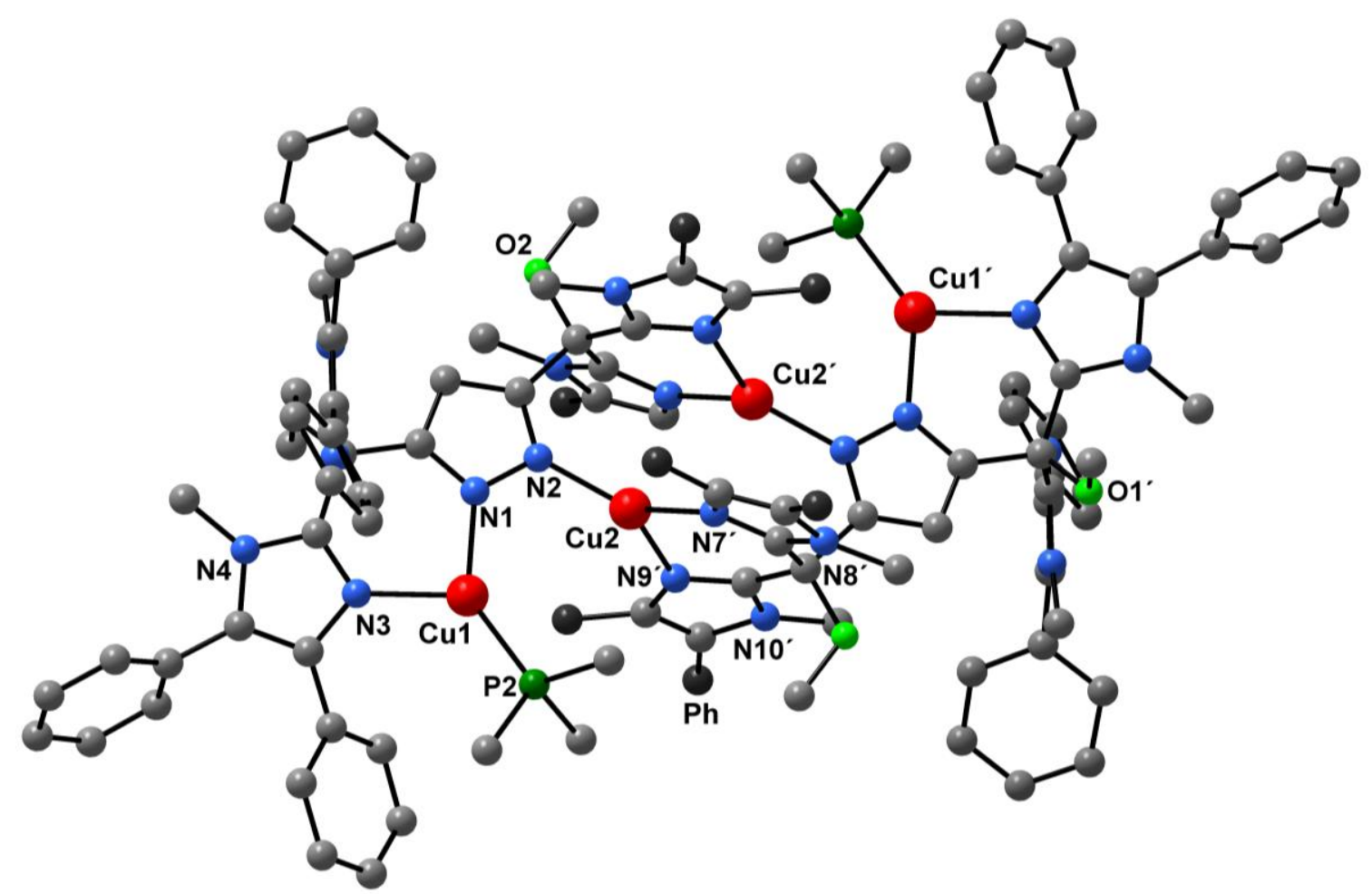

Abbildung 34: Festkörperstruktur des Dikations von 16 (Für bessere Übersichtlichkeit sind alle H-Atome nicht dargestellt und die Phenylringe der vier mittleren Imidazoleinheiten nur durch dunkelgraue Kugeln angedeutet).

Jedes der vier Kupfer(l)-lonen ist trigonal-planar koordiniert. Die äußeren Metallionen sind jeweils von einem Pyrazol- und einem Imidazolstickstoffatom koordiniert. Die dritte Koordinationsstelle wird vom Phosphoratom des Trimethylphosphins besetzt. Es ergibt sich eine Cu1-P2-Bindung von 2.1759(16) Å. Diese Bindungslänge liegt damit in dem typischen Bereich derartiger Bindungen. Ardizzoia et al. fanden für den $\operatorname{Kupfer}(\mathrm{I})$ - 
Komplex XV Abstände von 2.1877(7) ̊̊ für die Cu1-P1-Bindung und 2.1863(7) ̊̊ für die Cu2-P2-Bindung. Für den mononuklearen Kupfer(I)-Komplex XVI konnten Badiei et. al. einen Cu-P-Abstand von 2.161(1) A ermitteln (Abbildung 35). ${ }^{[125,126]}$

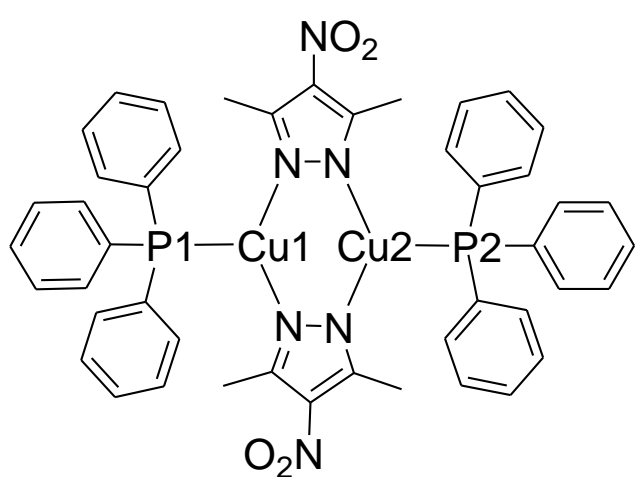

XV

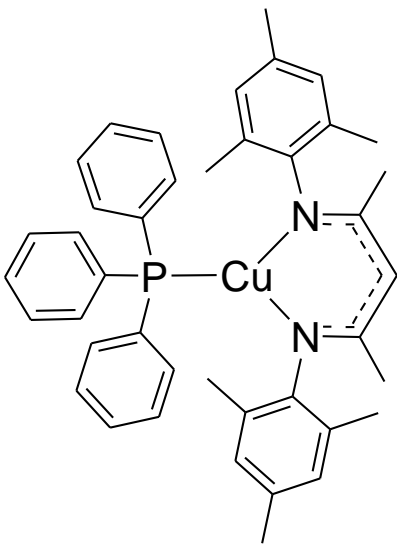

XVI

Abbildung 35: Kupfer(I)-Komplexe mit trigonal planar koordinierten $\mathrm{Cu}^{\prime}$ und Phosphin als Co-Ligand.

Durch den engen $\mathrm{N}-\mathrm{Cu}-\mathrm{N}-$ Winkel von $91.54^{\circ}$ sind die beiden Winkel zum Phosphoratom auf $127.70^{\circ}$ bzw. $140.19^{\circ}$ aufgeweitet (Tabelle 8). Aus der Tatsache, dass die beiden großen Winkel zum $\mathrm{PMe}_{3}$-Molekül sich so stark unterscheiden, sieht man, dass der Co-Ligand etwas nach außen gedrängt wird. Ein Imidazolarm ist, wie schon bei allen Kupfer(II)-Komplexen, nicht an der Koordination zum Metallion beteiligt und nach hinten weggedreht. Die beiden inneren Kupferzentren binden an ein Pyrazolstickstoffatom und werden außerdem von zwei Imidazolringen des jeweils anderen Liganden koordiniert. Dadurch werden die beiden inneren Metallionen in einem Abstand von 3.325(1) ^ zueinander gehalten. Die pyrazolverbrückten Kupfer(I)-Ionen sind 3.505(1) $\AA$ voneinander entfernt. Dieser Wert liegt damit geringfügig über den Distanzen, die für alle Kupfer(II)-Komplexe erhalten wurden. Aufgrund der anders gearteten Bindungssituation werden diese Kupfer(I)-Ionen etwas weiter voneinander separiert. 


\begin{tabular}{|c|c|c|c|}
\hline \multicolumn{4}{|c|}{ Abstände $[\AA ̊]$} \\
\hline Cu1-N1 & $1.972(4)$ & Cu2-N2 & $1.934(4)$ \\
\hline Cu1-N3 & $2.050(4)$ & Cu2-N7' & $1.998(4)$ \\
\hline Cu1-P2 & $2.1759(16)$ & Cu2-N9' & $2.012(4)$ \\
\hline Cu1'.Cu2 & $3.505(1)$ & $\mathrm{Cu} 2{ }^{\prime \prime} \mathrm{Cu} 2^{\prime}$ & $3.325(1)$ \\
\hline \multicolumn{4}{|c|}{ Winkel $\left[^{\circ}\right]$} \\
\hline N1-Cu1-N3 & $91.54(16)$ & N2-Cu2-N7' & $133.97(16)$ \\
\hline N1-Cu1-P2 & $140.19(12)$ & N2-Cu2-N9' & $131.63(16)$ \\
\hline N3-Cu1-P2 & $127.70(13)$ & $\mathrm{N} 7^{\prime}-\mathrm{Cu} 2-\mathrm{N} 9^{\prime}$ & $94.41(16)$ \\
\hline
\end{tabular}

Tabelle 8: Ausgewählte Bindungslängen (in Å) und Bindungswinkel (in º) von 16.

Auch bei den inneren Metallzentren ist der $\mathrm{N}^{\mathrm{Im}}-\mathrm{Cu2}-\mathrm{N}^{\mathrm{Im}}$-Winkel mit $94.41^{\circ}$ kleiner als in einer idealen trigonal-planaren Geometrie. Dadurch sind auch hier die zwei anderen Winkel zum N-Donor des Pyrazols auf $131.63^{\circ}$ bzw. $133.97^{\circ}$ aufgeweitet. Allerdings ist der Unterschied zwischen beiden Werten nicht so deutlich wie im Fall der äußeren Kupfer(I)-lonen. Alle drei N-Cu-Bindungen sind mit 1.934(4) $\AA$ bis 2.012(4) $\AA$ in dem erwarteten Bereich solcher Bindungen, wobei die $\mathrm{N}^{\mathrm{Im}}$-Cu-Bindungen geringfügig länger sind als die $\mathrm{N}^{\mathrm{Pz}}$-Cu-Bindung. Dies lässt sich mit der negativen Ladung im Pyrazolring begründen.

Nimmt man von einer Lösung der Kristalle des Kupfer(I)-Komplexes 16 erneut ein ESI-Massenspektrum auf, erhält man ein nahezu identisches Ergebnis. Auch hier findet man wieder Signale bei $\mathrm{m} / \mathrm{z}=1363.6$ für $\left[\mathrm{L}^{3} \mathrm{Cu}_{2}\left(\mathrm{PMe}_{3}\right)_{2}\right]^{+}, \mathrm{m} / \mathrm{z}=1287.4$ für $\left[\mathrm{L}^{3} \mathrm{Cu}_{2}\left(\mathrm{PMe}_{3}\right)\right]^{+}, \mathrm{m} / \mathrm{z}=1223.5$ für $\left[\mathrm{L}^{3} \mathrm{Cu}\left(\mathrm{PMe}_{3}\right)\right]^{+}$und $\mathrm{m} / \mathrm{z}=1147.4$ für $\left[\mathrm{L}^{3} \mathrm{Cu}\right]^{+}$. Außerdem konnte durch ein hochaufgelöstes ESI-Massenspektrum die getroffene Zuordnung eindeutig bestätigt werden. In Abbildung 36 ist ein Ausschnitt dieses hochaufgelösten ESI-Massenspektrums von 16 gezeigt. 

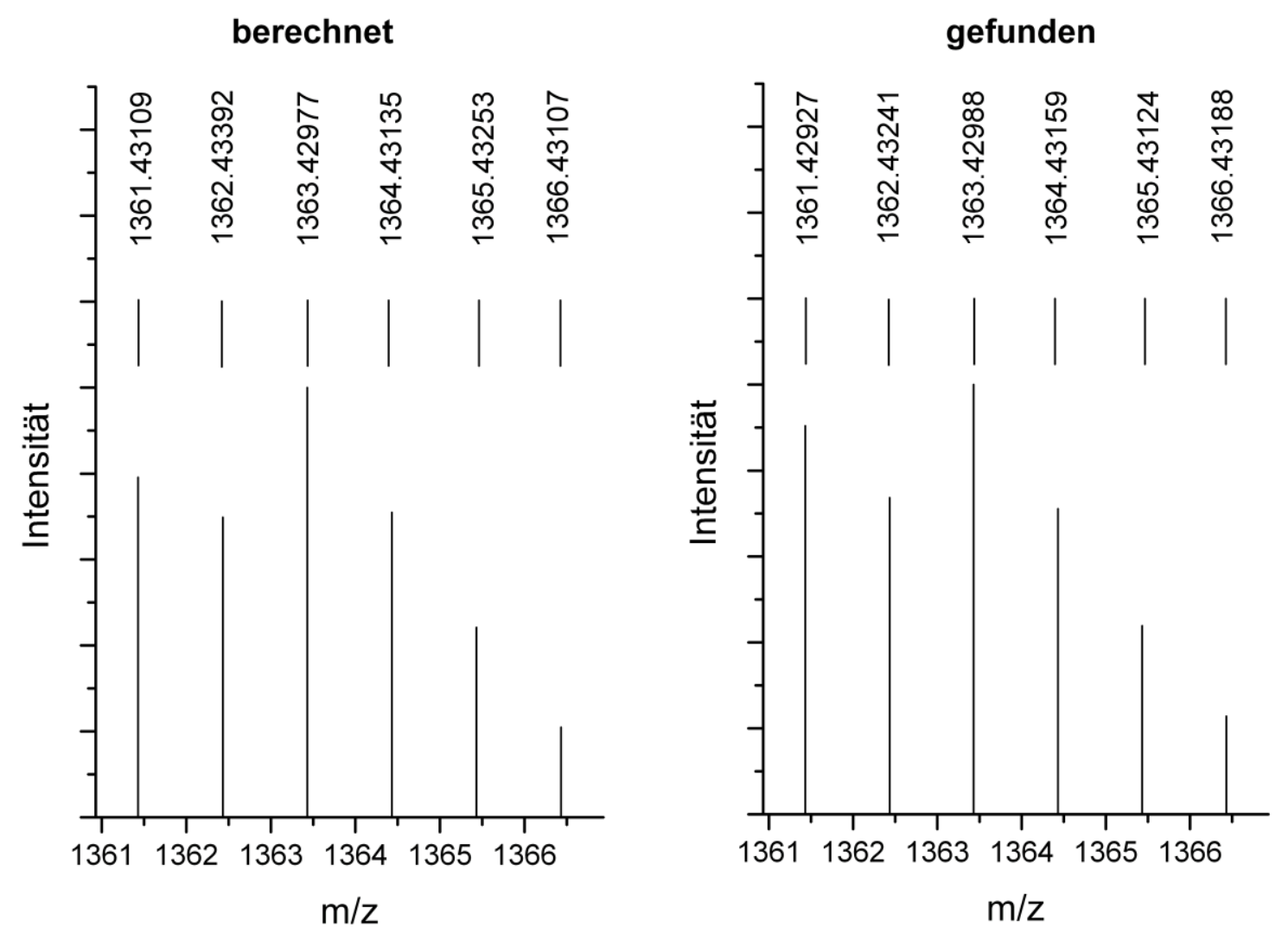

Abbildung 36: Ausschnitt aus dem HR-ESI-Massenspektrum von 16 in $\mathrm{CH}_{3} \mathrm{CN}$. Gezeigt ist die berechnete und experimentell gefundene Isotopenverteilung für das lon $\left[\mathrm{L}^{3} \mathrm{Cu}_{2}\left(\mathrm{PMe}_{3}\right)_{2}\right]^{+}$.

Wie oben bereits angedeutet eignen sich diverse Isonitrile ebenfalls als mögliche Co-Liganden für die Synthese stabiler Kupfer(I)-Komplexe. Aufgrund des partiell negativ geladenen Kohlenstoffatoms der Nitril-Funktion ergeben sich gute Donoreigenschaften derartiger Verbindungen und somit stabile Kupfer(I)-Komplexe.

Reaktionen für die Darstellung von Isonitrilen sind oftmals mit dem Einsatz sehr giftiger Ausgangsverbindungen verbunden. Ein Beispiel dafür ist die Synthese von Ethylisocyanid aus Ethyliodid, Silbercyanid und Kaliumcyanid in wässriger Lösung (Schema 21). ${ }^{[127]}$

$$
\widehat{\mathrm{I}}+\mathrm{AgCN}+2 \mathrm{KCN} \longrightarrow \widehat{\mathrm{NC}}+\mathrm{KAg}(\mathrm{CN})_{2}+\mathrm{KI}
$$

Schema 21: Synthese von Ethylisonitril 
Auch die Synthese von Methylisonitril aus N-Methylformamid, Chinolin und p-Toluolsulfonsäurechlorid ist nicht ohne Gefahr, da das Isonitril zum Einen toxisch ist und zum Anderen die destillative Reinigung hinter einer Schutzscheibe durchgeführt werden muss. ${ }^{[128]}$ Damit die Gefahren minimiert werden können, wurden die Komplexversuche mit käuflichen Isonitrilen in der Glovebox durchgeführt.

Zu den käuflichen Isonitrilen gehören unter anderem n-Butylisonitril und 2,6-Dimethylphenylisonitril. Analog zur oben beschriebenen Synthese von 16 wurde auch für diese Co-Liganden verfahren. Das aromatische Isonitril liegt bei Raumtemperatur als Feststoff vor und muss deshalb in Acetonitril gelöst werden, bevor es zur Reaktionslösung zugegeben wird. Da lediglich der eingesetzte Co-Ligand variiert, ist es nicht sehr überraschend, dass die nach Kristallisation aus Dichlormethan/Diethylether erhaltenen Strukturen der Komplexe 17 und 18 der von Verbindung 16 stark ähneln. Die bei der Röntgenstrukturanalyse erhaltene Kristallstruktur für $\mathbf{1 7}$ ist in Abbildung 37 und die Struktur von 18 ist in Abbildung 38 gezeigt.

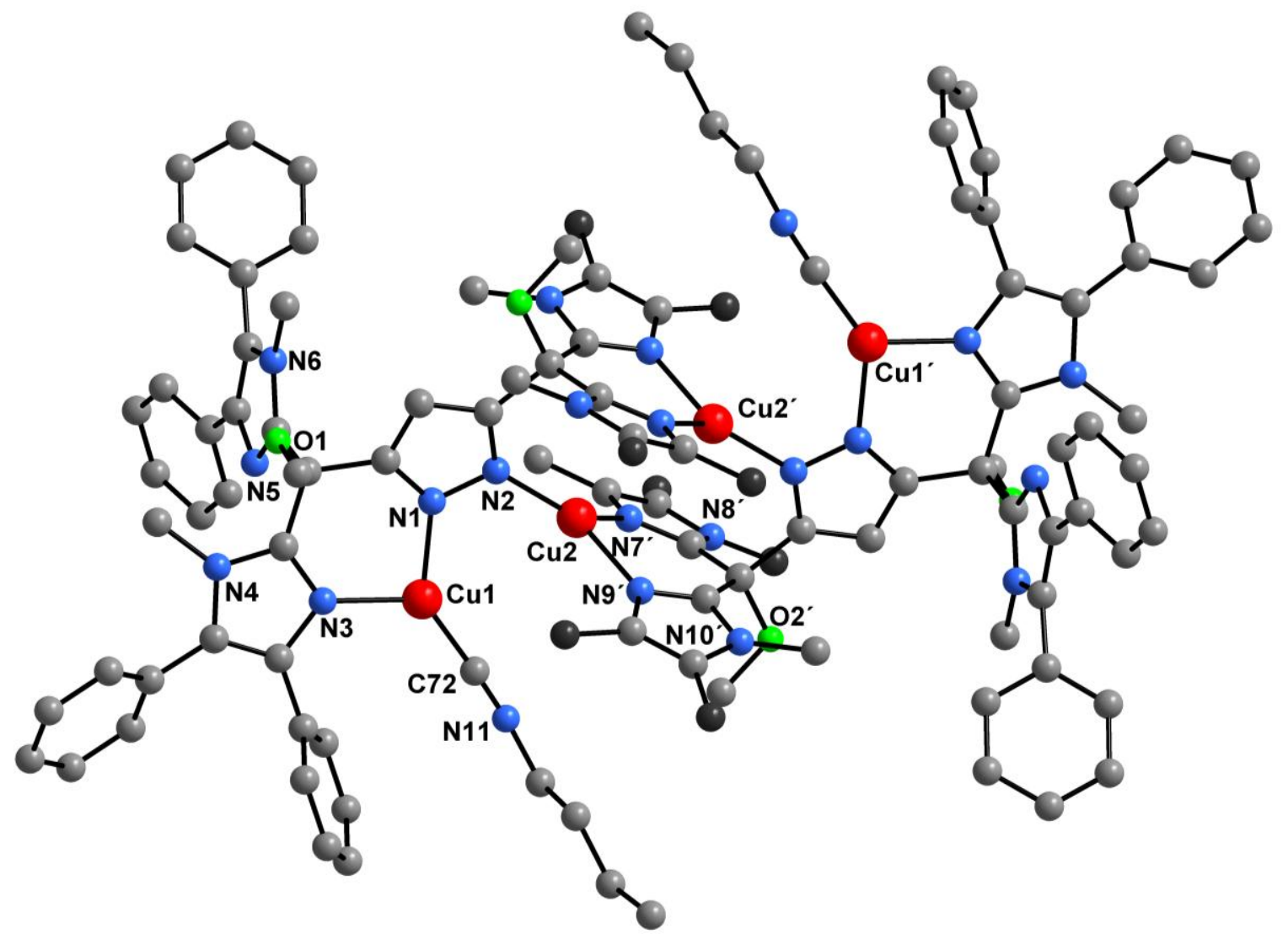

Abbildung 37: Festkörperstruktur des Dikations von 17 (Für bessere Übersichtlichkeit sind die Phenylringe der vier mittleren Imidazoleinheiten nur durch dunkelgraue Kugeln angedeutet und alle H-Atome nicht dargestellt). 


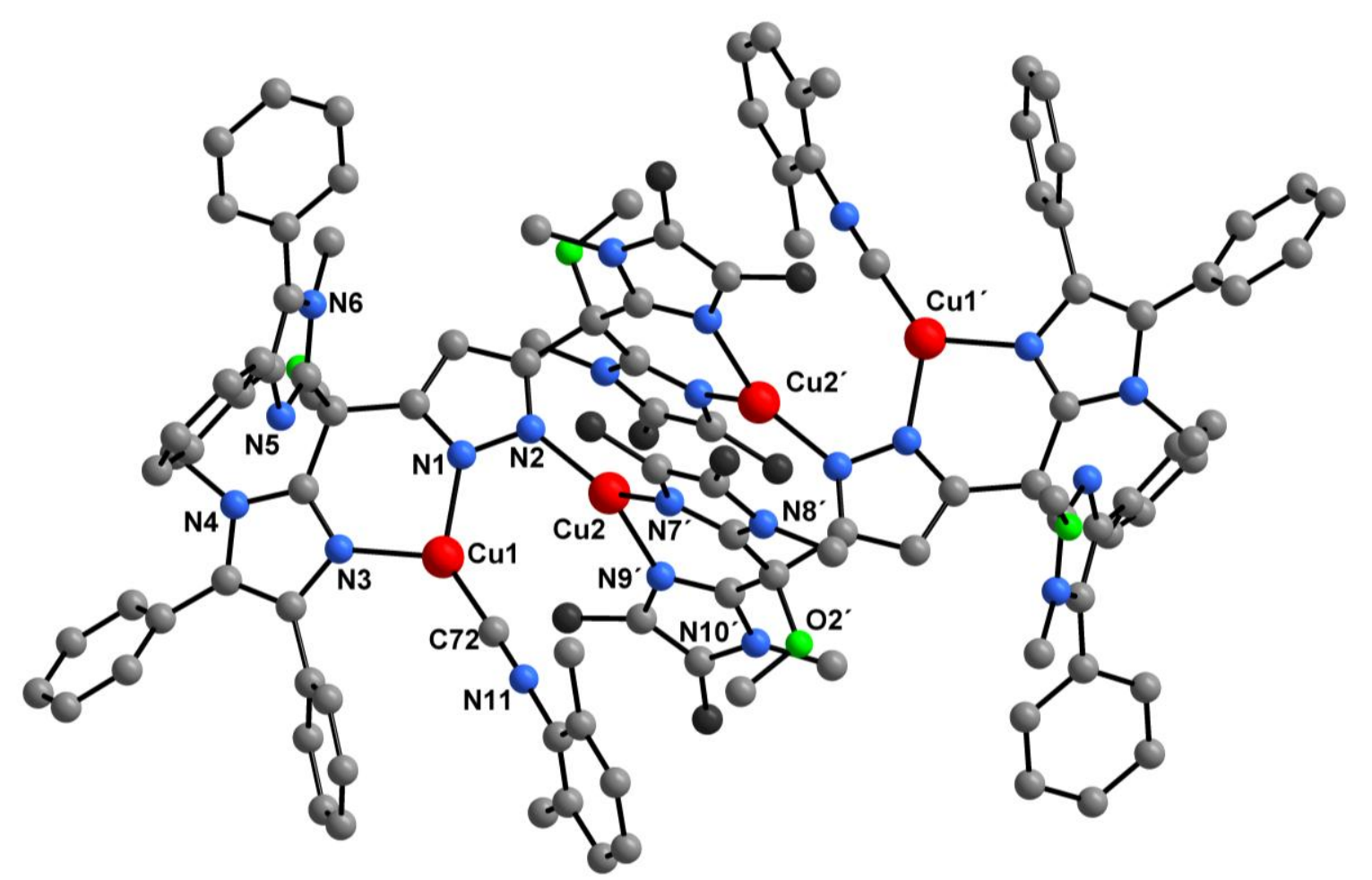

Abbildung 38: Festkörperstruktur des Dikations von 18 (Zur besseren Übersichtlichkeit sind die Phenylringe der vier mittleren Imidazoleinheiten nur durch dunkelgraue Kugeln angedeutet du alle $\mathrm{H}$-Atome nicht dargestellt)

In beiden Verbindungen $\mathbf{1 7}$ und $\mathbf{1 8}$ sind alle vier Kupfer(l)-lonen trigonal planar von den entsprechenden Donoratomen umgeben. Die Position des Phosphins in 16 wird nun von dem Kohlenstoffatom der Isonitrileinheit eingenommen.

In der Literatur sind bereits einige Kupfer(I)-Isonitril-Komplexe kristallografisch aufgeklärt. Darunter sind sowohl Komplexe, in denen das Kupfer(I)-Ion trigonal planar

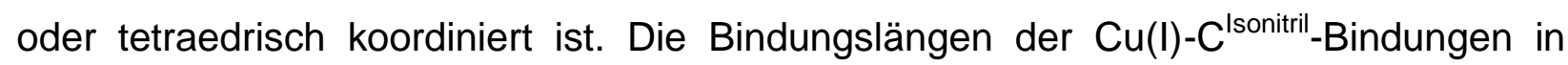
Komplexen mit tetraedrisch koordinierten Kupfer(I)-lonen sind um ca. $0.1 \AA$ länger als die für analoge Verbindungen mit trigonal planar koordinierten Kupfer(I)-lonen. Dies entspricht den Erwartungen, da die Elektronendichte über mehr Bindungspartner verteilt ist. In Abbildung 39 sind exemplarisch die literaturbekannten Kupfer(I)-IsonitrilKomplexe $\mathbf{X V I I},{ }^{[129]} \mathbf{X V I I I ,}{ }^{[126]} \mathbf{X I X}^{[130]}, \mathbf{X X}^{[125]}$ und $\mathbf{X X I}$ mit den dazugehörigen Bindungslängen zu den Isonitrilen gezeigt. 
<smiles></smiles>

XVII

$d\left(\mathrm{Cu}-\mathrm{C}^{\text {Isonitril }}\right)=1.849(3) \AA$

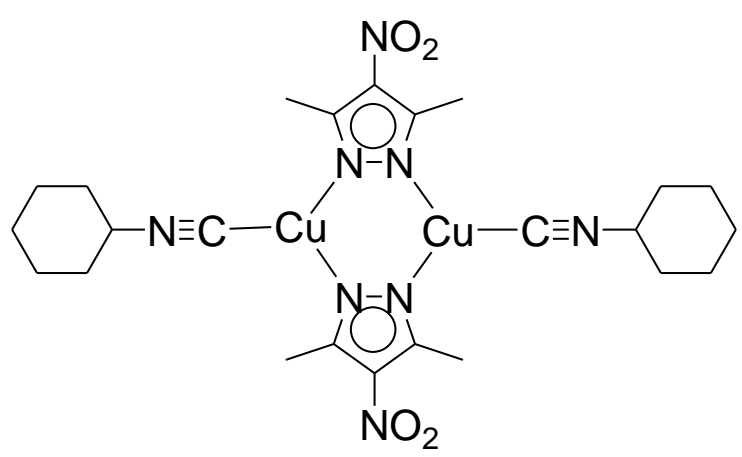

XVIII

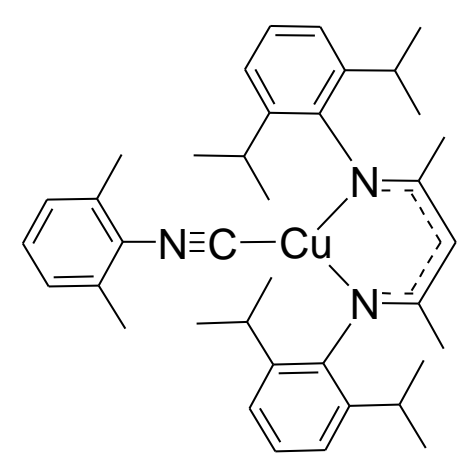

XIX

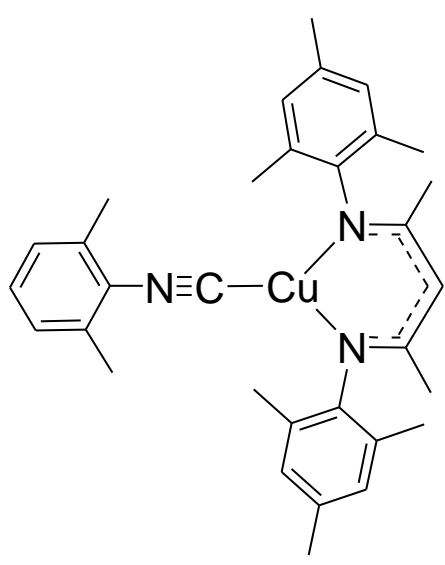

$\mathbf{X X}$

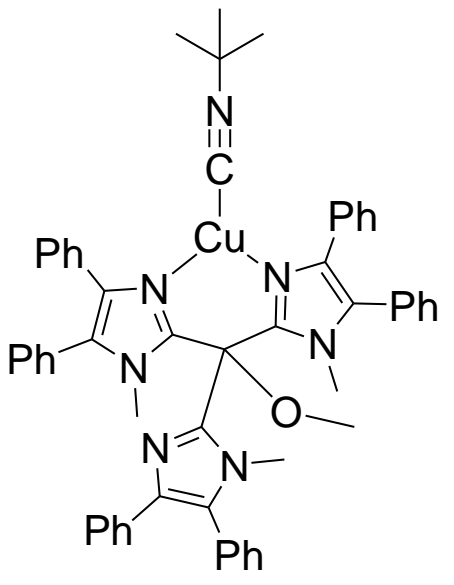

$\mathbf{X X I}$

$d\left(\mathrm{Cu}-\mathrm{C}^{\text {|sonitril }}\right)=1.817(2) \AA \quad d\left(\mathrm{Cu}-\mathrm{C}^{\text {Isonitril }}\right)=1.830(3) \AA \quad d\left(\mathrm{Cu}-\mathrm{C}^{\text {lsonitril }}\right)=1.824(2) \AA$

Abbildung 39: Literaturbekannte Kupfer(I)-Isonitril-Komplexe mit trigonal planarer Koordination.

Anhand der in Tabelle 9 zusammengefassten Bindungswinkel und -längen für $\mathbf{1 7}$ und 18 ist ersichtlich, dass die Cu1-C72-Bindung mit 1.823(4) $\AA$ ( $n$-Butylisocyanid) bzw. 1.818(2) $\AA$ (2,6-Dimethylphenylisonitril) viel kürzer ist, als alle anderen Bindungen zu den Kupfer(I)-Zentren, die für $\mathbf{1 7}$ und $\mathbf{1 8}$ gefunden wurden. Bei einem Vergleich mit bisher bekannten Verbindungen, die solch eine Einheit beinhalten, fällt auf, dass es sich um eine der Kürzesten überhaupt handelt. ${ }^{[126,129-136]}$ 


\begin{tabular}{|c|c|c|}
\hline \multicolumn{3}{|c|}{ Abstände $[\AA ̊]$} \\
\hline $1.942(3)$ & Cu1-N1 & $1.9547(16)$ \\
\hline $2.001(3)$ & Cu1-N3 & $1.9838(18)$ \\
\hline $1.823(4)$ & Cu1-C72 & $1.818(2)$ \\
\hline $1.927(3)$ & Cu2-N2 & $1.9129(17)$ \\
\hline $2.002(3)$ & Cu2-N7' & $2.0029(18)$ \\
\hline $2.024(3)$ & Cu2-N9' & $1.9993(17)$ \\
\hline $3.3656(6)$ & Cu1 $\cdots$ Cu2 & $3.2752(4)$ \\
\hline $3.2742(8)$ & Cu2'ㄷ Cu2' & $3.2878(6)$ \\
\hline \multicolumn{3}{|c|}{ Winkel $\left[^{\circ}\right]$} \\
\hline $92.71(11)$ & N1-Cu1-N3 & $91.96(7)$ \\
\hline $139.21(14)$ & N1-Cu1-C72 & $136.59(9)$ \\
\hline $128.05(14)$ & N3-Cu1-C72 & $131.25(8)$ \\
\hline $135.66(11)$ & N2-Cu1-N7' & $131.07(7)$ \\
\hline $132.31(11)$ & N2-Cu1-N9' & $136.24(7)$ \\
\hline $92.00(11)$ & N7'-Cu1-N9' & $92.66(7)$ \\
\hline
\end{tabular}

Tabelle 9: Ausgewählte Bindungslängen (in $\AA$ ) und Bindungswinkel (in ) von 17 und 18.

Für Komplexverbindungen, die Isonitril enthalten, ist die IR-Spektroskopie ein probates Mittel zur Analyse. Die CN-Dreifachbindung des Isonitrils kann anhand der sehr intensiven Bande zwischen $2100 \mathrm{~cm}^{-1}$ und $2200 \mathrm{~cm}^{-1}$ recht eindeutig identifiziert werden, da in der Regel kaum Absorptionen in diesem Bereich des IR-Spektrums auftreten. Für die oben gezeigten Verbindungen $\mathbf{X V I I}, \mathbf{X I X}, \mathbf{X X}$ und $\mathbf{X X \mathbf { I }}$ sind in Tabelle 10 die IR-Absorptionen der CN-Dreifachbindung des Isonitrils zu sehen.

\begin{tabular}{ccccc} 
Komplex & $\mathbf{X V I I}$ & $\mathbf{X I X}$ & $\mathbf{X X}$ & $\mathbf{X X I}$ \\
\hline$\tilde{v}\left[\mathrm{~cm}^{-1}\right]$ & 2170 & 2126 & 2121 & 2186 \\
\hline
\end{tabular}

Tabelle 10: IR-Absorptionen für ausgewählte Kupfer(I)-Isonitril-Komplexe. 
Die IR-Spektren für $\mathbf{1 7}$ und $\mathbf{1 8}$ sind in Abbildung 40 zu sehen. Es fällt vor allem der deutliche Unterschied für die Absorption der CN-Funktion des Isonitrils auf $\left(2196 \mathrm{~cm}^{-1}\right.$ und $2155 \mathrm{~cm}^{-1}$ ). Wie auch bei den in Tabelle 10 gezeigten Werten ergeben sich größere Werte für die Wellenzahl, wenn ein aliphatisches Isocyanid koordiniert ist.
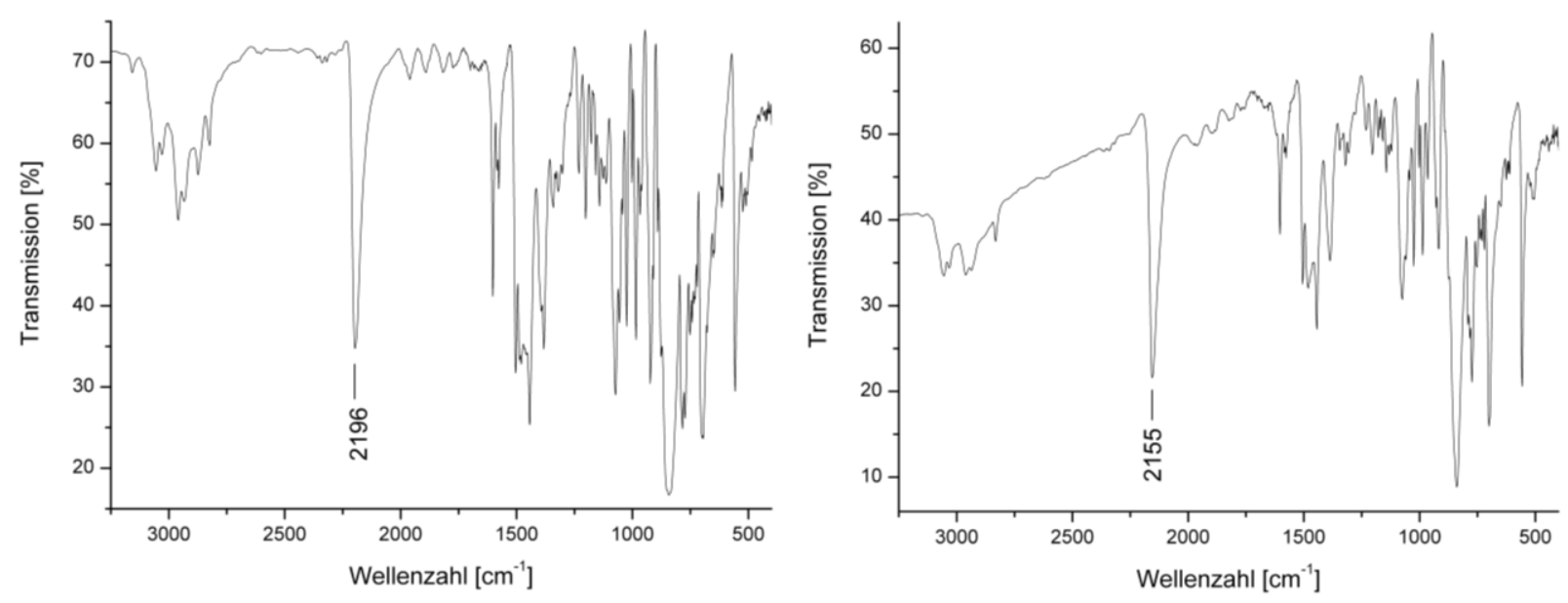

Abbildung 40: IR-Spektren von 17 (links) und 18 (rechts) als KBr-Presslinge.

Aufgrund der größeren Wellenzahl im Fall des aliphatischen Isonitrils lässt sich ableiten, dass für die Anregung der $\mathrm{C} \equiv \mathrm{N}$-Bindung etwas mehr Energie benötigt wird als für die analoge Bindung des substituierten Phenylisonitrils. Diese Bindung muss demnach in 17 etwas kürzer sein. Die Bestätigung liefert die Analyse der Strukturdaten. Für das aliphatische Isonitril lässt sich eine $C \equiv N$-Bindungslänge von 1.146(5) $\AA$ finden und die des aromatischen Isonitrils ist mit 1.165(3) $\AA$ etwas länger. Die $C \equiv N$-Bindung ist für das $n$-Butylisonitril geringfügig elektronenreicher, was für mehr $\pi$-Rückbindung vom Kupfer(I)-Ion zum Isonitril spricht. Jedoch wird es sicherlich entscheidend sein, dass für 2,6-Dimethylphenylisonitril aufgrund des konjugierten Systems die $\pi$-Elektronen über das gesamte System delokalisiert sind.

Die Absorption der C $\equiv N$-Bindung des freien $n$-Butylisonitrils ist bei $\tilde{v}_{\mathrm{CN}}=2149 \mathrm{~cm}^{-1} \mathrm{zu}$ finden und bei $\tilde{v}_{\mathrm{CN}}=2121 \mathrm{~cm}^{-1}$ kann man eine sehr intensive Absorption für die $\mathrm{C} \equiv \mathrm{N}$ Bindung des freien 2,6-Dimethylphenylisonitrils identifizieren. Es lassen sich somit eine große Differenz $\left(47 \mathrm{~cm}^{-1}\right)$ für das aliphatische Isonitril und ein kleinerer Unterschied im Fall des aromatischen Isonitrils $\left(34 \mathrm{~cm}^{-1}\right)$ ausmachen. Ohne Kenntnis der kristallografischen Daten für beide freien Isonitrile ist es schwierig, eine genaue Aussage über die 
$\mathrm{C} \equiv \mathrm{N}$-Bindungslänge zu treffen. Das hier verwendete aliphatische Isonitril ist zudem eine Flüssigkeit bei Raumtemperatur und dadurch eine Strukturbestimmung sehr schwierig. Sowohl für das aliphatische als auch das aromatische Isonitril kommt es zu einer hypsochromen Verschiebung. Im Fall des aromatischen Isonitrils beträgt diese ca. $35 \mathrm{~cm}^{-1}$ und für das alphatische Isonitril ist die Verschiebung etwa $47 \mathrm{~cm}^{-1}$. Diese Beobachtungen stehen im Einklang mit den Ergebnissen für Kupfer(I)-Komplexe mit terminal gebundenem Isonitril, die als Zwei-Elektronen $\sigma$-Donor fungieren und nur eine geringe $\pi$-Rückbindung aufbauen. ${ }^{[137-140]}$ Die Eigenschaft von Isonitrilen als $\pi$-Akzeptor zu wirken hängt von der Natur des am Stickstoffatom gebundenen Restes ab. Die Rückbindung erfolgt in zwei orthogonale $\pi^{\star}$-Orbitale der CミN-Bindung. ${ }^{[141]}$

Analog zu Komplex 16 lassen sich 17 und 18 gut mit ESI-Massenspektrometrie analysieren. Für $\mathbf{1 7}$ zählen zu den erhaltenen Fragmenten: $\mathrm{m} / \mathrm{z}=1375.6$ für $\left[\mathrm{L}^{3} \mathrm{Cu}_{2}\left({ }^{n} \mathrm{BuNC}\right)_{2}\right]^{+}, \mathrm{m} / \mathrm{z}=1292.3$ für $\left[\mathrm{L}^{3} \mathrm{Cu}_{2}\left({ }^{n} \mathrm{BuNC}\right)\right]^{+}$und 1147.5 für das Fragment $\left[\mathrm{L}^{3} \mathrm{Cu}\right]^{+}$. Die Bestätigung der Zuordnung ergibt sich durch Aufnahme eines hochaufgelösten ESI-Spektrums (Abbildung 41).
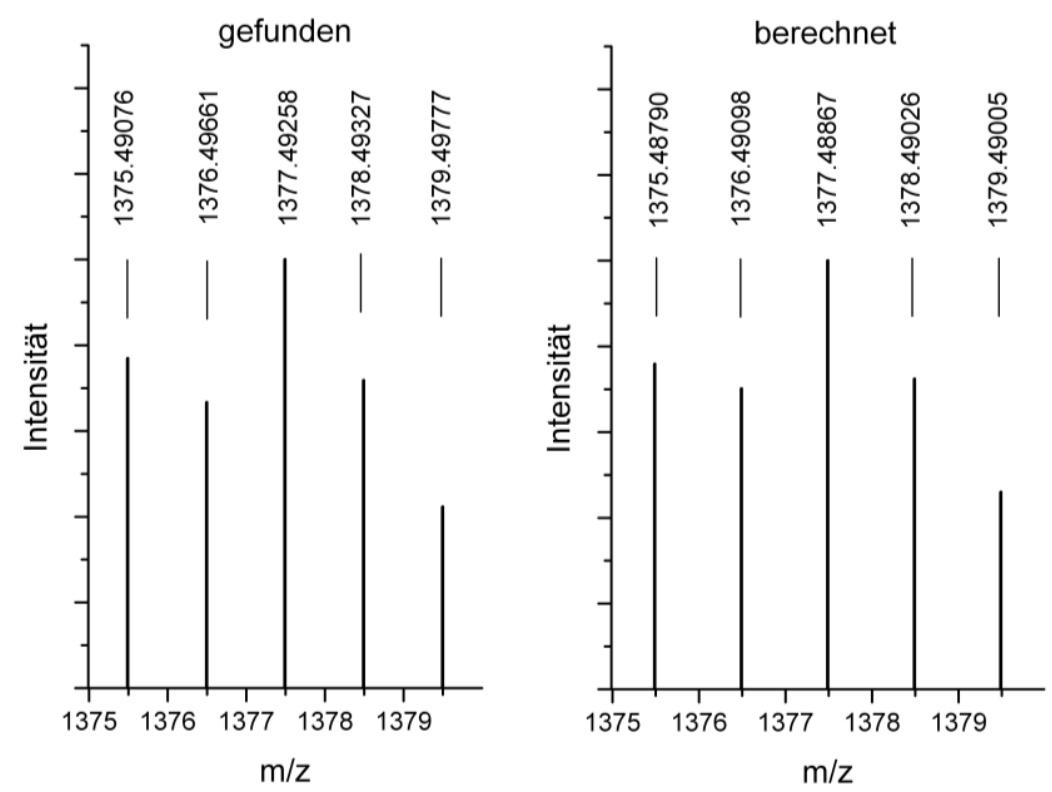

Abbildung 41: Ausschnitt aus dem HR-ESI-Massenspektrum von 17 in $\mathrm{CH}_{3} \mathrm{CN}$. Gezeigt ist die berechnete und experimentell gefundene Isotopenverteilung für das Ion $\left[\mathrm{L}^{3} \mathrm{Cu}_{2}\left({ }^{n} \mathrm{BuNC}\right)_{2}\right]^{+}$. 
Die berechneten Isotopenmuster stimmen für $\left[\mathrm{L}^{3} \mathrm{Cu}_{2}\left({ }^{n} \mathrm{BuNC}\right)_{2}\right]^{+}$und $\left[\mathrm{HL}^{3} \mathrm{Cu}_{2}\left({ }^{n} \mathrm{BuNC}\right)_{2}\right]^{2+}$ sehr gut mit den gefundenen Werten überein. In Abbildung 41 ist das Ergebnis für das Fragment $\left[\mathrm{L}^{3} \mathrm{Cu}_{2}\left({ }^{n} \mathrm{BuNC}\right)_{2}\right]^{+}$gezeigt.

Von 18 ist unter anderem ein hochauflösendes ESI-Massenspektrum aufgenommen worden. Dabei konnte ein Peak bei $m / z=1340.41943$ beobachtet werden. Die einzelnen Signale dieses Peaks haben einen Unterschied von jeweils $m / z=0.5$. Dies spricht für eine zweifach geladene Spezies. Durch Simulation des Isotopenmusters konnte schließlich dieser Peak dem Fragment $\left[\mathrm{L}^{3} \mathrm{Cu}_{2}\left(\mathrm{C}_{6} \mathrm{H}_{3}\left(\mathrm{CH}_{3}\right)_{2}\right)\right]_{2}{ }^{2+}$ zugeschrieben werden. Es handelt sich dabei um den Komplex 18 abzüglich beider $\mathrm{PF}_{6}$-Gegenionen. Damit konnte erstmal ein Hinweis erhalten werden, dass der vierkernige Kupfer(I)Komplex auch in Lösung vorliegt. Die einfach geladenen Fragmente mit kleinen Molmassen entstehen durch den Zerfall des Komplexes bei der lonisation der Probe im Massenspektrometer. Aufgrund der sehr großen Molmasse der Komplexe 16 bis 18 von etwa $2000 \mathrm{~g}^{\prime} \mathrm{mol}^{-1}$ ist es schwierig, diese im Massenspektrometer zu detektieren. Offensichtlich entstehen bei der Ionisation aller Proben der Kupfer(I)-Komplexe 16 bis 18 bevorzugt die einfach geladenen Spezies des zweikernigen Komplexes.

Bei allen bisher vorgestellten Kupfer(I)-Komplexen ist auffällig, dass sie immer ein Verhältnis Ligand:Kupfer:Co-Ligand von 1:2:1 beinhalten. Und das, obwohl ein Verhältnis von 1:2:2 eingesetzt wurde. Da die Versuche mehrmals durchgeführt wurden, ist auszuschließen, dass es sich um einen Fehler beim Einwiegen der Edukte handelt. Möglicherweise liegt ein Gleichgewicht bei der Komplexbildung vor. Damit die Co-Liganden an beide Kupferionen gebunden werden können und somit dinukleare Kupfer(I)-Komplexe entstehen, wie in Schema 20 gezeigt, kann man die Menge an Co-Ligand von zwei auf vier Äquivalente (Verhältnis Ligand:Kupfer:Co-Ligand von 1:2:4) erhöhen. Setzt man zur Komplexbildung vier statt zwei Äquivalente Trimethylphosphin zu und bringt den Komplexansatz mit $\mathrm{HL}^{1}$ zum Kristallisieren aus Dichlormethan/Diethylether, so erhält man ebenfalls fast farblose Kristalle. Sie sind für die Röntgenstrukturanalyse geeignet und ergeben die in Abbildung 42 gezeigte Struktur von Koordinationsverbindung 19. 


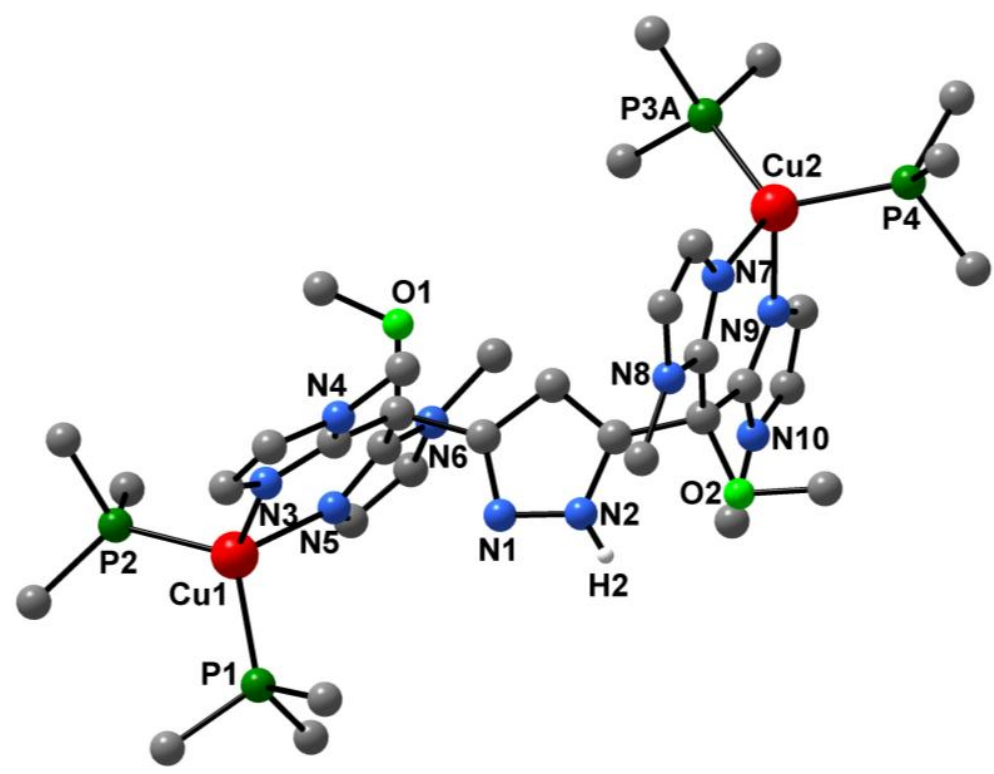

Abbildung 42: Festkörperstruktur des Dikations von 19 (Wegen der Übersichtlichkeit wurden alle $\mathrm{H}$-Atome bis auf das NH-Proton nicht abgebildet).

Dieser Komplex von Ligand $\mathrm{HL}^{1}$ enthält zwei Kupfer(I)-Ionen und insgesamt 4 Moleküle Trimethylphosphin. Desweiteren ist auffällig, dass der Pyrazolring das NH-Proton noch trägt, obwohl während der Reaktion ein Äquivalent Base zugegeben wurde. Beide Kupferzentren sind vom Pyrazolring weggedreht und es entsteht eine Distanz von 10.546(1) ^ zwischen den Metallionen. Die Koordination der Kupfer(I)-lonen erfolgt daher von beiden Imidazolringen jeder Ligandseite, außerdem sind noch zwei Moleküle Trimethylphosphin an jedes Kupferzentrum gebunden. Es entsteht somit eine verzerrt tetraedrische Koordinationsumgebung. In Tabelle 11 sind einige interessante und wichtige Bindungslängen und -winkel für diese Verbindung aufgelistet.

\begin{tabular}{llll}
\multicolumn{4}{c}{ Abstände $[\AA]$} \\
\hline Cu1-N3 & $2.038(5)$ & Cu2-N7 & $2.018(5)$ \\
Cu1-N5 & $2.044(5)$ & Cu2-N9 & $2.061(5)$ \\
Cu1-P2 & $2.2106(18)$ & Cu2-P3A & $2.205(2)$ \\
Cu1-P1 & $2.2263(17)$ & Cu2-P4 & $2.2568(18)$ \\
Cu1 $\cdots$ Cu2 & $10.546(1)$ & &
\end{tabular}




\begin{tabular}{llll}
\multicolumn{4}{c}{ Winkel $\left[^{\circ}\right]$} \\
\hline N3-Cu1-N5 & $88.45(18)$ & N7-Cu2-N9de & $88.61(18)$ \\
N3-Cu1-P2 & $115.67(16)$ & N7-Cu2-P3A & $120.86(16)$ \\
N5-Cu1-P2 & $112.84(13)$ & N9-Cu2-P3A & $103.37(14)$ \\
N3-Cu1-P1 & $105.86(15)$ & N7-Cu2-P4 & $112.31(15)$ \\
N5-Cu1-P1 & $111.07(14)$ & N9-Cu2-P4 & $106.37(15)$ \\
P2-Cu1-P1 & $118.81(8)$ & P3A-Cu2-P4 & $118.65(8)$ \\
\hline
\end{tabular}

Tabelle 11: Ausgewählte Bindungslängen (in Å) und Bindungswinkel (in º) von 19.

Als Alternative zum üblichen Gebrauch von $\mathrm{KO}^{t} \mathrm{Bu}$ als Base wurde Tris(triphenylphosphan)-Kupfer(I)-Phenolat eingesetzt. Hierbei sollte das Phenolation als interne Base wirken und das Pyrazol unter Bildung von Phenol deprotonieren. Danach wäre eine Koordination des Kupfer(I)-lons an die N-Donoratome möglich.

Es fand eine Umsetzung des Liganden $\mathrm{HL}^{1}$ mit dem oben beschriebenen Kupfer(I)-Phenolat und einem Äquivalent Tetrakis(acetonitril)-Kupfer(I)-Perchlorat in Acetonitril statt. Die nach der Kristallisation des Komplexes 20 aus Dichlormethan-Diethylether erhaltene Struktur ist in Abbildung 43 dargestellt. Es ist ein vierkerniger Kupferkomplex mit zwei Ligandmolekülen entstanden. Zwei der Kupfer-Ionen liegen in der Oxidationsstufe +2 vor und die anderen beiden Kupfer-Ionen besitzen die Oxidationsstufe +1 . Letztere werden jeweils von zwei Molekülen Triphenylphosphan stabilisiert. Hier liegt eine stark verzerrte Tetraedergeometrie vor.

Neben den zwei Triphenylphosphanmolekülen werden die Kupfer(I)-Ionen von zwei Imidazolringen einer Ligandseite koordiniert. Wie anhand der Werte in Tabelle 12 ersichtlich ist, liegen die Winkel im Tetraeder zwischen $89.85^{\circ}$ für den N3-Cu2-N5-Winkel und $118.42^{\circ}$ für den P1-Cu2-P2-Winkel. 


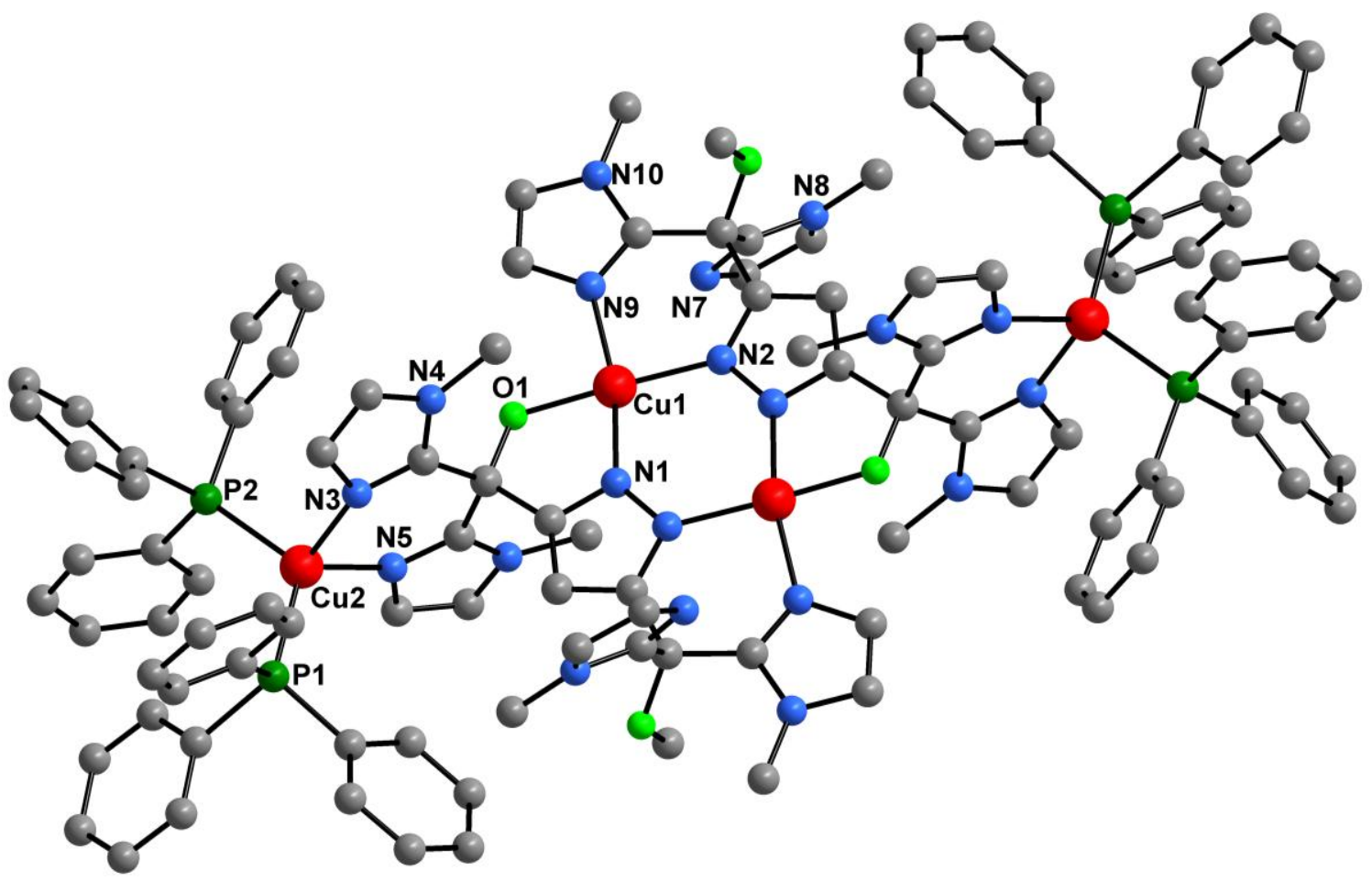

Abbildung 43: Festkörperstruktur des Dikations von 20 (Wegen der Übersichtlichkeit sind keine H-Atome dargestellt).

Die inneren Kupferionen sind während der Synthese von der Oxidationsstufe +1 in die Oxidationsstufe +2 oxidiert worden. Beide Kupfer-lonen sind quadratisch planar koordiniert und werden von zwei Pyrazolringen in einem Abstand von 3.8788(8) $\AA$ überbrückt. Die dritte und vierte Koordinationsstelle wird von einem N-Donor des Imidazolringes und von einem O-Donor des gespaltenen Methylethers gebildet. Während der Komplexsynthese ist auf einer Seite des Liganden die Etherfunktion gespalten worden. Es ist dabei eine Alkoholateinheit entstanden, die letztendlich an das Kupfer(II)-Ion koordiniert. Das Sauerstoffdonoratom und das koordinierte Imidazolstickstoffatom stehen jeweils um ca. $13^{\circ}$ aus der Kupfer-Pyrazol-Ebene heraus. Alle vier Cu1-Donor-Bindungen sind mit $1.91 \AA$ bis $1.97 \AA$ in etwa gleich lang. Aufgrund der sehr geringen Menge, die von diesem Komplex in kristalliner Form erhalten wurde, war es nicht möglich den Mechanismus zur partiellen Oxidation der Kupfer(I)-Ionen zu untersuchen. 


\begin{tabular}{|c|c|c|c|}
\hline \multicolumn{4}{|c|}{ Abstände $[\AA]]$} \\
\hline Cu1-N1 & $1.974(3)$ & Cu2-N3 & $2.044(3)$ \\
\hline Cu1-N2' & $1.948(3)$ & Cu2-N5 & $2.033(3)$ \\
\hline Cu1-N9' & $1.974(3)$ & Cu2-P1 & $2.2479(11)$ \\
\hline Cu1-O1 & $1.910(2)$ & Cu2-P2 & $2.2739(10)$ \\
\hline Cu1'Cu1' & $3.8788(8)$ & Cu1 $\cdots$ Cu2 & $6.3997(6)$ \\
\hline $\mathrm{Cu} 2{ }^{\prime \prime} \mathrm{Cu} 2^{\prime}$ & $14.650(1)$ & & \\
\hline \multicolumn{4}{|c|}{ Winkel $\left[^{\circ}\right]$} \\
\hline O1-Cu1-N2' & $170.70(12)$ & N5-Cu2-N3 & $89.85(12)$ \\
\hline N1-Cu1-N9' & $165.87(12)$ & P1-Cu2-P2 & $118.42(4)$ \\
\hline N9'-Cu1-N2' & $90.66(12)$ & N3-Cu2-P2 & $105.83(9)$ \\
\hline O1-Cu1-N1 & $82.99(11)$ & N5-Cu2-P2 & $116.48(10)$ \\
\hline $\mathrm{N} 2{ }^{\prime}-\mathrm{Cu} 1-\mathrm{N} 1$ & $100.18(11)$ & N3-Cu2-P1 & 112.53(9) \\
\hline O1-Cu1-N9' & $87.72(11)$ & N5-Cu2-P2 & $109.67(9)$ \\
\hline
\end{tabular}

Tabelle 12: Ausgewählte Bindungslängen (in $\AA$ ) und Bindungswinkel (in º) von 20. 
Mit dem Ligand $\mathrm{HL}^{3}$ ist es gelungen pyrazolatverbrückte Kupfer(l)-Komplexe zu synthetisieren und strukturell wie auch spektroskopisch aufzuklären. Dabei sind $\mathrm{mit}_{\mathrm{HL}}{ }^{3}$ ausschließlich vierkernige Komplexverbindungen erhalten worden. Wie auch schon bei den Kupfer(II)-Komplexen ist ein Strukturmotiv sehr dominant. Es bilden sich stets Verbindungen mit vier trigonal planar koordinierten Kupfer(I)-Ionen. An zwei Kupfer(I)Ionen ist je ein Co-Ligand, wie Trimethylphosphin, ${ }^{n}$ Butylisonitril oder 2,6-Dimethylphenylisonitril, koordiniert. Die beiden anderen beiden Metallionen weisen eine $\{\mathrm{N}\}_{3}-$ Koordination auf.

In Arbeiten von Borovik und Sorrell wird die Synthese eines Kupfer(I)-Komplexes mit einem Tris(imidazolyl)methan-Derivat beschrieben. Die Festkörperstruktur des Komplexes zeigt ein Dimer mit zwei trigonal planar koordinierten Kupfer(I)-Ionen. ${ }^{[20]}$ Ganz ähnlich sind die Ergebnisse, die Zhou bei Verwendung von Tris(1-Methyl-4,5diphenylimidazolyl)methan erhalten hat. Wenn der Ligand mit Kupfer(I)-Salzen umgesetzt wird und kein Co-Ligand beteiligt ist, entsteht ebenfalls der dimere Kupfer(I)Komplex mit trigonal planarer Koordination. Nachdem sie ${ }^{t} \mathrm{BuNC}$ als stabilisierenden Co-Liganden zugegeben hatten, beobachten sie verschiedene Produkte, die abhängig sind von der Natur des Restes am Brückenkopf-Kohlenstoff-Atom. Befindet sich dort eine Methoxy-Einheit, bildet sich erneut eine trigonal planare Koordination um das Kupfer(I)-Ion. Diese im Festkörper gefundene Struktur scheint in Lösung nicht zu existieren. Stattdessen findet ein schneller Austausch der Imidazol-Ringe statt. Handelt es sich bei dem vierten Substituenten am quartären Kohlenstoffatom um ein Wasserstoff-Atom, bildet sich ein vierfach koordinierter mononuklearer Kupfer(I)Komplex. ${ }^{[25]}$ Alle Imidazolringe koordinieren an das Kupferion und die tetraedrische Koordination wird durch die Isonitril-Einheit komplettiert.

Die Bildung der Komplexe 16 bis 18 wird sicherlich in mehreren Schritten ablaufen. So ist es denkbar, dass der deprotonierte Ligand zunächst zwei Kupfer(I)-Ionen bindet und freie Koordinationsstellen mit Lösungsmittel-Molekülen besetzt sind. Von der Reaktionslösung aufgenommene ${ }^{1} \mathrm{H}$ - und ${ }^{13} \mathrm{C}$-Spektren weisen auf dynamische Prozesse in dieser Lösung hin. Es ist möglich, diese Prozesse zu verlangsamen, wenn man die Messtemperatur absenkt. Vollständig einfrieren lassen sich diese in Acetonitril jedoch nicht, da unterhalb von -25 bis $-30{ }^{\circ} \mathrm{C}$ wegen der steigenden Viskosität der 
Lösung. Die Möglichkeit zu einem anderen Lösungsmittel zu wechseln ist ausgeschlossen, da sich der resultierende $\operatorname{Kupfer}(\mathrm{I})$-Komplex nicht ausreichend lösen würde. Aufgrund der freien Drehbarkeit zwischen dem C-Atom in 3- bzw. 5-Position des Pyrazols und dem benachbarten Brückenkopf-C-Atom ist es durchaus vorstellbar, dass es einen permanenten Wechsel der koordinierten Imidazolringe gibt. Da dies auf beiden Seiten simultan oder versetzt erfolgen kann, ergeben sich viele Kupfer(I)-Komplexe, die jeweils geringfügig verschiedene ${ }^{1} \mathrm{H}$ - und ${ }^{13} \mathrm{C}$-NMR-Spektren ergeben sollten.

Im ${ }^{1} \mathrm{H}-\mathrm{NMR}$-Spektrum kristalliner Proben von 16 bis 18 sind einige Signale etwas breiter als in den Spektren der freien Liganden. Außerdem sind mehr als zwei Signale zwischen 3.10 ppm und 3.70 ppm für die OMe- und NMe-Funktionen zu erkennen. Die Phenylgruppen ergeben ein Multiplett zwischen 7.00 ppm und 7.50 ppm. Außerdem gibt es noch drei weitere Signale im Bereich von 6.40 ppm bis 7.00 ppm, die für 16 relativ breit und für $\mathbf{1 7}$ erstaunlich scharf sind. Mittels $\mathrm{C}-\mathrm{H}$-Korrelation konnte eindeutig bestätigt werden, dass es sich um Signale eines Aromaten handelt. Die ${ }^{13} \mathrm{C}-\mathrm{NMR}$ Spektren von kristallinen Proben ergeben jeweils zwei Signale für die NMe-, OMe- und COMe-Einheiten. Dabei fällt auf, dass die Signale für eine funktionelle Gruppe nicht im Verhältnis 1:1 erhalten werden, sondern eher 2:1. Es müssen somit zwei verschiedene Verbindungen nebeneinander vorliegen.

Für die Koordination eines Metallions an alle drei Donoratome einer facial tridentaten Koordinationstasche ist es sicherlich nötig, weichere Donoratome am Ligand anzubringen. Dafür könnte sich zum Beispiel Schwefel eignen. Unter Beibehaltung des während dieser Arbeit synthetisierten Ligandsystems sollte es möglich sein anstelle der Imidazolringe schwefelhaltige Heterozyklen wie Thiazol oder andere substituierte Ringe wie Benzothiazol anzubringen. Daraus resultieren Liganden wie XXII und XXIII, die ein hartes und ein weiches Donoratom beinhalten (Abbildung 44). 


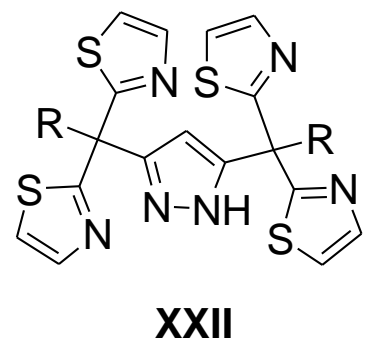<smiles>[R]c1csc(C([R])(c2cc(C([R])(c3nc([R7])sc3[R])c3nc([R])sc3[R7])n[nH]2)c2nc([R7])sc2[R])n1</smiles>

\section{XXIII}

Abbildung 44: Mögliche Ligandsysteme für dinukleare Kupfer-Komplexe.

Je nach eingesetztem Metallsalz können sich die verschiedensten Komplexverbindungen bilden. Vielleicht ist auf diese Weise eher möglich dinukleare Kupfer(I)-Komplexe herzustellen, die sich im Rahmen katalytischer Studien einsetzen lassen. Auch bei diesem System müsste es möglich sein, analog zu Schema 19 die Seitenarme um eine Methylenbrücke zu erweitern, damit das ganze System flexibler wird.

Unter Verwendung des hier genutzten Ligandsystems ist der Versuch die OH-Funktion zu reduzieren ganz reizvoll. Möglicherweise ergeben sich ähnliche Ergebnisse wie von Zhou beobachtet und es entsteht eine dinuklearer Kupfer(I)-Komplex. 


\subsection{Darstellung der Nickel(II)-Komplexe}

Für die Synthese der Nickel(II)-Komplexe wurde ebenfalls der Ligand in einem Lösungsmittel gelöst, $\mathrm{KO}^{t} \mathrm{Bu}$ als Base und zwei Äquivalente des entsprechenden Nickel(II)-Salzes hinzugefügt. Auch hier sollte wieder das erste Äquivalent Base zum Deprotonieren des Liganden dienen und das Zweite durch Deprotonierung eines Äquivalents des Kristallwassers eine OH-Brücke zwischen den Nickelatomen generieren.

Bei Verwendung des Liganden $\mathrm{HL}^{1}$ in Verbindung mit Nickel(II)-perchlorat entstand der vierkernige Komplex 21 (Abbildung 45). Alle Nickel-Ionen sind quadratisch planar koordiniert. Auch hier tritt wieder das gleiche Koordinationsmuster wie bei den Kupfer(II)-Komplexen 11a bis 13 auf. An ein Nickel-Ion binden ein Stickstoffatom des Pyrazolkerns und zwei N-Donoren von zwei Imidazolringen. Die Imidazolringe stammen jedoch von zwei verschiedenen Liganden, sodass es erneut zu einer Verschränkung der Liganden kommt. Es entsteht eine rechteckige Anordnung der vier Nickelzentren. Zwei Nickelatome, die von einem Pyrazolring verbrückt werden, sind außerdem durch eine $\mathrm{OH}$-Brücke verknüpft. Beide Nickelatome werden somit in einem Abstand von 3.267(1) $\AA$ gehalten (Tabelle 13). Dieser Wert ist geringfügig niedriger als die Cu-Cu-Abstände in den schon gezeigten Komplexen 8 bis 13.

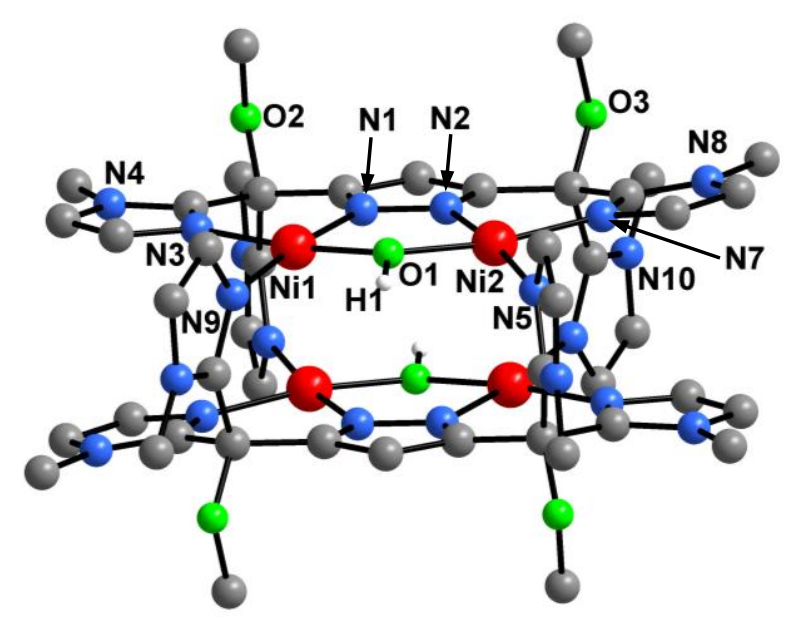

Abbildung 45: Festkörperstruktur des Kations von 21 (Wegen der Übersichtlichkeit sind alle H-Atome außer den $\mathrm{OH}$-Protonen weggelassen). 


\begin{tabular}{|c|c|c|c|}
\hline \multicolumn{4}{|c|}{ Abstände $[\AA ̊]$} \\
\hline Ni1-N1 & $1.866(5)$ & $\mathrm{Ni2}-\mathrm{N} 2$ & $1.863(6)$ \\
\hline Ni1-N3 & $1.890(6)$ & Ni2-N7 & $1.896(6)$ \\
\hline $\mathrm{Ni1}-\mathrm{O} 1$ & $1.851(5)$ & $\mathrm{Ni2}-\mathrm{N} 5^{\prime}$ & $1.903(6)$ \\
\hline Ni1-N9' & $1.897(5)$ & Ni2-O1 & $1.845(5)$ \\
\hline $\mathrm{Ni} 1{ }^{\cdots} \mathrm{Ni} 2$ & $3.267(1)$ & & \\
\hline \multicolumn{4}{|c|}{ Winkel $\left[^{\circ}\right]$} \\
\hline N9'-Ni1-N1 & $173.8(2)$ & $\mathrm{N} 5^{\prime}-\mathrm{Ni2}-\mathrm{N} 2$ & $173.2(3)$ \\
\hline O1-Ni1-N9' & $89.2(2)$ & N2-Ni2-N7 & $91.7(3)$ \\
\hline O1-Ni1-N1 & $86.7(2)$ & O1-Ni2-N5' & $89.1(2)$ \\
\hline O1-Ni1-N3 & $173.9(2)$ & O1-Ni2-N2 & $86.8(2)$ \\
\hline O4-Ni1-N3 & $92.4(3)$ & O1-Ni2-N7 & $171.3(2)$ \\
\hline N9'-Ni1-N3 & $92.1(2)$ & N5'-Ni2-N7 & $93.2(3)$ \\
\hline N1-O1-Ni2 & $124.75(11)$ & & \\
\hline
\end{tabular}

Tabelle 13: Ausgewählte Bindungslängen (in $\AA$ ) und Bindungswinkel (in º) von 21.

Dem allgemeinen Schema der Komplexsynthese folgend wurde der Ligand $\mathrm{HL}^{2}$ mit Nickel(II)-Nitrat Hexahydrat umgesetzt. Nach der Kristallisation konnte jedoch nicht die übliche Zusammensetzung des Komplexes beobachtet werden. Stattdessen wurde eine 3:2-Stöchiometrie von Metall zu Ligand in der Verbindung 22 erhalten. Wie in der Abbildung $46 \mathrm{zu}$ erkennen ist, wird das mittlere Nickelkation oktaedrisch von sechs Stickstoffatomen koordiniert und liegt auf dem Inversionszentrum des Komplexes. Die sechs N-Donoren werden von zwei Liganden zu gleichen Teilen bereitgestellt. Sie setzen sich aus zwei Stickstoffatomen des Pyrazolkerns und vier N-Donoren der Imidazolringe zusammen. In diesem Komplex ist es erstmals gelungen, beide $\mathrm{N}$-Donoren der Imidazolringe und das Stickstoffatom des Pyrazolkerns an ein und das selbe Metallion zu binden. 


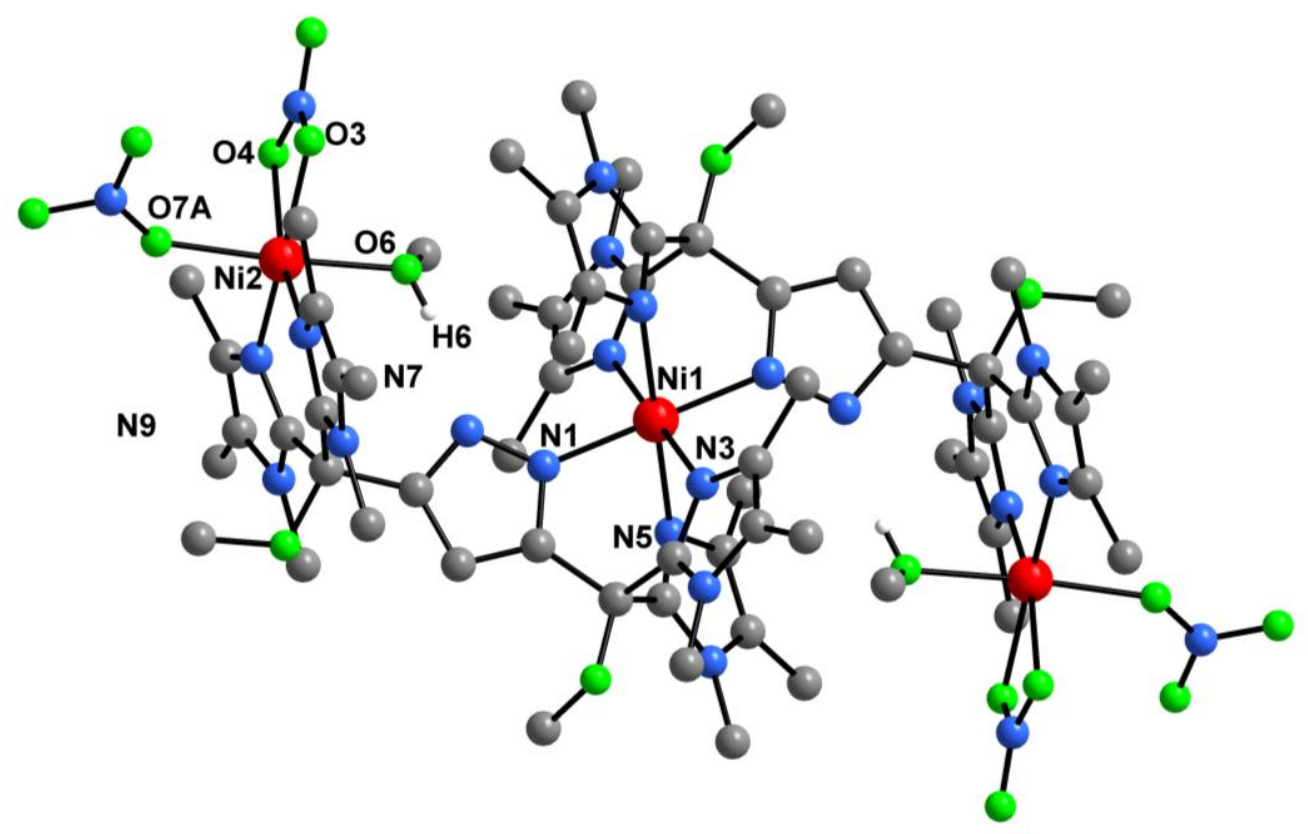

Abbildung 46: Festkörperstruktur von 22 (Wegen der Übersichtlichkeit sind alle H-Atome außer den $\mathrm{OH}-$ Protonen weggelassen).

Wie auch anhand der in Tabelle 14 gezeigten Werte zu sehen ist, handelt es sich um einen nahezu perfekten Oktaeder. Die basalen $\mathrm{Ni}-\mathrm{N}^{\mathrm{Im}}$-Bindungen sind gleich lang, nur die axiale $\mathrm{Ni}-\mathrm{N}^{\mathrm{Pz}}$-Bindung ist mit 2.033(4) $\AA$ um ca. $0.1 \AA$ kürzer. Die gegenüberliegenden N-Donoren liegen jeweils auf einer Gerade, die genau durch das Metallion geht. Nur die basale Ebene ist leicht verkippt (ca. $5^{\circ}$ aus der Senkrechten).

Die beiden äußeren Nickelatome (Ni2) sind ebenfalls oktaedrisch umgeben. Es sind jeweils ein Methanolmolekül und zwei Nitrationen koordiniert, so dass eine neutrale Verbindung entsteht. Ein Nitration stellt zwei O-Donoren zur Verfügung, die einen sehr geringen O-Ni-O-Winkel von $60.64^{\circ}$ aufspannen. Dies führt zu einer verzerrten Oktaedergeometrie. Anhand der Bindungslängen und -winkel in Tabelle 14 ist zu erkennen, dass beide $\mathrm{N}-\mathrm{Ni2}$-Bindungen geringfügig kürzer als alle N-Ni1-Bindungen sind. Die O-Ni-Bindungen sind etwas länger als die N-Ni-Bindungen, liegen aber im zu erwartenden Bereich für derartige Bindungen. 


\begin{tabular}{|c|c|c|c|}
\hline \multicolumn{4}{|c|}{ Abstände $[\AA ̊]$} \\
\hline Ni1-N1 & $2.033(4)$ & Ni2-O3 & $2.115(3)$ \\
\hline Ni1-N3 & $2.144(4)$ & $\mathrm{Ni2}-\mathrm{N} 7$ & $2.018(4)$ \\
\hline Ni1-N5 & $2.138(4)$ & $\mathrm{Ni2}-\mathrm{O} 6$ & $2.120(3)$ \\
\hline $\mathrm{Ni} 1{ }^{\cdots} \mathrm{Ni} 2$ & $6.6565(7)$ & $\mathrm{Ni2}-\mathrm{O} 4$ & $2.153(4)$ \\
\hline $\mathrm{Ni} 2 \cdots \mathrm{Ni} 2$ & $13.313(1)$ & Ni2-N9 & $2.009(4)$ \\
\hline & & $\mathrm{Ni2}-\mathrm{O} 7 \mathrm{~A}$ & $2.095(4)$ \\
\hline \multicolumn{4}{|c|}{ Winkel $\left[^{\circ}\right]$} \\
\hline $\mathrm{N} 1{ }^{\prime}-\mathrm{Ni1}-\mathrm{N} 1$ & $180.000(1)$ & O7A-Ni(2)-O6 & $172.90(16)$ \\
\hline N3'-Ni1-N3 & $180.000(1)$ & N9-Ni(2)-N7 & $91.27(15)$ \\
\hline N5'-Ni1-N5 & $180.000(1)$ & N7-Ni(2)-O7A & $96.12(17)$ \\
\hline N1-Ni1-N3 & $85.94(14)$ & N9-Ni(2)-O7A & $91.48(17)$ \\
\hline $\mathrm{N} 1^{\prime}-\mathrm{Ni1}-\mathrm{N} 3$ & $94.06(14)$ & N9-Ni(2)-O3 & $166.17(15)$ \\
\hline N1-Ni1-N5 & $84.95(14)$ & $\mathrm{N9}-\mathrm{Ni}(2)-\mathrm{O} 4$ & $105.78(15)$ \\
\hline $\mathrm{N} 1^{\prime}-\mathrm{Ni1}-\mathrm{N} 5$ & $95.05(14)$ & $\mathrm{N} 7-\mathrm{Ni}(2)-\mathrm{O} 3$ & $102.14(15)$ \\
\hline N3-Ni1-N5 & $86.77(14)$ & $\mathrm{N} 7-\mathrm{Ni}(2)-\mathrm{O} 4$ & $162.57(15)$ \\
\hline \multirow[t]{2}{*}{ N3'-Ni1-N5 } & $93.23(14)$ & $\mathrm{O} 4-\mathrm{Ni}(2)-\mathrm{O} 6$ & $86.24(14)$ \\
\hline & & O4-Ni(2)-O3 & $60.64(14)$ \\
\hline
\end{tabular}

Tabelle 14: Ausgewählte Bindungslängen (in $\AA$ ) und Bindungswinkel (in º) von 22.

Der Komplex 22 ist nach der Kristallisation aus Methanol/Diethylether jedoch sehr schlecht in Methanol löslich, so dass ein UV/Vis-Spektrum nur in DMF aufgenommen werden kann. Es ergibt sich eine sehr schwache Absorption bei $639 \mathrm{~nm}$ $\left(\varepsilon=32 \mathrm{~mol}^{-1} \cdot \mathrm{l} \mathrm{cm}^{-1}\right)$. Alle anderen Absorptionsbanden konnten wegen der Überlagerung mit Lösungsmittelsignalen nicht beobachtet werden. 
Mit Nickel(II)-chlorid Hexahydrat sind zudem Komplexe von $\mathrm{HL}^{2}$ und $\mathrm{HL}^{5}$ erhalten worden. Komplex 23 bildet sich, wenn man den mit $\mathrm{KO}^{t} \mathrm{Bu}$ deprotonierten Liganden mit 2 Äquivalenten Nickelchlorid umsetzt. Nach Kristallisation aus Methanol/ Diethylether konnten braune Kristalle von 23 erhalten werden (Abbildung 47).

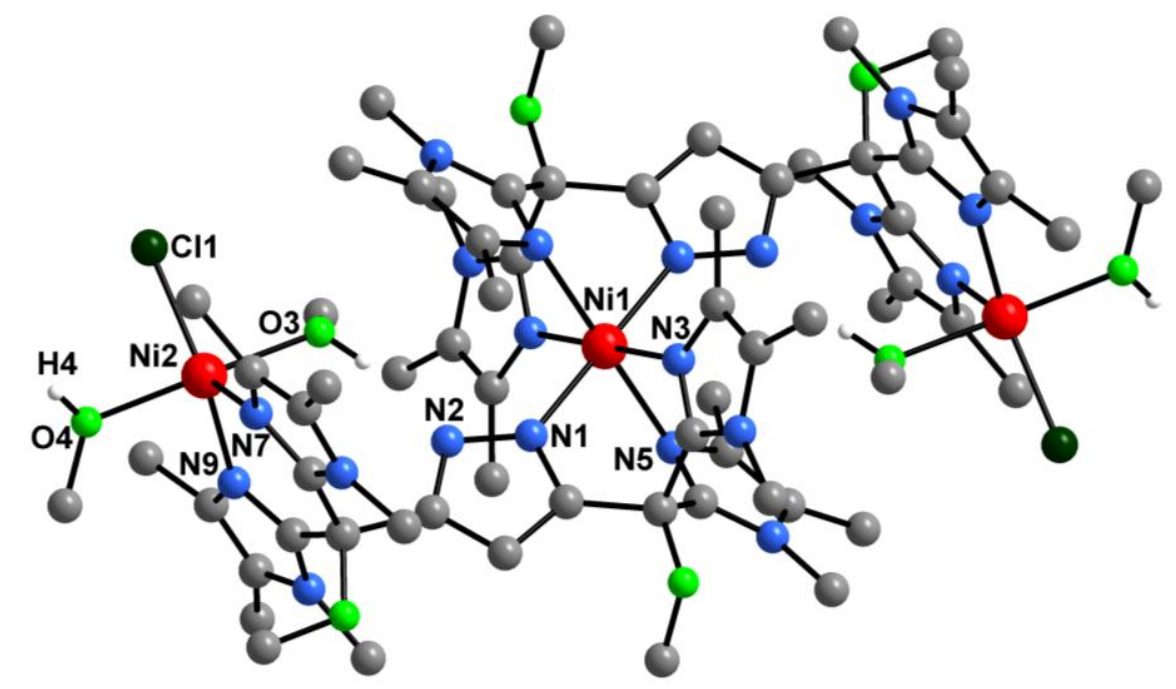

Abbildung 47: Festkörperstruktur des Kations von 23 (Wegen der Übersichtlichkeit sind alle H-Atome außer den $\mathrm{OH}-$ Protonen weggelassen).

Aus der Kristallstruktur von 23 ist ersichtlich, dass das Metall:Ligand-Verhältnis erneut 3:2 beträgt. Das innere Nickel(II)-Ion wird wiederum oktaedrisch von sechs StickstoffDonoren koordiniert. Analog zu Verbindung 22 sind diese Donoratome von zwei Pyrazol- und vier Imidazolringen. Wieder sind die beiden $\mathrm{Ni}-\mathrm{N}^{\mathrm{Pz}}$-Bindungen etwas verkürzt gegenüber den $\mathrm{Ni}-\mathrm{N}^{\mathrm{Im}}$-Bindungen, so dass ein geringfügig gestauchter Oktaeder entsteht.

Im Vergleich mit 22 ändert sich die Koordinationsumgebung der beiden äußeren Nickelkationen. Aufgrund der fünffachen Koordination durch ein Chloridion, zwei Sauerstoffatome der als Co-Liganden koordinierten Methanol-Moleküle und zwei Stickstoffatome der Imidazolringe ergibt sich eine stark verzerrte trigonale Bipyramide als Koordinationspolyeder $(\tau=0.43)$. Dabei sind allerdings die $\mathrm{N}-\mathrm{Ni2}-\mathrm{Cl}$-Winkel stark unterschiedlich mit $145.29(12)^{\circ}$ und 123.95(12) ${ }^{\circ}$. Das UV/Vis-Spektrum von 23 in Methanol zeigt eine sehr schwache Absorption bei $726 \mathrm{~nm}\left(\varepsilon=44 \mathrm{~mol}^{-1} \cdot \mathrm{cm}^{-1}\right)$, sowie weitere Banden bei $426 \mathrm{~nm}\left(\varepsilon=151 \mathrm{~mol}^{-1} \cdot \mathrm{cm}^{-1}\right), 239 \mathrm{~nm}\left(\varepsilon=52095 \mathrm{~mol}^{-1} \cdot \mathrm{lcm}{ }^{-1}\right)$ und $203 \mathrm{~nm}\left(\varepsilon=80838 \mathrm{~mol}^{-1} \cdot \mathrm{l} \cdot \mathrm{cm}^{-1}\right)$. 
Verwendet man den Liganden $\mathrm{HL}^{5}$ zur Komplexbildung mit Nickelchlorid in Methanol lassen sich nach abschließender Kristallisation aus Methanol/Diethylether hell grün gefärbte Kristalle von $\mathbf{2 4}$ erhalten werden, die für eine röntgenografische Strukturaufklärung geeignet waren (Abbildung 48). Es muss jedoch angemerkt werden, dass die Qualität der Kristalle nicht ausreichend war, um eine hohe Qualität der Struktur zu erzielen. Deshalb kann auch keine Aussage über die genaue Position der $\mathrm{OH}$-Protonen getroffen werden.

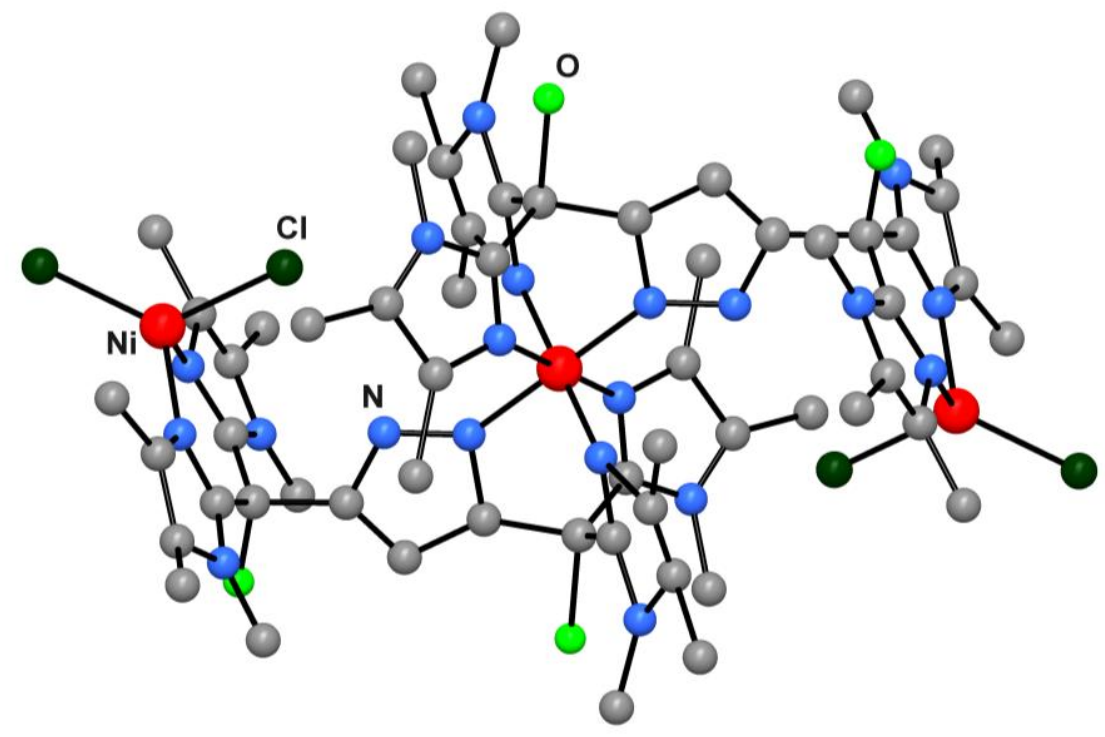

Abbildung 48: Festkörperstruktur von 24 (Wegen der Übersichtlichkeit sind alle H-Atome nicht dargestellt).

Trotz der schlechteren Qualität kann man auch in diesem Fall erneut das für diese Liganden offenbar typische Bindungsmuster erkennen. Das Ligand:Metall-Verhältnis von 2:3 tritt hier genauso wieder auf, wie sich auch die oktaedrische Koordination um das mittlere Nickel(II)-lon wiederholt. Jeder Ligand stellt sowohl ein Pyrazolstickstoffatom, als auch zwei Imidazolstickstoffatome zur Koordination bereit. Die beiden äußeren Nickel-Ionen sind tetraedrisch von zwei Imidazolstickstoffatomen und zwei Chlorid-lonen koordiniert.

Bei wiederholter Durchführung der Komplexbildung mit Ligand $\mathrm{HL}^{4}$ und Nickelchlorid kam es bei der Kristallisation aus Methanol/Diethylether zur Bildung von rotbraunen 
anstelle von hellgrünen Kristallen, die ebenfalls für die Röntgenstrukturanalyse geeignet waren. Dabei ist die in Abbildung 49 dargestellte Struktur des Nickelkomplexes 25 erhalten worden.

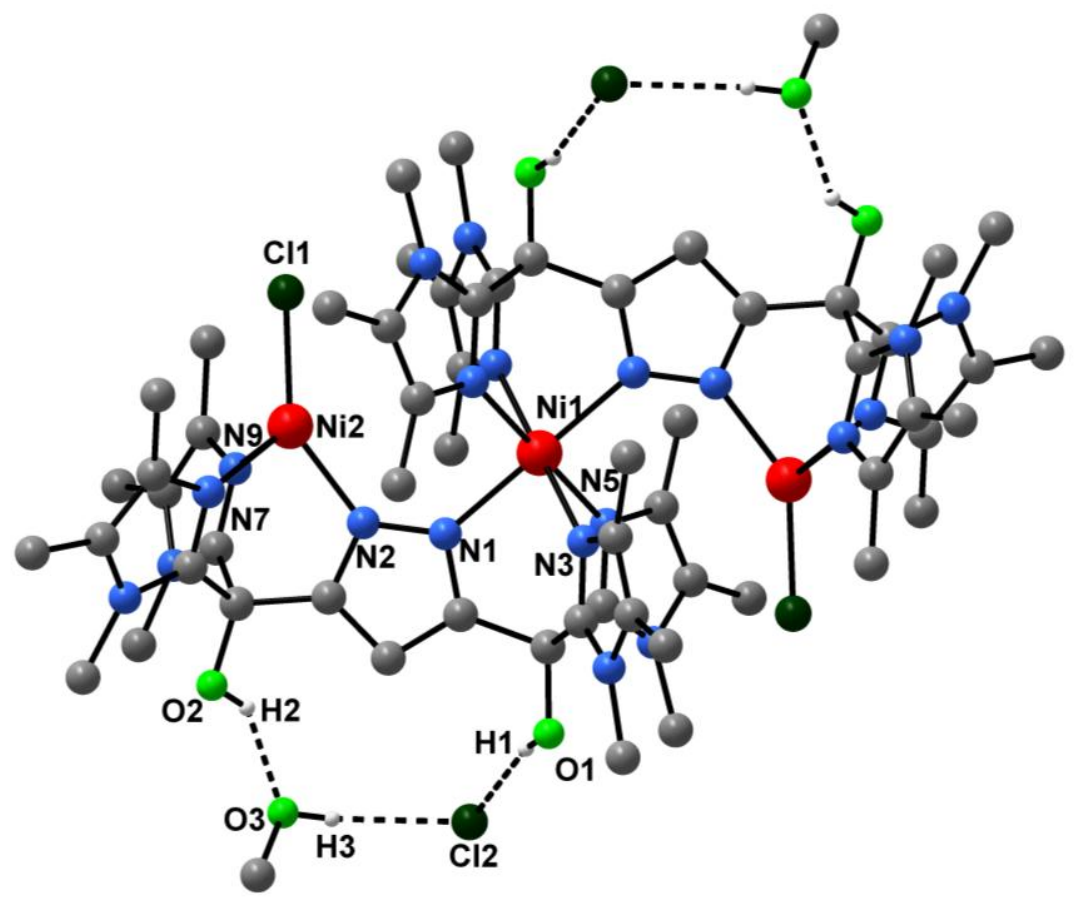

Abbildung 49: Struktur des Kations von 25 (Wegen der Übersichtlichkeit sind nur die H-Atome der $\mathrm{OH}$-Gruppen dargestellt).

Ganz analog zu Komplexen 22 bis $\mathbf{2 4}$ ist das mittlere Nickel(II)-lon oktaedrisch von sechs Stickstoff-Donoren koordiniert (Abbildung 50). Die Bindungslängen sind nahezu

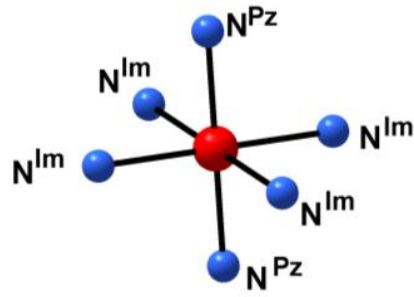

Abbildung 50: Oktaedrische Koordination des mittleren Nickel-lons. identisch, bis auf die der $\mathrm{N}^{\mathrm{Pz}}$-Ni-Bindung, die etwas verkürzt ist. Auch hier ist die basale Ebene um ca. $5^{\circ}$ verkippt. Auch sind die äußeren Nickel-Ionen tetraedrisch koordiniert. Doch im Gegensatz zu Verbindung 24 sind die koordinierenden Atome nicht zwei N-Atome und zwei Chlorid-Ionen, sondern nur ein Chlorid-Ion und drei N-Donoren. Diese stammen alle aus einer Hälfte des Liganden - ein Pyrazolstickstoffatom und zwei Imidazolstickstoffatome. Das aus der Koordination zum Nickelzentrum verdrängte Chlorid-lon ist in diesem Komplex über 
Wasserstoffbrückenbindung zusammen mit einem Molekül Methanol zwischen zwei $\mathrm{OH}-$ Funktionen des Liganden gebunden.

Es ist zum ersten Mal gelungen zwei Metalle in beide Taschen des Liganden zu binden, ohne dass sich ein Imidazolring wegdreht und somit nicht an der Koordination beteiligt ist. In Komplex 26 sind erstmals alle fünf Heterozyklen des Liganden an der Koordination zweier pyrazolverbrückter Nickel(II)-lonen beteiligt. Es tritt hier ein neues interessantes Strukturmotiv zum ersten Mal auf. Ein Ligand ermöglicht auf einer Seite eine tetraedrische Koordination und auf der anderen Seite des Liganden wird das Nickel-lon oktaedrisch umgeben. Es ergibt sich somit ein viel größerer Abstand zwischen zwei pyrazolverbrückten Nickel-Ionen als in allen anderen erhaltenen Metall-Komplexen mit derartigen Liganden. In Verbindung 25 wird ein Ni-Ni-Abstand von 4.1001(4) $\AA$ erhalten. Damit ist dieser mindestens $0.72 \AA$ größer als alle pyrazolverbrückten Distanzen, die während dieser Arbeit gefunden wurden.

In Tabelle 18 sind einige interessante Bindungslängen und -winkel für die beiden Chloro-Komplexe 23 und 25 aufgelistet.

\begin{tabular}{|c|c|c|}
\hline \multicolumn{3}{|c|}{ Abstände $[\AA]]$} \\
\hline $2.026(4)$ & $\mathrm{Ni1}-\mathrm{N} 1$ & $2.042(2)$ \\
\hline $2.138(4)$ & Ni1-N3 & $2.127(2)$ \\
\hline \multirow[t]{2}{*}{$2.133(4)$} & Ni1-N5 & $2.132(2)$ \\
\hline & Ni2-N2 & $2.132(2)$ \\
\hline $1.999(4)$ & Ni2-N7 & $2.022(2)$ \\
\hline $1.993(4)$ & Ni2-N9 & $2.013(2)$ \\
\hline $2.103(3)$ & $\mathrm{Ni2}-\mathrm{O} 3$ & \\
\hline $2.078(4)$ & $\mathrm{Ni2}-\mathrm{O} 4$ & \\
\hline $2.2700(14)$ & $\mathrm{Ni2}-\mathrm{Cl} 1$ & $2.1891(7)$ \\
\hline $6.5676(8)$ & $\mathrm{Ni} 1{ }^{\cdots} \mathrm{Ni} 2$ & $4.1001(4)$ \\
\hline $13.135(2)$ & $\mathrm{Ni}{ }^{\cdots}{ }^{\cdots} \mathrm{Ni} 2^{\prime}$ & $8.2000(7)$ \\
\hline
\end{tabular}




\begin{tabular}{|c|c|c|}
\hline \multicolumn{3}{|c|}{ Winkel $\left[^{\circ}\right]$} \\
\hline $180.000(1)$ & $\mathrm{N} 1^{\prime}-\mathrm{Ni1}-\mathrm{N} 1$ & $91.96(7)$ \\
\hline $180.000(1)$ & N3'-Ni1-N3 & $180.000(1)$ \\
\hline $180.0(2)$ & $\mathrm{N} 5{ }^{\prime}-\mathrm{Ni} 1-\mathrm{N} 5$ & $180.000(8)$ \\
\hline $86.05(15)$ & N1-Ni1-N3 & $180.000(1)$ \\
\hline $93.95(15)$ & $\mathrm{N} 1{ }^{\prime}-\mathrm{Ni1}-\mathrm{N} 3$ & $85.86(9)$ \\
\hline $84.61(15)$ & N1-Ni1-N5 & $94.14(9)$ \\
\hline $95.39(15)$ & $\mathrm{N} 1{ }^{\prime}-\mathrm{Ni} 1-\mathrm{N} 5$ & $85.61(8)$ \\
\hline $87.14(15)$ & N3-Ni1-N5 & $94.39(8)$ \\
\hline $92.86(15)$ & N3'-Ni1-N5 & $84.47(9)$ \\
\hline $171.27(16)$ & O4-Ni2-O3 & \\
\hline $90.58(16)$ & N9-Ni2-N7 & $88.67(9)$ \\
\hline $123.29(12)$ & N7-Ni2-Cl1 & $115.24(7)$ \\
\hline $145.29(12)$ & N9-Ni2-Cl1 & $115.76(7)$ \\
\hline $86.24(15)$ & N9-Ni2-O3 & \\
\hline $89.25(17)$ & N9-Ni2-O4 & \\
\hline $90.70(14)$ & N7-Ni2-O3 & \\
\hline $96.83(16)$ & N7-Ni2-O4 & \\
\hline $89.65(10)$ & O3-Ni2-Cl1 & \\
\hline \multirow[t]{4}{*}{$89.81(12)$} & $\mathrm{O} 4-\mathrm{Ni2}-\mathrm{Cl} 1$ & \\
\hline & N2-Ni2-N7 & $88.87(9)$ \\
\hline & N2-Ni2-N9 & $89.08(10)$ \\
\hline & $\mathrm{N} 2-\mathrm{Ni2}-\mathrm{Cl} 1$ & $144.42(7)$ \\
\hline
\end{tabular}

Tabelle 16: Vergleich ausgewählter Bindungslängen (in $\AA$ ) und -winkel (in º) von 23 und 25.

Die drei Strukturen $\mathbf{2 3}$ bis $\mathbf{2 5}$ kann man im weitesten Sinne als lonisationsisomere zu verstehen. Auch wenn der Ligand im Falle von 23 zwei Methoxygruppen anstelle der $\mathrm{OH}-F u n k t i o n e n$ in $\mathbf{2 4}$ und $\mathbf{2 5}$ trägt, sollte es möglich sein alle drei Komplexe in Zusammensetzung zu setzen. Ausgehend von Komplex 24 entsteht durch Abspaltung 
eines Chloridions von den äußeren Nickel(II)-lonen die Verbindung 23. Gleichzeitig wird die $\mathrm{Ni}-\mathrm{N}^{\mathrm{Pz}}$-Bindung gebildet und es bildet sich erneut ein tetraedrisch koordiniertes Nickelion. Erfolgt anschließend noch ein Angriff eines Methanol-Moleküls an das Nickel(II)-lon wird die Ni-NPz-Bindung wieder gespalten. Es bildet sich zunächst ein vierfach koordiniertes Nickelzentrum, das durch ein weiteres Molekül Methanol in die verzerrte trigonal bipyramidale Anordnung in $\mathbf{2 3}$ überführt wird (Schema 21).

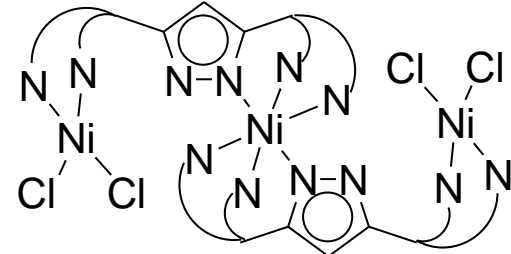

23

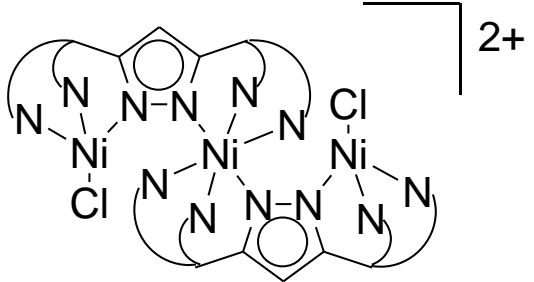

25

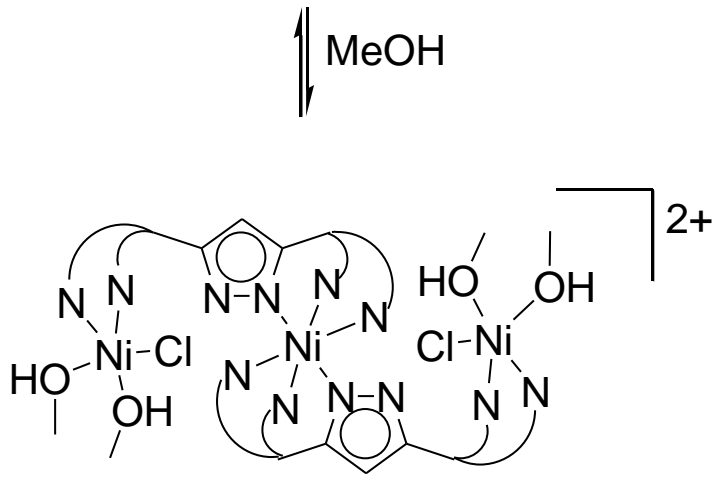

24

Schema 21: Ionisationsisomere 23, 24 und 25.

Um zu überprüfen, ob diese Umwandlung von $\mathbf{2 5}$ in $\mathbf{2 3}$ und $\mathbf{2 4}$ möglich ist, sind jedoch genauere Untersuchungen notwendig. So wäre es durchaus denkbar, dass das Gleichgewicht zwischen $\mathbf{2 3}$ und $\mathbf{2 5}$ mit der Konzentration von Chloridionen beeinflussen kann. Da diese in $\mathbf{2 3}$ an das Metallion gebunden sind, sollte bei Zugabe von Chloridionen zum Gleichgewicht zwischen 23 und 25 die Bildung von 23 gefördert werden. Die Untersuchung des Gleichgewichts von 25 und 24 ist etwas schwieriger, da beide Komplexe gut in Methanol löslich sind und nur Methanol abgespalten wird. Eine genaue Aufklärung des gesamten Prozesses ist nicht durchgeführt worden. 
Die erhaltenen Strukturen zeigen, dass es möglich ist, ein Nickel(II)-Ion in der Bindungstasche einer Ligandhälfte durch alle drei N-Donoren zu fixieren. Dabei entsteht eine tetraedrische Koordination, wenn ein Co-Ligand an das Metallzentrum bindet. Koordinieren sogar drei Co-Liganden bzw. ein dreizähniger Co-Ligand an das Nickel-Ion, kann sich eine oktaedrische Koordinationsumgebung ausbilden. Als chelatisierende Liganden könnten sich zum Beispiel 2,2'-Bipyridin oder auch das Acetylacetonat-Ion eignen. Damit Nickel-Komplexe mit solchen Co-Liganden gebildet werden können (Abbildung 51), muss der Ligand mit einer Base deprotoniert und dann mit zwei Äquivalenten eines Nickel(II)-Salzes umgesetzt werden. Abschließend müssen noch zwei Äquivalente des entsprechenden Co-Liganden zugesetzt werden.
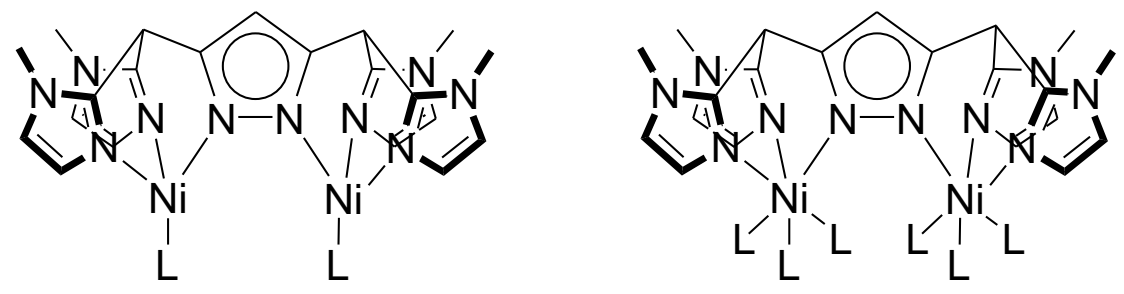

Abbildung 51: Schematische Darstellung der gewünschten Nickel-Komplexe mit tetraedrischer (links) oder oktaedrischer (rechts) Koordinationsgeometrie.

Setzte man 2,2'-Bipyridin zum Komplexansatz zu, erfolgte ein Farbumschlag von braun nach grün. Dies lies auf eine veränderte Koordination um das Metall schließen. Nach Kristallisation aus Acetonitril/Diethylether sind violett gefärbte Kristalle entstanden. Die röntgenographische Strukturbestimmung lieferte die in Abbildung 52 gezeigte Struktur für 26.

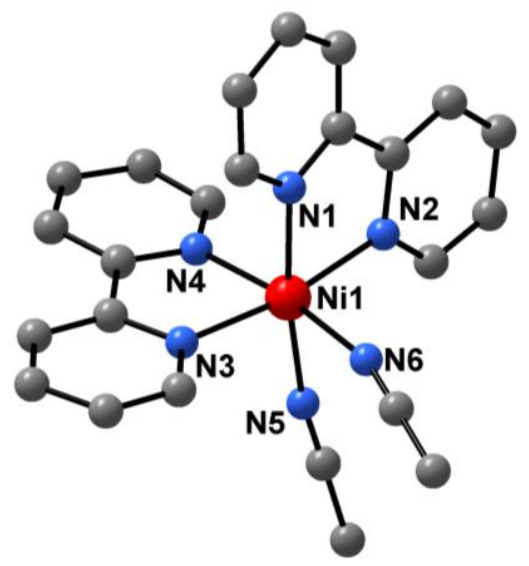

Abbildung 52: Struktur des Kations von 26 (Alle H-Atome wurden wegen der Übersichtlichkeit nicht dargestellt). 
Es wird ersichtlich, dass der zugesetzte Co-Ligand 2,2'-Bipyridin ein sehr guter Chelatbildner für das Nickel(II)-lon ist und die Verbindung keinen Pyrazolat-Liganden mehr enthält. Es bildet sich ein oktaedrischer Komplex mit sechs N-Donoren am NickelIon. Darunter sind zwei N-Atome von Acetonitril-Molekülen und zwei Bipyridin-Liganden stellen ebenfalls je zwei Stickstoffatome für die Koordination zur Verfügung. Einige Bindungslängen und -winkel sind in Tabelle 17 aufgelistet. Die erhaltenen Werte liegen in dem erwarteten Bereich solcher Systeme.

\begin{tabular}{|c|c|c|c|}
\hline \multicolumn{4}{|c|}{ Abstände $[\AA ̊]$} \\
\hline Ni1-N1 & $2.081(2)$ & Ni1-N2 & $2.079(2)$ \\
\hline Ni1-N3 & $2.087(2)$ & Ni1-N4 & $2.080(2)$ \\
\hline Ni1-N5 & $2.096(2)$ & Ni1-N6 & $2.090(3)$ \\
\hline \multicolumn{4}{|c|}{ Winkel $\left[^{\circ}\right]$} \\
\hline N2-Ni1-N3 & $170.36(9)$ & N2-Ni1-N1 & $79.10(9)$ \\
\hline N4-Ni1-N6 & $170.92(9)$ & N4-Ni1-N3 & $78.97(9)$ \\
\hline N1-Ni1-N5 & $172.00(10)$ & N6-Ni1-N5 & $84.45(10)$ \\
\hline N1-Ni1-N3 & $93.98(9)$ & $\mathrm{N} 2-\mathrm{Ni1}-\mathrm{N} 4$ & $94.17(9)$ \\
\hline
\end{tabular}

Tabelle 17: Ausgewählte Bindungslängen (in $\AA$ ) und Bindungswinkel (in º) von 26.

Das ESI-Massenspektrum dieses Komplexes zeigt Signale bei $\mathrm{m} / \mathrm{z}=469.0$ für $\left[\mathrm{Ni}(\text { bipy })_{2}\left(\mathrm{ClO}_{4}\right)\right]^{+}, \mathrm{m} / \mathrm{z}=370.1$ für $\left[\mathrm{Ni}(\text { bipy })_{2}\right]^{+}, \mathrm{m} / \mathrm{z}=313.0$ für $\left[\mathrm{Ni}(\text { bipy })\left(\mathrm{ClO}_{4}\right)\right]^{+}$und $\mathrm{m} / \mathrm{z}=214.0$ für $[\mathrm{Ni}(\text { bipy })]^{+}$. Außerdem lässt sich ein Signal bei $\mathrm{m} / \mathrm{z}=1039.0$ erkennen, dass dem Dimer $\left[\left\{\mathrm{Ni}(\text { bipy })_{2}\left(\mathrm{ClO}_{4}\right)\right\}_{2}\left(\mathrm{ClO}_{4}\right)\right]^{+}$entspricht. Die Isotopenmuster von all diesen Signalen zeigen sehr gute Übereinstimmungen mit den berechneten Werten. 
Verwendet man anstelle des Bipyridins Acetylaceton als potentiellen Co-Liganden, sollte es möglich sein, die freien Koordinationsstellen am Nickel-Ion mit den Sauerstoffatomen des Diketonats zu besetzen. Nachdem die Reaktion durchgeführt und Acetylaceton zugefügt wurde, konnten nach Kristallisation aus Methanol/Diethylether türkisfarbene Kristalle erhalten werden. Die röntgenographische Strukturbestimmung lieferte die in Abbildung 53 dargestellte Struktur der Komplexverbindung 27.

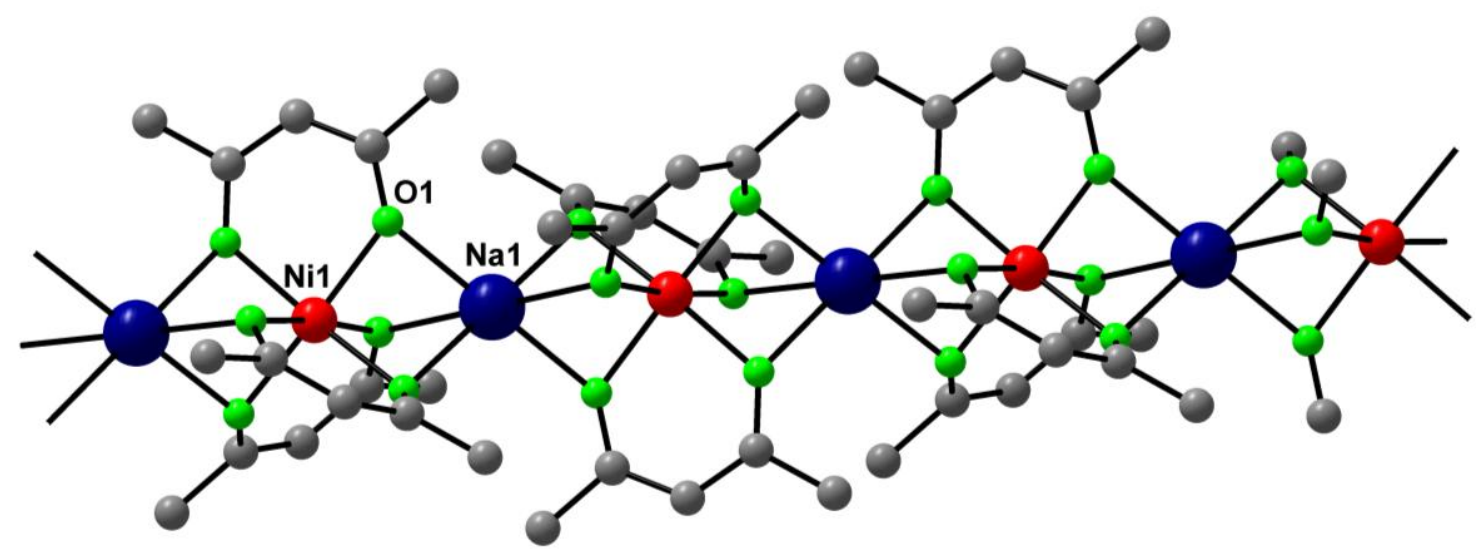

Abbildung 53: Struktur von 27 (Wegen der Übersichtlichkeit sind die H-Atome nicht dargestellt).

Auch in diesem Fall ist kein Pyrazol-Ligand an das Metallzentrum koordiniert. Stattdessen haben sich drei Acetylacetonat-Ionen an das Nickel(II)-lon angelagert. Jedes Nickelion ist oktaedrisch von sechs O-Donoren umgeben. Die Ni-O-Bindungen sind jeweils 2.0338(9) $\AA$ lang und der O-Ni-O-Winkel, der von zwei Atomen eines Acetylacetonats mit dem Nickelzentrum gebildet wird, beträgt $89.73(5)^{\circ}$. Die einzelnen $\mathrm{Ni}(\mathrm{acac})_{3}$-Einheiten sind über Natriumionen miteinander verknüpft. Dadurch entstehen neutrale $\mathrm{Na}\left[\mathrm{Ni}(\mathrm{acac})_{3}\right]$-Einheiten. In Tabelle 18 sind einige interessante Bindungslängen und -winkel abgebildet. 


\begin{tabular}{|c|c|c|c|}
\hline \multicolumn{4}{|c|}{ Abstände $[\AA]$} \\
\hline $\mathrm{Ni} 1 \cdots \mathrm{Na} 1$ & $2.91817(18)$ & Ni1-O1 & $2.0338(9)$ \\
\hline $\mathrm{Na1}-\mathrm{O} 1$ & $2.3218(9)$ & & \\
\hline \multicolumn{4}{|c|}{ Winkel $\left[^{\circ}\right]$} \\
\hline O1'-Ni1-O1 & $86.48(4)$ & $\mathrm{Na} 1^{\prime \cdots} \mathrm{Ni} 2 \cdots \mathrm{Na} 1$ & 180.0 \\
\hline $\mathrm{O} 1^{\prime}-\mathrm{Ni1}{ }^{\cdots} \mathrm{Na}^{\prime}{ }^{\prime}$ & $127.72(3)$ & $\mathrm{O} 1^{\prime \prime}-\mathrm{Ni} 1{ }^{\cdots} \mathrm{Na}^{\prime}$ & $52.28(3)$ \\
\hline O1-Na1-O1' & $73.76(4)$ & $\mathrm{O} 1{ }^{\prime \prime}-\mathrm{Na} 1-\mathrm{O} 1$ & $106.24(4)$ \\
\hline $\mathrm{O} 1-\mathrm{Na} 1 \cdots \mathrm{Ni} 1$ & $43.86(2)$ & $\mathrm{O} 1^{\prime}-\mathrm{Na} 1{ }^{\cdots} \mathrm{Ni} 1$ & $136.14(2)$ \\
\hline
\end{tabular}

Tabelle 18: Ausgewählte Bindungslängen (in $\AA$ ) und Bindungswinkel (in º) von 27.

Diese Verbindung ist literaturbekannt ${ }^{[142]}$ und lässt sich sehr einfach darstellen. Durch Reaktion von Natronlauge mit Acetylaceton in Methanol entsteht zunächst NatriumAcetylacetonat, welches dann mit Nickel(II)-Chlorid das oben gezeigte Produkt ergibt. In der Literatur wurde diese Verbindung jedoch nur als türkisfarbenes Pulver erhalten. Die Struktur konnte in dieser Arbeit erstmals aufgeklärt werden. Daraus ist ersichtlich, dass es sich bei Verbindung 27 nicht um einen mononuklearen anionischen Komplex mit Natrium-Kationen als Gegenionen handelt. Vielmehr kommt es zur Ausbildung von kettenartigen Strukturen. Bei gezielter Synthese erhält man geeignete Kristalle durch Kristallisation aus Wasser/Methanol.

Betrachtet man die Struktur der Verbindung 27 genauer, fällt im Vergleich zu allen anderen Substanzen dieser Arbeit eine hohe Symmetrie auf. Alle Natrium- und Nickelionen liegen auf einer Linie. Legt man diese übereinander, so entsteht die in Abbildung 54 gezeigte Gestalt des Komplexes. Die Acetylacetonat-Ionen ordnen sich wie die Schaufelräder eines Propellers um diese Linie an. Die

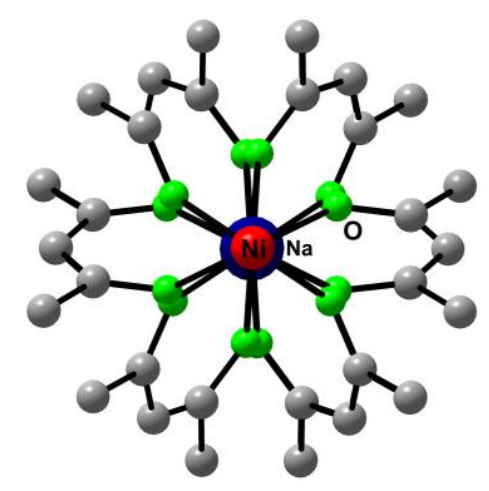
Acetylacetonat-Einheiten von benachbarten $\left[\mathrm{Ni}(\mathrm{acac})_{3}\right]^{-}$-Fragmenten sind um ca. $60^{\circ}$ gegeneinander verdreht. Dadurch stehen alle acac-Einheiten auf Lücke. 
Es ist gelungen das Koordinationsverhalten des Liganden gegenüber Nickel zu untersuchen. Dabei war auffällig, dass es bis auf einen Fall nicht zu den erwarteten Zusammensetzungen der Nickel(II)-Komplexe gekommen ist. Doch im Gegensatz zu allen Kupfer(II)- und Kupfer(I)-Komplexen konnte mehrmals die erwünschte Koordinationsgeometrie für ein Metallion erhalten werden. Erstmals ist es gelungen, beide Imidazolringe und ein Pyrazolstickstoffatom an das selbe Metallion, hier Nickel(II)-lon, zu binden. Aus der dadurch entstandenen facial tridentaten Koordination entsteht durch Koordination von drei weiteren Donoratomen die gezeigte Oktaedergeometrie.

Die Koordinationschemie des Nickels kann sehr vielseitig sein. Das Nickel(II)-lon ist in der Lage, die verschiedensten Koordinationsgeometrien zu ermöglichen. Es sind nahezu alle Koordinationsmuster für ein vierfach (tetraedrisch, quadratisch planar), fünffach (trigonal bipyramidal, quadratisch pyramidal) oder sechsfach (oktaedrisch) koordiniertes Nickel-Ion möglich. Daraus lässt sich eine Vielzahl möglicher Übergänge konstruieren, die bei den unterschiedlichsten Wellenlängen absorbieren.

So beobachtet man für oktaedrisch koordinierte Nickel(II)-Ionen sehr schwache Absorptionen bei 620 bis $690 \mathrm{~nm} \cdot{ }^{[143,144]}$ Für andere Komplexe, die oktaedrisch koordinierte Nickel(II)-lonen enthalten, sind zwei charakteristische Absorptionen beobachtet worden. Zum Einen handelt es sich dabei um eine Bande zwischen 800 und $870 \mathrm{~nm}$ und die zweite Bande liegt zwischen 950 und $1050 \mathrm{~nm}$. Diese tritt jedoch nicht in allen Komplexen auf. ${ }^{[145,146]}$ Daneben treten noch weitere Absorptionsbanden auf, die durch LMCT's und $\pi$ - $\pi^{*}$-Übergänge zurückzuführen sind. Für Komplexverbindungen mit tetraedrisch koordinierten Nickel(II)-Ionen ergibt sich im UV/Vis-Spektrum eine intensive Bande bei etwa 650 bis $760 \mathrm{~nm}$ für den d-d-Übergang. In einigen Fällen kann man auch das breite Absorptionssignal zwischen bei 770 bis $840 \mathrm{~nm}$ für einen LMCT identidifiziert werden kann. ${ }^{[147,148]}$ Außerdem findet man in allen Komplexen zusätzlich die ligandabhängigen Banden für $\pi-\pi^{*}$-Übergänge.

Da die entsprechenden Banden für die verschiedenen Fälle sehr ähnlich sind, ist es sehr schwierig, die aufgenommenen UV/Vis-Spektren von 22 bis 25 richtig zu interpretieren, da stets neben dem idealen oktaedrischen Koordinationspolyeder ein zweites Koordinationsmuster für die beiden äußeren Nickel(II)-Ionen auftritt. Die Verbindung 22 bildet am aufgrund der bidentaten Koordinationsweise eines Nitrat-Ions 
einen verzerrten Oktaeder für die beiden äußeren Nickel(II)-Ionen. Für 22 kann man eine sehr schwache Absorptionsbande bei $639 \mathrm{~nm}$ mit $\varepsilon=32 \mathrm{~mol}^{-1} \cdot \mathrm{r} \mathrm{cm}^{-1}$. Dies ist wie oben bereits erwähnt charakteristisch für oktaedrische Nickel(II)-Komplexe.

Anhand eines Vergleichs von UV/Vis-Spektren der trinuklearen Nickel(II)-Komplexe, die in Lösung und als Feststoff gemessen wurden, können Aussagen über die vorliegende Struktur in Lösung getroffen werden. In Abbildung 55 sind die verschiedenen UV/VisSpektren gezeigt.

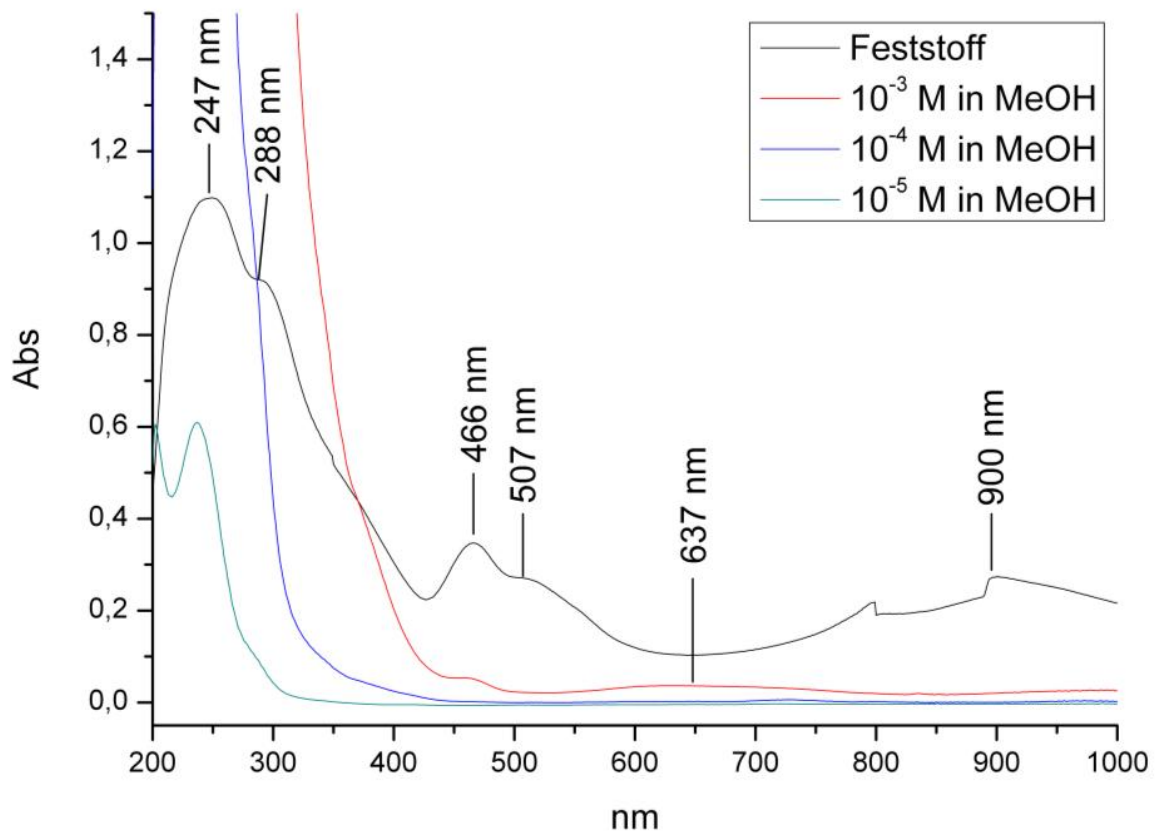

Abbildung 55: Vergleich des Festkörper-UV/Vis-Spektrums mit den in Lösung gemessenen UV/VisSpektren von 24. (Die zwei Sprünge in der Kurve des Festkörper-UV/Vis-Spektrums sind auf Wechsel des Gitters im Messgerät zurückzuführen.)

Dabei ist deutlich zu erkennen, dass es neben den eher unbedeutenden Veränderungen im Bereich von $200 \mathrm{~nm}$ bis ca. $600 \mathrm{~nm}$ einen gravierenden Shift der Absorption gibt. Die in konzentrierter Lösung zu sehende Bande bei $637 \mathrm{~nm}$ ist im Festkörper nicht zu sehen. Dafür hat sich eine Absorption bei $900 \mathrm{~nm}$ gebildet. Die Absorption bei $637 \mathrm{~nm}$ kann zweifelsfrei für oktaedrisch koordiniertes Nickel(II)-lon angesehen werden, da für den komplett oktaedrisch koordinierten trinuklearen Nickel(II)-Komplex 22 ebenfalls nur eine schwache Absorption zwischen 500 nm und $1000 \mathrm{~nm}$ gefunden wird. 


\section{Cyclovoltammetrie}

Das von Ligand $\mathrm{HL}^{1}$ aufgenommene Cyclovoltammogramm zeigt einen Reduktionspeak -1.57 V. Im weiteren Verlauf erkennt man ein sehr schwaches Signal für einen möglichen Reoxidationsprozess. Die Kriterien für einen reversiblen Prozess sind jedoch nicht erfüllt. Der Unterschied zwischen dem Reduktions- und Oxidationspotential ist mit $0.13 \mathrm{~V}$ zu groß. Außerdem sind die Peakflächen für beide Prozesse sehr verschieden, so dass hier auf jeden Fall von einer irreversiblen elektrochemischen Umwandlung gesprochen werden muss.

Für alle Kupfer(II)-Komplexe zeigen die Ergebnisse der cyclovoltammetrischen Untersuchungen ebenfalls irreversible Reduktionsprozesse von $\mathrm{Cu}(\mathrm{II}) \mathrm{zu} \mathrm{Cu}(\mathrm{I})$. Diese Tatsache ist nicht verwunderlich, da mit der Umwandlung eines Kupfer(II)-Ions in eine Kupfer(I)-Spezies eine strukturelle Änderung der Koordinationsumgebung verbunden ist. So ändert sich nicht nur die Anzahl der Donoratome am Metall von 5 für die Oxidationsstufe +ll zu 3 bzw. 4 für Kupfer(I)-lonen, sondern auch die Koordinationsgeometrien sind vollkommen verschieden. Während man für Kupfer(II)Ionen in der Regel quadratisch pyramidale Anordnungen um das Metallzentrum findet, treten bevorzugt trigonal planare oder tetraedrische Koordinationsgeometrien für Kupfer(I)-Ionen auf. Außerdem haben die harten O-Liganden nur eine geringe Affinität zum weichen Kupfer(I)-lon, so dass man daraus ableiten kann, dass die $\mathrm{OH}$-Brücke im Verlaufe der Reduktion abdissoziieren wird und auch eine veränderte Koordination der Imidazolringe auftreten wird.

Für den zweikernigen Kupfer(II)-Komplex 8 ist das in Abbildung 56 dargestellte Cyclovoltammogramm erhalten worden. Es sind zwei Peaks zu erkennen, die einer Reduktion zugeordnet werden müssen. Die Potentiale dafür liegen bei $-2.01 \mathrm{~V}$ bzw. $-2.75 \mathrm{~V}$. Außerdem enthält das Spektrum zwei weitere Peaks bei $-0.45 \mathrm{~V}$ und $+0.99 \mathrm{~V}$, die Oxidationsprozessen zuzuschreiben sind. Es gibt zwei mögliche Interpretationen für die gefundenen Signale im Reduktionsbereich. Zum Einen kann die erste Bande bei $-2.01 \mathrm{~V}$ das Resultat einer Einelektronen-Reduktion zum gemischtvalenten $\mathrm{Cu}^{\prime} \mathrm{Cu}^{\prime \prime}-$ Komplex sein, die eine strukturelle Veränderung des Liganden bewirkt. 


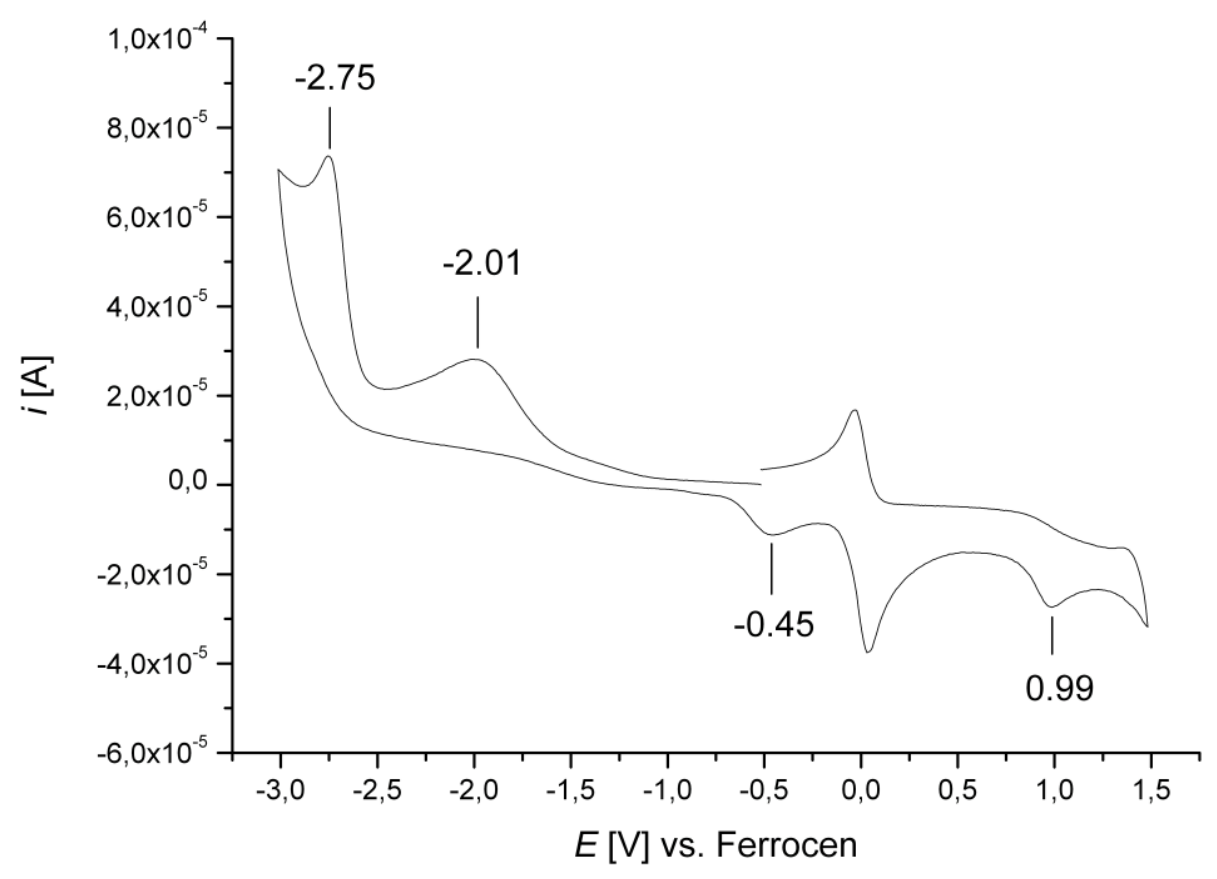

Abbildung 56: Cyclovoltammogramm von 8 in $\mathrm{CH}_{3} \mathrm{CN}\left(\mathrm{N}\left({ }^{n} \mathrm{Bu}\right)_{4} \mathrm{PF}_{6}, 100 \mathrm{mV} / \mathrm{s}\right)$; Potenzial in $\mathrm{V}$ vs. Ferrocen

Daraus resultiert der ungewöhnlich weit verschobene Reduktionspeak bei $-2.75 \mathrm{~V}$, dem eine weitere Einelektronen-Reduktion zur $\mathrm{Cu}^{\prime} \mathrm{Cu}$ '-Verbindung zugeordnet werden muss. Zum Zweiten ist es aber auch möglich, dass der erste Peak die Umwandlung des dinuklearen Kupfer(II)-Komplexes zur Cu'Cu'-Spezies wiedergibt und das zweite Signal von einer Reduktion des Liganden herrührt. Da es eine derartige Bande im freien Liganden nicht gibt, ist diese Möglichkeit eher unwahrscheinlich.

Eine mögliche Erklärung für das Auftreten des Signals bei $-2.75 \mathrm{~V}$ war zunächst die Zersetzung des Acetonitrils. Dies erfolgt jedoch erst bei noch niedrigerem Potential, so dass der damit verbundene Prozess mit dem Komplex 8 in Zusammenhang stehen muss. Möglicherweise ist dieser Peak das Resultat der Reduktion des zweiten Kupfer(II)-Ions zu Cu(I). Diese Oxidationsstufe kann sehr gut durch die Koordination des Acetonitrils stabilisiert werden, wie man an den verschiedenen stabilen $\left[\mathrm{Cu}^{\prime}(\mathrm{MeCN})_{4}\right]$-Salzen sehen kann. Die Untersuchung derartiger Phänomene war allerdings nicht Bestandteil dieser Arbeit, sie sind daher nicht genauer untersucht worden. 
Die Signale für oxidative Vorgänge bei $-0.45 \mathrm{~V}$ und $+0.99 \mathrm{~V}$ lassen sich auf ähnliche Weise interpretieren, wie die der Reduktionsprozesse. Auch hier ist es möglich, dass beide Kupfer(I)-Ionen in einem Schritt zu Cull oxidiert werden und anschließend der Ligand einer Oxidation unterliegt. Genauso gut ist es aber auch möglich, dass hier eine schrittweise Oxidation der Metallionen von $\mathrm{Cu}^{\prime} \mathrm{Cu}^{\prime} \mathrm{zu} \mathrm{Cu}^{\prime \prime} \mathrm{Cu}^{\prime}$ stattfindet und das zweite Signal die Oxidation zu CullCul repräsentiert. Diese These lässt sich mit einem Cyclovoltammogramm

Damit eine bessere Zuordnung für die ablaufenden Prozesse getroffen werden kann, sind außerdem noch Messungen von $0 \mathrm{~V}$ bis $-2 \mathrm{~V}$ und von $0 \mathrm{~V}$ bis 2,5 $\mathrm{V}$ durchgeführt wurden. Dabei wurden ausschließlich Cyclovoltammogramme erhalten, die irreversible Prozesse zeigen. Zum Teil gibt es mehrere Peaks für Reduktionsprozesse, die aber wiederum stark unterschiedliche Potentialflächen aufweisen. Zum anderen ist es nicht möglich Signale für die dazugehörigen Reoxidationsprozesse zu identifizieren, da deren Intensität sehr klein ist oder aber im Potentialbereich der Reduktionsprozesse kein dazugehöriges Signal für die Reoxidation zu finden ist.

Die im Rahmen dieser Arbeit synthetisierten Kupfer(l)-Komplexe sind nicht cyclovoltammetrisch analysiert worden. Obwohl die Koordinationsgeometrien sehr unterschiedlich sind, müsste sich trotzdem ein Vergleich mit den Potentialen für Kupfer(II)-Komplexe treffen lassen. Desweiteren sollte es möglich sein, eine Aussage zur Stabilität der isolierten Kupfer(I)-Komplexe vorzunehmen.

Stellt man einen Vergleich der Reduktionspotentiale mit bekannten Kupfer(II)Komplexen an, so findet man für Kupfer-Pyrazolat-Komplexe mit verschiedenen Aminseitenarmen Potenziale von $-0.53 \mathrm{~V}$ und $-0.90 \mathrm{~V}$ für $E_{p}{ }^{\text {red, } 1}$ und für $E_{p}{ }^{\text {red,2 }}-1.03 \mathrm{~V}$ und -1.14 V. ${ }^{[103]}$ Die Aminseitenarme unterscheiden sich in der Anzahl der StickstoffDonoren und der Größe der sich bildenden Chelatringe. Auch für Kupfer(II)-Komplexe von anderen Liganden sind ähnliche Potentiale gefunden worden. ${ }^{[149-151]}$

Die im Rahmen der Arbeit vorgestellten Kupfer(II)-Komplexe weisen Potentiale im Bereich von $-1.61 \mathrm{~V}$ bis $-2.75 \mathrm{~V}$ auf. Das spricht dafür, dass der Ligand eine sehr große Stabilität für Kupfer(II)-Komplexe aufweist. Dies bedeutet allerdings nicht, dass es unmöglich ist, stabile Kupfer(I)-Komplexe zu synthetisieren. 


\section{Potentiometrische Messungen}

Die hier diskutierten Messungen sind alle im Arbeitskreis von Prof. Dr. Etelka Farkas durch Edit Csapo an der Universität Debrecen (Ungarn) durchgeführt worden.

Alle potentiometrischen Messungen sind bei $25^{\circ} \mathrm{C}$ und einer lonenstärke von $0.2 \mathrm{M}$ $(\mathrm{KCl})$ durchgeführt worden. Die Titrationen des freien Liganden $\mathrm{HL}^{1}$ erfolgten ausgehend von $\mathrm{pH} 2$ bis $\mathrm{pH} 11$ mit $\mathrm{KOH}$ als Titrationsmittel. Die vier erhaltenen Dissoziationskonstanten $\left(\mathrm{p} K_{\mathrm{a}}\right)$ sind in Tabelle 19 zusammengefasst. Dabei ergibt sich für jede Protonierung eines Imidazolringes eine separate Dissoziationskonstante. Es ist auffällig, das der Unterschied zwischen der ersten und zweiten sowie dritten und vierten Konstante mit 1.13 bzw. 1.12 Einheiten sehr ähnlich ist. Nur die Differenz zwischen der zweiten und dritten Protonierungsstufe ist mit 2.36 Einheiten wesentlich größer. Dies lässt sich damit erklären, dass zunächst ein Imidazolring jeder Ligandhälfte protoniert wird. Die Protonierung des jeweils zweiten Imidazolringes erfolgt bei geringerem $\mathrm{pH}$-Wert und die Protonierung des Pyrazolrings kann bei dieser Messung nicht beobachtet werden, da diese bei pH-Werten kleiner als 2 erfolgt.

\begin{tabular}{lllll}
\hline & {$\left[\mathrm{H}_{5} \mathrm{~L}\right]^{4+}$} & {$\left[\mathrm{H}_{4} \mathrm{~L}\right]^{3+}$} & {$\left[\mathrm{H}_{3} \mathrm{~L}\right]^{2+}$} & {$\left[\mathrm{H}_{2} \mathrm{~L}\right]^{+}$} \\
\hline $\mathrm{p} K_{\mathrm{a}}$ & $1.41(1)$ & $2.54(1)$ & $4.80(1)$ & $5.92(1)$ \\
\hline
\end{tabular}

Tabelle 19: Dissoziationskonstanten $\left(\mathrm{p} K_{\mathrm{a}}\right)$ von Ligand $\mathrm{HL}^{1}$ (Standardabweichungen sind in Klammern angegeben).

Die potentiometrischen Titrationen des Liganden unter Zusatz von Kupfer(II)-, Nickel(II)und Zink(II)-Chlorid erfolgten zwischen pH 2 und pH 12 mit zwei verschiedenen MetallLigand- Verhältnissen (1:1 und 2:1). Die Ergebnisse sind in Abbildung 57 dargestellt. Es ist ein deutlicher Unterschied zwischen den einzelnen Metallsalzen zu erkennen. Die verschiedenen Metallionen werden unterschiedlich von dem Liganden koordiniert und es sollten sich deshalb andere Komplexbildungskonstanten ergeben.

In jeder einzelnen Grafik ist die Titration des freien Liganden $\mathrm{HL}^{1}$ in schwarz dargestellt. Die roten Linien repräsentieren die Titration eines Systems, dass ein Ligand:Metall- 
Verhältnis von 1:1 enthält. Die Titration der Systeme mit Ligand:Metall-Verhältnis 1:2 sind durch die blauen Linien dargestellt.
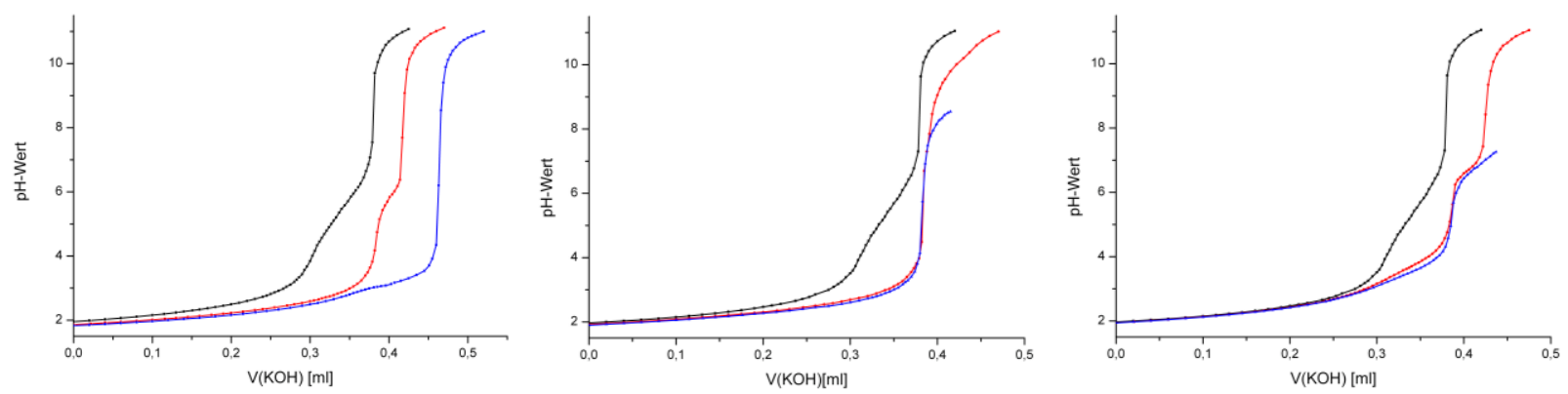

Abbildung 57: $\mathrm{pH}$-potentiometrische Titrationskurven von $\mathrm{HL}^{1}\left(\mathrm{c}=1.65^{\cdot} 10^{-3} \mathrm{M}\right) \mathrm{mit} \mathrm{Cu}$ (II)-Salz (links), $\mathrm{Ni}(\mathrm{II})-\mathrm{Salz}$ (Mitte) und Zn(II)-Salz (rechts).

\subsection{Potentiometrie von Kupfer(II)-Komplexen}

Die Auswertung der Titrationskurven des Systems $\mathrm{HL}^{1} / \mathrm{Cu}^{2+}$ (Abbildung 58) hat ergeben, dass zunächst bei niedrigem $\mathrm{pH}$-Wert freies $\mathrm{Cu}$ (II) neben der mononuklearen Spezies $\left[\mathrm{HL}^{1} \mathrm{Cu}\right]^{2+}$ vorliegt. Beide Spezies liegen zu gleichen Anteilen vor und nehmen bis $\mathrm{pH}=5$ auf einen Anteil von $0 \%$ ab. Von $\mathrm{pH}=2$ steigt der Anteil der dinuklearen Spezies $\left[\mathrm{L}^{1}{ }_{-\mathrm{H}} \mathrm{Cu}_{2}\right]^{2+}$ bis etwa $\mathrm{pH}=5$ auf $100 \%$ an. Diese Spezies bleibt bis $\mathrm{zu} \mathrm{pH}=10$ die einzig feststellbare Verbindung.

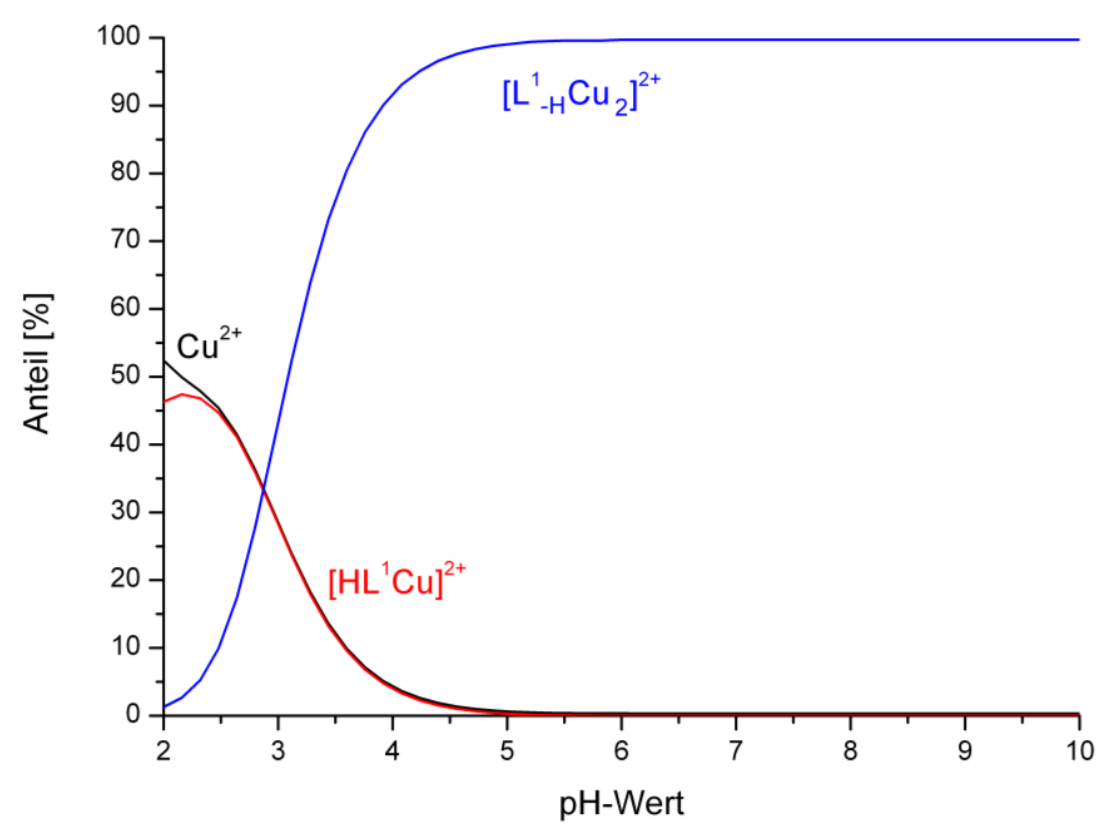

Abbildung 58: Speziesverteilung des Systems $\mathrm{HL}^{1}: \mathrm{Cu}^{\prime \prime}\left(I=0.2 \mathrm{M}(\mathrm{KCl}), T=25^{\circ} \mathrm{C}, \mathrm{C}_{\text {Ligand }}=1.7 \cdot 10^{-3} \mathrm{M}\right)$. 
Zudem lässt sich das Cu":Ligand-System per pH-Wert abhängiger UV/VisSpektroskopie untersuchen. In Abbildung 59 sind die Titrationsergebnisse für das System Cu": $\mathrm{HL}^{1}$ (1:1) dargestellt. Die Aufnahme mehrerer Spektren im Bereich von $\mathrm{pH}=1.9$ bis $\mathrm{pH}=10.5$ zeigt eine deutliche Verschiebung der Absorptionsbande bei $750 \mathrm{~nm}$ für $\mathrm{pH}=1.9$ zu $620 \mathrm{~nm}$ für $\mathrm{pH}=10.5$.

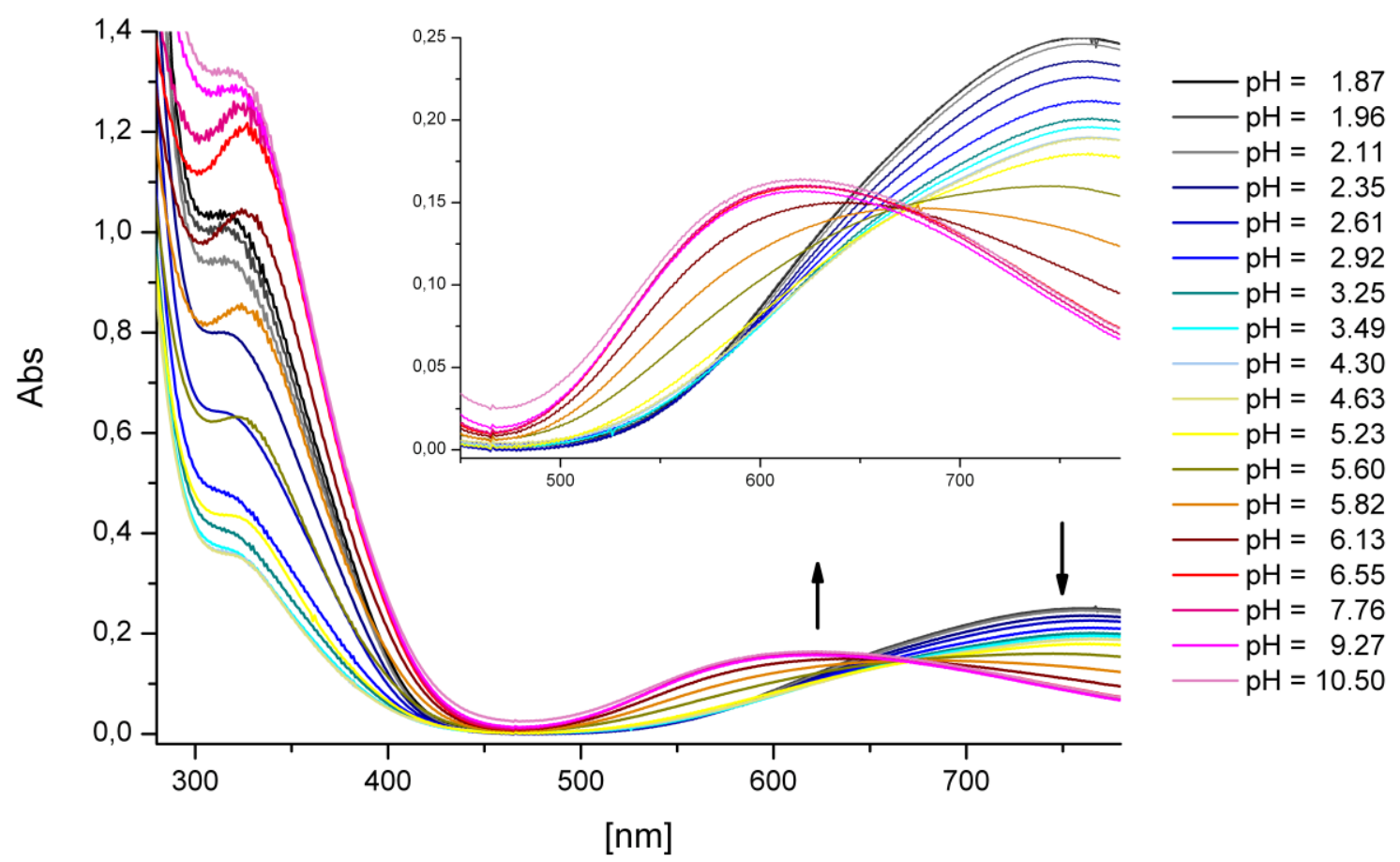

Abbildung 59: UV/Vis-Spektren für das $\mathrm{Cu}^{\prime \prime}-\mathrm{HL}^{1}$-System (1:1) als Funktion des $\mathrm{pH}-$ Wertes $\left(\mathrm{C}_{\text {Ligand }}=1.51 \cdot 10^{-3} \mathrm{M}\right)$.

Die Absorption bei $750 \mathrm{~nm}$ ist somit der mononuklearen Spezies zuzuschreiben. Demzufolge muss die Absorption bei $620 \mathrm{~nm}$ zum dinuklearen Kupfer(II)-Komplex $\left[\mathrm{L}^{1}{ }_{\mathrm{H}} \mathrm{Cu}_{2}\right]^{2+}$ gehören. Dazwischen existiert ein Übergangsbereich bei $\mathrm{pH}=5.8$ bis $\mathrm{pH}=6.6$. Vergleicht man diese Ergebnisse mit der erhaltenen Speziesverteilung, fällt ein Unterschied auf. So sollte der Übergangsbereich laut Speziesverteilung zwischen $\mathrm{pH}=3$ und $\mathrm{pH}=5$ liegen. Eine mögliche Ursache könnte in der Bildung der $\mathrm{OH}$-Brücke liegen. Bei sehr niedrigen pH-Werten kann diese eventuell durch eine Chlorid-Brücke ausgetauscht werden.

$\mathrm{Da}$ anhand der bisher erhaltenen Ergebnisse der potentiometrischen Messungen erkennbar ist, dass die Bildung einer dinuklearen Kupferspezies stark bevorzugt wurde, 
ist für ein System $\mathrm{Cu}^{\prime \prime}: \mathrm{HL}^{1}$ (2:1) ebenfalls $\mathrm{pH}-$ Wert abhängige UV/Vis-Spektren gemessen worden. Der Verlauf von $\mathrm{pH}=1.83$ bis $\mathrm{pH}=10.42$ ist in Abbildung 60 gezeigt. Die Abfolge der einzelnen UV/Vis-Spektren unterscheidet sich von denen für das System Cu":HL (1:1). So beobachtet man in dem 2:1-System einen Wechsel der Absorption von $630 \mathrm{~nm}$ auf $570 \mathrm{~nm}$ in einem pH-Wert-Bereich von 3.02 bis 3.30 . Diese Werte stimmen sehr gut mit den Ergebnissen der Speziesverteilung überein. So sollten die Absorptionen bei etwa $630 \mathrm{~nm}$ durch die mononukleare Spezies verursacht werden und die dinukleare Spezies quadratisch pyramidal bzw. quadratisch planare Koordinationsmuster $\left[\mathrm{L}^{1}{ }_{\mathrm{H}} \mathrm{Cu}_{2}\right]^{2+}$ weist eine starke Absorption bei ca. $570 \mathrm{~nm}$ auf.

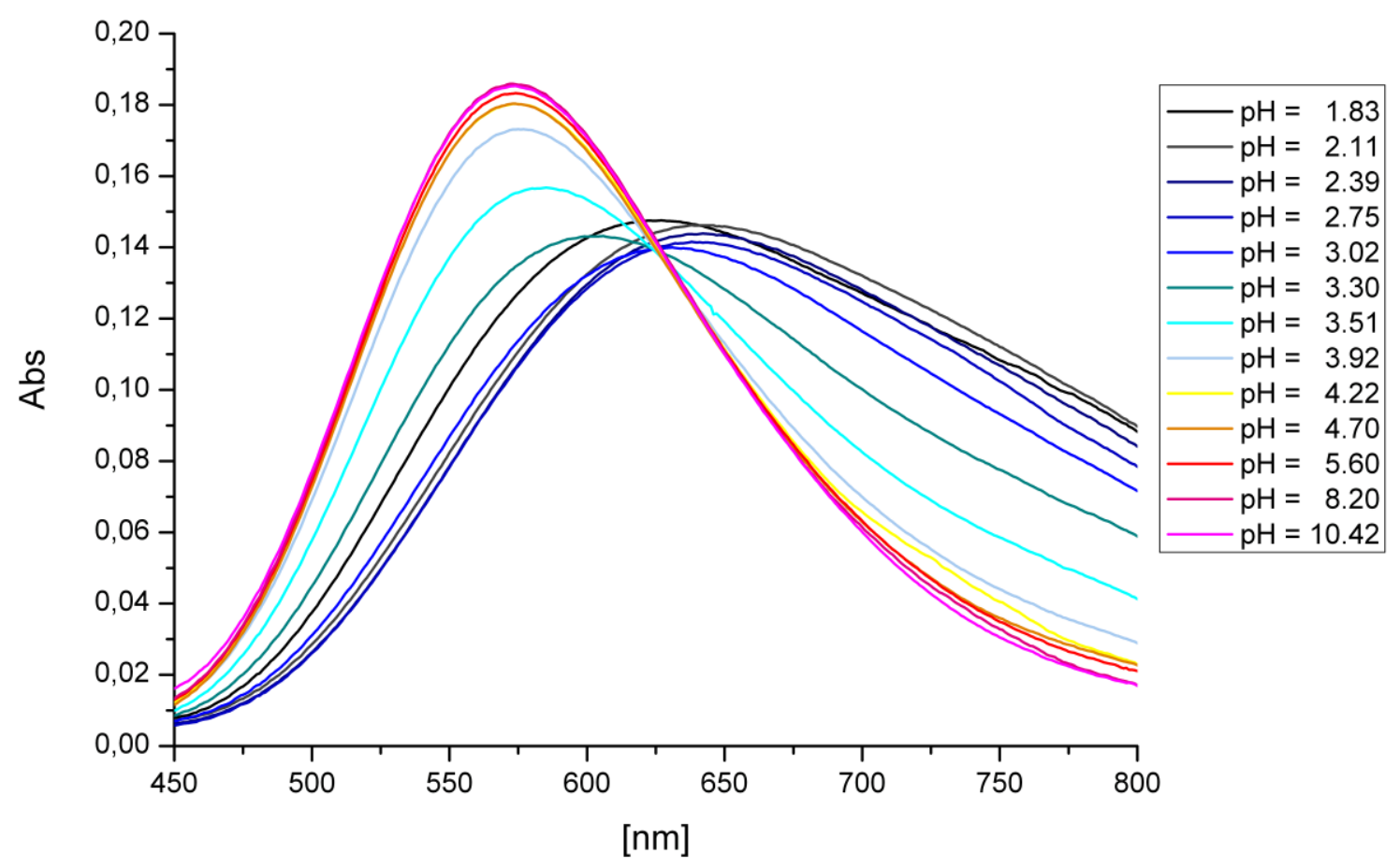

Abbildung 60: UV/Vis-Spektren für das $\mathrm{Cu}^{\prime \prime}-\mathrm{HL}^{1}$-System (2:1) als Funktion des $\mathrm{pH}$-Wertes $\left(\mathrm{C}_{\text {Ligand }}=1.51 \cdot 10^{-3} \mathrm{M}\right)$.

Aufgrund der sehr guten Übereinstimmung mit den UV/Vis-Spektren der Kupfer(II)Komplexe $\mathbf{8}$ bis $\mathbf{1 3}$ ist es möglich, Strukturvorschläge für die Spezies aus Abbildung 61 zu treffen. Wie bereits in Abschnitt 4.1 besprochen wurde, ähneln sich die in Lösung aufgenommenen UV/Vis-Spektren mit denen des als Feststoff vermessenen Komplexes sehr stark. Deshalb kann man davon ausgehen, dass in Lösung die gleiche Komplexstruktur vorliegt, wie sie mittels Röntgenstrukturanalyse bestimmt wurde. 
Die Spezies $\left[\mathrm{HL}^{1} \mathrm{Cu}\right]^{2+}$ entspricht der Koordination eines Kupfer(II)-Ions an den Liganden. Dabei ist das $\mathrm{NH}$-Proton des Pyrazolrings noch vorhanden und die Koordination kann ausschließlich über das zweite Stickstoffatom des Pyrazols und über die Imidazolstickstoffatome erfolgen. Für die über einen sehr breiten $\mathrm{pH}$-Bereich dominante Spezies $\left[\mathrm{L}^{1}{ }_{-H} \mathrm{Cu}_{2}\right]^{2+}$ kann analog $\mathrm{zu}$ den in Kapitel 4.1. vorgestellten Ergebnissen die in Abbildung 60 gezeigte Formel angenommen werden.

Methlgr
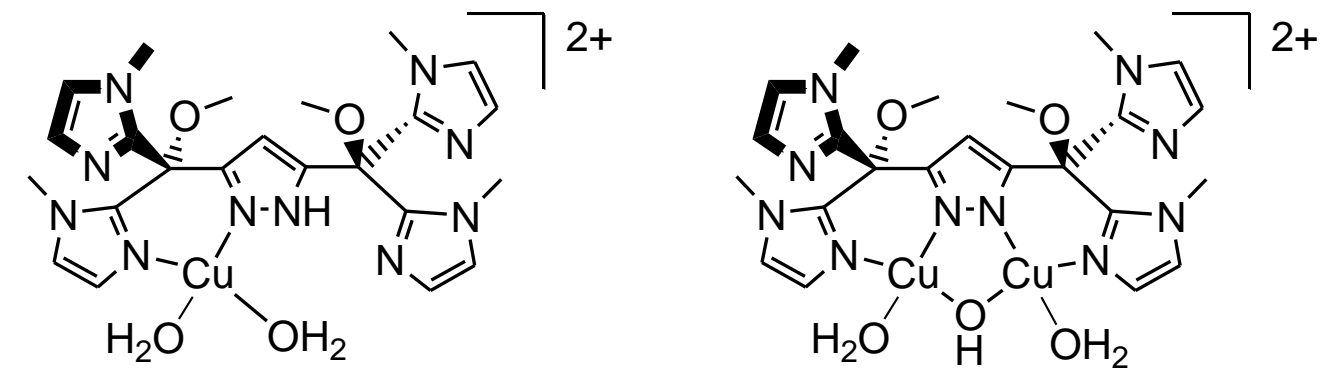

Abbildung 60: Strukturvorschläge für $\left[\mathrm{HL}^{1} \mathrm{Cu}\right]^{2+}$ (links) und $\left[\mathrm{L}_{-H}^{1} \mathrm{Cu}_{2}\right]^{2+}$ (rechts).

Alle Kupfer(II)-Komplexe, die im Rahmen dieser Arbeit strukturell aufgeklärt werden konnten, haben ein $\mathrm{Cu}^{\prime \prime}:$ Ligand-Verhältnis von 2:1. Die bevorzugte Bildung von dinuklearen Einheiten konnte mit den potentiometrischen Messungen bestätigt werden. Allerdings ist es nicht möglich, eine Unterscheidung zwischen dem Monomer $\left[\mathrm{L}^{1}{ }_{-H} \mathrm{Cu}_{2}\right]^{2+}$ und dem Dimer $\left[\mathrm{L}^{1} \cdot{ }_{\mathrm{H}} \mathrm{Cu}_{2}\right]_{2}{ }^{4+}$ zu vollziehen.

Um die erhaltenen Ergebnisse zu untermauern, wurde der Ligand $\mathrm{HL}^{1}$ mit $\mathrm{Kupfer}$ (II)Chlorid in Wasser zur Reaktion gebracht. Es entsteht eine blaue Lösung, die einen pH-Wert von etwa 3.2 aufweist. Durch Zugabe von Natronlauge ist es möglich, den pH-Wert der Lösung auf ca. 8 einzustellen. Nachdem das Lösungsmittel im Vakuum entfernt wurde, ist von dem resultierenden Rohprodukt ein FAB-Massenspektrum aufgenommen worden. Darin sind zahlreiche Peaks im Bereich von $\mathrm{m} / \mathrm{z}=253$ bis $\mathrm{m} / \mathrm{z}=1329 \mathrm{zu}$ finden. Eine Identifizierung des Fragments $\left[\mathrm{L}^{1}{ }_{-} \mathrm{Cu}_{2}\right]^{2+}$ in Form von $\left[\mathrm{L}^{1} \mathrm{Cu}_{2}(\mathrm{OH})(\mathrm{Cl})\right]^{+}$oder $\left[\mathrm{L}^{1} \mathrm{Cu}_{2}(\mathrm{Cl})_{2}\right]^{+}$konnte nicht erfolgen. Das Spektrum zeigt lediglich deutliche Signale bei $\mathrm{m} / \mathrm{z}=252.9$ sowie $\mathrm{m} / \mathrm{z}=539.2$ für $\left[\mathrm{HL}^{1} \mathrm{Cu}\right]^{+}$. Daneben sind im Bereich von $\mathrm{m} / \mathrm{z}=1078$ bis $\mathrm{m} / \mathrm{z}=1425$ einige Peaks $z u$ finden, die tetranuklearen Spezies zugesprochen werden müssen. Dazu zählen unter anderem die Signale bei $\mathrm{m} / \mathrm{z}=1292.2$ für $\left[\mathrm{L}_{2} \mathrm{Cu}_{4} \mathrm{Cl}_{2}(\mathrm{O})\right]^{+}$und bei $\mathrm{m} / \mathrm{z}=1327.2$ für $\left[\mathrm{L}^{1}{ }_{2} \mathrm{Cu}_{4} \mathrm{Cl}_{3}(\mathrm{O})\right]^{+}$. Die Bildung des Dimers der dinuklearen Einheit kann zum einen im Massenspektrometer erfolgen. 
Allerdings sind in Abschnitt 4.1. zahlreiche tetranukleare Kupfer(II)-Komplexe vorgestellt worden, die als Dimer einer dinuklearen Einheit aufgefasst werden müssen. Deshalb ist $\mathrm{zu}$ vermuten, dass auch in dieser Reaktion ein tetranuklearer Kupfer(II)-Komplex gebildet wurde. Mittels FAB-Massenspektrometrie kann somit auch das Auftreten einer dinuklearen Kupfer(II)-Spezies und dessen Dimer bestätigt werden.

\subsection{Untersuchung der Nickel(II)-Komplexe}

Im Fall des Systems $\mathrm{HL}^{1} / \mathrm{Ni}^{\mathrm{Il}}$ erlauben die Titrationskurven die Berechnung der Stabilitätskonstanten einiger mononuklearer Spezies (Abbildung 61). Dazu zählen $\left[\mathrm{H}_{2} \mathrm{~L}^{1} \mathrm{Ni}\right]^{3+},\left[\mathrm{HL}^{1} \mathrm{Ni}^{2+},\left[\mathrm{L}^{1} \mathrm{Ni}\right]^{+}\right.$und $\left[\mathrm{L}_{-}^{1}{ }_{-H} \mathrm{Ni}\right]$. Freies $\mathrm{Ni}^{1 /}$ liegt bei sehr niedrigen $\mathrm{pH}-$ Werten in Anteilen bis zu $60 \%$ vor. Bei $\mathrm{pH}=4$ kann aber bereits kein freies $\mathrm{Ni}(\mathrm{II})$ mehr festgestellt werden. In Abhängigkeit des pH-Wertes bilden sich protonierte bzw. deprotonierte Komplexe. Ausgehend von $\mathrm{pH}=2$ steigt der Anteil von $\left[\mathrm{H}_{2} \mathrm{~L}^{1} \mathrm{Ni}\right]^{3+}$ bis $\mathrm{pH}=2.5$ an und nimmt dann bis $\mathrm{pH}=4.5$ auf einen Anteil von $0 \%$ ab. Dabei handelt es sich um eine Verbindung, in der der Pyrazolring noch das $\mathrm{NH}$-Proton trägt. Außerdem könnte noch ein Imidazolring protoniert sein. $\left[\mathrm{HL}^{1} \mathrm{Ni}\right]^{2+}$ ist über einen großen pH-Bereich (ca. 3 bis 8 ) die vorherrschende Spezies.

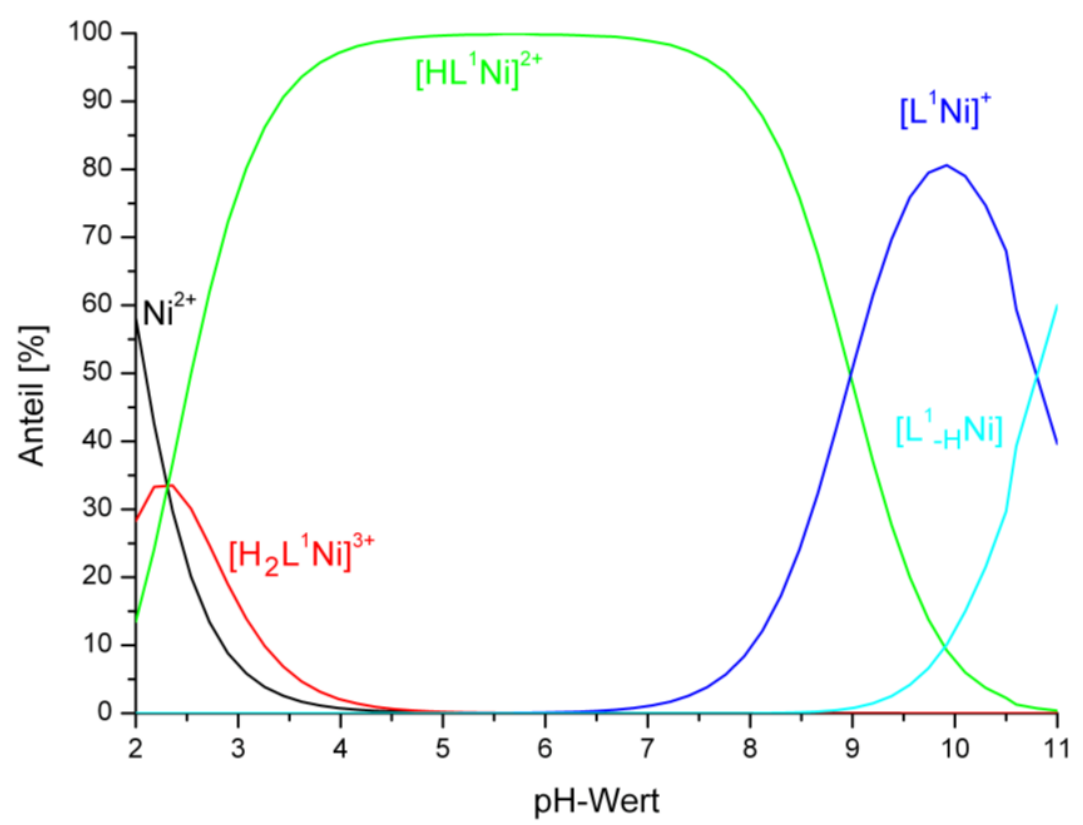

Abbildung 61: Speziesverteilung des Systems $\mathrm{HL}^{1} / \mathrm{Ni}^{\prime \prime}\left(I=0.2 \mathrm{M}(\mathrm{KCl}), T=25^{\circ} \mathrm{C}, \mathrm{C}_{\text {Ligand }}=1.7 \cdot 10^{-3} \mathrm{M}\right)$. 
Ab einem pH-Wert von etwa 9 überwiegt eine Spezies, die einen deprotonierten Ligand und ein Nickel(II)-Ion enthält. Oberhalb eines pH-Wertes von ca. 10 nimmt der Anteil dieser Spezies ab und es entsteht bevorzugt eine Verbindung, die einer weiteren Deprotonierung unterliegt.

Die Resultate der pH-abhängigen UV/Vis-Titration für das System Ni"/HL ${ }^{1}$ (1:1) im $\mathrm{pH}$-Bereich von 1.9 bis 10.9 sind in Abbildung 62 zusammengefasst. Hierin ist klar zu erkennen, dass bei sehr niedrigen $\mathrm{pH}-$ Werten (1.9 bis 2.3) die UV/Vis-Spektren bei ca. $640 \mathrm{~nm}$ eine deutliche Absorptionsbande aufweisen. Ein Vergleich mit der ermittelten Speziesverteilung lässt den Schluss zu, dass die Absorption der Spezies $\left[\mathrm{H}_{2} \mathrm{~L}^{1} \mathrm{Ni}\right]^{3+}$ zugeordnet werden muss. Die darauffolgende Absorption bei steigenden pH-Werten stammt damit von den danach auftretenden Spezies $\left[\mathrm{HL}^{1} \mathrm{Ni}\right]^{2+}$ und $\left[\mathrm{L}^{1} \mathrm{Ni}\right]^{+}$. Da kaum ein Unterschied beim Wechsel der einzelnen Spezies nicht zu erkennen ist, lässt sich ableiten, dass die Koordinationsumgebung in beiden Verbindungen sehr ähnlich sein muss.

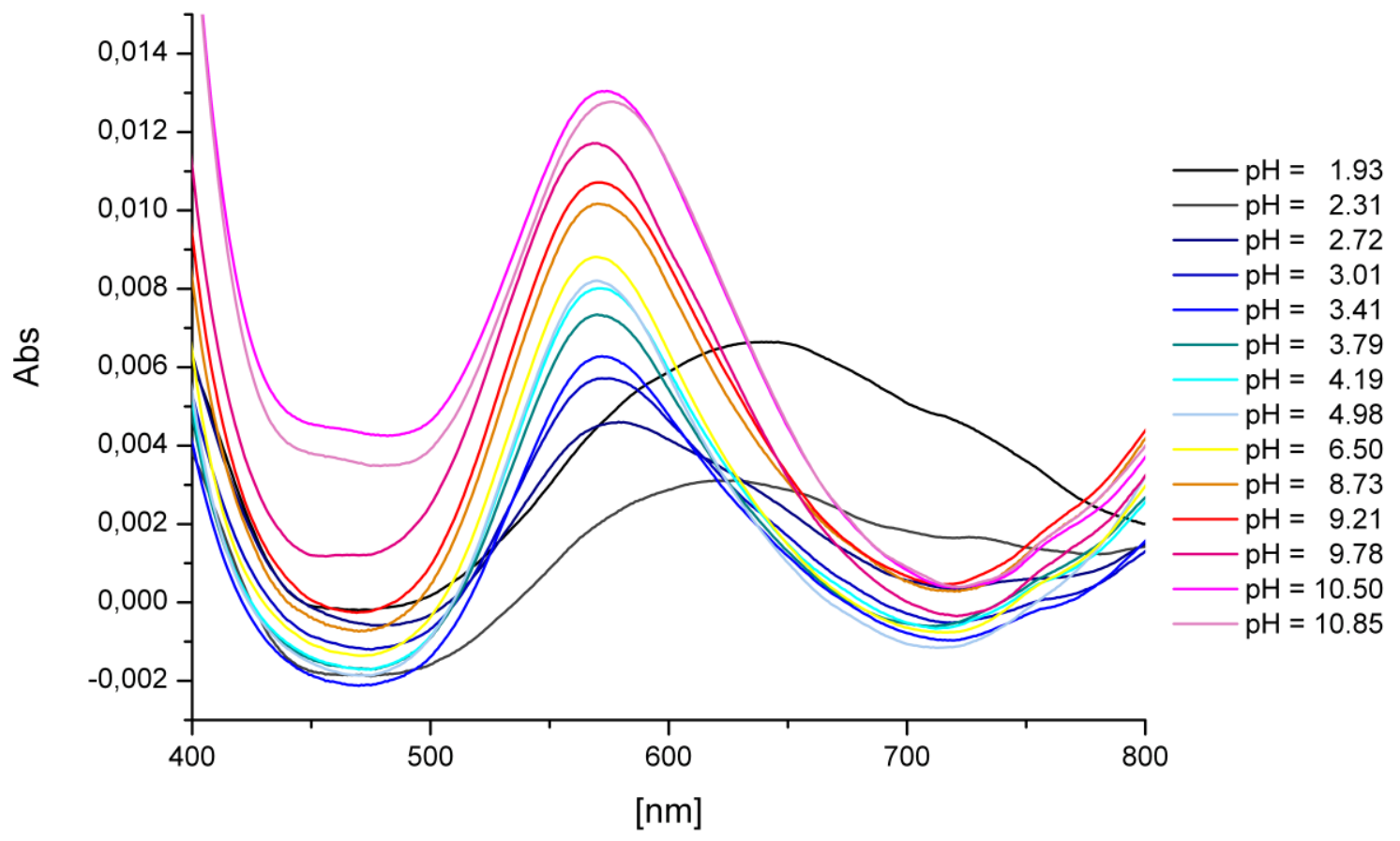

Abbildung 62: UV/Vis-Spektrum für das $\mathrm{Ni}^{\mathrm{I}}-\mathrm{HL}^{1}$-System (1:1) als Funktion des $\mathrm{pH}-$ Wertes $\left(c_{\text {Ligand }}=1.36 \cdot 10^{-3} \mathrm{M}\right)$. 
Auch zur Spezies [ $\left.\mathrm{L}^{1}{ }_{-H} \mathrm{Ni}\right]$ besteht sehr große Ähnlichkeit. Das Absorptionsmaximum bei $568 \mathrm{~nm}$ verschiebt sich ab einem $\mathrm{pH}=10.5$ auf ca. $574 \mathrm{~nm}$. Oberhalb dieses $\mathrm{pH}-$ Wertes liegt vorrangig die doppelt deprotonierte Spezies vor. Da der Unterschied aber nur $6 \mathrm{~nm}$ beträgt, ist es wahrscheinlich, dass die Koordinationsgeometrie unverändert bleibt und nur ein Co-Ligand am Nickel-lon ausgetauscht wird. Außerdem ist es denkbar, dass ein Imidazol vom Nickelzentrum abdissoziiert und dafür ein Wassermolekül zusätzlich koordiniert.

Mögliche Strukturen für alle diese Spezies sind in Abbildung 63 aufgezeigt. Anhand der Speziesverteilung kann nicht erkannt werden, von welcher Position ein Proton abstrahiert wird. Aus diesem Grund ist die Nomenklatur angepasst. Dabei meint $\mathrm{HL}$ den freien Liganden in seiner neutralen Form. $\mathrm{H}_{2} \mathrm{~L}$ ist die protonierte Form und $\mathrm{L}$ symbolisiert den deprotonierten Zustand des Liganden. Die Bezeichnung $L_{-H}$ bedeutet, dass der deprotonierte Ligand vorliegt und dem System ein weiteres Proton entzogen wurde. Dies kann normalerweise nicht aus dem Ligand stammen. Dafür kommt in wässrigen Systemen zum Beispiel die Deprotonierung von Wasser zum Hydroxid-Ion in Frage.
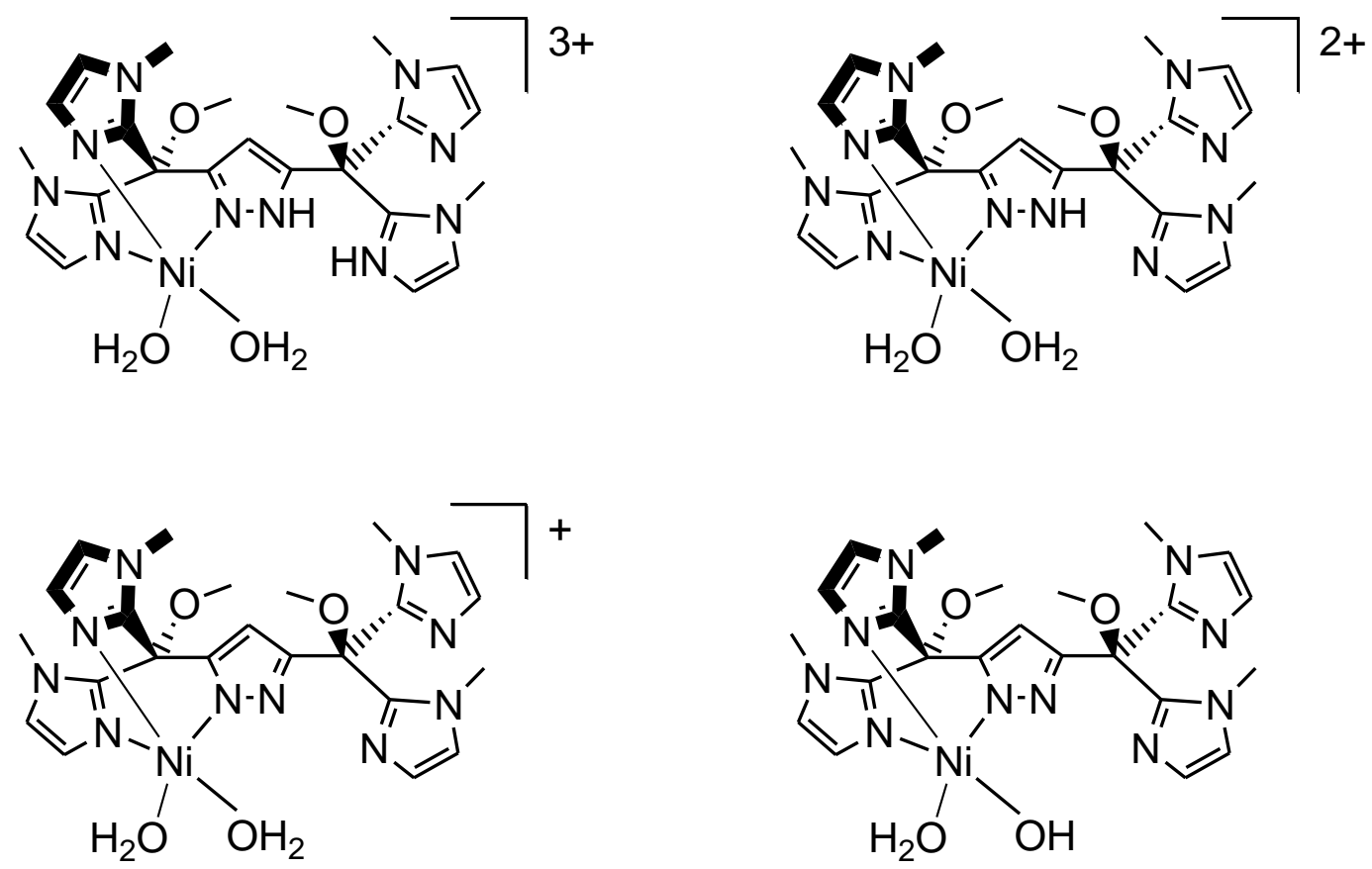

Abbildung 63: Strukturvorschläge für die beobachteten Spezies $\left[\mathrm{H}_{2} \mathrm{~L}^{1} \mathrm{Ni}\right]^{3+}$ (oben links), $\left[\mathrm{HL}^{1} \mathrm{Ni}\right]^{2+}$ (oben rechts), $\left[\mathrm{L}^{1} \mathrm{Ni}\right]^{+}$(unten links) und $\left[\mathrm{L}^{1}{ }_{-\mathrm{H}} \mathrm{Ni}\right]$ (unten rechts). 
Auch für dieses System ist eine analoge Umsetzung des Liganden $\mathrm{HL}^{1}$ mit Nickel(II)chlorid in wässriger Lösung durchgeführt worden. Die resultierende schwach graublau gefärbte Reaktionslösung weist einen pH-Wert von etwa 5.4 auf. Bei diesem pH-Wert sollte die Spezies $\left[\mathrm{HL}^{1} \mathrm{Ni}\right]^{2+}$ vorliegen. Durch Aufnahme eines FAB-Massenspektrums des nach Entfernen des Lösungsmittels entstandenen Rohproduktes kann nicht überprüft werden, welcher Protonierungsgrad vorliegt. Allerdings lässt sich eine Aussage treffen, ob es sich um mono- oder dinukleare Nickel(II)-Komplexe handelt. So findet man einige Peaks im Bereich von $\mathrm{m} / \mathrm{z}=265$ bis $\mathrm{m} / \mathrm{z}=1447$. Bei $\mathrm{m} / \mathrm{z}=1103.2$ ist der intensivste Peak zu finden, der der Spezies $\left[\mathrm{L}_{2}^{1} \mathrm{Ni}_{2} \mathrm{Cl}\right]^{+}$zugeordnet werden kann. Wie bereits bei den potentiometrischen Messungen des Systems $\mathrm{Cu}^{\prime \prime}: \mathrm{HL}^{1}$ erwähnt wurde, ist eine Unterscheidung zwischen $\left[\mathrm{L}^{1} \mathrm{Ni}\right]^{+}$und $\left[\mathrm{L}^{1} \mathrm{Ni}_{2}{ }_{2}^{2+}\right.$ nicht möglich.

\subsection{Charakterisierung der Zink(II)-Komplexe}

Das Kupfer(I)-lon besitzt die gleiche Elektronenkonfiguration $\left(\mathrm{d}^{10}\right)$ wie das $\mathrm{Zn}(\mathrm{II})$-lon. Die in der Regel gefundenen Koordinationsgeometrien (oktaedrisch, trigonal bipyramidal) ähneln aber eher denen von $\mathrm{Cu}^{\prime \prime}$ oder $\mathrm{Ni}^{\prime \prime}$. Die Ergebnisse der potentiometrischen Messungen sollten somit denen von Ni sehr ähnlich sein.

Aus diesem Grund sind ebenfalls Titrationskurven des Systems $\mathrm{HL}^{1} / \mathrm{Zn}^{\prime \prime}$ aufgenommen worden. Anhand der in Abbildung 64 gezeigten Speziesverteilung lässt sich erkennen, dass wie schon für Nickel(II)-lonen vorwiegend mononukleare Spezies wie $\left[\mathrm{H}_{3} \mathrm{~L}^{1} \mathrm{Zn}\right]^{4+}$, $\left[\mathrm{H}_{2} \mathrm{~L}^{1} \mathrm{Zn}\right]^{3+}$, [HL $\left.\mathrm{L}^{1} \mathrm{Zn}\right]^{2+}$ und $\left[\mathrm{L}^{1} \mathrm{Zn}\right]^{+}$vorliegen. Wie schon für die beiden anderen Metalllonen liegt bei niedrigem pH-Werten ein großer Anteil an freiem Zink(II)-lonen vor. Außerdem existieren bei niedrigen $\mathrm{pH}$-Wert zwei Spezies mit protonierten Liganden in geringen prozentualen Anteilen. $\mathrm{Ab} \mathrm{pH}=4$ liegt vor allem eine Spezies vor, die aus einem Zink(II)-Ion und dem Liganden besteht. Oberhalb eines pH-Wertes von etwa 6.8 wird der Ligand offensichtlich deprotoniert und mehrheitlich eine einfachgeladene Spezies gebildet. 


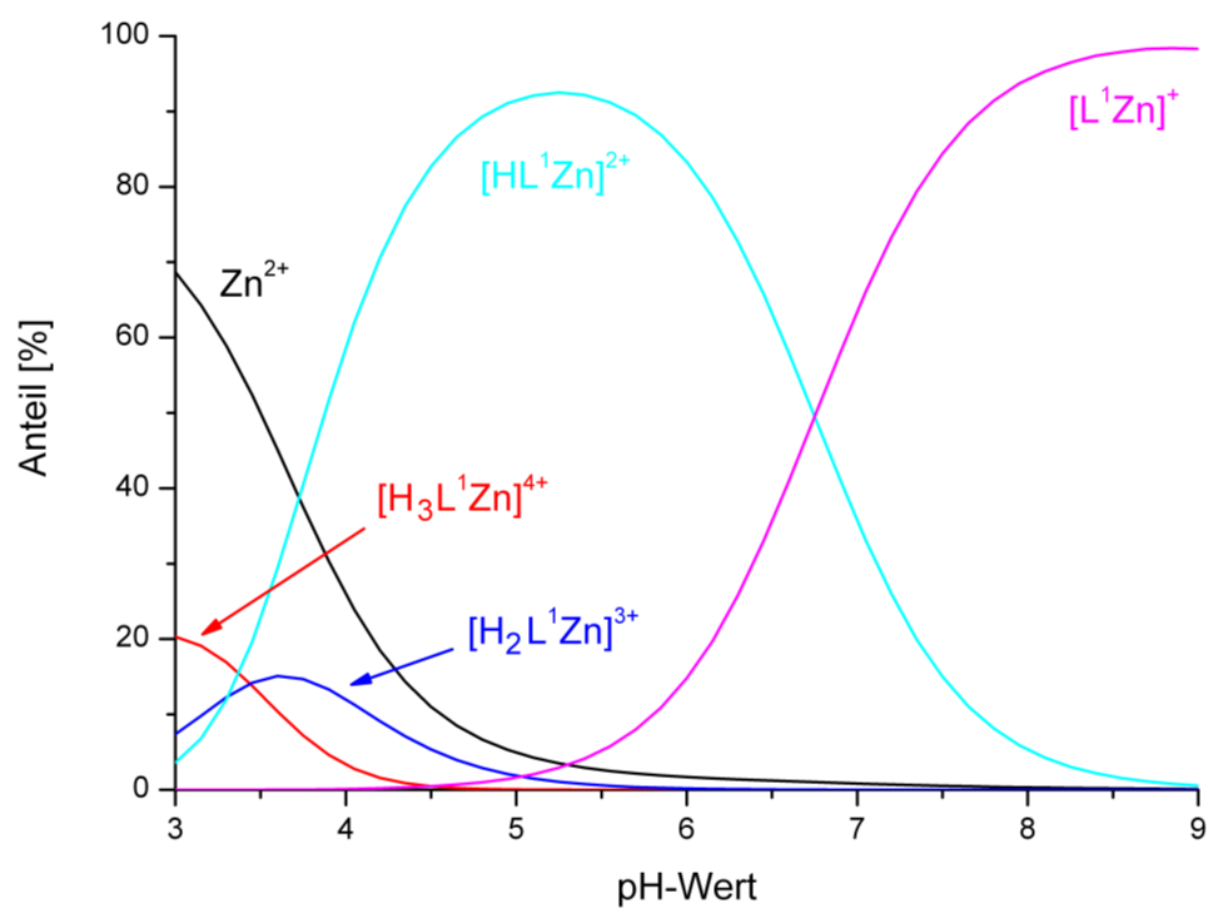

Abbildung 64: Speziesverteilung des Systems $\mathrm{HL}^{1} / \mathrm{Zn}^{\prime \prime}\left(I=0.2 \mathrm{M}(\mathrm{KCl}), T=25^{\circ} \mathrm{C}, \mathrm{C}_{\text {Ligand }}=1.7 \cdot 10^{-3} \mathrm{M}\right)$.

Auch für diese verschiedenen Spezies können verschiedene Strukturen vorgeschlagen werden. So wird offensichtlich Schritt für Schritt der Ligand deprotoniert, bis in der letzten Spezies, die beobachtet werden konnte, auch der Pyrazolring deprotoniert vorliegen sollte (Abbildung 65).
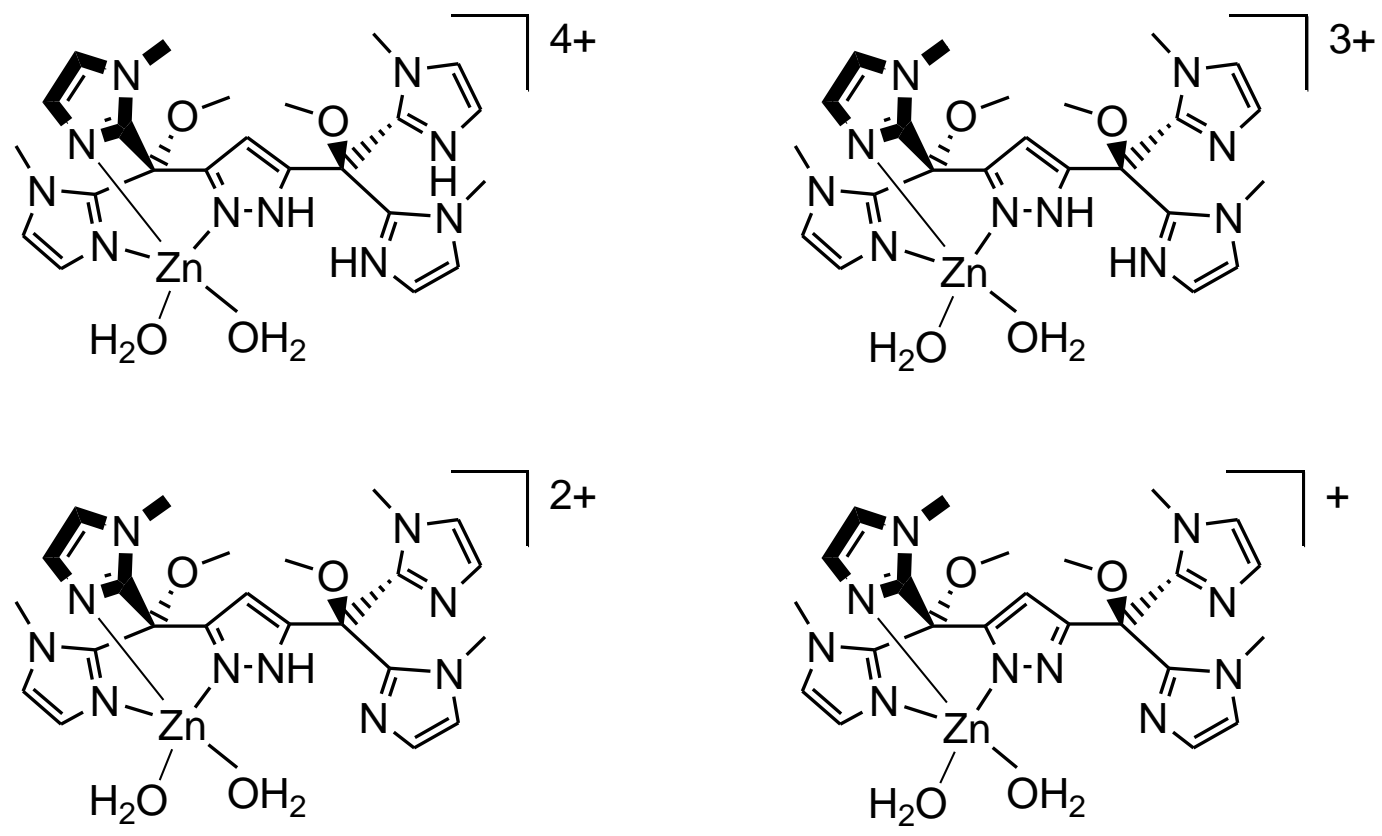

Abbildung 65: Strukturvorschläge für $\left[\mathrm{H}_{3} \mathrm{~L}^{1} \mathrm{Zn}\right]^{4+}$ (oben links), $\left[\mathrm{H}_{2} \mathrm{~L}^{1} \mathrm{Zn}\right]^{3+}$ (oben rechts), $\left[\mathrm{HL}^{1} \mathrm{Zn}\right]^{2+}$ (unten links), $\left[\mathrm{L}^{1} \mathrm{Zn}\right]^{+}$(unten rechts). 
Auch für dieses System ist durch die Umsetzung des Liganden $\mathrm{HL}^{1}$ mit Zink(II)-chlorid in wässriger Lösung das Ergebnis der potentiometrischen Titration überprüft worden. Die Reaktionslösung weist einen $\mathrm{pH}$-Wert von 5.65 auf. Es sollte vorrangig die Spezies $\left[\mathrm{HL}^{1} \mathrm{Zn}\right]^{2+}$ vorliegen. Das FAB-Massenspektrum zeigt eine Vielzahl von Signalen im Bereich von $\mathrm{m} / \mathrm{z}=313$ bis $\mathrm{m} / \mathrm{z}=1390$. Jedoch lassen sich davon nicht alle eindeutig entsprechenden Fragmenten zuordnen. Die Spezies $\left[\mathrm{HL}^{1} \mathrm{ZnCl}\right]^{+}$kann mit dem Signal bei $\mathrm{m} / \mathrm{z}=575.1$ identifiziert werden. Dieses Ergebnis entspricht dem erwarteten Strukturvorschlag von Abbildung 65 (unten links). Die koordinierten Wassermoleküle sind durch ein Chlorid-Ion ausgetauscht. Dadurch trägt die Spezies keine doppelte Ladung und ist sehr gut zu detektieren. Daneben lassen sich zwei weitere Peaks mit den dazugehörigen Spezies in Verbindung bringen. Das Signal bei $\mathrm{m} / \mathrm{z}=1117.3$ lässt sich der Spezies $\left[\mathrm{L}_{2}^{1} \mathrm{ZnCl}\right]^{+}$zuordnen und der Peak bei $\mathrm{m} / \mathrm{z}=1253.1$ ist der Spezies $\left[\mathrm{L}_{2}^{1} \mathrm{Zn}_{3} \mathrm{Cl}_{3}\right]^{+}$zuzuschreiben. Die Spezies entspricht dem Dimer des Strukturvorschlages aus Abbildung 65 unten rechts. Es ist für Übergangsmetalle wie Nickel, Kupfer oder Zink bekannt, dass sich zweifach pyrazolatverbrückte dinukleare Metallkomplexe bilden können. ${ }^{[152-158]}$

Mit Kupfer(II)-Ionen bildet sich hautsächlich eine dinukleare Spezies $\left[\mathrm{L}^{1}{ }_{\mathrm{H}} \mathrm{Cu}_{2}\right]^{2+}$, die zwischen $\mathrm{pH}=4$ bis $\mathrm{pH}=10$ die einzig vorkommende Spezies darstellt.

Im Gegensatz werden sowohl mit Zink(II)- als auch mit Nickel(II)-lonen in Gegenwart von $\mathrm{HL}^{1}$ vorrangig mononukleare Komplexe gebildet. Eine weitere Gemeinsamkeit ist das Auftreten von Spezies, in denen der Ligand einfach oder sogar doppelt protoniert ist. Während aber die Spezies $\left[\mathrm{HL}^{1} \mathrm{Ni}^{2+}\right.$ zwischen $\mathrm{pH}=4$ und $\mathrm{pH}=7$ vorliegt, ist die gleiche Spezies im Fall von $\mathrm{Zn}$ "l lediglich zwischen $\mathrm{pH} 4.5$ und $\mathrm{pH}=6$ die Hauptspezies. Dafür liegt die Spezies $\left[\mathrm{L}^{1} \mathrm{Zn}\right]^{+}$über einen größeren pH-Bereich ( 7.5 bis 9 ) vor als die entsprechende Nickel-Spezies (9.5 bis 10.3). Wie man auch an den Zahlenwerten erkennt, liegt der $\mathrm{pH}$-Bereich der Spezies $\left[\mathrm{L}^{1} \mathrm{Zn}\right]^{+}$etwas weiter Richtung neutral bis schwach sauren pH-Werten. Diese Tatsache liegt vor allem an der Natur des Zink(II)lons. Es ist lewis-acide und kann somit Substrate und Co-Liganden polarisieren. So ist der pKs-Wert für $\left[\mathrm{Zn}\left(\mathrm{H}_{2} \mathrm{O}\right)_{6}\right]^{2+}$ etwa 10 , hingegen er in einigen enzymatischen Systemen bis auf 6 abnimmt. ${ }^{[32]}$ 
Aus allen drei Titrationen können für die entsprechenden Spezies die

Stabilitätskonstanten $(\log \beta)$ ermittelt werden. Diese sind in Tabelle 20 aufgelistet.

\begin{tabular}{lccc}
\hline & $\mathrm{Cu}(\mathrm{II})$ & $\mathrm{Ni}(\mathrm{II})$ & $\mathrm{Zn}(\mathrm{II})$ \\
\hline$\left[\mathrm{H}_{3} \mathrm{~L}^{1} \mathrm{M}\right]^{4+}$ & - & - & $13.26(7)$ \\
{$\left[\mathrm{H}_{2} \mathrm{~L}^{1} \mathrm{M}\right]^{3+}$} & - & $12.14(3)$ & $9.82(9)$ \\
{$\left[\mathrm{HL}^{1} \mathrm{M}\right]^{2+}$} & $11.45(8)$ & $9.82(2)$ & $6.51(3)$ \\
{$\left[\mathrm{L}^{1} \mathrm{M}\right]^{+}$} & - & $0.84(6)$ & $-0.24(6)$ \\
{$\left[\mathrm{L}_{-H}^{1} \mathrm{M}\right]$} & - & $-9.98(8)$ & - \\
{$\left[\mathrm{L}^{1}{ }_{-H} \mathrm{M}_{2}\right]^{2+}$} & $8.4(1)$ & - & - \\
\hline
\end{tabular}

Tabelle 20: Stabilitätskonstanten $(\log \beta)$ für $\mathrm{Cu}(\mathrm{II})-\mathrm{Ni}(\mathrm{II})-$ und $\mathrm{Zn}(\mathrm{II})-$ Komplexe des Liganden $\mathrm{HL}^{1}$, bei $T=25^{\circ} \mathrm{C}, I=0.2 \mathrm{M}(\mathrm{KCl})$ (Standardabweichungen sind in Klammern angegeben). 


\section{Reaktivitätsuntersuchungen der Kupfer(I)-Komplexe}

Kupfer(I)-Komplexe lassen sich mit einer Vielzahl von Reagenzien umsetzen, die als Oxidationsmittel wirken. Am besten ist die Reaktion zahlreicher Kupfer(I)-Komplexe mit Sauerstoff untersucht worden. Es wurden dabei die verschiedensten Möglichkeiten der $\mathrm{O}_{2}$-Anbindung an die Kupfer-lonen gefunden (vgl. Seite 15 Abbildung 7). Diese Addukte lassen sich mit diversen Substraten umsetzen. Es gibt aber auch noch andere kleine Moleküle, die für eine Umsetzung mit den in dieser Arbeit synthetisierten Kupfer(I)Komplexen geeignet sein sollten. Dazu zählen zum Beispiel Schwefel oder Kohlendioxid. Der Vorteil von Kohlendioxid ist, dass es gasförmig vorliegt und kaum Störsignale während der Messung liefert. Wenn während der Reaktion die Kupfer(I)Ionen oxidiert werden und Kupfer(II)-Komplexe entstehen, verfärben sich die Reaktionslösungen in der Regel nach grün oder blau. Falls die auftretenden Zwischenstufen zudem gefärbt sind, wie bei einem violett gefärbten Peroxo-Komplex, lassen sich die Veränderungen während einer solchen Substratumsetzung besonders gut mittels der UV/Vis-Spektroskopie verfolgen. Anhand der gebildeten Banden kann eine Aussage über gebildete Zwischenstufen und Reaktionsprodukte getroffen werden. Die Untersuchungen bezüglich der Reaktivität der synthetisierten Kupfer(I)-Komplexe gegenüber $\mathrm{O}_{2}$ und $\mathrm{CO}_{2}$ sind mit einem mobilen UV/Vis-Spektrometer durchgeführt worden. Da mit gewöhnlichen Quarz-Glas-Küvetten keine inerten Bedingungen erreicht werden können, ist für derartige Messungen auf eine UV/Vis-Sonde (Abbildung 66) zurückgegriffen worden. Die Messungen werden in dem abgebildeten Glasgefäß mit seitlichen Abgängen und einem Schliff durchgeführt. Der linke Anschluss ist notwendig, um Vakuum oder Stickstoff anzulegen und um zu gewährleisten, dass die Reaktion unter inerten Bedingungen abläuft. Mit dem Hahn auf der rechten Seite ist es möglich, das entsprechende Gas zuzugeben, nachdem das Messgefäß evakuiert wurde. Die Messsonde taucht in die Lösung ein und über ein Lichtleiterkabel wird die gemessene Absorption

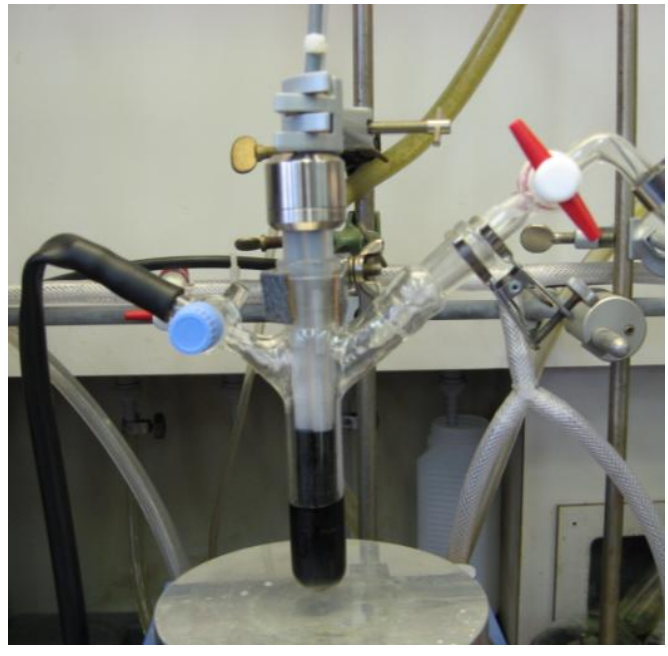

Abbildung 66: Messanordnung zur Aufnahme von UV/VisSpektren mittels Sonde. 
an das UV/Vis-Spektrometer geleitet und die Werte mittels eines Computers ausgewertet.

Zunächst wurde der Kupfer(I)-Komplex gemäß der in Schema 20 aufgeführten Reaktionsgleichung in situ ohne weiteren Co-Liganden hergestellt und nicht isoliert. Nachdem vor jeder Messreihe der Background bestimmt wurde, ist eine Probe des präparierten Kupfer(I)-Komplexes in Acetonitril unter Stickstoff in die Messzelle gegeben und die UV/Vis-Sonde eingesetzt worden. Anschließend wurde das Gefäß evakuiert, so dass das in einem Schlenkkolben befindliche Gas durch Öffnen eines Hahnes in die Messzelle strömen kann. So konnten bei jeder Messung in etwa $20 \mathrm{ml}$ Gas zugegeben werden. Dies entspricht für ein ideales Gas einer Stoffmenge von etwa $8.9 \mathrm{mmol}$. Je nachdem welche Konzentration verwendet wurde, entspricht diese Stoffmenge einem 4- bis 20 fachen Überschuss an Gas. Somit sollte gewährleistet, sein, dass eine vollständige Umsetzung der Kupfer(I)-Komplexe erfolgen kann. Abschließend wurde die Messung gestartet. Dafür wurde im Setup des Messprogrammes eingestellt, dass die Messungen bei $1000 \mathrm{~nm}$ gestartet und bis $200 \mathrm{~nm}$ die Absorption gemessen wurden. Außerdem muss noch eingestellt werden, wie häufig und mit welchem zeitlichen Abstand die Messungen aufgenommen werden sollen. Die einzelnen Messzeiten und -intervalle sind bei den jeweiligen Auswertungen angegeben.

\subsection{Kohlendioxid}

In Abbildung 67 sind die erhaltenen Messergebnisse für die Reaktion des aus Ligand $\mathrm{HL}^{1}$ hergestellten Kupfer(I)-Komplexes mit $\mathrm{CO}_{2}$ dargestellt. Zu Beginn der Reaktion erhält man von der orangebraun gefärbten Lösung ein UV/Vis-Spektrum, das im Bereich von $1000 \mathrm{~nm}$ bis $600 \mathrm{~nm}$ kein Maximum erkennen lässt. Erst bei Wellenlängen kleiner als 600 ist ein schwacher Anstieg auszumachen, der ab etwa $480 \mathrm{~nm}$ stark zunimmt und bei $370 \mathrm{~nm}$ eine Absorption > 1 erreicht. Da für diesen Bereich das Lambert-Beer-Gesetz nicht mehr ausreichend exakt gilt, sind diese Werte nicht dargestellt. Außerdem erfolgen bei Wellenlängen $<370 \mathrm{~nm}$ vor allem $\pi$ - $\pi^{*}$-Übergänge des Liganden. Diese sind für die hier angestellten Untersuchungen nicht interessant. 


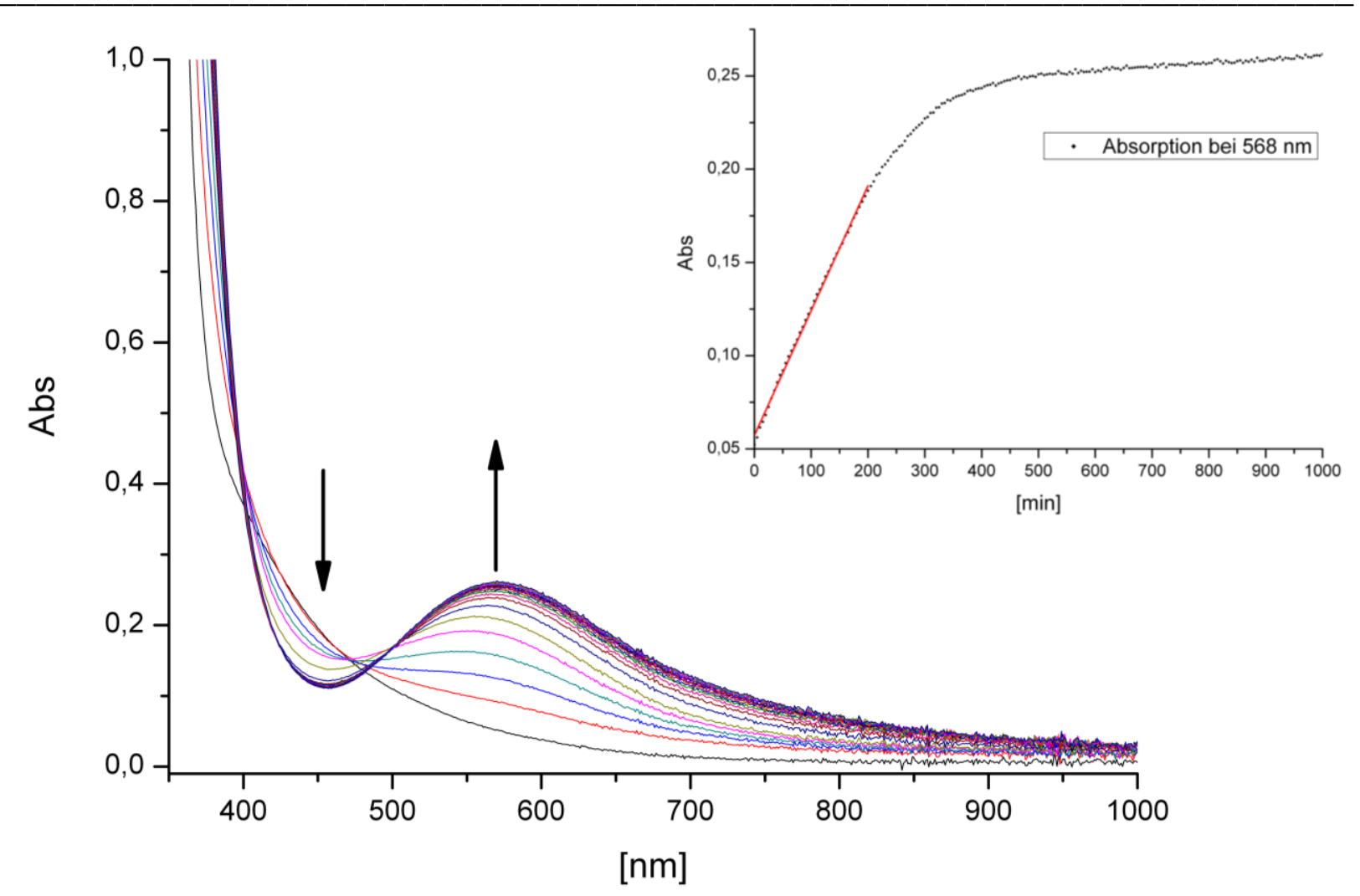

Abbildung 67: UV/Vis-Spektren für die Reaktion eines Kupfer(I)-Komplexes von $\mathrm{HL}^{1}$ mit $\mathrm{CO}_{2}$ bei RT und $0.02 \mathrm{M}$ in $\mathrm{CH}_{3} \mathrm{CN}$ (Nur jede zehnte Messung ist abgebildet).

Mit zunehmendem Fortschritt der Reaktion bildet sich eine Bande bei $568 \mathrm{~nm}$ $\left(\varepsilon=130 \mathrm{~mol}^{-1} \cdot \mathrm{lcm}^{-1}\right)$ aus und der Anstieg der Kurve ab etwa $480 \mathrm{~nm}$ verschwindet. Dafür bildet sich in diesem Bereich ein Minimum aus und ab Wellenlängen $<440 \mathrm{~nm}$ steigt die Absorption erneut sehr stark an und man erhält bis zur Messgrenze von $200 \mathrm{~nm}$ durchweg Absorptionen > 1. Desweiteren fallen zwei Punkte dieser Messreihe auf, in denen sich fast alle Kurven schneiden. Ein Punkt liegt bei ca. $480 \mathrm{~nm}$ und der zweite Punkt dieser Art ist bei etwa $400 \mathrm{~nm}$. Diese Punkte nennt man isosbestische Punkte. Nach ca. 10 bis 11 Stunden ist die Reaktion abgeschlossen und es hat sich eine blauviolette Lösung gebildet.

Trägt man die Absorption des Maximums bei $568 \mathrm{~nm}$ als Funktion der Zeit auf, erhält man den in Abbildung 67 (rechts) gezeigten Verlauf. Zunächst gibt es einen nahezu linearen Anstieg bis 190 Minuten. Danach flacht die Kurve stetig ab, ehe sie ab etwa 550 Minuten parallel zur x-Achse verläuft. Mit dem Programm ORIGIN ist es möglich, den Anstieg und damit auch ein Maß für die Anfangsgeschwindigkeit zu ermitteln. Die rote Linie stellt die bestmögliche Anpassung dar. 
Durch Dampfdiffusion von Diethylether in die blauviolette Komplexlösung ist es möglich, blauviolette Kristalle zu erhalten. Diese sind für eine Röntgenstrukturanalyse geeignet und die daraus erhaltene Struktur des vierkernigen Kupferkomplexes 28 ist in Abbildung 68 gezeigt.

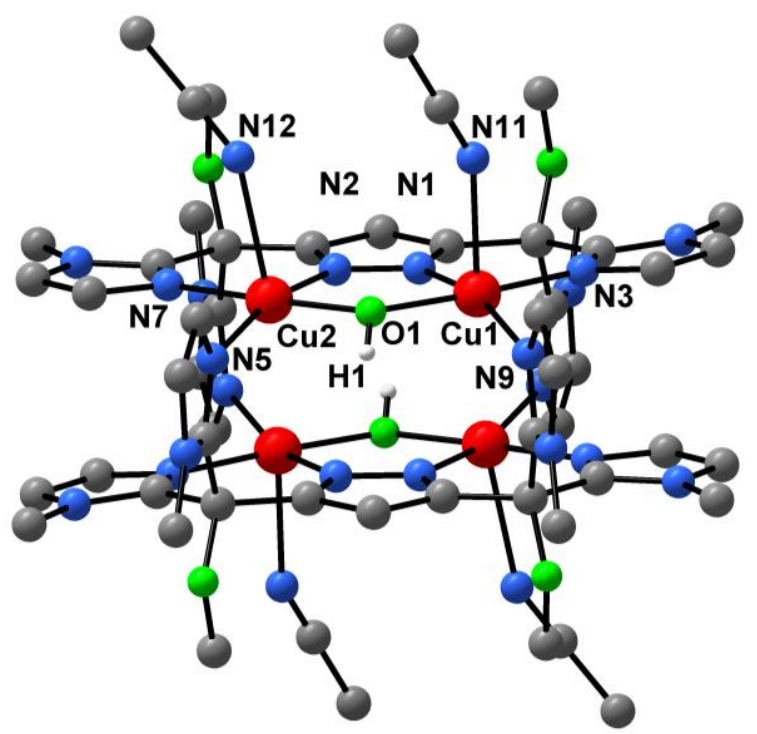

Abbildung 68: Struktur des Tetrakations von 28 (Außer den Protonen der OH-Gruppen sind alle $\mathrm{H}$-Atome wegen der Übersichtlichkeit nicht dargestellt).

Es ist deutlich zu erkennen, dass sich hier das schon in Kapitel 4.1. vorgestellte Strukturmotiv wiederholt. Der Pyrazol-Kern verbrückt zwei Kupfer(II)-lonen und hält diese in einem Abstand von 3.3797(7) ^̊ voneinander entfernt. Außerdem bildet sich eine Hydroxidbrücke zwischen beiden Kupfer-lonen. Es entsteht dabei ein Cu1-O1-Cu2-Winkel von 125.14(13) ${ }^{\circ}$. Jedes Kupferion ist quadratisch pyramidal von den Liganden umgeben. Die $\tau$-Werte von 0.20 für Cu1 und 0.17 für Cu2 belegen diese Koordinationsumgebung. Dabei wird bei allen vier Kupfer-lonen die axiale Koordinationsstelle von Acetonitril ausgefüllt. Die Bindungen der Kupferzentren zu den Stickstoff-Donoren des Lösungsmittels sind mit 2.149(3) $\AA$ bzw. 2.487(3) $\AA$ deutlich länger als die der apikalen Bindungen. Aufgrund der Jahn-Teller-Verzerrung handelt es sich um schwache Wechselwirkungen zum Metall-Ion. Weitere Bindungslängen und -winkel sind in Tabelle 21 aufgelistet. 


\begin{tabular}{llll}
\multicolumn{3}{c}{ Abstände [Å] } \\
\hline Cu1-N1 & $1.978(3)$ & Cu2-N2 & $1.973(3)$ \\
Cu1-N3 & $1.975(3)$ & Cu2-N7 & $1.968(3)$ \\
Cu1-O1 & $1.911(2)$ & Cu2-N5' & $2.023(3)$ \\
Cu1-N9' & $2.035(3)$ & Cu2-O1 & $1.897(2)$ \\
Cu1-N11 & $2.419(3)$ & Cu2-N12 & $2.487(3)$ \\
Cu1'-Cu2 & $3.3797(7)$ & & \\
& & & \\
\hline N9'-Cu1-N1 & $163.32(11)$ & N5'-Cu2-N2 & $165.97(11)$ \\
N3-Cu1-N9' & $95.51(11)$ & N2-Cu2-N7 & $89.75(11)$ \\
O1-Cu1-N1 & $86.68(10)$ & O1-Cu2-N5' & $88.95(10)$ \\
O1-Cu1-N3 & $175.37(11)$ & O1-Cu2-N2 & $86.82(10)$ \\
O1-Cu1-N9' & $89.12(10)$ & O1-Cu2-N7 & $176.54(11)$ \\
N1-Cu1-N3 & $88.85(11)$ & N5'-Cu2-N7 & $94.49(11)$ \\
O1-Cu1-N11 & $86.85(11)$ & O1-Cu2-N12 & $97.04(11)$ \\
N3-Cu1-N11 & $92.66(11)$ & N7-Cu2-N12 & $82.88(12)$ \\
N1-Cu1-N11 & $98.95(11)$ & N2-Cu2-N12 & $97.11(12)$ \\
N9'-Cu1-N11 & $96.92(11)$ & N5'-Cu2-N12 & $96.69(12)$ \\
\hline Cu1-O1-Cu2 & $125.14(13)$ & & \\
\hline
\end{tabular}

Tabelle 21: Ausgewählte Bindungslängen (in $\AA$ ) und Bindungswinkel (in ${ }^{\circ}$ ) von 28.

Die OH-Brücke konnte sich durch im Diethylether vorhandene Spuren von Wasser oder durch Feuchtigkeit in der Umgebungsluft bilden.

Führt man die gleiche Reaktion mit der doppelten Komplexkonzentration ( $\mathrm{c}=0.04 \mathrm{M}$ in $\mathrm{CH}_{3} \mathrm{CN}$ ) durch und verfolgt diese ebenfalls mittels UV/Vis-Spektroskopie, erhält man 
Kurven, die denen in Abbildung 67 sehr ähnlich sind. Der einzige Unterschied ist die beobachtete Absorption. Aufgrund der höheren Konzentration werden Absorptionen von etwa 0.5 erhalten. Außerdem ist die Reaktion des Kupfer(I)-Komplexes mit Kohlendioxid bei $5{ }^{\circ} \mathrm{C}$ untersucht. So sollte überprüft werden, ob die durchgeführte Reaktion temperaturabhängig ist. In Abbildung 69 sind die zeitlichen Verläufe aller drei Reaktionen dargestellt.

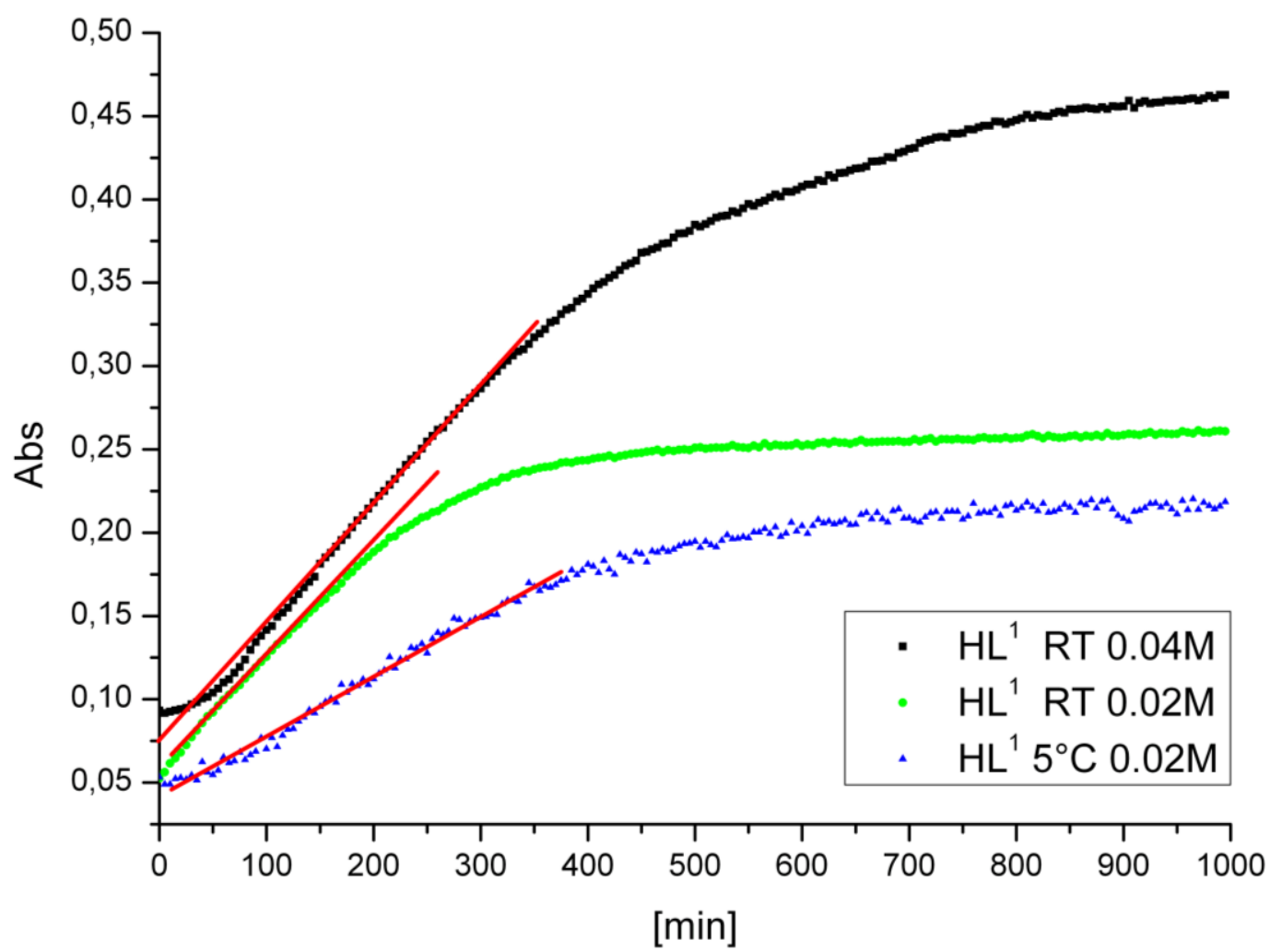

Abbildung 69: Zeitlicher Absorptionsverlauf der UV/Vis-Spektren für die Reaktion eines Kupfer(I)Komplexes von $\mathrm{HL}^{1}$ mit $\mathrm{CO}_{2}$ bei $\mathrm{RT}$ und $0.04 \mathrm{M}$ (schwarz), RT und 0.02M (grün) sowie $5{ }^{\circ} \mathrm{C}$ und $0.02 \mathrm{M}$ (blau) in $\mathrm{CH}_{3} \mathrm{CN}$.

Es fällt auf, dass die Werte für den bei $5^{\circ} \mathrm{C}$ durchgeführten Versuch eine größere Streuung aufweisen. Das liegt daran, dass die Absorption korrigiert werden musste. Bei Raumtemperatur steigt die Absorption über die gesamte Zeit im Bereich von $900 \mathrm{~nm}$ bis $1000 \mathrm{~nm}$ kaum an. Im Gegensatz ist bei $5^{\circ} \mathrm{C}$ ein langsamer Anstieg der Absorption in diesem Bereich zu erkennen. Damit eine gute Aussage zur Reaktivität getroffen werden kann wurde eine Korrektur der Absorption vorgenommen. Dazu wurde die Absorption bei $1000 \mathrm{~nm}$ zu einem bestimmten Zeitpunkt ermittelt und die Differenz zur Absorption 
bei dieser Wellenlänge zum Zeitpunkt $t=0$ errechnet. Diese erhaltene Differenz ist von der Absorption bei $568 \mathrm{~nm}$ zu einem bestimmten Zeitpunkt subtrahiert worden (Gleichung 1). Daraus resultiert die in Abbildung 69 gezeigte blaue Kurve für den zeitlichen Verlauf bei $568 \mathrm{~nm}$.

$$
A_{\text {korr }}=A_{t}(568 n m)-\left[A_{t}(1000 n m)-A_{t=0}(1000 n m)\right]
$$

Die Reaktionsverfolgung wurde nach etwa 17 Stunden beendet und die erhaltene blauviolette Lösung unter Stickstoff in einen Schlenkkolben überführt und das Lösungsmittel im Hochvakuum entfernt. Das IR-Spektrum des resultierenden Feststoffs liefert eine sehr scharfe Bande bei $1648 \mathrm{~cm}^{-1}$. Ein mögliches Produkt der Reaktion eines Kupfer(I)-Komplexes mit Kohlendioxid ist das Oxalat-Ion. Vergleicht man die Lage des Signals mit einfachen Salzen der Oxalsäure, fällt beispielsweise mit Kupfer(II)Oxalat eine sehr gute Übereinstimmung für die erhaltene Wellenzahl auf. Das IRSpektrum von Kupfer(II)-Oxalat zeigt eine intensive Bande bei $1644 \mathrm{~cm}^{-1}$. Diese muss der $\mathrm{C}=\mathrm{O}$-Schwingung des Oxalat-Ions zugeschrieben werden. Auch die IR-Spektren anderer Salze der Oxalsäure wie Kaliumoxalat oder Natriumoxalat weisen charakteristische Banden bei $1640 \mathrm{~cm}^{-1}$ bzw. $1590 \mathrm{~cm}^{-1}$ auf. Sowohl das aufgenommene das FAB- wie auch das ESI-Massenspektrum lassen keine Aussage über eine vorliegende Struktur zu. In beiden Spektren kann nur das Signal bei $\mathrm{m} / \mathrm{z}=603.0$ dem Fragment $\left[\mathrm{L}^{1} \mathrm{Cu}_{2}\right]^{2+}$ sicher zugeordnet werden.

Stellt man den Kupfer(I)-Komplex mit Ligand $\mathrm{HL}^{2}$ her und untersucht die $\mathrm{CO}_{2}$-Reaktion mittels UV/Vis-Spektroskopie, erhält man die in Abbildung 70 (links) gezeigten Graphen.
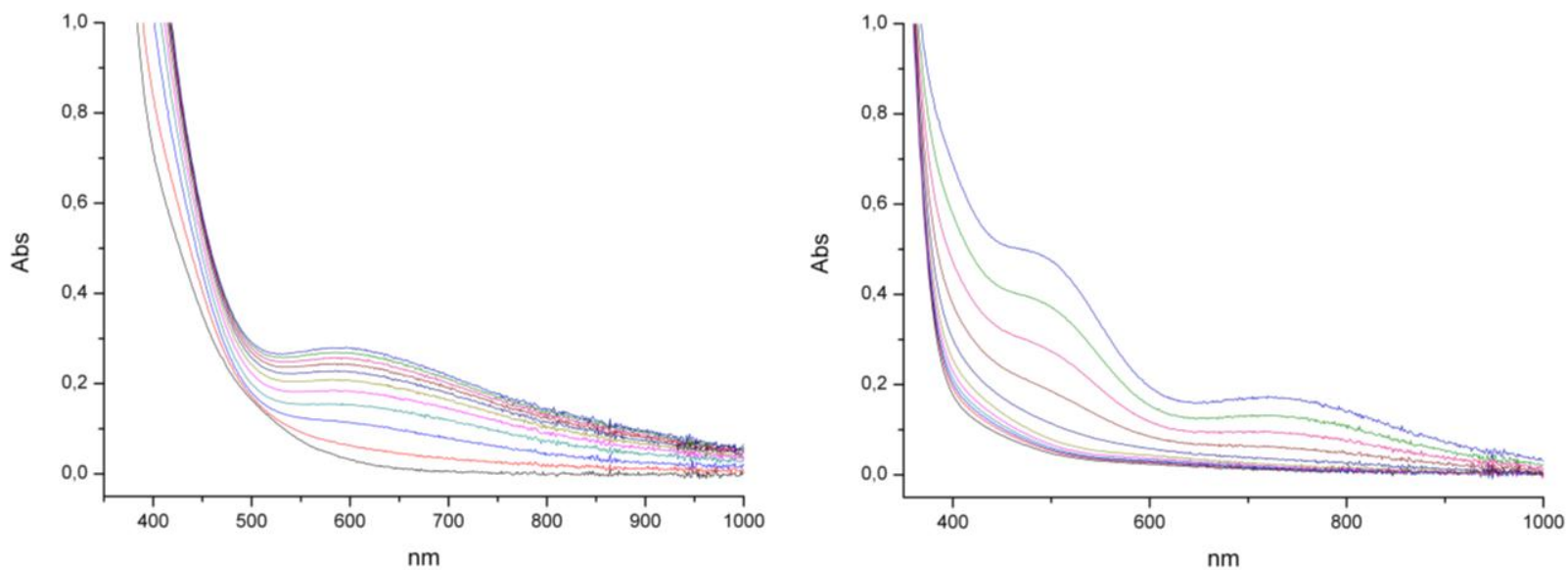

Abbildung 70: UV/Vis-Spektren für die Reaktion eines Kupfer(I)-Komplexes von $\mathrm{HL}^{2}$ mit $\mathrm{CO}_{2}$ bei RT und $0.04 \mathrm{M}$ in $\mathrm{CH}_{3} \mathrm{CN}$ (links) sowie $\mathrm{HL}^{3}$ mit $\mathrm{CO}_{2}$ bei RT und $0.02 \mathrm{M}$ in $\mathrm{CH}_{3} \mathrm{CN}$ (rechts). 
Der Verlauf differiert von den schon gezeigten Spektren für $\mathrm{HL}^{1}$. Es bildet sich eine Bande bei $596 \mathrm{~nm}$, die jedoch nicht so große Absorptionen hervorruft, wie eine Lösung von $\mathrm{HL}^{1}$ mit gleicher Konzentration. Auch konnte die Bildung des Minimums bei etwa $450 \mathrm{~nm}$ nicht festgestellt werden. Wie auch schon beim Versuch mit $\mathrm{HL}^{1}$ ist die Reaktion nach etwa 17 Stunden nicht vollständig abgeschlossen, so dass keine konstante Absorption nach dieser Zeit beobachtet werden kann.

Von der resultierenden grünen Lösung ist das Lösungsmittel entfernt und ein IR-Spektrum aufgenommen worden. Es ist eine schwache Bande bei $1617 \mathrm{~cm}^{-1} \mathrm{zu}$ erkennen, die eventuell auf das Vorhandensein von Oxalat-Ionen schließen lässt.

Mit Kenntnis der Ergebnisse der Umsetzung des Kupfer(I)-Komplexes von $\mathrm{HL}^{1}$ mit Kohlendioxid ist es nicht verwunderlich, dass die Reaktion vollständig abläuft, wenn man die Komplex-Konzentration halbiert.

In Abbildung 70 (rechts) sind außerdem die UV/Vis-Spektren dargestellt, die für die Reaktion einer 0.02 molaren Lösung eines Kupfer(I)-Komplexes von $\mathrm{HL}^{3}$ mit Kohlendioxid erhalten wurden. Es ergibt sich ein Spektrum, das nach der Reaktion zwei Banden bei etwa $480 \mathrm{~nm}$ und $730 \mathrm{~nm}$ zeigt. Die Bande bei $480 \mathrm{~nm}$ besitzt kein gut ausgeprägtes Maximum, lässt sich aber aufgrund der relativ großen Absorptionen sehr gut für die zeitliche Auftragung der Absorption verwenden (Abbildung 71). Daraus kann man erkennen, dass für den sterisch anspruchsvollen Liganden $\mathrm{HL}^{3}$ die Absorption sehr lange konstant ist, ehe sie nach etwa 550 Minuten sehr schnell linear ansteigt. Nach etwa 1000 Minuten ist dennoch

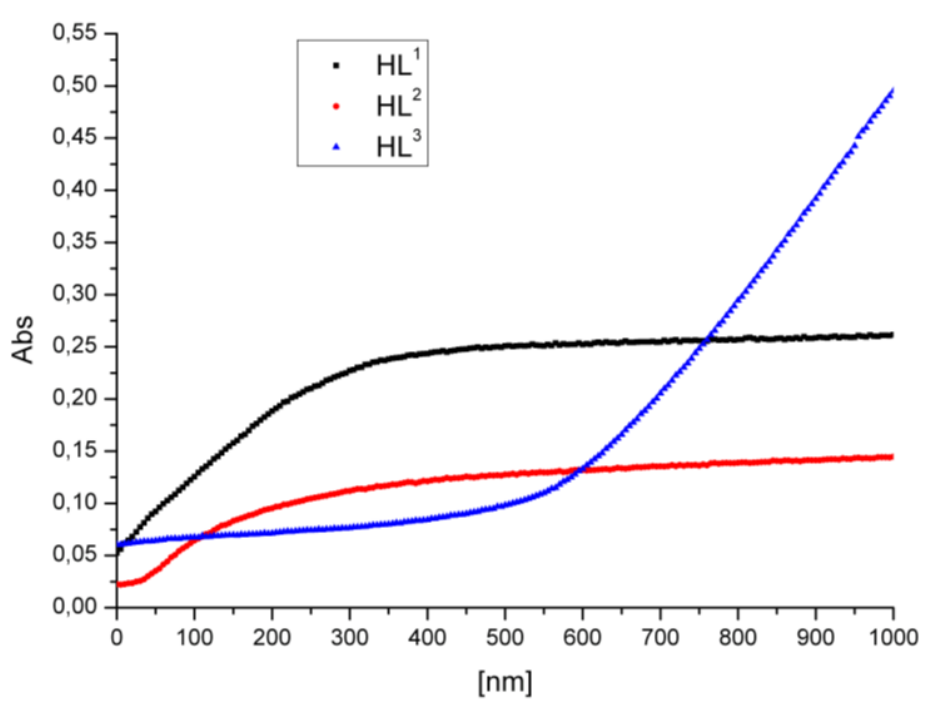

Abbildung 71: Vergleich der Absorptions-Zeit-Verläufe für Umsetzungen von $\mathrm{CO}_{2}$ mit $\mathrm{Kupfer}(\mathrm{I})$ Komplexen $\left(0.02 \mathrm{M}^{2} \mathrm{CH}_{3} \mathrm{CN}\right)$ von $\mathrm{HL}^{1}$ (schwarz), $\mathrm{HL}^{2}$ (rot) und $\mathrm{HL}^{3}$ (blau). 
kein Reaktionsende abzusehen. Vergleicht man die einzelnen Absorptions-Zeit-Verläufe für alle drei Liganden miteinander, ist auffällig, dass sich $\mathrm{HL}^{1}$ und $\mathrm{HL}^{3}$ sehr ähnlich sind. Die absoluten Werte für die Absorptionen können nicht direkt miteinander verglichen werden, da die Wellenlängen der Absorptionsmaxima etwas variiert. Dennoch kann man den sehr ähnlichen Kurvenverlauf von $\mathrm{HL}^{1}$ und $\mathrm{HL}^{2}$ erkennen. In beiden Fällen ist die Reaktion nach etwa 400 Minuten beendet und die Absorption bleibt annähernd konstant.

Unter der Annahme, dass für alle Liganden $\left(\mathrm{HL}^{1}, \mathrm{HL}^{2}\right.$ und $\left.\mathrm{HL}^{3}\right)$ die Reaktion ähnlich abläuft, können die erhaltenen Kuren miteinander verglichen werden. Die Reaktion des Liganden $\mathrm{HL}^{1}$ läuft offensichtlich schneller als für die Umsetzung mit $\mathrm{HL}^{2}$. Dies wird vor allem an den veränderten Substitutionsmustern der Imidazol-Ringe begründet sein. Zum Einen kommt ein erhöhter sterischer Anspruch zum Tragen, aber auch elektronische Einflüsse der Methylgruppen am Rückgrat des Imidazols sind zu berücksichtigen (Tabelle 22).

\begin{tabular}{cccc} 
Ligand & CKomplex $\left[\mathrm{mol}^{-1} \mathrm{I}^{-1}\right]$ & $T[\mathrm{~K}]$ & Anstieg zu Beginn der Reaktion $\left[\mathrm{nm}^{-1}\right]$ \\
\hline $\mathrm{HL}^{1}$ & 0.02 & 298 & $0.00067 \pm 5.0373 \cdot 10^{-6}$ \\
$\mathrm{HL}^{1}$ & 0.04 & 298 & $0.00071 \pm 6.4313 \cdot 10^{-6}$ \\
$\mathrm{HL}^{1}$ & 0.02 & 278 & $0.00035 \pm 3.7721 \cdot 10^{-6}$ \\
$\mathrm{HL}^{3}$ & 0.04 & 298 & $0.00045 \pm 4.6453 \cdot 10^{-6}$ \\
$\mathrm{HL}^{3}$ & 0.02 & 298 & $0.00047 \pm 0.00001$ \\
\hline
\end{tabular}

Tabelle 22: Darstellung der erhaltenen Parameter für die Umsetzung mit $\mathrm{CO}_{2}$.

Eine Auswertung der Anfangsgeschwindigkeit für diese Reaktionen mit $\mathrm{HL}^{3}$ nicht sinnvoll, da die Absorption zunächst über einen größeren Zeitraum konstant bleibt. Vergleicht man die restlichen Reaktionen miteinander, so fällt auf, dass eine Konzentrationsänderung keinen signifikanten Einfluss auf die Geschwindigkeit hat. Im Fall von $\mathrm{HL}^{1}$ erhöht sich der Wert für den Anstieg geringfügig bei Erhöhung der Konzentration. Das Gegenteil tritt für $\mathrm{HL}^{2}$ ein. Ein minimaler Anstieg der Geschwindigkeit ist bei Halbierung der Komplex-Konzentration zu beobachten. Dieser Unterschied ist allerdings eher zu vernachlässigen. Lässt man die Konzentration der 
Komplex-Lösung konstant und senkt die Temperatur um $20 \mathrm{~K}$, wird die Geschwindigkeit der Reaktion nahezu halbiert.

Außerdem fällt auf, dass die Reaktion von Kupfer(I)-Komplexen mit Ligand $\mathrm{HL}^{2}$ wesentlich langsamer erfolgt als die gleiche Reaktion mit Kupfer(I)-Komplexen von $\mathrm{HL}^{1}$.

\subsection{Sauerstoff}

Auch die Umsetzungen der Kupfer(I)-Komplexe mit Sauerstoff lassen sich sehr gut per UV/Vis-Spektroskopie verfolgen. Während für die Umsetzung mit Kohlendioxid ein Messintervall von 5 Minuten ausreichend war, muss hier der Abstand zwischen den Messungen auf eine Minute verkürzt werden, um ausreichend viele Messpunkte in der ersten Phase der Reaktion zu sammeln. Die UV/Vis-Verfolgung für die Reaktion einer $0.04 \mathrm{M}$ Komplexlösung von $\mathrm{HL}^{1}$ in Acetonitril lieferte das in Abbildung 72 gezeigte Spektrum.

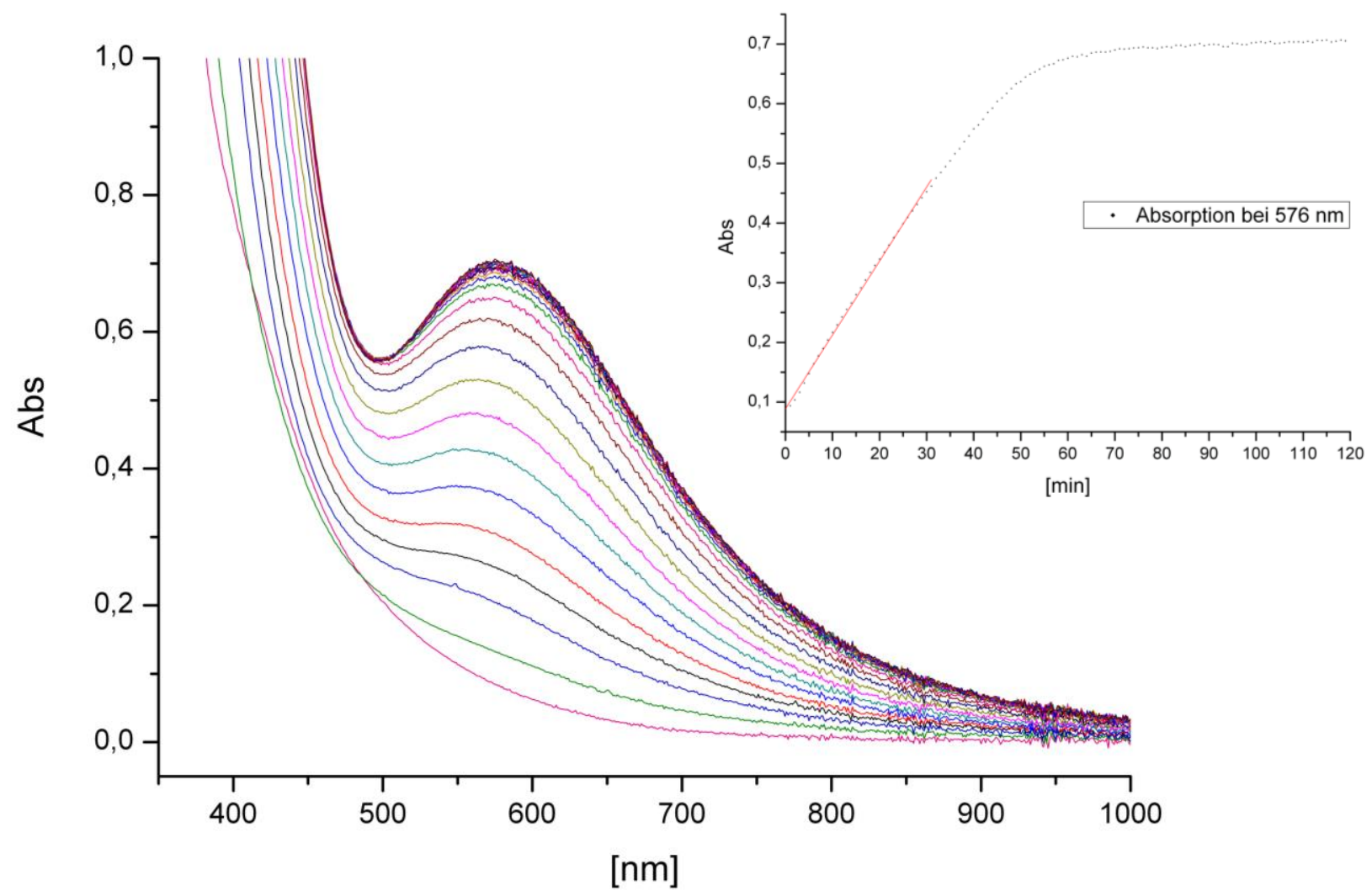

Abbildung 72: UV/Vis-Spektren für die Reaktion eines Kupfer(I)-Komplexes von $\mathrm{HL}^{1}$ mit $\mathrm{O}_{2}$ bei $\mathrm{RT}$ und $0.04 \mathrm{M}$ in $\mathrm{CH}_{3} \mathrm{CN}$ (Nur jede fünfte Kurve ist abgebildet). 
Mit zunehmender Reaktionsdauer bildet sich eine Bande bei $576 \mathrm{~nm}$ $\left(\varepsilon=177 \mathrm{~mol}^{-1} \cdot \mathrm{lcm}^{-1}\right)$, die einem d-d-Übergang des Kupfer(II)-Komplexes zugeschrieben werden muss. Der ebenfalls in Abbildung 72 gezeigte zeitliche Verlauf macht deutlich, dass die Absorption bereits nach ca. 70 Minuten konstant bleibt. Daraus lässt sich schließen, dass die Umsetzung zu dieser Zeit abgeschlossen ist.

$\mathrm{Ob}$ es für diese Reaktion möglich ist durch Variierung der Komplexkonzentration die Reaktionsgeschwindigkeit zu beeinflussen, ist durch Halbierung der Konzentration der Kupfer(I)-Komplexlösung überprüft worden. Der aus den UV/Vis-Spektren abgeleitete zeitliche Verlauf der Absorption sieht der in Abbildung 72 gezeigten Kurve sehr ähnlich. Der einzig markante Unterschied ist die geringere Absorption, die aufgrund der halbierten Konzentration bedingt ist.

Desweiteren ist die Untersuchung der Reaktivität von $\mathrm{HL}^{1}$ gegenüber Sauerstoff bei einer weiteren Temperatur durchgeführt worden. Dazu wurde die gefüllte Messzelle in einem Kühlbad auf $5^{\circ} \mathrm{C}$ temperiert und alle 2 Minuten erneut ein UV/Vis-Spektrum aufgenommen. Die Ergebnisse der Titration ähneln dem bei Raumtemperatur erhaltenen Verlauf für dieselbe Konzentration (0.02 M). In Abbildung 73 sind alle Ergebnisse für die O2-Untersuchung zusammengefasst.

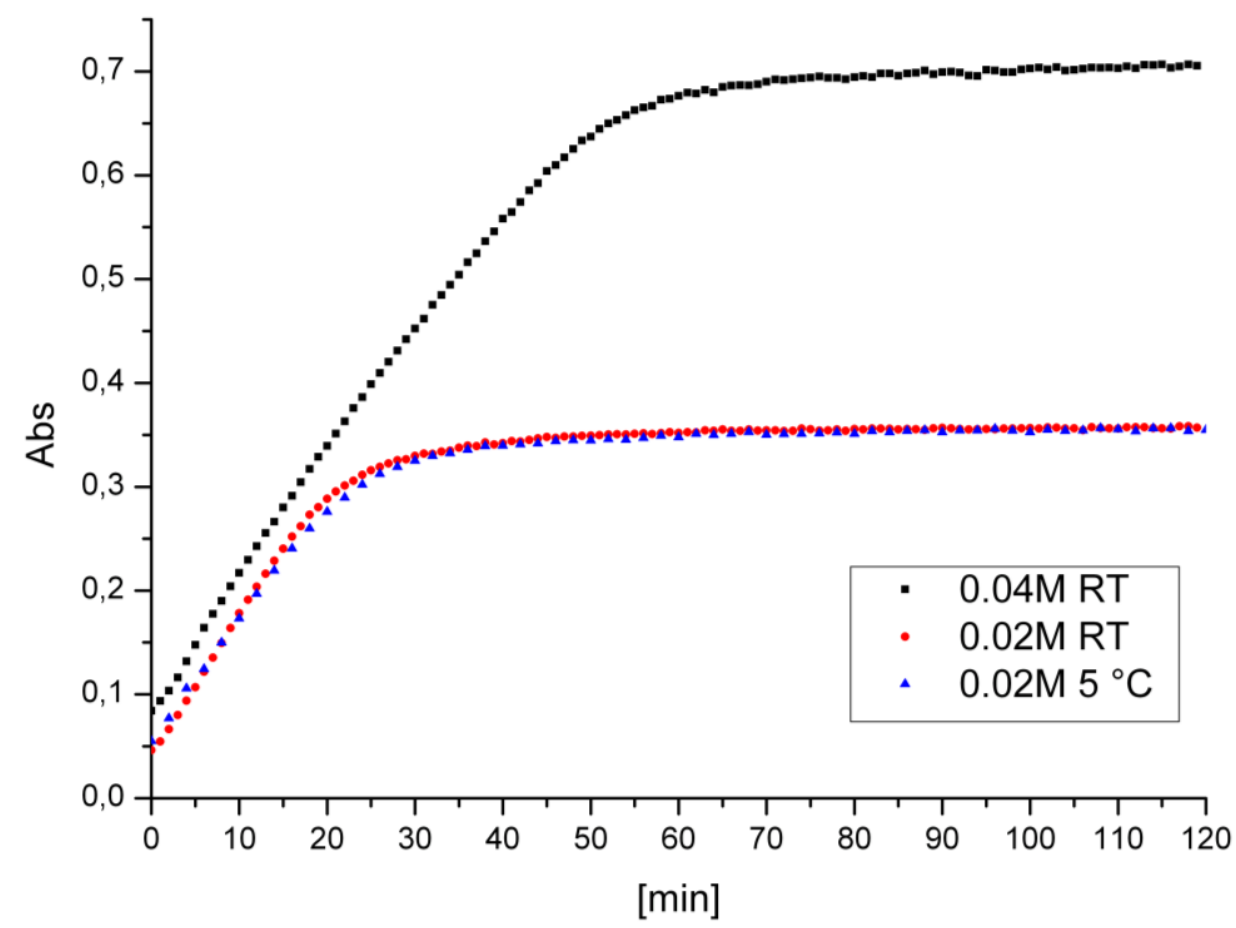

Abbildung 73: Vergleich der zeitlichen Verläufe der Reaktionen von $\mathrm{HL}^{1}$ mit $\mathrm{O}_{2}$. 
Es ist klar zu erkennen, dass alle drei Messungen nahezu identisch aussehen. Der anfängliche Anstieg kann als Maß für die Reaktionsgeschwindigkeit angesehen werden. Dieser ist für alle drei Messreihen identisch. Die erwartete Verlangsamung der Reaktion durch die Verringerung der Temperatur ist für diese Umsetzung nicht eingetreten.

Die Umsetzung mit Sauerstoff sind auch mit den Kupfer(I)-Komplexen untersucht worden, die aus der Verwendung der Liganden $\mathrm{HL}^{2}$ und $\mathrm{HL}^{3}$ für die Komplexsynthese resultieren. Die erhaltenen Messergebnisse sind in Abbildung 74 präsentiert. Das Aussehen der Messkurven unterscheidet deutlich von denen für $\mathrm{HL}^{1}$. Im Fall von $\mathrm{HL}^{2}$ steigt die Absorption während der Reaktion über den gesamten Wellenlängenbereich an, so dass das Absorptionsmaximum bei etwa $650 \mathrm{~nm}$ nur schlecht auszumachen ist. Dieses Phänomen ist auch schon bei der Reaktion mit $\mathrm{CO}_{2}$ aufgetreten, konnte dort aber mit einer Absorptionskorrektur behoben werden. Dieses Verfahren führte für die Reaktion mit $\mathrm{O}_{2}$ zu keinem besseren Aussehen des zeitlichen Verlaufes der Absorption bei $650 \mathrm{~nm}$.
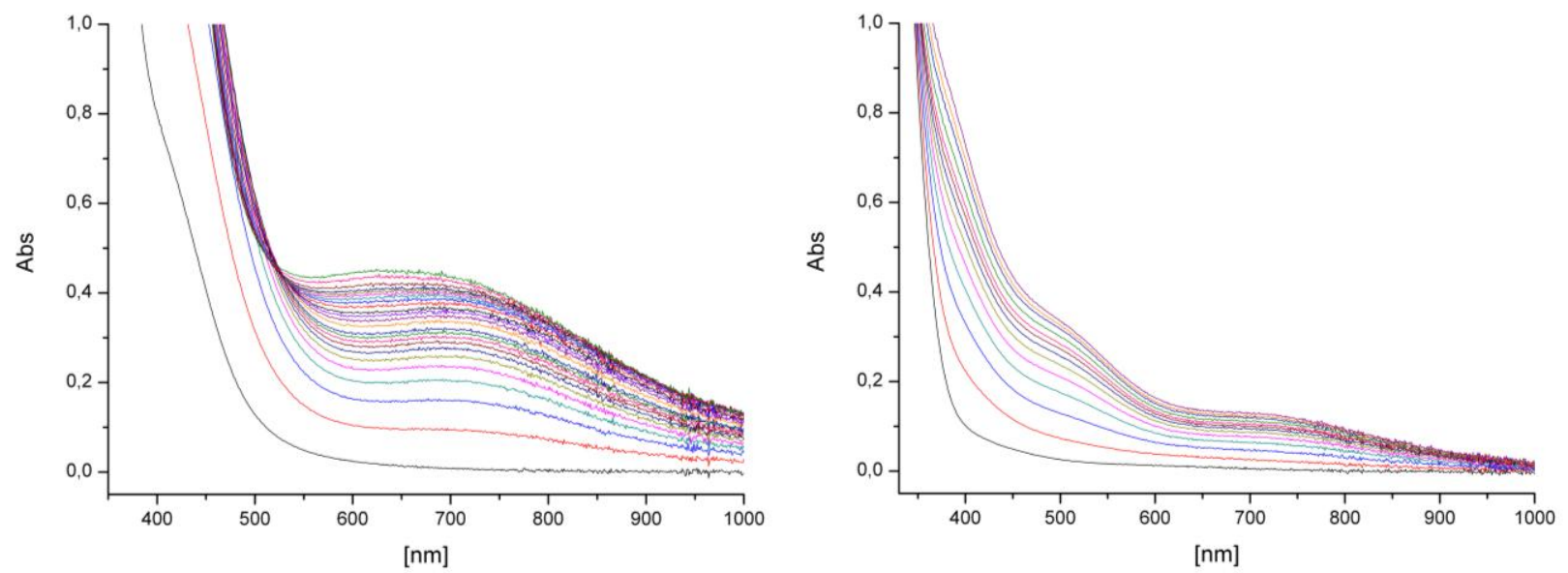

Abbildung 74: UV/Vis-Spektren für die Reaktion eines Kupfer(I)-Komplexes von $\mathrm{HL}^{2}$ mit $\mathrm{O}_{2}$ bei RT und $0.04 \mathrm{M}$ (links) sowie von $\mathrm{HL}^{3}$ mit $\mathrm{O}_{2}$ bei RT und $0.01 \mathrm{M}$ (rechts) in $\mathrm{CH}_{3} \mathrm{CN}$. Für eine bessere Übersichtlichkeit wurden nicht alle Kurven der Reaktionsverfolgung abgebildet.

Für den mit $\mathrm{HL}^{3}$ hergestellten Kupfer(I)-Komplex hat das UV/Vis-Spektrum ein anderes Aussehen. Schon nach kurzer Reaktionszeit bildet sich eine Schulter bei etwa $500 \mathrm{~nm}$ sowie eine schwache Absorption bei etwa $710 \mathrm{~nm}$. Die Schulter ist dennoch für das Erstellen eines zeitlichen Verlaufes geeignet. Die Sauerstoff-Umsetzung verläuft für dieses System sehr schnell. Bereits nach etwa 35 Minuten ist die Absorption konstant, was für eine abgeschlossene Reaktion spricht. 
Für einen Vergleich aller Sauerstoff-Umsetzungen der Kupfer(I)-Komplexe untereinander bestimmt man erneut den Anstieg zu Beginn der Reaktion. Umso größer der dafür erhaltene Wert ist, desto schneller läuft die Reaktion ab. Wie schon für die Komplexe von $\mathrm{HL}^{1}$ erwähnt wurde ähneln sich die Graphen der zeitlichen Verläufe sehr. Die ermittelten Anstiege sind fast identisch, so dass die Reaktionsgeschwindigkeit weder temperaturabhängig, noch von der Konzentration des Kupfer(I)-Komplexes abhängig ist (Tabelle 23).

Die Anfangsgeschwindigkeit für die Sauerstoff-Reaktion mit dem aus $\mathrm{HL}^{2}$ gebildeten Kupfer(I)-Komplex ist nur halb so groß wie für $\mathrm{HL}^{1}$. Etwas unerwartet ist jedoch, dass die Anfangsgeschwindigkeit für Kupfer(I)-Komplexe mit $\mathrm{HL}^{3}$ bei den untersuchten Konzentrationen stark verschieden ist. Die Reaktion mit einer $0.01 \mathrm{M}$ Lösung des Kupfer(I)-Komplexes von $\mathrm{HL}^{3}$ läuft etwa doppelt so schnell ab, wie für $\mathrm{HL}^{1}$. Eine weitere Beschleunigung der Reaktion wird erreicht, wenn die Konzentration auf $0.02 \mathrm{moll}^{-1}$ erhöht wird. Diese Reaktion geschieht etwa fünfmal schneller als die Reaktion des Kupfer-Komplexes von $\mathrm{HL}^{1}$ mit der gleichen Konzentration.

\begin{tabular}{cccc} 
Ligand & $C_{\text {Komplex }}\left[\mathrm{mol}^{-1} \mathrm{l}^{-1}\right]$ & $T[\mathrm{~K}]$ & Anstieg zu Beginn der Reaktion $\left[\mathrm{nm}^{-1}\right]$ \\
\hline $\mathrm{HL}^{1}$ & 0.02 & 298 & $0.01239 \pm 0.0001$ \\
$\mathrm{HL}^{1}$ & 0.04 & 298 & $0.01273 \pm 0.00017$ \\
$\mathrm{HL}^{1}$ & 0.02 & 278 & $0.01126 \pm 0.00018$ \\
$\mathrm{HL}^{2}$ & 0.04 & 298 & $0.00621 \pm 0.00038$ \\
$\mathrm{HL}^{3}$ & 0.01 & 298 & $0.02357 \pm 0.00101$ \\
$\mathrm{HL}^{3}$ & 0.02 & 298 & $0.06289 \pm 0.00584$ \\
\hline
\end{tabular}

Tabelle 23: Auflistung der erhaltenen Parameter für die Reaktionen mit Sauerstoff.

Stellt man einen Vergleich der Anstiege zu Beginn der Reaktion mit Sauerstoff mit denen für die $\mathrm{CO}_{2}$-Umsetzung, fällt auf, dass die $\mathrm{O}_{2}$-Reaktionen in etwa um den Faktor 20 schneller ablaufen. Im Fall von $\mathrm{HL}^{1}$ ist die $\mathrm{O}_{2}$-Reaktion nicht konzentrations- aber scheinbar temperaturabhängig. Für die $\mathrm{O}_{2}$-Reaktion konnte keine signifikante Temperatur- und Konzentrationsabhängigkeit im Rahmen der hier durchgeführten Messungen festgestellt werden. 


\section{Magnetische Eigenschaften der Komplexe}

In jüngster Zeit hat sich der Molekulare Magnetismus als ein Bereich der modernen Naturwissenschaften etabliert, der sich mit der Entwicklung neuer magnetischer Materialien auf molekularer Basis beschäftigt. Diese sollen in Zukunft herkömmliche Materialien wie Metalle, Metalloxide oder Legierungen in zahlreichen Anwendungen ersetzen. ${ }^{[159]}$ Die Untersuchung magnetischer Eigenschaften von Metallkomplexen kann zu wichtigen Erkenntnissen auf dem Weg dahin führen.

Für die Untersuchung der magnetischen Eigenschaften wurde ein MPMS-5S-SQUID Magnetometer (Superconducting Quantum Interference Device) der Firma Quantum-Design verwendet. SQUIDs sind die empfindlichsten heute verfügbaren, Detektoren für magnetische Felder. Ihre Funktion beruht auf dem physikalischen Effekt der Supraleitung und der Verdrängung von Magnetfeldern aus einem Supraleiter. Dabei wird der magnetische Fluss in eine Spannung umgewandelt. ${ }^{[160]}$ Das im Rahmen dieser Arbeit verwendete Gerät ermöglicht Messungen bei Temperaturen zwischen $1.7 \mathrm{~K}$ und $400 \mathrm{~K}$ und bei homogenen Magnetfeldern bis zu $5 \mathrm{~T}$.

\subsection{Probenvorbereitung und Magnetische Messungen}

Für die magnetischen Messungen wurden die kristallinen Komplexe pulverisiert. Normalerweise ist es möglich, derartige Messungen mit Probenmengen von $10 \mathrm{mg}$ bis $20 \mathrm{mg}$ durchzuführen. Bei den hier vorliegenden Komplexen ist die Molmasse relativ groß und das magnetische Moment der Probe sehr klein. Die optimale Einwaage der zu untersuchenden Verbindungen liegt daher bei 40-65 mg Substanz. Die pulverförmigen Proben wurden in handelsüblichen Gelatinekapseln (Größe 4; $5 \mathrm{~mm}$ Durchmesser) in einigen Fällen so präpariert (Abbildung 72), dass eine kugelförmige Probengeometrie entstand. Der obere Teil (kleinerer Durchmesser) wurde gekürzt, um den diamagnetischen Beitrag der Kapsel zu verringern. ${ }^{[161]}$ Die
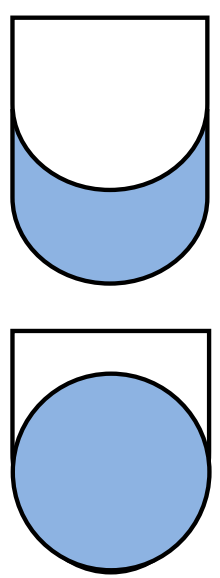

Abbildung 75: Probe (blau) in der Gelatinekapsel. 
Gelatinekapsel wurde in einem Kunststoffröhrchen (Strohhalm) fixiert, in einem Schlenkgefäß zum Messgerät transportiert und am Probenhalter des SQUID-Magnetometers befestigt.

Bei allen Verbindungen wurde die magnetische Suszeptibilität bei zwei Feldern zwischen 1000 Oe und 5000 Oe im Temperaturbereich zwischen $300 \mathrm{~K}$ bzw. $295 \mathrm{~K}$ und $2 \mathrm{~K}$ gemessen. Damit für jede Probe das beste SQUID-Signal erreicht werden konnte, musste die Feldstärke probenabhängig geringfügig variiert werden. Mit der Suszeptibilitätsmessung bei zwei Feldern kann man zum einen wissenschaftlich relevante Phänomene (z.B. Ferromagnetismus) erforschen, aber auch die Reinheit der Probe überprüfen. Molekülbasierende Verbindungen und niederdimensionale Systeme, wie zum Beispiel die in dieser Arbeit untersuchten Verbindungen, besitzen in der Regel keine signifikante Feldabhängigkeit. Die manchmal beobachtete Feldabhängigkeit der Suszeptibilität war verursacht durch geringe Spuren von Verunreinigungen, die sich durch Umkristallisieren nicht entfernen ließen.

\subsection{Auswertung von Suszeptibilitätsmessungen}

Für die Auswertung der temperaturabhängigen magnetischen Messungen müssen die erhaltenen Rohdaten zuerst um die Werte des Probenhalters (Gelatinekapsel) korrigiert werden. Für die Ermittlung der molaren Suszeptibilität benötigt man die genaue Einwaage, das Molekulargewicht und die angelegte Feldstärke. Dann wird die molare Suszeptibilität um den diamagnetischen Beitrag der untersuchten Probe korrigiert, der sowohl aus experimentellen Daten entsprechender diamagnetischer Verbindungen als auch aus den Inkrementen bestimmt werden kann (Verwendung der Pascal-Konstanten bzw. der Inkrementmethode nach Haberditzl). ${ }^{[162,163]}$ 


\subsection{Anpassung an die experimentellen Daten}

Mittels Gleichung 2 kann aus der erhaltenen Suszeptibilität $\chi_{M}$ und der Messtemperatur $T$ das effektive magnetische Moment $\mu_{\text {eff }}$ berechnet werden.

$$
\mu_{\text {eff }}=\sqrt{\frac{3 k}{N \beta^{2}}} \sqrt{\chi_{M} T}=2.83 \sqrt{\chi_{M} T}
$$

(Gleichung 2)

Der dabei aus dem Experiment erhaltene Wert wird mit dem sogenannten "spin-only“ Wert, einem theoretisch erwarteten Wert, verglichen. Dieser "spin-only“ Wert für Verbindungen mit Wechselwirkungen zwischen paramagnetischen Zentren lässt sich nach Gleichung 3 ausrechnen.

$$
\mu_{\text {eff }}=g \sqrt{S(S+1)} \mu_{B}
$$

Dabei ist $\mathrm{S}$ der Gesamtspin. Für den Fall von beispielsweise zwei paramagnetischen Zentren ohne Wechselwirkungen ergibt sich der "spin-only“ Wert nach folgender Gleichung (Gleichung 4):

$$
\mu_{\text {eff }}=g \sqrt{S_{1}\left(S_{1}+1\right)+S_{2}\left(S_{2}+1\right)} \mu_{B}
$$

Die Anpassung an die Daten der magnetischen Messungen wurde mit dem Programm juI $X^{[164]}$ durchgeführt. juIX simuliert auf der Basis eines Hamiltonoperators molare magnetische Suszeptibilitäten für paramagnetische Systeme von bis zu vier Spins mit isotropen Austauschwechselwirkungen und lokalen axialen und rhombischen Nullfeldwechselwirkungen. In Gleichung 5 ist ein Beispiel des Heisenberg-Dirac-vanVleck-Hamiltonoperators mit zusätzlichem Term für die Zeeman-Aufspaltung abgebildet.

$$
\hat{H}=-2 J \hat{S}_{1} \hat{S}_{2}+g \mu_{B} B\left(\hat{S}_{1}+\hat{S}_{2}\right)
$$


Die Zeeman-Wechselwirkung wird richtungsabhängig berücksichtigt und für Pulverproben entweder über die drei kanonischen Hauptachsen oder über ein 16-Punkte Lebedev-Gitter gemittelt. Die Diagonalisierung der Hamiltonmatrix erfolgt numerisch mit der LAPACK Routine ZHEEV. Magnetische Momente werden durch numerische Ableitung der Energien nach dem Feld berechnet. Einzelne oder mehrere Parameter der Hamiltonoperatoren können mit der Simplexroutine AMOEBA von NUMERICAL RRECIPIES automatisch optimiert werden, wobei paarweise Korrelation von beliebigen Variablen möglich ist. Für Visualisierungszwecke können Energiediagramme geplottet werden und für systematische Fehlerbetrachtungen können Fehler-Kontourplots oder Fehlerflächen für beliebige Variablen-Paare berechnet werden.

Als Ergebnis werden dabei der Austauschparameter $J$ sowie der $g$-Faktor, der Molanteil der Verunreinigungen $\rho$ und der Beitrag des temperaturunabhängigen Paramagnetismus TIP ermittelt. Unter Verunreinigung versteht man in diesem Zusammenhang einen sehr kleinen Anteil paramagnetischer Spezies in der Probe. Es wird vorausgesetzt, dass die Verunreinigung Curie-Verhalten zeigt, dieselbe molare Masse und denselben $g$-Faktor wie die untersuchte Verbindung hat. Um den TIP zu berücksichtigen, wird zu Gleichung 3 ein additives Glied hinzugefügt.

\subsection{Ergebnisse der magnetischen Messungen}

Die temperaturabhängigen Messdaten für die magnetische Suszeptibilität $\chi_{M}$ sowie das Produkt $\chi_{M} T$ für den zweikernigen Kupfer(II)-Komplex 8 ist in Abbildung 76 dargestellt. Für 8 wird ein $\chi_{M} T$-Wert von $0.20 \mathrm{~cm}^{3} \cdot \mathrm{K}^{\prime} \mathrm{mol}^{-1}\left(1.26 \mu_{B}\right)$ bei Raumtemperatur beobachtet. Dieser Wert liegt weit entfernt vom "spin-only“ Wert für zwei ungekoppelte $S=1 / 2$ Kupfer(II)-Ionen $\left(2.63 \mu_{B}\right.$ für $\left.g=2.15\right)$ und nimmt exponentiell bis $2 \mathrm{~K}$ ab. Dieses Verhalten deutet auf antiferromagnetischen Austausch hin ( $S=0$ Gundzustand). Die Daten der Suszeptibilitätsmessungen von 8 wurden im Temperaturbereich von $295 \mathrm{~K}$ bis $2 \mathrm{~K}$ unter Verwendung des Programms $j u l X$ angepasst. Die durchgezogene Linie in Abbildung 32 repräsentiert die beste Anpassung für 8. Als Parameter ergaben sich $g=2.15$ (fixiert) und $J=-327.3 \mathrm{~cm}^{-1}\left(\rho=0.2 \%\right.$ (fixiert), $\left.T I P=2.5 \cdot 10^{-4} \mathrm{~cm}^{3} \cdot \mathrm{mol}^{-1}\right)$. Diese 
Parameter bestätigen neben der oben erwähnten $\chi_{M} T$-Wert-Differenz bei Raumtemperatur eine sehr starke antiferromagnetische Kopplung.

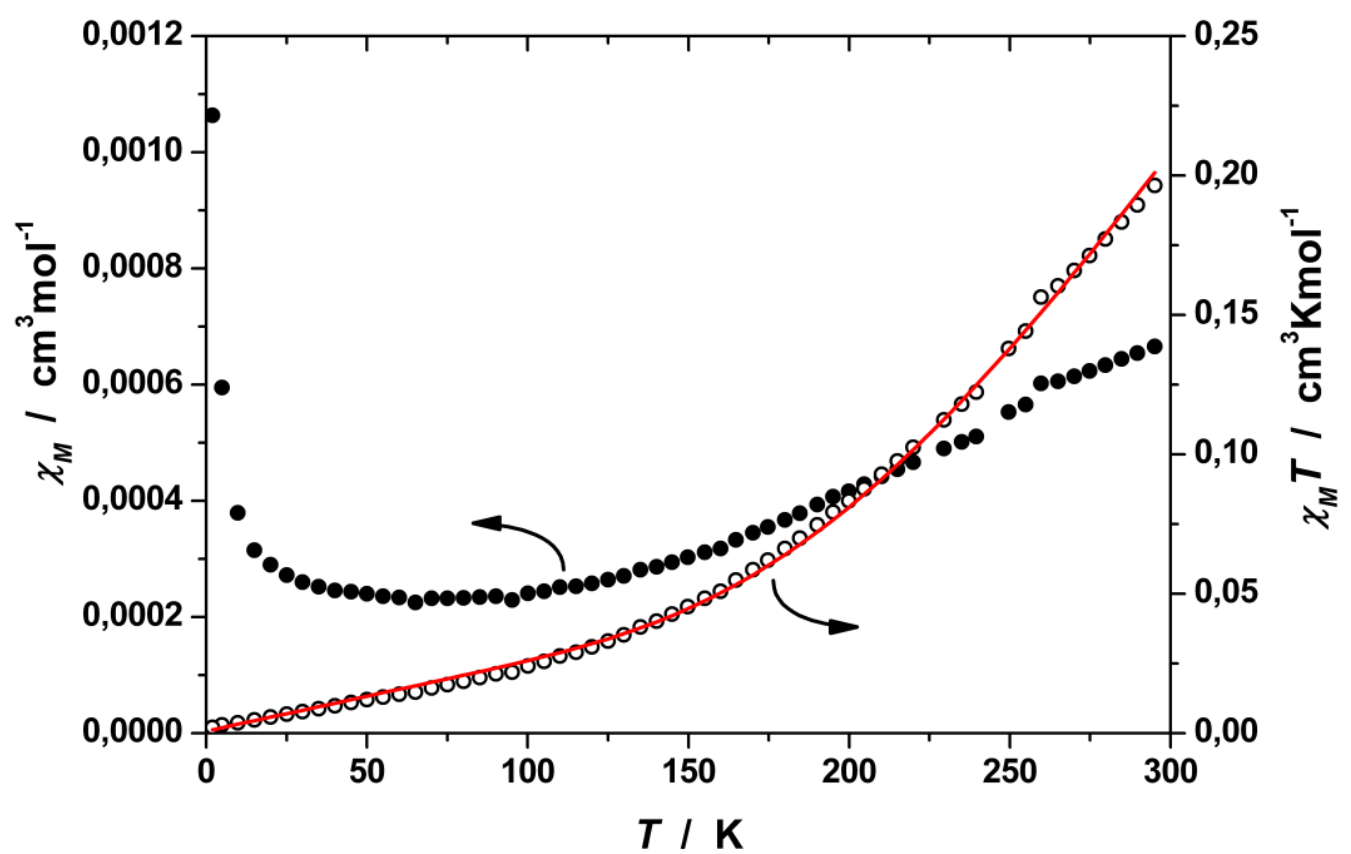

Abbildung 76: Temperaturabhängigkeit von $\chi_{M}$ und $\chi_{M} T$ für 8 (Die rote Linie repräsentiert die beste Anpassung).

In Abbildung 77 sind die temperaturabhängigen Messdaten für die magnetische Suszeptibilität $\chi_{M}$ sowie das Produkt $\chi_{M} T$ für den stufenförmigen Komplex 10 mit vier Kupfer(II)-Ionen dargestellt. Für 10 wird ein $\chi_{M} T$-Wert von $0.35 \mathrm{~cm}^{3} \cdot \mathrm{K}^{\prime} \cdot \mathrm{mol}^{-1}\left(1.67 \mu_{B}\right)$ bei Raumtemperatur beobachtet. Es gibt somit eine große Differenz zu dem „spin-only“ Wert für zwei ungekoppelte $S=1 / 2 \operatorname{Kupfer(II)-Ionen~}\left(2.63 \mu_{B}\right.$ für $\left.g=2.15\right)$. Auch für den stufenförmigen Kupfer(II)-Komplex 10 nimmt das Produkt $\chi_{M} T$ exponentiell bis $2 \mathrm{~K}$ ab. Dieses Verhalten deutet auf antiferromagnetischen Austausch hin ( $S=0$ Gundzustand). 


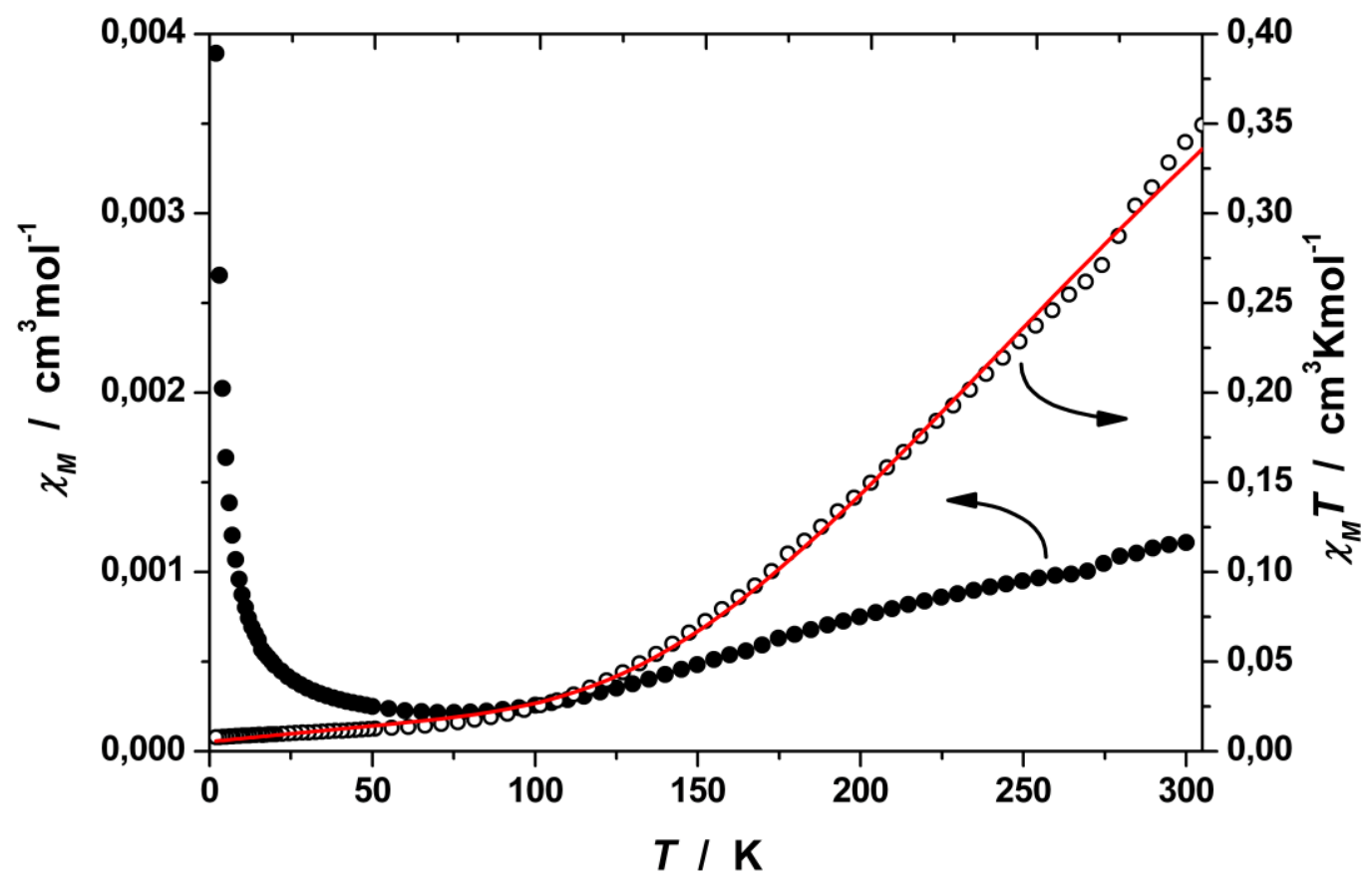

Abbildung 77: Temperaturabhängigkeit von $\chi_{M}$ und $\chi_{M} T$ für 10 (Die rote Linie repräsentiert die beste Anpassung).

Die Daten der Suszeptibilitätsmessungen von 10 wurden im Temperaturbereich von $300 \mathrm{~K}$ bis $2 \mathrm{~K}$ unter Verwendung des Programms julX angepasst. Dabei muss angemerkt werden, dass der vierkernige Kupferkomplex 10 in zwei zweikernige Komplexe zerlegt wird, um die Anpassung durchzuführen. Dies ist erlaubt, da zwischen den Kupferzentren Cu2 und Cu3 eine Brücke besteht, die keine magnetische Kopplung vermittelt. Eine Kopplung durch den Raum würde, wenn sie tatsächlich stattfinden sollte, eine sehr geringe Kopplungskonstante liefern, so dass dieses Vorgehen eine gute und realistische Näherung ist. Der Kopplungspfad der existierenden Brücke ist zu lang und $J_{2}$ somit vernachlässigbar klein (Schema 21).

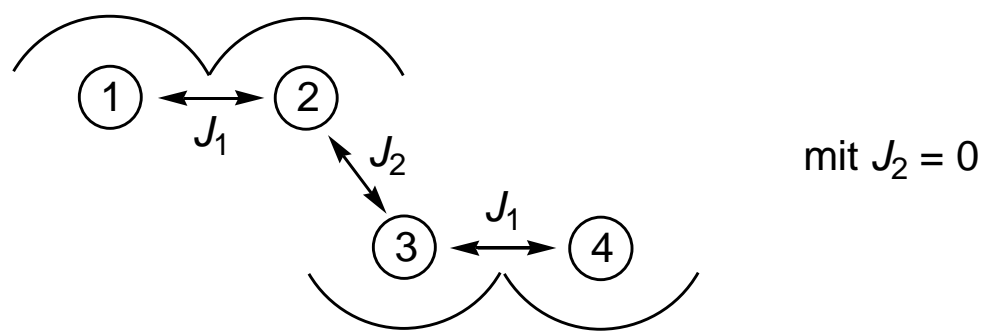

Schema 21: Kopplungsschema von 10. 
Die durchgezogene Linie in Abbildung 77 repräsentiert die beste Anpassung für 10. Als Parameter ergaben sich $g=2.15$ (fixiert) und $J_{1}=-233.2 \mathrm{~cm}^{-1}(\rho=1.4 \%$ (fixiert), $\left.T I P=1.8 \cdot 10^{-4} \mathrm{~cm}^{3} \cdot \mathrm{mol}^{-1}\right)$. Daraus kann man, wie auch aus dem Unterschied des $\chi_{M} T$-Wertes zum "spin-only" Wert bei Raumtemperatur, ebenfalls eine sehr starke antiferromagnetische Kopplung folgern.

Für den Komplex 11a sind in Abbildung 79 die temperaturabhängigen Messdaten für die magnetische Suszeptibilität $\chi_{M}$ sowie das Produkt $\chi_{M} T$ dargestellt. Für 11a wird ein $\chi_{M} T$-Wert von $0.21 \mathrm{~cm}^{3} \cdot \mathrm{K} \cdot \mathrm{mol}^{-1}\left(1.30 \mu_{B}\right)$ bei Raumtemperatur beobachtet. Dieser Wert liegt wiederum weit entfernt vom "spin-only“ Wert für zwei ungekoppelte $S=1 / 2$ Kupfer(II)-Ionen $\left(2.63 \mu_{B}\right.$ für $\left.g=2.15\right)$, was auf eine starke antiferromagnetische Kopplung hindeutet. $\chi_{M} T$ nimmt für diesen Komplex exponentiell ab. Dieses Verhalten deutet ebenfalls auf antiferromagnetischen Austausch hin ( $S=0$ Grundzustand). Die Daten der Suszeptibilitätsmessungen von 10 wurden im Temperaturbereich von $295 \mathrm{~K}$ bis $2 \mathrm{~K}$ unter Verwendung des Programms juIX angepasst. Dabei gilt auch für diese Verbindung, dass der vierkernige Kupferkomplex 11a für die Anpassung in zwei dinukleare Komplexe zerlegt wird. Es besteht ebenfalls keine vermittelnde Brücke zwischen Cu1 und Cu4 bzw. Cu2 und Cu3, die eine signifikante

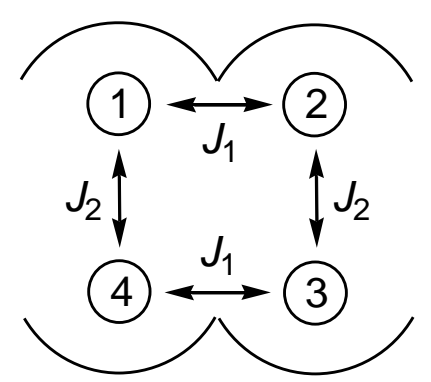

Schema 22: Kopplungsschema für 11a, 12, 13 und 28. Kopplung zwischen den zweikernigen pyrazolatverbrückten Einheiten ermöglichen würde (Schema 19). Aus diesem Grund kann die Kopplungskonstante $J_{2}$ als null festgesetzt werden. Ein Blick auf die in Kapitel 4.1. vorgestellten Komplexstrukturen

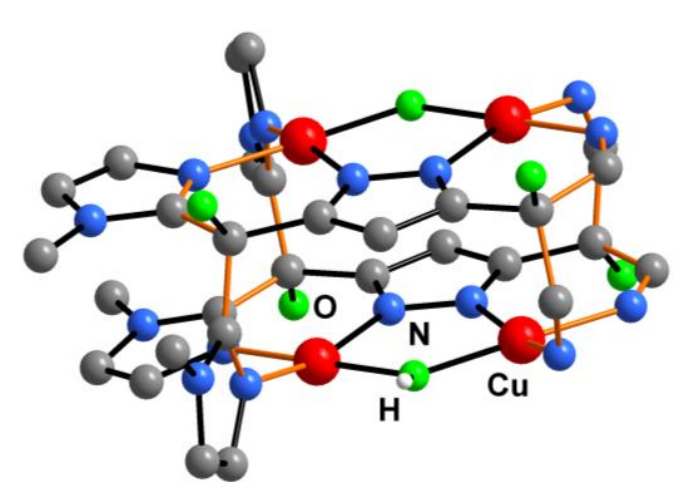

Abbildung 78: Kopplungspfad (orange) für 11a. zeigt, dass Cu1 und Cu4 bzw. Cu2 und Cu3 jeweils über zwei fünfatomige Brücken miteinander verknüpft sind (Abbildung 78). Der mögliche Kopplungspfad bildet sich dabei vom koordinierenden $\mathrm{N}$-Donor eines Imidazolringes über das zentrale C-Atom zum N-Donor des zweiten Imidazolringes zwischen zwei Kupferionen aus. 
Erneut repräsentiert die durchgezogene Linie in Abbildung 79 die beste Anpassung für 11a. Als Parameter ergaben sich $g=2.15$ (fixiert) und $J_{1}=-371.1 \mathrm{~cm}^{-1}(\rho=4.6 \%$ (fixiert), $\quad T I P=3.8 \cdot 10^{-4} \mathrm{~cm}^{3} \cdot \mathrm{mol}^{-1}$ ). Dies bestätigt ebenfalls eine sehr starke antiferromagnetische Kopplung.

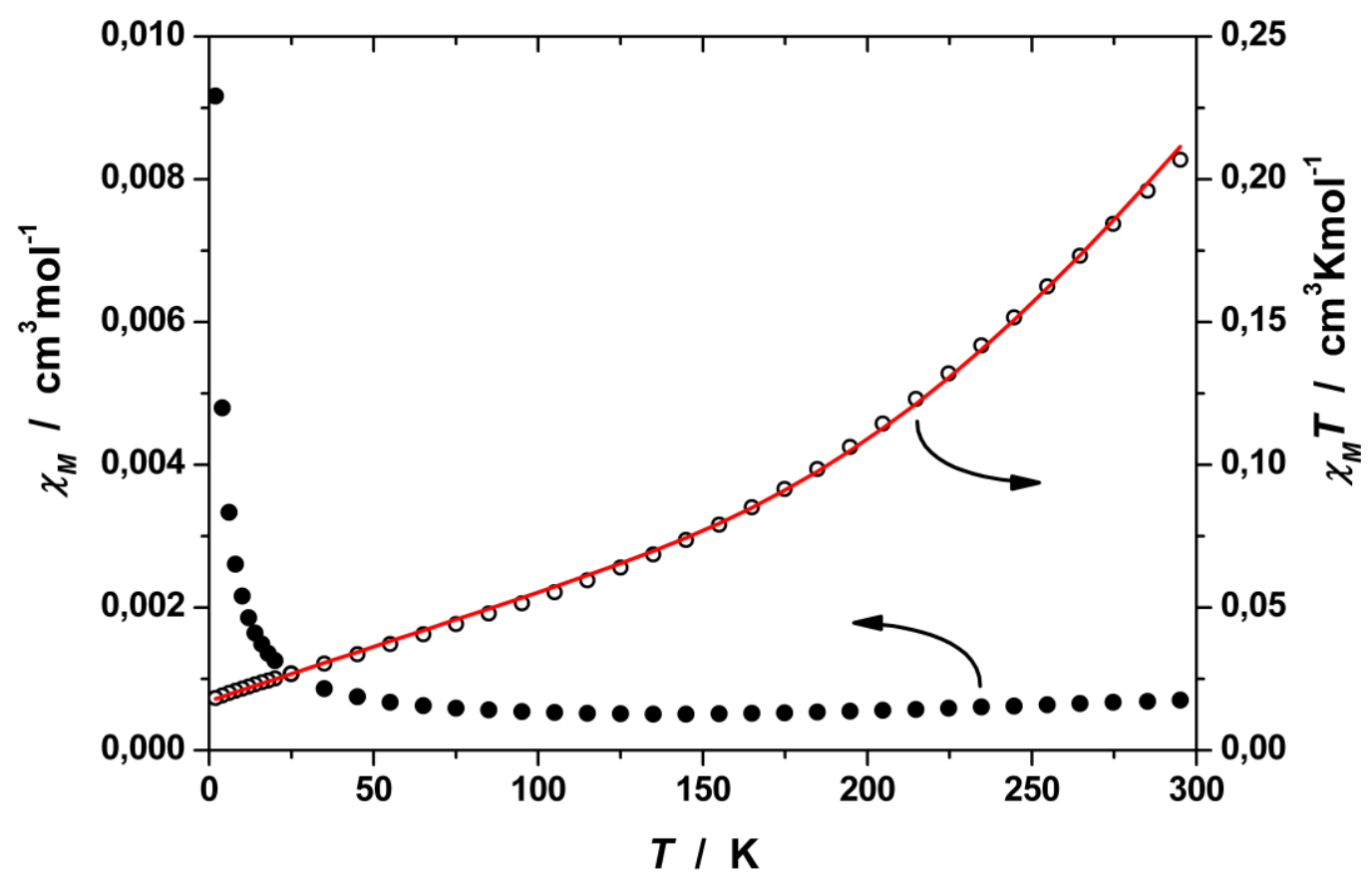

Abbildung 79: Temperaturabhängigkeit von $\chi_{M}$ und $\chi_{M} T$ für 11a (Die rote Linie repräsentiert die beste Anpassung).

Mit den Komplexen 12, 13 und 28 sind ebenfalls magnetochemische Messungen durchgeführt worden. Die Gestalt dieser Komplexverbindungen ähnelt sehr stark der Verbindung 11a. Unterschiedlich sind dabei nur die in axialen Positionen koordinierten Co-Liganden. Zum Teil sind diese Stellen mit Lösungsmitteln oder Gegenionen besetzt. Es gibt aber auch Kupfer(II)-Ionen mit freien axialen Koordinationsstellen, so dass hier quadratisch planare Koordinationsumgebungen um das Kupfer(II)-lon vorliegen. Die temperaturabhängigen Messdaten für die magnetische Suszeptibilität $\chi_{M}$ sowie das Produkt $\chi_{M} T$ sind für die vierkernigen Komplexe 12, 13 und 28 dargestellt. Für alle drei Verbindungen sind ähnliche $\chi_{M} T$-Werte von etwa $0.21 \mathrm{~cm}^{3} \cdot \mathrm{K}^{\prime} \cdot \mathrm{mol}^{-1}\left(1.30 \mu_{B}\right)$ bei Raumtemperatur erhalten worden. Für zwei ungekoppelte $S=1 / 2$ Kupfer(II)-lonen ist der theoretisch erwartete „spin-only“ Wert $2.63 \mu_{B}$ für $g=2.15$ und damit recht weit von dem erhaltenen Messwerten entfernt, was erneut auf eine starke antiferromagnetische Kopplung hindeutet. Der Verlauf der Messkurven von $\chi_{M} T$ gegen $T$ ist fast identisch. 
$\chi_{M} T$ nimmt stets exponentiell ab und das magnetische Moment sinkt ab etwa $120 \mathrm{~K}$ nur noch sehr langsam bis auf 0 (für 12 sinkt der Wert auf ca. 0.00025 ab). Dieses Verhalten deutet auf antiferromagnetischen Austausch hin ( $S=0$ Grundzustand). Die Daten der Suszeptibilitätsmessungen von 12 wurden im Temperaturbereich von $295 \mathrm{~K}$ bis $2 \mathrm{~K}$ unter Verwendung des Programms juIX angepasst. Erneut repräsentiert die

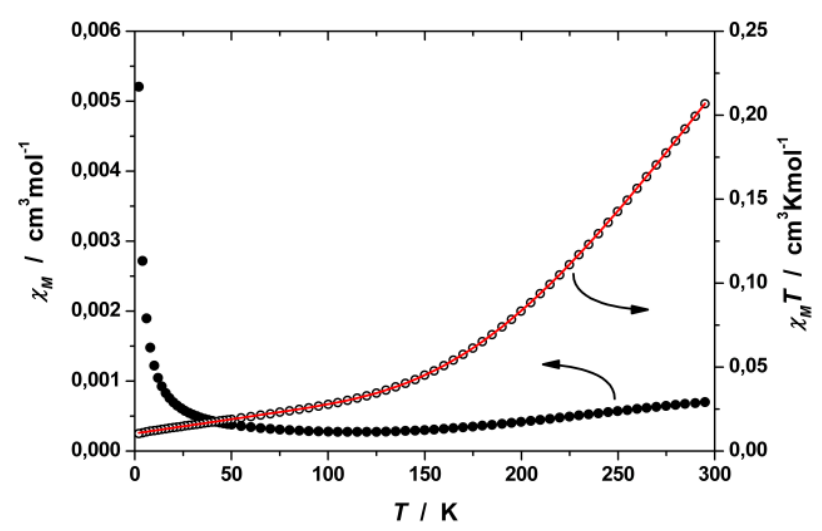
durchgezogene Linie in Abbildung 80 (oben) die beste Anpassung für 12. Als Parameter ergaben sich $g=2.15$ und $\quad J_{1}=-306.9 \mathrm{~cm}^{-1} \quad(\rho=2.8 \%$ (fixiert), $T I P=1.7 \cdot 10^{-4} \mathrm{~cm}^{3} \cdot \mathrm{mol}^{-1}$ ).

Da für die Komplexe 13 und 28 oberhalb von $250 \mathrm{~K}$ bzw. $270 \mathrm{~K}$ einige Messpunkte aufgrund des geringen magnetischen Moments der Probe

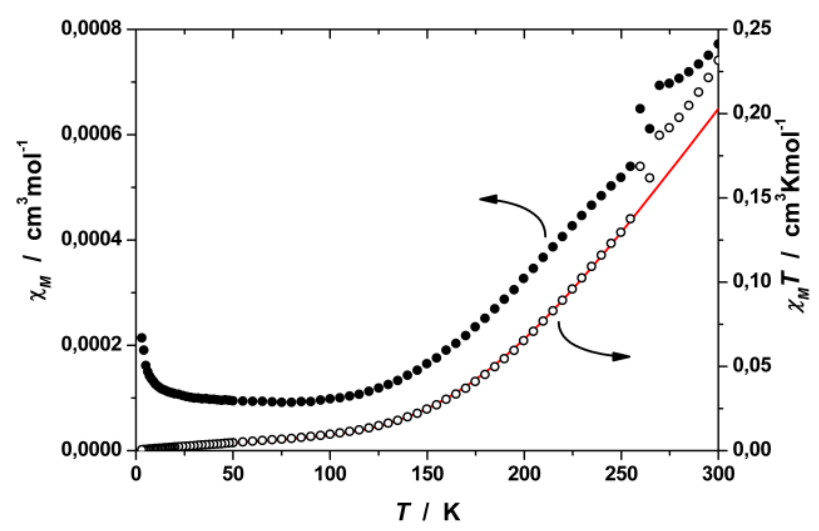
etwas von der idealen Messkurve abweichen, wurde hier die Anpassung nur für den Bereich von $250 \mathrm{~K}$ bzw. $270 \mathrm{~K}$ durchgeführt. Die roten Linien geben die besten Anpassungen für $\mathbf{1 3}$ $\begin{array}{lllll}\text { (Abbildung } 80 & \text { Mitte) } & \text { und } & \mathbf{2 8}\end{array}$ (Abbildung 80 unten) wieder. Es ergaben sich für Verbindung $\mathbf{1 3}$ die

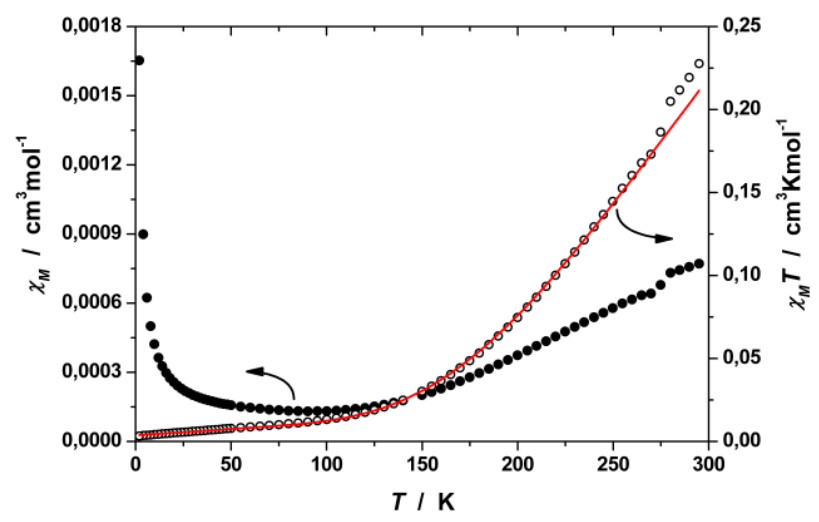

Abbildung 80: Temperaturabhängigkeit von $\chi_{M}$ und $\chi_{M} T$ für 12 (oben), 13 (Mitte) und 28 (unten) (Die roten Linien repräsentieren die besten Anpassungen).

Parameter $g=2.15 \quad$ (fixiert) und $J_{1}=-291.0 \mathrm{~cm}^{-1}(\rho=0.2 \%$ (fixiert), TIP $\left.=0.8 \cdot 10^{-4} \mathrm{~cm}^{3} \cdot \mathrm{mol}^{-1}\right)$ und für den Kupfer(II)-Komplex 28 die Parameter $g=2.15$ (fixiert) und $J_{1}=-281.4 \mathrm{~cm}^{-1}$ $(\rho=0.7 \% \quad$ (fixiert), TIP $\left.=0.8 \cdot 10^{-4} \mathrm{~cm}^{3} \cdot \mathrm{mol}^{-1}\right)$. Für alle drei hier zusammengefassten Komplexe ergeben sich sehr starke antiferromagnetische Kopplungen. 
Bei der Ermittlung der Anpassungskurven sind die vierkernigen Komplexverbindungen in zwei dinukleare Komplexe zerlegt worden, da es zwischen Cu1 und Cu4 bzw. Cu2 und Cu3 keine vermittelnde Brücke für einen signifikanten Austausch geben sollte. Als Beleg für die Richtigkeit dieser Annahme ist exemplarisch für Komplexverbindung 12 untersucht worden, inwieweit sich die Kopplungskonstanten $J_{1}$ und $J_{2}$ (Schema 22) gegenseitig beeinflussen. Dafür wurden für $J_{1}\left(-260 \mathrm{~cm}^{-1}\right.$ bis $\left.-360 \mathrm{~cm}^{-1}\right)$ und $J_{2}\left(-50 \mathrm{~cm}^{-1}\right.$ bis $\left.50 \mathrm{~cm}^{-1}\right)$ beliebige Startwerte festgelegt. Nach Berechnung des Programms julX veränderte sich $J_{1}$ dabei stets auf $-310 \mathrm{~cm}^{-1}$ und der Wert für $J_{2}$ zeigte keine Veränderung gegenüber dem eingegebenen Startwert. Egal ob für $J_{2}$ positive oder negative Werte angenommen wurden, das Ergebnis für $J_{1}$ war stets das Gleiche. Aus der in Abbildung 81 gezeigten Korrelation der beiden Kopplungskonstanten $J_{1}$ und $J_{2}$ mit der Qualität der entsprechenden Anpassung kann man erkennen, dass die Kopplungskonstante $J_{1}$ konstant bleibt, wenn man $J_{2}$ variiert. Ein Zahlenwert für Qualität der Anpassung (Goodness of fit) von 0 entspricht in dem verwendeten Programm julX einer sehr guten Anpassung mit sehr geringem Fehler. Würde zwischen beiden dinuklearen pyrazolatverbrückten Einheiten eine Kopplung bestehen, wären beide

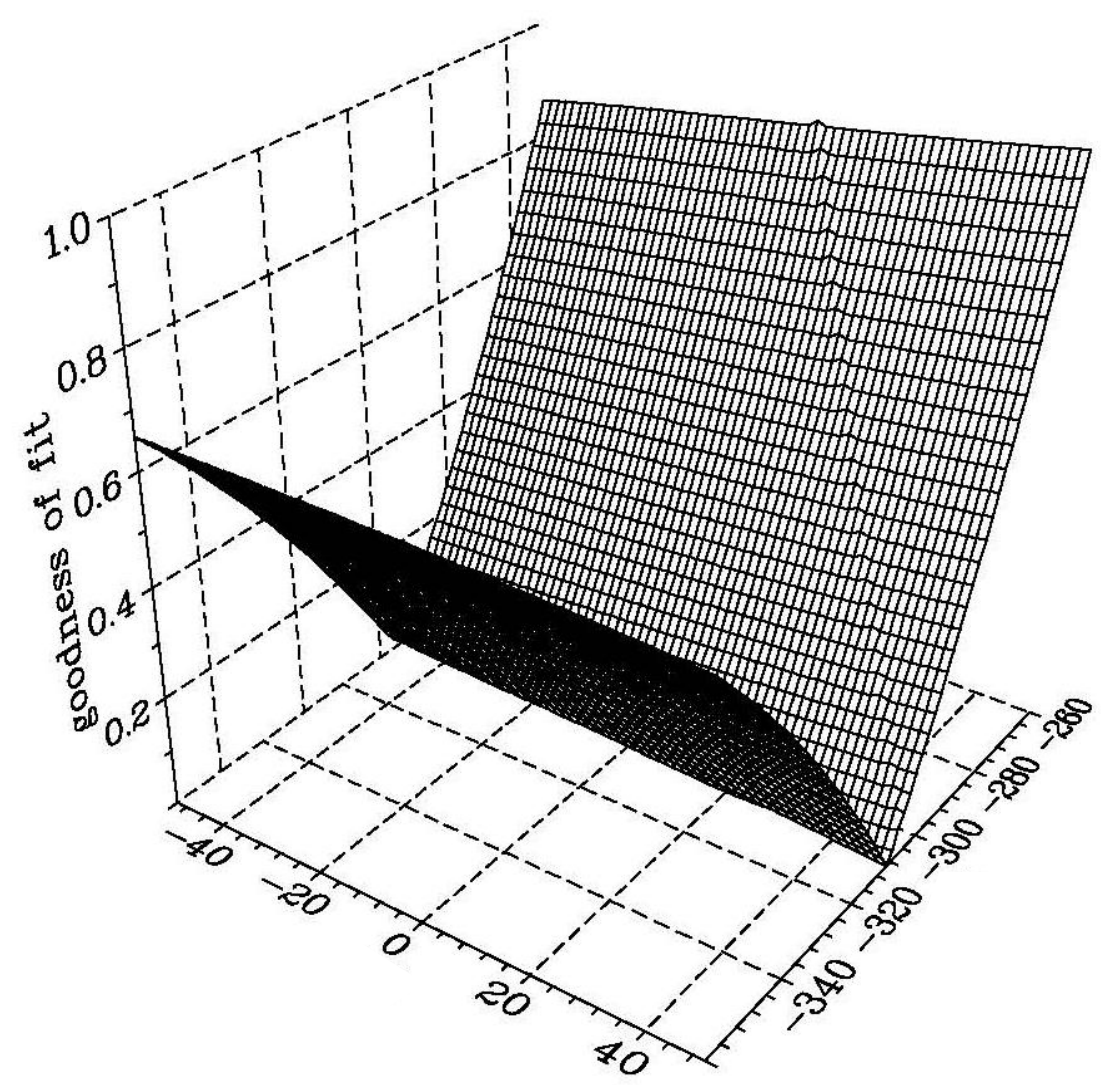

Abbildung 81: Korrelation zwischen $J_{1}$ und $J_{2}$ in Abhängigkeit von der Qualität der Anpassung für 12. 
Kopplungskonstanten von einander abhängig, so dass es nur eine Möglichkeit geben sollte für eine sehr gute Anpassung an die experimentell gefundenen Daten. Die daraus resultierende Grafik sieht wie ein trichterförmiges Gebilde aus. Die Lage des Minimums in der Darstellung würde den beiden Kopplungskonstanten entsprechen.

In der nachfolgenden Tabelle (Tabelle 24) sind die wichtigsten Parameter der magnetischen Messungen zusammengefasst. Neben dem $g$-Wert, der Kopplungskonstante $J$ und dem TIP ist auch die paramagnetische Verunreinigung $\rho$ gezeigt.

Die paramagnetische Verunreinigung $\rho$ ist für den Anstieg der $\chi_{M}$-Werte im Bereich sehr kleiner Temperaturen $(<10 \mathrm{~K})$ verantwortlich, jedoch ist die Größe von $\rho$ für alle Komplexe im Rahmen des üblichen Toleranzbereiches.

\begin{tabular}{cccccc}
\hline Komplex & Feldstärke [Oe] & $g$ & $J_{1}\left[\mathrm{~cm}^{-1}\right]$ & $\rho[\%]$ & $T I P\left[\mathrm{~cm}^{3} \cdot \mathrm{mol}^{-1}\right]$ \\
\hline $\mathbf{1 2}$ & 3000 & 2.15 & -306.9 & 2.8 & $1.7 \cdot 10^{-4}$ \\
$\mathbf{1 3}$ & 2000 & 2.15 (fixiert) & -291.0 & 0.2 & $0.8 \cdot 10^{-4}$ \\
$\mathbf{1 0}$ & 5000 & 2.15 (fixiert) & -233.2 & 1.4 & $1.8 \cdot 10^{-4}$ \\
$\mathbf{8}$ & 2000 & 2.15 (fixiert) & -327.3 & 0.2 (fixiert) & $2.5 \cdot 10^{-4}$ \\
$\mathbf{2 8}$ & 5000 & 2.15 (fixiert) & -281.4 & 0.7 & $0.8 \cdot 10^{-4}$ \\
$\mathbf{1 1 a}$ & 2000 & 2.15 (fixiert) & -371.1 & 4.6 & $3.8 \cdot 10^{-4}$ \\
\hline
\end{tabular}

Tabelle 24: Magnetische Parameter für die Komplexe 8, 10, 11a, 12, 13 und 28.

In Tabelle 25 sind für alle Kupfer(II)-Komplexe die gefundenen Kopplungskonstanten den wichtigsten strukturellen Parametern gegenüber gestellt. Wenn intramolekulare Kopplungen stattfinden sollen, müssen bestimmte Voraussetzungen erfüllt werden. So sind zum Beispiel die Torsionswinkel (Cu-N-N-Cu) entscheidend, da ein starkes Verdrehen eines Kupferzentrums aus der Ebene, die durch beide PyrazolStickstoffatome und das zweite Kupferion aufgespannt wird, zu schlechter Überlappung der entsprechenden Orbitale führt. Im Falle des größten Torsionswinkels in Komplex 10 
wird die kleinste Kopplungskonstante gefunden. Stellt man die zunehmenden Kopplungskonstanten den entsprechenden Torsionswinkeln gegenüber, so erhält man nicht die erwartete lineare Abhängigkeit. So passen z. B. die Werte von Komplex 11a nicht in das Schema.

\begin{tabular}{ccccc} 
Komplex & $\ll(\mathrm{Cu}-\mathrm{O}-\mathrm{Cu})\left[{ }^{\circ}\right]$ & $\ll(\mathrm{Cu}-\mathrm{N}-\mathrm{N})\left[{ }^{\circ}\right]$ & $\varangle(\mathrm{Cu}-\mathrm{N}-\mathrm{N}-\mathrm{Cu})\left[^{\circ}\right]$ & $J_{1}\left[\mathrm{~cm}^{-1}\right]$ \\
\hline $\mathbf{8}$ & $124.88(10)$ & $118.21(15)$ & $-15.54(24)$ & -327.3 \\
$\mathbf{1 0}$ & $119.8(5)$ & $118.1(11)$ & $-26.2(14)$ & -233.2 \\
$\mathbf{1 1 a}$ & $125.1(2)$ & $121.0(3)$ & $-1.98(53)$ & -371.1 \\
$\mathbf{1 2}$ & $125.3(2)$ & $120.9(4)$ & $0.3(6)$ & -306.9 \\
$\mathbf{1 3}$ & $124.752(4)$ & $120.861(5)$ & 2.51 & -291.0 \\
$\mathbf{2 8}$ & $125.143(7)$ & $120.953(9)$ & $-1.824(16)$ & -281.4 \\
\hline
\end{tabular}

Tabelle 25: Zusammenstellung einiger wichtiger Parameter.

Der ungewöhnlich große Wert für die Kopplungskonstante $J_{1}=-371.1 \mathrm{~cm}^{-1}$ in 11 a resultiert vermutlich aus geringen Verunreinigungen in der Probe. Bei Betrachtung der Wertepaare fällt auf, dass der Torsionswinkel nicht allein für die Größe der Kopplung entscheidend sein kann. Ein Blick auf die Cu-O-Cu-Winkel zeigt ebenfalls keine eindeutige Abhängigkeit. Für den kleinsten Wert findet man auch die geringste Kopplungskonstante. Betrachtet man die sehr ähnlichen Cu-O-Cu-Winkel von Verbindung 12 und 28 bzw. 8 und 13, findet man erstaunlicherweise große Unterschiede für die Kopplungskonstanten. Diese Differenzen liegen bei $36.3 \mathrm{~cm}^{-1} \mathrm{bzw}$. $25.5 \mathrm{~cm}^{-1}$. Hatfield und Hodgson haben für Hydroxyverbrückte Kupfer(II)-Komplexe eine Korrelation des $\mathrm{Cu}-\mathrm{OH}-\mathrm{Cu}-W i n k e l s(\alpha)$ mit der erhaltenen Kopplungskonstante $(\mathcal{J})$ erstellt. Die ungefähre Größenordnung der Kopplungskonstante $J$ kann demnach mittels Gleichung 6 berechnet werden.

$$
2 J\left[\mathrm{~cm}^{-1}\right]=-74 \cdot \alpha[\mathrm{Grad}]+7270
$$

Demnach müsste für 8 eine Kopplungskonstante von $J=-985 \mathrm{~cm}^{-1}$ auftreten. Für alle anderen Systeme lassen sich aufgrund der großen Ähnlichkeit nahezu identische Werte ermitteln. Damit unterscheiden sich die berechneten Parameter von den experimentell 
bestimmten Werten um etwa Faktor 3. Diese Korrelation ist somit nicht für derartige Systeme einsetzbar.

Mit den oben aufgelisteten $\mathrm{Cu}-\mathrm{N}-\mathrm{N}-$ Winkeln lassen sich die großen Unterschiede zwischen den Kopplungskonstanten ebenso wenig erklären. Offenbar lassen sich anhand struktureller Daten keine genauen Vorhersagen über die Größe der intramolekularen Kopplung treffen. In den Komplexverbindungen 8 und $\mathbf{1 0}$ sind die Cu-N-N-Winkel fast identisch und trotzdem liegen die Kopplungskonstanten um $96.1 \mathrm{~cm}^{-1}$ auseinander.

Vor allem die Komplexe 12, 13 und $\mathbf{2 8}$ haben den gleichen Aufbau und sollten somit einen guten Vergleich der Werte zulassen und eine Korrelation der Werte ermöglichen. Jedoch kann hier keine eindeutige Korrelation zwischen strukturellen Parametern und den magnetischen Werten getroffen werden, da die antiferromagnetische Kopplung sehr stark ist. Vermutlich kann die Größe der Kopplung auch etwas von der Koordinationsumgebung um die Kupferzentren beeinflusst werden. Auch die Auswirkungen, die Verunreinigungen auf das Messergebnis haben, sind nicht zu vernachlässigen. Wie an den Werten für $\rho$ zu erkennen ist, sind diese Zahlen für 12, 13 und 28 kleiner als für 11a.

Molekularer Magnetismus ist ein interessantes und vielseitiges Gebiet und so ist es nicht verwunderlich, dass sich zum Vergleich mit den hier erhaltenen Werten eine Vielzahl von Publikationen finden lassen, die einen Vergleich der experimentell erhaltenen Werten zulassen. ${ }^{[165-167]}$ Eine kleine Auswahl soll hier gegeben werden. Thompson et. al. entwickelten die Liganden XXIV und XXV und stellten Kupfer(II)Komplexe damit her. Beide Kupfer(II)-Ionen koppeln mit einer Kopplungskonstante von $J=-160 \mathrm{~cm}^{-1}$ bis $-400 \mathrm{~cm}^{-1}$ (Abbildung 82). ${ }^{[168,169]}$

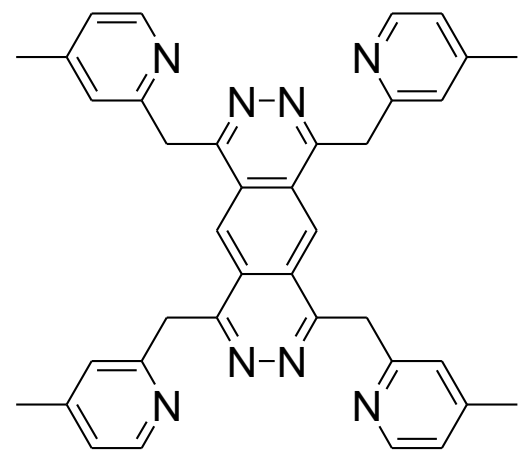

XXIV

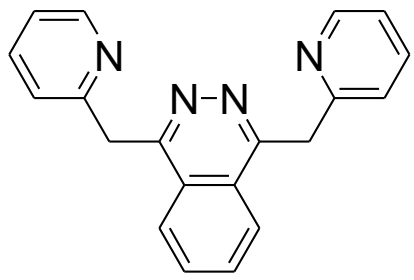

XXV

Abbildung 82: Verwendete Liganden für Kupfer(II)-komplexe. 
Für den trinuklearen Nickel(II)-Komplex 25 sind die temperaturabhängigen Messergebnisse für die magnetische Suszeptibilität $\chi_{M}$ sowie das Produkt $\chi_{M} T$ in Abbildung 83 dargestellt. Bei Raumtemperatur kann ein $\chi_{M} T$-Wert von $4.17 \mathrm{~cm}^{3} \mathrm{~K} \cdot \mathrm{mol}^{-1}$ $\left(5.78 \mu_{B}\right)$ abgelesen werden. Dieser Zahlenwert liegt recht nahe an dem theoretisch erwarteten "spin-only“ Wert für drei ungekoppelte $S=1$ Nickel(II)-lonen (5.38 $\mu_{B}$ für $g=2.20)$. Der Landé-Faktor von 2.20 ergibt sich als Mittelwert der drei erhaltenen $g$-Werte $\left(g_{1}=g_{3}=2.24\right.$ und $\left.g_{2}=2.11\right)$. Der Verlauf des Produktes von $\chi_{M} T$ ist mit abnehmender Temperatur nahezu konstant bis etwa $50 \mathrm{~K}$. Es gibt nur einen ganz leichten Abfall der $\chi_{M} T$-Werte von $4.17 \mathrm{~cm}^{3} \cdot \mathrm{K} \cdot \mathrm{mol}^{-1}$ auf $3.82 \mathrm{~cm}^{3} \cdot \mathrm{K}^{\cdot} \cdot \mathrm{mol}^{-1}$. Dies liegt an am Vorhandensein des temperaturunabhängigen Paramagnetismus $\left(T I P=16.2 \cdot 10^{-4} \mathrm{~cm}^{3} \cdot \mathrm{mol}^{-1}\right)$. Unterhalb von $50 \mathrm{~K}$ steigt die Kurve sehr schnell an und erreicht bei $2 \mathrm{~K}$ einen $\chi_{M} T$-Wert von $7.76 \mathrm{~cm}^{3} \cdot \mathrm{K} \cdot \mathrm{mol}^{-1}\left(7.88 \mu_{B}\right)$. Für einen ferromagnetischen Austausch ( $S=3$ Grundzustand) lässt sich ein „spin-only" Wert von $7.62 \mu_{B}$ (für $g=2.20$ ) errechnen. Damit stimmt der experimentell erhaltene Wert sehr gut mit dem theoretisch möglichen Wert überein. Im Temperaturbereich von $295 \mathrm{~K}$ bis $2 \mathrm{~K}$ wurden die Daten der Suszeptibilitätsmessung von 25 unter Verwendung des Programms juIX angepasst. Dabei wurde der Spin-Hamiltonian-Operator mit ZeemanTerm (Gleichung 7) angewendet.

$$
\hat{H}=-2 J_{12} \hat{S}_{1} \hat{S}_{2}-2 J_{23} \hat{S}_{2} \hat{S}_{3}+g \mu_{\mathrm{B}} \mathrm{B} \sum_{i} \mathrm{~S}_{\mathrm{zi}} \quad \text { (Gleichung 7) }
$$

Wie schon in den anderen Abbildungen repräsentiert die rote Linie die beste Anpassung. 


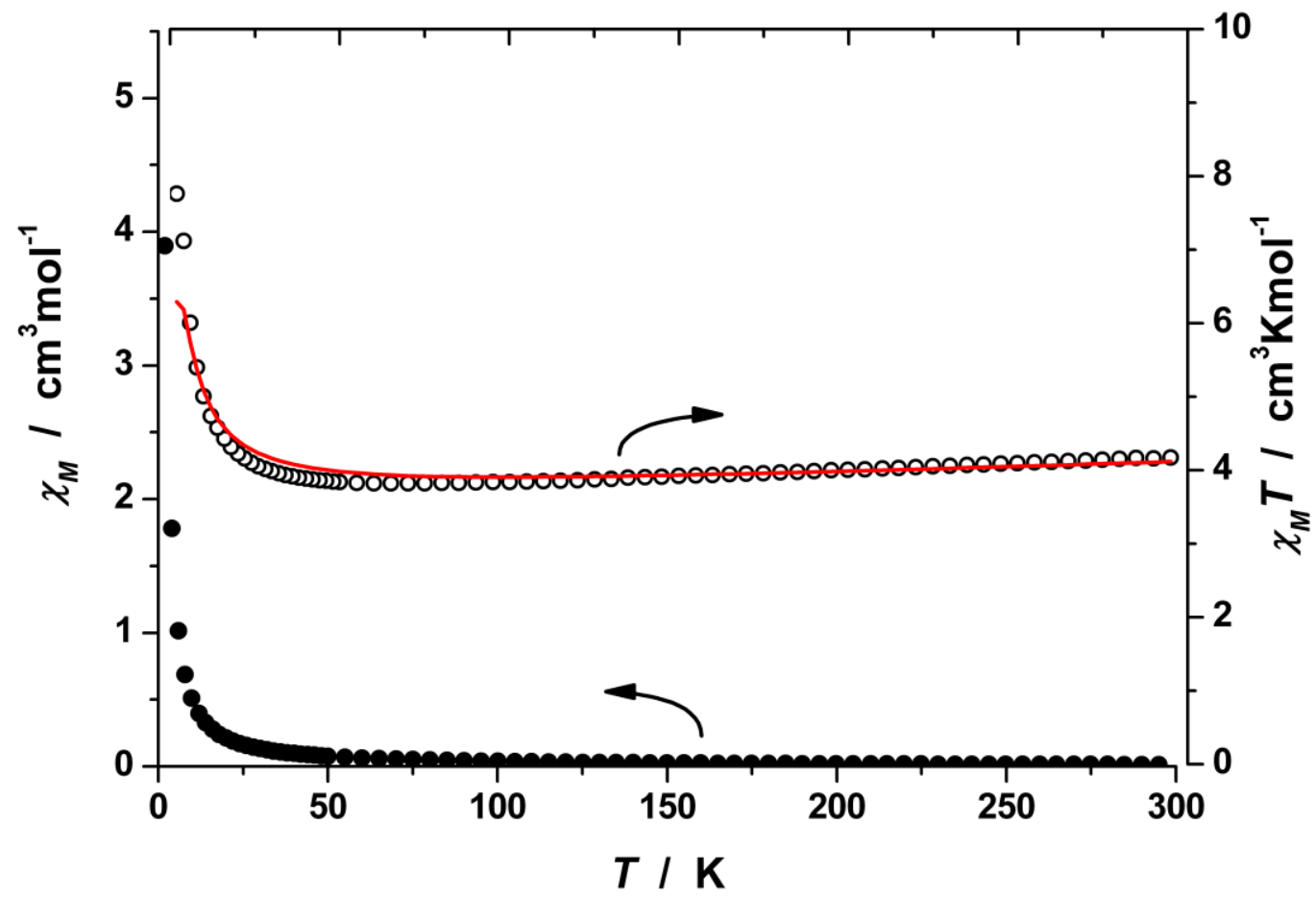

Abbildung 83: Temperaturabhängigkeit von $\chi_{M}$ und $\chi_{M} T$ für 25 (Die rote Linie repräsentiert die beste Anpassung).

Die daraus erhaltenen Parameter sind $g_{1}=g_{3}=2.24$ und $g_{2}=2.11$, sowie $J_{12}=J_{23}=$ $1.76 \mathrm{~cm}^{-1}\left(\rho=1.0 \%\right.$ (fixiert), $\left.T I P=16.2 \cdot 10^{-4} \mathrm{~cm}^{3} \cdot \mathrm{mol}^{-1}\right)$. Auch daraus lässt sich die schwache ferromagnetische Kopplung erkennen. Das hier zutreffende Kopplungsschema ist in Schema 23 abgebildet.

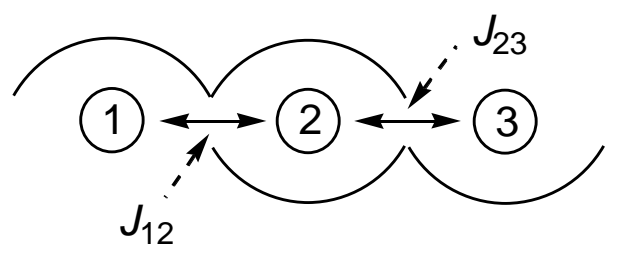

Schema 23: Kopplungsschema für 25.

Hiermit ist es erstmals gelungen, eine ferromagnetische Kopplung zwischen zwei Nickel(II)-Ionen über eine Pyrazolatbrücke aufzubauen. Dies lässt sich mit der unterschiedlichen Koordinationsumgebung der Nickelionen begründen. Es konnte mit diesem Komplex ein komplett neues Strukturmotiv aufgezeigt werden. In der Literatur sind bereits eine Reihe trinuklearer Nickelkomplexe mit verbrückender Pyrazol-, Pyridazin- oder Triazoleinheit beschrieben. ${ }^{[170-177]}$ Darin findet man jedoch 
ausschließlich Nickelzentren, die oktaedrisch umgeben sind. Das zentrale Metallion ist dabei mit den beiden äußeren Nickel(II)-Ionen über drei entsprechende Heterozyklen oder über zwei Heterozyklen und ein Anion verbrückt. Die drei übrigen Koordinationsstellen der äußeren Metallionen sind durch Lösungsmittelmoleküle oder Anionen belegt. Es ergeben sich immer schwache bis mittelstarke antiferromagnetische Kopplungen mit Werten für $J=-0.24 \mathrm{~cm}^{-1}$ bis $-10.3 \mathrm{~cm}^{-1} \cdot{ }^{[170,171,175,177]}$ Außerdem wird ein symmetrischer trinuklearer Nickel(II)-Komplex beschrieben, in dem zwei Nickel(II)-Ionen über zwei Triazoleinheiten und ein exogene Thiocyanat-Einheit verbrückt sind. Dadurch entstehen auch wieder drei oktaedrisch koordinierte Nickelzentren mit einer ferromagnetischen Kopplung die durch das Thiocyanation vermittelt werden muss. ${ }^{[143]}$

Um das hier auftretende Phänomen zu erklären, ist es notwendig, die magnetisch relevanten Orbitale zu betrachten. Das Nickel(II)-Ion hat die Elektronenkonfiguration $d^{8}$. Die Aufspaltungen der d-Orbitale für den Oktaeder- sowie den Tetraeder-Fall sind in Abbildung 84 dargestellt.

$t_{2}$

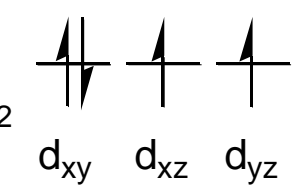

tetraedrisch
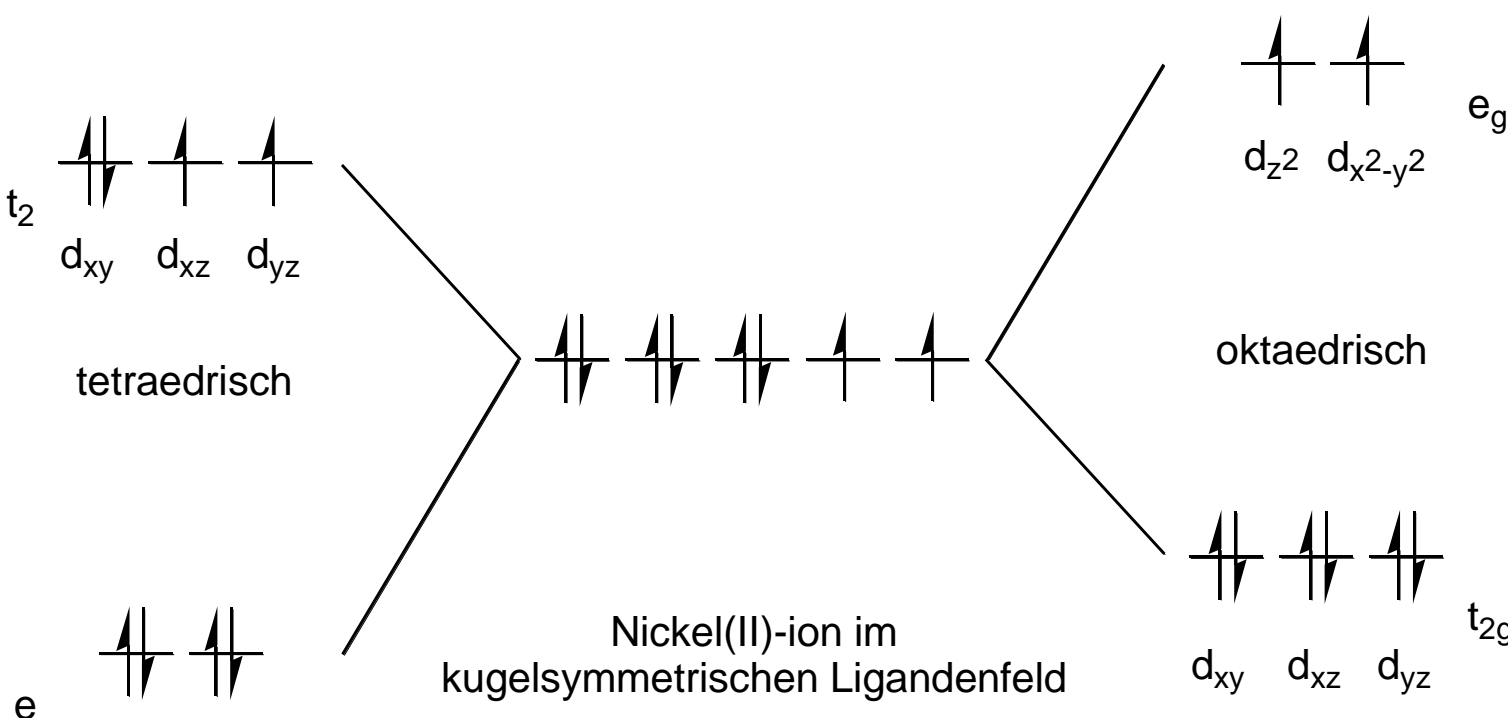
kugelsymmetrischen Ligandenfeld

$d_{x^{2}-y^{2}}$

oktaedrisch

$$
d_{z^{2}} \quad d_{x^{2}-y^{2}}
$$

Abbildung 84: Aufspaltung der d-Orbitale für tetraedrische (links) und oktaedrische Koordinationsumgebung (rechts) eines Nickel(II)-ions.

Darin ist ersichtlich, dass beim Oktaeder die relevanten Orbitale das $d_{x^{2}-y^{2}-O r b i t a l}$ und das $d_{z^{2}}$-Orbital sind. Im Tetraeder-Fall handelt es sich dabei um die $d_{x y^{-}}, d_{x z^{-}}$und $d_{y z}$-Orbitale. Damit die intramolekulare Kopplung über die Pyrazolat-Einheit erklärt 
werden kann, muss man das Konzept der "Orthogonalen Orbitale“ von O. Kahn ${ }^{[178]}$ berücksichtigen. Dieses Konzept basiert auf der Tatsache, dass sich die Größe einer magnetischen Kopplung $(J)$ aus einer ferromagnetischen Kopplungskontante $\left(J_{F}\right)$ und einer Kopplungskonstante für die antiferromagnetische Kopplung $\left(J_{\mathrm{AF}}\right)$ additiv zusammensetzt. Sind die Orbitale mit den ungepaarten Elektronen orthogonal zueinander ist die Überlappung nicht mehr gegeben und $J_{A F}$ sinkt auf null. Dadurch kommt die viel kleinere $J_{F}$ zum Tragen und es resultiert ein ferromagnetisch gekoppeltes System. Wie aus Abbildung $84 \mathrm{zu}$ erkennen ist, befinden sich die ungepaarten Elektronen von Komplex 25 in Orbitalen mit unterschiedlicher Gestalt. Die Orbitale des $t_{2 g}$-Satzes liegen zwischen den Achsen eines Koordinatensystems. Im Gegensatz dazu, befinden sich die Orbitale des $e_{g}$-Satzes auf den Achsen eines Koordinatensystems. Beide Orbital-Sets stehen senkrecht zueinander und eine Überlappung ist nicht möglich. Es ergibt sich ausschließlich die hier gefundene ferromagnetische Kopplung mit $J=+1.76 \mathrm{~cm}^{-1}$.

Ferromagnetische Kopplungen können auch in heterobimetallischen Komplexen mit geeigneter Brücke zwischen zwei oktaedrisch koordinierten Metallionen auftreten. Die Elektronenkonfiguration der verwendeten Metalle, sollte dafür so sein, dass die ungepaarten Elektronen eines Metalls im $e_{g}$-Satz und die ungepaarten Elektronen des zweiten Metallions im $t_{2 g}$-Satz zu finden sind. Daraus ergibt sich z. B. die Verwendung eines frühen und eines späten Übergangsmetalls. Die Synthese derartiger Komplexe sollte jedoch von einem unsymmetrischen Liganden ausgehen, da sich sonst bei Verwendung zweier verschiedener Metalle in der Regel eine statistische Verteilung der erhaltenen Komplexverbindungen ergibt. In dem hier vorgestellten Komplex handelt es sich aber um einen symmetrischen Ligand und ebenso um das gleiche Metallion. Bisher gibt es keine literaturbekannten Beispiele für Systeme mit derartiger Koordinationsgeometrie und außerdem ist dies das erste Beispiel für eine ferromagnetische Kopplung, die durch eine Pyrazolat-Einheit vermittelt wird. 


\section{Zusammenfassung}

Im Rahmen der vorliegenden Arbeit ist ein Satz neuartiger Pyrazolatliganden mit zwei facial-tridentaten Koordinationstaschen hergestellt worden. Die Synthese der sechs neuen Liganden erfolgte in Anlehnung an Literaturvorschriften für Bisarylsysteme. Die Darstellung erfolgte über die in Schema 24 gezeigte Reaktionsfolge.

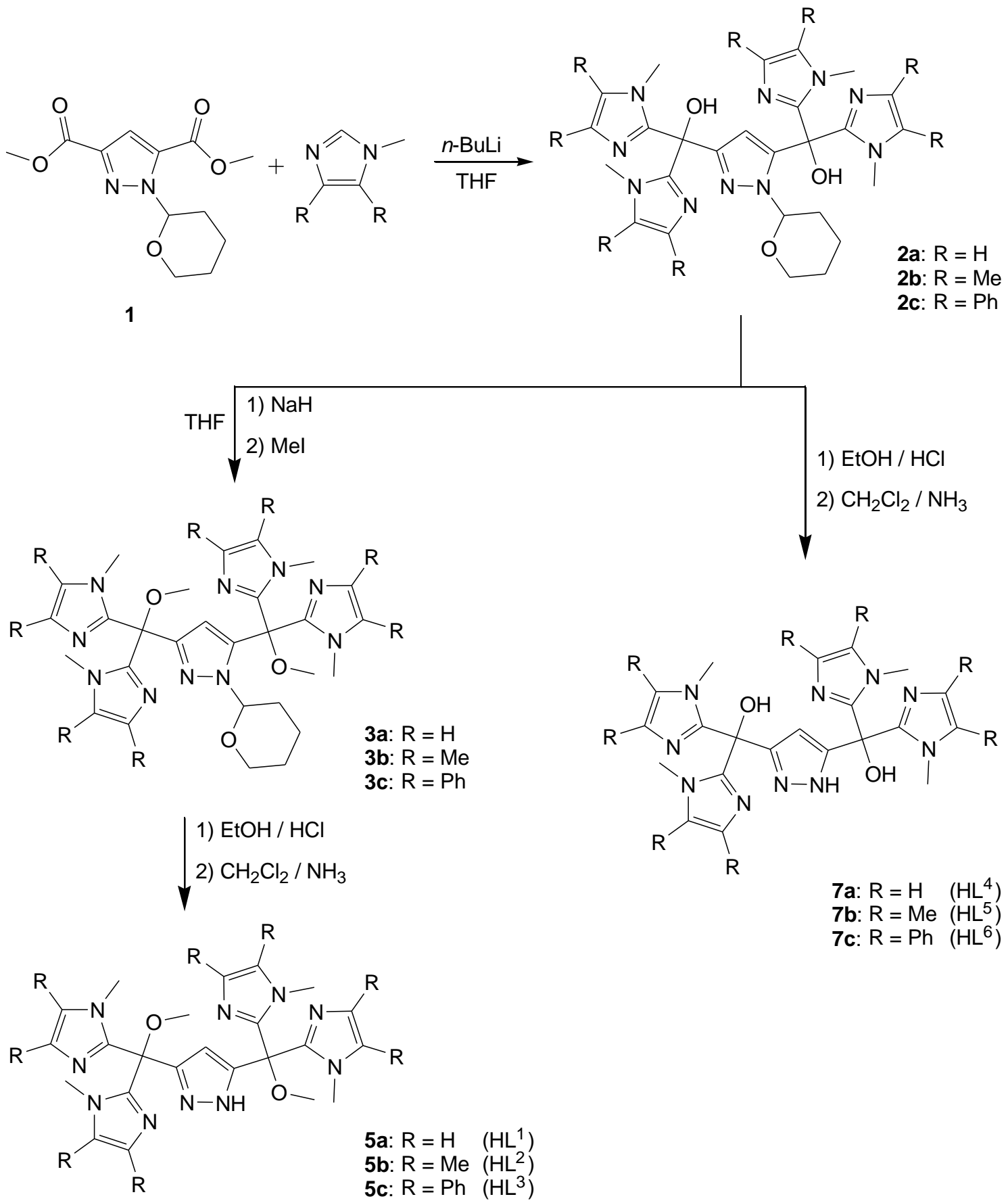

Schema 24: Synthese der neuartigen Pyrazolat-Liganden $\mathrm{HL}^{1}$ bis $\mathrm{HL}^{6}$. 
Ausgehend vom Thp-geschützten 3,5-Pyrazoldicarbonsäuremethylester (1) lassen sich zum einen die Liganden $\mathrm{HL}^{1}, \mathrm{HL}^{2}$ und $\mathrm{HL}^{3}$ herstellen. Führt man die Veretherung der $\mathrm{OH}$-Funktionen nicht durch, gelangt man zu den Liganden $\mathrm{HL}^{4}, \mathrm{HL}^{5}$ und $\mathrm{HL}^{6}$.

Mit diesen Liganden war es möglich mit verschiedenen Kupfer(II)-Salzen die Kupfer(II)Komplexe 8 bis $\mathbf{1 5}$ herzustellen und die Struktur kristallographisch zu ermitteln. Durch Deprotonierung des Pyrazolrings konnten zwei Kupfer-lonen an ein Ligand-Molekül gebunden werden. Dabei lässt sich in allen synthetisierten Komplexverbindungen das gleiche Strukturmotiv finden. Zwei Kupferzentren sind durch einen Pyrazolring und eine Hydroxid-Brücke verbrückt, so dass die Kupfer(II)-Ionen einen Abstand von $3.29 \AA$ bis $3.41 \AA$ aufweisen. Die einzelnen Cu1-Cu2-Abstände der Komplexe 8 bis 15 sind in Tabelle 26 zusammengefasst. Darin ist zu sehen, dass für beide Komplexe (14 und 15) mit dem Liganden $\mathrm{HL}^{2}$ die größten Cu1-Cu2-Abstände gefunden worden.

\begin{tabular}{cc} 
Komplex & Cu1-Cu2-Abstand $[\AA]]$ \\
\hline $\mathbf{8}$ & $3.3367(5)$ \\
$\mathbf{9}$ & $3.3268(9)$ \\
$\mathbf{1 0}$ & $3.2945(8)$ \\
$\mathbf{1 1 a}$ & $3.3918(9)$ \\
$\mathbf{1 1 b}$ & $3.3731(5)$ \\
$\mathbf{1 2}$ & $3.377(1)$ \\
$\mathbf{1 3}$ & $3.3641(4)$ \\
$\mathbf{1 4}$ & $3.4094(8)$ \\
$\mathbf{1 5}$ & $3.3440(5)$ \\
\hline
\end{tabular}

Tabelle 26: Cu1-Cu2-Abstände für die Kupfer(II)-Komplexe 8 bis 15.

Die beiden Kupfer(II)-Komplexe 8 und 15 enthalten zwei Kupfer-lonen (Abbildung 85). Die Koordination des jeweiligen Liganden ist identisch. An jedes Kupferzentrum binden jeweils ein N-Donor des Pyrazol- und ein Stickstoffatom des Imidazolrings. Auf jeder Seite des Liganden ist ein Imidazolring weggedreht und steht nicht für die Koordination 
zur Verfügung. Im Gegensatz zu dem acetathaltigen Komplex 8 ist in Verbindung 15 diese Koordination durch die Protonierung des weggedrehten Imidazolrings bedingt. Ein weiterer Unterschied ist die Art der Co-Liganden an den Kupferzentren. Sind es in $\mathbf{8}$ Acetat-Ionen, die ein- bzw. zweizähnig an das Kupfer(II)-Ion binden, wird jedes Kupferzentren in $\mathbf{1 5}$ von zwei Acetonitril koordiniert und es entsteht ebenfalls eine quadratisch pyramidale Anordnung.

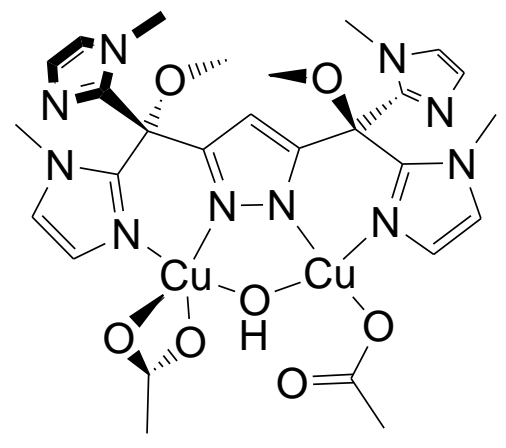

8

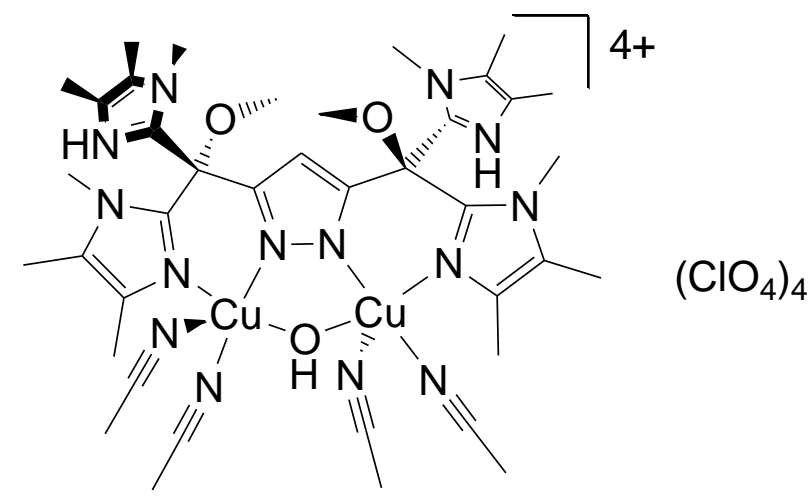

15

Abbildung 85: Zweikernige Kupfer(II)-Komplexe 8 und 15.

Desweiteren sind die Strukturen der vierkernigen Kupfer(II)-Komplexe 9 bis 14 kristallographisch aufgeklärt worden. Die Komplexverbindung 10 weist ein anderes Koordinationsmuster als die restlichen vierkernigen Komplexe auf. Die Anordnung der Kupfer(II)-lonen ist stufenförmig (Abbildung 86). Der freie Imidazolring einer Seite koordiniert an ein Kupferzentrum einer zweiten dinuklearen Einheit.

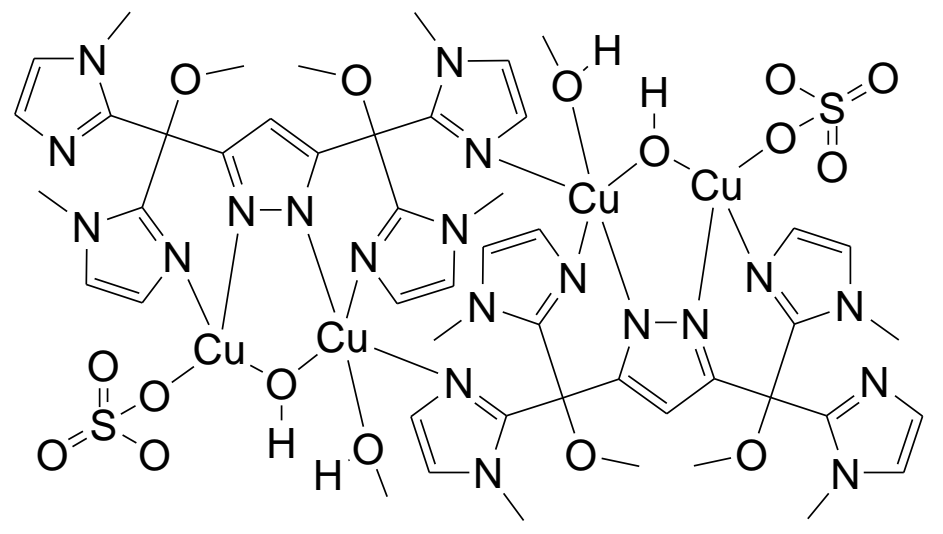

10

Abbildung 86: Schematische Darstellung des stufenförmigen Kupfer(II)-Komplexes 10. 
Die Kupfer(II)-Ionen in den restlichen Kupfer(II)-Komplexen sind rechteckig angeordnet. Die Imidazolringe, die in $\mathbf{8}$ und $\mathbf{1 0}$ an kein Kupferion gebunden sind, binden nun an ein benachbartes Kupferion und es bilden sich rechteckige Strukturen aus. In Abbildung 87 ist dies schematisch gezeigt.

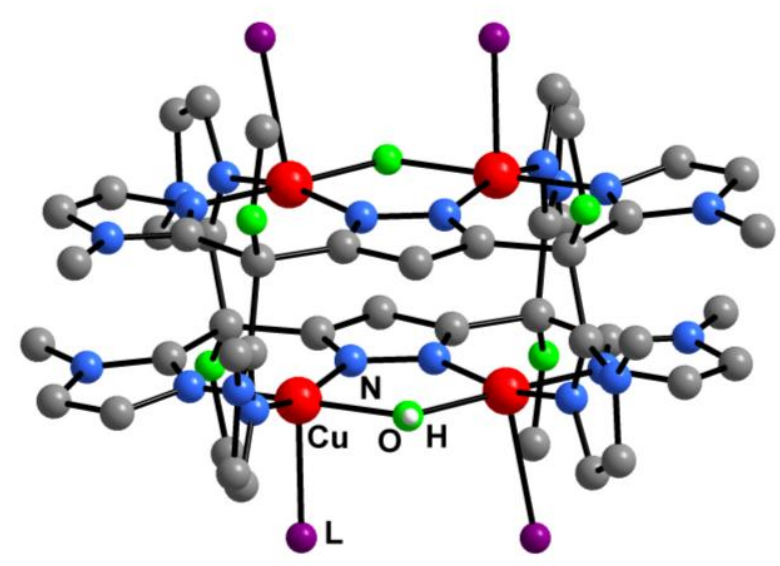

Abbildung 87: Schematische Darstellung der rechteckigen tetranuklearen Kupfer(II)-Komplexe ( $L$ steht für beliebige Co-Liganden - Gegenionen oder Lösungsmittel).

Zwischen den zwei Ebenen, bestehend aus dem Pyrazolring mit einem Imidazolring jeder Ligandseite, gibt es einen Abstand von etwa 3.3 A. Seitlich wird dieser Hohlraum durch die sich gegenüberstehenden Imidazolringe begrenzt. Die Abmessungen dieses Hohlraums sind in Tabelle 27 aufgelistet.

\begin{tabular}{ccc} 
Komplex & $\begin{array}{c}\text { Abstand zwischen den } \\
\text { Pyrazol-Ebenen }[\AA]\end{array}$ & $\begin{array}{c}\text { Seitliche Ausdehnung } \\
{[\AA]}\end{array}$ \\
\hline 11a & 3.20 & 6.55 \\
11b & 3.29 & 6.57 \\
12 & 3.29 & 6.54 \\
13 & 3.32 & 6.52 \\
14 & 3.30 & 6.61 \\
\hline
\end{tabular}

Tabelle 27: Dimensionen des Hohlraumes in den tetranuklearen Komplexen 11a bis 14. 
Die Suszeptibilitätsmessungen aller Kupfer(II)-Komplexe zeigen eine starke antiferromagnetische Kopplung zwischen zwei pyrazolatverbrückten Kupfer-Ionen. Anhand der gefunden Kopplungskonstanten von $-233.2 \mathrm{~cm}^{-1}$ bis $-371.1 \mathrm{~cm}^{-1}$ lässt sich diese Tatsache belegen.

Neben den zahlreichen Kupfer(II)-Komplexen konnten die Strukturen einiger Kupfer(I)Komplexe kristallographisch aufgeklärt werden (Abbildung 88). Dabei ist deutlich geworden, dass die zwei Koordinationstaschen des Liganden offenbar keine tetraedrische Umgebung für ein Kupfer(I)-lon bereitstellen. Stattdessen klappt ein Imidazolring erneut nach hinten weg und es bildet sich stets eine trigonal planare Koordinationsgeometrie für die Kupfer(I)-Ionen der Komplexe 16, 17 und 18. Die drei Komplexe unterscheiden sich nur in der Art des koordinierten Co-Liganden.

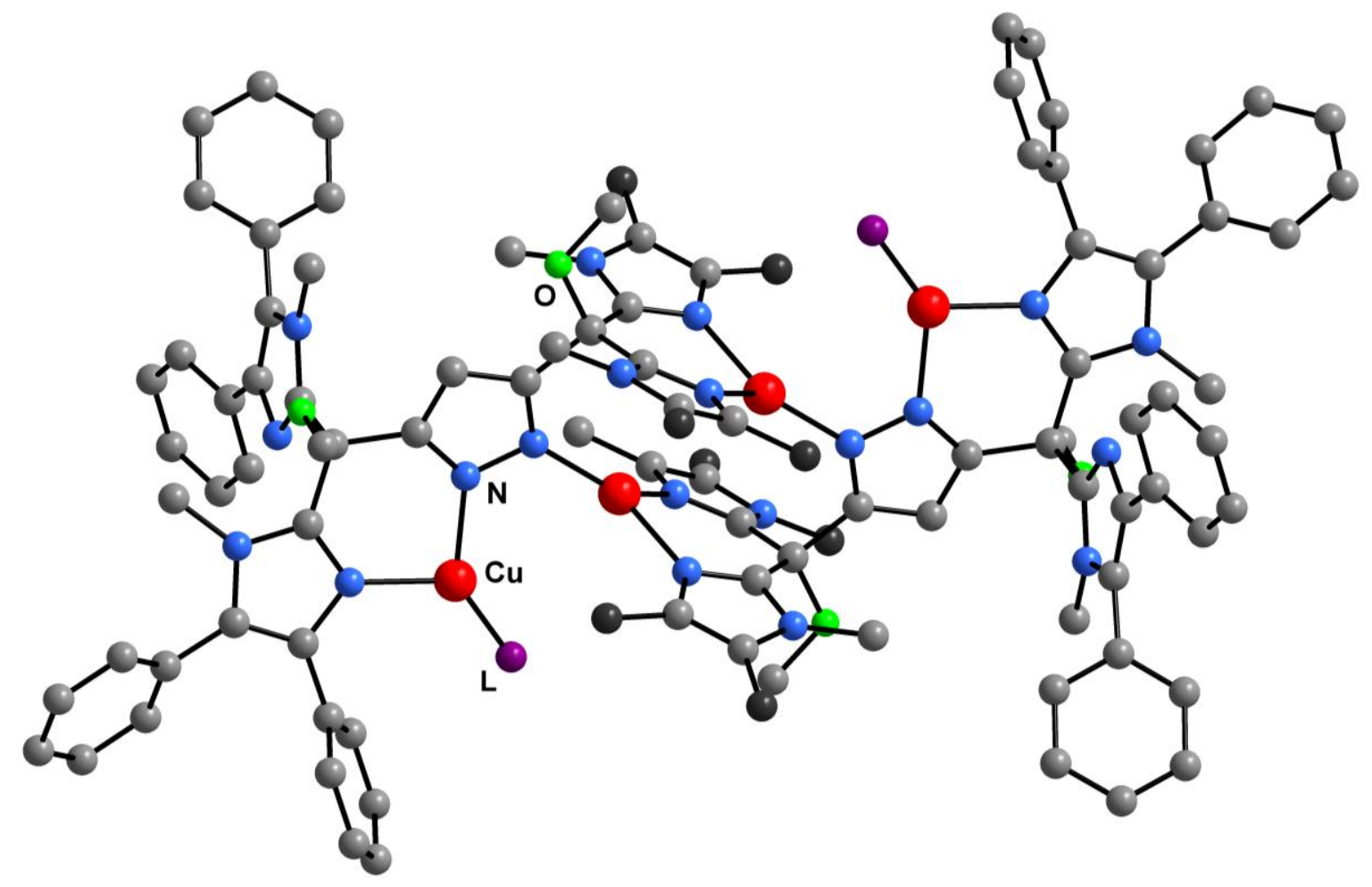

Abbildung 88: Schematische Darstellung der erhaltenen Kupfer(I)-Komplexe ( $L$ steht für CoLiganden wie $n$-Butylisonitril, 2,6-Dimethylphenylisonitril oder $\mathrm{PMe}_{3}$ ).

Der phenylsubstituierte Ligand $\mathrm{HL}^{3}$ lieferte bei der Synthese kristalliner $\mathrm{Kupfer}(\mathrm{I})$ Komplexe die besten Ergebnisse. Aufgrund der Anwesenheit von Co-Liganden sind die erhaltenen Verbindungen luftstabile Feststoffe. Deren Lösungen sind jedoch sehr 
empfindlich, so dass auf strikten Ausschluss von Sauerstoff in absoluten Lösungsmitteln geachtet werden muss.

Die hohe Reaktivität gegenüber Sauerstoff konnte auch bei den UV/Visspektroskopischen Verfolgungen der Reaktion eines Kupfer(I)-Komplexes mit Sauerstoff beobachtet werden. Es kam zur Oxidation des Kupfer(I)-Ions, was sich sehr gut anhand der sich bildenden Absorptionsbande bei etwa $580 \mathrm{~nm}$ bis $700 \mathrm{~nm}$ beobachtet werden kann. Die erfolgten Umsetzungen verlaufen wesentlich schneller als die Reaktionen mit Kohlendioxid. So zeigt ein mit $\mathrm{HL}^{3}$ synthetisierter Kupfer(I)-Komplex nach etwa 50 Minuten nahezu eine vollständige Umwandlung zur entsprechenden Kupfer(II)-Verbindung. Eine Umsetzung mit Kohlendioxid unter gleichen Reaktionsbedingungen zeigt eine beginnende Reaktion nach ca. 300 Minuten bis 350 Minuten. Weiterhin lässt sich anmerken, dass das Ende der Reaktion auch nach 17 Stunden noch nicht erreicht ist.

Als Folgeprodukt der Reaktion eines Kupfer(I)-Komplexes mit Sauerstoff konnte der vierkernige Kupfer(II)-Komplex 12 erhalten werden. Eine Aussage über die Anbindung des Sauerstoffs an den Kupfer(I)-Komplex lässt sich anhand der Spektren und des kristallisierten Folgeprodukts 12 nicht treffen. Anhand von IR-Spektren, die von den Rohprodukten nach der Umsetzung mit $\mathrm{CO}_{2}$ aufgenommen wurden, lässt sich vermuten, dass in einer Redoxreaktion beide Kupfer(I)-Ionen zu Kupfer(II)-Ionen oxidiert werden und dadurch eine Reduktion des $\mathrm{CO}_{2}$ zum Oxalat-Ion stattfindet. Ein Indiz für das Vorhandensein eines Oxalat-Ions im Komplex ist der Peaks bei $1650 \mathrm{~cm}^{-1}$. Eine nachfolgende Kristallisation der Reaktionslösung führte jedoch zur Hydroxoverbrückten Kupfer(II)-Verbindung 28. Die OH-Brücke muss durch Spuren von Wasser generiert worden sein. Das bedeutet aber auch, dass ein Austausch des möglicherweise gebundenen Oxalat-lons gegen die $\mathrm{OH}$-Einheit stattgefunden haben muss. Die Aufnahme eines IR-Spektrums von 28 beweist, dass das Signal bei $1650 \mathrm{~cm}^{-1}$ nicht aus dem $\mathrm{OH}$-verbrückten Komplex stammt.

Auch die dargestellten Nickel(II)-Komplexe zeigten interessante Eigenschaften. So unterscheiden sich die Strukturen der erhaltenen Nickel(II)-Komplexe deutlich von denen aller Kupfer-Komplexe. In den Komplexverbindungen 22 bis 25 tritt nicht das 
übliche Ligand:Metall-Verhältnis von 1:2 auf. Stattdessen findet man ein Ligand:MetallVerhältnis von 2:3 (Abbildung 89). Dabei ist das mittlere der drei Nickel(II)-lonen stets oktaedrisch von sechs Stickstoff-Donoren umgeben. Die beiden äußeren Nickel-Ionen weisen verschiedene Koordinationsgeometrien auf. So treten hierfür oktaedrische 22 oder trigonal bipyramidale Strukturen auf 23.

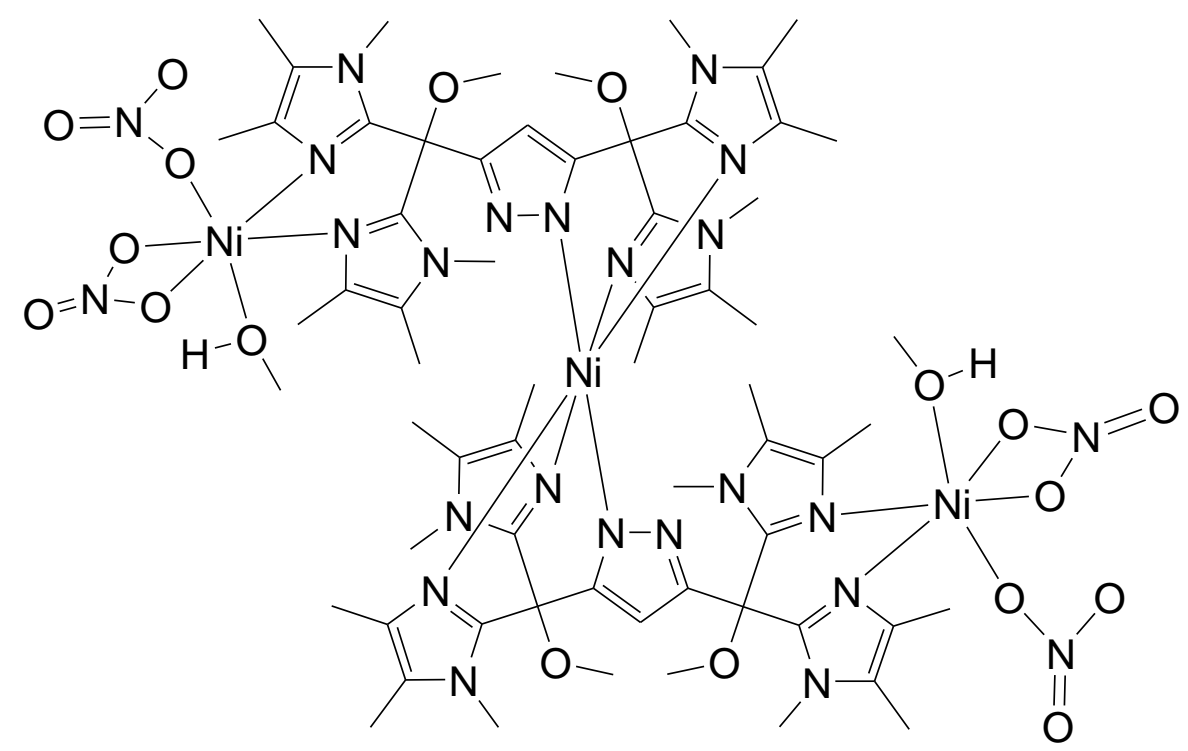

Abbildung 89: Schematische Darstellung der trinuklearen Nickel(II)-Komplexe am Beispiel von 22.

Aber auch tetraedrische Koordinationsumgebung wird in den zwei Komplexen 24 und 25 beobachtet.

Bei den Verbindungen 23, 24 und $\mathbf{2 5}$ handelt es sich formal um lonisationsisomere, die sich durch Koordination und Abdissoziieren von Methanol oder Chlorid-Ionen ineinander überführen lassen sollten. Eine Überprüfung dieses Vorgangs steht noch aus.

Der Unterschied zwischen $\mathbf{2 4}$ und $\mathbf{2 5}$ ist die Verbrückung der Nickel(II)-Ionen in $\mathbf{2 5}$. Der Pyrazolring verbrückt beide Nickel(II)-lonen und hält diese somit in einem Abstand von 4.1001(4) § zueinander. Das äußere Nickel(II)-Ion besitzt eine tetraedrische $\mathrm{N}_{3} \mathrm{Cl}$-Koordination, wobei alle drei Stickstoff-Donoren erstmals von einer Ligandseite stammen. Da das innere Nickel-lon oktaedrisch umgeben ist, kann man erstmals eine ferromagnetische Kopplung zweier Metall-lonen über einen Pyrazolring beobachten. Die Kopplungskonstante von $J=+1.76 \mathrm{~cm}^{-1}$ weist auf eine schwache ferromagnetische Kopplung hin. 


\section{Experimenteller Teil}

\subsection{Allgemeines}

\section{Warnung}

Perchloratsalze von Übergangsmetallkomplexen können explosiv sein! Obwohl während dieser Arbeit keine Probleme auftraten, sollten diese mit entsprechender Vorsicht gehandhabt werden!

\section{Apparaturen}

Es wurden konventionelle Glasapparaturen oder Schlenkkolben verwendet. Sofern nötig, wurden die Apparaturen unter Verwendung eines Heißluftföns ausgeheizt, mehrmals im Ölpumpenvakuum evakuiert und mit trockenem und sauerstofffreiem Stickstoff (Trocknung durch Sicapent der Firma Merck und Desoxygenierung durch einen CuO-Katalysator R3-11 der Firma BASF) gespült.

\section{Lösungsmittel}

Sofern notwendig wurden die Reaktionen in frisch absolutierten und destillierten Lösungsmitteln durchgeführt. Folgende Trocknungsmittel kamen zum Einsatz:

Dichlormethan

Diethylether

Ethanol

Tetrahydrofuran
Calciumhydrid

Kaliumhydroxid und anschließend Natrium/Kalium Natrium und anschließend Molsieb $3 \AA$

Kalium

\section{NMR-Spektren}

Die NMR-Spektren wurden mit Geräten des Typs Bruker Avance $500\left({ }^{1} \mathrm{H}: 500.13 \mathrm{MHz}\right.$, $\left.{ }^{13} \mathrm{C}: 125.77 \mathrm{MHz}\right), 300\left({ }^{1} \mathrm{H}: 300.13 \mathrm{MHz},{ }^{13} \mathrm{C}: 75.47 \mathrm{MHz}\right)$ und $200\left({ }^{1} \mathrm{H}: 200.13 \mathrm{MHz}\right.$, ${ }^{13} \mathrm{C}$ : $50.32 \mathrm{MHz}$ ) aufgenommen. Die angegebenen Werte der chemischen Verschiebung $\delta$ beziehen sich auf den internen Standard durch die Lösungsmittel $\mathrm{CDCl}_{3}\left({ }^{1} \mathrm{H}: \delta=7.24 \mathrm{ppm},{ }^{13} \mathrm{C}: 77.0 \mathrm{ppm}\right), \mathrm{d}_{6}$-Dimethylsulfoxid $\left({ }^{1} \mathrm{H}: \delta=2.49 \mathrm{ppm}\right.$, $\left.{ }^{13} \mathrm{C}: 39.5 \mathrm{ppm}\right)$, und $\mathrm{d}_{3}$-Acetonitril $\left({ }^{1} \mathrm{H}: \delta=1.94\right.$ ppm, $\left.{ }^{13} \mathrm{C}: 1.3 \mathrm{ppm}\right)$ relativ zu externen 
Tetramethylsilan (jeweils $\delta=0 \mathrm{ppm}$ ). Die ${ }^{13} \mathrm{C}$-NMR-Spektren wurden ${ }^{1} \mathrm{H}$-entkoppelt aufgenommen. Abkürzungen: $\mathrm{s}=$ Singulett, $\mathrm{d}=$ Dublett, $\mathrm{t}=$ Triplett, sept $=$ Septett, $\mathrm{m}=$ Multiplett, $\mathrm{br}=$ breites Singulett.

Zur Zuordnung der Signale wurden, wenn nötig, zusätzlich DEPT und H-C-COSY Experimente durchgeführt.

\section{Massenspektrometrie}

Die Massenspektren wurden mit den Geräten Finnigan MAT 8200 und Finnigan MAT 95 aufgenommen. lonisierungsmethode für die El-Massenspektren war Ionenstoß-Ionisation bei $70 \mathrm{eV}$. Bei Fast-Atom-Bombardement-(FAB)-Experimenten wurde die zu messende Substanz in eine Matrix aus 3-Nitrobenzylalkohol (3-NBA) eingelagert und durch Beschuss mit Cäsium-Kationen ionisiert. Für Elektronen-Spraylonisations-(ESI)-Messungen wurden die Proben im jeweils angegebenen Lösungsmittel gelöst und anschließend mit einem Finnigan MAT LCQ ESI-Spektren oder Applied Biosystems API 2000 aufgenommen. Hochaufgelöste ESIMassenspektren wurden ebenfalls mit einem Finnigan MAT LCQ gemessen. Isotopensimulationen wurden mit dem Programm Molecular Weight Calculator durchgeführt.

\section{Elementaranalyse}

Die CHN-Elementaranalysen erfolgten durch das Mikroanalytische Labor des Institutes für Anorganische Chemie der Universität Göttingen mit einem CHN-O-Rapid der Firma Elementar.

\section{Infrarotspektroskopie}

Die Infrarotspektren wurden auf einem Digilab Excalibur Spektrometer aufgenommen. Feststoffe wurden als KBr-Presslinge gemessen. Die Lage der Banden ist in Wellenzahlen angegeben $\left(\tilde{v}\right.$ in $\left.\mathrm{cm}^{-1}\right)$. Die Intensitäten sind wie folgt abgekürzt: $\mathrm{w}=$ schwach, $\mathrm{m}=$ mittel, $\mathrm{s}=$ stark, $\mathrm{vs}=$ sehr stark, $\mathrm{br}=$ breit .

\section{UV-Vis-Spektroskopie}

Die UV/Vis-Spektren wurden an einem Varian Cary-5000 Spektrometer in Küvetten aus Quarzglas mit einer Schichtdicke von $1 \mathrm{~cm}$ aufgenommen. 
Die UV/Vis-spektrophotometrischen Reaktionsverfolgungen sind an einem Varian Cary Bio 50 mittels einer Ganz-Quarz-Tauchsonde von Hellma, die über ein Lichtleiter-Kabel an das Spektrometer angeschlossen ist, durchgeführt worden.

\section{Schmelz- und Zersetzungspunkte}

Die Schmelz- und Zersetzungspunkte wurden an einem OptiMelt MPA 100 der Firma Stanford Research Systems bestimmt. Hierfür wurden die Substanzen in Schmelzpunktbestimmungsröhrchen gefüllt und mit einer Heizrate von $5 \mathrm{~K} \cdot \mathrm{min}^{-1}$ erwärmt. Die Bestimmung der Schmelz- und Zersetzungstemperaturen erfolgte mittels der zugehörigen Software.

\section{Inertgasarbeiten}

Alle Arbeiten zur Herstellung von Kupfer(I)-Komplexen wurden in einer Glovebox der Firma MBRAUN mit angeschlossenem Solvent Purification System (SPS) durchgeführt. So konnte in einer Stickstoffatmosphäre mit $<0.1 \mathrm{ppm}$ Wasser und $<0.1 \mathrm{ppm}$ Sauerstoff gearbeitet werden.

\section{Röntgenstrukturanalysen}

Die Bestimmung der Elementarzellen und die Sammlung der Messdaten erfolgte an einem STOE/IPDS II - Gerät mit graphitmonochromatisierter Mo-K $\mathrm{K}_{\alpha}$-Strahlung $(\lambda=0.711 \AA$ ). Die Messwerte wurden bezüglich Lorentz- und Polarisationseffekten korrigiert. Zusätzlich erfolgten Absorptionskorrekturen. Lösung und Verfeinerung wurden mit den Programmen SHELXS-97 $7^{[179]}$ und SHELXL-97 ${ }^{[180]}$ durchgeführt.

\section{Magnetochemie}

Die Suszeptibilitäts-Messungen erfolgten an einem Quantum-Design-MPMS-5SSQUID-Magnetometer ausgestattet mit einem 5 Tesla Magnet im Bereich von $2 \mathrm{~K}$ bis $295 \mathrm{~K}$. Die Proben wurden in Gelatinekapseln präpariert und in einem nicht magnetischen Probenhalter für die Messung fixiert. Für die diamagnetischen Korrekturen wurden nach Korrektur des Probenhalters (incl. Gelatinekapsel) die aus den Inkrementen ermittelten Werte verwendet. ${ }^{[162,163]}$ Die Anpassung der Messkurven erfolgte mit dem Programm julX. ${ }^{[164]}$ 


\section{Reagentien}

Die ständig im Labor benötigten Chemikalien waren bei der Chemikalienausgabe des Anorganisch-chemischen Institutes der Universität Göttingen erhältlich. Folgende Reagentien wurden über die angegebenen Firmen bezogen:

Benzoin

$n$-Butyllitium in $n$-Hexan (1.6 M)

$n$-Butylisonitril

2,6-Dimethylphenylisonitril

Formamid

Kalium-tert-butylat

Kupferacetat Hydrat

Kupfernitrat Trihydrat

Kupferperchlorat Hexahydrat

Kupfersulfat Pentahydrat

Kupfertetrafluoroborat Tetrahydrat

Natriumhydrid

Nickelchlorid Hexahydrat

Nickelnitrat Hexahydrat

$N$-Methylimidazol

Methyliodid

Trimethylphosphin in THF (1.0 M)
Aldrich

Fluka

Acros

Fluka

Acros

Aldrich

Merck

Merck

Aldrich

Merck

Aldrich

Aldrich

Acros

Acros

Aldrich

Aldrich

Aldrich

Cyclooctadienyl-kupfer(I)-chlorid ${ }^{[181,182]}$

4,5-Diphenyl-1-methylimidazol ${ }^{[114]}$

Dimethyl-1-(tetrahydropyran-2-yl)-pyrazol-3,5-dicarboxylat ${ }^{[112]}$

Tetrakis(acetonitril)kupfer(I)perchlorat, Tetrakis(acetonitril)kupfer(I)hexafluorophoshat, Tetrakis(acetonitril)kupfer(I)tetrafluoroborat und Tetrakis(acetonitril)kupfer(I)triflat ${ }^{[183]}$ Tris(triphenylphoshan)kupfer(I)-phenolat ${ }^{[184]}$

1,4,5-Trimethylimidazol ${ }^{[113]}$ 


\subsection{Darstellung der Liganden}

\subsubsection{Ligandsynthese $\mathrm{HL}^{1}$}

10.2.1.1. 3,5-Bis-[bis-(1-methylimidazol-2-yl)-hydroxymethyl]-1-(tetrahydropyran-2-yl)pyrazol (2a)<smiles>COC(=O)c1cc(C(=O)OC)n(P)n1</smiles>

1

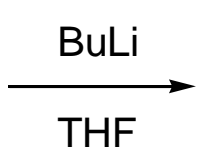

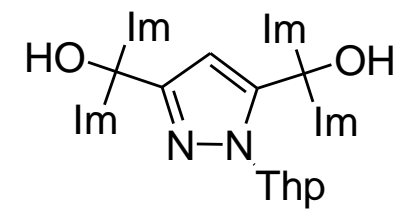

$2 a$

Bei Raumtemperatur löst man $8.0 \mathrm{ml}(8.21 \mathrm{~g}, 100.0 \mathrm{mmol}) \mathrm{N}$-Methylimidazol in $450 \mathrm{ml}$ absolutem THF unter $\mathrm{N}_{2}$-Schutzgas. Anschließend werden vorsichtig $69.0 \mathrm{ml}$ (110.0 mmol) einer 1.6 M Lösung von $n$-BuLi in $n$-Hexan unter Rühren zugetropft. Nachdem man die Reaktionslösung zwei weitere Stunden rühren ließ, gibt man eine Lösung von $6.70 \mathrm{~g}$ (25.0 mmol) 1-(Tetrahydropyran-2-yl)-pyrazol-3,5-dicarbonsäuremethylester (1) in $200 \mathrm{ml}$ absolutem THF zu. Die Reaktionsmischung wird weitere 16 Stunden bei Raumtemperatur gerührt. Im Anschluss gibt man zum Quenchen der Reaktion $100 \mathrm{ml}$ gesättigte $\mathrm{NH}_{4} \mathrm{Cl}$-Lösung dazu, wobei sich die Lösung von graugelb nach orangegelb verfärbt. Im Vakuum wird die Lösung zur Trockne eingeengt und der Rückstand in $200 \mathrm{ml}$ ammoniakalischer Dichlormethanlösung aufgenommen. Danach wird die organische Phase über $\mathrm{MgSO}_{4}$ getrocknet und das Lösungsmittel im Vakuum entfernt. Abschließend wird das Rohprodukt im Hochvakuum getrocknet und man erhält 2a als weißen Feststoff.

Summenformel

Molgewicht

Ausbeute

${ }^{1} \mathrm{H}-\mathrm{NMR}\left(\mathrm{CDCl}_{3}\right)$
$\mathrm{C}_{26} \mathrm{H}_{32} \mathrm{~N}_{10} \mathrm{O}_{3}$

$532.61 \mathrm{~g}^{\prime} \mathrm{mol}^{-1}$

$7.17 \mathrm{~g} \mathrm{(14} \mathrm{mmol,} 54 \%)$.

6.70-6.85 (m, 8 H, H $\left.\mathrm{H}^{\mathrm{Im} 4}, \mathrm{H}^{\mathrm{Im} 5}\right), 5.79\left(\mathrm{~s}, 1 \mathrm{H}, \mathrm{H}^{\mathrm{Pz} 4}\right), 5.35(\mathrm{dd}$, $\left.1 \mathrm{H},{ }^{3} \mathrm{~J}=9.6 \mathrm{~Hz},{ }^{3} \mathrm{~J}=1.8 \mathrm{~Hz}, \mathrm{H}^{\mathrm{Thp}}\right), 3.55-3.65(\mathrm{~m}, 1 \mathrm{H}$, $\mathrm{H}^{\text {Thp3 }}$ ), $3.54\left(\mathrm{~s}, 3 \mathrm{H}, \mathrm{NCH}_{3}\right), 3.37\left(\mathrm{~s}, 3 \mathrm{H}, \mathrm{NCH}_{3}\right), 3.35(\mathrm{~s}, 3 \mathrm{H}$, $\left.\mathrm{NCH}_{3}\right), 3.27\left(\mathrm{~s}, 3 \mathrm{H}, \mathrm{NCH}_{3}\right), 3.00-3.20\left(\mathrm{~m}, 1 \mathrm{H}, \mathrm{H}^{\text {Thp3}}\right)$, 


\begin{tabular}{|c|c|}
\hline & $\begin{array}{l}\left.\text { 2.05-2.20 (m, } 1 \mathrm{H}, \mathrm{H}^{\mathrm{Thp} 6}\right), 1.70-1.90\left(\mathrm{~m}, 2 \mathrm{H}, \mathrm{H}^{\text {Thp5 }}, \mathrm{H}^{\text {Thp6 }}\right) \text {, } \\
1.35-1.50\left(\mathrm{~m}, 2 \mathrm{H}, \mathrm{H}^{\mathrm{Thp} 4}, \mathrm{H}^{\mathrm{Thp5}}\right), 1.25-1.35\left(\mathrm{~m}, 1 \mathrm{H}, \mathrm{H}^{\text {Thp4 }}\right) .\end{array}$ \\
\hline${ }^{13} \mathrm{C}-\mathrm{NMR}\left(\mathrm{CDCl}_{3}\right)$ & 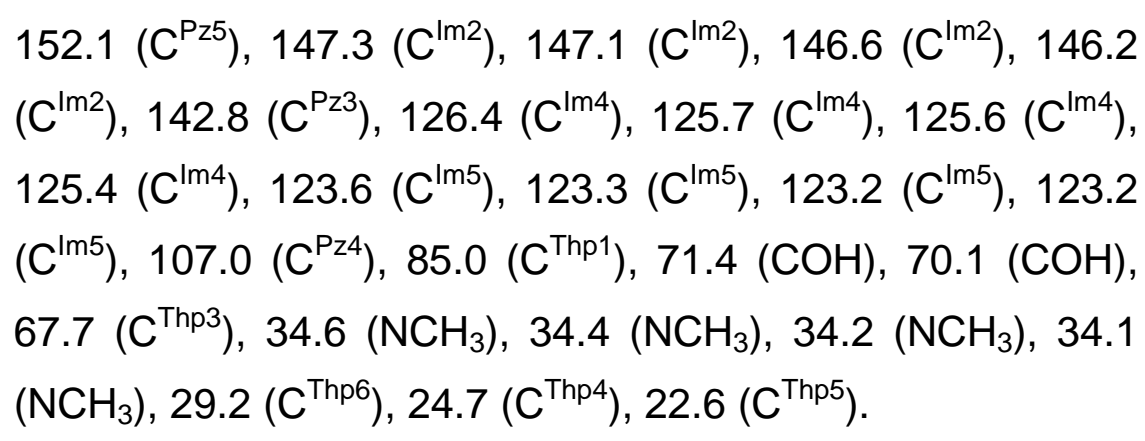 \\
\hline $\mathrm{MS}\left(\mathrm{FAB}^{+}\right) m / z(\%)$ & $\begin{array}{l}533\left(52,[\mathrm{M}+\mathrm{H}]^{+}\right), 451\left(7,[\mathrm{M}+\mathrm{H}-\mathrm{Im}]^{+}\right), 449\left(10,[\mathrm{M}+\mathrm{H}-\mathrm{Thp}]^{+}\right), \\
367\left(18,[\mathrm{M}+\mathrm{H}-\mathrm{Thp}-\mathrm{Im}]^{+}\right), 285\left(100[\mathrm{M}+\mathrm{H}-\mathrm{Thp}-2 \mathrm{Im}]^{+}\right), 83(14, \\
\left.[\mathrm{Im}+\mathrm{H}]^{+}\right) .\end{array}$ \\
\hline $\mathrm{IR}(\mathrm{KBr}) \tilde{v}\left(\mathrm{~cm}^{-1}\right)$ & $\begin{array}{l}3350 \text { (br), } 3109 \text { (s), } 2949 \text { (s), } 2858 \text { (s), } 2735 \text { (br), } 1641 \\
(\mathrm{~m}), 1525 \text { (m), } 1488 \text { (vs), } 1469 \text { (s), } 1404 \text { (s), } 1350 \text { (s), } 1320 \\
(\mathrm{~m}), 1282 \text { (vs), } 1251 \text { (m), } 1205 \text { (s), } 1131 \text { (s), } 1083 \text { (s), } 1041 \\
\text { (s), } 1007 \text { (s), } 946 \text { (vs), } 896 \text { (s), } 875 \text { (s), } 820 \text { (m), } 736 \text { (br), } \\
553 \text { (w), } 461(w) .\end{array}$ \\
\hline Schmelzpunkt & $130^{\circ} \mathrm{C}$ (Zersetzung). \\
\hline Elementaranalyse & $\begin{array}{l}\text { für } 2 \mathrm{a} \cdot\left(2 \mathrm{THF}+0.5 \mathrm{H}_{2} \mathrm{O}\right) \text { : } \\
\text { ber.: C 59.54, H 7.20, N 20.42, } \\
\text { gef.: C 59.63, H 7.09, N 20.43. }\end{array}$ \\
\hline
\end{tabular}

10.2.1.2. 3,5-Bis-[bis-(1-methylimidazol-2-yl)-methoxymethyl]-1-(tetrahydropyran-2-yl)pyrazol (3a)<smiles>CCC(O)(c1cc(C(O)([AlH2])C(C)C)n([PbH2])n1)C(C)(C)C</smiles>

$2 a$

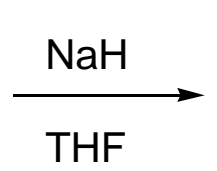

THF

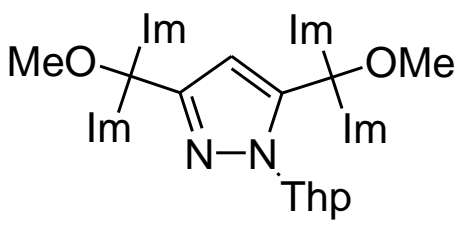

$3 a$

$\mathrm{Zu}$ einer Suspension von $0.37 \mathrm{~g}(15.3 \mathrm{mmol}) \mathrm{NaH}$ in $200 \mathrm{ml}$ absolutem THF gibt man bei Raumtemperatur und unter $\mathrm{N}_{2}$-Schutzgas $3.69 \mathrm{~g}(6.9 \mathrm{mmol}) \mathbf{2 a}$, gelöst in $100 \mathrm{ml}$ absolutem THF, und lässt zwei Stunden rühren. Danach werden $0.95 \mathrm{ml}(15.3 \mathrm{mmol})$ 
Methyliodid zugesetzt und über Nacht gerührt. Zum Quenchen der Reaktion gibt man $50 \mathrm{ml}$ einer halbgesättigten $\mathrm{NaCl}$-Lösung. Im Vakuum werden alle leichtflüchtigen Bestandteile entfernt. Die resultierende wässrige Phase wird mit $\mathrm{CHCl}_{3}(5 \times 50 \mathrm{ml})$ extrahiert und die vereinigten organischen Phasen über $\mathrm{MgSO}_{4}$ getrocknet. Nach Entfernen des Lösungsmittels im Vakuum erhält man einen weißen Feststoff von $\mathbf{3 a}$.

Summenformel

Molgewicht

Ausbeute

${ }^{1} \mathrm{H}-\mathrm{NMR}\left(\mathrm{CDCl}_{3}\right)$

${ }^{13} \mathrm{C}-\mathrm{NMR}\left(\mathrm{CDCl}_{3}\right)$

MS $\left(\mathrm{FAB}^{+}\right) \mathrm{m} / \mathrm{z}(\%)$

$\operatorname{IR}(\mathrm{KBr}) \tilde{v}\left(\mathrm{~cm}^{-1}\right)$

Schmelzpunkt
$\mathrm{C}_{28} \mathrm{H}_{36} \mathrm{~N}_{10} \mathrm{O}_{3}$

$560.66 \mathrm{~g} \mathrm{~mol}^{-1}$

$3.32 \mathrm{~g}(5.9 \mathrm{mmol}, 86 \%)$.

6.80-7.00 (m, $\left.8 \mathrm{H}, \mathrm{H}^{\mathrm{Im} 4}, \mathrm{H}^{\mathrm{Im} 5}\right), 6.44\left(\mathrm{~s}, 1 \mathrm{H}, \mathrm{H}^{\mathrm{Pz} 4}\right), 5.49$ (dd, $\left.1 \mathrm{H},{ }^{3} \mathrm{~J}=8.1 \mathrm{~Hz},{ }^{3} \mathrm{~J}=1.8 \mathrm{~Hz}, \mathrm{H}^{\mathrm{Thp} 2}\right), 3.60-3.70(\mathrm{~m}, 1 \mathrm{H}$, $\mathrm{H}^{\text {Thp6 }}$ ), 3.51 (s, $3 \mathrm{H}, \mathrm{NCH}_{3}$ ), $3.45\left(\mathrm{~s}, 6 \mathrm{H}, \mathrm{NCH}_{3}\right), 3.39(\mathrm{~s}, 3 \mathrm{H}$, $\left.\mathrm{NCH}_{3}\right), 3.36\left(\mathrm{~s}, 3 \mathrm{H}, \mathrm{OCH}_{3}\right), 3.32\left(\mathrm{~s}, 3 \mathrm{H}, \mathrm{OCH}_{3}\right), \quad 3.25-3.35$ $\left(\mathrm{m}, 1 \mathrm{H}, \mathrm{H}^{\text {Thp } 6}\right), 2.10-2.20\left(\mathrm{~m}, 1 \mathrm{H}, \mathrm{H}^{\text {Thp3 }}\right), 1.75-1.85(\mathrm{~m}, 1 \mathrm{H}$, $\left.\mathrm{H}^{\text {Thp4 }}\right), 1.60-1.70\left(\mathrm{~m}, 1 \mathrm{H}, \mathrm{H}^{\text {Thp3 }}\right), 1.40-1.50\left(\mathrm{~m}, 2 \mathrm{H}, \mathrm{H}^{\text {Thp4 }}\right.$, $\left.\mathrm{H}^{\text {Thp5 }}\right)$, 1.25- $1.35\left(\mathrm{~m}, 1 \mathrm{H}, \mathrm{H}^{\text {Thp5 }}\right)$.

$149.5\left(C^{\text {Pz5 }}\right) 145.8\left(C^{\operatorname{lm} 2}\right), 145.64\left(C^{\operatorname{lm} 2}\right), 144.2\left(C^{\mathrm{lm} 2}\right), 144.1$ $\left(C^{\mathrm{Im} 2}\right), 140.0\left(C^{\mathrm{Pz} 3}\right), 126.8\left(C^{\mathrm{Im} 4}\right), 126.7\left(\mathrm{C}^{\mathrm{Im} 4}\right), 126.6\left(\mathrm{C}^{\mathrm{Im} 4}\right)$, $126.5\left(C^{\operatorname{lm} 4}\right), 123.8\left(C^{\operatorname{lm} 5}\right), 123.8\left(C^{\operatorname{lm} 5}\right), 123.2\left(C^{\operatorname{lm} 5}\right), 123.1$ $\left(\mathrm{C}^{\mathrm{Im} 5}\right), \quad 108.8 \quad\left(\mathrm{C}^{\mathrm{Pz} 4}\right), \quad 85.3 \quad\left(\mathrm{C}^{\mathrm{Thp} 2}\right), 78.5\left(\mathrm{COCH}_{3}\right), \quad 77.2$ $\left(\mathrm{COCH}_{3}\right), 67.4\left(\mathrm{C}^{\text {Thp } 6}\right), 54.2\left(\mathrm{OCH}_{3}\right), 53.8\left(\mathrm{OCH}_{3}\right), 34.7$ $\left(\mathrm{NCH}_{3}\right), 34.7\left(\mathrm{NCH}_{3}\right), 34.6\left(\mathrm{NCH}_{3}\right), 34.5\left(\mathrm{NCH}_{3}\right), 29.6$ $\left(\mathrm{C}^{\text {Thp3 }}\right), 24.9\left(\mathrm{C}^{\text {Thp5 }}\right), 22.9\left(\mathrm{C}^{\text {Thp4 }}\right)$.

$583\left(40,[\mathrm{M}+\mathrm{Na}]^{+}\right), 561\left(100,[\mathrm{M}+\mathrm{H}]^{+}\right), 547\left(22,\left[\mathrm{M}+\mathrm{H}-\mathrm{CH}_{3}\right]^{+}\right)$, $477\left(40,[\mathrm{M}+\mathrm{H}-\mathrm{Thp}]^{+}\right), 349\left(26,\left[\mathrm{M}+\mathrm{H}-\mathrm{Thp}-\mathrm{Im}-\mathrm{OCH}_{3}-\mathrm{CH}_{3}\right]^{+}\right)$. 3393 (br), 3107 (s), 2943 (vs), 2858 (s), 2576 (w), $2486(w)$, $2187(w), 2031(w), 1905(w), 1677(m), 1638(m), 1522(m)$, $1485(\mathrm{~s}), 1403(\mathrm{~s}), 1354(\mathrm{~m}), 1319(\mathrm{~m}), 1282(\mathrm{~s}), 1251(\mathrm{~m})$, $1206(\mathrm{~s}), 1181(\mathrm{~m}), 1132(\mathrm{~s}), 1082(\mathrm{br}), 1031(\mathrm{~s}), 1007(\mathrm{~s})$, 969 (s), $940(\mathrm{~m}), 907$ (s), 873 (s), $821(\mathrm{~m}), 747$ (br), $661(\mathrm{~m})$, $593(w), 551(w), 460(w)$.

$121^{\circ} \mathrm{C}$ (Zersetzung). 
Elementaranalyse

für $3 a \cdot 1.3 \mathrm{CH}_{2} \mathrm{Cl}_{2}$ :

ber.: C 52.44, H 5.80, N 20.87,

gef.: C 52.57, H 6.00, N 20.68.

10.2.1.3. 3,5-Bis-[bis-(1-methylimidazol-2-yl)-methoxymethyl]-pyrazol

Hydrochlorid (4a)<smiles>COC([InH])(c1ccccc1)c1cc(C(C)(C)C(C)(C)C)nn1P</smiles>

$3 a$

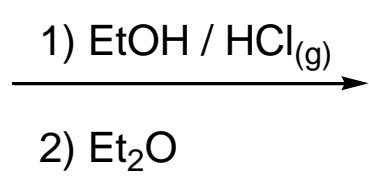

2) $\mathrm{Et}_{2} \mathrm{O}$<smiles>COC(c1ccccc1)(c1ccccc1)c1cc(C([AlH2])(c2ccccc2)c2ccccc2)n[nH]1</smiles>

$4 a$

$14.66 \mathrm{~g}(26.2 \mathrm{mmol})$ von $3 \mathrm{a}$ werden in $100 \mathrm{ml}$ ethanolischer $\mathrm{HCl}$ aufgenommen und die Reaktionslösung über Nacht gerührt. Für das Ausfällen des Hydrochlorides gibt man $400 \mathrm{ml}$ Diethylether dazu. Der helle Niederschlag wird durch eine Fritte abgesaugt, mit $\mathrm{Et}_{2} \mathrm{O}$ gewaschen und im Hochvakuum getrocknet. Man erhält 4a als weißen Feststoff.

\begin{tabular}{|c|c|}
\hline Summenformel & $\mathrm{C}_{23} \mathrm{H}_{29} \mathrm{~N}_{10} \mathrm{O}_{2} \mathrm{Cl}$ \\
\hline Molgewicht & $513.01 \mathrm{~g}^{\cdot} \mathrm{mol}^{-1}$ \\
\hline Ausbeute & $14.02 \mathrm{~g}(25.5 \mathrm{mmol}, 98 \%)$ \\
\hline 1H-NMR (DMSO) & $\begin{array}{l}7.73\left(\mathrm{~d}, 4 \mathrm{H},{ }^{3} \mathrm{~J}=1.6 \mathrm{~Hz}, \mathrm{H}^{\mathrm{Im} 4}\right), 7.48\left(\mathrm{~d}, 4 \mathrm{H},{ }^{3} \mathrm{~J}=1.6 \mathrm{~Hz},\right. \\
\left.\mathrm{H}^{\mathrm{Im} 5}\right), 6.89\left(\mathrm{~s}, 1 \mathrm{H}, \mathrm{H}^{\mathrm{Pz} 4}\right), 3.53\left(\mathrm{~s}, 12 \mathrm{H}, \mathrm{NCH}_{3}\right), 3.23(\mathrm{~s}, 6 \mathrm{H} \text {, } \\
\left.\mathrm{OCH}_{3}\right) .\end{array}$ \\
\hline${ }^{13} \mathrm{C}-\mathrm{NMR}$ (DMSO) & $\begin{array}{l}140.1\left(\mathrm{C}^{\mathrm{Im} 2}\right), 126.5\left(\mathrm{C}^{\mathrm{Im} 4}\right), 121.7\left(\mathrm{C}^{\mathrm{Im} 4}\right), 108.7\left(\mathrm{C}^{\mathrm{Pz} 4}\right), 74.8 \\
\left(\mathrm{COCH}_{3}\right), 53.3\left(\mathrm{OCH}_{3}\right), 35.3\left(\mathrm{NCH}_{3}\right) .\end{array}$ \\
\hline $\mathrm{MS}\left(\mathrm{FAB}^{+}\right) \mathrm{m} / z(\%)$ & $\begin{array}{l}477 \quad\left(100, \quad[\mathrm{M}-\mathrm{Cl}]^{+}\right), \quad 463 \quad\left(16, \quad\left[\mathrm{M}-\mathrm{Cl}-\mathrm{CH}_{3}\right]^{+}\right), \quad 413 \quad(7, \\
\left.\left[\mathrm{M}-\mathrm{Cl}-2 \mathrm{OCH}_{3}\right]^{+}\right), 349\left(9,\left[\mathrm{M}-\mathrm{Cl}-\mathrm{CH}_{3}-\mathrm{Im}\right]^{+}\right) .\end{array}$ \\
\hline $\mathrm{IR}(\mathrm{KBr}) \tilde{v}\left(\mathrm{~cm}^{-1}\right)$ & $\begin{array}{l}3400(\mathrm{br}), 3322(\mathrm{br}), 3118(\mathrm{~s}), 3083(\mathrm{~s}), 2928(\mathrm{~s}), 2836(\mathrm{br}), \\
2734(\mathrm{br}), 1758(\mathrm{w}), 1648(\mathrm{~m}), 1590(\mathrm{~s}), 1525(\mathrm{~s}), 1454(\mathrm{~m}), \\
1382(\mathrm{~s}), 1321(\mathrm{~s}), 1294(\mathrm{~s}), 1279(\mathrm{~m}), 1205(\mathrm{~m}), 1130(\mathrm{~m}), \\
1087(\mathrm{vs}), 1038(\mathrm{~m}), 966(\mathrm{~m}), 941(\mathrm{~m}), 893(\mathrm{~m}), 855(\mathrm{~m}), 774 \\
(\mathrm{~s}), 714(\mathrm{~m}), 677(\mathrm{~m}), 622(\mathrm{w}), 567(\mathrm{~m}), 532(\mathrm{~m}) .\end{array}$ \\
\hline
\end{tabular}


Schmelzpunkt

Elementaranalyse $163^{\circ} \mathrm{C}$ (Zersetzung).

für $4 \mathrm{a} \cdot 4 \mathrm{HCl}$ :

ber.: C 41.93, H 5.05, N 21.26,

gef.: C 41.41, H 5.31, N 20.72.

10.2.1.4. 3,5-Bis-[bis-(1-methylimidazol-2-yl)-methoxymethyl]-pyrazol (5a)

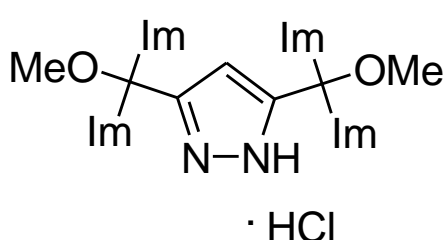

$4 a$

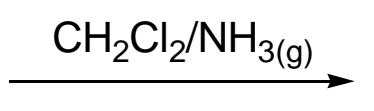

$5 a$

$14.02 \mathrm{~g} \quad(25.5 \mathrm{mmol})$ des Hydrochlorides $4 \mathbf{4 a}$ suspendiert man in $100 \mathrm{ml}$ ammoniakalischer Dichlormethanlösung und lässt die Lösung über Nacht rühren. Der feine Niederschlag wird abfiltriert und das Lösungsmittel über $\mathrm{MgSO}_{4}$ getrocknet. Danach werden alle flüchtigen Bestandteile im Vakuum entfernt und das Rohprodukt wird durch zehnstündige Soxhlet-Extraktion mit $1000 \mathrm{ml}$ Diethylether gereinigt. Man erhält einen weißen Feststoff von $\mathbf{5 a}$.

\begin{tabular}{|c|c|}
\hline Summenformel & $\mathrm{C}_{23} \mathrm{H}_{28} \mathrm{~N}_{10} \mathrm{O}_{2}$ \\
\hline Molgewicht & $476.55 \mathrm{~g}^{\cdot} \mathrm{mol}^{-1}$ \\
\hline Ausbeute & $5.92 \mathrm{~g}(12.3 \mathrm{mmol}, 48 \%)$ \\
\hline${ }^{1} \mathrm{H}-\mathrm{NMR}\left(\mathrm{CDCl}_{3}\right)$ & $\begin{array}{l}6.89\left(\mathrm{~d}, 4 \mathrm{H},{ }^{3} \mathrm{~J}=1.2 \mathrm{~Hz}, \mathrm{H}^{\mathrm{Im} 4}\right), 6.77\left(\mathrm{~d}, 4 \mathrm{H},{ }^{3} \mathrm{~J}=1.2 \mathrm{~Hz},\right. \\
\left.\mathrm{H}^{\mathrm{Im} 5}\right), 6.29\left(\mathrm{~s}, 1 \mathrm{H}, \mathrm{H}^{\mathrm{Pz} 4}\right), 3.35\left(\mathrm{~s}, 12 \mathrm{H}, \mathrm{NCH}_{3}\right), 3.15(\mathrm{~s}, 6 \mathrm{H} \text {, } \\
\left.\mathrm{OCH}_{3}\right) .\end{array}$ \\
\hline${ }^{13} \mathrm{C}-\mathrm{NMR}\left(\mathrm{CDCl}_{3}\right)$ & $\begin{array}{l}145.0\left(\mathrm{C}^{\mathrm{Im} 2}\right), 126.3\left(\mathrm{C}^{\mathrm{Im} 4}\right), 123.0\left(\mathrm{C}^{\mathrm{Im} 5}\right), 106.3\left(\mathrm{C}^{\mathrm{Pz} 4}\right), 52.4 \\
\left(\mathrm{OCH}_{3}\right), 33.9\left(\mathrm{NCH}_{3}\right) .\end{array}$ \\
\hline MS (EI) m/z (\%) & $\begin{array}{l}476\left(5,[\mathrm{M}]^{+}\right), 461\left(38,\left[\mathrm{M}-\mathrm{CH}_{3}\right]^{+}\right), 446\left(48,\left[\mathrm{M}-2 \mathrm{CH}_{3}\right]^{+}\right), 429 \\
\left(32,\left[\mathrm{M}^{-} \mathrm{CH}_{3}-\mathrm{OCH}_{3}\right]^{+}\right), 413 \quad\left(5, \quad[\mathrm{M}-2 \mathrm{OCH}]^{+}\right), \quad 365 \quad(6, \\
\left.\left[\mathrm{M}-\mathrm{OCH}_{3}-\mathrm{Im}\right]^{+}\right), 83\left(100,[\mathrm{Im}+\mathrm{H}]^{+}\right) .\end{array}$ \\
\hline $\mathrm{IR}(\mathrm{KBr}) \tilde{v}\left(\mathrm{~cm}^{-1}\right)$ & $\begin{array}{l}3104(\mathrm{~s}), 2950(\mathrm{~s}), 2827(\mathrm{~s}), 1636(\mathrm{w}), 1522(\mathrm{~m}), 1486(\mathrm{vs}), \\
1403(\mathrm{~s}), 1347(\mathrm{~m}), 1281(\mathrm{vs}), 1229(\mathrm{~m}), 1186(\mathrm{~m}), 1128(\mathrm{~s}),\end{array}$ \\
\hline
\end{tabular}


1074 (vs), 1013 (m), 970 (s), 907 (m), 892 (s), 819 (w), 752 (m), $729(\mathrm{~m}), 685(\mathrm{~m}), 558(\mathrm{w}), 464(\mathrm{w})$.

Schmelzpunkt

$167^{\circ} \mathrm{C}$ (Zersetzung).

Elementaranalyse

für 5a:

ber.: C 57.97, H 5.92, N 29.39,

gef.: C 57.44, H 6.18, N 28.70. 


\subsubsection{Ligandsynthese $\mathrm{HL}^{2}$}

10.2.2.1. 3,5-Bis-[bis-(1,4,5-trimethylimidazol-2-yl)-hydroxymethyl]-1-(tetrahydropyran2-yl)-pyrazol (2b)<smiles>COC(=O)c1cc(C(=O)OC)n(Pc2ccccc2)n1</smiles>

1<smiles>Cc1ncn(C)c1C</smiles>

THF

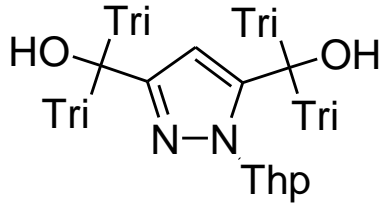

$2 b$

Bei Raumtemperatur löst man $25.92 \mathrm{~g} \quad$ (235.3 mmol) frisch sublimiertes 1,4,5-Trimethylimidazol in $400 \mathrm{ml}$ absolutem THF unter $\mathrm{N}_{2}$-Schutzgas. Anschließend werden vorsichtig $169 \mathrm{ml}(270.6 \mathrm{mmol})$ einer 1.6 M Lösung von $n$-BuLi in $n$-Hexan unter Rühren zugetropft. Nachdem die gelborange gefärbte Reaktionslösung eine weitere Stunde gerührt wurde, fügt man eine Lösung von $15.78 \mathrm{~g}$ (58.8 mmol) 1-Tetrahydropyran-2-yl-pyrazol-3,5-dicarbonsäuremethylester (1) in $250 \mathrm{ml}$ absolutem THF hinzu. Die rot gefärbte Suspension wird über Nacht bei Raumtemperatur gerührt. Im Anschluss gibt man zum Quenchen der Reaktion $80 \mathrm{ml}$ einer gesättigten $\mathrm{NH}_{4} \mathrm{Cl}$ Lösung dazu, wobei sich eine gelbe Lösung mit weißem Niederschlag bildet. Im Vakuum wird die Lösung bis zur Trockene eingeengt und der gelbe Rückstand in $400 \mathrm{ml}$ ammoniakalischer Dichlormethanlösung aufgenommen. Die Lösung wird filtriert, über $\mathrm{MgSO}_{4}$ getrocknet und nach Entfernen des Lösungsmittels sowie Trocknen im Hochvakuum erhält man $\mathbf{2 b}$ als gelben Feststoff.

Summenformel

Molgewicht

Ausbeute

${ }^{1} \mathrm{H}-\mathrm{NMR}\left(\mathrm{CDCl}_{3}\right)$
$\mathrm{C}_{34} \mathrm{H}_{48} \mathrm{~N}_{10} \mathrm{O}_{3}$

$644.83 \mathrm{~g} \cdot \mathrm{mol}^{-1}$

$37.37 \mathrm{~g}(57.95 \mathrm{mmol}, 98 \%)$.

6.40 (br, $1 \mathrm{H}, \mathrm{OH}), 5.88\left(\mathrm{~s}, 1 \mathrm{H}, \mathrm{H}^{\mathrm{Pz} 4}\right), 5.85$ (br, $\left.1 \mathrm{H}, \mathrm{OH}\right)$, $5.32\left(\mathrm{dd}, 1 \mathrm{H}, \mathrm{H}^{\mathrm{Thp}},{ }^{3} \mathrm{~J}=10.0 \mathrm{~Hz},{ }^{3} \mathrm{~J}=2.2 \mathrm{~Hz}\right), 3.55-3.65(\mathrm{~m}$, $1 \mathrm{H}, \mathrm{H}^{\mathrm{Thp6}}$ ), 3.40 (s, $3 \mathrm{H}, \mathrm{NCH}_{3}$ ), $3.26\left(\mathrm{~s}, 3 \mathrm{H}, \mathrm{NCH}_{3}\right), 3.18$ (s, $\left.3 \mathrm{H}, \mathrm{NCH}_{3}\right), 3.13\left(\mathrm{~s}, 4 \mathrm{H}, \mathrm{NCH}_{3}, \mathrm{H}^{\mathrm{Thp} 6}\right), 2.10-2.20(\mathrm{~m}, 1 \mathrm{H}$, 
${ }^{13} \mathrm{C}-\mathrm{NMR}\left(\mathrm{CDCl}_{3}\right)$

${ }^{1} \mathrm{H}-\mathrm{NMR}(\mathrm{DMSO})$

${ }^{13}$ C-NMR (DMSO)

$\mathrm{MS}\left(\mathrm{FAB}^{+}\right) \mathrm{m} / \mathrm{z}(\%)$

$\mathrm{IR}(\mathrm{KBr}) \tilde{v}\left(\mathrm{~cm}^{-1}\right)$

Schmelzpunkt
$\left.\mathrm{H}^{\text {Thp3 }}\right)$, 2.00-2.10 (m, $\left.24 \mathrm{H}, \mathrm{CH}_{3}\right), 1.75-1.90\left(\mathrm{~m}, 1 \mathrm{H}, \mathrm{H}^{\text {Thp4}}\right)$, 1.65-1.75 (m, $\left.1 \mathrm{H}, \mathrm{H}^{\text {Thp3 }}\right)$, 1.25-1.55 (m, $\left.3 \mathrm{H}, \mathrm{H}^{\text {Thp4 }}, \mathrm{H}^{\text {Thp5 }}\right)$. $152.7\left(C^{\mathrm{Pz} 5}\right), 145.0\left(\mathrm{C}^{\mathrm{Im} 2}\right), 144.8\left(\mathrm{C}^{\mathrm{Im} 2}\right), 144.4\left(\mathrm{C}^{\mathrm{lm} 2}\right), 143.9$ $\left(\mathrm{C}^{\mathrm{Im} 2}\right), 142.8\left(\mathrm{C}^{\mathrm{Pz} 3}\right), 130.9\left(\mathrm{C}^{\mathrm{Im} 4}\right), 130.2\left(\mathrm{C}^{\mathrm{Im} 4}\right), 130.0\left(\mathrm{C}^{\mathrm{Im} 4}\right)$, $129.9\left(C^{\operatorname{lm} 4}\right), 125.2\left(C^{\operatorname{lm} 5}\right), 124.9\left(C^{\operatorname{lm} 5}\right), 124.8\left(C^{\operatorname{lm} 5}\right), 124.7$ $\left(\mathrm{C}^{\mathrm{Im} 5}\right), 107.5\left(\mathrm{C}^{\mathrm{Pz} 4}\right), 84.7\left(\mathrm{C}^{\mathrm{Thp} 2}\right), 71.0(\mathrm{COH}), 69.8(\mathrm{COH})$, $67.4\left(\mathrm{C}^{\text {Thp6 }}\right), 31.8\left(\mathrm{NCH}_{3}\right), 31.5\left(\mathrm{NCH}_{3}\right), 31.4\left(\mathrm{NCH}_{3}\right), 31.4$ $\left(\mathrm{NCH}_{3}\right), 29.1\left(\mathrm{C}^{\text {Thp3 }}\right), 24.7\left(\mathrm{C}^{\text {Thp5 }}\right), 22.7\left(\mathrm{C}^{\text {Thp4 }}\right), 12.5\left(\mathrm{CH}_{3}\right)$, $12.4\left(\mathrm{CH}_{3}\right), 12.4\left(\mathrm{CH}_{3}\right), 12.3\left(\mathrm{CH}_{3}\right), 8.8\left(\mathrm{CH}_{3}\right), 8.8\left(\mathrm{CH}_{3}\right), 8.7$ $\left(\mathrm{CH}_{3}\right), 8.7\left(\mathrm{CH}_{3}\right)$.

7.00 (br, $1 \mathrm{H}, \mathrm{OH}), 6.30(\mathrm{br}, 1 \mathrm{H}, \mathrm{OH}), 6.06\left(\mathrm{~s}, 1 \mathrm{H}, \mathrm{H}^{\mathrm{Pz} 4}\right.$ ), $5.15\left(\mathrm{dd}, 1 \mathrm{H}, \mathrm{H}^{\mathrm{Thp} 2},{ }^{3} \mathrm{~J}=10.1 \mathrm{~Hz},{ }^{3} \mathrm{~J}=1.7 \mathrm{~Hz}\right), 3.49(\mathrm{~m}, 1 \mathrm{H}$, $\mathrm{H}^{\text {Thp6 }}$ ), $3.31\left(\mathrm{~s}, 3 \mathrm{H}, \mathrm{NCH}_{3}\right), 3.29\left(\mathrm{~s}, 3 \mathrm{H}, \mathrm{NCH}_{3}\right), 3.25(\mathrm{~s}, 3 \mathrm{H}$, $\mathrm{NCH}_{3}$ ), $3.19\left(\mathrm{~s}, 3 \mathrm{H}, \mathrm{NCH}_{3}\right), 2.92\left(\mathrm{~m}, 1 \mathrm{H}, \mathrm{H}^{\text {Thp6 }}\right), 1.92-2.10$ (m, $\left.25 \mathrm{H}, \mathrm{CH}_{3}, \mathrm{H}^{\text {Thp3 }}\right), 1.70-1.80\left(\mathrm{~m}, 1 \mathrm{H}, \mathrm{H}^{\text {Thp4}}\right), 1.60-1.70$ $\left(\mathrm{m}, 1 \mathrm{H}, \mathrm{H}^{\text {Thp3 }}\right), 1.29\left(\mathrm{~m}, 3 \mathrm{H}, \mathrm{H}^{\text {Thp4 }}, \mathrm{H}^{\text {Thp5 }}\right)$.

$152.1\left(C^{\mathrm{Pz} 3}\right), 145.7\left(\mathrm{C}^{\mathrm{Im} 2}\right), 145.4\left(\mathrm{C}^{\mathrm{Im} 2}\right), 144.8\left(\mathrm{C}^{\mathrm{Im} 2}\right), 144.7$ $\left(C^{\operatorname{lm} 2}\right), 143.5\left(C^{\text {Pz5 }}\right), 129.8\left(C^{\operatorname{lm} 5}\right), 129.1\left(C^{\operatorname{lm} 5}\right), 128.9\left(C^{\operatorname{lm} 5}\right)$, $128.8\left(C^{\operatorname{lm} 5}\right), 124.6\left(C^{\operatorname{lm} 4}\right), 124.5\left(C^{\operatorname{lm} 4}\right), 124.4\left(C^{\operatorname{lm} 4}\right), 124.3$ $\left(\mathrm{C}^{\mathrm{Im} 4}\right), \quad 105.6 \quad\left(\mathrm{C}^{\mathrm{Pz} 4}\right), 84.4 \quad\left(\mathrm{C}^{\mathrm{Thp} 2}\right), 71.2\left(\mathrm{COCH}_{3}\right), 70.9$ $\left(\mathrm{COCH}_{3}\right), 66.7\left(\mathrm{C}^{\mathrm{Thp} 6}\right), 31.7\left(\mathrm{NCH}_{3}\right), 31.4\left(\mathrm{NCH}_{3}\right), 31.2$ $\left(\mathrm{NCH}_{3}\right), 31.0\left(\mathrm{NCH}_{3}\right), 29.1\left(\mathrm{C}^{\text {Thp3}}\right), 24.5\left(\mathrm{C}^{\text {Thp5 }}\right), 22.6\left(\mathrm{C}^{\text {Thp4 }}\right)$, $12.4\left(\mathrm{CH}_{3}\right), 12.4\left(\mathrm{CH}_{3}\right), 12.3\left(\mathrm{CH}_{3}\right), 12.3\left(\mathrm{CH}_{3}\right), 8.5\left(\mathrm{CH}_{3}\right)$, $8.4\left(\mathrm{CH}_{3}\right), 8.4\left(\mathrm{CH}_{3}\right), 8.3\left(\mathrm{CH}_{3}\right)$.

$645\left(86,[\mathrm{M}+\mathrm{H}]^{+}\right), \quad 535\left(10,[\mathrm{M}+\mathrm{H}-\mathrm{Tri}]^{+}\right), 341$ (100, $\left.[\mathrm{M}+\mathrm{H}-2 \text { Tri-thp }]^{+}\right), 247\left(10,\left[\mathrm{C}(\mathrm{OH})(\text { Tri })_{2}\right]^{+}\right), 111\left(56,[\text { Tri+H }]^{+}\right)$. 3398 (br), 2942 (s), 2921 (s), 2861 (s), 2737 (m), 1728 (s), $1636(\mathrm{~m}), 1602$ (s), 1444 (vs), 1402 (s), 1371 (s), 1301 (m), $1252(\mathrm{~m}), 1220(\mathrm{~m}), 1205(\mathrm{~m}), 1181(\mathrm{~m}), 1135(\mathrm{~m}), 1083(\mathrm{~s})$, 1061 (s), 1043 (s), 1008 (m), 929 (w), 907 (m), 876 (m), 823 (w), $770(\mathrm{~m}), 730(\mathrm{~s}), 688(\mathrm{w}), 562(\mathrm{w}), 459(\mathrm{w})$. $150{ }^{\circ} \mathrm{C}$ (Zersetzung). 
Elementaranalyse

für $\mathbf{2 b} \cdot 0.6 \mathrm{CH}_{2} \mathrm{Cl}_{2}$ :

ber.: C 59.73, H 7.13, N 20.13,

gef.: C 59.87, H 7.38, N 19.94.

10.2.2.2. 3,5-Bis-[bis-(1,4,5-trimethylimidazol-2-yl)-methoxymethyl]-1-(tetrahydropyran2 -yl)-pyrazol (3b)

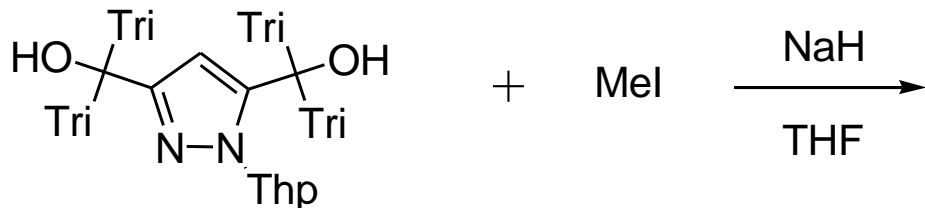

2b

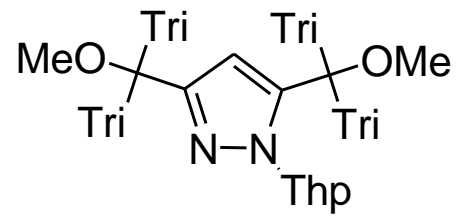

$3 \mathbf{b}$

$\mathrm{Zu}$ einer Suspension von $1.70 \mathrm{~g}(70.6 \mathrm{mmol}) \mathrm{NaH}$ in $250 \mathrm{ml}$ absolutem THF gibt man bei Raumtemperatur und unter $\mathrm{N}_{2}$-Schutzgas $18.50 \mathrm{~g}(28.2 \mathrm{mmol}) \mathbf{2 b}$, gelöst in $50 \mathrm{ml}$ absolutem THF, und lässt die rote Suspension zwei weitere Stunden rühren. Danach werden $4.9 \mathrm{ml}$ (70.6 mmol) Methyliodid zugesetzt und über Nacht rühren gelassen. Zum Quenchen der orange gefärbten Reaktionslösung gibt man $60 \mathrm{ml}$ einer halbgesättigten $\mathrm{NaCl}$-Lösung. Alle flüchtigen organischen Bestandteile werden am Rotationsverdampfer entfernt, die wässrige Phase mit $\mathrm{CHCl}_{3}(7 \times 60 \mathrm{ml})$ extrahiert und die vereinigten organischen Phasen erneut mit demineralisiertem Wasser $(5 \times 40 \mathrm{ml})$ ausgeschüttelt. Die organische Phase wird anschließend über $\mathrm{MgSO}_{4}$ getrocknet. Nach Entfernen des Lösungsmittels im Vakuum und Trocknen im Hochvakuum erhält man einen gelben, schaumartigen Feststoff von $\mathbf{3 b}$.

Summenformel

Molgewicht

Ausbeute

${ }^{1} \mathrm{H}-\mathrm{NMR}\left(\mathrm{CDCl}_{3}\right)$
$\mathrm{C}_{36} \mathrm{H}_{52} \mathrm{~N}_{10} \mathrm{O}_{3}$

$672.88 \mathrm{~g} \mathrm{~mol}^{-1}$

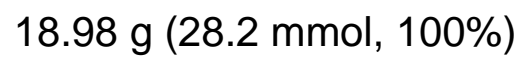

$6.39\left(\mathrm{~s}, 1 \mathrm{H}, \mathrm{H}^{\mathrm{Pz} 4}\right), 5.55\left(\mathrm{dd}, 1 \mathrm{H}, \mathrm{H}^{\mathrm{Thp} 2},{ }^{3} \mathrm{~J}=4.3 \mathrm{~Hz}\right.$, $\left.{ }^{3} \mathrm{~J}=1.4 \mathrm{~Hz}\right)$, 3.58-3.71 $\left(\mathrm{m}, 1 \mathrm{H}, \mathrm{H}^{\mathrm{Thp} 3}\right), 3.30\left(\mathrm{~s}, 3 \mathrm{H}, \mathrm{NCH}_{3}\right)$, $3.28\left(\mathrm{~s}, 3 \mathrm{H}, \mathrm{OCH}_{3}\right), 3.26\left(\mathrm{~s}, 3 \mathrm{H}, \mathrm{OCH}_{3}\right), 3.23(\mathrm{~d}, 1 \mathrm{H}$, $\left.\mathrm{H}^{\text {Thp3 }}\right), 3.21\left(\mathrm{~s}, 3 \mathrm{H}, \mathrm{NCH}_{3}\right), 3.18\left(\mathrm{~s}, 3 \mathrm{H}, \mathrm{NCH}_{3}\right), 3.11(\mathrm{~s}, 3 \mathrm{H}$, 
$\left.\mathrm{NCH}_{3}\right), 1.88-2.20\left(\mathrm{~m}, 26 \mathrm{H}, \mathrm{CH}_{3}, \mathrm{H}^{\text {Thp6 }}\right), 1.62-1.84(\mathrm{~m}, 1 \mathrm{H}$, $\left.H^{\text {Thp5 }}\right), 1.21-1.57\left(\mathrm{~m}, 3 \mathrm{H}, \mathrm{H}^{\text {Thp5 }}, \mathrm{H}^{\text {Thp4 }}\right)$.

${ }^{13} \mathrm{C}-\mathrm{NMR}\left(\mathrm{CDCl}_{3}\right)$

$150.4\left(C^{\mathrm{Pz} 5}\right), 143.1\left(\mathrm{C}^{\mathrm{Im} 2}\right), 143.0\left(\mathrm{C}^{\mathrm{Im} 2}\right), 141.8\left(\mathrm{C}^{\mathrm{Im} 2}\right), 141.6$ $\left(C^{\mathrm{Im} 2}\right), 140.0\left(C^{\mathrm{Pz} 3}\right), 131.0\left(C^{\mathrm{Im} 4}\right), 130.9\left(C^{\mathrm{Im} 4}\right), 130.7\left(\mathrm{C}^{\mathrm{Im} 4}\right)$, $130.6\left(C^{\operatorname{lm} 4}\right), 124.8\left(C^{\operatorname{lm} 5}\right), 124.7\left(C^{\operatorname{lm} 5}\right), 124.3\left(C^{\operatorname{lm} 5}\right), 124.2$ $\left(\mathrm{C}^{\mathrm{Im} 5}\right), \quad 108.7\left(\mathrm{C}^{\mathrm{Pz} 4}\right), \quad 85.0 \quad\left(\mathrm{C}^{\mathrm{Thp} 2}\right), 78.2\left(\mathrm{COCH}_{3}\right), 77.2$ $\left(\mathrm{COCH}_{3}\right), 67.5\left(\mathrm{C}^{\text {Thp } 6}\right), 53.7\left(\mathrm{OCH}_{3}\right), 53.2\left(\mathrm{OCH}_{3}\right), \quad 32.0$ $\left(\mathrm{NCH}_{3}\right), 31.9\left(\mathrm{NCH}_{3}\right), 31.6\left(\mathrm{NCH}_{3}\right), 31.5\left(\mathrm{NCH}_{3}\right), 29.5$ $\left(\mathrm{C}^{\text {Thp3 }}\right), 24.8\left(\mathrm{C}^{\text {Thp5 }}\right), 22.7\left(\mathrm{C}^{\text {Thp4 }}\right), 12.9\left(\mathrm{CH}_{3}\right), 12.8\left(\mathrm{CH}_{3}\right)$, $12.7\left(\mathrm{CH}_{3}\right), 12.6\left(\mathrm{CH}_{3}\right), 8.9\left(\mathrm{CH}_{3}\right), 8.9\left(\mathrm{CH}_{3}\right), 8.8\left(\mathrm{CH}_{3}\right), 8.8$ $\left(\mathrm{CH}_{3}\right)$.

MS (FAB $\left.{ }^{+}\right) \mathrm{m} / \mathrm{z}(\%)$

$687.3\left(53,\left[\mathrm{M}+\mathrm{CH}_{3}\right]^{+}\right), 673.4\left(100,[\mathrm{M}+\mathrm{H}]^{+}\right), 525.3(54$, $\left[\mathrm{M}-2 \mathrm{OCH}_{3}-\mathrm{Thp}^{+}\right), 433.2\left(34\right.$, [M-2Tri-thp] $\left.^{+}\right)$.

$\mathrm{IR}(\mathrm{KBr}) \tilde{v}\left(\mathrm{~cm}^{-1}\right)$

3423 (br), 2942 (s), 2939 (vs), 2860 (s), 1693 (m), 1647 (m), 1600 (s), 1442 (vs), 1398 (vs), 1354 (m), 1300 (m), 1251 $(\mathrm{m}), 1205(\mathrm{~m}), 1135(\mathrm{~m}), 1082(\mathrm{~s}), 1038(\mathrm{~s}), 1010(\mathrm{~m}), 947$ $(\mathrm{m}), 906(\mathrm{~m}), 874(\mathrm{~m}), 820(\mathrm{~m}), 763(\mathrm{~m}), 729(\mathrm{~s}), 698(\mathrm{~m})$, $558(w)$.

Schmelzpunkt $133^{\circ} \mathrm{C}$ (Zersetzung).

Elementaranalyse

für $3 \mathbf{b} \cdot 0.6 \mathrm{CHCl}_{3}$ :

ber.: C 59.05, H 7.12, N 18.81, gef.: C 59.41, H 7.47, N 18.56. 
10.2.2.3. 3,5-Bis-[bis-(1,4,5-trimethylimidazol-2-yl)-methoxymethyl]-pyrazol Hydrochlorid (4b)

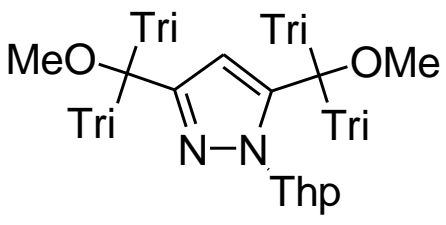

$3 b$

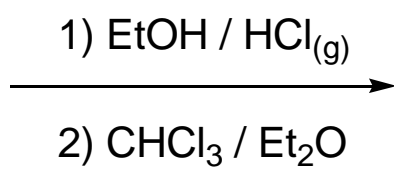

2) $\mathrm{CHCl}_{3} / \mathrm{Et}_{2} \mathrm{O}$<smiles>[3H]C([3H])([3H])c1cc(C([3H])([3H])OC)n[nH]1</smiles>

$4 b$

$11.13 \mathrm{~g}$ (16.6 mmol) von 3b werden in $80 \mathrm{ml}$ ethanolischer $\mathrm{HCl}$ aufgenommen und die Reaktionslösung 2 Stunden gerührt. Nachdem das Lösungsmittel im Vakuum entfernt wurde, wird der Rückstand in $50 \mathrm{ml} \mathrm{CHCl}_{3}$ aufgenommen und langsam in $600 \mathrm{ml} \mathrm{Et} 2 \mathrm{O}$ getropft. Der ausgefallene hellgelbe Niederschlag wird abgesaugt und im Hochvakuum getrocknet. Man erhält 4b als weißgelben Feststoff.

Summenformel
Molgewicht
Ausbeute
${ }^{1} \mathrm{H}-\mathrm{NMR}$ (DMSO)
${ }^{13} \mathrm{C}-\mathrm{NMR}$ (DMSO)

MS $\left(\mathrm{FAB}^{+}\right) \mathrm{m} / \mathrm{z}(\%)$

$\mathrm{IR}(\mathrm{KBr}) \tilde{v}\left(\mathrm{~cm}^{-1}\right)$

Schmelzpunkt
$\mathrm{C}_{31} \mathrm{H}_{45} \mathrm{~N}_{10} \mathrm{O}_{2} \mathrm{Cl}$

$625.22 \mathrm{~g}^{\prime} \mathrm{mol}^{-1}$

$10.00 \mathrm{~g}$, (16.0 mmol, $97 \%)$.

$7.34\left(\mathrm{~s}, 1 \mathrm{H}, \mathrm{H}^{\mathrm{Pz} 4}\right), 3.56\left(\mathrm{~s}, 12 \mathrm{H}, \mathrm{NCH}_{3}\right), 3.33(\mathrm{~s}, 6 \mathrm{H}$, $\left.\mathrm{OCH}_{3}\right), 2.31\left(\mathrm{~s}, 12 \mathrm{H}, \mathrm{CH}_{3}\right), 2.23\left(\mathrm{~s}, 12 \mathrm{H}, \mathrm{CH}_{3}\right)$.

$140.2\left(\mathrm{C}^{\mathrm{Pz} 3+5}\right), 135.9\left(\mathrm{C}^{\operatorname{lm} 2}\right), 130.7\left(\mathrm{C}^{\operatorname{lm} 4}\right), 126.1\left(\mathrm{C}^{\operatorname{lm} 5}\right), 109.7$ $\left(\mathrm{C}^{\mathrm{Pz} 4}\right), 74.1\left(\mathrm{COCH}_{3}\right), 54.0\left(\mathrm{OCH}_{3}\right), 33.2\left(\mathrm{NCH}_{3}\right), 9.1\left(\mathrm{CH}_{3}\right)$, $8.4\left(\mathrm{CH}_{3}\right)$.

$589\left(100,[\mathrm{M}-\mathrm{Cl}]^{+}\right)$.

3485 (br), 3059 (m), 2943 (m), 2877 (m), 2751 (m), 1648 (vs), 1520 (s), 1445 (s), 1381 (w), 1323 (m), 1262 (m), 1225 (m), 1201 (w), $1170(w), 1083$ (s), 1033 (m), 996 (w), 959 $(\mathrm{w}), 895(\mathrm{~m}), 751(\mathrm{~m}), 563(\mathrm{~m})$.

$192{ }^{\circ} \mathrm{C}$ (Zersetzung). 
10.2.2.4. 3,5-Bis-[bis-(1,4,5-trimethylimidazol-2-yl)-methoxymethyl]-pyrazol (5b)

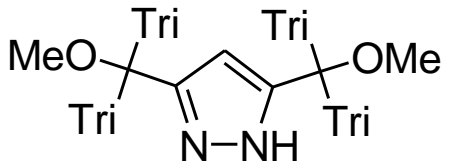

$\cdot \mathrm{HCl}$

$4 b$

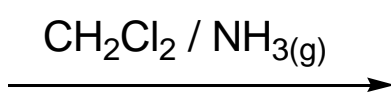

$5 b$

$5.10 \mathrm{~g}$ (8.2 mmol) des Hydrochlorides 4b suspendiert man in $150 \mathrm{ml}$ ammoniakalischer Dichlormethanlösung und lässt die Lösung über Nacht rühren. Der entstandene feine Niederschlag wird abfiltriert und das Lösungsmittel über $\mathrm{MgSO}_{4}$ getrocknet. Anschließend wird die organische Phase mit demineralisiertem Wasser $(5 \times 40 \mathrm{ml})$ extrahiert und die organische Phase über $\mathrm{MgSO}_{4}$ getrocknet. Zum Schluss werden alle flüchtigen Bestandteile im Vakuum entfernt und nach Trocknen im Hochvakuum erhält man einen braunen Feststoff von $\mathbf{5 b}$.

Summenformel

Molgewicht

Ausbeute

${ }^{1} \mathrm{H}-\mathrm{NMR}\left(\mathrm{CDCl}_{3}\right)$

${ }^{13} \mathrm{C}-\mathrm{NMR}\left(\mathrm{CDCl}_{3}\right)$

MS (El) $m / z(\%)$

$\mathrm{IR}(\mathrm{KBr}) \tilde{v}\left(\mathrm{~cm}^{-1}\right)$

Schmelzpunkt
$\mathrm{C}_{31} \mathrm{H}_{44} \mathrm{~N}_{10} \mathrm{O}_{2}$

$588.76 \mathrm{~g}^{\prime} \mathrm{mol}^{-1}$

$3.13 \mathrm{~g} \mathrm{(5.3} \mathrm{mmol,} 65 \%)$.

$6.21\left(\mathrm{~s}, 1 \mathrm{H}, \mathrm{H}^{\mathrm{Pz} 4}\right), 3.31\left(\mathrm{~s}, 12 \mathrm{H}, \mathrm{NCH}_{3}\right), 3.03$ (s, $6 \mathrm{H}$, $\left.\mathrm{OCH}_{3}\right), 2.01\left(\mathrm{~s}, 12 \mathrm{H}, \mathrm{CH}_{3}\right), 1.95\left(\mathrm{~s}, 12 \mathrm{H}, \mathrm{CH}_{3}\right)$.

$146.50\left(\mathrm{C}^{\mathrm{Pz} 3+5}\right), 142.30\left(\mathrm{C}^{\mathrm{Im} 2}\right), 130.53\left(\mathrm{C}^{\operatorname{lm} 4}\right), 124.71\left(\mathrm{C}^{\mathrm{Im} 5}\right)$, $106.23\left(\mathrm{C}^{\mathrm{Pz} 4}\right), 75.80\left(\mathrm{COCH}_{3}\right), 52.12\left(\mathrm{OCH}_{3}\right), 31.03\left(\mathrm{NCH}_{3}\right)$, $12.31\left(\mathrm{CH}_{3}\right), 8.66\left(\mathrm{CH}_{3}\right)$.

$588\left(5,[\mathrm{M}]^{+}\right), 573\left(10,\left[\mathrm{M}-\mathrm{CH}_{3}\right]^{+}\right), 558\left(2,\left[\mathrm{M}-2 \mathrm{CH}_{3}\right]^{+}\right), 541$ (12, $\left.\left[\mathrm{M}-\mathrm{CH}_{3}-\mathrm{OCH}_{3}\right]^{+}\right), \quad 525\left(2, \quad\left[\mathrm{M}-2 \mathrm{OCH}_{3}\right]^{+}\right), \quad 313$ (24, $\left.\left[\mathrm{M}-\mathrm{CH}_{3}-\mathrm{C}(\mathrm{Im})_{2} \mathrm{OCH}_{3}\right]^{+}\right), 110\left(100,[\mathrm{Tri}]^{+}\right)$.

3255 (br), 2921 (s), 2862 (m), $2825(\mathrm{~m}), 1722(\mathrm{~m}), 1635(\mathrm{~m})$, 1601 (s), 1542 (m), 1476 (s), 1443 (s), 1398 (s), 1301 (m), 1262 (s), 1231 (m), 1131 (m), 1075 (vs), 1013 (s), 949 (m), 899 (s), 802 (s), 747 (vs), 659 (m).

$132{ }^{\circ} \mathrm{C}$ (Zersetzung). 


\subsubsection{Ligandsynthese $\mathrm{HL}^{3}$}

10.2.3.1. 3,5-Bis-[bis-(4,5-diphenyl-1-methylimidazol-2-yl)-hydroxymethyl]-1(tetrahydropyran-2-yl)-pyrazol (2c)<smiles>COC(=O)c1cc(C(=O)OC)n([PbH2])n1</smiles>

1<smiles>Cn1cnc(-c2ccccc2)c1-c1ccccc1</smiles>
Thp

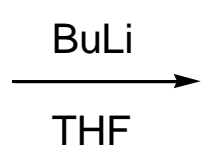

THF

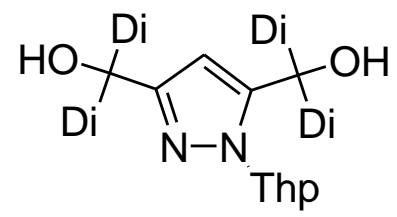

2c

$44.30 \mathrm{~g} \quad(189.0 \mathrm{mmol})$ des 4,5-Diphenyl-1-methylimidazols werden in $1000 \mathrm{ml}$ Tetrahydrofuran suspendiert und $130.0 \mathrm{ml} n$-Butyllithium $(208.0 \mathrm{mmol}, 1.6 \mathrm{M}$ in $n$-Hexan) langsam zugetropft, wobei eine Farbänderung von gelb zu dunkelbraun auftritt. Die Lösung wird 1.5 Stunden bei Raumtemperatur gerührt. Nach Zutropfen von $12.68 \mathrm{~g} \mathrm{(47.3} \mathrm{mmol)} \mathrm{1-Tetrahydropyran-2-yl-pyrazol-3,5-dicarbonsäuremethylester} \mathrm{(1),}$ gelöst in $300 \mathrm{ml}$ absolutem Tetrahydrofuran, wird die Reaktionslösung über Nacht gerührt. Die braune, fast klare Lösung wird anschließend mit $50 \mathrm{ml}$ halbgesättigter $\mathrm{NH}_{4} \mathrm{Cl}$-Lösung versetzt, woraus eine orange-bräunliche, klare Lösung resultiert. Im Vakuum werden alle flüchtigen Bestandteile entfernt und das Rohprodukt in $400 \mathrm{ml}$ ammoniakalischer Dichlormethanlösung aufgenommen. Die Lösung wird filtriert, über $\mathrm{MgSO}_{4}$ getrocknet und das Lösungsmittel im Vakuum entfernt. Nach Trocknen im Hochvakuum wird 2c als gelber Feststoff erhalten.

\begin{tabular}{|c|c|}
\hline Summenformel & $\mathrm{C}_{74} \mathrm{H}_{64} \mathrm{~N}_{10} \mathrm{O}_{3}$ \\
\hline Molgewicht & $1141.39 \mathrm{~g} \cdot \mathrm{mol}^{-1}$ \\
\hline Ausbeute & $48.90 \mathrm{~g}$ (42.8 mmol, $91 \%)$ \\
\hline${ }^{1} \mathrm{H}-\mathrm{NMR}\left(\mathrm{CDCl}_{3}\right)$ & $\begin{array}{l}\left.\text { 7.05-7.50 (m, } 10 \mathrm{H}, \mathrm{H}^{\mathrm{Ph}}\right), 6.34(\mathrm{~s}, 1 \mathrm{H}, \mathrm{OH}), 6.81(\mathrm{~s}, 1 \mathrm{H}, \\
\mathrm{OH}), 6.16\left(\mathrm{~s}, 1 \mathrm{H}, \mathrm{H}^{\mathrm{Pz} 4}\right), 5.56\left(\mathrm{dd}, 1 \mathrm{H}, \mathrm{H}^{\text {Thp2 },}{ }^{3} \mathrm{~J}=10 \mathrm{~Hz},\right. \\
\left.{ }^{3} \mathrm{~J}=1.9 \mathrm{~Hz}\right), 3.75\left(\mathrm{~m}, 1 \mathrm{H}, \mathrm{H}^{\mathrm{Thp} 6}\right), 3.57\left(\mathrm{~s}, 3 \mathrm{H}, \mathrm{NCH}_{3}\right), \\
3.39\left(\mathrm{~s}, 3 \mathrm{H}, \mathrm{NCH}_{3}\right), 3.35\left(\mathrm{~s}, 3 \mathrm{H}, \mathrm{NCH}_{3}\right), 3.30(\mathrm{~s}, 3 \mathrm{H}, \\
\left.\mathrm{NCH}_{3}\right), 3.17\left(\mathrm{~m}, 1 \mathrm{H}, \mathrm{H}^{\text {Thp6 }}\right), 2.34\left(\mathrm{~m}, 1 \mathrm{H}, \mathrm{H}^{\text {Thp3}}\right), 1.87(\mathrm{~m}, \\
\left.3 \mathrm{H}, 2 \mathrm{H}^{\text {Thp3 }}, \mathrm{H}^{\text {Thp4 }}\right), 1.35\left(\mathrm{~m}, 2 \mathrm{H}, \mathrm{H}^{\text {Thp5 }}\right) .\end{array}$ \\
\hline
\end{tabular}


${ }^{13} \mathrm{C}-\mathrm{NMR}\left(\mathrm{CDCl}_{3}\right)$

$\mathrm{MS}\left(\mathrm{FAB}^{+}\right) \mathrm{m} / z(\%)$

$\operatorname{IR}(\mathrm{KBr}) \tilde{v}\left(\mathrm{~cm}^{-1}\right)$

Schmelzpunkt

Elementaranalyse
$152.6\left(C^{q}\right), \quad 146.8\left(C^{q}\right), \quad 146.8\left(C^{q}\right), \quad 146.7\left(C^{q}\right), \quad 145.9\left(C^{q}\right)$, $142.0\left(C^{9}\right), 135.7\left(C^{9}\right), 135.1\left(C^{9}\right), 134.0\left(C^{9}\right), 133.7\left(C^{9}\right)$, $134.6\left(C^{9}\right), 134.4\left(C^{9}\right), 131.9\left(C^{q}\right), 131.4\left(C^{q}\right), 131.4\left(C^{q}\right)$, $131.3\left(C^{\mathrm{q}}\right), 130.8\left(C^{\mathrm{q}}\right), 131.1\left(C^{\mathrm{Ph}}\right), 131.0\left(C^{\mathrm{Ph}}\right), 131.0\left(C^{\mathrm{Ph}}\right)$, $131.0\left(C^{P h}\right), 130.8\left(C^{q}\right), 129.0\left(C^{P h}\right), 128.9\left(C^{P h}\right), 128.9$ $\left(C^{\mathrm{Ph}}\right), 128.6\left(\mathrm{C}^{\mathrm{Ph}}\right), 128.6\left(\mathrm{C}^{\mathrm{Ph}}\right), 128.0\left(\mathrm{C}^{\mathrm{Ph}}\right), 127.9\left(\mathrm{C}^{\mathrm{Ph}}\right)$, $127.9\left(C^{\mathrm{Ph}}\right), \quad 126.6\left(\mathrm{C}^{\mathrm{Ph}}\right), \quad 126.5\left(\mathrm{C}^{\mathrm{Ph}}\right), \quad 126.1\left(\mathrm{C}^{\mathrm{Ph}}\right)$, $126.0\left(\mathrm{C}^{\mathrm{Ph}}\right), \quad 126.0\left(\mathrm{C}^{\mathrm{Ph}}\right), \quad 108.7\left(\mathrm{C}^{\mathrm{Pz} 4}\right), \quad 85.0\left(\mathrm{C}^{\mathrm{Thp}}\right)$, $71.7(\mathrm{COH}), \quad 70.3(\mathrm{COH}), \quad 68.0\left(\mathrm{C}^{\mathrm{Thp} 6}\right), \quad 33.2\left(\mathrm{NCH}_{3}\right)$, $32.7\left(\mathrm{NCH}_{3}\right), \quad 32.6\left(\mathrm{NCH}_{3}\right), \quad 32.5\left(\mathrm{NCH}_{3}\right), \quad 29.3\left(\mathrm{C}^{\mathrm{Thp} 5}\right)$, $24.8\left(\mathrm{C}^{\text {Thp3}}\right), 22.8\left(\mathrm{C}^{\text {Thp4 }}\right)$.

$1141.7\left(30,[\mathrm{M}+\mathrm{H}]^{+}\right), 1057.5\left(3,[\mathrm{M}+\mathrm{H}-\mathrm{Thp}]^{+}\right), 589.3(100$, $\left[\mathrm{M}+\mathrm{H}-\mathrm{Thp}-2 \mathrm{Di}^{+}\right), 235.2\left(66,[\mathrm{Di}+\mathrm{H}]^{+}\right)$.

3367 (br), 3056 (s), 2947 (s), 2858 (m), 1954 (w), 1889 (w), 1810 (w), 1772 (w), 1602 (vs), 1505 (vs), 1472 (m), 1443 (s), $1387(\mathrm{~m}), 1353(\mathrm{~m}), 1319(\mathrm{~m}), 1250(\mathrm{~m}), 1234(\mathrm{~m}), 1205$ $(\mathrm{m}), 1178(\mathrm{~m}), 1139(\mathrm{~s}), 1071(\mathrm{~m}), 1059(\mathrm{~s}), 1041(\mathrm{~s}), 1025$ (s), $1006(\mathrm{~m}), 970(\mathrm{~s}), 907(\mathrm{~s}), 874(\mathrm{~m}), 820(\mathrm{w}), 773(\mathrm{vs})$, $720(\mathrm{~m}), 694$ (vs), $647(\mathrm{~m})$.

$179{ }^{\circ} \mathrm{C}$ (Zersetzung).

für $2 \mathrm{c} \cdot 0.4 \mathrm{CH}_{2} \mathrm{Cl}_{2}$ :

ber.: C 76.03, H 5.56, N 11.92,

gef.: C 76.22, H 5.80, N 11.81 . 
10.2.3.2. 3,5-Bis-[bis-(4,5-Diphenyl-1-methylimidazol-2-yl)-methoxymethyl]-1(tetrahydropyran-2-yl)-pyrazol (3c)

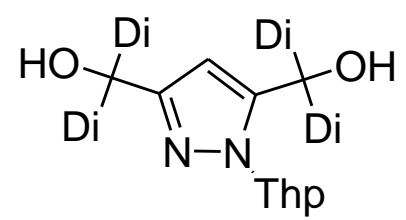

2c<smiles></smiles>

3c

In $150 \mathrm{ml}$ Tetrahydrofuran werden $10.00 \mathrm{~g}$ (8.8 mmol) 2c gelöst. Zu der klaren, gelben Lösung gibt man $0.53 \mathrm{~g}(22.0 \mathrm{mmol})$ Natriumhydrid. Nach ca. einer Stunde werden bei Raumtemperatur $1.37 \mathrm{ml}(3.11 \mathrm{~g}, 22.0 \mathrm{mmol})$ Methyliodid zugetropft, und die dunkel gefärbte Lösung über Nacht gerührt. Nach Zugabe von $60 \mathrm{ml}$ einer halbgesättigten Natriumchlorid-Lösung wird das Lösungsmittel im Vakuum entfernt. Anschließend wird die wässrige Phase mit Chloroform $(3 \times 50 \mathrm{ml})$ extrahiert und die vereinten organischen Phasen über $\mathrm{MgSO}_{4}$ getrocknet. Nach Entfernen des Lösungsmittels im Vakuum und abschließendem Trocknen im Hochvakuum erhält man 3c als gelben Feststoff.

Summenformel

Molgewicht

Ausbeute

${ }^{1} \mathrm{H}-\mathrm{NMR}\left(\mathrm{CDCl}_{3}\right)$

${ }^{13} \mathrm{C}-\mathrm{NMR}\left(\mathrm{CDCl}_{3}\right)$
$\mathrm{C}_{76} \mathrm{H}_{68} \mathrm{~N}_{10} \mathrm{O}_{3}$

$1169.45 \mathrm{~g}^{\prime} \mathrm{mol}^{-1}$

$9.42 \mathrm{~g}(8.1 \mathrm{mmol}, 92 \%)$.

7.05-7.50 (m, $\left.10 \mathrm{H}, \mathrm{H}^{\mathrm{Ph}}\right), 6.85\left(\mathrm{~s}, 1 \mathrm{H}, \mathrm{H}^{\mathrm{Pz} 4}\right), 5.75(\mathrm{~m}, 1 \mathrm{H}$, $\mathrm{H}^{\text {Thp2 }}$ ), $3.79\left(\mathrm{~s}, 3 \mathrm{H}, \mathrm{NCH}_{3}\right), 3.72\left(\mathrm{~m}, 1 \mathrm{H}, \mathrm{H}^{\text {Thp6 }}\right), 3.66(\mathrm{~s}$, $\left.3 \mathrm{H}, \mathrm{NCH}_{3}\right), 3.62\left(\mathrm{~s}, 6 \mathrm{H}, \mathrm{OCH}_{3}, \mathrm{NCH}_{3}\right), 3.47$ (s, $6 \mathrm{H}, \mathrm{OCH}_{3}$, $\left.\mathrm{NCH}_{3}\right), 3.17\left(\mathrm{~m}, 1 \mathrm{H}, \mathrm{H}^{\text {Thp6 }}\right), 2.45\left(\mathrm{~m}, 1 \mathrm{H}, \mathrm{H}^{\text {Thp3 }}\right), 1.87(\mathrm{~m}$, $\left.3 \mathrm{H}, \mathrm{H}^{\text {Thp3 }}, \mathrm{H}^{\text {Thp4 }}\right), 1.30\left(\mathrm{~m}, 2 \mathrm{H}, \mathrm{H}^{\text {Thp5 }}\right)$.

$149.4\left(C^{\mathrm{Pz} 5}\right), 145.6\left(\mathrm{C}^{\mathrm{Im} 2}\right), 145.3\left(\mathrm{C}^{\mathrm{Im} 2}\right), 144.0\left(\mathrm{C}^{\mathrm{Im} 2}\right), 143.5$ $\left(C^{\operatorname{lm} 2}\right), 140.7 \quad\left(C^{P z 3}\right), 135.7,135.6,135.5,135.1,135.0$, $135.0,134.8,131.5,131.5,131.2,131.0,130.9,130.9$, 128.9 , 128.8, 128.5, 128.4, 128.0, 127.9, 127.8, 127.7, 126.5, 126.4, 125.9, 125.8, 125.7, $110.0\left(\mathrm{C}^{\mathrm{Pz} 4}\right), 85.3\left(\mathrm{C}^{\mathrm{Thp} 2}\right)$, $79.1\left(\mathrm{COCH}_{3}\right), 78.1\left(\mathrm{COCH}_{3}\right), 68.0\left(\mathrm{C}^{\mathrm{Thp} 6}\right), 54.5\left(\mathrm{OCH}_{3}\right)$, $54.2\left(\mathrm{OCH}_{3}\right), 33.0\left(\mathrm{NCH}_{3}\right), 32.9\left(\mathrm{NCH}_{3}\right), 32.8\left(\mathrm{NCH}_{3}\right), 32.6$ $\left(\mathrm{NCH}_{3}\right), 29.7\left(\mathrm{C}^{\mathrm{Thp} 3}\right), 24.9\left(\mathrm{C}^{\mathrm{Thp} 5}\right), 22.8\left(\mathrm{C}^{\mathrm{Thp} 4}\right)$. 
$\mathrm{MS}\left(\mathrm{FAB}^{+}\right) \mathrm{m} / \mathrm{z}(\%)$

$\mathrm{IR}(\mathrm{KBr}) \tilde{v}\left(\mathrm{~cm}^{-1}\right)$

Schmelzpunkt

Elementaranalyse
$1191.7\left(92,[\mathrm{M}+\mathrm{Na}]^{+}\right), 1169.8\left(100,[\mathrm{M}+\mathrm{H}]^{+}\right), 1085.6(27$, $\left.[\mathrm{M}-\mathrm{thp}+\mathrm{H}]^{+}\right), \quad 993.5 \quad\left(31, \quad[\mathrm{M}-\mathrm{thp}-\mathrm{Ph}-\mathrm{O}]^{+}\right), \quad 805.3 \quad$ (38, $\left.\left[\mathrm{M}-\text { thp-Di-OCH}{ }_{3}-\mathrm{OH}\right]^{+}\right), 247.0\left(82,\left[\mathrm{Di}+\mathrm{CH}_{2}\right]^{+}\right)$.

$3054(\mathrm{~m}), 2939(\mathrm{~m}), 2857(\mathrm{~m}), 1953(\mathrm{w}), 1888(\mathrm{w}), 1810(\mathrm{w})$, 1756 (w), 1689 (w), 1602 (s), 1576 (m), 1505 (s), 1443 (s), $1384(\mathrm{~m}), 1353(\mathrm{w}), 1319(\mathrm{~m}), 1236(\mathrm{~m}), 1205(\mathrm{~m}), 1179(\mathrm{~m})$, 1131 (m), 1072 (s), 1025 (s), 968 (m), 907 (m), 890 (m), 874 (m), 844 (w), 819 (w), 773 (vs), 722 (m), 695 (s), 647 (w), $613(w), 523(\mathrm{~m})$.

$230{ }^{\circ} \mathrm{C}$ (Zersetzung).

für 3c:

ber.: C 78.06, H 5.86, N 11.98, gef.: C 77.59, H 6.15, N 11.85 .

10.2.5.3. 3,5-Bis-[bis-(4,5-diphenyl-1-methylimidazol-2-yl)-methoxymethyl]-pyrazol Hydrochlorid (4c)

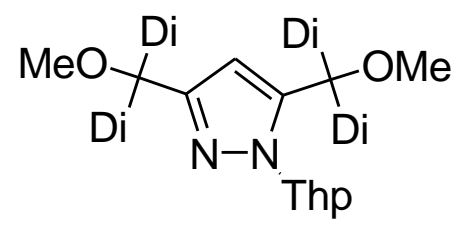

3c

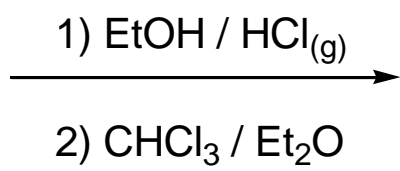

2) $\mathrm{CHCl}_{3} / \mathrm{Et}_{2} \mathrm{O}$

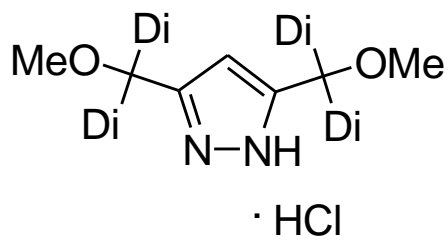

4c

$9.42 \mathrm{~g}$ (8.1 mmol) von 3c werden in $50 \mathrm{ml}$ ethanolischer $\mathrm{HCl}$ gelöst und über Nacht bei Raumtemperatur gerührt. Das Lösungsmittel wird im Vakuum entfernt und der Rückstand in $50 \mathrm{ml} \mathrm{CHCl}_{3}$ aufgenommen. Diese Lösung tropft man vorsichtig in $600 \mathrm{ml}$ Diethylether. Den weißen Niederschlag saugt man durch eine Fritte ab und nach Trocknen im Hochvakuum erhält man einen weißen Feststoff von 4c.

Summenformel

Molgewicht

Ausbeute
$\mathrm{C}_{71} \mathrm{H}_{61} \mathrm{~N}_{10} \mathrm{O}_{2} \mathrm{Cl}$

$1121.79 \mathrm{~g}^{\prime} \mathrm{mol}^{-1}$

$8.94 \mathrm{~g}(8.0 \mathrm{mmol}, 99 \%)$. 
${ }^{1} \mathrm{H}-\mathrm{NMR}(\mathrm{DMSO})$

${ }^{13} \mathrm{C}-\mathrm{NMR}$ (DMSO)

MS $\left(\mathrm{FAB}^{+}\right) \mathrm{m} / \mathrm{z}(\%)$

$\mathrm{IR}(\mathrm{KBr}) \tilde{v}\left(\mathrm{~cm}^{-1}\right)$

Schmelzpunkt

Elementaranalyse
7.34-7.57 (m, $\left.40 \mathrm{H}, \mathrm{CH}^{\mathrm{Ph}}\right), 7.20\left(\mathrm{~s}, 1 \mathrm{H}, \mathrm{CH}^{\mathrm{Pz} 4}\right), 3.49(\mathrm{~s}, 6 \mathrm{H}$, $\left.\mathrm{OCH}_{3}\right), 3.38\left(\mathrm{~s}, 12 \mathrm{H}, \mathrm{NCH}_{3}\right)$.

$144.3\left(C^{\text {Pz3 }+5}\right), 142.1\left(C^{\operatorname{lm} 2}\right), 132.5\left(C^{i-P h}\right), 132.4\left(C^{i-P h}\right), 131.1$ $\left(C^{\mathrm{o} / \mathrm{m}-\mathrm{Ph}}\right), 130.3\left(\mathrm{C}^{\mathrm{Im} 4}\right), 129.8\left(\mathrm{C}^{\mathrm{p}-\mathrm{Ph}}\right), 129.2\left(\mathrm{C}^{\mathrm{o} / \mathrm{m}-\mathrm{Ph}}\right), 128.3$ $\left(C^{\mathrm{o} / \mathrm{m}-\mathrm{Ph}}\right), 127.9\left(\mathrm{C}^{\mathrm{p}-\mathrm{Ph}}\right), 127.7\left(\mathrm{C}^{\mathrm{lm} 5}\right), 127.4\left(^{\mathrm{o} / \mathrm{m}-\mathrm{Ph}}\right), 110.3$ $\left(\mathrm{C}^{\mathrm{Pz} 4}\right), 79.4\left(\mathrm{COCH}_{3}\right), 53.4\left(\mathrm{OCH}_{3}\right), 33.3\left(\mathrm{NCH}_{3}\right)$.

$1085.5\left(100,[\mathrm{M}-\mathrm{Cl}]^{+}\right), 805.2\left(9,\left[\mathrm{M}-\mathrm{Cl}-\mathrm{Di}-3 \mathrm{CH}_{3}\right]^{+}\right), 543.1$ (16, $\left.[\mathrm{M}-\mathrm{Cl}+\mathrm{H}]^{2+}\right), 235.0\left(24,[\mathrm{Di}+\mathrm{H}]^{+}\right)$.

3402 (br), 3040 (s), 2963 (s), $1632(\mathrm{~m}), 1596(\mathrm{~m}), 1503(\mathrm{~m})$, $1477(\mathrm{~m}), 1445(\mathrm{~s}), 1418(\mathrm{~m}), 1323(\mathrm{~m}), 1289(\mathrm{~m}), 1233(\mathrm{w})$, $1183(\mathrm{w}), 1075$ (s), 1025 (s), $978(\mathrm{~m}), 902(\mathrm{~m}), 770(\mathrm{~s}), 696$ (vs).

$145^{\circ} \mathrm{C}$ (Zersetzung).

für $4 \mathbf{c} \cdot(4.9 \mathrm{HCl}+0.4 \mathrm{EtOH})$ :

ber.: C 65.39, H 5.22, N 10.62, Cl 15.86,

gef.: C 64.98, H 5.67, N 10.95, Cl 15.70.

10.2.5.4. 3,5-Bis-[bis-(4,5-diphenyl-1-methylimidazol-2-yl)-methoxymethyl]-pyrazol (5c)<smiles>[2H]C([2H])(OC)c1cc(C([2H])([2H])OC)[nH]n1</smiles>

$4 c$

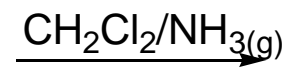

$5 c$

$57.73 \mathrm{~g}(52.7 \mathrm{mmol})$ des $4 \mathrm{c}$ wird mit $600 \mathrm{ml}$ ammoniakalischem Dichlormethan versetzt und 4 Stunden gerührt. Nach Abfiltrieren des ausgefallenen Feststoffes wird das Lösungsmittel im Vakuum entfernt, das Produkt in wenig Dichlormethan aufgenommen und in $300 \mathrm{ml} n$-Hexan getropft. Der ausgefallene weiße Niederschlag wird durch eine Fritte abgesaugt und das weiße Rohprodukt erneut in $200 \mathrm{ml}$ Dichlormethan gelöst. Nach Entfernen des Lösungsmittels und Trocknen im Hochvakuum erhält man so einen braunen Feststoff von $\mathbf{5 c}$. 


\begin{tabular}{|c|c|}
\hline Summenformel & $\mathrm{C}_{71} \mathrm{H}_{60} \mathrm{~N}_{10} \mathrm{O}_{2}$ \\
\hline Molgewicht & $1085.33 \mathrm{~g} \mathrm{~mol}^{-1}$ \\
\hline Ausbeute & $31.95 \mathrm{~g}$ (30.2 mmol, $58 \%)$. \\
\hline${ }^{1} \mathrm{H}-\mathrm{NMR}\left(\mathrm{CDCl}_{3}\right)$ & $\begin{array}{l}13.70(\mathrm{~s}, 1 \mathrm{H}, \mathrm{NH}), 7.05-7.50\left(\mathrm{~m}, 40 \mathrm{H}, \mathrm{H}^{\mathrm{Ph}}\right), 6.87(\mathrm{~s}, 1 \mathrm{H} \text {, } \\
\left.\mathrm{H}^{\mathrm{Pz} 4}\right), 3.55\left(\mathrm{~s}, 6 \mathrm{H}, \mathrm{OCH}_{3}\right), 3.41\left(\mathrm{~s}, 12 \mathrm{H}, \mathrm{NCH}_{3}\right) .\end{array}$ \\
\hline${ }^{13} \mathrm{C}-\mathrm{NMR}\left(\mathrm{CDCl}_{3}\right)$ & $\begin{array}{l}144.9\left(\mathrm{C}^{\mathrm{Pz} 3+5}\right), \quad 135.8\left(\mathrm{C}^{\mathrm{q}}\right), 134.6\left(\mathrm{C}^{\mathrm{q}}\right), \quad 131.1\left(\mathrm{C}^{\mathrm{q}}\right), 131.0 \\
\left(\mathrm{C}^{\mathrm{o} / \mathrm{m}-\mathrm{Ph}}\right), 128.9\left(\mathrm{C}^{\mathrm{o} / \mathrm{m}-\mathrm{Ph}}\right), 128.5\left(\mathrm{C}^{\mathrm{p}-\mathrm{Ph}}\right), 127.9\left(\mathrm{C}^{\mathrm{o} / \mathrm{m}-\mathrm{Ph}}\right), 126.8 \\
\left(\mathrm{C}^{\mathrm{o} / \mathrm{m}-\mathrm{Ph}}\right), \quad 126.0\left(\mathrm{C}^{\mathrm{p}-\mathrm{Ph}}\right), \quad 108.3 \quad\left(\mathrm{C}^{\mathrm{pz} 4}\right), \quad 76.4 \quad\left(\mathrm{COCH}_{3}\right), \\
53.4\left(\mathrm{OCH}_{3}\right), 33.5\left(\mathrm{NCH}_{3}\right) .\end{array}$ \\
\hline $\mathrm{MS}\left(\mathrm{FAB}^{+}\right) \mathrm{m} / \mathrm{z}(\%)$ & $\begin{array}{l}1085.4\left(100,[\mathrm{M}+\mathrm{H}]^{+}\right), 1069.3\left(35,[\mathrm{M}+\mathrm{H}-\mathrm{Me}]^{+}\right), 993.3(21, \\
\left.[\mathrm{M}+\mathrm{H}-\mathrm{Ph}-\mathrm{Me}]^{+}\right), 805.2 \quad\left(16, \quad[\mathrm{M}+\mathrm{H}-\mathrm{Di}-3 \mathrm{Me}]^{+}\right), 247.1(41, \\
\left.\left[\mathrm{Di}+\mathrm{CH}_{2}\right]^{+}\right) .\end{array}$ \\
\hline $\mathrm{IR}(\mathrm{KBr}) \tilde{v}\left(\mathrm{~cm}^{-1}\right)$ & $\begin{array}{l}3244(\mathrm{br}), 3055(\mathrm{~m}), 2948(\mathrm{~m}), 2826(\mathrm{~m}), 1951(\mathrm{w}), 1886(\mathrm{w}), \\
1809(\mathrm{w}), 1602(\mathrm{~s}), 1504(\mathrm{~s}), 1442(\mathrm{~s}), 1384(\mathrm{~m}), 1318(\mathrm{w}), \\
1266(\mathrm{w}), 1234(\mathrm{w}), 1126(\mathrm{w}), 1072(\mathrm{~s}), 1024(\mathrm{~m}), 968(\mathrm{~m}), \\
902(\mathrm{~m}), 816(\mathrm{w}), 773(\mathrm{vs}), 745(\mathrm{~m}), 720(\mathrm{~m}), 694(\mathrm{vs}), 522 \\
(\mathrm{~m}) .\end{array}$ \\
\hline Schmelzpunkt & $161^{\circ} \mathrm{C}$ (Zersetzung). \\
\hline Elementaranalyse & für $5 \mathrm{c} \cdot 0.2 \mathrm{CH}_{2} \mathrm{Cl}_{2}$ : \\
\hline & ber.: C 77.58, H 5.58, N 12.71, \\
\hline & gef.: C 77.57, H 5.43, N 12.69. \\
\hline
\end{tabular}




\subsubsection{Ligandsynthese $\mathrm{HL}^{4}$}

10.2.4.1. 3,5-Bis-[bis-(1-methylimidazol-2-yl)-hydroxymethyl]-pyrazol Hydrochlorid (6a)

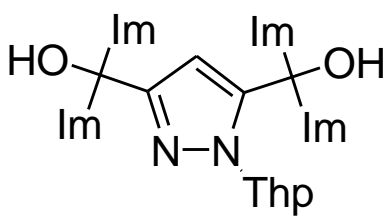

2a

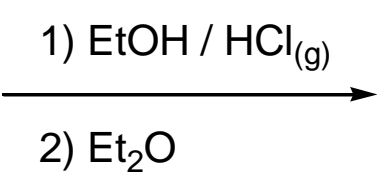

2) $\mathrm{Et}_{2} \mathrm{O}$<smiles>CC(C)C(O)([AlH2])c1cc(C(O)(c2ccccc2)C(C)(C)C)[nH]n1</smiles>

$6 a$

$19.60 \mathrm{~g}$ (36.8 mmol) von $2 \mathrm{a}$ werden in $150 \mathrm{ml}$ ethanolischer $\mathrm{HCl}$ aufgenommen und die Reaktionslösung über Nacht gerührt. Zur Ausfällung des Hydrochlorides gibt man $500 \mathrm{ml}$ Diethylether zur Mischung. Der weiße Niederschlag wird durch eine Fritte abgesaugt, mit $\mathrm{Et}_{2} \mathrm{O}$ gewaschen und im Hochvakuum getrocknet. Man erhält einen weißen Feststoff von 6a.

\begin{tabular}{|c|c|}
\hline Summenformel & $\mathrm{C}_{21} \mathrm{H}_{25} \mathrm{~N}_{10} \mathrm{O}_{2} \mathrm{Cl}$ \\
\hline Molgewicht & $484.95 \mathrm{~g} \cdot \mathrm{mol}^{-1}$ \\
\hline Ausbeute & $17.80 \mathrm{~g}(36.7 \mathrm{mmol}, 99 \%)$ \\
\hline 1H-NMR (DMSO) & $\begin{array}{l}7.83\left(\mathrm{~d}, 4 \mathrm{H},{ }^{3} \mathrm{~J}=1.7 \mathrm{~Hz}, \mathrm{H}^{\mathrm{Im} 4}\right), 7.53\left(\mathrm{~d}, 4 \mathrm{H},{ }^{3} \mathrm{~J}=1.7 \mathrm{~Hz},\right. \\
\left.\mathrm{H}^{\mathrm{Im5}}\right), 6.73\left(\mathrm{~s}, 1 \mathrm{H}, \mathrm{H}^{\mathrm{Pz} 4}\right), 3.68\left(\mathrm{~s}, 12 \mathrm{H}, \mathrm{NCH}_{3}\right) .\end{array}$ \\
\hline${ }^{13} \mathrm{C}-\mathrm{NMR}$ (DMSO) & $\begin{array}{l}145.1\left(\mathrm{C}^{\mathrm{Pz} 3+5}\right), 141.6\left(\mathrm{C}^{\mathrm{Im} 2}\right), 126.7\left(\mathrm{C}^{\mathrm{Im} 4}\right), 120.1\left(\mathrm{C}^{\mathrm{Im} 5}\right), 106.5 \\
\left(\mathrm{C}^{\mathrm{Pz} 4}\right), 69.0(\mathrm{COH}), 36.1\left(\mathrm{NCH}_{3}\right) .\end{array}$ \\
\hline $\mathrm{MS}\left(\mathrm{FAB}^{+}\right) \mathrm{m} / z(\%)$ & $\begin{array}{l}449\left(100,[\mathrm{M}-\mathrm{Cl}]^{+}\right), \quad 367 \quad\left(18, \quad[\mathrm{M}-\mathrm{Cl}-\mathrm{Im}]^{+}\right), \quad 285 \quad(45, \\
\left.[\mathrm{M}-\mathrm{Cl}-2 \mathrm{Im}]^{+}\right), 83\left(38,\left[\mathrm{Im}+\mathrm{H}^{+}\right) .\right.\end{array}$ \\
\hline $\mathrm{IR}(\mathrm{KBr}) \tilde{v}\left(\mathrm{~cm}^{-1}\right)$ & $\begin{array}{l}3392(\mathrm{br}), 3118(\mathrm{~s}), 3047(\mathrm{~s}), 2966(\mathrm{~s}), 2819(\mathrm{~s}), 2741(\mathrm{~s}), \\
1723(\mathrm{w}), 1639(\mathrm{w}), 1591(\mathrm{~m}), 1520(\mathrm{~s}), 1455(\mathrm{~m}), 1384(\mathrm{~s}), \\
1312(\mathrm{~s}), 1279(\mathrm{~m}), 1210(\mathrm{~m}), 1161(\mathrm{w}), 1118(\mathrm{~m}), 1072(\mathrm{~s}), \\
1016(\mathrm{w}), 924(\mathrm{~m}), 885(\mathrm{~m}), 769(\mathrm{~s}), 720(\mathrm{~m}), 621(\mathrm{w}) .\end{array}$ \\
\hline Schmelzpunkt & $180^{\circ} \mathrm{C}$ (Zersetzung). \\
\hline Elementaranalyse & $\begin{array}{l}\text { für } 6 \mathbf{a} \cdot(3.9 \mathrm{HCl}+0.5 \mathrm{EtOH}) \text { : } \\
\text { ber.: C } 40.64, \mathrm{H} 4.95, \mathrm{~N} 21.54 \text {, } \\
\text { gef.: C 40.55. H 5.35, N 21.82. }\end{array}$ \\
\hline
\end{tabular}


10.2.4.2. Synthese von 3,5-Bis-[bis-(1-methylimidazol-2-yl)-hydroxymethyl]-pyrazol (7a)<smiles>CC(C)C(O)(c1cc(C(O)(c2ccccc2)C(C)(C)C)[nH]n1)C(C)(C)C</smiles>

$\cdot \mathrm{HCl}$

$6 a$

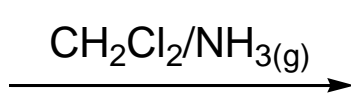

$7 a$

$17.80 \mathrm{~g}$ (36.7 mmol) des Hydrochlorides $6 \mathrm{a}$ werden in $250 \mathrm{ml}$ ammoniakalischer Dichlormethanlösung gelöst und für 3 Stunden bei Raumtemperatur gerührt. Das ausgefallene Ammoniumchlorid wird abfiltriert und die Lösung über $\mathrm{MgSO}_{4}$ getrocknet. Anschließend werden alle flüchtigen Bestandteile im Vakuum entfernt und man erhält 7a als weißen Feststoff.

Summenformel

Molgewicht

Ausbeute

${ }^{1} \mathrm{H}-\mathrm{NMR}$ (DMSO)

${ }^{13} \mathrm{C}-\mathrm{NMR}$ (DMSO)

MS (El) $m / z(\%)$

$\mathrm{IR}(\mathrm{KBr}) \tilde{v}\left(\mathrm{~cm}^{-1}\right)$

Schmelzpunkt
$\mathrm{C}_{21} \mathrm{H}_{24} \mathrm{~N}_{10} \mathrm{O}_{2}$

$448.49 \mathrm{~g}^{\prime} \mathrm{mol}^{-1}$

$3.38 \mathrm{~g}(7.6 \mathrm{mmol}, 21 \%)$.

12.52 (br, $1 \mathrm{H}, \mathrm{NH}), 7.11\left(\mathrm{~s}, 4 \mathrm{H}, \mathrm{H}^{\mathrm{Im} 4}\right), 6.73\left(\mathrm{~s}, 4 \mathrm{H}, \mathrm{H}^{\operatorname{Im} 5}\right)$, $5.80\left(\mathrm{~s}, 1 \mathrm{H}, \mathrm{H}^{\mathrm{Pz} 4}\right), 3.39$ (s, $\left.12 \mathrm{H}, \mathrm{NCH}_{3}\right)$.

$147.59\left(\mathrm{C}^{\mathrm{Pz} 3+5}\right), 125.02\left(\mathrm{C}^{\operatorname{lm} 4}\right), 123.62\left(\mathrm{C}^{\mathrm{Im} 5}\right), 103.59\left(\mathrm{C}^{\mathrm{Pz} 4}\right)$, $70.93(\mathrm{COH}), 33.93\left(\mathrm{NCH}_{3}\right)$.

$448\left(1,[\mathrm{M}]^{+}\right), 366\left(4,[\mathrm{M}-\mathrm{Im}]^{+}\right), 285\left(4,[\mathrm{M}-2 \mathrm{Im}]^{+}\right), 82(100$, $\left.[\mathrm{Im}]^{+}\right)$.

3418 (br), 3134 (s), 2924 (s), 1708 (m), 1637 (m), 1527 (w), 1487 (s), 1403 (s), 1283 (s), 1197 (m), $1129(\mathrm{~m}), 1038$ (s), 1021 (m), 937 (s), 898 (vs), 820 (w), 759 (m), 723 (w), 682 (w), $551(w), 448(w)$.

$154{ }^{\circ} \mathrm{C}$ (Zersetzung). 


\subsubsection{Ligandsynthese $\mathrm{HL}^{5}$}

10.2.5.1. 3,5-Bis-[bis-(1,4,5-trimethylimidazol-2-yl)-hydroxymethyl]-pyrazol Hydrochlorid (6b)

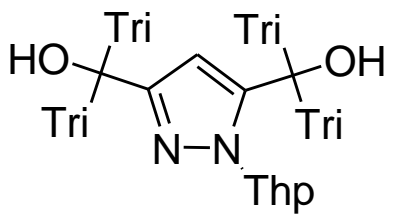

2b

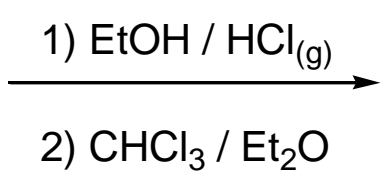

2) $\mathrm{CHCl}_{3} / \mathrm{Et}_{2} \mathrm{O}$

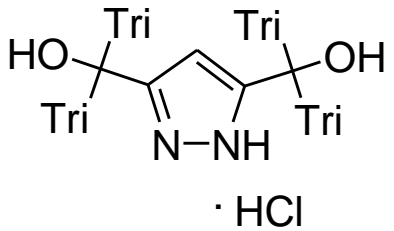

$6 b$

$18.55 \mathrm{~g}(28.8 \mathrm{mmol})$ von $\mathbf{2 b}$ werden in $50 \mathrm{ml}$ ethanolischer $\mathrm{HCl}$ aufgenommen und die Reaktionslösung über Nacht gerührt. Im Vakuum werden alle flüchtigen Bestandteile entfernt und der Rückstand in $100 \mathrm{ml} \mathrm{CHCl}_{3}$ aufgenommen. Für das Ausfällen des Hydrochlorides tropft man diese Chloroformlösung unter Rühren in $700 \mathrm{ml}$ Diethylether. Der entstandene weiße, flockige Niederschlag wird durch eine Fritte abgesaugt und im Hochvakuum getrocknet. Man erhält so einen hellgrauen Feststoff von $\mathbf{6 b}$.

Summenformel

Molgewicht

Ausbeute

${ }^{1} \mathrm{H}-\mathrm{NMR}$ (DMSO)

${ }^{13} \mathrm{C}-\mathrm{NMR}$ (DMSO)

MS $\left(\mathrm{FAB}^{+}\right) \mathrm{m} / \mathrm{z}(\%)$

$\mathrm{IR}(\mathrm{KBr}) \tilde{v}\left(\mathrm{~cm}^{-1}\right)$

Schmelzpunkt
$\mathrm{C}_{29} \mathrm{H}_{41} \mathrm{~N}_{10} \mathrm{O}_{2} \mathrm{Cl}$

$597.17{\mathrm{~g} \cdot \mathrm{mol}^{-1}}^{-1}$

$16.83 \mathrm{~g}(28.2 \mathrm{mmol}, 98 \%)$.

12.50-15.50 (br, $2 \mathrm{H}, \mathrm{NH}), 9.56(\mathrm{br}, 2 \mathrm{H}, \mathrm{OH}), 7.05(\mathrm{~s}, 1 \mathrm{H}$, $\mathrm{H}^{\mathrm{Pz} 4}$ ), $3.56\left(\mathrm{~s}, 12 \mathrm{H}, \mathrm{NCH}_{3}\right), 2.26\left(\mathrm{~s}, 12 \mathrm{H}, \mathrm{CH}_{3}\right), 2.21$ (s, $12 \mathrm{H}, \mathrm{CH}_{3}$ ).

$138.5\left(\mathrm{C}^{\mathrm{Im} 2}\right), 133.1\left(\mathrm{C}^{\mathrm{Im} 4}\right), 125.3\left(\mathrm{C}^{\mathrm{Im5}}\right), 107.5\left(\mathrm{C}^{\mathrm{Pz} 4}\right), 68.2$ $(\mathrm{COH}), 33.2\left(\mathrm{NCH}_{3}\right), 8.9\left(\mathrm{CH}_{3}\right), 8.3\left(\mathrm{CH}_{3}\right)$.

$561\left(100,[\mathrm{M}]^{+}\right), 451\left(6,\left[\mathrm{M}-\mathrm{Tri}^{+}\right), 341\left(68,[\mathrm{M}-2 \text { Tri }]^{+}\right), 110\right.$ (77, [Tri $\left.]^{+}\right)$.

3408 (br), 3051 (vs), 2960 (vs), 2886 (s), 2752 (s), 2084 (br), 1649 (vs), 1520 (s), 1444 (s), 1385 (m), 1323 (m), 1264 (m), $1224(\mathrm{~m}), 1192(\mathrm{~m}), 1074(\mathrm{~m}), 1018(\mathrm{w}), 996(\mathrm{w}), 898(\mathrm{~m})$, $830(\mathrm{~m}), 749(\mathrm{~m}), 566(\mathrm{w})$.

$198^{\circ} \mathrm{C}$ (Zersetzung). 
10.2.5.2. 3,5-Bis-[bis-(1,4,5-trimethylimidazol-2-yl)-hydroxymethyl]-pyrazol (7b)<smiles>[3H]C([3H])([3H])c1cc(C([3H])(O)[17F])n[nH]1</smiles>

$\cdot \mathrm{HCl}$

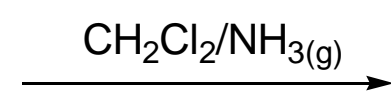

$7 b$

$16.83 \mathrm{~g}$ (28.2 mmol) des Hydrochlorides $6 \mathrm{~b}$ werden in $150 \mathrm{ml}$ ammoniakalischer Dichlormethanlösung gelöst und über Nacht gerührt. Die Reaktionslösung wird filtriert und über $\mathrm{MgSO}_{4}$ getrocknet. Danach wird das Lösungsmittel im Vakuum entfernt, das Produkt in wenig Dichlormethan aufgenommen und in $400 \mathrm{ml} n$-Hexan getropft. Der ausgefallene weiße Niederschlag wird durch eine Fritte abgesaugt und nach abschließendem Trocknen im Hochvakuum erhält man 7b als hellgrauen Feststoff.

\footnotetext{
Summenformel

$\mathrm{C}_{29} \mathrm{H}_{39} \mathrm{~N}_{10} \mathrm{O}_{2}$

Molgewicht

$560.71 \mathrm{~g}^{\prime} \mathrm{mol}^{-1}$

Ausbeute

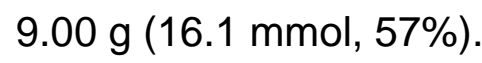

${ }^{1} \mathrm{H}-\mathrm{NMR}$ (DMSO)

$5.92\left(\mathrm{~s}, 1 \mathrm{H}, \mathrm{H}^{\mathrm{Pz} 4}\right), 3.18$ (s, $\left.12 \mathrm{H}, \mathrm{NCH}_{3}\right), 1.99$ (s, $\left.24 \mathrm{H}, \mathrm{CH}_{3}\right)$.

${ }^{13} \mathrm{C}-\mathrm{NMR}(\mathrm{DMSO})$

$148.7\left(\mathrm{C}^{\mathrm{Pz} 3+5}\right), 129.4\left(\mathrm{C}^{\operatorname{lm} 4}\right), 125.5\left(\mathrm{C}^{\mathrm{Im} 5}\right), 104.7\left(\mathrm{C}^{\mathrm{Pz} 4}\right), 69.9$ $(\mathrm{COH}), 31.5\left(\mathrm{NCH}_{3}\right), 11.7\left(\mathrm{CH}_{3}\right), 8.6\left(\mathrm{CH}_{3}\right)$.

MS (El) $m / z(\%)$

$560\left(5,[\mathrm{M}]^{+}\right), 450\left(36,\left[\mathrm{M}-\mathrm{Tri}^{+}\right), 340\left(36,\left[\mathrm{M}-2\right.\right.\right.$ Tri $\left.^{+}\right), 110$ $\left(100,\left[\right.\right.$ Tri $\left.^{+}\right)$.

$\mathrm{IR}(\mathrm{KBr}) \tilde{v}\left(\mathrm{~cm}^{-1}\right)$

3452 (br), 3098 (br), 2954 (m), 2923 (m), 2860 (m), 2761 (m), 1717 (s), 1671 (s), 1603 (m), 1445 (m), 1404 (m), 1301 (m), $1223(\mathrm{w}), 1138(\mathrm{w}), 1056(\mathrm{~m}), 997(\mathrm{~m}), 905(\mathrm{~s}), 806(\mathrm{~m})$, $765(w), 731(w), 653(w), 567(w)$.
} 


\subsubsection{Ligandsynthese $\mathrm{HL}^{6}$}

10.2.6.1. 3,5-Bis-[bis-(4,5-Diphenyl-1-methylimidazol-2-yl)-hydroxymethyl]-pyrazol Hydrochlorid (6c)

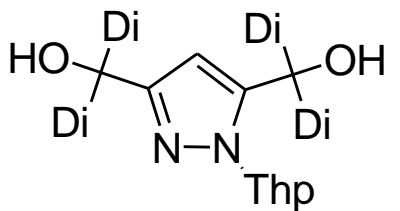

2c

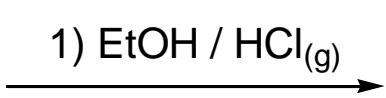

2) $\mathrm{MeOH} / \mathrm{Et}_{2} \mathrm{O}$

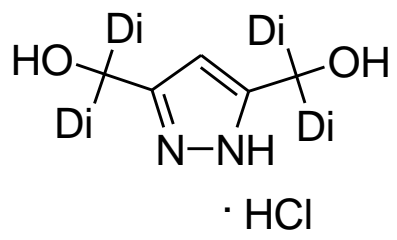

$6 c$

$11.32 \mathrm{~g}(9.9 \mathrm{mmol}) \mathbf{2 c}$ werden mit $50 \mathrm{ml}$ ethanolischer $\mathrm{HCl}$ versetzt und 5 Stunden bei Raumtemperatur gerührt. Das Lösungsmittel wird im Vakuum entfernt und der Rückstand in $20 \mathrm{ml}$ Methanol aufgenommen. Diese Lösung wird unter Rühren in $400 \mathrm{ml}$ Diethylether getropft. Der weiße flockige Niederschlag wird abgesaugt und im Hochvakuum getrocknet. Es wird $\mathbf{6 c}$ als hellgrauer Feststoff erhalten.

\begin{tabular}{|c|c|}
\hline Summenformel & $\mathrm{C}_{69} \mathrm{H}_{56} \mathrm{~N}_{10} \mathrm{O}_{2} \mathrm{Cl}$ \\
\hline Molgewicht & $1093.73 \mathrm{~g} \cdot \mathrm{mol}^{-1}$ \\
\hline Ausbeute & 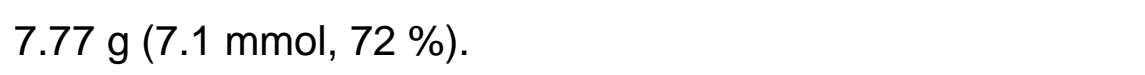 \\
\hline ํH-NMR (DMSO) & $\begin{array}{l}\left.\text { 7.21-7.55 (m, } 40 \mathrm{H}, \mathrm{H}^{\mathrm{Ph}}\right), 7.07\left(\mathrm{~s}, 1 \mathrm{H}, \mathrm{H}^{\mathrm{Pz} 4}\right), 3.50(\mathrm{~s}, 12 \mathrm{H} \text {, } \\
\left.\mathrm{NCH}_{3}\right) .\end{array}$ \\
\hline${ }^{13} \mathrm{C}-\mathrm{NMR}$ (DMSO) & 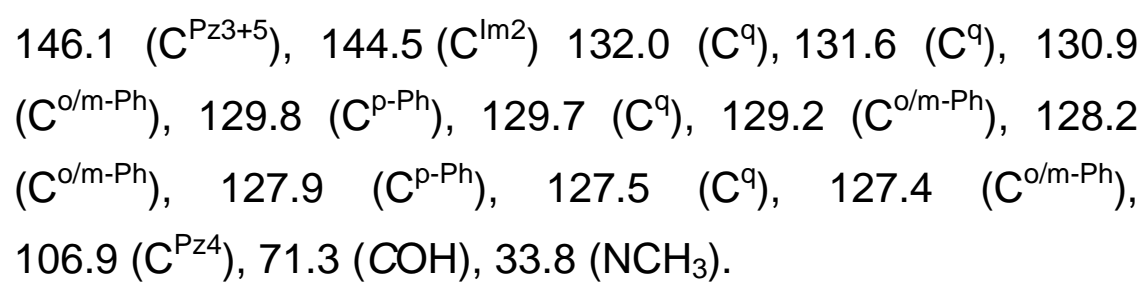 \\
\hline $\mathrm{MS}\left(\mathrm{FAB}^{+}\right) m / z(\%)$ & $\begin{array}{l}1057.5\left(100,[\mathrm{M}-\mathrm{Cl}]^{+}\right), 823.3\left(13, \quad[\mathrm{M}-\mathrm{Cl}-\mathrm{Di}]^{+}\right), \quad 589.2(97, \\
\left.[\mathrm{M}-\mathrm{Cl}-2 \mathrm{Di}]^{+}\right), 235.1\left(100,[\mathrm{Di}+\mathrm{H}]^{+}\right) .\end{array}$ \\
\hline $\mathrm{IR}(\mathrm{KBr}) \tilde{v}\left(\mathrm{~cm}^{-1}\right)$ & $\begin{array}{l}3407(\mathrm{br}), 3054(\mathrm{~m}), 2961(\mathrm{~m}), 2726(\mathrm{~m}), 1955(\mathrm{w}), 1827 \\
(\mathrm{br}), 1632(\mathrm{w}), 1596(\mathrm{w}), 1504(\mathrm{~m}), 1478(\mathrm{~m}), 1445(\mathrm{~s}), 1420 \\
(\mathrm{w}), 1324(\mathrm{w}), 1286(\mathrm{w}), 1232(\mathrm{w}), 1184(\mathrm{w}), 1126(\mathrm{w}), 1074 \\
(\mathrm{~m}), 1025(\mathrm{~m}), 972(\mathrm{w}), 902(\mathrm{~m}), 770(\mathrm{~s}), 697(\mathrm{vs}), 611(\mathrm{w}), \\
520(\mathrm{~m}) .\end{array}$ \\
\hline
\end{tabular}


Schmelzpunkt

Elementaranalyse $181^{\circ} \mathrm{C}$ (Zersetzung).

für $6 c \cdot 4.0 \mathrm{HCl}$ :

ber.: C 68.88, H 5.03, N 11.64,

gef.: C 68.78, H 5.13, N 11.60 .

10.2.6.2. 3,5-Bis-[bis-(4,5-Diphenyl-1-methylimidazol-2-yl)-hydroxymethyl]-pyrazol (7c)

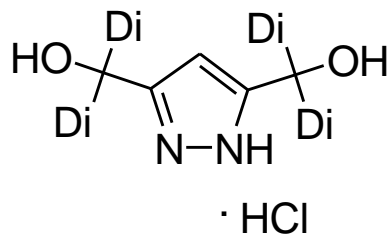

6c

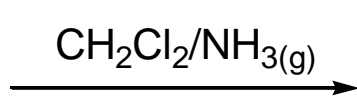

7c

$7.77 \mathrm{~g}(7.1 \mathrm{mmol}) 6 \mathrm{c}$ werden mit $150 \mathrm{ml}$ ammoniakalischem Dichlormethan versetzt und bei Raumtemperatur über Nacht gerührt. Das ausgefallene Ammoniumchlorid wird abfiltriert und die Lösung über $\mathrm{MgSO}_{4}$ getrocknet. Nach Entfernen des Lösungsmittels im Vakuum und Trocknen des Produktes im Hochvakuum erhält man einen hellgelben Feststoff von 7c.

Summenformel

Molgewicht

Ausbeute

${ }^{1} \mathrm{H}-\mathrm{NMR}\left(\mathrm{CDCl}_{3}\right)$

${ }^{13} \mathrm{C}-\mathrm{NMR}\left(\mathrm{CDCl}_{3}\right)$

$M S\left(F A B^{+}\right) \mathrm{m} / \mathrm{z}(\%)$

$\mathrm{IR}(\mathrm{KBr}) \tilde{v}\left(\mathrm{~cm}^{-1}\right)$
$\mathrm{C}_{69} \mathrm{H}_{55} \mathrm{~N}_{10} \mathrm{O}_{2}$

$1057.27 \mathrm{~g}^{-1} \mathrm{~mol}^{-1}$

$4.66 \mathrm{~g}(4.4 \mathrm{mmol}, 62 \%)$

7.10-7.45 (m, $\left.40 \mathrm{H}, \mathrm{H}^{\mathrm{Ph}}\right) 6.41\left(\mathrm{~s}, 1 \mathrm{H}, \mathrm{H}^{\mathrm{Pz} 4}\right), 3.31(\mathrm{~s}, 12 \mathrm{H}$, $\left.\mathrm{NCH}_{3}\right)$.

$146.3\left(C^{\text {im2 }}\right), \quad 135.2\left(C^{q}\right), \quad 134.2\left(C^{q}\right), 131.5\left(C^{q}\right), 130.9$ $\left(\mathrm{C}^{\mathrm{o} / \mathrm{m}-\mathrm{Ph}}\right), 130.5\left(\mathrm{C}^{\mathrm{q}}\right), 128.9\left(\mathrm{C}^{\mathrm{o} / \mathrm{m}-\mathrm{Ph}}\right), 128.7\left(\mathrm{C}^{\mathrm{p}-\mathrm{Ph}}\right), 127.9$ $\left(C^{\mathrm{o} / \mathrm{m}-\mathrm{Ph}}\right), \quad 126.5 \quad\left(\mathrm{C}^{\mathrm{o} / \mathrm{m}-\mathrm{Ph}}\right), \quad 126.2 \quad\left(\mathrm{C}^{\mathrm{p}-\mathrm{Ph}}\right), \quad 104.7\left(\mathrm{C}^{\mathrm{Pz} 4}\right)$, $70.1(\mathrm{COH}), 32.2\left(\mathrm{NCH}_{3}\right)$.

$1057.4\left(93,[\mathrm{M}+\mathrm{H}]^{+}\right), \quad 823.3 \quad\left(14, \quad[\mathrm{M}-\mathrm{Di}]^{+}\right), \quad 589.2(100$, $\left.[\mathrm{M}-2 \mathrm{Di}]^{+}\right), 235.2\left(76,[\mathrm{Di}+\mathrm{H}]^{+}\right)$.

3339 (br), $3167(\mathrm{~m}), 3052(\mathrm{~m}), 2951(\mathrm{~m}), 1955(\mathrm{w}), 1889(\mathrm{w})$, $1810(w), 1638(w), 1602(s), 1559(w), 1504(s), 1442(s)$, $1383(\mathrm{~m}), 1319(\mathrm{w}), 1265(\mathrm{w}), 1230(\mathrm{w}), 1130(\mathrm{~m}), 1058(\mathrm{~s})$, 
1025 (s), 966 (s), 899 (s), 772 (vs), 730 (m), 694 (vs), 522 (w).

Schmelzpunkt

$197^{\circ} \mathrm{C}$.

Elementaranalyse

für $7 \mathrm{c} \cdot 0.2 \mathrm{CH}_{2} \mathrm{Cl}_{2}$ :

ber.: C 77.44, H 5.20, N 13.05,

gef.: C 77.23, H 5.22, N 13.06 . 


\subsection{Komplexsynthesen}

\subsubsection{Darstellung von Kupfer(II)-Komplexen}

10.3.1.1. $\left[\mathrm{L}^{1} \mathrm{Cu}_{2}(\mathrm{OH})(\mathrm{OAc})_{2}\right](8)$

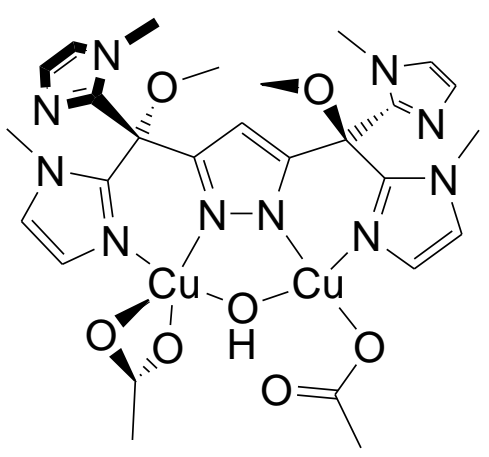

Zu einer Lösung von $1.00 \mathrm{~g}(2.09 \mathrm{mmol}) \mathrm{HL}^{1}$ in $50 \mathrm{ml}$ Methanol werden unter Rühren zwei Äquivalente $(471 \mathrm{mg}, 4.18 \mathrm{mmol}$ ) KO'Bu zugegeben. Nach etwa 15 Minuten gibt man zwei Äquivalente (838 mg, $4.18 \mathrm{mmol}) \mathrm{Cu}(\mathrm{OAc})_{2} \mathrm{H}_{2} \mathrm{O}$ dazu und lässt die Reaktionslösung über Nacht bei Raumtemperatur rühren. Anschließend entfernt man das Lösungsmittel im Vakuum, nimmt den Rückstand in $20 \mathrm{ml}$ Wasser auf und reinigt das Rohprodukt säulenchromatographisch an Sephadex SP C25. Den Rückstand der tiefblauen Fraktion nimmt man in $20 \mathrm{ml}$ Dichlormethan auf, filtriert und überschichtet diese Lösung in einem Schlenkrohr mit $80 \mathrm{ml}$ n-Hexan. Die erhaltenen blauvioletten Kristalle sind analysenrein und für die Röntgenstrukturanalyse geeignet.

$\begin{array}{ll}\text { Summenformel } & \mathrm{C}_{27} \mathrm{H}_{34} \mathrm{Cu}_{2} \mathrm{~N}_{10} \mathrm{O}_{7} \\ \text { Molgewicht } & 737.72 \mathrm{~g} / \mathrm{mol} \\ \text { Ausbeute } & 341 \mathrm{mg}(0.46 \mathrm{mmol}, 23 \%) \\ \mathrm{IR}(\mathrm{KBr}) \tilde{\mathrm{v}}\left(\mathrm{cm}^{-1}\right) & 3406(\mathrm{br}), 3137(\mathrm{~s}), 3051(\mathrm{~m}), 2956(\mathrm{~s}), 2830(\mathrm{w}), 2544(\mathrm{w}), \\ & 2360(\mathrm{w}), 2303(\mathrm{w}), 1611(\mathrm{vs}), 1499(\mathrm{~m}), 1383(\mathrm{~s}), 1326(\mathrm{~m}), \\ & 1279(\mathrm{~m}), 1227(\mathrm{w}), 1185(\mathrm{w}), 1159(\mathrm{w}), 1136(\mathrm{w}), 1072(\mathrm{~s}), \\ & 1013(\mathrm{w}), 978(\mathrm{~m}), 903(\mathrm{~m}), 849(\mathrm{w}), 806(\mathrm{w}), 754(\mathrm{~m}), 725 \\ & (\mathrm{~m}), 696(\mathrm{~m}), 671(\mathrm{~m}), 466(\mathrm{w}) . \\ & 1141.1\left(63,\left[(\mathrm{HL})_{2} \mathrm{Cu}_{3}\right]^{+}\right), 719.0\left(12,\left[\mathrm{~L}^{1} \mathrm{Cu}_{2}(\mathrm{OAc})_{2}\right]^{+}\right), 690.9 \\ & \left(34,\left[\mathrm{~L}^{1} \mathrm{Cu}_{2}(\mathrm{HCOO})\right]^{+}\right), 659.9\left(46,\left[\mathrm{~L}^{1} \mathrm{Cu}_{2}(\mathrm{OAc})\right]^{+}\right), 601.0(100, \\ & \left.\left[\mathrm{L}^{1} \mathrm{Cu}_{2}\right]^{+}\right), 539.0\left(29,\left[\mathrm{HL}{ }^{1} \mathrm{Cu}\right]^{+}\right) .\end{array}$




$\begin{array}{ll}\text { UV/Vis }\left(\mathrm{CH}_{2} \mathrm{Cl}_{2}\right) \lambda(\varepsilon) & 559(120), 332 \text { (2860), } 230 \text { (36000). } \\ \text { UV/Vis }(\mathrm{KBr}) \lambda & 593,337,239 . \\ \text { Elementaranalyse } & \text { für } 8 \cdot 1.65 \mathrm{CH}_{2} \mathrm{Cl}_{2} \\ & \text { ber.: C } 39.20, \mathrm{H} 4.28, \mathrm{~N} 15.96 \\ & \text { gef.: C 39.14, H 4.49, N 15.71. }\end{array}$

10.3.1.2. $\left[\left\{\mathrm{L}^{1} \mathrm{Cu}_{2}(\mathrm{OAc})_{2}\right\}_{2} \mathrm{O}\right](9)$

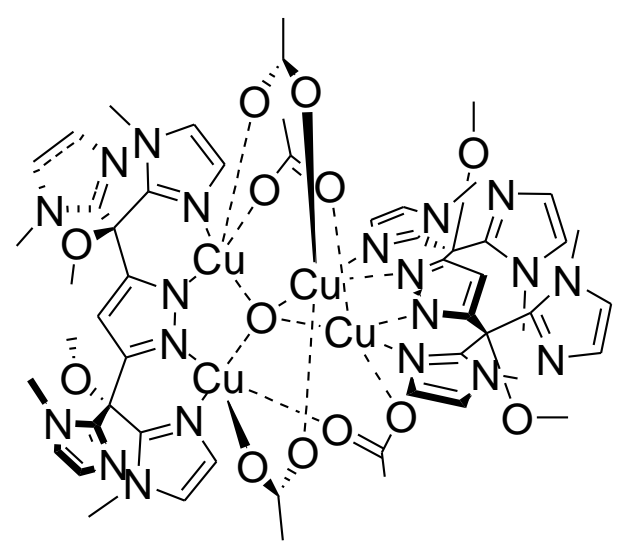

Zu einer Lösung von $300 \mathrm{mg}(0.63 \mathrm{mmol}) \mathrm{HL}^{1}$ in $50 \mathrm{ml}$ Methanol werden unter Rühren zwei Äquivalente (141 mg, $1.26 \mathrm{mmol}$ ) $\mathrm{KO}^{\mathrm{t} B u}$ zugegeben. Nach etwa 15 Minuten gibt man zwei Äquivalente (251 mg, $1.26 \mathrm{mmol}) \mathrm{Cu}(\mathrm{OAc})_{2} \cdot \mathrm{H}_{2} \mathrm{O}$ dazu und lässt die Reaktionslösung 5 Stunden bei Raumtemperatur rühren. Anschließend entfernt man das Lösungsmittel im Vakuum, nimmt den Rückstand in $20 \mathrm{ml}$ Dichlormethan auf, filtriert und entfernt erneut das Lösungsmittel im Vakuum. Danach wird der dunkelblaue Feststoff in Toluol $(70 \mathrm{ml})$ suspendiert und Eisessig $(18 \mu \mathrm{l}, 0.32 \mathrm{mmol})$ zugegeben. Man erhitzt mit Wasserabscheider zum Sieden. Nach etwa 3 Stunden filtriert man die heiße Suspension, trocknet den türkisfarbenen Feststoff im Hochvakuum und schleust das Rohprodukt in die Glovebox ein. Dort wird der Feststoff in wenig Dichlormethan gelöst und mit $n$-Hexan überschichtet. Es bildet sich eine türkisfarbener Feststoff mit ganz wenigen Kristallen, die für die Röntgenstrukturanalyse geeignet sind.

Summenformel

Molgewicht
$\mathrm{C}_{54} \mathrm{H}_{66} \mathrm{Cu}_{4} \mathrm{~N}_{20} \mathrm{O}_{13}$

$1457.43 \mathrm{~g} / \mathrm{mol}$ 
$\mathrm{IR}(\mathrm{KBr}) \tilde{v}\left(\mathrm{~cm}^{-1}\right)$

10.3.1.3. $\left[\mathrm{L}^{1} \mathrm{Cu}_{2}(\mathrm{OH})\left(\mathrm{SO}_{4}\right)(\mathrm{MeOH})\right]_{2}(\mathbf{1 0})$

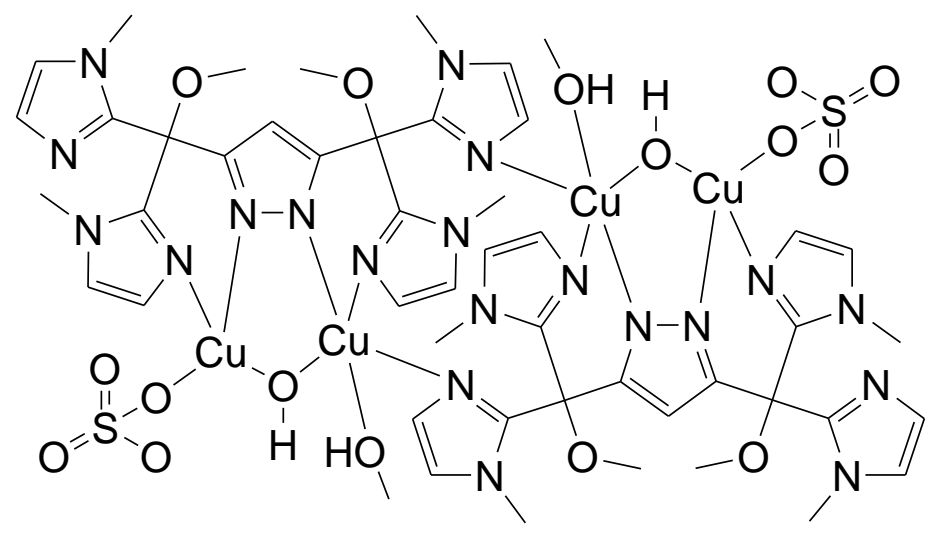

Zu einer Lösung von $250 \mathrm{mg}(0.53 \mathrm{mmol}) \mathrm{HL}^{1}$ in $50 \mathrm{ml}$ Methanol werden unter Rühren zwei Äquivalente (118 mg, $1.05 \mathrm{mmol}$ ) KO'Bu zugegeben. Nach etwa 15 Minuten gibt man zwei Äquivalente (262 mg, $1.05 \mathrm{mmol}) \mathrm{Cu}\left(\mathrm{SO}_{4}\right)_{2} \cdot 5 \mathrm{H}_{2} \mathrm{O}$ dazu und lässt die Reaktionslösung $5 \mathrm{~h}$ bei Raumtemperatur rühren. Anschließend entfernt man das Lösungsmittel im Vakuum. Den blauen Rückstand nimmt man in $12 \mathrm{ml}$ Methanol auf und filtriert in ein Reagenzglas. Das Reagenzglas stellt man in ein mit $50 \mathrm{ml}$ Diethylether gefülltes Schlenkrohr. Die erhaltenen blauen Kristalle sind analysenrein und für die Röntgenstrukturanalyse geeignet.

Summenformel

Molgewicht

Ausbeute

$\mathrm{IR}(\mathrm{KBr}) \tilde{v}\left(\mathrm{~cm}^{-1}\right)$
$\mathrm{C}_{48} \mathrm{H}_{64} \mathrm{Cu}_{4} \mathrm{~N}_{20} \mathrm{O}_{16} \mathrm{~S}_{2}$

$1495.47 \mathrm{~g} / \mathrm{mol}$

$41.4 \mathrm{mg}(0.03 \mathrm{mmol}, 10 \%)$

3400 (br), $3128(w), 2954(w), 2933(w), 2826(m), 1644(b r)$,

$1544(w), 1489(m), 1457(w), 1440(w), 1350(m), 1279(m)$, 
1163 (s), 1134 (s), 1072 (s), 1027 (s), 978 (m), 947 (m), 910 (m), $802(w), 762(w), 728(m), 613(m), 554(w)$.

$\mathrm{MS}\left(\mathrm{FAB}^{+}\right) \mathrm{m} / \mathrm{z}(\%)$ $1413\left(45,\left[\mathrm{~L}_{2}^{1} \mathrm{Cu}_{4}(\mathrm{OH})\left(\mathrm{SO}_{4}\right)_{2}\right]^{+}\right), 1396\left(56,\left[\mathrm{~L}_{2}^{1} \mathrm{Cu}_{4}\left(\mathrm{SO}_{4}\right)_{2}\right]^{+}\right)$, $1302\left(18,\left[\mathrm{~L}_{2}^{1} \mathrm{Cu}_{2}\left(\mathrm{SO}_{4}\right)_{2}(\mathrm{MeOH})\right]^{+}\right), 699\left(100,\left[\mathrm{~L}^{1} \mathrm{Cu}_{2}\left(\mathrm{SO}_{4}\right)\right]^{+}\right)$.

UV/Vis $(\mathrm{MeOH}) \lambda(\varepsilon)$ 228 (44400), 328 (4920), 580 (211).

UV/Vis (KBr) $\lambda$ 582, 335, 238.

Elementaranalyse für $10 \cdot 8 \mathrm{H}_{2} \mathrm{O}$ ber.: C 35.07, H 4.61, N 17.78 gef.: C 35.46, H 4.33, N 17.49 .

10.3.1.4. $\left[\mathrm{L}^{1} \mathrm{Cu}_{2}(\mathrm{OH})\left(\mathrm{NO}_{3}\right)(\mathrm{MeOH})\right]_{2}\left(\mathrm{NO}_{3}\right)_{2}(\mathbf{1 1} \mathbf{a})$

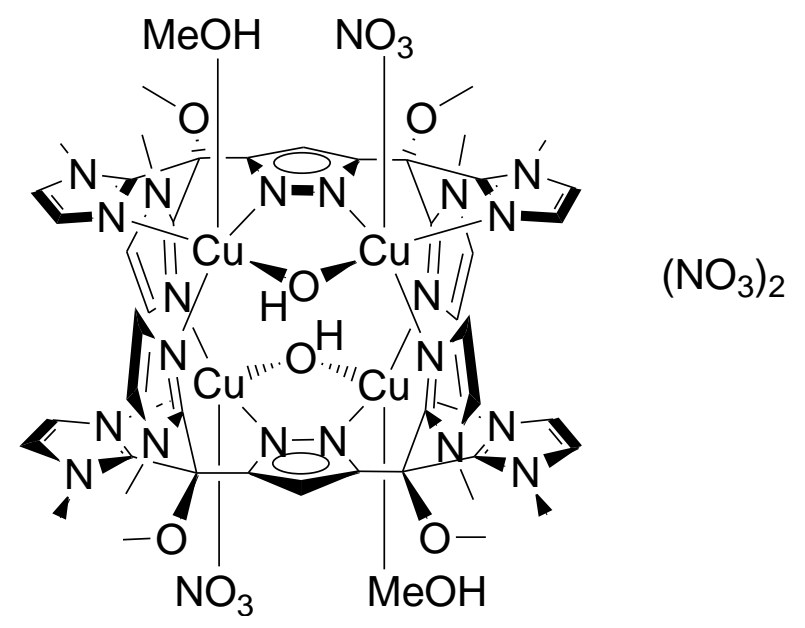

$\mathrm{Zu}$ einer Lösung von $250 \mathrm{mg}(0.53 \mathrm{mmol}) \mathrm{HL}^{1}$ in $40 \mathrm{ml}$ Methanol werden unter Rühren zwei Äquivalente (118 mg, $1.05 \mathrm{mmol}$ ) KO'Bu zugegeben. Nach etwa 15 Minuten gibt man zwei Äquivalente (254 mg, $1.05 \mathrm{mmol}) \mathrm{Cu}\left(\mathrm{NO}_{3}\right)_{2} \cdot 3 \mathrm{H}_{2} \mathrm{O}$ dazu und lässt die Reaktionslösung 5 Stunden bei Raumtemperatur rühren. Anschließend entfernt man das Lösungsmittel im Vakuum. Den blauen Rückstand nimmt man in $12 \mathrm{ml}$ Methanol auf und filtriert in ein Reagenzglas. Die durch Dampfdiffusion mit Diethylether erhaltenen blauen Kristalle sind analysenrein und für die Röntgenstrukturanalyse geeignet.

Summenformel

Molgewicht
$\mathrm{C}_{48} \mathrm{H}_{64} \mathrm{Cu}_{4} \mathrm{~N}_{24} \mathrm{O}_{20}$

$1551.37 \mathrm{~g} / \mathrm{mol}$ 


$\begin{array}{ll}\text { Ausbeute } & 95 \mathrm{mg}(0.06 \mathrm{mmol}, 23 \%) \\ \mathrm{IR}(\mathrm{KBr}) \tilde{v}\left(\mathrm{~cm}^{-1}\right) & 3430(\mathrm{br}), 3127(\mathrm{~s}), 2956(\mathrm{~m}), 2937(\mathrm{~m}), 2834(\mathrm{~m}), 1635(\mathrm{~s}), \\ & 1546(\mathrm{w}), 1486(\mathrm{~m}), 1377(\mathrm{vs}), 1351(\mathrm{w}), 1278(\mathrm{w}), 1225(\mathrm{~m}), \\ & 1159(\mathrm{w}), 1082(\mathrm{~s}), 1042(\mathrm{~m}), 977(\mathrm{~s}), 910(\mathrm{~s}), 827(\mathrm{~m}), 766 \\ & (\mathrm{~m}), 732(\mathrm{~m}), 684(\mathrm{~m}), 557(\mathrm{w}) . \\ & 1282 \quad\left(11, \quad\left[\mathrm{~L}_{2} \mathrm{Cu}_{4}(\mathrm{OH})\left(\mathrm{NO}_{3}\right)\right]^{+}\right), \\ \mathrm{MS}\left(\mathrm{FAB}^{+}\right) \mathrm{m} / \mathrm{z}(\%) & \left.\left[\mathrm{L}_{2} \mathrm{Cu}_{3}(\mathrm{OH})\left(\mathrm{NO}_{3}\right)_{2}(\mathrm{MeOH})_{2}\right]^{+}\right), \\ & \left.\left[\mathrm{L}_{2}{ }_{2} \mathrm{Cu}_{3}(\mathrm{OH})_{2}\left(\mathrm{NO}_{3}\right)_{2}(\mathrm{MeOH})_{2}\right]^{+}\right) . \\ & 330(5200), 578(238) .\end{array}$

10.3.1.5. $\left[\mathrm{L}^{1} \mathrm{Cu} 2(\mathrm{OH})\left(\mathrm{NO}_{3}\right)\left(\mathrm{H}_{2} \mathrm{O}\right)\right]_{2}\left(\mathrm{NO}_{3}\right)_{2}(\mathbf{1 1 b})$

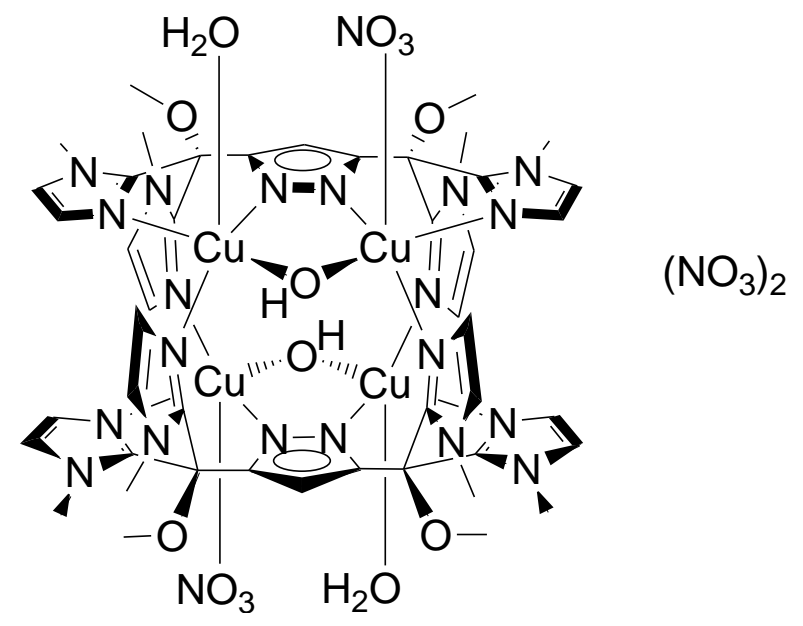

$\mathrm{Zu}$ einer Lösung von $1.00 \mathrm{~g}(2.10 \mathrm{mmol}) \mathrm{HL}^{1}$ in $200 \mathrm{ml}$ Methanol werden unter Rühren zwei Äquivalente (471 mg, $4.20 \mathrm{mmol}$ ) $\mathrm{KO}^{\dagger} \mathrm{Bu}$ zugegeben. Nach etwa 15 Minuten gibt man zwei Äquivalente (1.01 g, $4.20 \mathrm{mmol}) \mathrm{Cu}\left(\mathrm{NO}_{3}\right)_{2} \cdot 3 \mathrm{H}_{2} \mathrm{O}$ dazu und lässt die Reaktionslösung 5 Stunden bei Raumtemperatur rühren. Anschließend entfernt man das Lösungsmittel im Vakuum. Den blauen Rückstand nimmt man in $20 \mathrm{ml}$ Wasser auf und lässt das Lösungsmittel sehr langsam verdampfen. Die erhaltenen blauen Kristalle sind analysenrein und für die Röntgenstrukturanalyse geeignet.

Summenformel

Molgewicht

Ausbeute
$\mathrm{C}_{46} \mathrm{H}_{60} \mathrm{Cu}_{4} \mathrm{~N}_{24} \mathrm{O}_{20}$

$1523.32 \mathrm{~g} / \mathrm{mol}$

$0.98 \mathrm{~g}(0.64 \mathrm{mmol}, 62 \%)$ 
$\operatorname{IR}(\mathrm{KBr}) \quad\left(\mathrm{cm}^{-1}\right)$

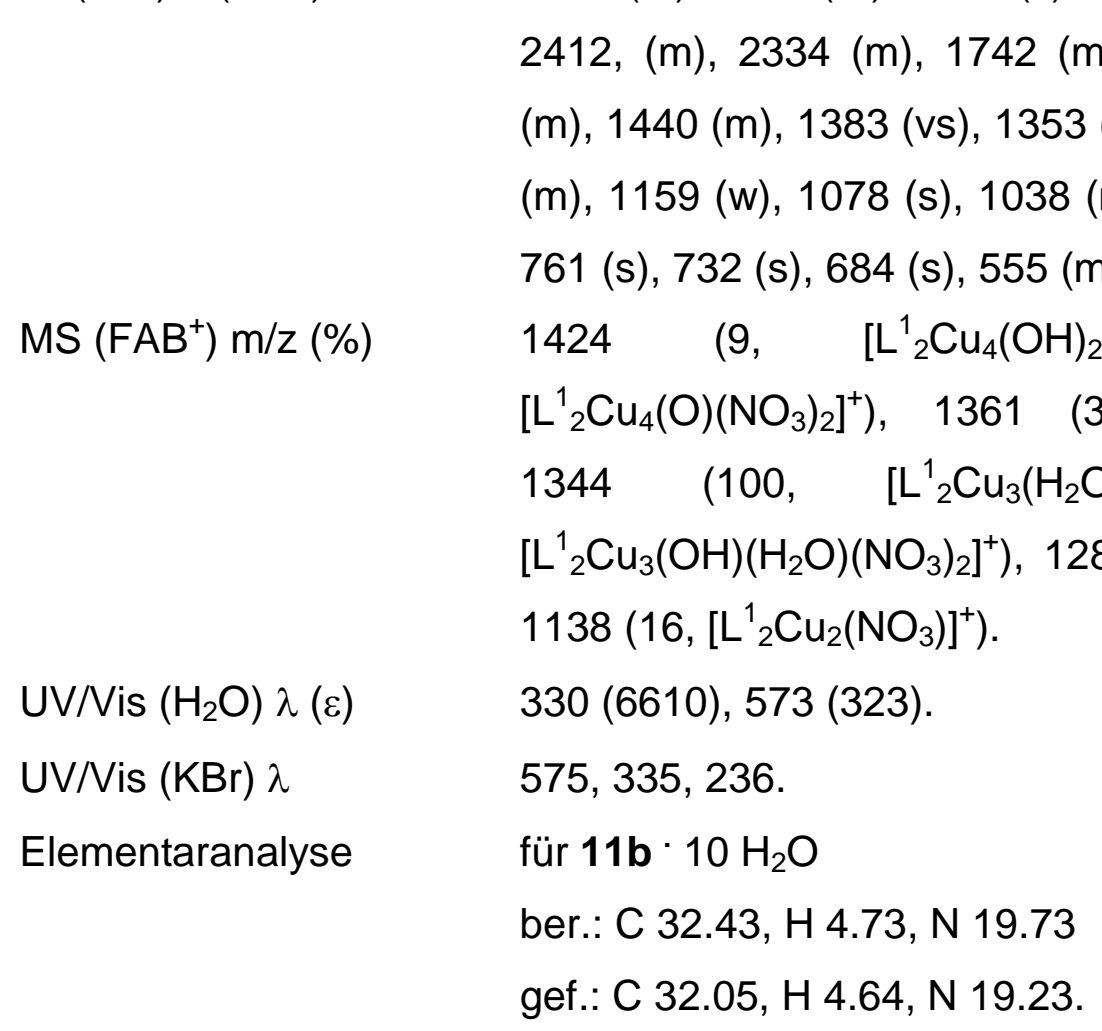

10.3.1.6. $\left[\mathrm{L}^{1} \mathrm{Cu}_{2}(\mathrm{OH})\left(\mathrm{ClO}_{4}\right)_{2}\right]_{2}(\mathbf{1 2})$

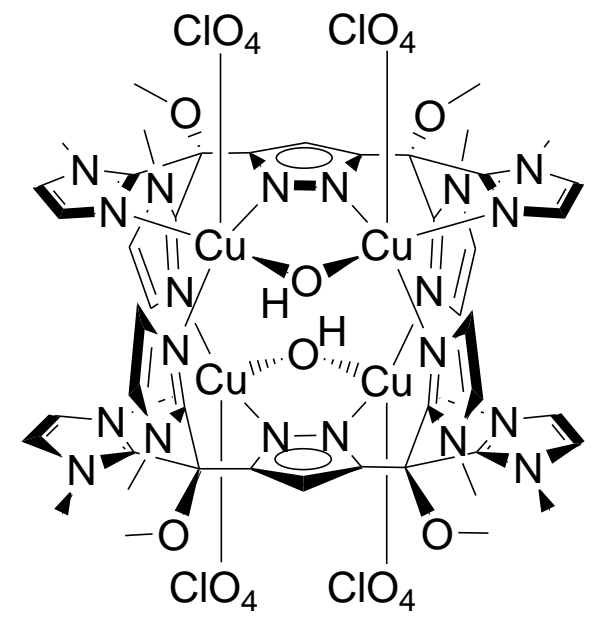

$\mathrm{Zu}$ einer Lösung von $225 \mathrm{mg}(0.47 \mathrm{mmol}) \mathrm{HL}^{1}$ in $40 \mathrm{ml}$ trockenem und entgasten Propionitril wird unter Schutzgas und Rühren ein Äquivalent (53 mg, $0.47 \mathrm{mmol}$ ) KO'Bu zugegeben. Nach etwa 15 Minuten entgast man die entstandene Reaktionslösung erneut und gibt zwei Äquivalente (309 mg, $0.94 \mathrm{mmol})\left[\mathrm{Cu}\left(\mathrm{CH}_{3} \mathrm{CN}\right)_{4}\right] \mathrm{ClO}_{4}$ hinzu. Nach 
ca. 30 Minuten hat sich die Lösung von hellgelb über tieflila nach blau verfärbt. Das Lösungsmittel wird im Hochvakuum entfernt und der verbleibende Rückstand in wenig Acetonitril aufgenommen. Nach Filtration schichtet man die grüne Lösung auf Chloroform. Die erhaltenen violetten Kristalle sind analysenrein und für die Röntgenstrukturanalyse geeignet.

$\mathrm{Zu}$ einer Lösung von $250 \mathrm{mg}(0.53 \mathrm{mmol}) \mathrm{HL}^{1}$ in $50 \mathrm{ml}$ Acetonitril werden unter Rühren zwei Äquivalente (118 mg, $1.05 \mathrm{mmol}$ ) KO'Bu zugegeben. Nach etwa 15 Minuten gibt man zwei Äquivalente (389 mg, $1.05 \mathrm{mmol}$ ) $\mathrm{Cu}\left(\mathrm{ClO}_{4}\right)_{2} \cdot 6 \mathrm{H}_{2} \mathrm{O}$ dazu und lässt die Reaktionslösung 5 Stunden bei Raumtemperatur rühren. Anschließend entfernt man das Lösungsmittel im Vakuum. Den blauen Rückstand nimmt man in $20 \mathrm{ml}$ Acetonitril auf, filtriert diese Lösung in ein Schlenkrohr und überschichtet vorsichtig mit $75 \mathrm{ml}$ Diethylether. Die erhaltenen blauen Kristalle sind analysenrein und für die Röntgenstrukturanalyse geeignet.

Summenformel

Molgewicht

Ausbeute

$\mathrm{IR}(\mathrm{KBr}) \tilde{v}\left(\mathrm{~cm}^{-1}\right)$

$\mathrm{MS}\left(\mathrm{FAB}^{+}\right) \mathrm{m} / \mathrm{z}(\%)$

UV/Vis $(\mathrm{MeCN}) \lambda(\varepsilon)$

UV/Vis $(\mathrm{KBr}) \lambda$

Elementaranalyse
$\mathrm{C}_{46} \mathrm{H}_{56} \mathrm{Cl}_{4} \mathrm{Cu}_{4} \mathrm{~N}_{20} \mathrm{O}_{22}$

$1637.07 \mathrm{~g} / \mathrm{mol}$

$50 \mathrm{mg}(0.03 \mathrm{mmol}, 6 \%)$

$3616(\mathrm{~m}), 3450(\mathrm{br}), 3130(\mathrm{~m}), 2965(\mathrm{~m}), 2879(\mathrm{w}), 2838(\mathrm{w})$, $2016(\mathrm{w}), 1623(\mathrm{w}), 1548(\mathrm{~m}), 1489(\mathrm{~s}), 1439(\mathrm{~m}), 1354(\mathrm{~m})$, 1288 (m), 1227 (m), 1089 (vs), 1042 (m), 978 (s), 910 (s), $801(\mathrm{w}), 756(\mathrm{~m}), 731(\mathrm{~m}), 684(\mathrm{~m}), 621$ (s), $560(\mathrm{w}), 483$ (W).

$1537.6 \quad\left(46, \quad\left[\mathrm{~L}_{2} \mathrm{Cu}_{4}(\mathrm{OH})_{2}\left(\mathrm{ClO}_{4}\right)_{3}\right]^{+}\right), \quad 719.1 \quad$ (100, $\left.\left[\mathrm{LCu}_{2}(\mathrm{OH})\left(\mathrm{ClO}_{4}\right)\right]^{+}\right)$.

566 (296), 336 (6350), 227 (84400).

579, 336, 237.

für $12 \cdot \mathrm{CHCl}_{3}$

ber.: C 32.10, H 3.38, N 15.93

gef.: C 32.06, H 3.45, N 15.86 . 
10.3.1.7. $\left[\mathrm{L}^{1} \mathrm{Cu}_{2}(\mathrm{OH})\left(\mathrm{BF}_{4}\right)\left(\mathrm{CHCl}_{3}\right)\right]_{2}\left(\mathrm{BF}_{4}\right)_{2}(\mathbf{1 3})$

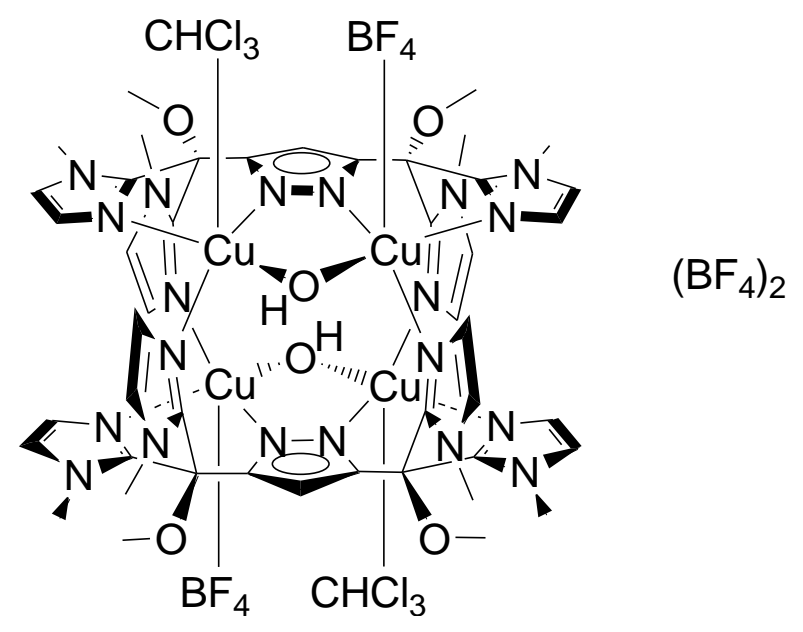

Zu einer Lösung von $250 \mathrm{mg}(0.53 \mathrm{mmol}) \mathrm{HL}^{1}$ in $50 \mathrm{ml}$ Methanol werden unter Rühren zwei Äquivalente (118 mg, $1.05 \mathrm{mmol}$ ) KO'Bu zugegeben. Nach etwa 15 Minuten gibt man zwei Äquivalente (327 mg, $1.05 \mathrm{mmol}) \mathrm{Cu}\left(\mathrm{BF}_{4}\right)_{2} \cdot 4 \mathrm{H}_{2} \mathrm{O}$ dazu und lässt die Reaktionslösung $5 \mathrm{~h}$ bei Raumtemperatur rühren. Anschließend entfernt man das Lösungsmittel im Vakuum. Den blauen Rückstand nimmt man in $20 \mathrm{ml}$ Acetonitril auf, filtriert und schichtet diese Lösung in einem Schlenkrohr vorsichtig auf $60 \mathrm{ml}$ Chloroform. Die erhaltenen blauen Kristalle sind analysenrein und für die Röntgenstrukturanalyse geeignet.

Summenformel

Molgewicht

Ausbeute

$\mathrm{IR}(\mathrm{KBr}) \tilde{v}\left(\mathrm{~cm}^{-1}\right)$

MS $\left(\mathrm{FAB}^{+}\right) \mathrm{m} / \mathrm{z}(\%)$

UV/Vis $(\mathrm{MeCN}) \lambda(\varepsilon)$

UV/Vis $(\mathrm{KBr}) \lambda$
$\mathrm{C}_{48} \mathrm{H}_{58} \mathrm{~B}_{4} \mathrm{Cl}_{6} \mathrm{Cu}_{4} \mathrm{~F}_{16} \mathrm{~N}_{20} \mathrm{O}_{6}$

$1825.17 \mathrm{~g} / \mathrm{mol}$

$62 \mathrm{mg}(0.03 \mathrm{mmol}, 5 \%)$

$3623(\mathrm{~m}), 3570(\mathrm{~m}), 3431(\mathrm{br}), 3156(\mathrm{~m}), 3136(\mathrm{~m}), 2959$

$(\mathrm{m}), 2939(\mathrm{~m}), 2836(\mathrm{~m}), 1634(\mathrm{~m}), 1548(\mathrm{~m}), 1492(\mathrm{~s}), 1440$

(m), $1355(\mathrm{~m}), 1290(\mathrm{~m}), 1228(\mathrm{~m}), 1163(\mathrm{~s}), 1061$ (vs), 977

(s), $909(\mathrm{~s}), 811(\mathrm{w}), 757(\mathrm{~m}), 731(\mathrm{~m}), 686(\mathrm{~m}), 630(\mathrm{w}), 560$ (w), $521(\mathrm{~m}), 480(\mathrm{w})$.

$1498.6 \quad\left(21, \quad\left[\mathrm{~L}_{2} \mathrm{Cu}_{4}(\mathrm{OH})_{2}\left(\mathrm{BF}_{4}\right)_{3}\right]^{+}\right), \quad 705.8$

$(54$, $\left.\left[\mathrm{LCu}_{2}(\mathrm{OH})\left(\mathrm{BF}_{4}\right)\right]^{+}\right)$.

561 (207), 334 (4890), 227 (67500).

575, 334, 237. 
Elementaranalyse

für 13

ber.: C 34.83, H 3.56, N 17.66

gef.: C 34.47, H 3.71, N 18.00 .

10.3.1.8. $\left[\mathrm{L}^{1} \mathrm{Cu}_{2}(\mathrm{OH})\left(\mathrm{CH}_{3} \mathrm{CN}\right)_{2}\right]_{2}\left(\mathrm{PF}_{6}\right)_{4}(\mathbf{2 8})$

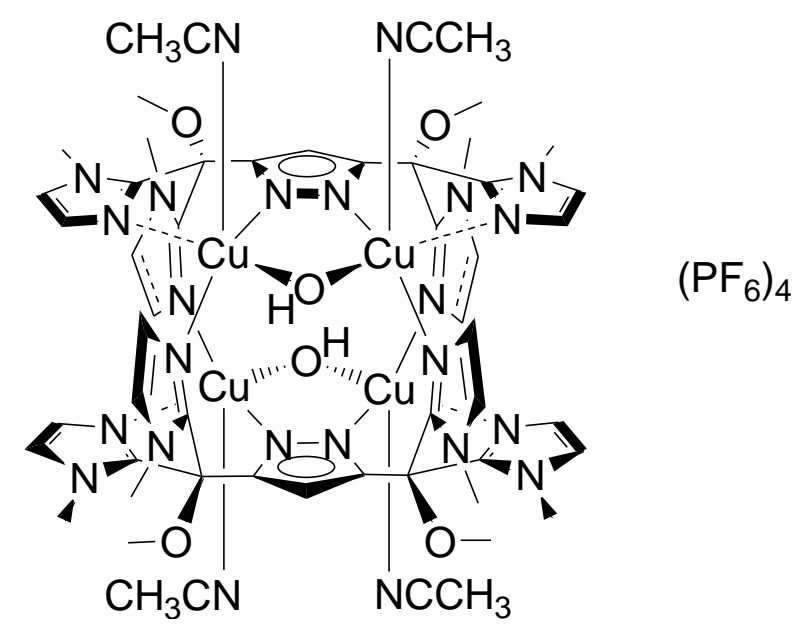

In der Glovebox werden $300 \mathrm{mg}(0.63 \mathrm{mmol}) \mathrm{HL}^{1}$ und ein Äquivalent $(71 \mathrm{mg}$, $0.63 \mathrm{mmol}) \mathrm{KO}^{\mathrm{t}} \mathrm{Bu}$ in $20 \mathrm{ml}$ Aceonitril gelöst. Nach etwa 10 Minuten gibt man eine Lösung von zwei Äquivalenten (470 mg, $1.26 \mathrm{mmol})\left[\mathrm{Cu}\left(\mathrm{CH}_{3} \mathrm{CN}\right)_{4}\right] \mathrm{PF}_{6}$ in $10 \mathrm{ml}$ Acetonitril dazu. Die Reaktionslösung wird 30 Minuten bei Raumtemperatur gerührt. Anschließend wird das Reaktionsgefäß ausgeschleust, Vakuum angelegt und mit $\mathrm{CO}_{2}$ unter Rühren belüftet. Die Lösung lässt man über Nacht rühren. Danach überführt man die Lösung in ein Schlenkrohr, überschichtet mit $20 \mathrm{ml}$ eines 1:1-Gemisches Acetonitril:Diethylether und abschließend noch mit $80 \mathrm{ml}$ Diethylether. Die erhaltenen violetten Kristalle sind analysenrein und für die Röntgenstrukturanalyse geeignet.

Summenformel

Molgewicht

Ausbeute

$\operatorname{IR}(\mathrm{KBr}) \tilde{v}\left(\mathrm{~cm}^{-1}\right)$
$\mathrm{C}_{54} \mathrm{H}_{68} \mathrm{Cu}_{4} \mathrm{~F}_{24} \mathrm{~N}_{24} \mathrm{O}_{6} \mathrm{P}_{4}$

$1983.33 \mathrm{~g} / \mathrm{mol}$

$230 \mathrm{mg}(0.12 \mathrm{mmol}, 37 \%)$

$3675(\mathrm{~m}), 3632(\mathrm{~m}), 3420(\mathrm{br}), 3175(\mathrm{~m}), 3150(\mathrm{~m}), 2946$

(m), $2834(\mathrm{~m}), 1627(\mathrm{~m}), 1548(\mathrm{~m}), 1488(\mathrm{~s}), 1439(\mathrm{~m}), 1354$

(m), $1288(\mathrm{~m}), 1226(\mathrm{~m}), 1162(\mathrm{~s}), 1087(\mathrm{vs}), 981(\mathrm{~s}), 911$ 
(s), 836 (vs), 758 (s), 730 (s), 685 (s), 630 (w), 557 (vs), 469 (w).

MS (FAB $) \mathrm{m} / \mathrm{z}(\%)$

UV/Vis $(\mathrm{MeCN}) \lambda(\varepsilon)$

Elementaranalyse
$1673.2 \quad\left(94, \quad\left[\mathrm{~L}_{2} \mathrm{Cu}_{4}\left(\mathrm{OH}_{2}\right)_{2}\left(\mathrm{PF}_{6}\right)_{3}\right]^{+}\right), \quad 1528.2$ $\left.\left[\mathrm{L}_{2} \mathrm{Cu}_{4}(\mathrm{OH})_{2}\left(\mathrm{PF}_{6}\right)_{2}\right]^{+}\right), \quad 1511.2 \quad\left(23, \quad\left[\mathrm{~L}_{2} \mathrm{Cu}_{4}(\mathrm{OH})\left(\mathrm{PF}_{6}\right)_{2}\right]^{+}\right)$, $1384.2\left(82,\left[\mathrm{~L}_{2} \mathrm{Cu}_{4}(\mathrm{OH})_{2}\left(\mathrm{PF}_{6}\right)\right]^{+}\right), 1239.1\left(56,\left[\mathrm{~L}_{2} \mathrm{Cu}_{4}(\mathrm{OH})_{2}\right]^{+}\right)$, 560 (295), 335 (6490), 232 (50100).

für $28-2.5 \mathrm{CH}_{3} \mathrm{CN}$

ber.: C 31.28, H 3.29, N 16.00,

gef.: C 30.98, H 3.49, N 15.76 .

\subsubsection{Darstellung von Kupfer(I)-Komplexen}

10.3.2.1. $\quad\left[\mathrm{L}^{1-\mathrm{Me}} \mathrm{Cu}\left\{\mathrm{Cu}\left(\mathrm{PPh}_{3}\right)_{2}\right\}\right]_{2}\left(\mathrm{ClO}_{4}\right)_{2}(\mathbf{2 0})$

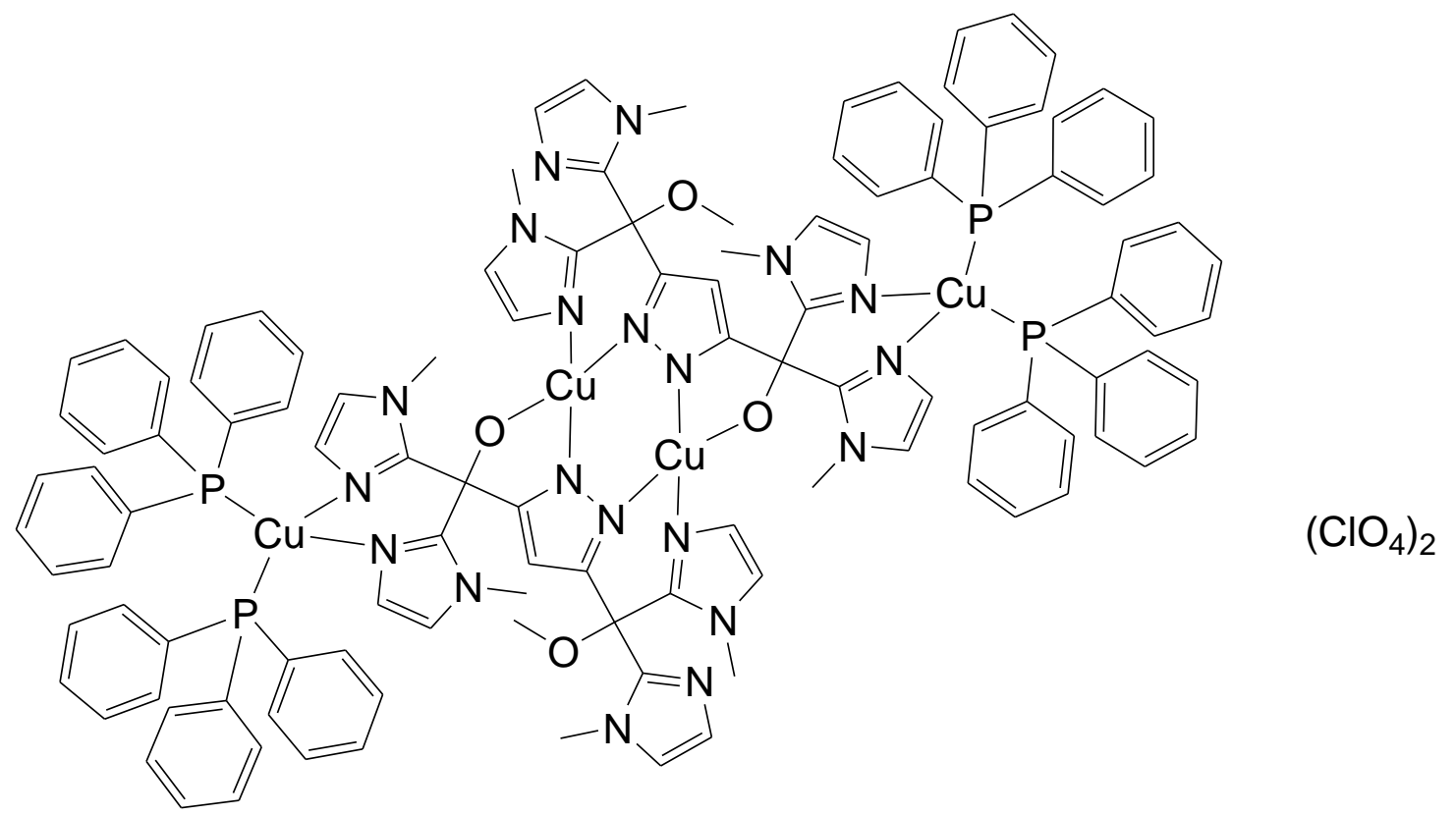

In einem $50 \mathrm{ml}$ Schlenkkolben werden $200 \mathrm{mg}(0.42 \mathrm{mmol}) \mathrm{HL}^{1}$ in $15 \mathrm{ml}$ Acetonitril gelöst und zu (396 mg, $0.42 \mathrm{mmol}$ ) Tris(triphenylphoshan)kupfer(l)-phenolat zugefügt. Anschließend gibt man noch ein Äquivalent $(137 \mathrm{mg}, 0.42 \mathrm{mmol})\left[\mathrm{Cu}(\mathrm{MeCN})_{4}\right] \mathrm{ClO}_{4}$, gelöst in $10 \mathrm{ml}$ Acetonitril, dazu und lässt die Lösung über Nacht rühren. Nach Entfernen des Lösungsmittels wird der Rückstand erneut in $7 \mathrm{ml}$ Dichlormethan 
aufgenommen und filtriert. Nach Dampfdiffusion mit Diethylether werden rote Kristalle erhalten, die für die Röntgenstrukturanalyse geeignet sind.

Summenformel

Molgewicht

Ausbeute
$\mathrm{C}_{116} \mathrm{H}_{108} \mathrm{Cu}_{4} \mathrm{~N}_{20} \mathrm{O}_{12} \mathrm{P}_{4} \mathrm{Cl}_{2}$

$2423.25 \mathrm{~g} / \mathrm{mol}$

$5.4 \mathrm{mg}(\mathrm{mmol}, \%)$

10.3.2.2. $\quad\left[\mathrm{L}^{3} \mathrm{Cu}_{2}\left(\mathrm{PMe}_{3}\right)\right]_{2}\left(\mathrm{PF}_{6}\right)_{2}(\mathbf{1 6})$

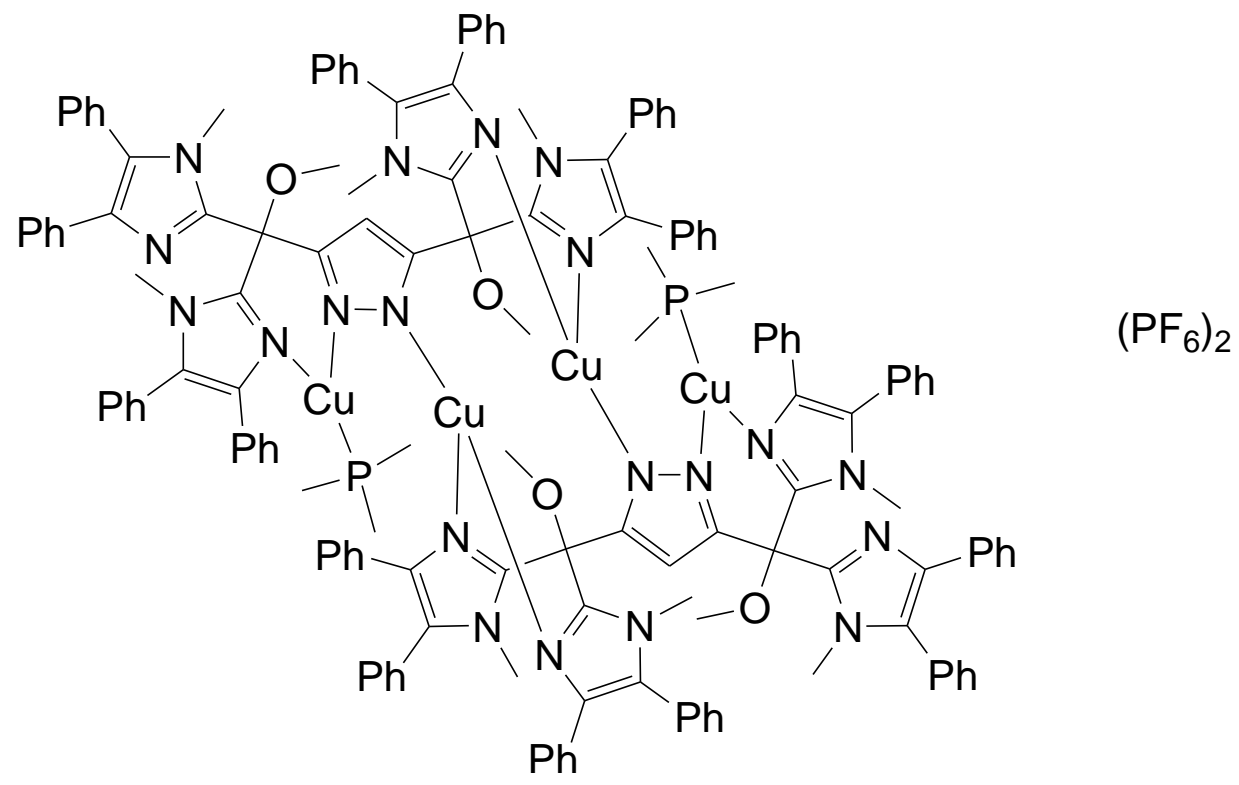

In einem $50 \mathrm{ml}$ Schlenkkolben werden $500 \mathrm{mg}(0.46 \mathrm{mmol}) \mathrm{HL}^{3}$ und $51 \mathrm{mg}(0.46 \mathrm{mmol})$ $\mathrm{KO} \mathrm{B}^{\mathrm{B} u}$ in $10 \mathrm{ml}$ THF gelöst. Anschließend gibt man eine Lösung von $343 \mathrm{mg}$ $(0.96 \mathrm{mmol})$ [ $\left.\mathrm{Cu}(\mathrm{MeCN})_{4}\right] \mathrm{PF}_{6}$, gelöst in $10 \mathrm{ml}$ Acetonitril, dazu und lässt die Lösung etwa 15 Minuten rühren. Danach werden noch zwei Äquivalente einer $1.0 \mathrm{M}$ Trimethylphosphinlösung in THF $(0.92 \mathrm{ml}, 0.92 \mathrm{mmol})$ zugefügt und die Lösung für weitere 15 Minuten gerührt. Nach Entfernen des Lösungsmittels wird der Rückstand erneut in $5 \mathrm{ml}$ Dichlormethan aufgenommen und filtriert. Nach Dampfdiffusion mit Diethylether werden farblose Kristalle erhalten. Diese sind analysenrein und für die Röntgenstrukturanalyse geeignet.

Summenformel

Molgewicht
$\mathrm{C}_{148} \mathrm{H}_{136} \mathrm{Cu}_{4} \mathrm{~N}_{20} \mathrm{O}_{4} \mathrm{P}_{4} \mathrm{~F}_{12}$

$2864.91 \mathrm{~g} / \mathrm{mol}$ 


\begin{tabular}{|c|c|}
\hline Ausbeute & $279.8 \mathrm{mg}$ (0.1 mmol, $42 \%$ ) \\
\hline $\mathrm{IR}(\mathrm{KBr}) \tilde{v}\left(\mathrm{~cm}^{-1}\right)$ & $\begin{array}{l}3352(\mathrm{br}), 3057(\mathrm{~m}), 2965(\mathrm{~m}), 2904(\mathrm{~m}), 2833(\mathrm{w}), 1963(\mathrm{w}), \\
1896(\mathrm{w}), 1825(\mathrm{w}), 1603(\mathrm{vs}), 1577(\mathrm{~m}), 1504(\mathrm{~s}), 1480(\mathrm{~s}), \\
1444(\mathrm{vs}), 1385(\mathrm{~s}), 1307(\mathrm{~m}), 1289(\mathrm{~m}), 1231(\mathrm{w}), 1201(\mathrm{w}), \\
1141(\mathrm{w}), 1127(\mathrm{w}), 1074(\mathrm{vs}), 1057(\mathrm{~s}), 1026(\mathrm{~s}), 1000(\mathrm{~m}), \\
986(\mathrm{~s}), 961(\mathrm{vs}), 917(\mathrm{vs}), 837(\mathrm{vs}), 788(\mathrm{~s}), 773(\mathrm{~s}), 729 \\
(\mathrm{~m}), 697(\mathrm{~s}), 651(\mathrm{w}), 557(\mathrm{vs}), 522(\mathrm{w}) .\end{array}$ \\
\hline${ }^{1} \mathrm{H}-\mathrm{NMR}\left(\mathrm{CD}_{3} \mathrm{CN}\right)$ & 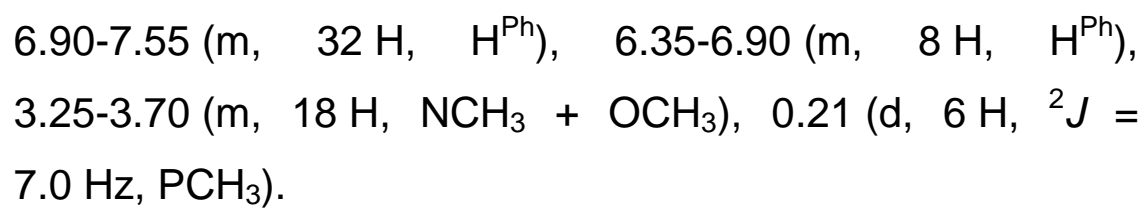 \\
\hline${ }^{13} \mathrm{C}-\mathrm{NMR}\left(\mathrm{CD}_{3} \mathrm{CN}\right)$ & 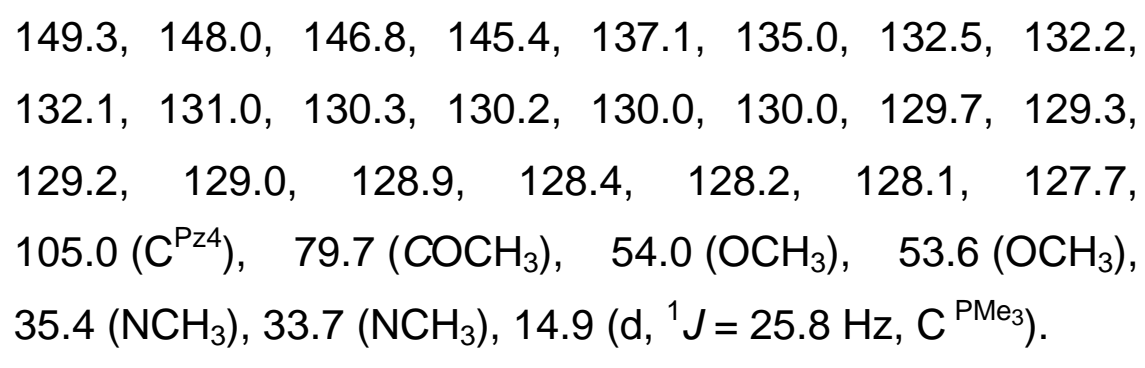 \\
\hline${ }^{31} \mathrm{P}-\mathrm{NMR}\left(\mathrm{CD}_{3} \mathrm{CN}\right)$ & $-44.8\left(\mathrm{br}, \mathrm{P}^{\mathrm{PMe}_{3}}\right),-46.7\left(\mathrm{~s}, \mathrm{P}^{\mathrm{PM}_{3}}\right),-144.2\left(\right.$ sept, $\left.\mathrm{P}^{\mathrm{PF}}{ }_{6}\right)$ \\
\hline MS (ESI) m/z (\%) & $\begin{array}{l}1363.6\left(13,\left[\mathrm{~L}^{3} \mathrm{Cu}_{2}\left(\mathrm{PMe}_{3}\right)_{2}\right]^{+}\right), 1287.4\left(24,\left[\mathrm{~L}^{3} \mathrm{Cu}_{2}\left(\mathrm{PMe}_{3}\right)\right]^{+}\right), \\
1223.5\left(6,\left[\mathrm{~L}^{3} \mathrm{Cu}\left(\mathrm{PMe}_{3}\right)\right]^{+}\right), 1147.4\left(100,\left[\mathrm{~L}^{3} \mathrm{Cu}\right]^{+}\right) .\end{array}$ \\
\hline \multirow[t]{6}{*}{$\begin{array}{l}\text { Hochauflösende Masse } \\
(\mathrm{ESI}, \mathrm{MeCN})\end{array}$} & $\begin{array}{l}\text { berechnet: }\left[\mathrm{L}^{3} \mathrm{Cu}_{2}\left(\mathrm{PMe}_{3}\right)_{2}\right]^{+} 1361.42927 \\
\text { gefunden: } 1361.43109\end{array}$ \\
\hline & $\begin{array}{l}\text { berechnet: }\left[\mathrm{L}^{3} \mathrm{Cu}_{2}\left(\mathrm{PMe}_{3}\right)\right]^{+} 1285.38509 \\
\text { gefunden: } 1285.38571\end{array}$ \\
\hline & berechnet: $\left[\mathrm{L}^{3} \mathrm{Cu}\left(\mathrm{PMe}_{3}\right)\right]^{+} 1223.46598$ \\
\hline & gefunden: 1223.46285 \\
\hline & berechnet: $\left[\mathrm{H}_{2} \mathrm{~L}^{3}\right]^{+} 1085.49785$ \\
\hline & gefunden: 1085.49329 \\
\hline \multirow[t]{3}{*}{ Elementaranalyse } & für $16 \cdot 5.6 \mathrm{CH}_{2} \mathrm{Cl}_{2}$ \\
\hline & ber.: C 55.19, H 4.50, N 8.38 \\
\hline & : 55.14, H 4.51, N 8.25. \\
\hline
\end{tabular}


10.3.2.3. $\quad\left[\mathrm{L}^{3} \mathrm{Cu}_{2}(n \mathrm{BuNC})\right]_{2}\left(\mathrm{PF}_{6}\right)_{2}(\mathbf{1 7})$

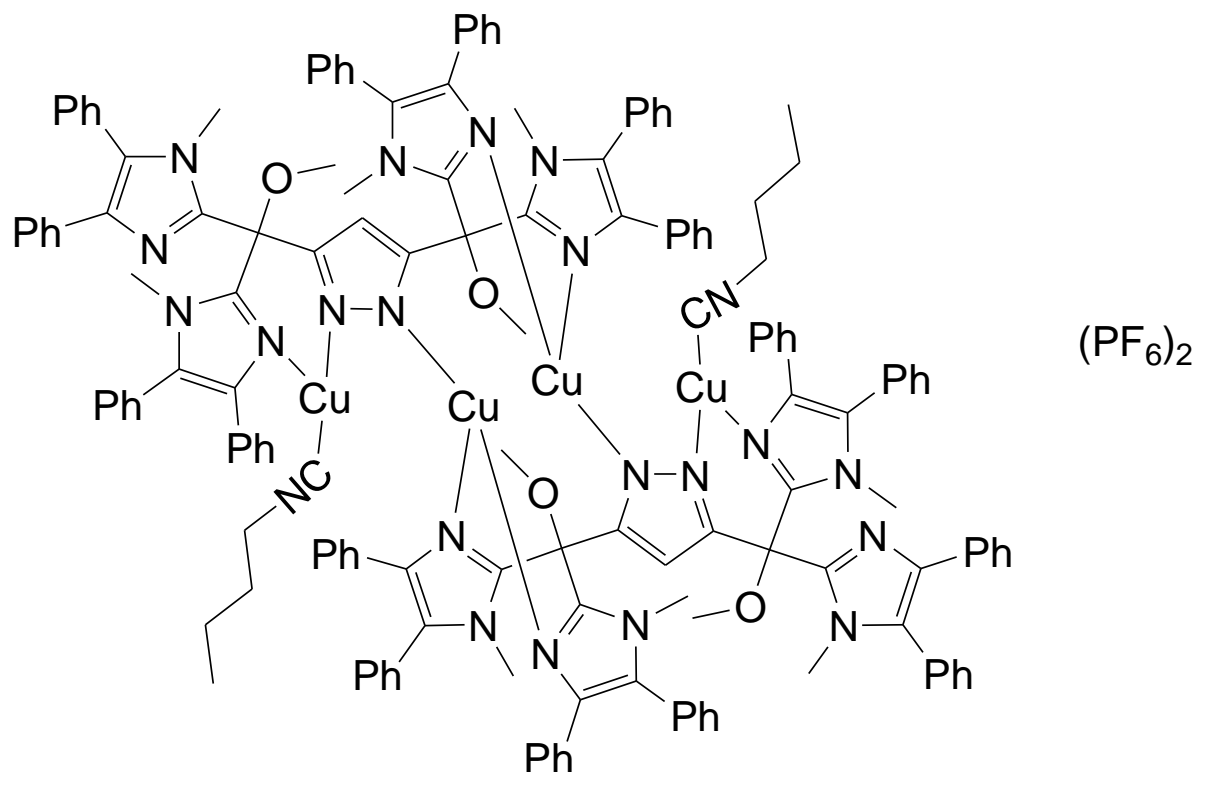

In einem $50 \mathrm{ml}$ Schlenkkolben werden $500 \mathrm{mg}(0.46 \mathrm{mmol}) \mathrm{HL}^{3}$ und $51 \mathrm{mg}(0.46 \mathrm{mmol})$ $\mathrm{KO}{ }^{\dagger} \mathrm{Bu}$ in $10 \mathrm{ml}$ THF gelöst. Anschließend gibt man eine Lösung von $343 \mathrm{mg}$ $(0.92 \mathrm{mmol})\left[\mathrm{Cu}(\mathrm{MeCN})_{4} \mathrm{PF}_{6}\right.$, gelöst in $10 \mathrm{ml}$ Acetonitril, dazu und lässt die Lösung etwa 15 Minuten rühren. Danach werden noch zwei Äquivalente $n$-Butylisocyanid ( $96 \mu$ l, $0.92 \mathrm{mmol}$ ) zugefügt und die Lösung für weitere 15 Minuten gerührt. Nach Entfernen des Lösungsmittels wird der Rückstand erneut in $5 \mathrm{ml}$ Dichlormethan aufgenommen und filtriert. Nach Dampfdiffusion mit Diethylether werden farblose Kristalle erhalten. Diese sind analysenrein und für die Röntgenstrukturanalyse geeignet.

Summenformel

Molgewicht

Ausbeute

$\operatorname{IR}(\mathrm{KBr}) \tilde{v}\left(\mathrm{~cm}^{-1}\right)$
$\mathrm{C}_{152} \mathrm{H}_{136} \mathrm{Cu}_{4} \mathrm{~N}_{22} \mathrm{O}_{4} \mathrm{P}_{2} \mathrm{~F}_{12}$

$2879.02 \mathrm{~g} / \mathrm{mol}$

$658.1 \mathrm{mg}(0.23 \mathrm{mmol}, 100 \%)$

$3055(\mathrm{~m}), 2965(\mathrm{~m}), 2960(\mathrm{~m}), 2874(\mathrm{~m}), 2833(\mathrm{~m}), 2196$

(vs), 1961 (w), $1890(w), 1816(w), 1773$ (w), 1603 (s), 1577

(m), 1504 (s), 1480 (s), 1443 (s), 1382 (s), 1341 (m), 1231

(w), 1202 (m), 1142 (w), 1073 (vs), 1024 (s), 985 (s), 964

(w), 921 (vs), 842 (vs), $784(\mathrm{~s}), 774$ (s), $729(\mathrm{~m}), 695$ (s), 613 (w), 557 (s).

${ }^{1} \mathrm{H}-\mathrm{NMR}\left(\mathrm{CD}_{3} \mathrm{CN}\right)$
7.10-7.50 (m, $\left.32 \mathrm{H}, \mathrm{H}^{\mathrm{Ph}}\right), 7.06\left(\mathrm{~s}, 1 \mathrm{H}, \mathrm{H}^{\mathrm{PZ} 4}\right), 7.50-7.00(\mathrm{~m}$, $\left.8 \mathrm{H}, \mathrm{H}^{\mathrm{Ph}}\right)$ 3.05-3.60 (m, $\left.18 \mathrm{H}, \mathrm{NCH}_{3}+\mathrm{OCH}_{3}\right)$, Protonen des 
Isonitril können nicht eindeutig zugeordnet werden, da sehr breite Signale.

${ }^{13} \mathrm{C}-\mathrm{NMR}\left(\mathrm{CD}_{3} \mathrm{CN}\right) \quad$ 148.6, 148.1, 146.7, 145.2, 137.3, 137.3, 134.7, 133.5, $132.8,132.7,132.1,132.0,130.8,130.1,130.1,130.0$, $130.0,129.9,129.7,129.6,129.5,129.4,129.3,129.2$, $129.0,128.9,128.8,128.4,128.3,128.2,128.1,128.0$, $104.4\left(\mathrm{C}^{\mathrm{Pz} 4}\right), \quad 80.9\left(\mathrm{COCH}_{3}\right), \quad 79.2\left(\mathrm{COCH}_{3}\right), \quad 54.0\left(\mathrm{OCH}_{3}\right)$, $52.9\left(\mathrm{OCH}_{3}\right), 42.2\left(\mathrm{C}^{\text {Isonitril-4 }}\right), 34.8\left(\mathrm{NCH}_{3}\right), 34.1\left(\mathrm{NCH}_{3}\right), 31.0$ $\left(C^{\text {lsonitril-3 }}\right), 20.1\left(C^{\text {Isonitril-2 }}\right), 13.4\left(C^{\text {lsonitril-1 }}\right)$.

$\mathrm{MS}(\mathrm{ESI}, \mathrm{MeCN}) \mathrm{m} / \mathrm{z}(\%) \quad 1377.6 \quad\left(15, \quad\left[\mathrm{~L}^{3} \mathrm{Cu}_{2}(n \mathrm{BuNC})_{2}\right]^{+}\right), \quad 1292.3 \quad$ (45, $\left.\left[\mathrm{L}^{3} \mathrm{Cu}_{2}(n \mathrm{BuNC})\right]^{+}\right), 1147.5\left(100,\left[\mathrm{~L}^{3} \mathrm{Cu}\right]^{+}\right)$.

Hochauflösende Masse berechnet: $\left[\mathrm{L}^{3} \mathrm{Cu}_{2}(n \mathrm{BuNC})_{2}\right]^{+} 1375.48790$ (ESI, MeCN) gefunden: 1375.49076 berechnet: $\left[\mathrm{HL}^{3} \mathrm{Cu}_{2}(n \mathrm{BuNC})_{2}\right]^{2+} 688.24759$ gefunden: 688.24901

Elementaranalyse für $17 \cdot 3.8 \mathrm{CH}_{2} \mathrm{Cl}_{2}$ ber.: C 58.28, H 4.58, N 9.60 gef.: C 58.41, H 4.63, N 9.58. 
10.3.2.4. $\left[\mathrm{L}^{3} \mathrm{Cu}_{2}\left(\mathrm{C}_{6} \mathrm{H}_{3}\left(\mathrm{CH}_{3}\right)_{2} \mathrm{NC}\right)\right]_{2}\left(\mathrm{PF}_{6}\right)_{2}(\mathbf{1 8})$

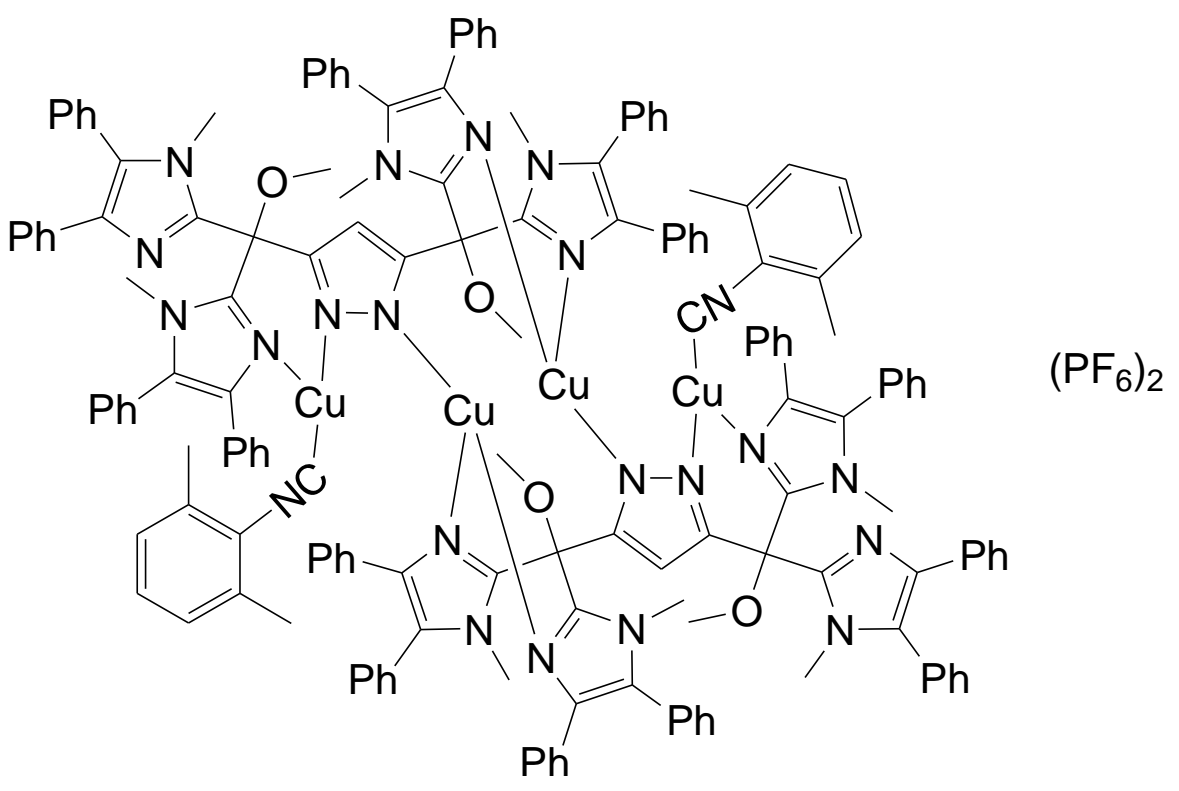

In einem $50 \mathrm{ml}$ Schlenkkolben werden $500 \mathrm{mg}(0.46 \mathrm{mmol}) \mathrm{HL}^{3}$ und $51 \mathrm{mg}(0.46 \mathrm{mmol})$ $\mathrm{KO}{ }^{\dagger} \mathrm{Bu}$ in $10 \mathrm{ml}$ THF gelöst. Anschließend gibt man eine Lösung von $343 \mathrm{mg}$ (0.92 mmol) $\left[\mathrm{Cu}(\mathrm{MeCN})_{4}\right] \mathrm{PF}_{6}$, gelöst in $10 \mathrm{ml}$ Acetonitril, dazu und lässt die Lösung etwa 15 Minuten rühren. Danach werden noch zwei Äquivalente 2,6-Dimethylphenylisocyanid (121 mg, $0.92 \mathrm{mmol}$ ), gelöst in $10 \mathrm{ml}$ Acetonitril, zugefügt und die Lösung für weitere 15 Minuten gerührt. Nach Entfernen des Lösungsmittels wird der Rückstand erneut in $5 \mathrm{ml}$ Dichlormethan aufgenommen und filtriert. Nach Dampfdiffusion mit Diethylether werden farblose Kristalle erhalten. Diese sind analysenrein und für die Röntgenstrukturanalyse geeignet.

Summenformel

Molgewicht

Ausbeute

$\operatorname{IR}(\mathrm{KBr}) \tilde{v}\left(\mathrm{~cm}^{-1}\right)$

${ }^{1} \mathrm{H}-\mathrm{NMR}\left(\mathrm{CD}_{3} \mathrm{CN}\right)$
$\mathrm{C}_{160} \mathrm{H}_{136} \mathrm{Cu}_{4} \mathrm{~N}_{22} \mathrm{O}_{4} \mathrm{P}_{2} \mathrm{~F}_{12}$

$2975.11 \mathrm{~g} / \mathrm{mol}$

$503.1 \mathrm{mg}(0.17 \mathrm{mmol}, 74 \%)$

$3055(\mathrm{~m}), 3032(\mathrm{~m}), 2960(\mathrm{~m}), 2832(\mathrm{~m}), 2155$ (vs), 1963 (w), $1898(w), 1823(w), 1773(w), 1603(s), 1577(m), 1504$ (s), 1480 (s), 1443 (vs), 1387 (s), 1320 (w), 1231 (w), 1204 (w), 1131 (w), 1074 (vs), 1025 (s), 987 (s), 965 (m), 917 (s), 839 (vs), 773 (s), 698 (vs), 557 (s), 506 (w).

7.10-7.50 (m, $\left.32 \mathrm{H}, \mathrm{H}^{\mathrm{Ph}}\right), 7.06\left(\mathrm{~s}, 1 \mathrm{H}, \mathrm{H}^{\mathrm{Pz} 4}\right), 7.50-7.00(\mathrm{~m}$, $\left.8 \mathrm{H}, \mathrm{H}^{\mathrm{Ph}}\right)$ 3.05-3.60 (m, $\left.18 \mathrm{H}, \mathrm{NCH}_{3}+\mathrm{OCH}_{3}\right)$, Protonen der 
Methylgruppen des Isonitrils können nicht eindeutig zugeordnet werden, da Überlagerung mit $\mathrm{CD}_{3} \mathrm{CN}$.

${ }^{13} \mathrm{C}-\mathrm{NMR}\left(\mathrm{CD}_{3} \mathrm{CN}\right) \quad 148.9,147.9,146.6,145.4,137.6,137.3,135.1,133.8$, $132.2,132.1,131.0,130.3,130.2,130.0,129.9,129.8$, $129.4,128.9,128.7,128.5,128.3,128.0,104.3\left(\mathrm{C}^{\mathrm{Pz} 4}\right)$, $79.4\left(\mathrm{COCH}_{3}\right), \quad 79.2\left(\mathrm{COCH}_{3}\right), \quad 54.2\left(\mathrm{OCH}_{3}\right), \quad 51.6\left(\mathrm{OCH}_{3}\right)$, $34.9\left(\mathrm{NCH}_{3}\right), 34.3\left(\mathrm{NCH}_{3}\right), 31.0\left(\mathrm{C}^{\text {Isonitril-3 }}\right), 18.6\left(\mathrm{C}^{\text {Me-Isonitril }}\right)$.

MS (ESI, MeCN) m/z (\%) $1473.7 \quad\left(7, \quad\left[\mathrm{~L}^{3} \mathrm{Cu}_{2}\left(\mathrm{C}_{6} \mathrm{H}_{3}\left(\mathrm{CH}_{3}\right)_{2} \mathrm{NC}\right)_{2}\right]^{+}\right), \quad 1342.8 \quad(15$, $\left.\left[\mathrm{L}^{3} \mathrm{Cu}_{2}\left(\mathrm{C}_{6} \mathrm{H}_{3}\left(\mathrm{CH}_{3}\right)_{2} \mathrm{NC}\right)\right]^{+}\right), 1238.5\left(15,\left[\mathrm{~L}^{3} \mathrm{Cu}_{2}(\mathrm{CN})\right]^{+}\right), 1147.6$ $\left(100,\left[\mathrm{~L}^{3} \mathrm{Cu}\right]^{+}\right)$.

Hochauflösende Masse berechnet: $\left[{ }^{3} \mathrm{Cu}_{2}\left(\mathrm{C}_{6} \mathrm{H}_{3}\left(\mathrm{CH}_{3}\right)_{2} \mathrm{NC}\right)\right]_{2}{ }^{2+} 1340.41439$ (ESI, MeCN) gefunden: 1340.41943 berechnet: $\left[\mathrm{L}^{3} \mathrm{Cu}\right]^{+} 1147.41915$ gefunden: 1147.41933 berechnet: $\left[\mathrm{H}_{2} \mathrm{~L}^{3}\right]^{+} 1085.49785$ gefunden: 1085.49687

Elementaranalyse für $18 \cdot 1.5 \mathrm{CH}_{2} \mathrm{Cl}_{2}$ ber.: C 62.52, H 4.52, N 9.93 gef.: C 62.39, H 4.59, N 10.04 . 


\subsubsection{Darstellung von Nickel(II)-Komplexen}

10.3.3.1. $\left[\mathrm{L}^{1} \mathrm{Ni}_{2}(\mathrm{OH})\right]_{2}\left(\mathrm{ClO}_{4}\right)_{4}(\mathbf{2 1})$

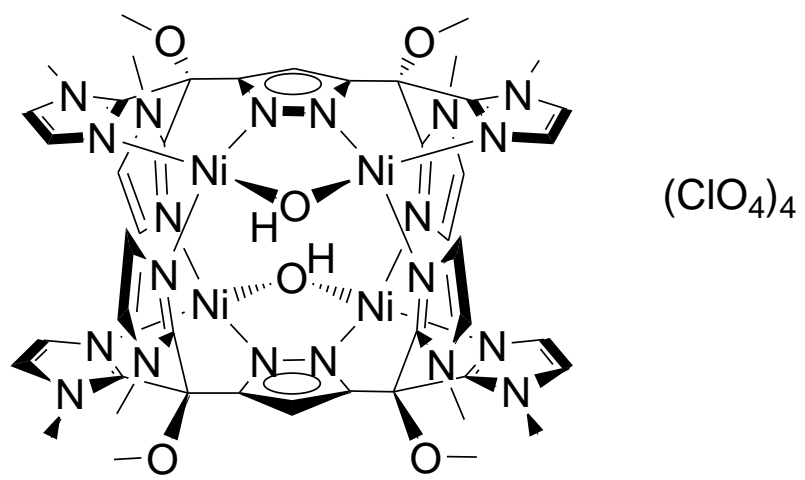

Zu einer Lösung von $400 \mathrm{mg}(0.84 \mathrm{mmol}) \mathrm{HL}^{1}$ in $50 \mathrm{ml}$ Methanol werden unter Rühren zwei Äquivalente (94 mg, $0.84 \mathrm{mmol}$ ) KO $\mathrm{K}^{\mathrm{Bu}}$ zugegeben. Nach etwa 15 Minuten gibt man zwei Äquivalente (614 mg, $1.68 \mathrm{mmol}) \mathrm{Ni}\left(\mathrm{ClO}_{4}\right)_{2} \cdot 6 \mathrm{H}_{2} \mathrm{O}$ sowie vier Äquivalente Trimethylphosphin $(1.0 \mathrm{M}$ in Toluol, $3.36 \mathrm{ml}, 3.36 \mathrm{mmol})$ dazu und lässt die Reaktionslösung 5 Stunden bei Raumtemperatur rühren. Anschließend entfernt man das Lösungsmittel im Vakuum, nimmt den Rückstand in $20 \mathrm{ml}$ Acetonitril auf, filtriert und überschichtet diese Lösung in einem Schlenkrohr mit $80 \mathrm{ml}$ Diethylether. Die erhaltenen roten Kristalle für die Röntgenstrukturanalyse geeignet.

Summenformel

Molgewicht

Ausbeute

$\mathrm{IR}(\mathrm{KBr}) \tilde{v}\left(\mathrm{~cm}^{-1}\right)$
$\mathrm{C}_{46} \mathrm{H}_{56} \mathrm{Cl}_{4} \mathrm{Ni}_{4} \mathrm{~N}_{20} \mathrm{O}_{22}$

$1617.64 \mathrm{~g} / \mathrm{mol}$

$16 \mathrm{mg}(0.01 \mathrm{mmol}, 3 \%)$

3460 (br), 3151 (m), 2964 (m), 2836 (w), 2254 (w), 2017 (w), $1550(\mathrm{~m}), 1505$ (s), $1491(\mathrm{~m}), 1436(\mathrm{~m}), 1384(\mathrm{w}), 1363$ (w), 1289 (s), 1229 (m), 1089 (br), 984 (m), 913 (m), 865 (w), 809 $(\mathrm{w}), 752(\mathrm{~m}), 686(\mathrm{~m}), 619(\mathrm{~m})$.

MS (ESI, MeCN) m/z (\%) $1517.2 \quad\left(5, \quad\left\{\left[\mathrm{~L}^{1} \mathrm{Ni}_{2}(\mathrm{OH})\left(\mathrm{ClO}_{4}\right)\right]_{2}\right\}^{+}\right), \quad 709.3$ $\left.\left[\mathrm{L}^{1} \mathrm{Ni}_{2}(\mathrm{OH})\left(\mathrm{ClO}_{4}\right)\right]^{+}\right)$. 
10.3.3.2. $\left[\left\{\mathrm{L}^{2} \mathrm{NiCl}(\mathrm{MeOH})_{2}\right\}_{2} \mathrm{Nij}\right] \mathrm{Cl}_{2}(\mathbf{2 3})$

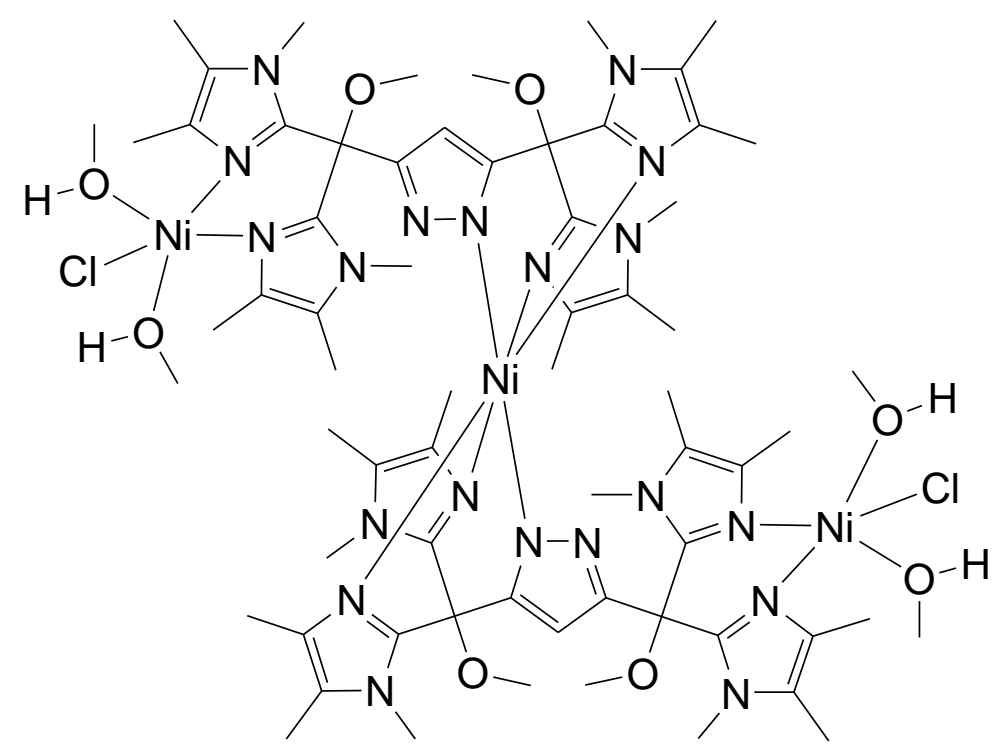

$(\mathrm{Cl})_{2}$

Zu einer Lösung von $500 \mathrm{mg}(0.85 \mathrm{mmol}) \mathrm{HL}^{2}$ in $50 \mathrm{ml}$ Methanol werden unter Rühren zwei Äquivalente $\left(95 \mathrm{mg}, 0.85 \mathrm{mmol}\right.$ ) $\mathrm{KO} \mathrm{B}^{\mathrm{B} u}$ zugegeben. Nach etwa 15 Minuten gibt man zwei Äquivalente (303 mg, $1.27 \mathrm{mmol}$ ) $\mathrm{NiCl}_{2} \cdot 6 \mathrm{H}_{2} \mathrm{O}$ dazu und lässt die Reaktionslösung 5 Stunden bei Raumtemperatur rühren. Anschließend entfernt man das Lösungsmittel im Vakuum, nimmt den Rückstand in $20 \mathrm{ml}$ Methanol auf, filtriert und überschichtet diese Lösung in einem Schlenkrohr zunächst mit $20 \mathrm{ml}$ einer 1:1-Mischung von Methanol:Diethylether und dann mit $80 \mathrm{ml}$ Diethylether. Die erhaltenen gelbbraunen Kristalle sind für die Röntgenstrukturanalyse geeignet.

Summenformel

Molgewicht

Ausbeute

$\mathrm{IR}(\mathrm{KBr}) \tilde{v}\left(\mathrm{~cm}^{-1}\right)$
$\mathrm{C}_{66} \mathrm{H}_{102} \mathrm{Cl}_{4} \mathrm{~N}_{20} \mathrm{Ni}_{3} \mathrm{O}_{8}+5 \mathrm{CH}_{3} \mathrm{OH}$

$1621.56 \mathrm{~g} / \mathrm{mol}$

$407.8 \mathrm{mg}(0.25 \mathrm{mmol}, 60 \%)$

$3393($ br), $2924(\mathrm{~m}), 2833(\mathrm{w}), 2566(\mathrm{w}), 1651(\mathrm{~m}), 1620(\mathrm{~m})$, (vs), $1445(\mathrm{~m}), 1410(\mathrm{~m}), 1340(\mathrm{w}), 1306(\mathrm{w}), 1224(\mathrm{~m})$, $1168(\mathrm{w}), 1114(\mathrm{~m}), 1076(\mathrm{~m}), 1033(\mathrm{~s}), 1024(\mathrm{~s}), 994(\mathrm{~m})$, $967(\mathrm{w}), 916(\mathrm{~s}), 879(\mathrm{~s}), 779(\mathrm{~m}), 739(\mathrm{w}), 521(\mathrm{w})$.

UV/Vis (MeOH) $\lambda(\varepsilon) \quad 726$ (44), 426 (151), 239 (52095), 203 (80838).

UV/Vis $(\mathrm{KBr}) \lambda \quad 706,415,242$. 
10.3.3.3. $\left[\left\{\mathrm{L}^{2} \mathrm{Ni}\left(\mathrm{NO}_{3}\right)_{2}(\mathrm{MeOH})\right\}_{2} \mathrm{Ni}\right](\mathbf{2 2})$

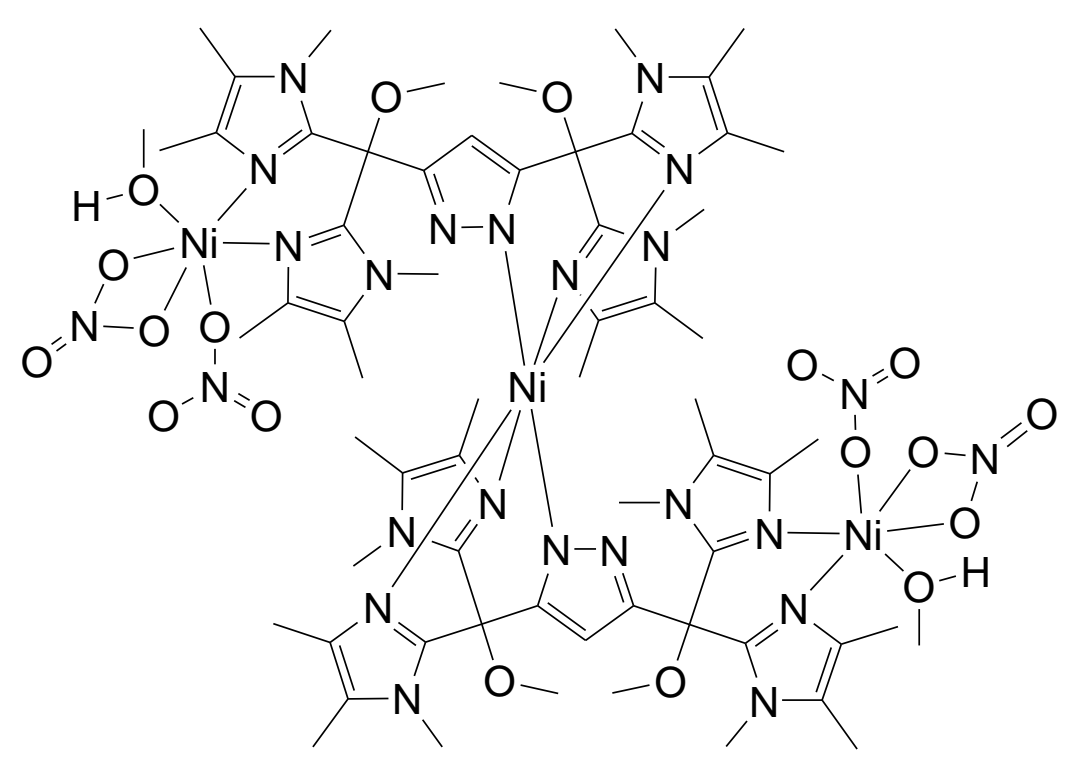

Zu einer Lösung von $300 \mathrm{mg}(0.51 \mathrm{mmol}) \mathrm{HL}^{2}$ in $50 \mathrm{ml}$ Methanol werden unter Rühren zwei Äquivalente $\left(57 \mathrm{mg}, 0.51 \mathrm{mmol}\right.$ ) $\mathrm{KO} \mathrm{B}^{\mathrm{B} u}$ zugegeben. Nach etwa 15 Minuten gibt man zwei Äquivalente (223 mg, $0.76 \mathrm{mmol}) \mathrm{Ni}\left(\mathrm{NO}_{3}\right)_{2} \cdot 6 \mathrm{H}_{2} \mathrm{O}$ dazu und lässt die Reaktionslösung 5 Stunden bei Raumtemperatur rühren. Anschließend entfernt man das Lösungsmittel im Vakuum, nimmt den Rückstand in $20 \mathrm{ml}$ Methanol auf, filtriert und überschichtet diese Lösung in einem Schlenkrohr zunächst mit $20 \mathrm{ml}$ einer 1:1-Mischung Methanol:Diethylether und danach mit $80 \mathrm{ml}$ Diethylether. Die erhaltenen grünen Kristalle sind für die Röntgenstrukturanalyse geeignet.

$\begin{array}{ll}\text { Summenformel } & \mathrm{C}_{64} \mathrm{H}_{94} \mathrm{~N}_{24} \mathrm{Ni}_{3} \mathrm{O}_{18}+2 \mathrm{Et}_{2} \mathrm{O} \\ \text { Molgewicht } & 1663.68 \mathrm{~g} / \mathrm{mol} \\ \text { Ausbeute } & 140.5 \mathrm{mg}(0.09 \mathrm{mmol}, 33 \%) \\ \mathrm{IR}(\mathrm{KBr}) \tilde{\mathrm{v}}\left(\mathrm{cm}^{-1}\right) & 3564(\mathrm{~s}), 3418(\mathrm{br}), 2934(\mathrm{~s}), 2827(\mathrm{w}), 2604(\mathrm{w}), 1892(\mathrm{br}), \\ & 1619(\mathrm{~s}), 1488(\mathrm{vs}), 1445(\mathrm{~s}), 1408(\mathrm{~s}), 1384(\mathrm{~s}), 1352(\mathrm{~s}), \\ & 1295(\mathrm{vs}), 1220(\mathrm{~m}), 1119(\mathrm{~m}), 1085(\mathrm{~m}), 1027(\mathrm{~m}), 995(\mathrm{~m}), \\ & 916(\mathrm{~s}), 826(\mathrm{~m}), 811(\mathrm{~m}), 784(\mathrm{~m}), 739(\mathrm{w}), 522(\mathrm{w}) . \\ \mathrm{MS}(\mathrm{ESI}, \mathrm{MeOH}) \mathrm{m} / \mathrm{z}(\%) & 1534.5 \quad\left(25, \quad\left[\left[\left\{\mathrm{~L}^{2} \mathrm{Ni}^{+}\left(\mathrm{NO}_{3}\right)\right\}_{2} \mathrm{NijNO}_{3}\right]^{+}, \quad 737.4 \quad(47,\right.\right. \\ & \left.\left[\left\{\mathrm{L}^{2} \mathrm{Ni}\left(\mathrm{NO}_{3}\right)\right\}_{2} \mathrm{Nii}\right]^{2+}\right), 645.4\left(100,\left[\mathrm{~L}^{2} \mathrm{Ni}\right]^{+}\right) . \\ \text {UV } / \mathrm{Vis}(\mathrm{DMF}) \lambda(\varepsilon) & 639(32) . \\ \text { UV } / \mathrm{Vis}(\mathrm{KBr}) \lambda & 644,345,245 .\end{array}$


10.3.3.4. $\quad\left[\left\{\mathrm{L}^{5} \mathrm{Ni}(\mathrm{Cl})\right\}_{2} \mathrm{Ni}\right](\mathrm{Cl})_{2}(25)$

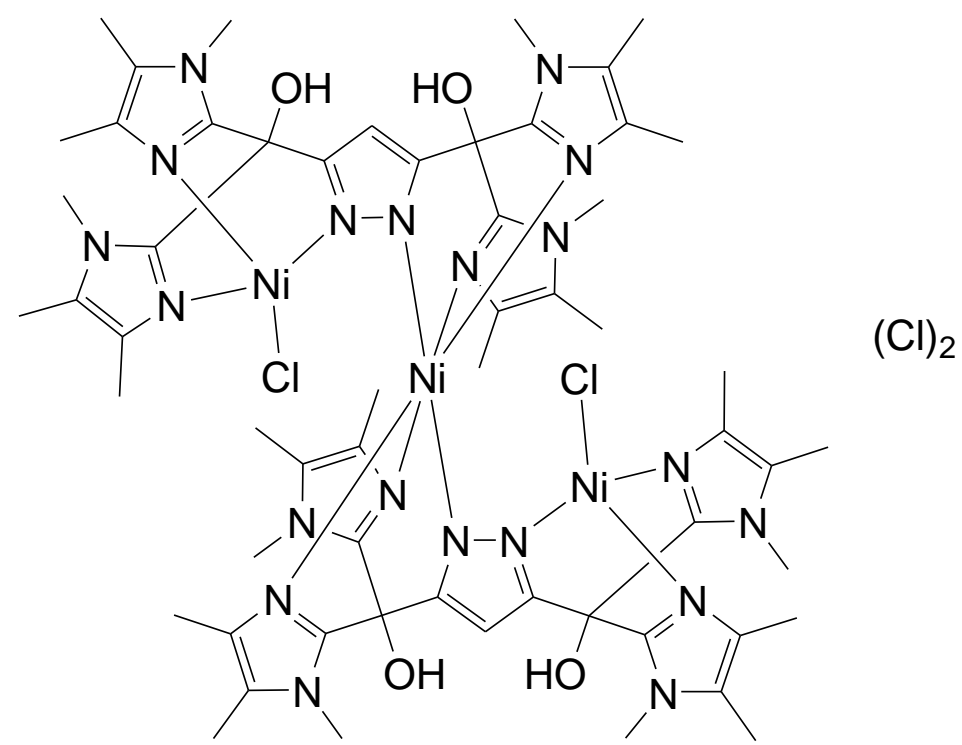

Zu einer Lösung von $600 \mathrm{mg}(1.07 \mathrm{mmol}) \mathrm{HL}^{5}$ in $50 \mathrm{ml}$ Methanol werden unter Rühren zwei Äquivalente (240 mg, $2.14 \mathrm{mmol}$ ) KOtBu zugegeben. Nach etwa 15 Minuten gibt man zwei Äquivalente (508 mg, $2.14 \mathrm{mmol}$ ) $\mathrm{NiCl}_{2} \cdot 6 \mathrm{H}_{2} \mathrm{O}$ dazu und lässt die Reaktionslösung 2 Stunden bei Raumtemperatur rühren. Anschließend entfernt man das Lösungsmittel im Vakuum, nimmt den Rückstand in $10 \mathrm{ml}$ Methanol auf, filtriert und überschichtet diese Lösung in einem Schlenkrohr zunächst mit $20 \mathrm{ml}$ eines 1:1-Gemisches Methanol:Diethylether und anschließend noch mit $60 \mathrm{ml}$ Diethylether. Die erhaltenen orangebraun gefärbten Kristalle sind für die Röntgenstrukturanalyse geeignet.

Summenformel

Molgewicht

Ausbeute

$\mathrm{IR}(\mathrm{KBr}) \tilde{v}\left(\mathrm{~cm}^{-1}\right)$
$\mathrm{C}_{58} \mathrm{H}_{78} \mathrm{Cl}_{4} \mathrm{~N}_{20} \mathrm{Ni}_{3} \mathrm{O}_{4}$

$1437.28 \mathrm{~g} / \mathrm{mol}$

$37 \mathrm{mg}(0.03 \mathrm{mmol}, 5 \%)$

3377 (br), 3209 (br), $2954(\mathrm{~m}), 2923(\mathrm{~m}), 1665(\mathrm{~s}), 1613(\mathrm{~s})$, 1496 (vs), 1444 (s), 1414 (s), $1363(\mathrm{~m}), 1311(\mathrm{~m}), 1226(\mathrm{~m})$, 1152 (vs), $1028(\mathrm{w}), 985(\mathrm{~m}), 906$ (vs), $871(\mathrm{w}), 790(\mathrm{~s}), 745$ $(\mathrm{m}), 684(\mathrm{w}), 448(\mathrm{w})$.

UV/Vis (MeCN) $\lambda(\varepsilon) \quad 637$ (36), 457 (54), 237 (61098), 203 (60307).

UV/Vis $(\mathrm{KBr}) \lambda \quad 897,507,466,289,248$. 
10.3.3.5. $\left[\mathrm{Ni}(\text { bipy })_{2}\left(\mathrm{CH}_{3} \mathrm{CN}\right)_{2}\right]\left(\mathrm{ClO}_{4}\right)_{2}(\mathbf{2 6})$

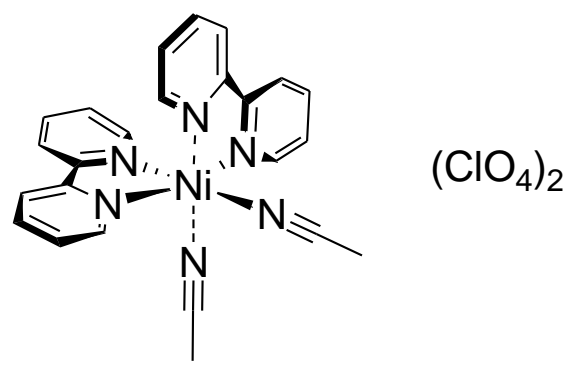

Zu einer Lösung von $400 \mathrm{mg}(0.84 \mathrm{mmol}) \mathrm{HL}^{1}$ in $50 \mathrm{ml}$ Methanol werden unter Rühren ein Äquivalent ( $47 \mathrm{mg}, 0.42 \mathrm{mmol}$ ) KOtBu zugegeben. Nach etwa 15 Minuten gibt man zwei Äquivalente $(251 \mathrm{mg}, 1.68 \mathrm{mmol}) \mathrm{Ni}\left(\mathrm{ClO}_{4}\right)_{2} 6 \mathrm{H}_{2} \mathrm{O}$ sowie zwei Äquivalente 2,2'Bipyridin (262 mg, $1.68 \mathrm{mmol}$ ) dazu und lässt die Reaktionslösung 5 Stunden bei Raumtemperatur rühren. Anschließend entfernt man das Lösungsmittel im Vakuum, nimmt den Rückstand in $20 \mathrm{ml}$ Acetonitril auf, filtriert und überschichtet diese Lösung in einem Schlenkrohr mit $80 \mathrm{ml}$ Diethylether. Die erhaltenen roten Kristalle sind analysenrein und für die Röntgenstrukturanalyse geeignet.

Zu einer Lösung von $2.00 \mathrm{~g}, 12.83 \mathrm{mmol}$ 2,2'-Bipyridin in $50 \mathrm{ml}$ Acetonitril wird unter Rühren ein halbes Äquivalent $(2.35 \mathrm{~g}, 6.41 \mathrm{mmol}) \mathrm{Ni}\left(\mathrm{ClO}_{4}\right)_{2} 6 \mathrm{H}_{2} \mathrm{O}$ zugegeben und lässt die Reaktionslösung 3 Stunden bei Raumtemperatur rühren. Anschließend entfernt man das Lösungsmittel im Vakuum, nimmt den Rückstand in $10 \mathrm{ml}$ Acetonitril auf, filtriert in ein Schlenkrohr und überschichtet diese Lösung zunächst mit $20 \mathrm{ml}$ eines 1:1Gemisches Acetonitril:Diethylether und dann mit $100 \mathrm{ml}$ Diethylether. Die erhaltenen violetten Kristalle sind analysenrein und für die Röntgenstrukturanalyse geeignet.

$\begin{array}{ll}\text { Summenformel } & \mathrm{C}_{24} \mathrm{H}_{22} \mathrm{Cl}_{2} \mathrm{~N}_{6} \mathrm{NiO}_{8} \\ \text { Molgewicht } & 652.09 \mathrm{~g} / \mathrm{mol} \\ \text { Ausbeute } & 2.41 \mathrm{~g}(3.70 \mathrm{mmol}, 58 \%) \\ \mathrm{IR}(\mathrm{KBr}) \tilde{v}\left(\mathrm{~cm}^{-1}\right) & 3245(\mathrm{~s}), 3210(\mathrm{~s}), 3118(\mathrm{~s}), 3085(\mathrm{~s}), 2995(\mathrm{~s}), 2934(\mathrm{~s}), \\ & 2314(\mathrm{vs}), 2284(\mathrm{vs}), 2019(\mathrm{vs}), 1602(\mathrm{vs}), 1572(\mathrm{vs}), 1494 \\ & (\mathrm{~m}), 1475(\mathrm{vs}), 1444(\mathrm{vs}), 1374(\mathrm{~m}), 1317(\mathrm{~s}), 1284(\mathrm{w}), 1249 \\ & (\mathrm{~s}), 1220(\mathrm{w}), 1096(\mathrm{vs}), 931(\mathrm{~m}), 809(\mathrm{w}), 763(\mathrm{vs}), 735(\mathrm{~s}), \\ & 655(\mathrm{~m}), 622(\mathrm{vs}), 442(\mathrm{~m}), 413(\mathrm{~m}) .\end{array}$


MS (FAB) $m / z(\%)$

$1039.0 \quad\left(4, \quad\left[\left\{\mathrm{Ni}(\text { bipy })_{2}\left(\mathrm{ClO}_{4}\right)\right\}_{2}\left(\mathrm{ClO}_{4}\right)\right]^{+}\right), \quad 469.0 \quad$ (100, $\left.\left[\mathrm{Ni}(\text { bipy })_{2}\left(\mathrm{ClO}_{4}\right)\right]^{+}\right), \quad 370.1 \quad\left(39, \quad\left[\mathrm{Ni}(\text { bipy })_{2}\right]^{+}\right), \quad 313.0 \quad(17$, $\left.\left[\mathrm{Ni}(\text { bipy })\left(\mathrm{ClO}_{4}\right)\right]^{+}\right), 214.0\left(51,[\mathrm{Ni}(\text { bipy })]^{+}\right)$.

UV/Vis $(\mathrm{MeCN}) \lambda(\varepsilon)$

Elementaranalyse
859 (9), 531 (9), 281 (24200), 244 (23200).

für 26

ber.: C 44.21, H 3.40, N 12.89,

gef.: C 43.73, H 3.33, N 12.61 .

10.3.3.6. $\mathrm{Na}\left[\mathrm{Ni}(\mathrm{acac})_{3}\right](27)$

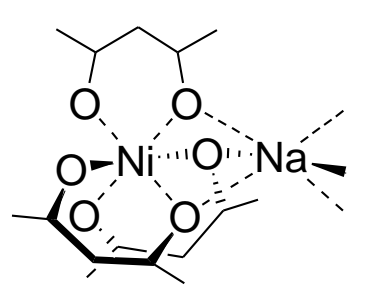

$\mathrm{Zu}$ einer Lösung von $400 \mathrm{mg}(0.84 \mathrm{mmol}) \mathrm{HL}^{1}$ in $100 \mathrm{ml}$ Methanol wird unter Rühren $\mathrm{KO}^{\mathrm{t}} \mathrm{Bu}$ (94 mg, $0.84 \mathrm{mmol}$ ) zugegeben. Nach etwa 15 Minuten gibt man je ein Äquivalent $\mathrm{Ni}(\mathrm{acac})_{2}(216 \mathrm{mg}, 0.84 \mathrm{mmol})$ und $\mathrm{Ni}\left(\mathrm{ClO}_{4}\right)_{2} \cdot 6 \mathrm{H}_{2} \mathrm{O}(307 \mathrm{mg}, 0.84 \mathrm{mmol})$ dazu und lässt die Reaktionslösung 5 Stunden bei Raumtemperatur rühren. Anschließend entfernt man das Lösungsmittel im Vakuum, nimmt den Rückstand in 20 $\mathrm{ml}$ Acetonitril auf, filtriert und überschichtet diese Lösung in einem Schlenkrohr mit $80 \mathrm{ml}$ Diethylether. Die erhaltenen türkis gefärbten Kristalle sind analysenrein und für die Röntgenstrukturanalyse geeignet.

$\mathrm{Zu}$ einer Lösung von $3.00 \mathrm{~g}, 30.00 \mathrm{mmol}$ Acetylaceton in $30 \mathrm{ml}$ Wasser wird unter Rühren $1.20 \mathrm{~g}$ (30.00 mmol) gemörsertes Natriumhydroxid zugegeben. Danach gibt man eine Lösung von $1.19 \mathrm{~g}(5.00 \mathrm{mmol}) \mathrm{NiCl}_{2} \cdot 6 \mathrm{H}_{2} \mathrm{O}$, gelöst in $20 \mathrm{ml}$ Wasser, zu. Die resultierende grüne Lösung wird mit $150 \mathrm{ml}$ Wasser verdünnt und 3 Stunden bei Raumtemperatur gerührt. Nachdem die Lösung filtriert wurde, lässt man das Lösungsmittel sehr langsam abdampfen. Die erhaltenen blau-grünen Kristalle sind analysenrein und für die Röntgenstrukturanalyse geeignet. 
Summenformel

Molgewicht

Ausbeute

$\mathrm{IR}(\mathrm{KBr}) \tilde{v}\left(\mathrm{~cm}^{-1}\right)$

MS (EI) $\mathrm{m} / \mathrm{z}(\%)$

Elementaranalyse
$\mathrm{C}_{15} \mathrm{H}_{21} \mathrm{NaNiO}_{6}$

$379.01 \mathrm{~g} / \mathrm{mol}$

$853.8 \mathrm{mg}(2.25 \mathrm{mmol}, 45 \%)$

3403 (br), 3240 (w), 3076 (w), $2989(\mathrm{~m}), 2925(\mathrm{~m}), 2449(\mathrm{w})$, 1959 (m), 1616 (vs), 1521 (vs), 1465 (vs), 1400 (vs), 1261 (s), 1200 (s), 1020 (vs), 934 (vs), 766 (vs), 661 (m), $591(\mathrm{~s}), 427(\mathrm{~m})$.

$256.0\left(100, \mathrm{Ni}(\mathrm{acac})_{2}\right), 241$ (78, Ni(acac $\left.)_{2}-\mathrm{Me}\right), 156.9$ (72, ( $\mathrm{Ni}(\mathrm{acac}))$.

für $27 \cdot 2.3 \mathrm{H}_{2} \mathrm{O}$

ber.: C 42.85, H 6.14,

gef.: C 42.52, H 5.64 . 


\subsection{Röntgenographischer Anhang}

\begin{tabular}{|c|c|c|}
\hline Verbindung & $2 a$ & 8 \\
\hline \multirow{2}{*}{ Formel } & $\mathrm{C}_{34} \mathrm{H}_{32} \mathrm{~N}_{10} \mathrm{O}_{3}$ & $\mathrm{C}_{27} \mathrm{H}_{34} \mathrm{Cu}_{2} \mathrm{~N}_{10} \mathrm{O}_{7}$ \\
\hline & $+\left(2 \mathrm{THF}+0.25 \mathrm{H}_{2} \mathrm{O}\right)$ & $+2 \mathrm{CH}_{2} \mathrm{Cl}_{2}$ \\
\hline$M\left[\mathrm{~g} \mathrm{~mol}^{-1}\right]$ & 681.33 & 907.57 \\
\hline Kristallgröße [mm] & $0.39 \times 0.34 \times 0.22$ & $0.51 \times 0.15 \times 0.13$ \\
\hline Kristallsystem & monoklin & triklin \\
\hline Raumgruppe & $P 2{ }_{1} / \mathrm{c}$ & $P-1$ \\
\hline$a[\AA]$ & $11.6344(8)$ & $11.9639(9)$ \\
\hline$b[\AA ̊]$ & $15.6169(7)$ & $12.2627(9)$ \\
\hline$c[\AA ̊ \AA]$ & $19.7941(13)$ & $14.4928(11)$ \\
\hline$\alpha\left[^{\circ}\right]$ & 90 & $112.812(6)$ \\
\hline$\beta\left[^{\circ}\right]$ & $96.224(5)$ & $104.426(6)$ \\
\hline$\gamma\left[^{\circ}\right]$ & 90 & $94.924(6)$ \\
\hline$V\left[\AA^{3}\right]$ & $3575.3(4)$ & $1858.1(2)$ \\
\hline$Z$ & 4 & 2 \\
\hline$\rho_{\text {ber. }}\left[\mathrm{g} \mathrm{cm}^{-3}\right]$ & 1.266 & 1.622 \\
\hline$\mu\left(\mathrm{Mo}-\mathrm{K}_{\alpha}\right)\left[\mathrm{mm}^{-1}\right]$ & 0.088 & 1.491 \\
\hline$F(000)$ & 1458 & 928 \\
\hline$\theta$ Bereich $\left[^{\circ}\right]$ & $1.66-24.77$ & $1.60-24.79$ \\
\hline$h k /$ Bereich & $\pm 13, \pm 18, \pm 23$ & $\pm 14,-13-14, \pm 16$ \\
\hline Gemessene Reflexe & 39634 & 16549 \\
\hline Unabhängige Reflexe $\left[R_{\text {int }}\right]$ & $6104[0.0894]$ & $6145[0.0438]$ \\
\hline Verfeinerte Parameter & 561 & 481 \\
\hline Goodness-of-fit & 1.002 & 1.032 \\
\hline$R 1(I>2 \sigma(I))$ & 0.0444 & 0.0290 \\
\hline wR2 (alle Daten) & 0.1025 & 0.0716 \\
\hline Restelektronendichte $\left[\mathrm{e} \AA^{-3}\right.$ ] & $0.247 /-0.247$ & $0.657 /-0.480$ \\
\hline
\end{tabular}




\begin{tabular}{|c|c|c|}
\hline Verbindung & 10 & $11 a$ \\
\hline \multirow{2}{*}{ Formel } & $\mathrm{C}_{48} \mathrm{H}_{64} \mathrm{Cu}_{4} \mathrm{~N}_{24} \mathrm{O}_{16} \mathrm{~S}_{2}$ & $\mathrm{C}_{48} \mathrm{H}_{64} \mathrm{Cu}_{4} \mathrm{~N}_{24} \mathrm{O}_{20}$ \\
\hline & $+5 \mathrm{MeOH}$ & $+\mathrm{Et}_{2} \mathrm{O}$ \\
\hline$M\left[\mathrm{~g} \mathrm{~mol}^{-1}\right]$ & 1655.68 & 1625.51 \\
\hline Kristallgröße [mm] & $0.49 \times 0.35 \times 0.26$ & $0.36 \times 0.32 \times 0.28$ \\
\hline Kristallsystem & triklin & monoklin \\
\hline Raumgruppe & $P-1$ & $P 2_{1} / n$ \\
\hline$a[\AA]$ & $10.8898(9)$ & $13.2857(10)$ \\
\hline$b[\AA$ & $12.1806(11)$ & $16.8144(15)$ \\
\hline$c[\AA]$ & $14.4307(12)$ & $14.6801(12)$ \\
\hline$\alpha\left[^{\circ}\right]$ & $98.065(7)$ & 90 \\
\hline$\beta\left[^{\circ}\right]$ & $90.051(7)$ & $101.659(6)$ \\
\hline$\gamma\left[^{\circ}\right]$ & $114.105(6)$ & 90 \\
\hline$V\left[\AA^{3}\right]$ & $1726.4(3)$ & $3211.7(5)$ \\
\hline$Z$ & 1 & 2 \\
\hline$\rho_{\text {ber. }}\left[\mathrm{g} \mathrm{cm}^{-3}\right]$ & 1.593 & 1.681 \\
\hline$\mu\left(\mathrm{Mo}^{-\mathrm{K}_{\alpha}}\right)\left[\mathrm{mm}^{-1}\right]$ & 1.362 & 1.402 \\
\hline$F(000)$ & 858 & 1676 \\
\hline$\theta$ Bereich $\left[{ }^{\circ}\right]$ & $1.85-24.58$ & $1.86-24.81$ \\
\hline hkl Bereich & $\pm 12, \pm 14, \pm 16$ & $-15-14, \pm 19,-16-17$ \\
\hline Gemessene Reflexe & 26247 & 16519 \\
\hline Unabhängige Reflexe [ $\left.R_{\text {int }}\right]$ & $5778[0.0524]$ & $5319[0.048]$ \\
\hline Verfeinerte Parameter & 469 & 465 \\
\hline Goodness-of-fit & 1.038 & 1.077 \\
\hline$R 1(I>2 \sigma(I))$ & 0.0494 & 0.0587 \\
\hline wR2 (alle Daten) & 0.1381 & 0.1525 \\
\hline Restelektronendichte $\left[\mathrm{e} \AA^{-3}\right]$ & $0.882 /-0.712$ & $1.416 /-0.721$ \\
\hline
\end{tabular}




\begin{tabular}{|c|c|c|}
\hline Verbindung & $11 b$ & 12 \\
\hline Formel & $\begin{array}{l}\mathrm{C}_{46} \mathrm{H}_{70} \mathrm{Cu}_{4} \mathrm{~N}_{24} \mathrm{O}_{25} \\
+5 \mathrm{H}_{2} \mathrm{O}\end{array}$ & $\begin{array}{l}\mathrm{C}_{46} \mathrm{H}_{56} \mathrm{Cl}_{4} \mathrm{Cu}_{4} \mathrm{~N}_{20} \mathrm{O}_{22} \\
+\mathrm{CHCl}_{3}\end{array}$ \\
\hline$M\left[\mathrm{~g} \mathrm{~mol}^{-1}\right]$ & 1703.50 & 1756.44 \\
\hline Kristallgröße [mm] & $0.50 \times 0.36 \times 0.23$ & $0.25 \times 0.15 \times 0.12$ \\
\hline Kristallsystem & triklin & triklin \\
\hline Raumgruppe & $P-1$ & $P-1$ \\
\hline$a[\AA]$ & $11.1590(8)$ & $10.7239(10)$ \\
\hline$b[\AA]$ & $13.0502(8)$ & $11.5828(10)$ \\
\hline$c[\AA$ & $13.3115(9)$ & $12.9912(12)$ \\
\hline$\alpha\left[^{\circ}\right]$ & $61.959(5)$ & $84.973(7)$ \\
\hline$\beta\left[^{\circ}\right]$ & $85.065(6)$ & $77.294(7)$ \\
\hline$\gamma\left[^{\circ}\right]$ & $77.567(5)$ & $79.899(7)$ \\
\hline$V\left[\AA^{3}\right]$ & $1670.57(19)$ & $1547.7(2)$ \\
\hline$Z$ & 1 & 1 \\
\hline$\rho_{\text {ber. }}\left[\mathrm{g} \mathrm{cm}^{-3}\right]$ & 1.693 & 1.884 \\
\hline$\mu\left(\mathrm{Mo}^{-\mathrm{K}_{\alpha}}\right)\left[\mathrm{mm}^{-1}\right]$ & 1.361 & 1.754 \\
\hline$F(000)$ & 880 & 890 \\
\hline$\theta$ Bereich $\left[^{\circ}\right]$ & $1.73-24.80$ & $1.61-24.74$ \\
\hline hkl Bereich & $\pm 13, \pm 15, \pm 15$ & $\pm 12,-11-13, \pm 15$ \\
\hline Gemessene Reflexe & 24271 & 15321 \\
\hline Unabhängige Reflexe [ $\left.R_{\text {int }}\right]$ & $5692[0.0495]$ & $5263[0.0724]$ \\
\hline Verfeinerte Parameter & 510 & 433 \\
\hline Goodness-of-fit & 1.036 & 1.030 \\
\hline$R 1(I>2 \sigma(I))$ & 0.0349 & 0.0676 \\
\hline wR2 (alle Daten) & 0.0922 & 0.1955 \\
\hline Restelektronendichte $\left[\mathrm{e} \AA^{-3}\right]$ & $0.784 /-0.589$ & $0.990 /-1.496$ \\
\hline
\end{tabular}




\begin{tabular}{|c|c|c|}
\hline Verbindung & 13 & 14 \\
\hline Formel & $\begin{array}{l}\mathrm{C}_{48} \mathrm{H}_{58} \mathrm{~B}_{4} \mathrm{Cl}_{6} \mathrm{Cu}_{4} \mathrm{~F}_{16} \mathrm{~N}_{20} \mathrm{O}_{6} \\
+2 \mathrm{MeCN}\end{array}$ & $\begin{array}{l}\mathrm{C}_{62} \mathrm{H}_{88} \mathrm{Cl}_{4} \mathrm{Cu}_{4} \mathrm{~N}_{20} \mathrm{O}_{22} \\
+4 \mathrm{MeCN}\end{array}$ \\
\hline$M\left[\mathrm{~g} \mathrm{~mol}^{-1}\right]$ & 1907.35 & 2025.70 \\
\hline Kristallgröße [mm] & $0.30 \times 0.27 \times 0.15$ & $0.43 \times 0.32 \times 0.27$ \\
\hline Kristallsystem & triklin & monoklin \\
\hline Raumgruppe & $P-1$ & $C 2 / c$ \\
\hline$a[\AA]$ & $11.0959(7)$ & $30.8090(16)$ \\
\hline$b[\AA$ & $12.0973(7)$ & $17.8627(7)$ \\
\hline$c[\AA$ & $14.3897(8)$ & $15.7729(9)$ \\
\hline$\alpha\left[^{\circ}\right]$ & $106.840(4)$ & 90 \\
\hline$\beta\left[^{\circ}\right]$ & $97.451(5)$ & $92.119(4)$ \\
\hline$\gamma\left[^{\circ}\right]$ & $101.749(5)$ & 90 \\
\hline$V\left[\AA^{3}\right]$ & $1772.91(18)$ & $8674.4(8)$ \\
\hline$Z$ & 1 & 4 \\
\hline$\rho_{\text {ber. }}\left[\mathrm{g} \mathrm{cm}^{-3}\right]$ & 1.786 & 1.551 \\
\hline$\mu\left(\mathrm{Mo} \mathrm{K}_{\alpha}\right)\left[\mathrm{mm}^{-1}\right]$ & 1.517 & 1.175 \\
\hline$F(000)$ & 960 & 4192 \\
\hline$\theta$ Bereich $\left[^{\circ}\right]$ & $1.51-24.79$ & $1.83-24.79$ \\
\hline hkl Bereich & $-11-13, \pm 14, \pm 16$ & $\pm 36,-21-20, \pm 18$ \\
\hline Gemessene Reflexe & 26542 & 25201 \\
\hline Unabhängige Reflexe [ $\left.R_{\text {int }}\right]$ & $6058[0.5697]$ & $7377[0.0409]$ \\
\hline Verfeinerte Parameter & 507 & 612 \\
\hline Goodness-of-fit & 1.051 & 1.090 \\
\hline$R 1(I>2 \sigma(I))$ & 0.0289 & 0.0491 \\
\hline wR2 (alle Daten) & 0.0763 & 0.1216 \\
\hline Restelektronendichte $\left[\mathrm{e} \AA^{-3}\right]$ & 0.739 / -0.936 & $0.750 /-0.560$ \\
\hline
\end{tabular}




\begin{tabular}{|c|c|c|}
\hline Verbindung & 15 & 16 \\
\hline \multirow{2}{*}{ Formel } & $\mathrm{C}_{39} \mathrm{H}_{58} \mathrm{Cl}_{4} \mathrm{Cu}_{2} \mathrm{~N}_{14} \mathrm{O}_{19}$ & $\mathrm{C}_{148} \mathrm{H}_{136} \mathrm{Cu}_{4} \mathrm{~F}_{12} \mathrm{~N}_{20} \mathrm{O}_{4} \mathrm{P}_{4}$ \\
\hline & $+2 \mathrm{MeCN}$ & $+\left(x \mathrm{CH}_{2} \mathrm{Cl}_{2}+\mathrm{y} \mathrm{Et}{ }_{2} \mathrm{O}\right)$ \\
\hline$M\left[\mathrm{~g} \mathrm{~mol}^{-1}\right]$ & 1377.98 & 2864.81 \\
\hline Kristallgröße [mm] & $0.42 \times 0.34 \times 0.30$ & $0.46 \times 0.41 \times 0.34$ \\
\hline Kristallsystem & triklin & triklin \\
\hline Raumgruppe & $P-1$ & $P-1$ \\
\hline$a[\AA]$ & $13.6533(7)$ & $14.3209(9)$ \\
\hline$b[\AA \AA]$ & $14.1468(7)$ & $15.9580(12)$ \\
\hline$c[\AA \AA]$ & $19.0068(9)$ & $20.0162(13)$ \\
\hline$\alpha\left[^{\circ}\right]$ & $70.754(4)$ & $72.230(5)$ \\
\hline$\beta\left[^{\circ}\right]$ & $89.772(4)$ & $85.991(5)$ \\
\hline$\gamma\left[^{\circ}\right]$ & $62.899(3)$ & $65.827(5)$ \\
\hline$V\left[\AA^{3}\right]$ & $3038.4(3)$ & $3966.2(5)$ \\
\hline$z$ & 2 & 1 \\
\hline$\rho_{\text {ber. }}\left[\mathrm{g} \mathrm{cm}^{-3}\right]$ & 1.506 & 1.199 \\
\hline$\mu\left(\mathrm{Mo}-\mathrm{K}_{\alpha}\right)\left[\mathrm{mm}^{-1}\right]$ & 0.957 & 0.638 \\
\hline$F(000)$ & 1424 & 1480 \\
\hline$\theta$ Bereich $\left[^{\circ}\right]$ & $1.70-24.83$ & $1.53-24.81$ \\
\hline$h k /$ Bereich & $-16-15, \pm 16, \pm 22$ & $\pm 16, \pm 18, \pm 23$ \\
\hline Gemessene Reflexe & 49417 & 47189 \\
\hline Unabhängige Reflexe $\left[R_{\text {int }}\right]$ & $10412[0.563]$ & $13494[0.1025]$ \\
\hline Verfeinerte Parameter & 789 & 899 \\
\hline Goodness-of-fit & 1.002 & 1.062 \\
\hline$R 1(I>2 \sigma(I))$ & 0.0345 & 0.0765 \\
\hline wR2 (alle Daten) & 0.0918 & 0.2096 \\
\hline Restelektronendichte $\left[\mathrm{e} \AA^{-3}\right]$ & $0.578 /-0.397$ & $1.637 /-0.852$ \\
\hline
\end{tabular}




\begin{tabular}{|c|c|c|}
\hline Verbindung & 17 & 18 \\
\hline Formel & $\begin{array}{l}\mathrm{C}_{152} \mathrm{H}_{136} \mathrm{Cu}_{4} \mathrm{~F}_{12} \mathrm{~N}_{20} \mathrm{O}_{4} \mathrm{P}_{2} \\
+\left(2 \mathrm{CH}_{2} \mathrm{Cl}_{2}+\mathrm{y} \mathrm{Et} \mathrm{t}_{2} \mathrm{O}\right)\end{array}$ & $\begin{array}{l}\mathrm{C}_{160} \mathrm{H}_{136} \mathrm{Cu}_{4} \mathrm{~F}_{12} \mathrm{~N}_{22} \mathrm{O}_{4} \mathrm{P}_{2} \\
+\left(2 \mathrm{CH}_{2} \mathrm{Cl}_{2}+2 \mathrm{Et}_{2} \mathrm{O}\right)\end{array}$ \\
\hline$M\left[\mathrm{~g} \mathrm{~mol}^{-1}\right]$ & 3345.26 & 3293.10 \\
\hline Kristallgröße [mm] & $0.48 \times 0.37 \times 0.23$ & $0.45 \times 0.32 \times 0.18$ \\
\hline Kristallsystem & triklin & triklin \\
\hline Raumgruppe & $P-1$ & $P-1$ \\
\hline$a[\AA]$ & $14.1772(6)$ & $14.7874(7)$ \\
\hline$b[\AA]$ & $16.0440(8)$ & $17.1318(8)$ \\
\hline$c[\AA$ & $20.8363(10)$ & 18.9073(8) \\
\hline$\alpha\left[^{\circ}\right]$ & $68.949(4)$ & $105.728(4)$ \\
\hline$\beta\left[^{\circ}\right]$ & $81.595(4)$ & 109.984(3) \\
\hline$\gamma\left[^{\circ}\right]$ & $64.366(3)$ & $102.964(2)$ \\
\hline$V\left[\AA^{3}\right]$ & $3987.5(3)$ & $4058.2(3)$ \\
\hline$Z$ & 1 & 1 \\
\hline$\rho_{\text {ber. }}\left[\mathrm{g} \mathrm{cm}^{-3}\right]$ & 1.393 & 1.347 \\
\hline$\mu\left(\mathrm{Mo}^{-\mathrm{K}_{\alpha}}\right)\left[\mathrm{mm}^{-1}\right]$ & 0.693 & 0.679 \\
\hline$F(000)$ & 1740 & 1704 \\
\hline$\theta$ Bereich $\left[^{\circ}\right]$ & $1.49-24.80$ & $1.52-24.83$ \\
\hline hkl Bereich & $\pm 16, \pm 18, \pm 24$ & $-16-17, \pm 20, \pm 22$ \\
\hline Gemessene Reflexe & 62446 & 61241 \\
\hline Unabhängige Reflexe [ $\left.R_{\text {int }}\right]$ & $13597[0.0854]$ & $13936[0.0577]$ \\
\hline Verfeinerte Parameter & 1000 & 974 \\
\hline Goodness-of-fit & 1.029 & 1.004 \\
\hline$R 1(I>2 \sigma(I))$ & 0.0496 & 0.0341 \\
\hline wR2 (alle Daten) & 0.1251 & 0.0717 \\
\hline Restelektronendichte $\left[\mathrm{e} \AA^{-3}\right]$ & $0.557 /-0.708$ & $0.341 /-0.331$ \\
\hline
\end{tabular}




\begin{tabular}{|c|c|c|}
\hline Verbindung & 19 & 20 \\
\hline Formel & $\begin{array}{l}\mathrm{C}_{35} \mathrm{H}_{64} \mathrm{Cu}_{2} \mathrm{~F}_{12} \mathrm{~N}_{10} \mathrm{O}_{2} \mathrm{P}_{6} \\
+\left(1-2 \mathrm{Et}_{2} \mathrm{O}\right)\end{array}$ & $\begin{array}{l}\mathrm{C}_{116} \mathrm{H}_{108} \mathrm{Cl}_{2} \mathrm{Cu}_{4} \mathrm{~N}_{20} \mathrm{O}_{12} \mathrm{P}_{4} \\
+\times \mathrm{CH}_{2} \mathrm{Cl}_{2}\end{array}$ \\
\hline$M\left[\mathrm{~g} \mathrm{~mol}^{-1}\right]$ & 1197.86 & 2423.16 \\
\hline Kristallgröße [mm] & $0.50 \times 0.24 \times 0.17$ & $0.47 \times 0.33 \times 0.18$ \\
\hline Kristallsystem & triklin & monoklin \\
\hline Raumgruppe & $P-1$ & $P 121 / c 1$ \\
\hline$a[\AA]$ & $12.1174(4)$ & $21.2433(8)$ \\
\hline$b[\AA]$ & $15.3513(5)$ & $11.0605(3)$ \\
\hline$c[\AA$ & $32.7259(10)$ & $25.1003(10)$ \\
\hline$\alpha\left[^{\circ}\right]$ & $94.227(3)$ & 90 \\
\hline$\beta\left[^{\circ}\right]$ & $95.319(2)$ & $95.564(3)$ \\
\hline$\gamma\left[^{\circ}\right]$ & $91.012(3)$ & 90 \\
\hline$V\left[\AA^{3}\right]$ & $6043.1(3)$ & $5864.6(4)$ \\
\hline$Z$ & 4 & 2 \\
\hline$\rho_{\text {ber. }}\left[\mathrm{g} \mathrm{cm}^{-3}\right]$ & 1.317 & 1.372 \\
\hline$\mu\left(\mathrm{Mo}^{-\mathrm{K}_{\alpha}}\right)\left[\mathrm{mm}^{-1}\right]$ & 0.638 & 0.883 \\
\hline$F(000)$ & 2464 & 2500 \\
\hline$\theta$ Bereich $\left[{ }^{\circ}\right]$ & $1.25-24.83$ & $1.63-24.76$ \\
\hline hkl Bereich & $-13-14, \pm 18, \pm 38$ & $\pm 24,-12-13, \pm 29$ \\
\hline Gemessene Reflexe & 120662 & 72948 \\
\hline Unabhängige Reflexe [ $\left.R_{\text {int }}\right]$ & $20739[0.0606]$ & $9962[0.0804]$ \\
\hline Verfeinerte Parameter & 1357 & 630 \\
\hline Goodness-of-fit & 1.084 & 1.050 \\
\hline$R 1(I>2 \sigma(I))$ & 0.0641 & 0.0492 \\
\hline wR2 (alle Daten) & 0.1789 & 0.1252 \\
\hline Restelektronendichte $\left[\mathrm{e} \AA^{-3}\right]$ & $1.481 /-1.166$ & 0.6739 / -0.432 \\
\hline
\end{tabular}




\begin{tabular}{|c|c|c|}
\hline Verbindung & 21 & 22 \\
\hline \multirow{2}{*}{ Formel } & $\mathrm{C}_{46} \mathrm{H}_{56} \mathrm{Cl}_{4} \mathrm{~N}_{20} \mathrm{Ni}_{4} \mathrm{O}_{22}$ & $\mathrm{C}_{64} \mathrm{H}_{94} \mathrm{~N}_{24} \mathrm{Ni}_{3} \mathrm{O}_{18}$ \\
\hline & $+\mathrm{MeCN}$ & $+2 \mathrm{Et}_{2} \mathrm{O}$ \\
\hline$M\left[\mathrm{~g} \mathrm{~mol}^{-1}\right]$ & 1658.80 & 1812.00 \\
\hline Kristallgröße [mm] & $0.18 \times 0.07 \times 0.04$ & $0.46 \times 0.32 \times 0.21$ \\
\hline Kristallsystem & triklin & triklin \\
\hline Raumgruppe & $P-1$ & $P-1$ \\
\hline$a[\AA]$ & $10.2686(13)$ & $10.6925(8)$ \\
\hline$b[\AA]$ & $11.8234(11)$ & $14.9953(10)$ \\
\hline$c[\AA$ & $13.1469(16)$ & $15.5762(11)$ \\
\hline$\alpha\left[^{\circ}\right]$ & $83.819(9)$ & $107.269(5)$ \\
\hline$\beta\left[^{\circ}\right]$ & $79.588(10)$ & $99.014(6)$ \\
\hline$\gamma\left[^{\circ}\right]$ & $77.921(9)$ & $108.081(5)$ \\
\hline$V\left[\AA^{3}\right]$ & $1531.1(3)$ & $2180.3(3)$ \\
\hline$Z$ & 1 & 1 \\
\hline$\rho_{\text {ber. }}\left[\mathrm{g} \mathrm{cm}^{-3}\right]$ & 1.799 & 1.380 \\
\hline$\mu\left(\mathrm{Mo}^{-\mathrm{K}_{\alpha}}\right)\left[\mathrm{mm}^{-1}\right]$ & 1.483 & 0.721 \\
\hline$F(000)$ & 850 & 958 \\
\hline$\theta$ Bereich $\left[^{\circ}\right]$ & $1.58-24.83$ & $1.58-24.81$ \\
\hline hkl Bereich & $-11-12, \pm 13, \pm 15$ & $\pm 12, \pm 17, \pm 18$ \\
\hline Gemessene Reflexe & 16061 & 22279 \\
\hline Unabhängige Reflexe $\left[R_{\text {int }}\right]$ & $5237[0.0902]$ & $7450[0.0643]$ \\
\hline Verfeinerte Parameter & 467 & 536 \\
\hline Goodness-of-fit & 1.002 & 1.064 \\
\hline$R 1(I>2 \sigma(I))$ & 0.0637 & 0.0689 \\
\hline wR2 (alle Daten) & 0.1373 & 0.2016 \\
\hline Restelektronendichte $\left[e \AA^{-3}\right]$ & $0.835 /-0.491$ & $2.140 /-1.067$ \\
\hline
\end{tabular}




\begin{tabular}{|c|c|c|}
\hline Verbindung & 23 & 25 \\
\hline \multirow{2}{*}{ Formel } & $\mathrm{C}_{66} \mathrm{H}_{102} \mathrm{Cl}_{4} \mathrm{~N}_{20} \mathrm{Ni}_{3} \mathrm{O}_{8}$ & $\mathrm{C}_{58} \mathrm{H}_{78} \mathrm{Cl}_{4} \mathrm{~N}_{20} \mathrm{Ni}_{3} \mathrm{O}_{4}$ \\
\hline & $+5 \mathrm{MeOH}$ & $+\left(2 \mathrm{MeOH}+\mathrm{Et}_{2} \mathrm{O}\right)$ \\
\hline$M\left[\mathrm{~g} \mathrm{~mol}^{-1}\right]$ & 1781.82 & 1575.54 \\
\hline Kristallgröße [mm] & $0.38 \times 0.24 \times 0.14$ & $0.36 \times 0.24 \times 0.18$ \\
\hline Kristallsystem & triklin & triklin \\
\hline Raumgruppe & $P-1$ & $P-1$ \\
\hline$a[\AA]$ & $10.6658(8)$ & $10.9805(7)$ \\
\hline$b[\AA]$ & $15.0222(137)$ & $12.4995(7)$ \\
\hline$c[\AA]$ & $15.5924(13)$ & $14.1221(8)$ \\
\hline$\alpha\left[^{\circ}\right]$ & $107.507(6)$ & $77.448(5)$ \\
\hline$\beta\left[^{\circ}\right]$ & $98.908(6)$ & $83.058(5)$ \\
\hline$\gamma\left[^{\circ}\right]$ & $108.519(6)$ & $79.083(5)$ \\
\hline$V\left[\AA^{3}\right]$ & $2170.8(3)$ & $1851.27(19)$ \\
\hline$Z$ & 1 & 1 \\
\hline$\rho_{\text {ber. }}\left[\mathrm{g} \mathrm{cm}^{-3}\right]$ & 1.363 & 1.413 \\
\hline$\mu\left(\mathrm{Mo}^{-\mathrm{K}_{\alpha}}\right)\left[\mathrm{mm}^{-1}\right]$ & 0.835 & 0.964 \\
\hline$F(000)$ & 944 & 828 \\
\hline$\theta$ Bereich $\left[^{\circ}\right]$ & $1.66-24.71$ & $1.48-24.85$ \\
\hline hkl Bereich & $-11-12, \pm 17, \pm 18$ & $\pm 12, \pm 14, \pm 16$ \\
\hline Gemessene Reflexe & 21500 & 29726 \\
\hline Unabhängige Reflexe [ $\left.R_{\text {int }}\right]$ & $7336[0.0694]$ & $6371[0.0649]$ \\
\hline Verfeinerte Parameter & 531 & 443 \\
\hline Goodness-of-fit & 1.002 & 1.007 \\
\hline$R 1(I>2 \sigma(I))$ & 0.0583 & 0.0380 \\
\hline wR2 (alle Daten) & 0.1539 & 0.1020 \\
\hline Restelektronendichte $\left[\mathrm{e} \AA^{-3}\right]$ & $1.163 /-1.306$ & $0.798 /-0.529$ \\
\hline
\end{tabular}




\begin{tabular}{|c|c|c|}
\hline Verbindung & 26 & 27 \\
\hline Formel & $\mathrm{C}_{24} \mathrm{H}_{22} \mathrm{Cl}_{2} \mathrm{~N}_{6} \mathrm{NiO}_{8}$ & $\mathrm{C}_{15} \mathrm{H}_{21} \mathrm{NaNiO}_{6}$ \\
\hline$M\left[\mathrm{~g} \mathrm{~mol}^{-1}\right]$ & 652.09 & 379.02 \\
\hline Kristallgröße [mm] & $0.26 \times 0.20 \times 0.16$ & $0.43 \times 0.32 \times 0.22$ \\
\hline Kristallsystem & triklin & trigonal \\
\hline Raumgruppe & $P-1$ & $R-3 c$ \\
\hline$a[\AA]$ & $10.1007(11)$ & $16.1421(9)$ \\
\hline$b[\AA ̊ \AA]$ & $10.2300(11)$ & $16.1421(9)$ \\
\hline$c[\AA$ & $15.4624(16)$ & $11.6727(7)$ \\
\hline$\alpha\left[^{\circ}\right]$ & $82.558(8)$ & 90 \\
\hline$\beta\left[^{\circ}\right]$ & $83.417(8)$ & 90 \\
\hline$\gamma\left[{ }^{\circ}\right]$ & $60.711(7)$ & 120 \\
\hline$V\left[\AA^{3}\right]$ & 1379.3(3) & $2634.0(3)$ \\
\hline$z$ & 2 & 6 \\
\hline$\rho_{\text {ber. }}\left[\mathrm{g} \mathrm{cm}^{-3}\right]$ & 1.570 & 1.434 \\
\hline$\mu\left(\mathrm{Mo}-\mathrm{K}_{\alpha}\right)\left[\mathrm{mm}^{-1}\right]$ & 0.956 & 1.154 \\
\hline$F(000)$ & 668 & 1188 \\
\hline$\theta$ Bereich $\left[^{\circ}\right]$ & $2.29-24.62$ & $3.78-27.31$ \\
\hline$h k /$ Bereich & $\pm 11, \pm 11,-18-17$ & $\pm 20, \pm 20,-15-12$ \\
\hline Gemessene Reflexe & 13933 & 13251 \\
\hline Unabhängige Reflexe $\left[R_{\text {int }}\right]$ & $4544[0.0825]$ & $666[0.0349]$ \\
\hline Verfeinerte Parameter & 372 & 38 \\
\hline Goodness-of-fit & 1.017 & 1.135 \\
\hline$R 1(I>2 \sigma(I))$ & 0.0376 & 0.0220 \\
\hline wR2 (alle Daten) & 0.0795 & 0.0596 \\
\hline Restelektronendichte $\left[\mathrm{e} \AA^{-3}\right]$ & $0.311 /-0.367$ & $0.233 /-0.282$ \\
\hline
\end{tabular}




\begin{tabular}{|c|c|}
\hline Verbindung & 28 \\
\hline \multirow{2}{*}{ Formel } & $\mathrm{C}_{54} \mathrm{H}_{68} \mathrm{Cu}_{4} \mathrm{~F}_{24} \mathrm{~N}_{24} \mathrm{O}_{6} \mathrm{P}_{4}$ \\
\hline & $+2 \mathrm{MeCN}$ \\
\hline$M\left[\mathrm{~g} \mathrm{~mol}^{-1}\right]$ & 2065.47 \\
\hline Kristallgröße [mm] & $0.43 \times 0.31 \times 0.24$ \\
\hline Kristallsystem & triklin \\
\hline Raumgruppe & $P-1$ \\
\hline$a[\AA]$ & $11.8201(9)$ \\
\hline$b[\AA ̊]$ & $13.2614(11)$ \\
\hline$c[\AA]$ & $14.3938(11)$ \\
\hline$\alpha\left[^{\circ}\right]$ & $99.536(6)$ \\
\hline$\beta\left[^{\circ}\right]$ & $107.032(6)$ \\
\hline$\gamma\left[^{\circ}\right]$ & $107.647(6)$ \\
\hline$V\left[\AA^{3}\right]$ & 1974.0(3) \\
\hline$Z$ & 1 \\
\hline$\rho_{\text {oer. }}\left[\mathrm{g} \mathrm{cm}^{-3}\right]$ & 1.738 \\
\hline$\mu\left(\mathrm{Mo}-\mathrm{K}_{\alpha}\right)\left[\mathrm{mm}^{-1}\right]$ & 1.267 \\
\hline$F(000)$ & 1044 \\
\hline$\theta$ Bereich $\left[^{\circ}\right]$ & $1.68-24.69$ \\
\hline$h k /$ Bereich & $\pm 13, \pm 15, \pm 16$ \\
\hline Gemessene Reflexe & 21662 \\
\hline Unabhängige Reflexe $\left[R_{\text {int }}\right]$ & $6648[0.0388]$ \\
\hline Verfeinerte Parameter & 557 \\
\hline Goodness-of-fit & 1.026 \\
\hline$R 1(I>2 \sigma(I))$ & 0.0364 \\
\hline wR2 (alle Daten) & 0.0904 \\
\hline Restelektronendichte [e $\AA^{-3}$ ] & $0.582 /-0.842$ \\
\hline
\end{tabular}




\section{Strukturverzeichnis}

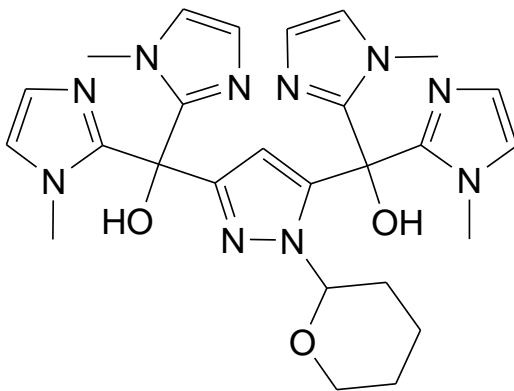

2a

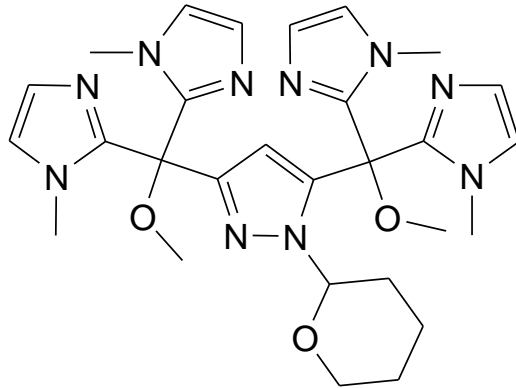

3a

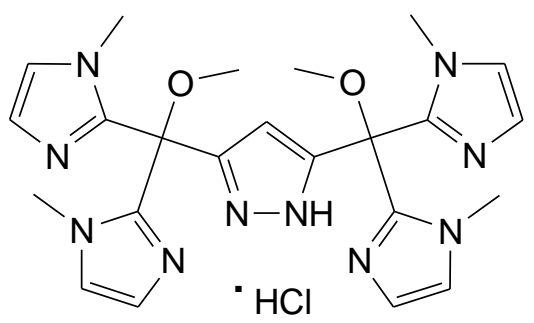

$4 a$

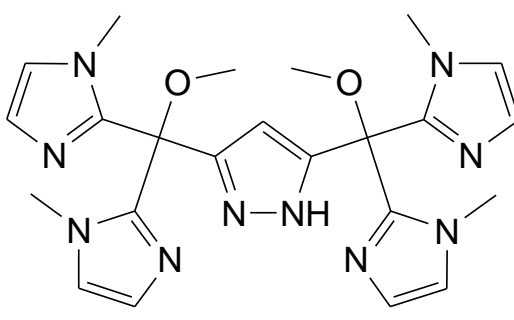

$5 a$

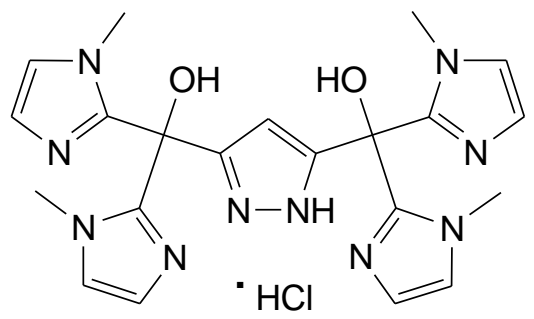

$6 a$

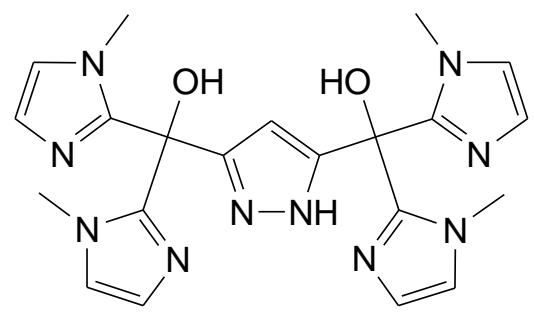

$7 a$

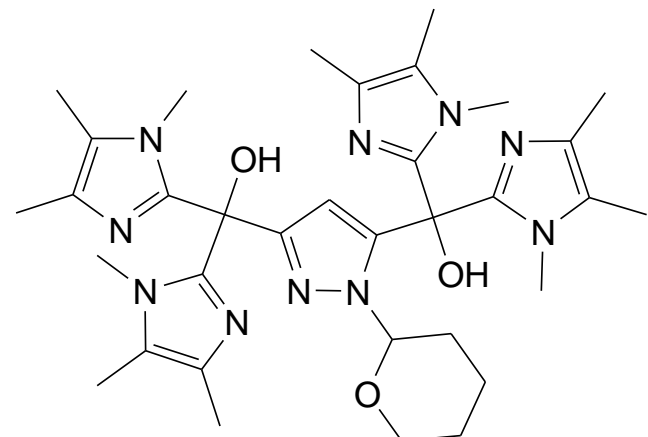

$2 b$

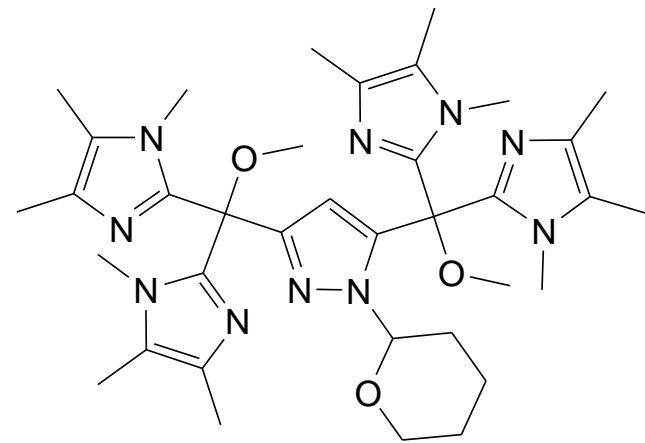

$3 b$ 


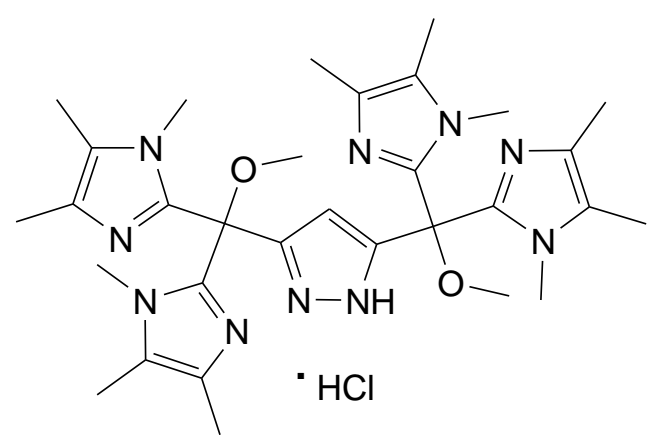

$4 b$

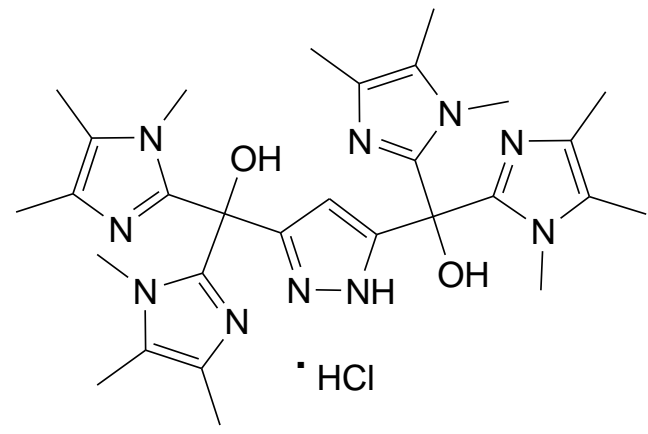

$6 b$

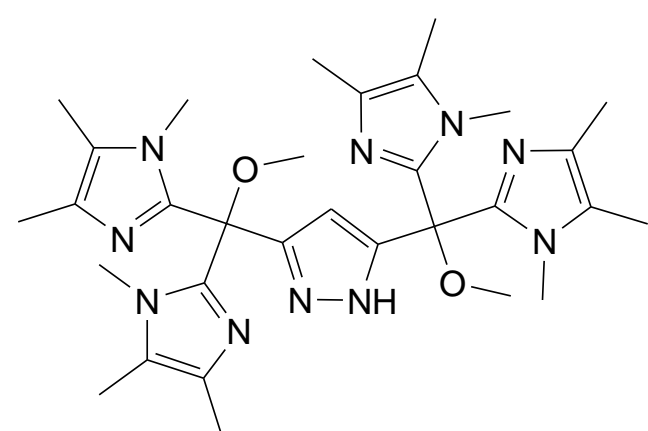

$5 b$

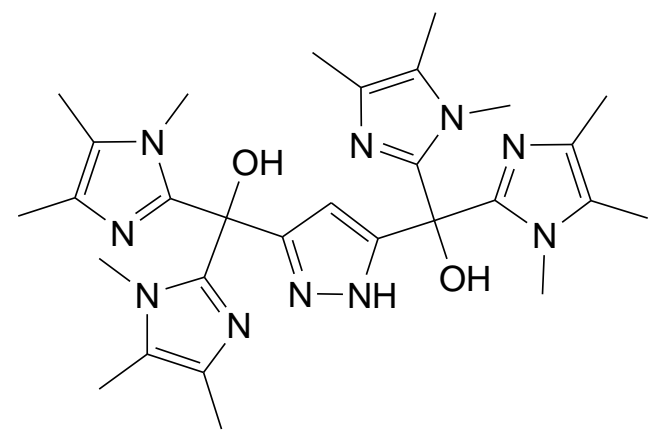

$7 b$

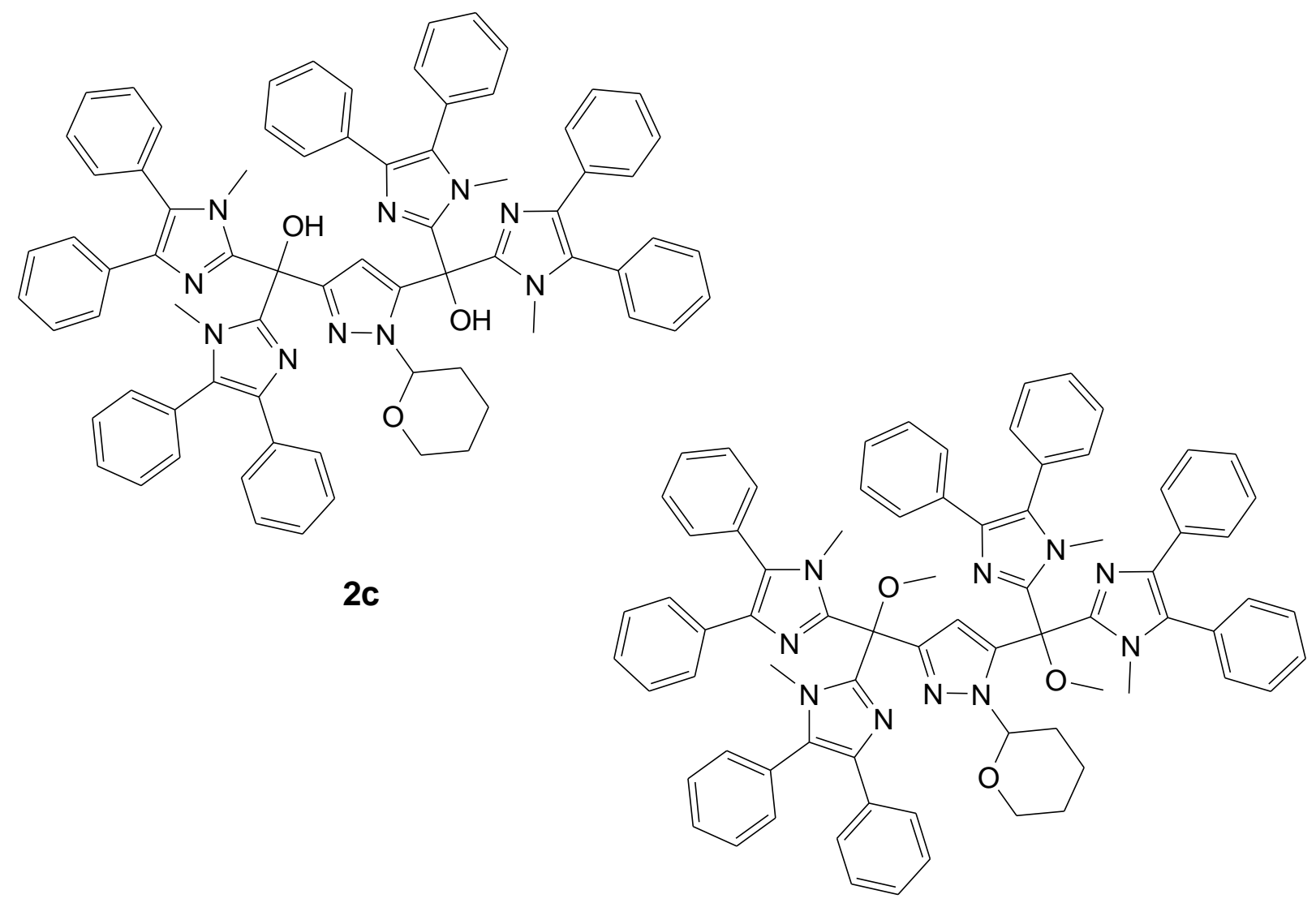

$3 c$ 

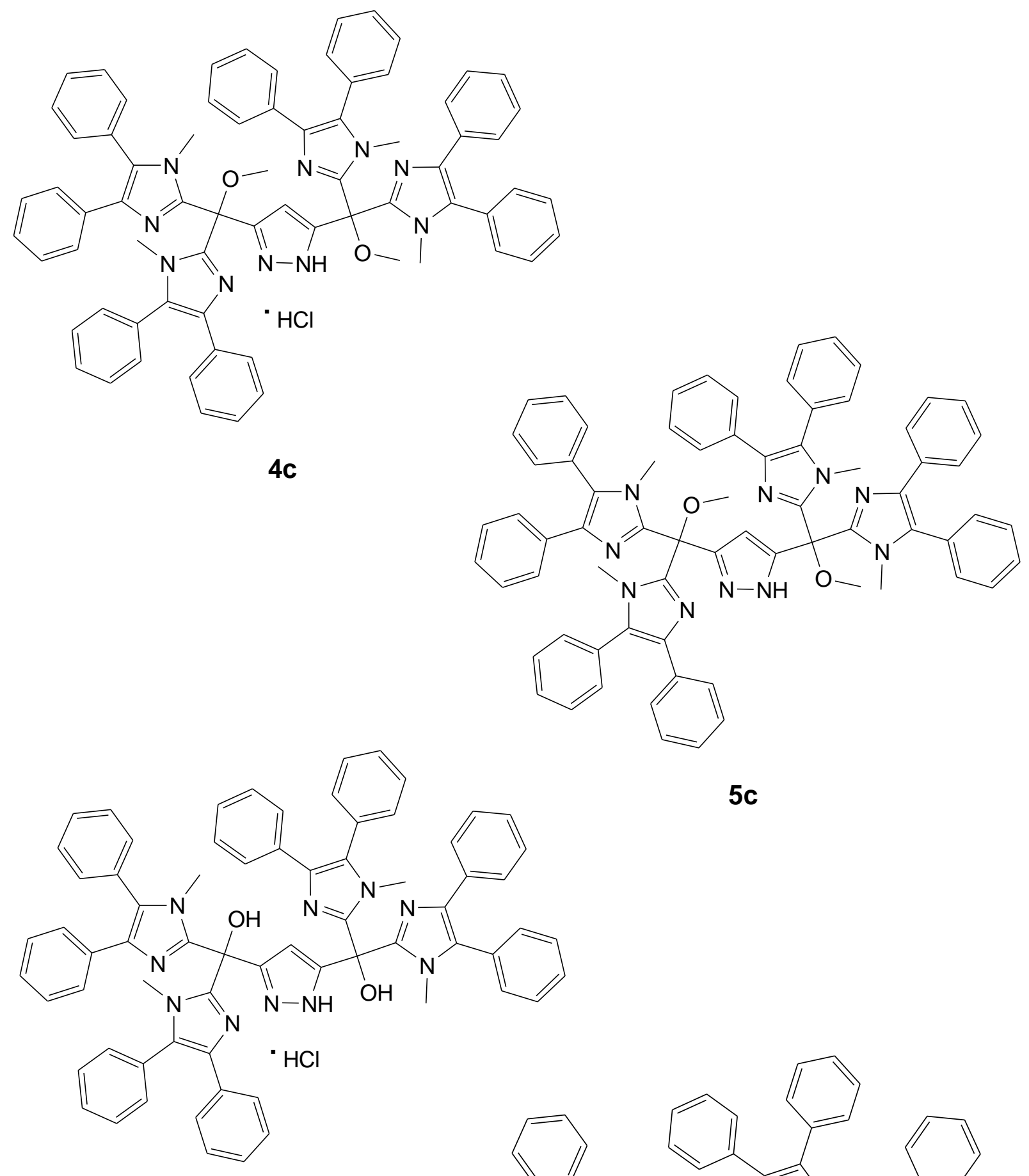

$6 c$

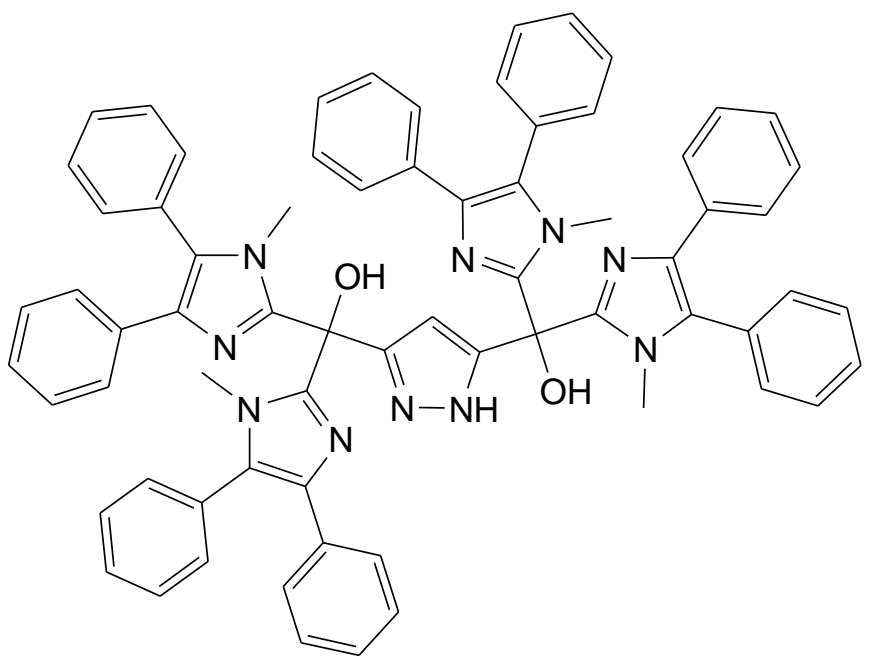

7c 


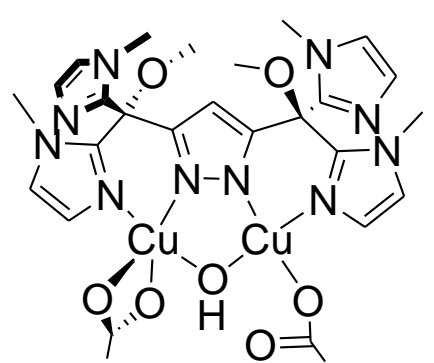

8

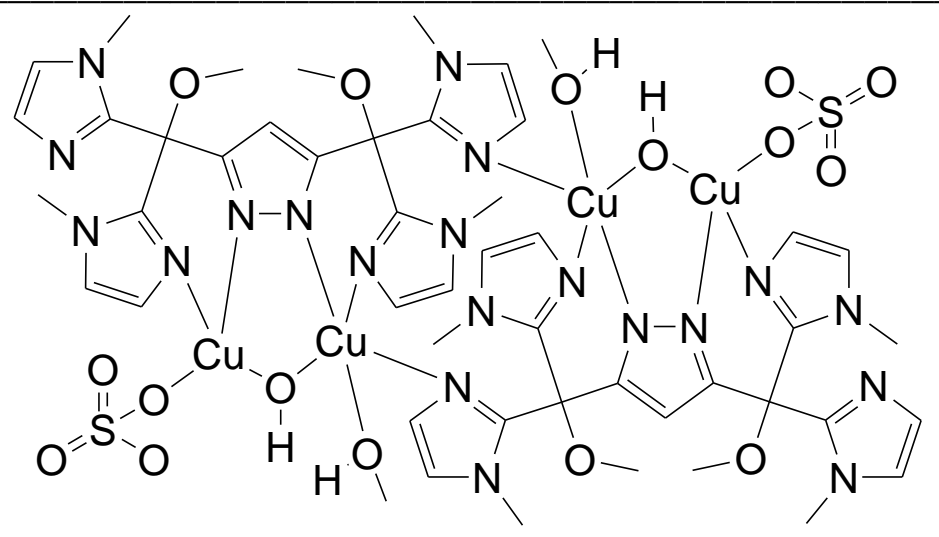

10

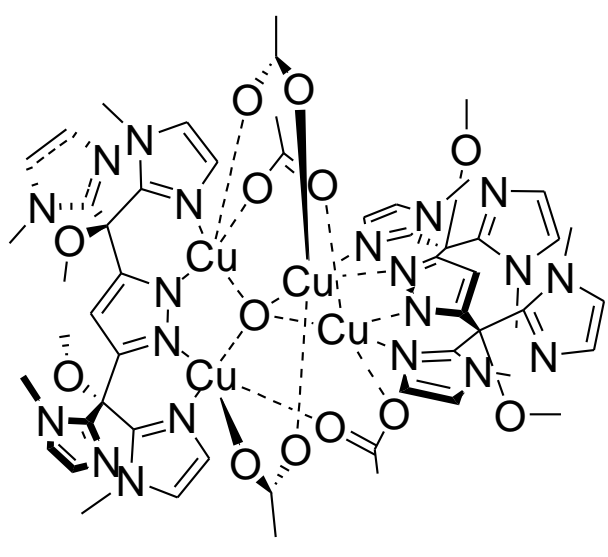

9

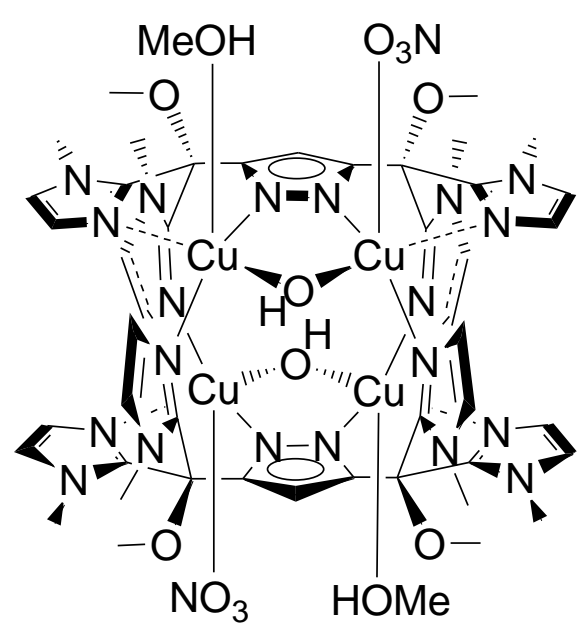

$11 a$

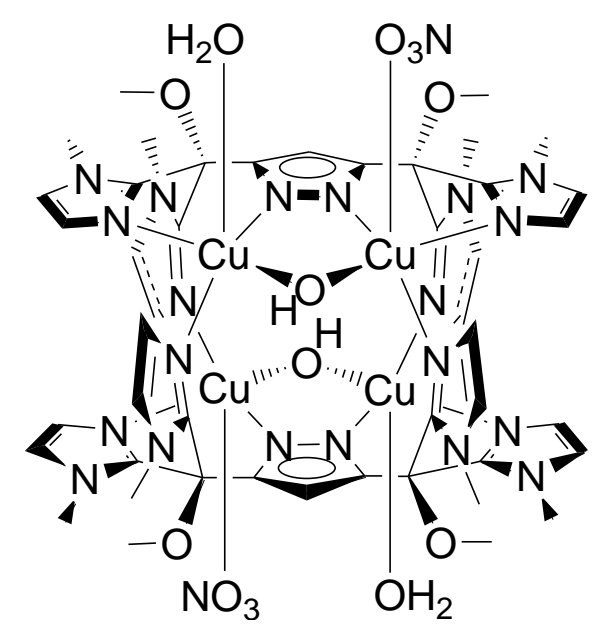

11b

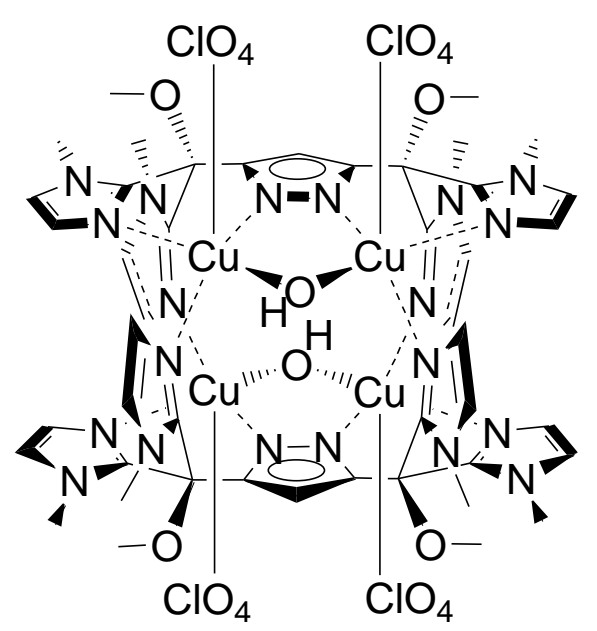

12 


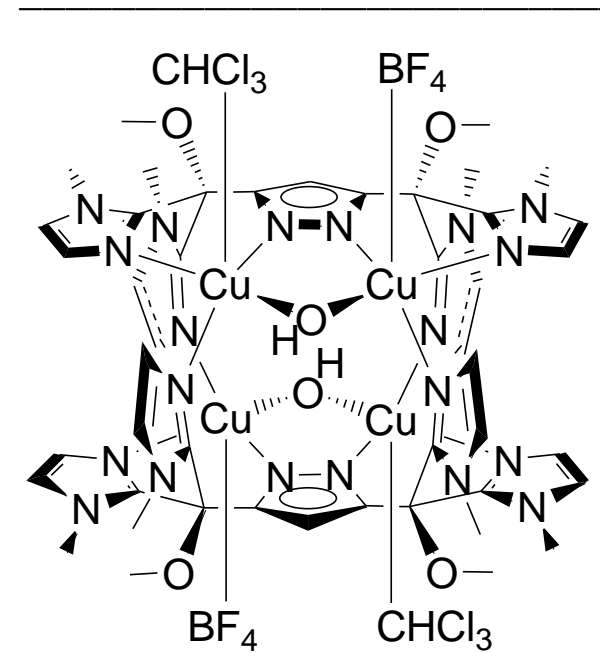

13

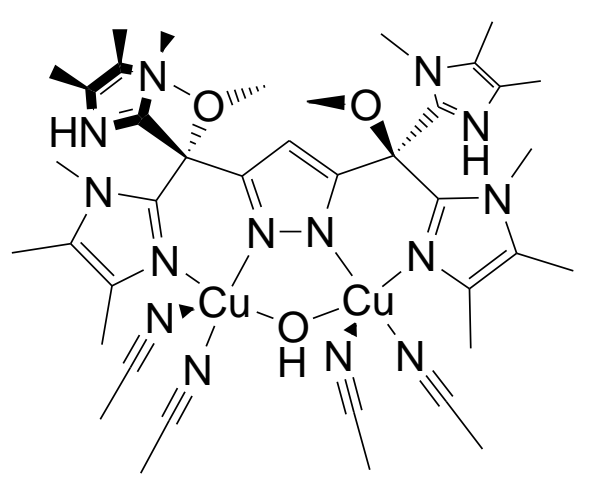

15

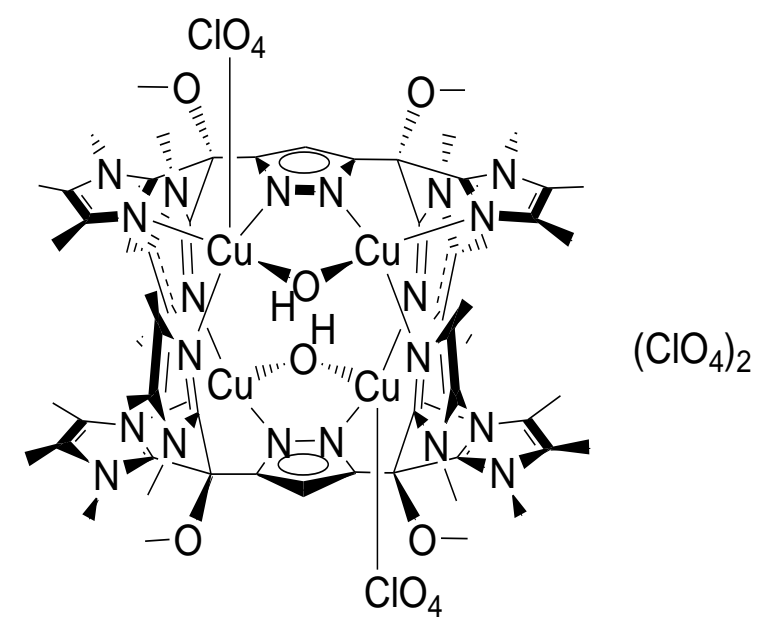

14

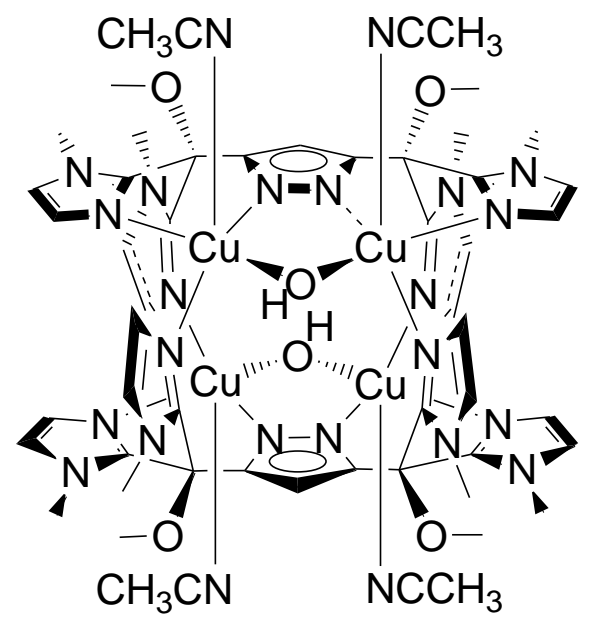

28

$\left(\mathrm{BF}_{4}\right)_{2}$

$\left(\mathrm{ClO}_{4}\right)_{4}$

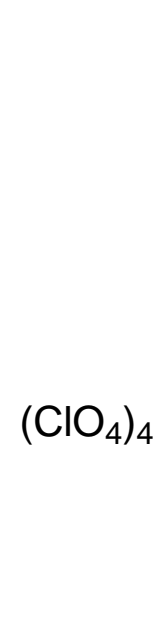

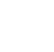

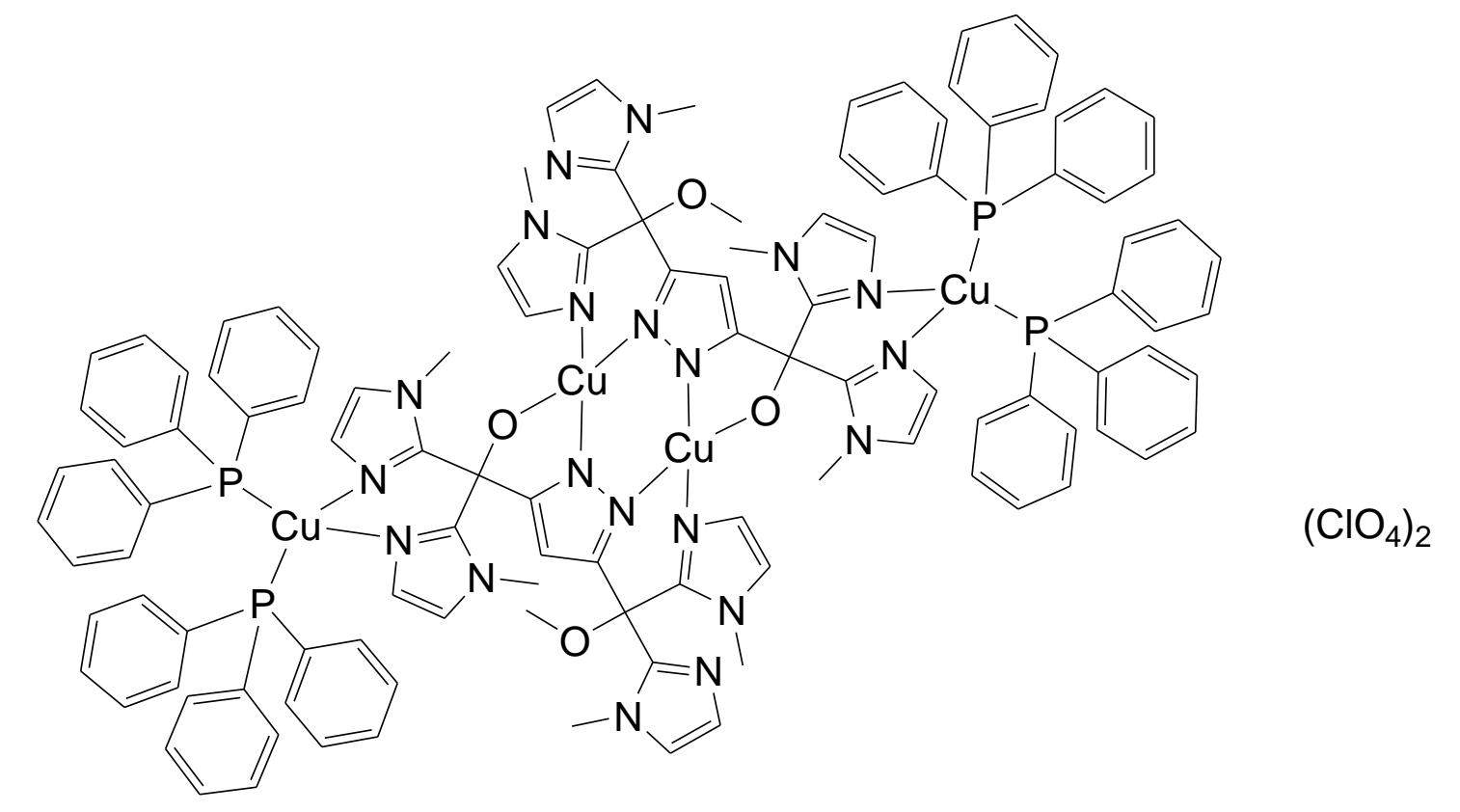




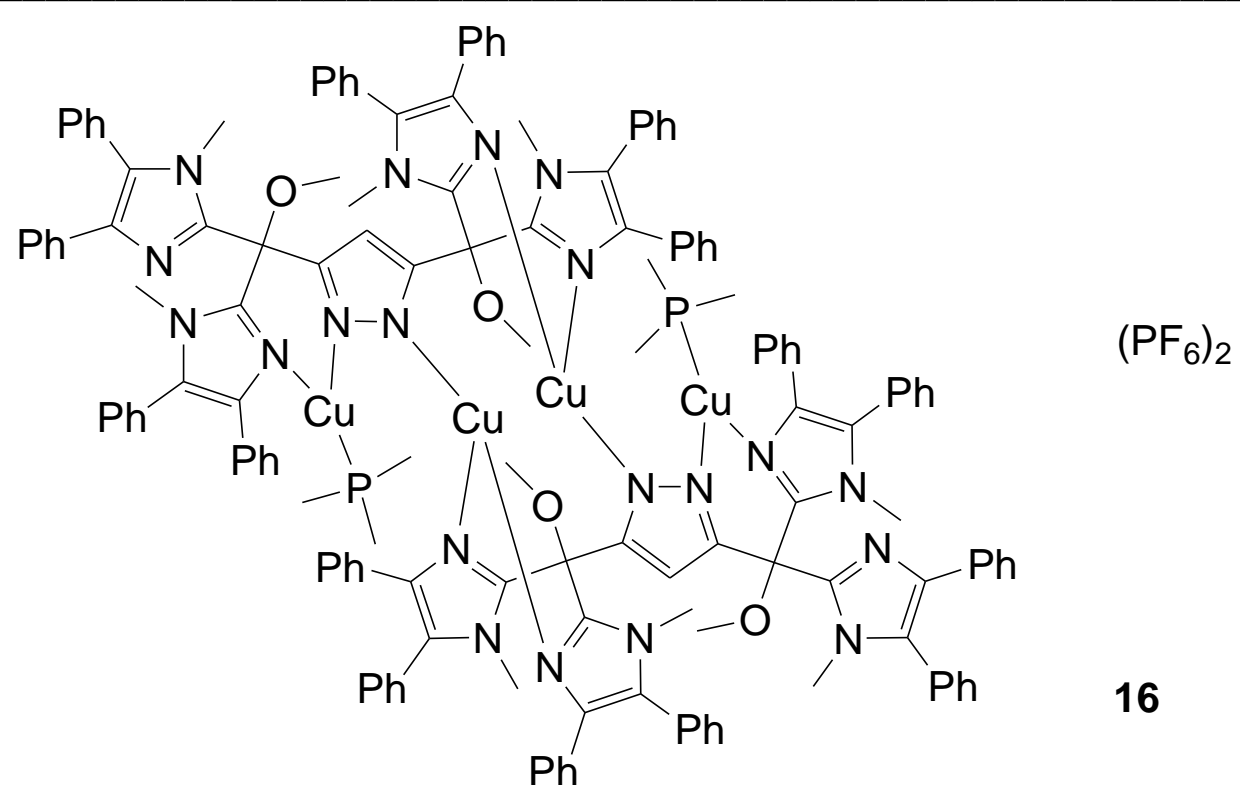

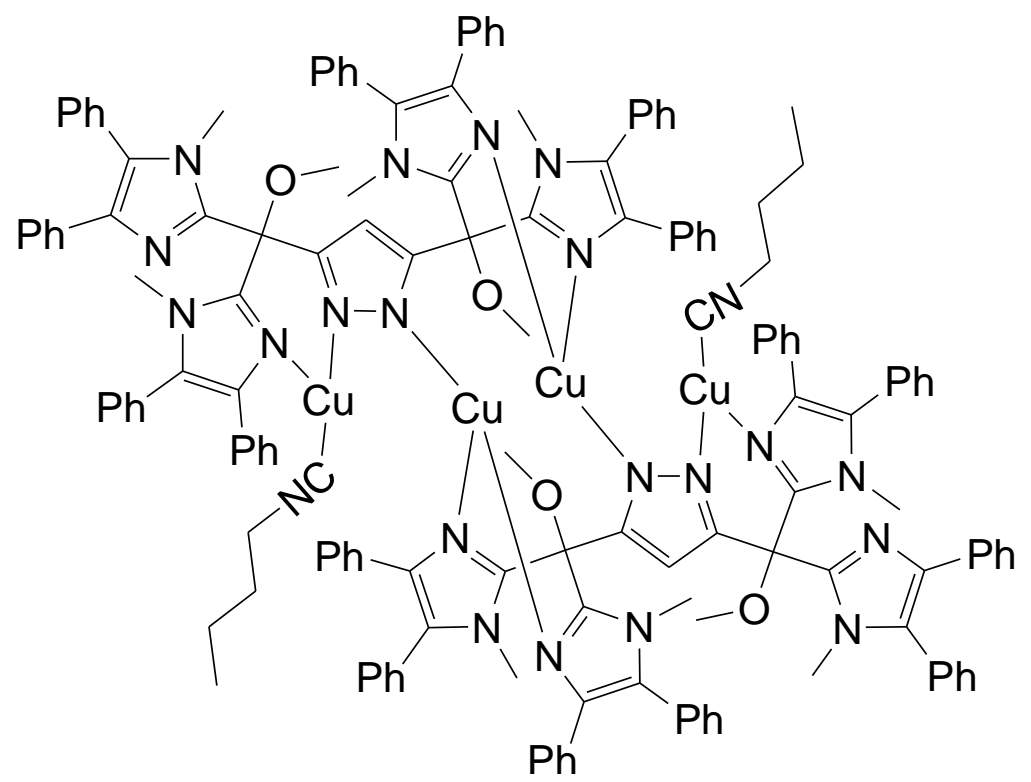

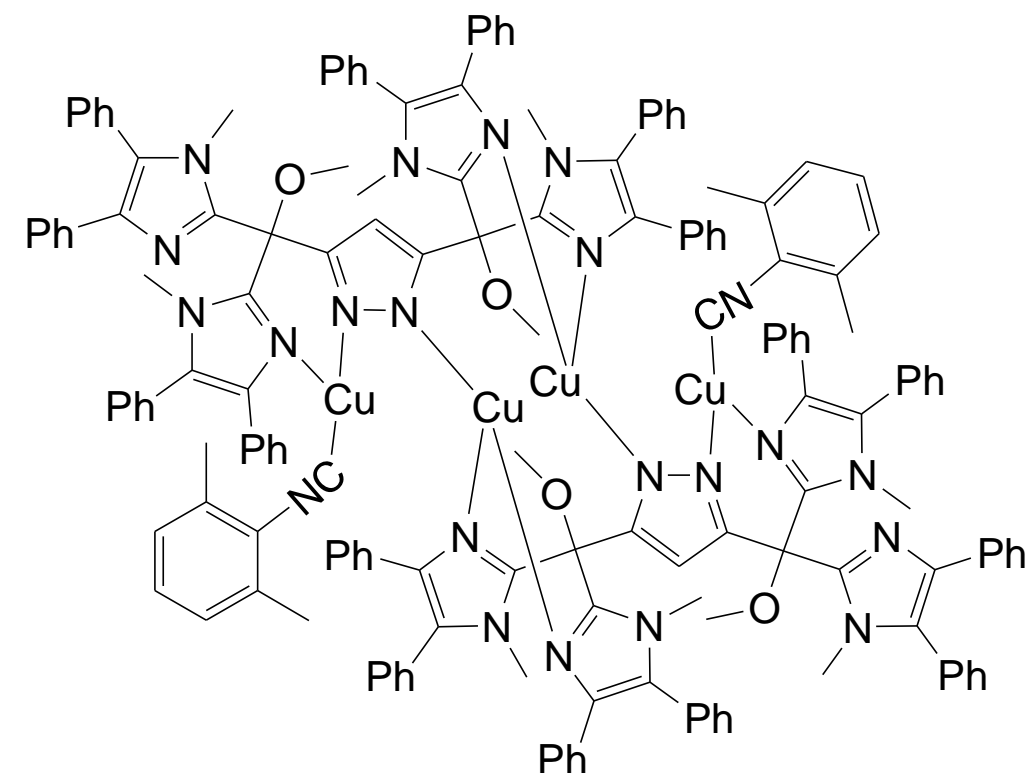




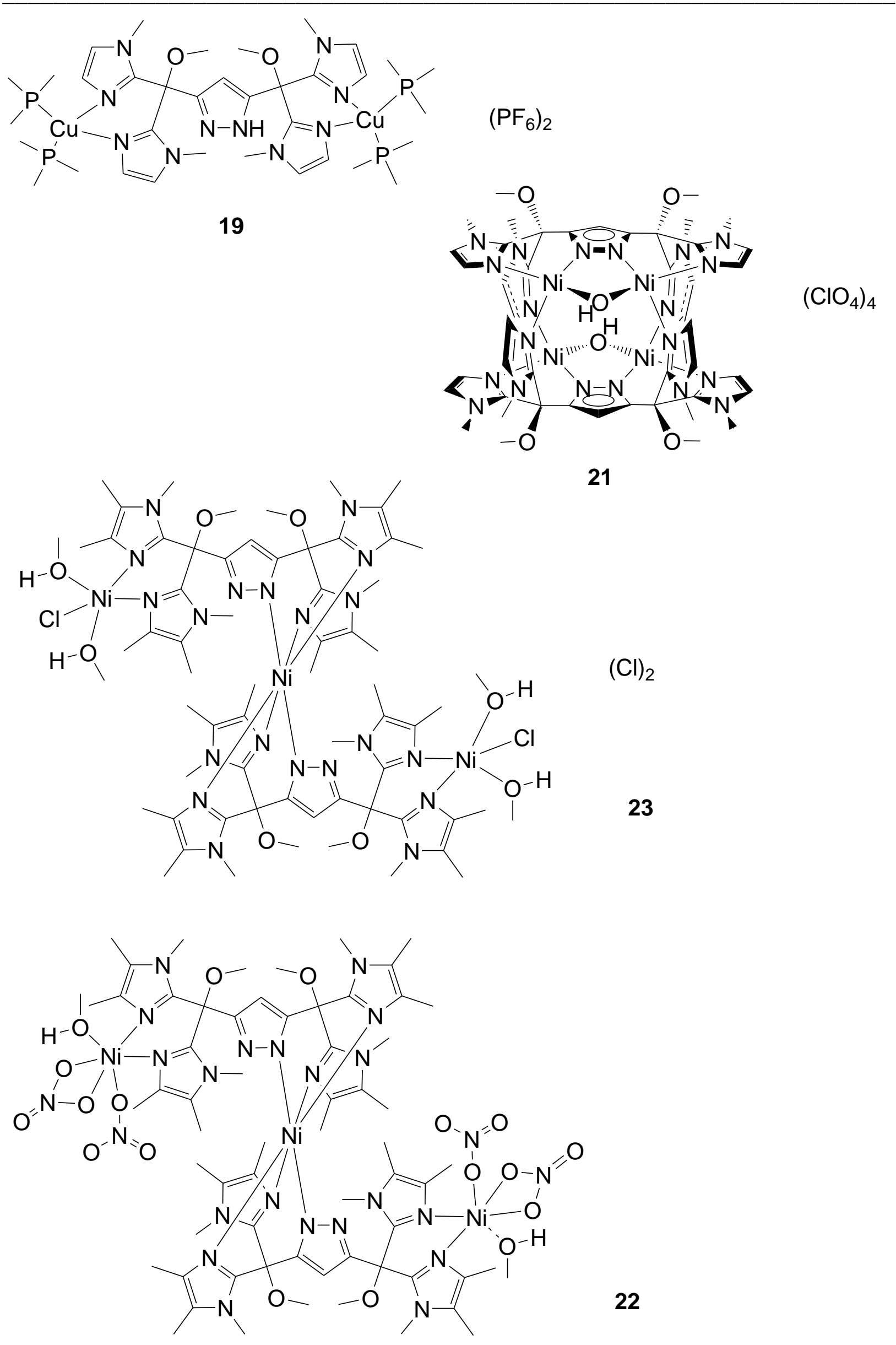




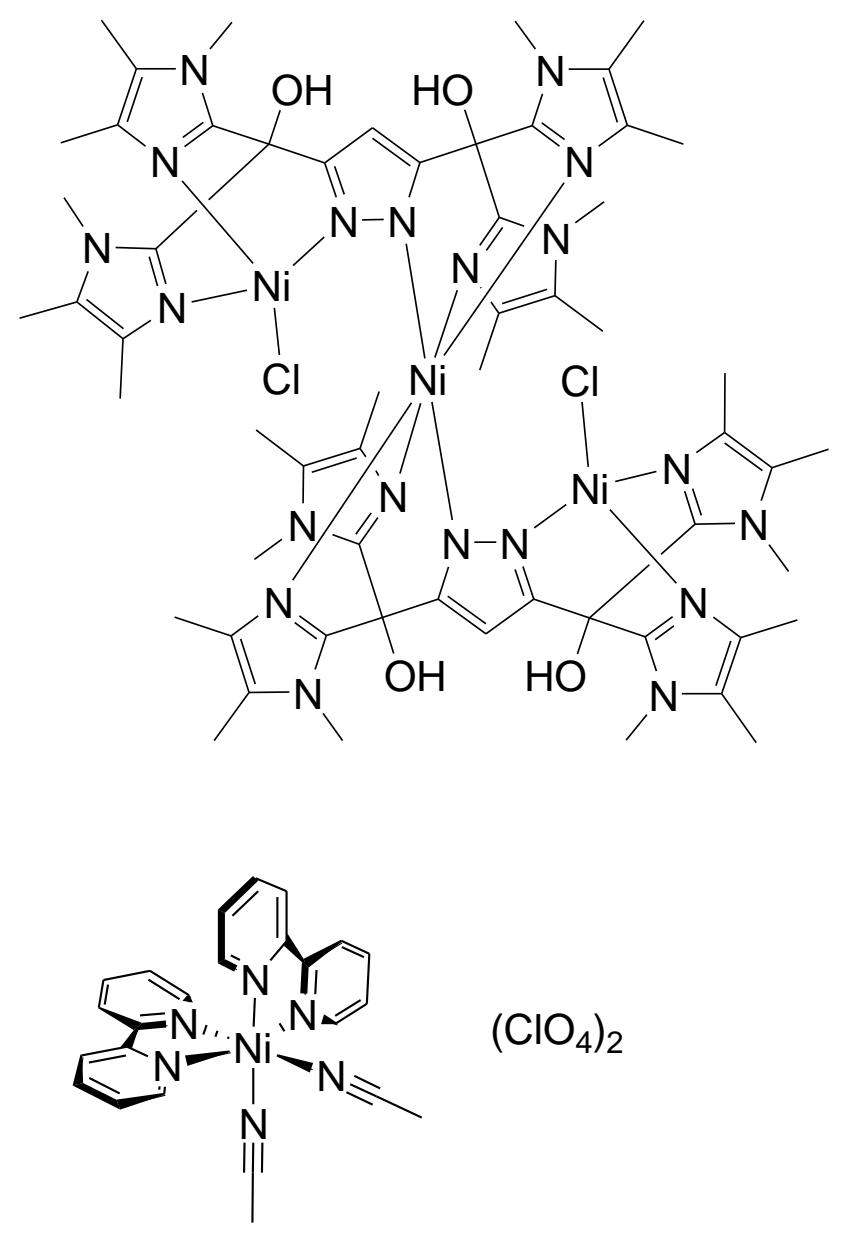

$(\mathrm{Cl})_{2}$

26

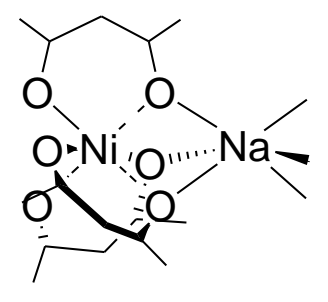

27 


\section{Literaturverzeichnis}

[1] J. Falbe, M. Regitz, RÖMPP Chemie Lexikon, Georg-Thieme-Verlag, Stuttgart, New York, 1990.

[2] P. Enhag, Encyclopedia of the elements, 1. Auflage, Wiley VCH-Verlag, Weinheim, 2004.

[3] C.E. Mortimer, U. Müller, Chemie - Das Basiswissen der Chemie, 7.Auflage, Georg-Thieme-Verlag, Stuttgart, 2001.

[4] A.F. Hollemann, E. Wiberg, Lehrbuch der Anorganischen Chemie, 101. Auflage, de Gruyter Verlag 1995.

[5] N.N. Greenwood, A. Earnshaw, Chemie der Elemente, 1. Auflage, Wiley-VCHVerlag, Weinheim, 1988.

[6] R.T. Morrison, R.N. Boyd, Lehrbuch der Organischen Chemie, 3. Auflage, VCH Verlag, Weinheim, New York, 1986.

[7] A.H. Lewin, T. Cohen, Tetrahedron Lett., 1965, 6, 4531.

[8] E. Pfeil, Angew. Chem., 1953, 65, 155.

[9] F. Meyer, H. Pritzkow, Angew. Chem., Int. Ed., 2000, 39, 2112.

[10] J. Ackermann, F. Meyer, E. Kaifer, H. Pritzkow, Chem. Eur. J., 2002, 8, 247.

[11] J. Ackermann, F. Meyer, H. Pritzkow, Inorg. Chim. Acta, 2004, 357, 3703.

[12] J. Ackermann, F. Meyer, H. Pritzkow, Z. Anorg. Allg. Chem., 2004, 630, 2627.

[13] B. Bauer-Siebenlist, F. Meyer, E. Farkas, D. Vidovic, J.A. Cuesta-Seijo, R. Herbst-Irmer, H. Pritzkow, Inorg. Chem., 2004, 43, 4189.

[14] G. Leibeling, S. Demeshko, B. Bauer-Siebenlist, F. Meyer, H. Pritzkow, Eur. J. Inorg. Chem., 2004, 2413.

[15] B. Bauer-Siebenlist, S. Dechert, F. Meyer, Chem. Eur. J., 2005, 11, 5343.

[16] B. Bauer-Siebenlist, F. Meyer, E. Farkas, D. Vidovic, S. Dechert, Chem. Eur. J., 2005, 11, 4349.

[17] F. Meyer, Eur. J. Inorg. Chem., 2006, 3789.

[18] A. Prokofieva, A.I. Prikhod'ko, A.E. Enyedy, E. Farkas, W. Maringgele, S.

Demeshko, S. Dechert, F. Meyer, Inorg. Chem., 2007, 46, 4298.

[19] F. Meyer, H. Pritzkow, Eur. J. Inorg. Chem., 2005, 2346.

[20] T.N. Sorrell, A.S. Borovik, J. Am. Chem. Soc., 1987, 109, 4255. 
[21] T. Rüther, K.J. Cavell, N. Braussaud, W. Skelton, A.H. White, J. Chem. Soc., Dalton Trans., 2002, 4684.

[22] R.T. Stibrany, J.A. Potenza, H.J. Schugar, Inorg. Chim. Acta, 1996, 243, 33.

[23] T.C. Higgs, M. Helliwell, E.J.L. McInnes, F.E. Mabbs, C.E. Harding, C.D. Garner, J. Chem. Soc., Dalton Trans., 1997, 927.

[24] L.P. Wu, Y. Yamagiwa, I. Ino, K. Sugimoto, T. Kuroda-Sowa, T. Kamikawa, M. Munakata, Polyhedron, 1999, 18, 2047.

[25] L. Zhou, D. Powell, K.M. Nicholas, Inorg. Chem., 2007, 46, 2316.

[26] C.E. Ruggiero, S.M. Carrier, W.E. Antholine, J.W. Whittaker, C.J. Cramer, W.B. Tolman, J. Am. Chem. Soc., 1993, 115, 11285.

[27] K. Fujisawa, T. Kobayashi, K. Fujita, N. Kitajima, Y. Moro-oka, Y. Miyashita, Y. Yamada, K.-i. Okamoto, Bull. Chem. Soc. Jpn., 2000, 73, 1797.

[28] I. Sylvestre, J. Wolowska, E.J.L. Mclnnes, C.A. Kilner, M. Halcrow, Inorg. Chim. Acta, 2005, 338, 1337.

[29] S. Dhar, P.A.N. Reddy, M. Nethaij, S. Mahadevan, M.K. Saha, A.R. Chakravarty, Inorg. Chem., 2002, 41, 3469.

[30] E. Riedel, Anorganische Chemie, 3. Auflage, de Gryuter, Berlin, New York, 1994.

[31] H.E.M. Christensen, L.S. Conrad, K.V. Mikkelsen, M.K. Nielsen, J. Ulstrup, Inorg. Chem., 1990, 29, 2808.

[32] W. Kaim, B. Schwederski, Bioanorganische Chemie, 4. Auflage, Teubner Studienbücher, Stuttgart, 2005.

[33] W. Kaim, J. Rall, Angew. Chem., 1996, 108, 47.

[34] W.E.B. Shepard, B.F. Anderson, D.A. Lewandoski, G.E. Norris, E.N. Baker, J. Am. Chem. Soc., 1990, 112, 7817.

[35] E.I. Solomon, M.J. Baldwin, M.D. Lowery, Chem. Rev., 1992, 92, 521.

[36] J.A. Guckert, M.D. Lowery, E.I. Solomon, J. Am. Chem. Soc., 1995, 117, 2817.

[37] W.S. McIntire, FASEB J., 1994, 8, 513.

[38] Y. Matoba, T. Kumagai, A. Yamamoto, H. Yoshitsu, M. Sugiyama, J. Biol. Chem., 2006, 281, 8981.

[39] H. Decker, T. Schweikardt, F. Tuczek, Angew. Chem., 2006, 118, 4658.

[40] A. Volbeda, W.G.J. Hol, J. Mol. Biol., 1989, 209, 249.

[41] B. Hazes, K.A. Magnus, C. Bonaventura, J. Bonaventura, Z. Dauter, K.H. Kalk, W.G.J. Hol, Protein Sci., 1993, 2, 597. 
[42] J.M. Brown, L. Powers, B. Kincaid, J.A. Larrabee, T.G. Spiro, J. Am. Chem. Soc., 1980, 102, 4210.

[43] G.L. Woolery, L. Powers, M. Winkler, E.I. Solomon, T.G. Spiro, J. Am. Chem. Soc., 1984, 106, 86.

[44] A. Messerschmidt, A. Rossi, R. Ladenstein, R. Huber, M. Bolognesi, G. Gatti, A. Marchesini, R. Petruzzelli, A. Finazzi-Agro, J. Mol. Biol., 1989, 206, 513.

[45] K.A. Magnus, B. Hazes, H. Ton-That, C. Bonaventura, J. Bonaventura, Proteins: Struct., Funct., Gen., 1994, 19, 302.

[46] M. Kodera, Y. Kajita, Y. Tachi, K. Katayama, K. Kano, S. Hirota, S. Fujinami, M. Suzuki, Angew. Chem., Int. Ed., 2004, 43, 334.

[47] P. Ghosh, Z. Tyeklar, K.D. Karlin, R.R. Jacobson, J. Zubieta, J. Am. Chem. Soc., 1987, 109, 6889.

[48] M. Kodera, K. Katayama, Y. Tachi, K. Kano, S. Hirota, S. Fujinami, M. Suzuki, J. Am. Chem. Soc., 1999, 121, 11006.

[49] N. Kitajima, K. Fujisawa, Y. Moro-oka, K. Toriumi, J. Am. Chem. Soc., 1989, 111, 8975.

[50] N. Kitajima, K. Fujisawa, C. Fujimoto, Y. Moro-oka, S. Hashimoto, T. Kitagawa, K. Toriumi, K. Tatsumi, A. Nakamura, J. Am. Chem. Soc., 1992, 114, 1277.

[51] K. Itoh, H. Hayashi, H. Furutachi, T. Matsumoto, S. Nagatomo, T. Tosha, S. Terada, S. Fujinami, M. Suzuki, T. Kitagawa, J. Am. Chem. Soc., 2005, 127, 5212.

[52] B.M.T. Lam, J.A. Halfen, V.G. Young, J.R. Hagadorn, P.L. Holland, A. Lledos, L. Cucurull-Sanchez, J.J. Novoa, S. Alvarez, W.B. Tolman, Inorg. Chem., 2000, 39, 4059.

[53] A.M. Reynolds, B.F. Gherman, C.J. Cramer, W.B. Tolman, Inorg. Chem., 2005, 44, 6989.

[54] N.W. Aboelella, E.A. Lewis, A.M. Reynolds, W.W. Brennessel, C.J. Cramer, W.B. Tolman, J. Am. Chem. Soc., 2002, 124, 10660.

[55] N.W. Aboelella, S.V. Kryatov, B.F. Gherman, W.W. Brennessel, V.G. Young, R. Sarangi, E.V. Rybak-Akimova, K.O. Hodgson, B. Hedman, E.I. Solomon, C.J. Cramer, W.B. Tolman, J. Am. Chem. Soc., 2004, 126, 16896.

[56] J. Reim, R. Werner, W. Haase, B. Krebs, Chem. Eur. J., 1998, 4, 289.

[57] Z. Hu, G.N. George, S.M. Gorun, Inorg. Chem., 2001, 40, 4812. 
[58] H.V. Obias, Y. Lin, N.N. Murthy, E. Pidcock, E.I. Solomon, M. Ralle, N.J. Blackburn, Y.M. Neuhold, A.D. Zuberbühler, K.D. Karlin, J. Am. Chem. Soc., 1998, 120, 12960.

[59] W.B. Tolman, Acc. Chem. Res., 1997, 30, 227.

[60] S. Mahapatra, J.A. Halfen, E.C. Wilkinson, G. Pan, X. Wang, V.G. Young, C.J. Cramer, L. Que, W.B. Tolman, J. Am. Chem. Soc., 1996, 118, 11555.

[61] S. Mahapatra, J.A. Halfen, E.C. Wilkinson, G. Pan, C.J. Cramer, L. Que Jr., W.B. Tolman, J. Am. Chem. Soc., 1995, 117, 8865.

[62] S. Mahapatra, J.A. Halfen, E.C. Wilkinson, L. Que, W.B. Tolman, J. Am. Chem. Soc., 1994, 116, 9785.

[63] E.I. Solomon, U.M. Sundaram, T.E. Machonkin, Chem. Rev., 1996, 96, 2563.

[64] S. Itoh, M. Taki, H. Nakao, P.L. Holland, W.B. Tolman, L. Que Jr, S. Fukuzumi, Angew. Chem., Int. Ed., 2000, 39, 398.

[65] L. Que Jr, W.B. Tolman, Angew. Chem., Int. Ed., 2002, 41, 1114.

[66] D.J.E. Spencer, N.W. Aboelella, A.M. Reynolds, P.L. Holland, W.B. Tolman, J. Am. Chem. Soc., 2002, 124, 2108.

[67] E. Pidcock, S. DeBeer, H.V. Obias, B. Hedman, K.O. Hodgson, K.D. Karlin, E.I. Solomon, J. Am. Chem. Soc., 1999, 121, 1870.

[68] D.J.E. Spencer, A.M. Reynolds, P.L. Holland, B.A. Jazdzewski, C. Duboc-Toia, L. Le Pape, S. Yokota, Y. Tachi, S. Itoh, W.B. Tolman, Inorg. Chem., 2002, 41, 6307.

[69] N. Kitajima, T. Katayama, K. Fujisawa, Y. Iwata, Y. Moro-oka, J. Am. Chem. Soc., 1993, 115, 7872.

[70] S. Mahapatra, S. Kaderli, A. Llobet, Y.M. Neuhold, T. Palanche, J.A. Halfen, V.G. Young, T.A. Kaden, L. Que, A.D. Zuberbuhler, W.B. Tolman, Inorg. Chem., 1997, $36,6343$.

[71] N. Kitajima, T. Koda, S. Hashimoto, T. Kitagiwa, Y. Moro-oka, J. Chem. Soc. Chem. Commun., 1988, 2, 151.

[72] N. Kitajima, Y. Moro-oka, J. Chem. Soc. Chem. Commun., 1993, 18, 2665.

[73] L.M. Mirica, M. Vance, D. Jackson Rudd, B. Hedman, K.O. Hodgson, E.I. Solomon, T.D.P. Stack, Science, 2005, 308, 1890.

[74] L.M. Mirica, D. Jackson Rudd, M.A. Vance, E.I. Solomon, K.O. Hodgson, B. Hedman, T.D.P. Stack, J. Am. Chem. Soc., 2006, 128, 2654. 
[75] E. Spodine, J. Manzur, Coord. Chem. Rev., 1992, 119, 171.

[76] E. Monzani, L. Quinti, A. Perotti, L. Casella, M. Gullotti, L. Randaccio, S. Geremia, G. Nardin, P. Faleschini, G. Tabbi, Inorg. Chem., 1998, 37, 553.

[77] E. Monzani, G. Battaini, A. Perotti, L. Casella, M. Gullotti, L. Santagostini, G. Nardin, L. Randaccio, S. Geremia, P. Zanello, G. Opromolla, Inorg. Chem., 1999, 38, 5359 .

[78] H. Ohi, Y. Tachi, S. Itoh, Inorg. Chem., 2006, 45, 10825.

[79] L. Casella, M. Gullotti, G. Pallanza, L. Rigoni, J. Am. Chem. Soc., 1988, 110, 4221.

[80] L. Casella, O. Carugo, M. Gullotti, S. Garofani, P. Zanello, Inorg. Chem., 1993, 32, 2056.

[81] K.D. Karlin, B.I. Cohen, R.R. Jacobson, J. Zubieta, J. Am. Chem. Soc., 1987, 109, 6194.

[82] P. Capdevielle, M. Maumy, Tetrahedron Lett., 1982, 23, 1573.

[83] F. Chioccara, P. Di Gennaro, G. La Monica, R. Sebastiano, B. Rindone, Tetrahedron, 1991, 47, 4429.

[84] L.M. Sayre, D.V. Nadkarni, J. Am. Chem. Soc., 1994, 116, 3157.

[85] L. Santagostini, M. Gullotti, E. Monzani, L. Casella, R. Dillinger, F. Tuczek, Chem. Eur. J., 2000, 6, 519.

[86] L.M. Mirica, M. Vance, D. Jackson Rudd, B. Hedman, K.O. Hodgson, E.I. Solomon, T.D. Stack, J. Am. Chem. Soc., 2002, 124, 9332.

[87] D.R. Yuan, J.M. Yan, C.Z. Yu, R.G. Xie, Chin. Chem. Lett., 2005, 16, 147.

[88] P.J. Baesjou, W.L. Driessen, G. Challa, J. Reedijk, J. Mol. Catal. A: Chem., 1996, 110, 195.

[89] M. Kodera, H. Shimakoshi, Y. Tachi, K. Katayama, K. Kano, Chem. Lett., 1998, 27, 441.

[90] R. Gupta, R. Mukherjee, Tetrahedron Lett., 2000, 41, 7763.

[91] K.D. Karlin, J.C. Hayes, Y. Gultneh, R.W. Cruse, J.W. McKown, J.P. Hutchinson, J. Zubieta, J. Am. Chem. Soc., 1984, 106, 2121.

[92] M.S. Nasir, B.I. Cohen, K.D. Karlin, J. Am. Chem. Soc., 1992, 114, 2482.

[93] E. Pidcock, H.V. Obias, C.X. Zhang, K.D. Karlin, E.I. Solomon, J. Am. Chem. Soc., 1998, 120, 7841.

[94] A.L. Gavrilova, B. Bosnich, Chem. Rev., 2004, 104, 349. 
[95] J.C. Röder, F. Meyer, E. Kaifer, Angew. Chem., 2002, 114, 2414.

[96] J.C. Röder, F. Meyer, H. Pritzkow, Organometallics, 2001, 20, 811.

[97] J.C. Röder, F. Meyer, H. Pritzkow, Zeitschrift für Naturforschung, 2002, 57b, 773.

[98] M. Alvarino Gil, Dissertation, Georg-August-Universität Göttingen 2006

[99] S. Demeshko, G. Leibeling, S. Dechert, F. Meyer, Dalton Trans., 2006, 3458.

[100] S. Demeshko, Dissertation, Georg-August-Universität Göttingen 2005

[101] S. Demeshko, G. Leibeling, W. Maringgele, F. Meyer, C. Mennerich, H.H. Klauss, H. Pritzkow, Inorg. Chem., 2005, 44, 519.

[102] G. Leibeling, S. Demeshko, S. Dechert, F. Meyer, Angew. Chem., 2005, 117, 7273.

[103] J. Ackermann, Dissertation, Universität Göttingen 2003

[104] J. Ackermann, S. Buchler, F. Meyer, C.R.Chimie, 2007, 10, 421.

[105] C.C. Tang, D. Davalian, P. Huang, R. Breslow, J. Am. Chem. Soc., 1978, 100, 3918.

[106] S. Takano, Y. Yano, W. Tagaki, Chem. Lett., 1981, 1177.

[107] R. Breslow, J.T. Hunt, R. Smiley, T. Tarnowski, J. Am. Chem. Soc., 1983, 105, 5337.

[108] S. Tamagaki, Y. Kanamaru, M. Ueno, W. Tagaki, Bull. Chem. Soc. Jpn., 1991, 64, 165.

[109] E.A. Kesicki, M.A. Derosch, L.H. Freeman, C.L. Walton, D.F. Harvey, W.C. Trogler, Inorg. Chem., 1993, 32, 5851.

[110] S.-I. Kondo, K. Shinbo, T. Yamaguchi, K. Yoshida, Y. Yano, J. Chem. Soc., Perkin Trans. 2, 2001, 128.

[111] K. Worm, F.Y. Chu, K. Matsumoto, M.D. Best, V. Lynch, E.V. Anslyn, Chem. Eur. J., 2003, 9, 741.

[112] J.C. Röder, F. Meyer, M. Konrad, S. Sandhöfner, E. Kaifer, H. Pritzkow, Eur. J. Org. Chem., 2001, 2001, 4479.

[113] F. Graf, L. Hupfer, DE 2940709 A1, 1981.

[114] L. Benisvy, private Information, MPI Mülheim, 2004.

[115] A.W. Addison, T. Nageswara Rao, J. Reedijk, J. van Rijn, G.C. Verschoor, J. Chem. Soc., Dalton Trans., 1984, 1349.

[116] Chemikalienkatalog der Fa. Merck KGaA, Darmstadt, 1999. 
[117] S. Das, G.P. Muthukumaragopal, S. Pal, S. Pal, New J. Chem., 2003, 27, 1102.

[118] Z.D. Matovic, V.D. Miletic, G. Samardzic, G. Pelosi, S. Ianelli, S. Trifunovic, Inorg. Chim. Acta, 2005, 358.

[119] H.-H. Yao, W.-T. Huang, J.-M. Lo, F.-L. Liao, P. Chaattopadhyay, J. Coord. Chem., 2005, 58, 975.

[120] K.D. Karlin, J.C. Hayes, S. Juen, J.P. Hutchinson, J. Zubieta, Inorg. Chem., 1982, 21, 4106.

[121] R.R. Jacobson, Z. Tyeklar, K.D. Karlin, J. Zubieta, Inorg. Chem., 1991, 30, 2035.

[122] H. Nagao, N. Komeda, M. Mukaida, M. Suzuki, K. Tanaka, Inorg. Chem., 1996, 35, 6809.

[123] D.M. Corsi, N.N. Murthy, V.G. Young, K.D. Karlin, Inorg. Chem., 1999, 38, 848.

[124] J. Reim, B. Krebs, J. Chem. Soc., Dalton Trans., 1997, 20, 3793.

[125] G.A. Ardizzoia, S. Cenini, G. La Monica, N. Masciocchi, A. Maspero, M. Moret, Inorg. Chem., 1998, 37, 4284.

[126] Y.M. Badiei, T.H. Warren, J. Organomet. Chem., 2005, 690, 5989.

[127] H.L. Jackson, B.C. McKusick, Org. Syntheses, Coll. Vol. 4, 438 (1963).

[128] R.E. Schuster, J.E. Scott, J. Casanova Jr., Org. Syntheses, 1966, 46, 75.

[129] G.A. Ardizzoia, E.M. Beccalli, G. La Monica, N. Masciocchi, M. Moret, Inorg. Chem., 1992, 31, 2706.

[130] B. A. Jazdzewski, P. L. Holland, M. Pink, V. G. Young, D. J. E. Spencer, W. B. Tolman, Inorg. Chem., 2001, 40, 6097.

[131] G.A. Ardizzoia, M.A. Angaroni, G. La Monica, N. Masciocchi, M. Moret, J. Chem. Soc., Dalton Trans., 1990, 2277.

[132] G.A. Ardizzoia, S. Cenini, G. La Monica, N. Masciocchi, M. Moret, Inorg. Chem., 1994, 33, 1458.

[133] H.V.R. Dias, H.L. Lu, J.D. Gorden, W. Jin, Inorg. Chem., 1996, 35, 2149.

[134] G.A. Ardizzoia, G. La Monica, A. Maspero, N. Masciocchi, M. Moret, Eur. J. Inorg. Chem., 1999, 1999, 1301.

[135] M. Costas, R. Xifra, A. Llobet, M. Sola, J. Robles, T. Parella, H. Stoeckli-Evans, M. Neuburger, Inorg. Chem., 2003, 42, 4456.

[136] B.A. Jazdzewski, A.M. Reynolds, P.L. Holland, V.G. Young Jr, S. Kaderli, A.D. Zuberbuhler, W.B. Tolman, J. Biol. Inorg. Chem., 2003, 8, 381. 
[137] F.A. Cotton, F. Zingales, J. Am. Chem. Soc., 1961, 83, 351.

[138] P.M. Treichel, Adv. Organomet. Chem., 1973, 11, 21.

[139] T. Tsuda, S.-I. Sanada, K. Ueda, T. Saegusa, Inorg. Chem., 1976, 15, 2329.

[140] E. Singleton, H.E. Oosthuizen, Adv. Organomet. Chem., 1983, 22, 209.

[141] C. Elschenbroich, A. Salzer, Organometallchemie, 3. Aufl., Teubner Verlag, Stuttgart, 1993.

[142] R.L. Lintvedt, L.L. Borer, D.P. Murtha, J.M. Kuszaj, M.D. Glick, Inorg. Chem., 1974, 13, 18.

[143] A.C. Fabretti, W. Malavasi, D. Gatteschi, R. Sessoli, J. Chem. Soc., Dalton Trans., 1991, 2331.

[144] N.-D. Sung, K.-S. Yun, T.-Y. Kim, K.-Y. Choi, M. Suh, J.-G. Kim, I.-H. Suh, J. Chin, Inorg. Chem. Commun., 2001, 4, 377.

[145] L. Gahan, T.W. Hambley, Transition Met. Chem., 1988, 13, 72.

[146] Z. Xu, L.K. Thompson, V.A. Milway, L. Zhao, T. Kelly, D.O. Miller, Inorg. Chem., 2003, 42, 2950.

[147] L.M. Venanzi, J. Nuc. Inorg. Chem., 1958, 8, 141.

[148] N.A. Eckert, E.M.R. Bones, R.J. Lachiotte, Inorg. Chem., 2003, 42, 1720 bis 1725.

[149] M. Halcrow, E.J.L. McInnes, F.E. Mabbs, I.J. Scowen, M. McPartlin, H.R. Powell, J.E. Davies, J. Chem. Soc., Dalton Trans., 1997, 4025.

[150] N. Sengottuvelan, D. Saravanakumar, S. Sridevi, V. Narayanan, M. Kandaswamy, Supramol. Chem., 2004, 16, 129.

[151] L. Li, N.N. Murthy, J. Telser, L.N. Zakharov, G.P.A. Yap, A.L. Rheingold, K.D. Karlin, S.E. Rokita, Inorg. Chem., 2006, 45, 7144.

[152] J. Casabó, J. Pons, K.S. Siddiqi, F. Teixidor, E. Molins, C. Miravitlles, J. Chem. Soc., Dalton Trans., 1989, 1401.

[153] F. Meyer, E. Kaifer, P. Kircher, K. Heinze, H. Pritzkow, Chem. Eur. J., 1999, 5, 1617.

[154] S. Bieller, A. Haghiri, M. Bolte, J.W. Bats, M. Wagner, H.-W. Lerner, Inorg. Chim. Acta, 2006, 359, 1559.

[155] F. Meyer, A. Jacobi, L. Zsolnai, Chemische Berichte, 1997, 130, 1441.

[156] G. Mezei, R.G. Raptis, Inorg. Chim. Acta, 2004, 357, 3279. 
[157] M. Du, S.-T. Chen, Y.-M. Guo, X.-H. Bu, J. Ribas, J. Mol. Struct., 2005, 737, 17.

[158] J. Teichgräber, G. Leibeling, S. Dechert, F. Meyer, Z. Anorg. Allg. Chem., 2005, 631, 2613.

[159] M. Verdaguer, Polyhedron, 2001, 20, 1115.

[160] S.J. Swithenby, Contemp. Phys., 1974, 15, 249

[161] G. Leibeling, Dissertation, Universität Jena 2003

[162] W. Haberditzl, Angew. Chem., 1966, 78, 277.

[163] W. Haberditzl, Magnetochemie, Akademie-Verlag, 1968.

[164] E. Bill, julX, Max-Planck-Institut für Bioanorganische Chemie, Mülheim/Ruhr.

[165] S.L. Mandal, L.K. Thompson, M.J. Newlands, J.-P. Charland, E.J. Gabe, Inorg. Chim. Acta, 1990, 178, 169.

[166] L. Chen, L.K. Thompson, J.N. Bridson, Inorg. Chem., 1993, 32, 2938.

[167] E. Spodine, A.M. Atria, G. Valenzuela, J. Jalocha, J. Manzur, A.M. Garcia, M.T. Garland, O. Pena, J.-Y. Saillard, J. Chem. Soc., Dalton Trans., 1999, 3029.

[168] L.K. Thompson, A.W. Hansoon, B.S. Ramaswamy, Inorg. Chem., 1984, 23, 2459.

[169] S.S. Tandon, S.L. Mandal, L.K. Thompson, R.C. Hynes, Inorg. Chem., 1992, 31, 2215.

[170] L. Antolini, A.C. Fabretti, D. Gatteschi, A. Giusti, R. Sessoli, Inorg. Chem., 1990, 29.

[171] J. Cano, G. De Munno, F. Lloret, M. Julve, Inorg. Chem., 2000, 39, 1611.

[172] G. Dong, D. Chun-ying, F. Chen-jie, M. Qing-jin, J. Chem. Soc., Dalton Trans., 2002, 834.

[173] O.G. Shakirova, D.Y. Naumov, Y.G. Shvedenkov, N.I. Alferova, G.V. Dolgushin, L.G. Lavrenova, J. Struct. Chem., 2003, 44, 642.

[174] S. Salameh, M. Abdul-Haj, M. Quirós, J.M. Salas, Eur. J. Inorg. Chem., 2005, 2779.

[175] B. Ding, L. Yi, W.-Z. Shen, P. Cheng, D.-Z. Liao, S.-P. Yan, Z.-H. Jiang, J. Mol. Struct., 2006, 784, 138.

[176] S. Yamada, M. Yasui, T. Nogami, T. Ishida, Dalton Trans., 2006, 1622. 
[177] F.-M. Nie, G. Leibeling, S. Demeshko, S. Dechert, F. Meyer, Eur. J. Inorg. Chem., 2007, 1233.

[178] O. Kahn, Molecular Magnetism, VCH-Verlag, New York, weinheim, 1993.

[179] G.M. Sheldrick, SHELXS-97, "Program for Crystal Structure Solution", Universität Göttingen, 1997.

[180] G.M. Sheldrick, SHELXL-97, "Program for Crystal Structure Refinement", Universität Göttingen, 1997.

[181] K.M. Chi, H.-K. Shin, M.J. Hampden-Smith, E.N. Duesler, T.T. Kodas, Polyhedron, 1991, 10, 2293.

[182] W.C. Baird Jr., J. Am. Chem. Soc., 1963, 85, 1009.

[183] G.J. Kubas, Inorg. Synth., 1990, 28, 68.

[184] K. Osakada, T. Takizawa, M. Tanaka, T. Yamamoto, J. Organomet. Chem., 1994, 473, 359. 


\section{POSTER}

7th European Biological Inorganic Chemistry Conference EUROBIC 7, Garmisch-Partenkirchen 2004

„Biomimetic Dinuclear Copper(II) Complexes with new Pyrazole Ligands“

Wissenschaftsforum Chemie

Ulm 2007

„Neuartige Pyrazolatliganden mit zwei facial tridentaten Koordinationstaschen als Gerüst für bioinspirierte Mehrkernkomplexe"

\section{VORTRÄGE}

Koordinationschemiker-Treffen

Kaiserslautern 2005

„Neue Pyrazolatliganden mit zwei facial-tridentaten Koordinationstaschen - erste Kupferkomplexe"

\section{Publikationen}

H. Müller, B. Bauer-Siebenlist, E. Csapo, S. Demeshko, S. Dechert, E. Farkas, F. Meyer, Inorg. Chem, in Bearbeitung.

H. Müller, S. Dechert, S. Demeshko, G. Leibeling, F. Meyer, J. Chem. Soc., Dalton Trans., in Bearbeitung. 


\section{DANKSAGUNG}

Zu allererst möchte ich mich ganz herzlich bei allen Mitgliedern des AK Meyer für die offenherzige Aufnahme in den Arbeitskreis bedanken. Ihr habt es möglich gemacht, dass ich mich von Anfang an wohl gefühlt habe und mit vollem Elan an dieses Projekt herangehen konnte. Außerdem möchte ich auf diesem Wege danke sagen für zwei wunderschöne AK-Fahrten. Sowohl die Sächsische Schweiz als auch der Elsass sind immer wieder eine Reise wert.

Prof. Dr. Franc Meyer danke ich für die Möglichkeit in seiner Gruppe zu promovieren, für das interessante Thema und die Diskussionsbereitschaft.

Bei Frau Jun.Prof. Dr. Carola Schulzke bedanke ich mich für das Korrekturlesen dieser Arbeit und dafür, dass sie als Oberassistentin des Chemie-Praktikums für Biologen stets ein offenes Ohr für Fragen und Probleme hatte.

Dr. W. Maringgele und Jörg Teichgräber danke ich für die Hilfe beim Aufbau des Labors 234 und beim Beschaffen der notwendigen Ausrüstung dafür. Außerdem stand mir Dr. W. Maringgele zu Beginn der Arbeit mit Rat und Tat zur Seite, wenn ich Fragen zur Synthese der Ligandvorstufen hatte.

Jörg Teichgräber danke ich für das Anfertigen der CV's (auch wenn die Messungen probenbedingt nicht so gut aussahen) sowie für die vielen kleinen Gespräche über Gott und die Welt (Man muss nicht immer über Chemie reden).

Für das Korrekturlesen dieser Arbeit möchte ich mich außerdem bei Ulrich Scheele, Sebastian Dechert und meiner Freundin Dani bedanken. Danke, dass ihr eure wertvolle Zeit für mich auch am Wochenende oder im Urlaub dafür geopfert habt.

Sebastian Dechert danke ich vor allem für das Anfertigen der Röntgenstrukturanalysen. Ich weiß, die Kristalle waren selten perfekt. Aber du hast es oft geschafft durch viel Geschick eine gute Messung daraus zu machen. Nicht zu vergessen, die gemeinsamen Fahrten mit dem Fahrrad nach Nikolausberg. Zu zweit macht es einfach viel mehr Spaß 
und ich konnte mich und insbesondere meine Kniegelenke etwas schonen. Allein nach Nikolausberg fahren ist sehr anstrengend, vor allem wenn man wie ich nicht langsam fahren will/kann auf dem Fahrrad.

Ulrich Scheele möchte ich für die anfängliche Hilfe und die Diskussionen über Chemie und imidazolspezifische Probleme danken. Außerdem bin ich dankbar, dass ich die Möglichkeit hatte, dass Arbeiten an der Glovebox kennenzulernen. Ganz besonders will ich mich bei dir für die zum Teil recht zeitaufwendige und nervige Zeit bedanken, in der wir zusammen die Glovebox betreut haben. Die Sorgen und Probleme, die uns MBRAUN mit den zwei Geräten teilweise bereitet haben, erscheinen viel kleiner, wenn man einen verlässlichen Partner hat, mit dem man diese gemeinsam lösen kann.

Tine Graef danke ich für die gemeinsame Zeit im Labor 234, auch wenn sie nicht immer reibungsfrei war. So macht es trotzdem viel mehr Spaß in Gemeinschaft zu arbeiten.

Miguel Alvariño Gil („El Matador“) danke ich für die vielen Gespräche die wir zusammen geführt haben. Ich war froh, dass ich jemand wie dich gefunden habe, der meine Leidenschaft für Sport teilt. Wer gewinnt, ist mir meistens egal, so lange es mit fairen Mitteln abläuft. Ganz besonders möchte ich mich bei dir für den super genialen Abend im Bremer Weserstadion bedanken. Es war für mich ein bisher einmaliges Erlebnis die Fussball Championsleague live zu sehen - und das auch noch im Fanblock von FC Barcelona! Echt spitze.

Ich möchte mich bei allen Abteilungspraktikanten (Conrad Weichbrodt, Jan-Nicklas Fricke, Gunther Speichert, Dirk Matthes, Anne Heinemann, Sven Stoller und Andreas Bögehold) bedanken, die mich mal mehr, mal weniger gut bei meinen Arbeiten unterstützt haben.

Für die gemeinsame Zeit im Chemiepraktikum für Biologen möchte ich mich außerdem bei allen Assistenten bedanken, ganz besonders bei Christine Schlicker.

Bei meiner Familie möchte ich mich ganz herzlich für die Unterstützung dieser Arbeit bedanken. Ihr habt mich aufgebaut, wenn es mal nicht so gut lief und ihr habt mich auch 
ständig vorwärts getrieben. Auch wenn es anfangs ein paar Probleme gab, bin ich jedesmal froh und glücklich, wenn ich euch wieder sehe und mich mal nicht nur am Telefon mit euch unterhalten kann. Danke für eure Unterstützung.

Danke an die NMR-, die MS-Abteilungen und dem Mikroanalytischen Labor des Instituts für Anorganische Chemie für die Analysen meiner Proben. Herrn Dr. Frauendorf danke ich für die HR-MS-Messungen. Herrn John danke ich zudem für die unkomplizierte und prompte Bearbeitung meiner Wünsche, insbesondere bei den $\mathrm{Cu}(\mathrm{I})$-Proben.

Dr. Julia und Dr. Marco („The hair cutter“) Klingele, Dr. Gilles Noel, Stamatia Katsiaouni, Nina Roth, Ulrich Scheele danke ich zum Einen für die vielen Gespräche abseits des Chemiealltags und zum Anderen dafür, dass ihr mich so nehmt wie ich bin. Ich bin glücklich, solche Freunde wie euch gefunden zu haben. Danke für alles, was ihr für mich getan habt.

Zuletzt möchte ich mich noch bei meiner Freundin Daniela („Dani“) Kühnel für die Liebe, ihr Vertrauen und die grenzenlose Unterstützung bei meiner Arbeit bedanken. Ich weiß, dass du vor allem in letzter Zeit viel zurückstecken und meine manchmal launische Art aushalten musstest. Ich danke dir auch für das dafür aufgebrachte Verständnis. 


\section{LEBENSLAUF}

Name

Geburtsdatum

Geburtsort

Familienstand

Staatsangehörigkeit

Schulbildung

Wehrdienst

Hochschulstudium

Promotion

Beschäftigungs-

verhältnisse
Holger Müller

08.05.1979

Karl-Marx-Stadt (jetzt Chemnitz)

ledig

deutsch

1985-1987 Polytechnische Oberschule „Arthur Becker“

1987-1990 Polytechnische Oberschule „Katja Niederkirchner“

1990-1992 Mittelschule „Rudolfschule“

1992-1997 Johann-Wolfgang-von-Goethe-Gymnasium

Abitur: 11.07.1997

1997-1998 Braunschweig

Chemie (Diplom) seit dem Wintersemester 1998/1999 an der Technischen Universität Chemnitz

Diplom-Vorprüfung: 29.09.2000

Diplom-Hauptprüfung: 17.09.2002

Diplomarbeit bei Prof. K. Banert an der TU Chemnitz

„Synthese von 5-Isocyanatopenta-2,3-diensäureestern durch sigmatrope Umlagerung“

Beginn: 01.10.2003 an der Georg-August-Universität Göttingen

Januar 2001 als studentische Hilfskraft an der TU Chemnitz Juli 2002 bis September 2002 als studentische Hilfskraft an der TU Chemnitz

seit Oktober 2003 als wissenschaftlicher Mitarbeiter an der Georg-August-Universität Göttingen 